\title{
$\therefore 0^{\circ}+0-20$
}

\section{3.

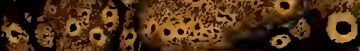

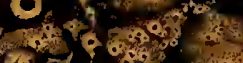

(a)

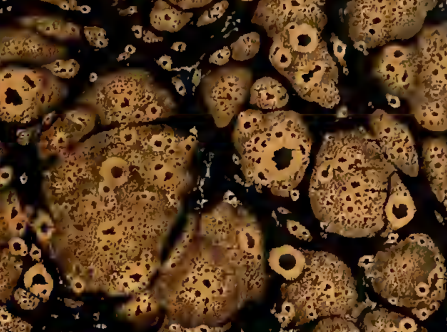

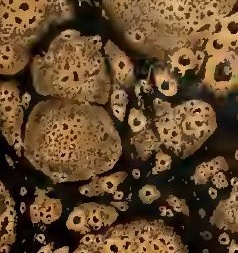

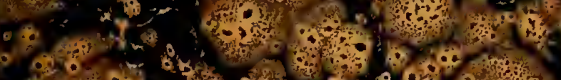

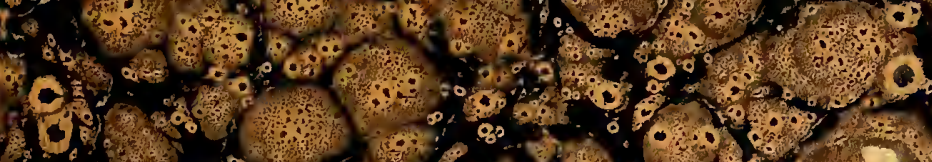

6.

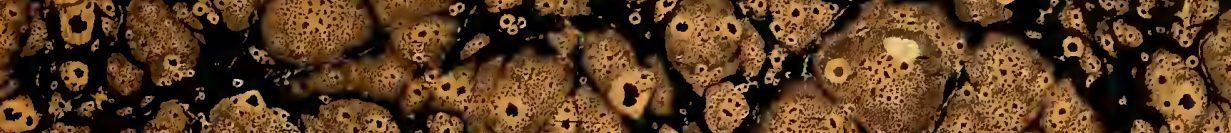

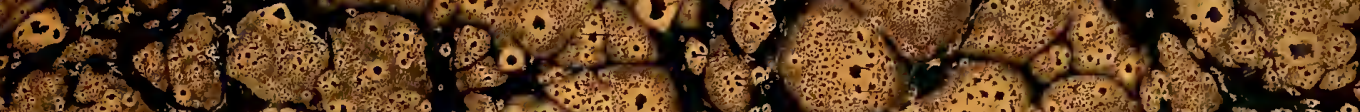

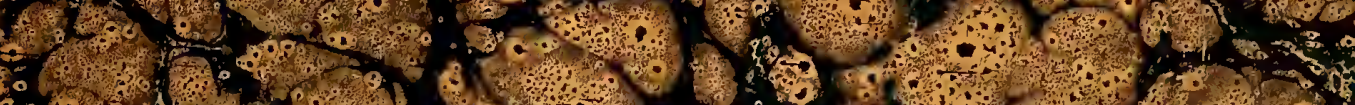

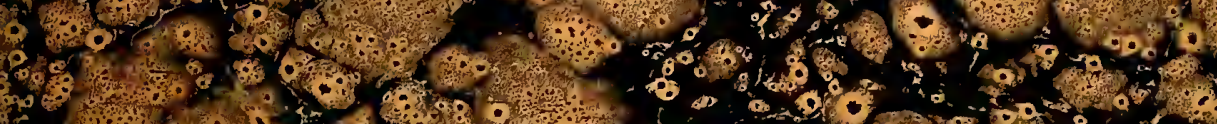

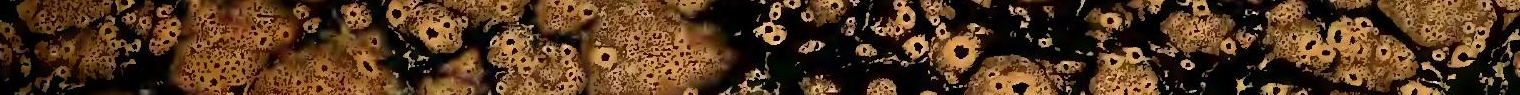

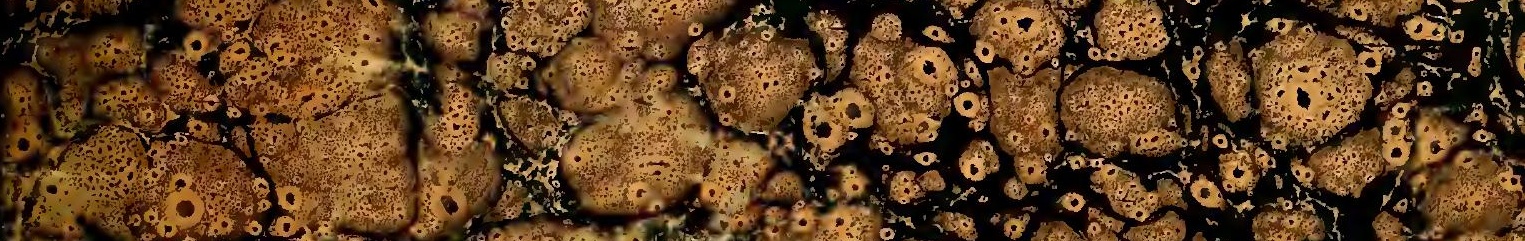

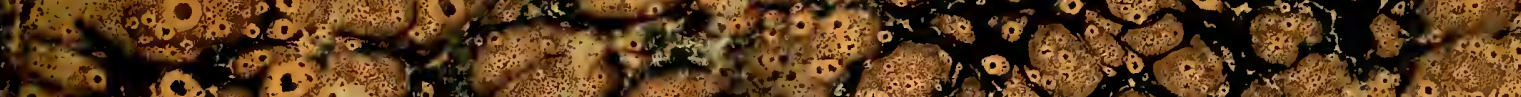

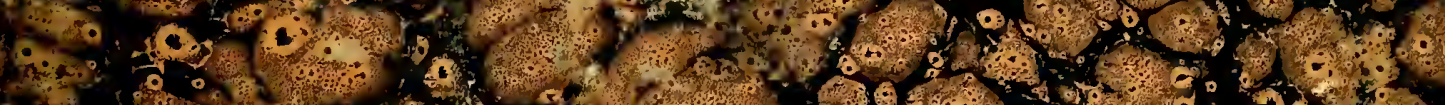

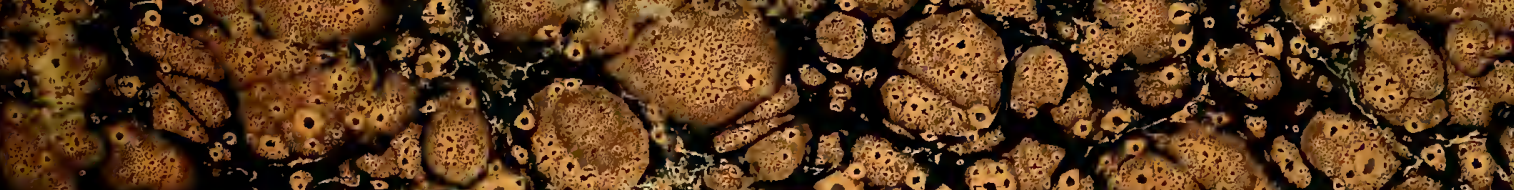

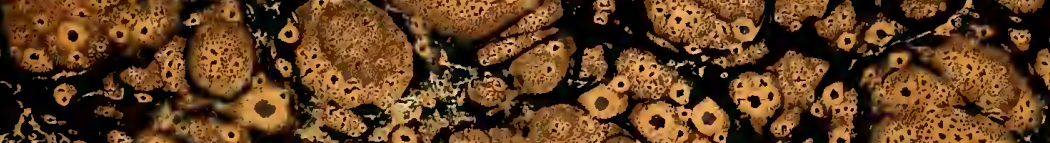
(5)

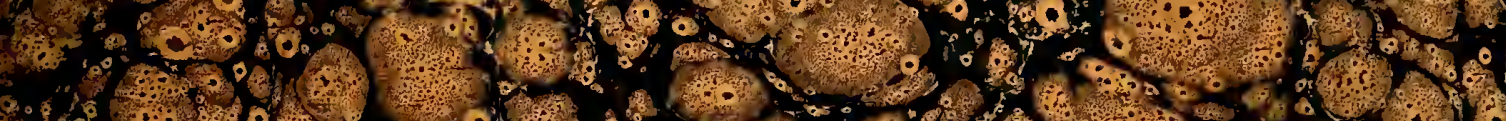

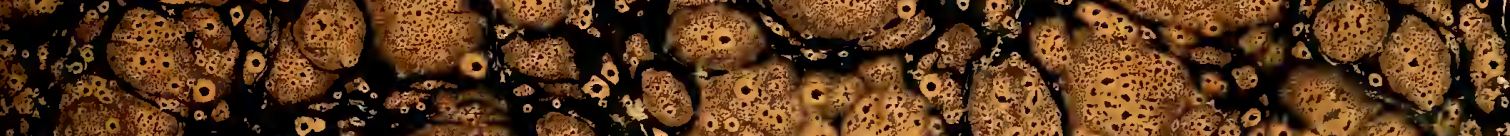

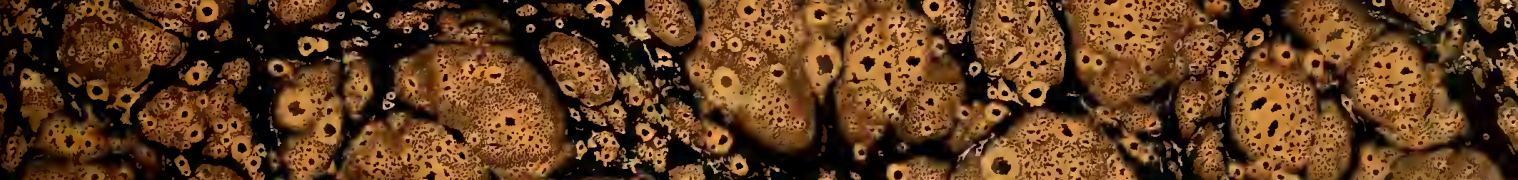
6.

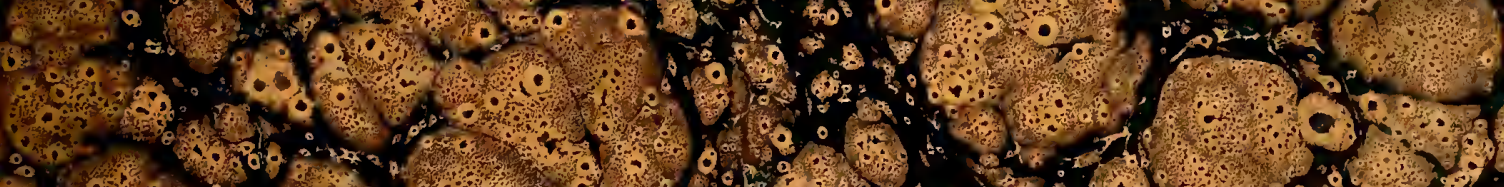

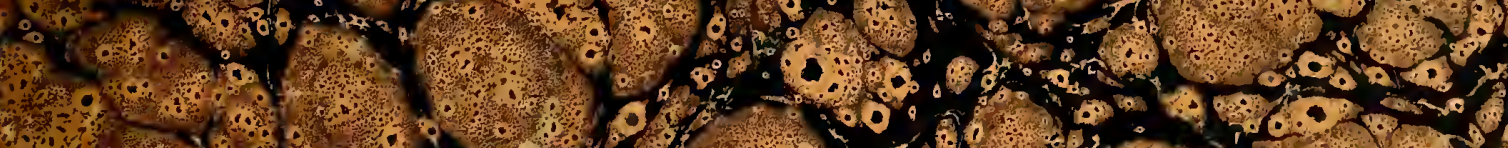

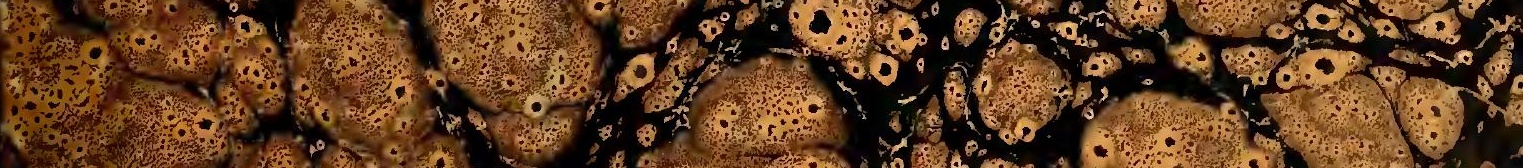

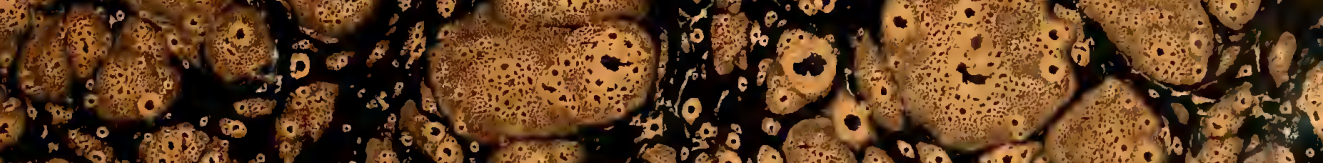

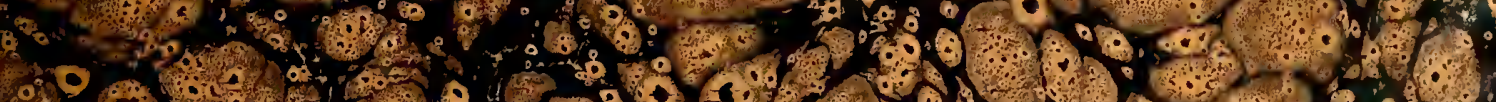

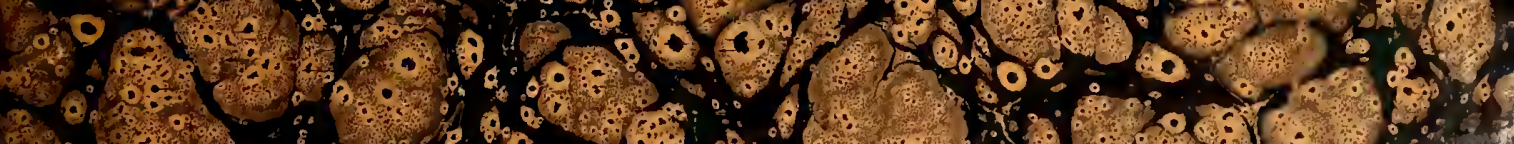




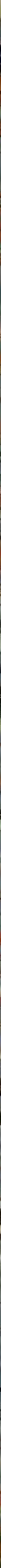




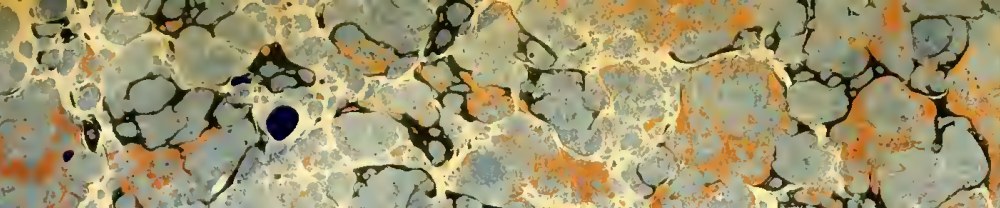

$\log ^{3}$

$1+e^{2}$

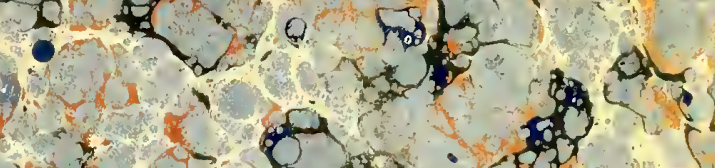

$3 x^{2}+2-3$

(

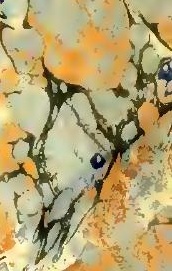

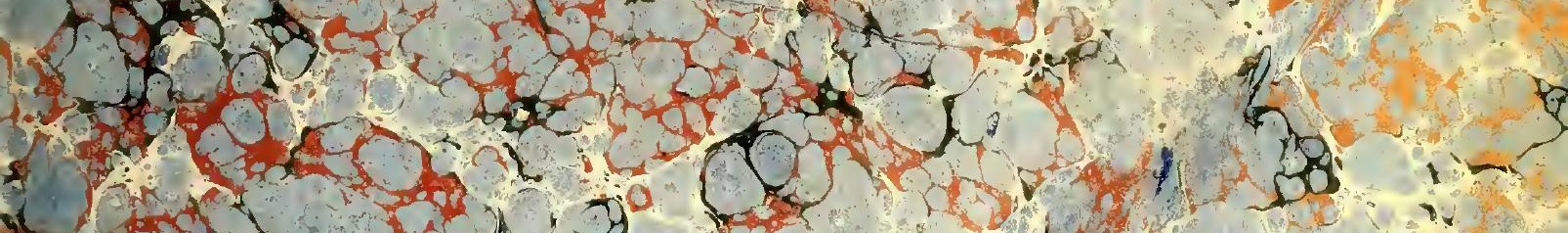

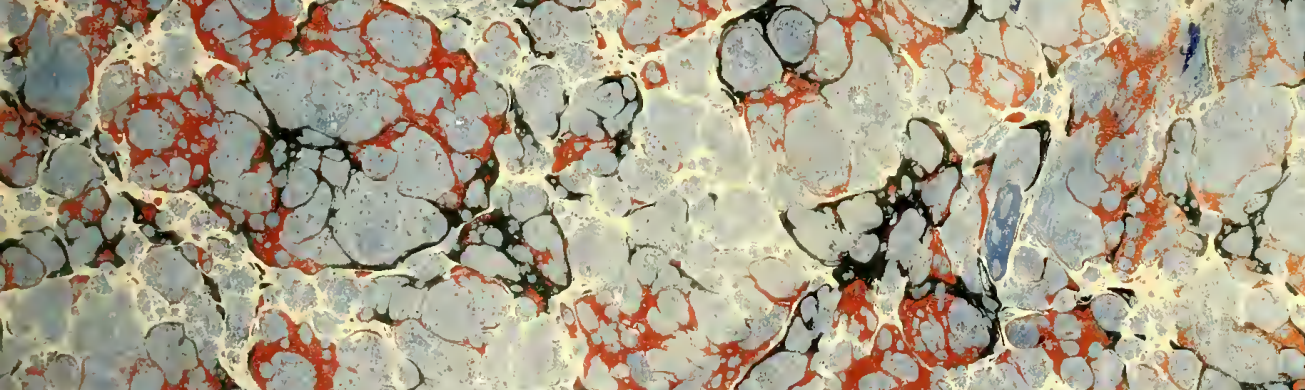

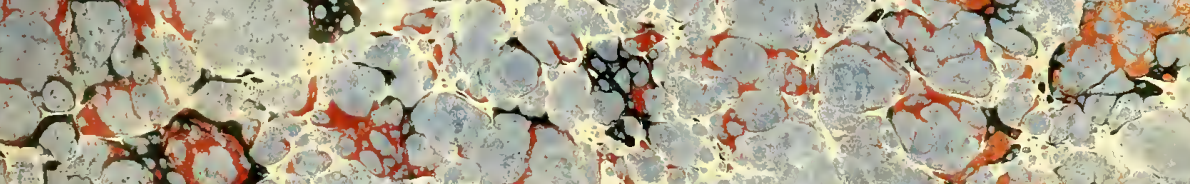

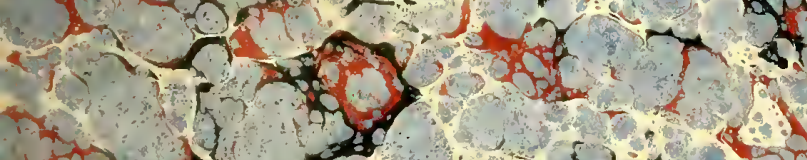

in 1

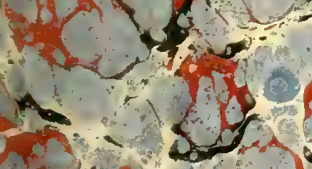

a

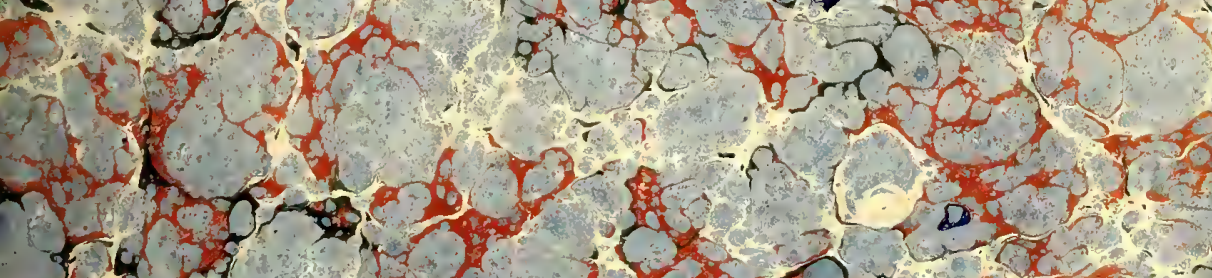

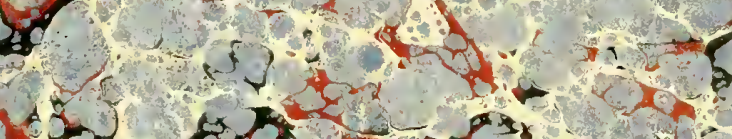

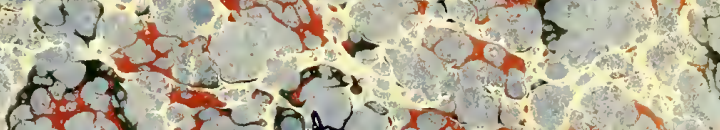

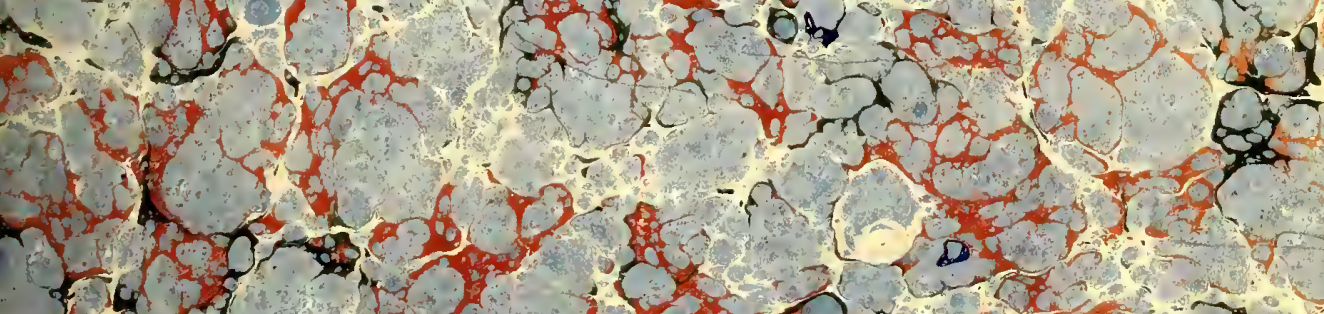

-

a.

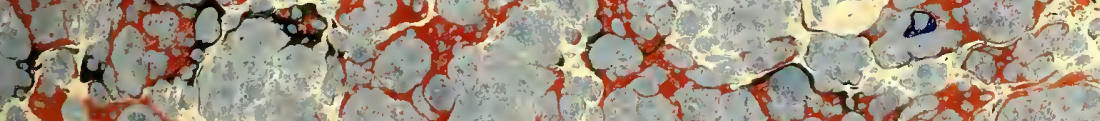

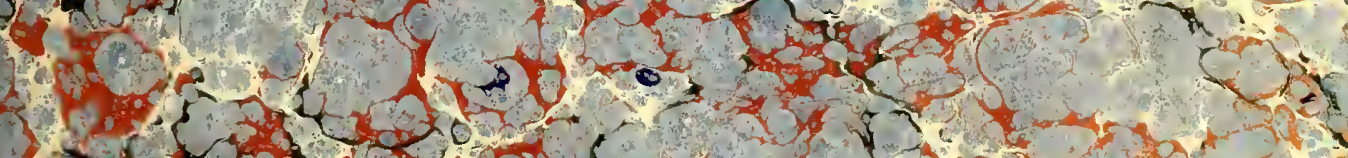

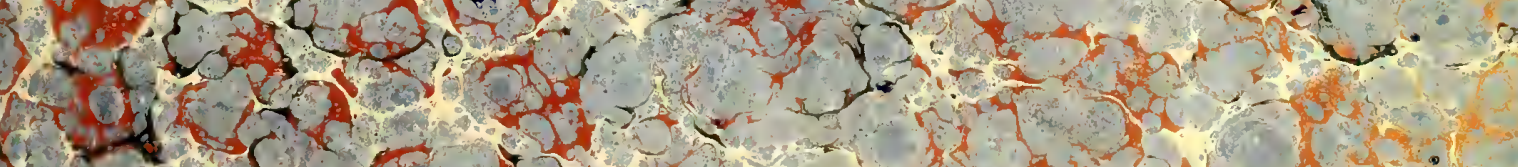

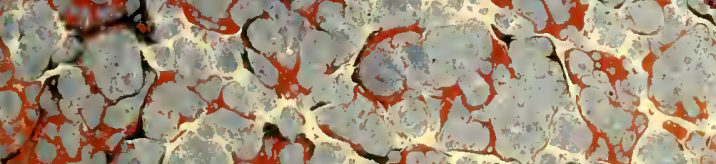

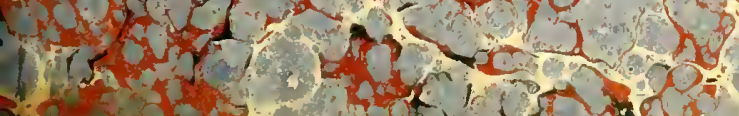





\section{STORIA ANTICA}

\section{DEL MESSICO}

CAVATA DA' MIGLIORI STORICI SPAGNUOLI,

E DA' MANOSCRITTI, E DALLE PITTURE ANTICHE DEGL' INDIANI:

\section{DIVISA IN DIECI LIBRI,}

E CORREDATA DI CARTE GEOGRAFICHE,

E DI VARIE FIGURE:

$\mathbf{E}$

\section{I S S E R T A Z IO N I}

Sulla Terra, fugli Animali, e fugli abitatori del Meffico,

O P E R A

$\begin{array}{lllllllll}D & E & L & L & A & B & A & T & E\end{array}$

D. FRANCESCO SAVERIO

C L A V I G E R O

00

T O M O III.

$26=20$

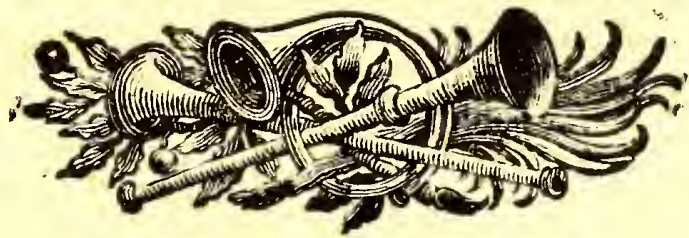

IN CESENA M DCCLXXX。

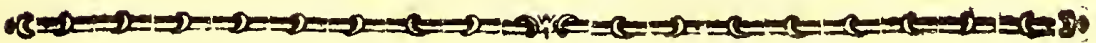

Per Gregorio Biasini all' Insegna di Pallade

Con Licenza de’ Superiori, 



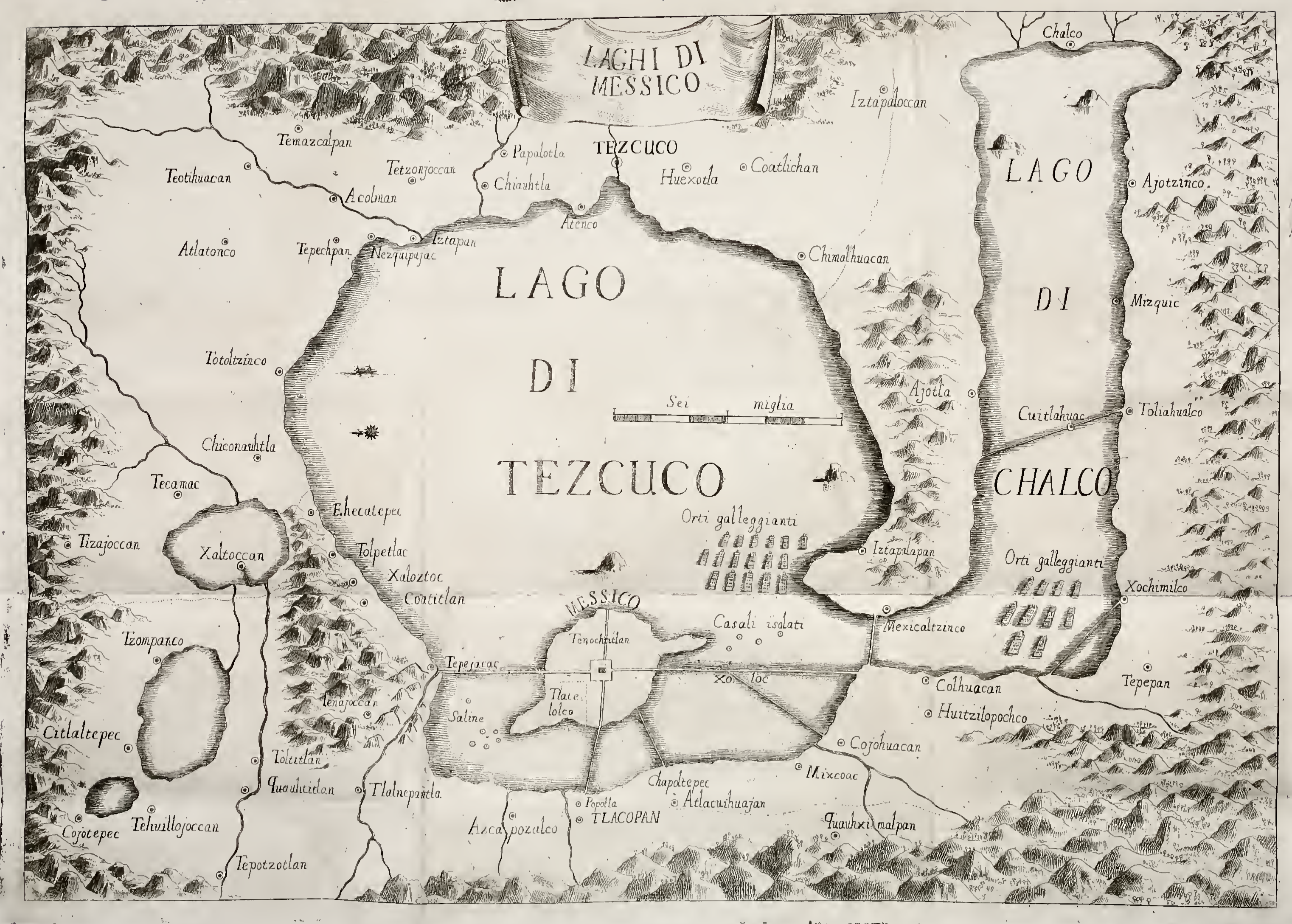




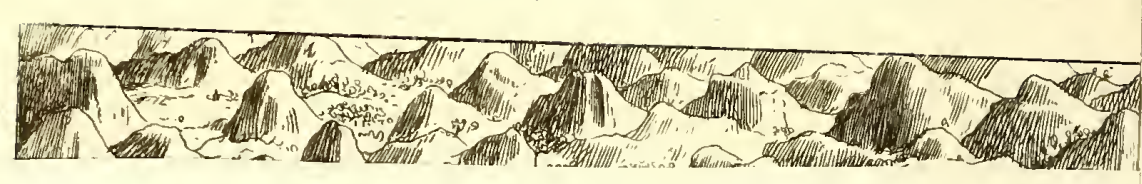




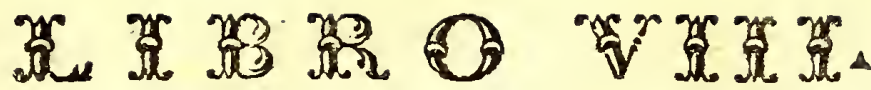

Arrivo degli Spagnuoli alle cofte d' Anabuac. Inquietudine, amo bafciate, e prefenti del Re Motezuma. Confederazione degli Spagnuoli co' Totonacbi, la loro guerra, ed alleanza co' Tla. fcallefi: la loro feverità verfo i Cbolullefi, e la loro folenne entrata in Mefseco. Notizia della celebre India: na Donna Marina. Fondazione della Veracroce, prima colonia degli Spagnuoli.
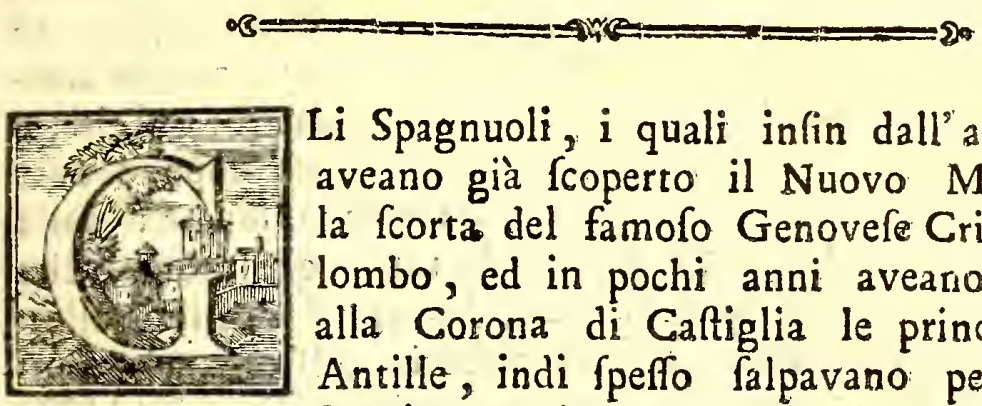

Li Spagnuoli, i quali infin dall' anno I 492 ; aveano già fcoperto il Nuovo Mondo lotto la fcorta del famofo Genovefe Criftofano Colombo, ed in pochi anni aveano cottopofte alla Corona di Caftiglia le principali ifole Antille, indi fpeffo falpavano per andare a fcoprir nuovi paefi, e barattar le bagatelle europee coll' oro americano. Tra gli altri falpò l' anno 1517 dal porto d' Ajaruco (oggidi Havana) Francefco Hernandez di Cordova con cento dieci foldati, e camminando verfo vaggj Ponente per configlio d'Antonio Alaminos, uno de' più ef.Spagnuoperti, e de' più famofi Piloti di quel tempo, e poi piegando liallecuverfo Mezzogiorno, foprirono ful principio di Marzo il ca- nahuac. po orientale della penifola di Jucatan, ch'effi appellarono Capo Cotocbe. Cofteggiarono una parte di quel paefe ammirando $i$ belli edifizj, e l'alte torri, che fi fcorgevano lungo. la colta, (d) gli abiti di diverfi colori, che porravano gl'Ia: A, 2 diani :

(a) Il Dott. Robertfon dice nel lib. 3., che gli Spagnuoli miferopiede aterra, ed inoltrandofi nel paefe ( di Jucatan) offervarono con iffupore delle gran cafe fabbricate di pietra. Cosi parla dove racconta il viaggio d'Hernandez; ma poche pagine dopo parlando del viaggio di Grijalva dice così: Molti villaggj erano jparfa lnngo la ccka, nei quali potevano ( gli Spagnuoli ) ravvifare delle cafe di pietra, che in difanza parevano bianche, e Juperbe. Nel salore dell' immagixazione fi figuravano bhe quefte foffero tante citta adorne ditorri, 
diani: oggetti non mai da loro veduti nel Nuovo Mondo:' LiB.VIII. I Jucatanefi dalla lor parte fi maravigliavano della grandezza, della forma, e dell' apparato de' vafcelli. In due luoghi, dove mifero il piede a terra gli Spagnuoli, ebbero due zuffe cogl'Indiani, nelle quali, ed in altri difagi, che ad effi fopravvennero, perdettero la metà de'loro Soldati, e lo fteffo Capitano ebbe dodici ferite, che fra pochi giorni gli cagionarono la morte. Ritornati però frettolofamente in Cuba, ravvivarono col loro ragguaglio, e con qualche oro, che portarono per moltra, involato da un tempio di Jucatan, la cupidigia di Didaco Velafquez, Conquîtatore già, ed allora Governatore di quell' Ifola : onde l'anno feguente mandò Giovanni di Grijalva fuo parente con quattro vafcelli,e dugento quaranta Soldati. Quefto Comandante dopo d'aver riconofciuta l'ifola di Cozumel, poche miglia lontana dalla colta orientale di Jucatan, cofteggiò tutto quel paefe, che v'è quinci infino al fume Panuco, barattando delle pallottoline di vetro, ed altre si fatte coluccie coll' oro, che tanto da loro fi bramava, e colle vettovaglie, di cui abbifo. gnavano.

Allorchè arrivarono a quell' ifoletta ch' effi appellarono S. Giovanni d'Ulisa, (b) poco più d'un miglio difcofta dal-

si, $e$ di cuppole. Fra tantí Storici del Meffico da me letti non ho trovato neppure uno, che abbia detto, che gli Spagnuoli s' immaginarono delle cuppole in Jucatan. Quefta immaginazione è fata del Robertfon, non di loro. Parve pure ad effi di vedere alte torri, e cafe ben grandi, ficcome infatti v'erano. I temrj di Jucatan, come quelli d'Anahuac, erano fabbricati per lo più a guifa di torri, ed erano molti ben alti. Berrial Diaz Autore finceriffimo, e teftimonio oculato di tutto quanto avvenne agli Spagnuoli in que'primi viaggi a Jucatan, dove parla dello sbarco che fecero nel primo lur viaggio nella cofta di Campecce, dice cosi : $C i$ conduffero ( gl'Indianı) a certe cafe affai grandi, :d affai ben fabbricate di pietra, e di calcina. Sicchè non folamente videro da lontano gli edifizj, ma s'accoftarono ad effi, e vi entrarono. Effendo poi ftato tantu famigliare a quelle Nazioni l'ufo della calcina, non è da maravigliare che foffe anche comune a loro l'ufanza d'imbiancar le cafe. Vedafi intorno a cid il lib. VII. della noftra Storia. Del refto io non poffo capire come polfa fembrar bianca da lontano una cala, che non è infatti bianca.

(b) Diedero a quell' ifoletta il nome di S. Giovanni; perchè vi approdarono 
La Spiaggia di Chalchiuhcuecan, i Governatori Mefficani di quelle cofte sbalorditi dal vedere de'vafcelli sì grandi, e de. Lis.VHII. gli uomini di si ftrana figura, e portamento, confultarono tra loro intorno a ciò, ch' era a farfi, e deliberarono di portarfi in perfona alla Corte per dar contezza al Re d' una cofa tanto ftraordinaria, $e$ per poter dargli una più compita idea, fecero in qualche maniera rapprefentar da' loro pittori i vafcelli, l'artiglieria, l'armi, gli abiti, e l'alpetto di quella nuova gente: e fenza indugio partirono per la Corte, dov' efpofero a bocca al Re tutto ciò, che v' era nella co.. fta, e gli prefentarono le pitture, ed alcune pallottoline di vetro, che aveano avute dagli Spagnuoli. Turboffi Motezu$m a$ in fentendo si fatta nuova; $m a$ per non precipitar la fua rifoluzione in un affare di tanto rilievo, tenne configlio con Cacamatzin Re d'Acolhuacan fuo nipote, con Cuitlahuatzin Signor d'Iztapalapan, fuo fratello, e con altri do. dici perfonagg fuoi configlieri ordinarj. Dopo una lunga conferenza conchiufero di comun parere, colui ch' era a quelle Ppiaggie approdato con un apparato si grande, altro non dover effere, ch' il Dio dell' aria Quetzalcoatl, già da tanti anni da loro afpettato; imperocchè correva tra quelle Nazioni, ficcome abbiam detto altrove, un' antica tradizione, che tal nume dopo d'efferfi colla fua vita innocente, e colla fingolare fua beneficenza procacciata la fima, e la venerazione de'popoli in Tollan, in Cholulla, e in Onohualco, era quivi fparito, avendo innanzi promeffo a loro di ritornarvi dopo qualche tempo per reggerli in pace, e renderli felici. I Re di que'paefi fi ftimavano luogotenenti di quel Dio, e depofitarj della Corona, la quale cedere a lui dovreb: bono,

sono il giorno del S. Precurfore, e perchè quefto era il nome del Comandante. Chiamaronla pure Ulua; perciocchè avendo ivi trovato due vittime umane di frefco facrificate; e addimandata per cenni la cagione di sì fatta inumanità, rifpoftro gl'Indiani addirando il paele di ponente, Acolbus, Acolbua, volendo fare incendere, ch' il facevano per ordine de'Mefficani, i quali, ficcone tutti que! della Valle mefficana, erano chiamati Acolhui da' popoli difcofti dalla lor Capitale. In quefta ifoletta v'è prefentemente una buona fortezza per difender l'entrata nel porto della Veracroce. 
bono, tofto che vi compariffe. Quefta immemorabile tradi Ers.VIII zione, varj contraffegni offervati da loro negli Spagnuoliconformi a quelli, che di Quetzalcoatl dava la loro mirologìa, la forprendente grandezza de' vafcelli paragonata con quella delle loro barche, o canoe, lo ftrepito, e la violenza dell'artiglieria tanto fomiglianti a quelle delle nubi, gl $^{\prime}$ induffe credere, non effere altro, ch'il Dio dell' aria, colui, che alle loro cofee era arrivato col terribile apparato di lampi, fulmini, e tuoni. Moffo da una tal credenza Motezuma ordinò a cin : que perfonaggj della fua Corte di portarfi incontanente a Chalchiuhcuecan' a congratularfi con quefta pretefa Divinità del felice di lei arrivo in quella terra a nome fuo, e di tutto il regno, e di portarle, qual omaggio, un gran prefente; ma prima $d$ ' inviarli, anticipò un ordine a' Governatori delle cofte di por delle fentinelle fulle alte montagne di Nathtlan, Quauhtla, Mictlan, e Tochtlan, acciocché offervaffero i movimen:ti dell' armata, e di tutto ciò che avveniffe mandaffero pron: to avvifo alla Corte. Gli Ambafciatori mefficani non pate. rono malgrado la fomma loro diligenza raggiugnere gli SpaEnuoli, quali terminato il loro commercio in quella fpiaggia continuarono marina marina la loro navigazione infin' al fiume di Panuco, là onde ritornarono a Cuba con dieci min. la zecchini in oro, parte acquiftato col baratto delle bagatelle, e parte da un ricco prefente fatto al Comandante da un Signore d'Onohualco.

Rincrebbe affai al Governatore di Cuba, che Grijalva 5,2, non aveffe piantata una colonia in quel nuovo paefe, che Carattere da tutti era rapprefentato il più ricco, e felice del mondo: cipali onde fece prontamente alleftire un altro più groffo arma: Conqui- mento, il cui comando pretefero a gara parecchj Coloni de Meffico. principali di quell' ifola; ma il Governatore per configlio di due fuoi confidenti lo commife a Ferdinando Cortès, uomo nobile, ed abbaftanza ricco per poter fopportare col fuo caa pitale, e coll' ajuto de' fuoi amict, una buona parte delle fpere dell' armata. Nacque in Medellin, piccola Città dell' Eitremadura, nell' anno I48.5. Per parte di Padre era Cortès, e Mon- 
e Monroi, e per parte di Madre Pizarro, ed Altamirano, effendofi in lui unito il fangue di que' quattro legnaggi, 'ch' LiB.VIrr. erano de' più chiari, e de' più antichi di quella Città. Nell' età di quattordici anni fu mandato da' fuoi genitori a Salamanca, acciocchè imparando in quella famof́a Univerfità la latinita, e la giurifprudenza, poteffe renderfi utile alla faa cafa ridotta alla povertà; ma non iftette guari, che il fuo genio militare lo diftolle dallo ftudio, e lo portò al Nuovo Mondo fulle tracce di molci illuftri giovani della Cua Nazio. ne. Accompagnò Didaco Velafquez nella conquifta dell' ifola di Cuba, dove fi procacciò de' beni, e fi conciliò grand' autorità. Era uomo di gran talento, affai accorto, corag. giofo, e deftro nell' efercizio dell' armi, fecondo nel trovar de'mezzi, ede' ripieghi per condurre a fine i fuoi progetti, fommamente ingegnofo nel farfi ubbidire, e rifpettare anche da'fuoi pari, magnanimo ne'fuoi difegni, e nelle fue azioni, cauto nell' operare, modefto nel parlare, coftante nelle fue intraprefe, e paziente nelle fue avverfità. II fuo zelo per la Religione non fu punto inferiore alla fua coftante, ed inviolabile fedeltà verfo il fuo Sovrano; ma lo fplendore di quelte, e d'altre buone qualità, che l'innalzarono alla clafe degli Eroi, venne eccliffato da alcune azioni non degne della gran. dezza del fuo animo. Lo fregolato amor delle donne l'inviluppò in alcune ree pratiche, e nel tempo addietro gli avea cagionato de'gravi difgufti, e perigli. Il troppo impegno, o fra oftinazione nelle fue imprefe, ed il timore di fraftornare la fua fortuna lo fecero talvolta mancare alla giuftizia , alla gratitudine, ed alla umanita; ma dove fu mai un Generale Conquiftatore formato nella fcuola del mondo, in cui le virtù non fi bilanciaffero co' vizj? Era Cortès di buona ftatura, e di corpo ben proporzionato, robufto, ed agile. Aveva il petto alquanto elevato, la barba negra, e gli occhj vivi, ed amorevoli. Tal è il ritratto del famofo Conquiltatore del Meffico che ci lafciarono i primi Storici, che il conobbero. $\left({ }^{*}\right)$

Tolto

(*) Nel ritratto đi Cortès, che noi daremo apprefró, ri rapprefenta quel Cosiquiftatoro guardando con occhi torvi per colpa dell' Incifore. 
Tofto ch' ei fi vide onorato colla carica di General LisVIII. dell' armata, fi mife a fare colla maggior diligenza turti i preparativi pal viaggio, e cominciò a trattarfi da gran Signore tanto nel fuo portamento, quants nel fuo fervizio, ben con: fapevole della virrù, che ha sì fatta comparfa d'abbagliare il volgo, e di conciliare autorita. Inalberò immediatamente 10 Atendardo Reale d'avanti alla fua cafa, e fese pubblicare un bando per tutta l'ifola per arrolar de' Soldati. Concorfero a gara a metterfi fotto il fuo comando anche uomini dei più cópicui di quel paefe tanto per la loro nafcita, quanto per i loro impieghi, ficcome Alfonfo Hernandez di Portocarrero, cugino del Conte di Medellin, Giovanni Velafquez di Leon, parente ftretto del Governatore, Didaco Ordaz, Francefco di Montejo, Francefco di Lugo, ed altri, che andremo nominando nel decorfo della Storia. Tra tutti meritano una particolar menzione Pietro d'Alvarado da Badajoz, Criftofano d'Olid da Baeza nell' Andaluzia, e Gonzalo di Sandoval da Medellin, per effere tati i primi Comandanti delle truppe impiegate in quella conquilta, e quei che fecero pil luminofa figura : tutti e tre guerrieri, affai coraggiofi, duri nelle fatiche della guerra, o periti nell'arte militare, ma d' un 'carattere affai diverfo. Alvarado era un giovane ben fatro, ed agiliffimo, biondo, graziofo, feftevole, popolare, portato pel. luffo, e per li paffatempi, avido dell' oro, di cui abbifognava per oftentar grandezza, e lecondo che affermano gli Autori antichi, poco fcrupolofo nel modo d'acquiftarlo, ed inumano, e violento in alcune delle fue tpedizioni. Olid era membruto, ombrofo, e doppio. Ambidue fervirono affai bene a Cor: rès nella conquifta; ma poi gli furono ingrati, ed ebbero un fine tragico. Alvarado morì nella Nuova Gallizia oppreflo da un Cavallo precipitato da una montagna. Olid fu da' fuoi nemici decapitato nella piazza di Naco nella Provincia d' Hon: duras. Sandoval giovane di buona nafcita appena aveva ventidue anni, allorchè s'arrolò nell' armata del fuo compatriota Cortès. Era di proporzionata ftatura, e di compleffione robu: fta, di pelo caftagno, e riccio, di voce forte, e groffa, di poche 


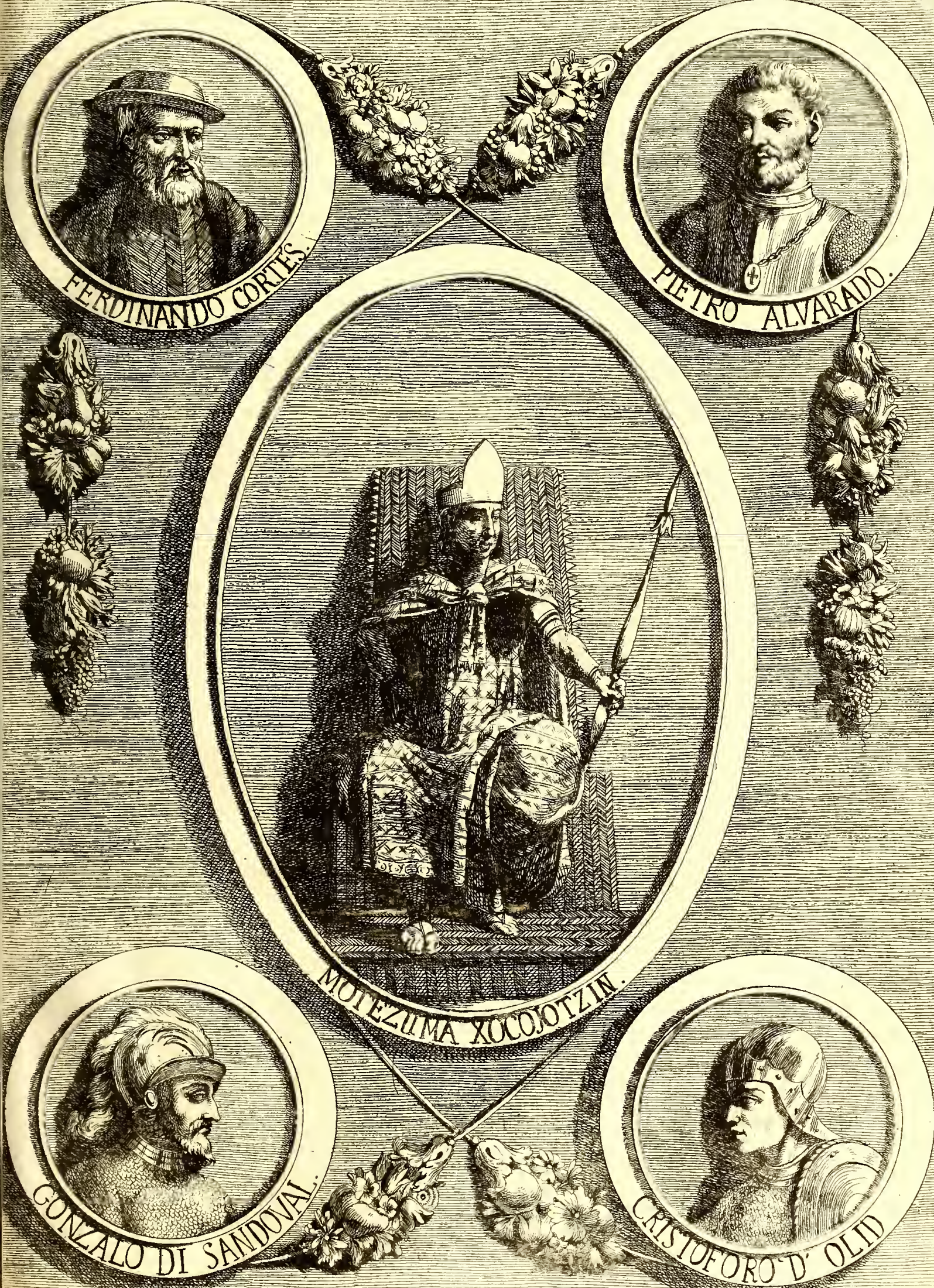



poche parole, e d'egregi fatti. A lui commife Cortés le pì̀ ardue, e pericolofe fpedizioni, ed in tutte riufci con onore .Lis.VIII. Nella guerra contro i Meficani fu Comandante d'una parte dell' elercito fpagnuolo, e nell' affedio della Capitale ebbe fotto i fuoi ordini più di trenta mila uomini, meritando fempre colla fua buona condotta la grazia del fuo Generale, il rifpetto de' fuoi Soldati, e l' amore degli ftefli nemici. Egli fondò la Colonia di Medellin nella colta di Chalchiuh. cuecan, e quella dello Spirito Santo ful fiume di Coatza. cualco. Fu Comandante del preitidio della Veracroce, e Go. vernatore qualche tempo del Meffico, ed in tutti i fuoi im. pieghi fece palefe la fua equità. Fu coltante ed affiduo nelIa fatica, ubbidiente, e fedele al fuo Generale, benigno verfo i fuoi foldati, umano $\left(^{*}\right)$ verfo i fuoi nemici, ed affatto libero dal comun contagio dell' avarizia. $E$ per dirlo in po. che parole, io non trovo in tutta la ferie de' Conquiftatori un uomo più compito, nè più degno di lode, mentre non Storia del Meffico Tom. Ill. B vi

$\left(^{*}\right)$ Il Dott. Robertion mette addoffo a Sandoval quello fpaventoro efempio di feverità fatto nei Panuchefi, allorchè gli Spagnuoli abbruciarono fer fanta Signori, e quattrocento Nobili fotto gli occhj dei loro figliuoli, e parenti, ed allega il teftimonio di Cortès, e di Gomara; ma Cortès non af ferma, che Sandoval faceffe quel gaftigo, neppure il nomina. Bernal Diaz la cui teftimonianza in quefto punto vale afiai più di quella del Gomara, dice, che avendo Sandoval vinti i Panuchefi, e fatti prigionieri venti $S_{i}$ gnori, ed alcune altre perfone ragguarderoli, fcriffe a Cortès rỉchiedendo la fua rifoluzione intorno ai prigionieri, e Cortès per far più giuftificata la loro condanna, commife il loro proceffo a Didaco d'Ocampo, Giudice di quella Provincia, il quale dopo aver fentita lis loro confeffione, gli cons dannò ad effer hruciati, ficcome fu afocuito. Bernal Diaz non efprime il numero dei rei puniti. Cortès dice, che furono bruciati quattrocento tra Signori e perfone principali. Un tal gaftigo fu fenz'altro ecceflivo, e crus dele; ma il Roberton, il quale ne fa tanti rimprover agli Spagnucli, dovrebbe per far manifelta la fua imparzialità, dichiarare i motivi, ch'ebbero coloro per incrudelire contro i Panuchefi. Quefti dopo di efferfi fottomeffi alla Corona di Spagna, fcoffero il giogo, e correndo all' armi mifero in ifcompiglio tutta quella Provincia; uccifero quattrocento Spagnuoli, fra $i$ quali bruciarono quaranta vivi in una cafa, e mangiarono gli altri. Quefti atroci delitti: non baftano a fcufar gli Spagnuoli; ma pur rendono men biafimevole la loro feverità. Il Robertfon leffe parimente preffo Gomara ed i misfatti dei Panuchefi, e la reverità degli Spagnuoli; ma efagera quefta, e tace quelli. 
- vi fu mai tra loro chi sì bene fapeffe accordare l'ardor gioLis.VIII.yanile colla prudenza, la bravura, e la intrepidezza colla umanità, la continenza col merito, e la modeftia colla felicità. Morì nella più frefca età in un luogo dell' Andaluzia effendo in cammino verfo la Corte di Spagna infeme con Cortès. Uomo veramente degno d'una miglior fortuna, e d'una vita più lưnga.

$\$, " 3$. Armata, Poichè furono fatti quafi tutti i preparativi pel viaggio; e viaggio il Governatore di Cuba rivocò per la fuggeftione, ed i ragdi Cortès giri de' rivali di Cortès la commeffione già data ad effo lui, ed anche diede ordine d'imprigionarlo; ma coloro che furono incaricati della cattura, non ebbero ardire di farla, vedendo tanti uomini riguardevoli, e coraggiofi impegnati nel foftenere il partito del nuovo loro Generale: ficchè Cortès, il quale non folamente avea (pefo ne' preparativi cutto il fuo capitale, ma eziandio s' era indebitato, ritenne a difpetto de' fuoi nemici la fua carica, ed avendo già tutto all' ordine, falpò dal porto d'Ajaruco a' Io. Febbrajo dell' anno I5Ig. L'armata era compofta d' undici vafcelli, di cinquecento otto foldati diftribuiti in undici compagnie, di cento nove uomini da mare, di fedici Cavalli, di dieci cannoni d'artiglie: ria, e di quattro falconetti. Navigarono fotto la direzione del Piloto Alaminos fin'all'ifola di Cozumel, dove recuperarono Girolamo Aguilar, Diacono Spagnuolo, il quale viaggiando alcuni anni addietro dal Darien all' ifola di S. Domenico, o fia Spagnuola, fece naufragio nelle cofte di Jucatan, e fu fatto fchiavo dagl' Indiani, ed ora confapevole dell'. arrivo degli Spagnuoli a Cozumel ottenne dal fuo Padrone la libertà, e s'aggregò all armata. Culla lunga pratica de Jucatanefi avea impararo la lingua maja, che vi fi parla: ond' ebbe pretfo Cortès l'impiego di turcimanno.

5. 4. Da Cozumel s'avanzarono colteggiando la penifola di Vittoria Jucatan fin'al fiume di Chiapa nella Provincia di Tabafco, degli Spa-pel quale fopra i battelli, ed i più piccoli naviglj s' inoltraTabafco. rono alquanto nel paefe infin' ad un palmeto, dove meffo piede a terra col pretefto di procacciarfi dell' acqua, e delle ver: Iova- 
tovaglie, s'avviarono verfo un groffo Villaggio quinci appena due miglia diftante, combattendo ognora con una folla $\mathrm{L}_{\mathrm{L}}$.VIII. $d^{2}$ Indiani, che con frecce, dardi, ed altre armi lor contra. ftavano il paffo, e fuperando gli fteccati, ch' effi aveano fatti per loro difefa. Rendurifi finalmente gli Spagnuoli padro. ni del Villaggio, quindi forrivano fpeffo per far delle fcorrerie ne'luoghi vicini, nelle quali ebbero alcune zuffe affai pericolofe finattantochè fi venne ad una battaglia campale, e decifiva a' di 25. Marzo . La battaglia î́ diede neile pianure di Centla, Villaggio poco difcolto dall' altro già men. tovato. L'efercito de.'Tabafchefi era molto fuperiore nel numero; ma a difpetto della loro moltitudine furono affatto vinti, mercè la miglior difciplina degli Spagnuoli, la fuperiorità delle loro armi, ed il terrore meffo fra gl' Indiani dalla grandezza, e dal fuoco de' cavalli. Ottocento Tabafchefi reftarono morti ful campo; degli Spagnuoli fu qualcuno uccifo; e più di feffanta feriti. Quefta vittoria fu il principio della felicità degli Spagnuoli, e per memoria vi fondarono poi una piccola citta col nome della Madonna della Vittoo ria, (c) la quale fu per lungo tempo la capitale di quella Provincia. Procurarono di giuftificare le loro oftilità colle iterate procefte, che prima di venire alle mani, fecero a' Tabafchefi, di non effere venuti in quel paefe come nemici a far verun male, ma foltanto come naviganti bifognofi a procacciarfi col baratto delle loro merci ciò, che aveano d'uopo per coutinuare il loro viaggio, alle quali protefte corrifpofero gl'Indiani con una tempefta di frecce, e di dardi o Prefe Cortès folenne poffeffo di quel paefe a nome del fus Sovrano con una ttrana ceremonia, ma confacevole alle ufanze, ed alle idee cavallerefche di quel fecolo. Imbracciò la rotella, e sfoderò la fpada, colla quale diede tre colpi in B. 2

un

(c) La Città della Vittoria fi fpopolò del tutto verfo la metà del recolo paffato a cagione delle frequenti invafioni degl' Inglefi. Fondoff poi in un fito più difcolto dal Mare un'altra piccola Città, che appellarono Villabermoja; ma la Capitale di quefa Provincia, dove rifiede il Governatore, è Ilacotlalpan. 
—un grand' albero, che era nel principal villaggio, proteftan: Lis.VIIIdo, che fe qualcuno aveffe ardire d'opporfi ad una tal pofo feffione, egli farebbe per difenderla con quella fpada.

Per raffodar vieppiù il dominio del fuo $\mathrm{Re}$, convocò ì Signori di quella Provincia, e lor perfuafe di rendergli ob. bedienza, e di riconofcerlo come loro legittimo Signore : c per imprimere una più vantaggiofa idea della poffanza del $\mathrm{Re}$, fece iananzi a loro fparare un cannone d'artiglieria, $e$ gl' induffe con certo inganno a credere, che il nitrito de'ca: valli foffe una moltra del loro fdegno contro i nemici degli Spagnuoli. Tutti parvero aderire alle propofizioni del vincitore, e afcoltarono con maraviglia, e con gradimente le prime verità della Religione Criftiana, che lor dichiaro per l'interprete Aguilar il P. Bartolommeo d' Olmedo, Religio: fo dotto, ed efemplare dell' Ordine della Madonna della Mer * cede, e Cappellano dell' armata. Prefentarono poi a Cortès in contraffegno della loro fommeffione alcune cofuccie d'oro; parecchie vefti di tela groffa (poichè altre non s' ufavano in quella Provincia,) e venti fchiave, le quali furono compar-

9. S: tite tra gli Uffiziali dell' armata . Fra quefte v'era una zi Notla fa-tella nobile, bella, di buon ingegno, e di molto fpirito, namofa In- tia di Painalla, Villaggio della Provincia mefficana di Coadiana tzacualco. (d) Suo Padre era itato Feudatario della Corona Marina, di Meffico, e Signore di parecchj luoghi. Effendo reftata vedova la Madre di lei, fi marito con un altro Nobile, da. cui

(d) In una Storia manofcritta, che v'era nclla libreria del Collegio di S. Fietro e S. Paolo deì Gefuiti di Meffico, fi leggeva, che Donna Marina era nata in Huilotla, Villaggió di Coatzacualco. Gomara feguito da Herrera, e da Torquemada dice, effere tata natia di Xxalixco, ed indi prefa da certi Mercanti Xicallanchefi, e trafportata alla loro patria; ma quefto è certamente falfo; poichè Xalixco è diftante da Xicallanco più dî novecento miglia, e non fí $f a$, e nè pure è verifimile, che vi fia mai ftato verun commercio fra quelle due Provincie tanto tra loro feparate. Bernal Diaz, il quale viffe lungo tempo in. Coarzacualco, e conobbe la madre; ed il fratello di Donna Marina, conferma la verità del noftro ragguaglio, e dice, effer cio certo, ed averlo fentito dalla ftefla Donna MarinaA ciò s'aggiunge la tradizione, che finora s'è confervata in Coatzacualco conforme a quello, che abbiamo detto. 
cui ebbe un figlio. L' amore, che portavano a quefto frutto del loro maritaggio, gli fpinfe all' iniquo configlio di fupporLis.VHr morta la primogenita, acciocchè ad effo lui andaffe tutta l'eredità. Or per farlo credere, la confegnarono clandeltinamente a certi Mercanti di Xicallanco, Città fituata fú confini di Tabafco, allorchè era morta la figlia d'una delle loro fchiave, nella cui morte fecero lo fteffo duolo, che fatto a. vrebbero, fe foffe itata veramente morta la loro figlia.I Xicallanchefi la diedero, o pur la vendettero a' Tabafchefi lor vicini, e quefti finalmente la prefentarono a Cortès affai lon. tano da penfare, che quella rara giovane foffe per fervir colla fua lingua alla conquilta di tutta quella terra. Sapeva bene, oltre alia lingua meffrcana propria del fuo paefe, la maja, che fi parlava in Jucatan, ed in Tabalco, e fra poco imparò altresì la fpagnuola. Inftruita prontamente ne' dogmi della Religione Criftiana $f u$ infieme colle altre fchiave folennemente battezzata col nome di Marina. (") Fu coltantemente fes dele agli Spagnuoli, e non poffono abbaltanza ftimarfi i fervizj lor farti da lei; poichè non folamente fu lo frumento delle lor negoziazioni co' Mefficani, co'Tlafcallefi, e coll'al. tre Nazioni d'Anahuac; ma lor falvò fpeffo la vita, avver. tendoli de' pericoli, che for fopraftavano, e additando la maniera di fchivarli. Accompagnò Cortès in turte le fue fpedizioni, fervendogli ognora da interprete, fovente da confi. gliere, e talvolta per la fua fventura da dama. Il figliuolo; che da effo lei ebbe quel Conquiltatore, fi chiamò Don Mars rino Cortès, Cavaliere dell'ordine militare di S. Giacomo. il quale per remerarj fofpeti di ribellione fu meffo alla tortura in Meffico l'anno I 568 , mettendo in non cale quegl'iniqui, e barbari Giudici gl' imparegoiabili fervizj farti da' geni. tori di quell' illutre reo al Re Cattolico, ed a tutta la $\mathrm{Na}$. zione

(*) I Mefficani adattando il nome di Marina al loro linguaggio l'appelratono Malintzir, onde venne il nome di Malingbe, con cui è conofciuta dagli Spagnuoli del Meftico. 
zione Spagnuola a (e) Dopo la conquilta fe maritò con un riLib.VIII guardevole Spagnuola appellato Giovanni di Xaramillo. Nel lungo, e malagevole viaggio, che fece in compagnia di Cortès alla Provincia d'Honduras. l'anno 1524 , ebbe occafione nel paffare per la fua patria di vedere la fua Madre ${ }_{2}$ ed il fuo fratello, i quali le fi prefentarono bagnati di lagrime, e cofternati, mentre temevano, che: trovandofi allora in si gran profperita colla: protezione degli Spagnuoli, non voleffe vendicar il torto fettole nella fua fanciullezza; ma ella gli accolle, e gli confortò con fomma amorevolezza, dando cosi a divedere, che la fua pietà, e la fua generofià non erano punto inferiori alle altre doti, di cui era tata dal Cielo fre giata. Non ci parve giufto il tralafciar quefti rifcontri d'una donna, che fu la prima Criftiana dell' Imperio Melficano, che fa nella foria: della Conquifta una figura tanto: lumino. $f a$, ed il cui nome è ftato, ed è prefentemente cotanto ce. lebre non meno preffo i Melficani, che preffo gli Spagnuoli. Rendutofi già Cortès frcuro della tranquillità di Tabafco, ed accorgendofi, che quefto non era paefe da poterfene cavar dell' oro, fi rifo!vette di continuar il fuo viaggio per cercarne un altro più ricco; ma accoltandofi la fefta delle palme, volle prima dare a'Tabafchefi una qualche idea della fantità della Religione Criftiana . Celebroffi quel giono la fanta Meffa col maggior apparato che fi potè, $f i$ benedif fero $\mathrm{i}$ rami, e fi fece una folenne proceffione colla mufica militare, alla quale intervennero sbalorditi, ed edificati que Gentili, reftando fin da quelt"ora ne' lor cuori quella femenza della grazia, ch'era per germogliare, e fruttificare in una: altra più convenevole ftagione.

Terminata quella funzione, e prefo congedo da'Signo.

(e) Quei che diedero la tortura a D. Martino Cortès, e mifero in prigione il Marchefe della Valle fuo frarello, furono, due formidabili Giudici mandati nel Meffico dal Re Filippo.II. Il principale di loro appellato Mugnox fece tali fragi, che moffo il Re dalle querele de'Mefficani il richiamo alla Corte, e gli fece una riprenfione cotanto fevera, e forte, che il di feguente fu trovato morto della paffione nella fua fedia a 
ri di Tabafco, fi rimife in viaggio l'armata, e camminan. do verfo Ponente dopo aver colteggiata la Provincia di Coa.Lib.VIII. tzacualco, e traveríata la bacca del frume di Papaloapan, entrò nel porto di S. Giovanni d' Ulua nel Giovedi Santo, 2 I Aprile. Appena aveano gettate l'ancore, che videro venir dalla cofta di Chalchiuhcuecan verlo la capitana due grandi canoe, fulle quali v'erano molti Mefficani mandati dal Governatore di quella colta per fapere chi erano quelli, che $v^{\prime}$ erano approdati in quella nuova armata, e che volevano, e per offerir loro turti i fuffidj, di cui abbifognaflero per la continuazione del loro viaggio: cio che fece pa. lefe la vigilanza di quel Governatore, e l'ofpitalità di quella Nazione. Venuti a bordo della capicana, e préentatifi a Cortès con maniere affai civili, gli elpofero la loro commeffrone per mezzo di Donna Marina, e d'Aguilar, mentre a ca. gione di non intenderfi allora da quefto il melícano, rè da quella lo .pagnuolo, era d'uopo in que' primi abboccamenti co' Meflicani l'adoperar tre lingue, e due interpreti. Donna Marira efponeva ad Aguilar in lingua maja ciò, che dicevano i Mefficani nel loro proprio linguaggio, ed Aguilar lo dichia rava in ifpagnuolo a Cortès. Quefto Generale accolfe corsefemente i Mefficani, e fapendo quanto folfero ftate a loro gradevoli l'anno avanti le bagatelle d'Europa, loro rifpofe, non effer egli per altro in quel paefe venuto, che per cornmerciare con loro, e per trattare col loro $\mathrm{Re}$ degli affari di fommo rilievo, e per obbligarli più fece ad effi guftar il vi. no di Spagna, e lor regalò alcune cofuccie, che credétte potere effere da loro gradite. $(f)$

II

(f) Torquemada dice, ch'effendo ftato avvifato Motezuma della nuova armata offervata gia dalle fentinelle pofte fulle montagne, fpedi fubito i fuoi Ambafciatori par riverire il pretefo Dio Quetzalcoatl, i quali portandofi con fomma celerita al porto di Chalchithcuecan, paffarono immediatamente a bordo della Capitana quello fteffo giorno, nel quale v'approda= rono gli Spagnuoli :-che Cortès avvertito dell'errore, e volendo prevalerfene, gli ricevè meffo a federe fopra un alto trono, che fece prontamente formare, dove fi lafciò adorare, veftire l' abito facerdotale di Quetzalcoatl, E fregiare il collo d'una collana di gemme, ed il capo d'una celata d'oro 
Il primo giorno di Pafqua, poichè aveano gli Spagnuō:

Lis.VIII.li meffo piede a terra, e sbarcato i loro cavalli, e la loro artiglieria, e s'erano fatte coll' ajuto degli fteffi Mefficani delle baracche di rami d'alberi in quella fpiaggia arenofa, dove prefentemente è la Città della Nuova Veracroce, vì arrivarono due Governatori di quella Colta appeilati $T$ eub. tlile, e Cuitlalpitoc $(g)$ con un gran feguito di fervitori; e fatte dall' una, e dall' altra parte le convenevoli ceremonie d'urbanità, o di rifpetto, prima d'inoltrarfi in verun difcorfo, volle Cortès non meno per felicitare la fua imprefa, che per dare a quegl'idolatri una qualche idea della noftra Religione, che fi celebraffe alla prefenza loro il fanto facrifizio della Meffa. Cantoffi pure colla maggior folennità polfibile, e quefta fu la prima, che fi celebrò ne'dominj de'. Mefficani. (b)

Invitolli poi a pranzar feco lui, e co' fuoi Capitani; pro-

tempeftata di gemme \&c.; ma quefto racconto è fenz' altro falfo. L'armata partì dal fiume di Tabafco il lunedi fanto, ed arrivò il giovedì al porto d'Ulùa. I monti di Tochtlan, e di Mictlan, donde fi potè più prefto offervar l'armata non fono diftanti dalla capitale meno di $300 \mathrm{miglia}$, nè quefta è diftante dal porto d'Ulùa meno di 220 : ficchè quantunque folfe ftata veduta l'armata quello feffo dì, che farpò da Tabafco, era impoffibile che arrivaffero gli Ambafciatori il giovedì ad Ulùa. Oltracciò non fi trova memoria di tal avvenimento preffo altro Autore; anzi dal raggua glio di Bernal Diaz fi vede, che tutto ciò è falfo, e che i Mefficani s'erano già accorti dell' errore, che lor cagionò la prima armata, che vi comparve.

(g) Bernal Diaz fcrife Tendile in vece di Teuhtlile, e Pitalpitoque in rece di Cuitlalpitoc. Herrera il chiama Pitalpitoe, ed il Solis, e il Robertion, che vollero emendarli, Tilevtns.

(b) Solis rinfaccia a Bernal Diaz, ed a Herrera l'aver affermato, fecon do ch'egli credette, che fi celebrò la meffa in Chalchiuhcuecan il Venerdì Santo. L'Autore della prefazione, che v'è nella ftampa d'Herrera del 1730, adopera una gran copia d'erudizione inopportuna, e nojofa per giufificar la pretefa celebrazione della meffa in quel fanto giorno-m ma con buona pace di quefto Autore, e del Solis, eglino non intefero bene il tefto di Bernal Diaz. Coftui foltanto dice nel cap. 38. ch'il Venerdi Santo sbarcarono i cavalli, e l'artiglieria., e facemmo, foggiunge, un altare, nel quale tofto fi diffe la meffa, Non dice, che quello fteffo giorno fecero l'altare ; anzi nel medefimo capo della fua ftoria afferma con parole affaichiare, che effo fi fece la Domenica, dappoi ch'era venuto il Governatos. Teubtlile a riverir Cortès: 
prōcūrando di conciliar con ofequ la lóto berievolenza. Tofto che fi levarono dalla tavola, gli conduffe in difparteLib. VIII. per efporre le fue pretenfioni. Diffe loro, ch'egli era fudo dito di Don Carlo d'Auftria il maggior Re dell' Oriente, la cui bontà, grandezza, e poffanza vanto colle più magnifiche efpreffioni, e foggiunfe, che quefto gran Monarca confapevole di quella terra, e del Signore che vi regnava, lo mandava a fargli a nome fuo una vifita, ed a comunicar. gli a bocca alcune cofe di gran rilievo, e che pero avreb. be caro di fapere, dove piacerebbe a lui di fentir l'amba. fciata. "Appena, rifpofe Teuhtlile, fiete arrivato in quefto "paefe, e fubito volete vedere il nofro Re? Ho afcoltato " con piacere ciò, che m'avete detto intorno alla grandez" za, ed alla bontà del voftro Sovrano, ma fappiate, che ") il noftro non è men buono, nè minor Re; anzi mi ma"raviglio; ch'effervi poffa al mondo un'altro più poffente "di lui; ma poichè voi l'affermate, io il farò fapere al "mio Sovrano, della cui bontà confido, che non folo avrà 3) piacere nel fentire si fatte nuove di quel gran Principe; 3) ma ancora onorerà il fuo Ambafciatore. Accettate frattan"to quefto regalo, che a nome fuo vi prefento," e ca. vando da una petlacalli, o fia caffa di canna inteffuta parecchi eccellenti lavori d'oro, gliene preíentò infieme con varie opere curiofe di penna, dieci fome di vefti fine di bambagia, ed una gran copia di vettovaglie. (i)

Accettò Cortes il prefente con fingolar dimoftrazione di gratitudine, e corrifpofe con cofe di poca valuta, ma da loro apprezzate $o$ : perchè erano affatto nuove in quel paefe, o pel loro apparente brillamento. Avea feco condotto Teuhtlile vari pittori, acciocchè tra loro compartendo i diverf Storia del Meffico Tom.III. ogget-

(i) Il Solis, ed il Robertion fanno Teuhtlile Generale delle armi, e lo privano del governo politico di quella cofta, laddove da Bernal Diaz, dal Gomara e da altri Storici antichi fappiamo, ch'egli era Governatore di Cuetlachtlan. Dicono ancora guegli Autori, che da principio s' oppofe Teuhtlile al difegno di Corrès di portarfi alla Corte; ma ci confta per la teftimonianza dei migliori Storici antichi, che quel Governatore non s'oppofe finattantocbè non ebbe un ordine poritivo dal fuo Re. 
oggetti, di cui fi componèva l'armata, poteffero in brieve Lib.VIII.copiarla tutta, e dar cosi al Re il piacere di veder cogli occhj fuoi ciò, che aveano a dirgli a bocca. Accortofi Cortès del loro intento ordinò per fornire a' Pittori un nuovo foggetto capace di far nell' animo del Re una impreffione più gagliarda, che la fua Cavallerìa correffe per la fpiaggia, e faceffe alcune evoluzioni militari, e che fi fparaffe ad un, ora tutta l'artiglieria: cid che fu offervato con quello : Aupore, che fi può immaginare, da'due Governatori, e dalla loro numerofa comitiva, la quale, fe diamo credenza al Gomara, non era meno di quattro mila uomini. Tra l'armi degli Spagnuoli offervò Teuhtlile una celata indorata; la quale, perchè era molto fomigliante ad un'altra, che aveva uno de' principali idoli di Mefsico, la dimandò a Cortès per farla vedere al Re, ed egli la concedette col patto di riaverla piena d'oro in polvere, fotto pretefto, che voleva vedere, fe l'oro, che fi cavava dalle miniere del Meffico, era come quello della fua patria. (k)

Tofto che furono terminate le pitture, fi congedd amo: revolmente Teuhtlile da Cortès, efibendofi di ritornar a lui fra pochi giorni colla rifpofta del fuo Sovrano; e lafciando in fuo luogo Cuitlalpitoc, acciocchè provvedeffe di tutto il bifognevole gli Spagnuoli, fe n'ando a Cuetlachrlan, luo. go della fua ordinaria refidenza; donde portò in perfona al. la Corte il ragguaglio, le pitture, ed il prefence del Generale fpagnuolo, come affermano Bernal Diaz, e Torquema. $\mathrm{da}$, o pure il mandò tutto, ficcome congettura il Solis, per le pofte, che v'erano fulle ftrade maeftre ognora pronte a far viaggio.

Ognuno può facilmente immaginar la grand' inquietudine, e perpleffita, in cui trovoffi Motezuma colla nuova di quell'

(k) Alcuni ftorici dicono, che Cortès per richiedere la celata piena d'oro fi prevalfe del pretefto di certa malattia di cuore, che pativano egli, ed $\mathbf{i}$ fuoi compagni, la quale diceva non poterfí guarire con altro rimedio, fe non con quel preziofo metallo; ma ciò poco importa per la foftanza del fatto. 
quell' armata, e coll' informazione più efatta del carattere di quegli ftranieri, del fuoco dei lor Cavalli, e della violenzaLib. VIII. diftruttiva delle loro armi. Come quegli ch' era tanto fuper- 5. 7. ftiziofo, fece confultare i fuoi Dei fulla loro pretenfione, e gli Inquiefu. rifpolto, per quel che dicono, che non accettaffe mai tudine di nella fua Corte quella nuova gente.$O$ aveffe in fatti quelt ma. oracolo, ficcome fi perfuafero parecchi Autori, dal Demonio, Prima il quale s'adoperaffe per tener chiuf tutti $\mathrm{i}$ paff al Vange- fciata, e lo, o liccome noi crediamo, dai Sacerdoti per loro interel prefente fe, o per quello di tutta la Nazione, Motezuma fi rifol- mandavette d'allora innanzi di. non ammettere gli Spagnuoli; ma to Cortès. per farlo con buona maniera e fecondo il fuo genio, mandò loro un'ambafciata con uni prefente degno veramente del. la fua regia magnificenza. L'Ambafciatore fu un grar perfonaggio della Corte, affai fomigliante tanto nella ftarura, quanto nelle fattezze al Generale Spagnuolo, ficcome ne fa fede un teftimonio oculato. $\left(^{*}\right)$ Appena erano paffati fette giorni da poichè fi congedò Teuhtile, che ritornò accompagnando I'Ambafciatore e conducendo feco più di cento uomini da foma, che portavano il prefente. (l). Tofto che fu l'Ambafciatore alla prefenza di Cortès, toccò colla mano la terra, e poi la portò alla bocca, giufta l'ufanza di quelle Nazioni, incensò $(m)$ il Generale, e gli altri UfiziaC 2 li,

(*) Bernal Diaz del Caftillo.

(l) Bernal Diaz appella queft Ambafciatore Quintalbor; ma cotal nome non è, nè può effer meflicano. Il Robertfon dice, che gli fteffi Uffiziali che aveano fin' allora trattato con Cortès, furono incaricati di portargli la rifpofta del Re, fenza far menzione dell' Ambafciatore; ma così Bernál Diaz, teftimonio oculato, come altri Storici Spagnuoli, ed Indiani affermano cid che noi diciamo. Solis attefo il corto intervallo di fette giorni, e la diftanza di fettanta leghe tra quel porto e la Capitale, non volle per fuaderfi, che vi veniffe allora l'A mbafciatore; ma avendo egli detto poco innanzi, che le pofte mefficane erano più diligenii delle Europee, non è da maravigliarfi, che in un giorno, o poco più, portaffero la nuova dell' Armaia alla Corte, e in quattro, o cinque giorni $\mathrm{n}^{\prime}$ andaffe in lettiga $\mathrm{l}^{\prime} \mathrm{Am}$ bafciatore fulle fpalle delle medefime pofte, come preffolorofi ufara. Poichè il fatto non è inverifimile, bifogna credere a Bernal Diaz, teftimonio oculato e fincero.

(m) L'incenfamento fatto agli Spagnuoli, benchè altro non foffe che un offe- 
li, ch' erano preffo a lui, gli falutd rifpet:ofamente, e mefo Lib.VIII.fofi a federe in una fedia, che gli porfe Cortès, pronunciò la fua aringa, la quale fi riduffe a congratularfi con quel Generale a nome del fuo $\operatorname{Re}$ del felice di lui arrivo in quel paefe, a fignificare il piacere, che fua Maeftà aveva avuto nel fapere, ch' erano capitati nel fuo regno degli uomini si prodi, e nel fentir le nuove, che gli portavano d'un $\mathrm{Mo}$. narca sì grande, ed a moftrargli quanto gradevole gli foffe ftato il regalo di lui; onde per moftra del fuo regio gradimento gli mandava quel prefente. Ciò detto fece diftendez fulla terra ftuoje fine di palma, e tele di bambagia, fulle quali fu pofto con buon ordine e fimmetria tutto il prefen: te. Quefto confifteva in molti lavori d'oro, e d'argento anto cor più ftimabili pel loro maravigliofo artifizio, che per la preziofa loro materia, tra i quali alcuni a veano incaftonate delle gemne ed altri erano figure di Lioni, di Tigri, di Scimie, e d'altri animali; in trenta fome, o balle di tele frniffime di bambagia di varj colori, ed in parte inteffute di vaghiffime penne, in parecchi lavori eccellenti di penna fregiati di molte figurine d'oro, e nella celata piena d'oro in polvere, come la voleva Cortès, la quale importava mille cinquecento zecchini; $\mathrm{ma}$ il più pregevole di sutto furono due grandi lamine fatte a guifa di ruote, una d'oro, e l'altra d'argento . Quella d'oro rapprefentante, fecondo quello che abbiam detto altrove, il fecolo mefficano, avea fcolpita nel mezzo l'immagine del fole, ed attorno diverfe figure di bafforilievo. La fua circonferenza era di trenta palmi toletani, ed il fuo valore di dieci mila zecchini. (n) Quella d'argento in cui era figurato $l^{\prime}$ anno meffieano, era ancor più grande con una Luna sel mezzo, circondata parimente di figure di baffo rilievo. Reo itaro.

offequio meramente civile, ed il nome di Teterctin (Signori o Cavalieri) con cui erano appellati, alquanto fimile a quello di Teteo (Dei,) li fece credere, ch'erano Aimati Dei da' Meflicani.

(n) V'è del divario preffo gli Autori intorno al valore delle lamine; ma io do maggior fede a Bernal Diaz teftimonio oculato, ch' il fapeva bene, come quegli che doveva aver la fua parte nel prefente di Motezuma. 
ftarono gli Spagnuoli non meno maravigliati, che contenti, di sì gran ricchezza." Quefto regalo, foggiunfe l' Ambalcia-L ib.VIII "tore parlando con Cortès, manda il mio Sovrano "per voi, "e per i voltri compagni, poichè pel voltro $R e$ v'indrizze"rà fra poco certe gemme di valore ineftimabile. Voi frat"tanto potrete trattenervi quanto vi piace in quefta fpiag. " gia per ripofare dalle fatiche di si lungo viaggio, e per ") provvedervi di quanto abbifognate per ritornare alla voltra " patria. Se qualche altra cofa di quelta terra volete pel "voltro Sovrano, fubito vi fi darà; ma per ciò che riguar" da la voltra pretenfione di portarvi alla Corte, fono inca", ricato di diffuadervi d'un si malagevole e pericolofo viag" gio, mentre vi farebbe d'uopo il camminare per deferti i"nabitabili, e per paefi di nemici." Cortès ricevette il prefente colle più grandi efpreffioni della fua gratitudine verfo la regia beneficenza, e corrifpole come porè; ma fenza ab. bandonare la fua richiefta, pregò l'Ambafciatore di rapprefentare al $\operatorname{Re} i$ pericoli, ed $i$ difag; da loro fofferti in sì lunga navigazione, ed il difpiacere, che avrebbe il fuo Sovrano nel vederfi delulo della fua fperanza: che del refto gli Spagnuoli erano di tal condizione, che nè le fatiche, nè i periglj erano capaci di diftornargli dalle loro imprefe. S'efibì l'A Ambafciacore a far ciò fapere al $\mathrm{Re}$, e prefe cortefemente congedo infieme con Teuhtlile, reftando Cuitlalpitoc con un gran numero di popolo in un cafale, che vi aveano formato di capanne, poco lontano dal campo degli Spagnuoli.

Ben vedeva Cortès in mezzo a tanta profperità, quanta fin'allora avea avuta, che non potea fuffiltere in quel fito; poichè oltre all'incomodo del caldo, ed alla importunità delle zanzare, che abbondano troppo in tutta quella fpiaggia, temeva qualche danno nelle fue navi dalla violenza della tramontana, a cui è molto efpolto quel cattivo porto: onde fpedi due vafcelli fotto il comando del Capitano Montejo, acciocchè camminando lungo la colta verfo Panuco, cercaffero un altro porto più ficuro. Ritornarcno fra po: 
chi giorni colla nuova d'aver trovato trenta fei miglia da Lib.VIIL. Ulua un porto fufficiente vicino ad una citta piantata fopra un luogo forte.

5. 8. Frattanto ritornò Teuhtlile al campo degli Spagnuoli, Prefen- e tirando in difparte Cortès cogl' interpreti, gli diffe, che
tediMotezuma il fuo Signore Motezuma avea gradito il nuovo prefente da pel Re lui mandatogli : che quello ch'egli allora gli mandava era Cattolico deftinato pel gran Re di Spagna: che gli defiderava una fomma felicità; ma del refto non gli mandaffe più meffag. gi, nè fi parlaffe più full' andare alla Corte. Il prefente pel $\operatorname{Re}$ Cattolico confifteva in parecchj lavori d'oro, che importavano mille cinquecento Zecchini, in dieci balle di ro. ba curioliffima di penna, ed in quattro gemme tanto dai. Mefficani ftimate, che per quello che affermo il medefimo Teuhtlile, ciafcuna valeva quattro fome d'oro. Penfava quel mal accorto Re d'obbligar colla fua liberalita gli Spagnuoli ad abbandonare quel paefe non avvedendofi, che l'amor dell' oro è un fúoco', che tanto più s' infiamma, quanto è più co. piofa l'efca, che gli fi porge. Rincrebbe affai a Cortès la ripulfa del Re; ma non perd fi diffolfe dal fuo penfiere, mentre la natural coftanza del fuo animo era davvantaggio f́pin. ta dall' allettamento della ricchezza .

Offervo Teuhtlile prima di congedarfi, che gli Spagnuoli nel fentire i tocchi della campana all' Avemaria s'inginocchiavano d'avanti ad una fanta Croce, e pieno di maraviglia addimandò, perchè adoravano quel legno. Prefe quindi occafione il P. Olmedo per dichiarargli i principali articoli della Religione Criftiana, e per rimproverargli il culto abbominevole degl' Idoli, e l'inumanità dei loro facrifizj; ma sì fatto difcorfo fu affatto infruttuofo, mentre non era ancor arrivato per quei Popoli il tempo della fantificazione.

Il di vegnente fi trovarono gli Spagnuoli sì abbando. nati dai Mefficani, che neppure uno fi lafciava vedere in tutta quella fpiaggia: effetto fenz' altro dell'ordine dato già čal Re di ritirare dal campo di quegli ftranieri e la gente deftinata al loro fervigio, e le vettovaglie, qualora perfiftef- 
fero nella loro temeraria rifoluzione: Una si fatta novità cagionò una gran cofternazione negli Spagnuoli, perchè ad Lib.VIL. ogni momento temevano, che piombaffe fopra il miferabil lor campo tutto il potere di quel valto Imperio: onde Cortès fece afficurar nei valcelli le loro provvigioni, e metter in arme le fue truppe per la difefa. Non v'è dubbio, che tanto in quefta, quanto in altre molte occafioni, che andremo rammentando nella Storia, potè agiatamente Motezuma disfare affatto quei pochi ftranieri, ch'erano per fargli tanto male; ma Iddio gli confervava, acciocchè foffero ftrumenti della fua giultizia, fervendofi de!l s loro armi per punire la fupertti. zione, la crudeltà, ed altri delitti, coi quali aveano quelle Nazioni provocato il fuo fdegno. Non vogliamo però giultificar l'intento, e la condotta dei Conquiftatori ; ma nè me. no poffiamo lafciar di riconofcere nella ferie della conquifta, a difpetto della incredulita, la mano di Dio, che andava preparando quell' Imperio alla fua rovina, e fi prevaleva degli Ateffi misfatti degli uomini per gli alti difegni della fua Provvi. denza.

In quefto medefimo giorno di tanta colternazione per gli Spagnuoli, ebbero pure un faggio della Providenza Divina. Aciatadel Due foldati, che facevano la guardia fuor del Campo, videro Signor di venir verfo di loro cinque Uomini, alquanto diverí dai Mefficani nei loro abiti, e nei loro ornamenti, i quali condorti Cempoalla, e al Generale Spagnuolo differo in mefficano, (poichè non era feguenze ivi chi intendeffe il lor proprio linguaggio,) ch'erano della nazione Totonaca, e mandati dal Signor di Cempoalla, Città diftante ventiquatıro miglia da quel luogo, per falutargli, per fapere chi erano e donde venivano, e per pregargli di portarfi a quella Città, dove farebbero bene accolti, foggiun. gendo, che non erano giunti prima al campo per paura dei Mefficani. Era il Signor di Cempoalla uno di quei Feudatarj, che vivevano impazienti fotto il giogo de' Mefficani. Confapevole della vittoria ottenuta dagli Spagnuoli in Tabafco, e del loro arrivo a quel porto, gli parve quefta la più bella occalio. ne per ifcuotere il giogo coll' ajuto d' uomini sí prodi. Cortès 
come quegli, che tulla piú bramavá; che si fatte alleanze Lib.VIII.per aumentar le fue forze, dopo d'efferfi abbaftanza informato dello ftato, e della condizione dei Totonachi, e dei torti che foffrivano dalla prepotenza dei Mefficani, rifpole ringraziando il Cempoallefe della fua cortesia, e prometten. do d'andare fenza indugio a trovarlo.

In fatti pubblicò fubito la fua partenza per Cempoalla; ma prima gli fu d'uopo fuperare gli oftacoli meffi dagli Iteffi fuoi Soldati. Alcuni parziali del Governatore di $\mathrm{Cu}$ ba, ormai Itanchi dagl' incomodi da loro tollerati, impauri. ti dai periglj, che prefentivano, e bramofi del ripofo, e della comodità delle loro cafe, fcongiurarono fortemente il Generale di ritornarfene in Cuba, efagerando la fcarfezza dei viveri, e la temerità d'una imprefa cotanto grande, quanto quella di opporre si piccolo numero di foldati alla gran poffanza del Re di Meffico, maffimamente dopo aver perduti in quei renai trentacinque uomini, parte per le ferite avute nella battaglia di Tabaíco, e parte a cagione dell' aria infalubre di quella fpiaggia. Cortès or con doni, or con pro. meffe, or con un poco di rigore opportunamente adoperato, or con altri mezzi inventati dal fuo raro ingegno, maneggió si beae gli animi, che non folo racchetò i malcontenti, e gl' induffe a reftarfene volentieri in quel doviziolo paefe; ma avanzandofi altresì nelle fue negoziazioni, ottenne che l'efercito a nome del $\mathrm{Re}$, e fenza veruna dipendenza dal Governatore di Cuba gli confermaffe il fupremo comando ranto politico, quanto militare, e che per le fpefe da lui fatte già nell' armata, e da farfi nell'avvenire, gli foffe d'allora in poi affe. gnata la quinta parte dell' oro, che foffe per acquiftarfi, cava. tane prima quella che al loro $\mathrm{Re}$ apparteneva. Fece inoltre creare i Magifrati, e tutte le cariche neceffarie per una colonia, che piantar voleva in quella cofta.

Avendo dunque fuperate cotali difficoltà, e prefe le mifure confacevoli all'efecuzione dei fuoi vafti progetti; fi mife in viaggio colle fue truppe. Il fuo intento non era quello fo. lamente di riftorare la fua gente travagliata affai in quella a. renofa 
renofa fpiaggia, e di cercar degli alleati, ma eziandio quel. lo di fcegliere un buon fito per la fondazione della Colo.Lib.VIII. nia, mentre Cempoalla era fulla ftrada per andare a Chia. huitztla, $\left({ }^{*}\right)$ nel cui diftretto era il nuovo porto trovato già dal Capitano Montejo. L'efercito con una parte dell'artiglieria marcio verfo Cempoalla in buon ordine, ed apparec. chiato a difenderfi, qualora affalito foffe, o dai Totonachi, della cui buona fede non erano ancor ficuri, o dai Meffica$\mathrm{ni}$, che fupponevano offefi dalla loro rifoluzione: difpolizio. ni da niun buon Generale ftimate foverchie, le quali non mai furono da Cortès trafcurate ancor nel tempo della più gran prolperità, utili ognora per mantener la difciplina militare, e per lo più neceffarie alla propria ficurezza. I Vafcelli s' incamminarono marina marina al porro di Chiahuitztla.

Tre miglia prima d'arrivare a Cempoalla vennero ad in. contrar l' efercito venti riguardevoli Cempoallefi, i quali prefentarono a Cortès un rinfrefco d'ananas e d'altre frutte del paefe, lo \{alutarono a nome del loro Signore, e domandarono fcufa, fe non era venuto egli feffo in perfona a cagione di trovarfi impedito. Entrarono nella citta ordinati in forma di battaglia, temendo qualche tradimento dagli abitanti. Un Cavalleggiere effendofi inoltrato infino alla piazza maggiore, ed avendo veduto un baftione del palazzo di quel Signore, il quale perchè era di frefco imbiancato, e ben fornito, rifplendeva col Sole, gli parve d'argento, e ritornò a tutta briglia per dar sì bella nuova al Generale. Si fatti inganni fono troppo frequenti in quelli, che hanno la mente ingombrata da qualche paffione gagliarda. Camminavano per le Atrade gli Spagnuoli non men giojofi, che maravigliati dal vedere quella Città la più grande che avea* no veduta fino allora nel nuovo Mondo, tanta folla di po. polo, e sì belli orti e giardini. Alcuni per la fua grandez.

Storia del Meffico Tom. III.

D

za

(*) Solis, e Robertfon danno a Chiabuitztla il nome di Quiabislan: il quale non è, nè può effer mefícano. 
za l'appellarono Siviglia, ed altri Villaviziofa per la fua Lib.VIIl.amenità. (o)

Quando giunfero al tempio maggiore, venne a ricevergli alla porta dell'atrio il Signor di quello ftato, il quale benchè inabile pel moto a cagione della fua fmifurata graffezza, era uomo accorto, e di buon ingegno. Dopo avergli falutati fecondo l'ufanza del paefe, e d'aver incenfato il Generale, prefe congedo promettendo di ritornare fubito che fi foffero ripofati dalle fatiche del viaggio. Alloggiò tutta la truppa Spagnuola in cerci edifizj grandi e belli, ch' erano dentro il recinto del tempio, o foffero a bella pofta fabbricati per alloggio dei foreftieri, ficcome quello ch' era nel tempio maggior di Meffico, o foffero deftinati per abitazione dei miniftri degl' Idoli . Quivi furono ben trattati, e provveduti di quanto abbifognavano a fpefe di quel Signore, il quale ritornò a loro dopo definare in una fedia portatile, o letriga ed accompagnato da gran Nobiltà. Nella conferenza fegreta, ch' ebbe con Cortès, vantò quefto Generale per mezzo dei fuoi interpreti la grandezza, e la poffanza del fuo fovrano, da cui era egli mandato in quel paefe, ed incaricato di parecchie commeffioni, di fommo rilievo, e tra l'altre di quella di dare ajuto alla inocenza oppreffa., Se però, foggiunfe, poffo in " qualche cofa fervirvi colla mia perfona, e colle mie trup. " pe, mel dite, poichè il farò volentieri." Nel fentire il Cempoallefe quefte efibizioni molto accortamente nel difcorfo introdotte, gittò un gran forpiro, il quale fu feguito da amare querele della fventura della fua Nazione. Gli diffe, ch' effendo ftati i Totonachi da tempo immemorabile liberi, e retti da Signori della lor propria Nazione, fi trovavano da pochi

(o) Non può dubitarfi della grandezza antica di Cempoalla attefo la teAtimonianza degli Autori, che la videro, e la ftenfione delle fue suine; ma non fi può far conto del computo che fa Torquemada, mentre nel lib. 3. cap. 5.fi numera da venticinque in trenta mila abitanti, in altro luogo cinquanta mila, e nell'indice del primo tomo cencinquanta mila. A Cempoalla accadde quel medefimo che ad altre Città del nuovo Mondo, cioè, che coll'infermità, e coi difagj del fecolo XVI. s'andó fcemando sin'a fpopolarfi affatto. 
pochi anni in quà fopraffatti dal pefante giogo dei Mefficani : che i Mefficani per l'oppotto da umili principj s'erano inal-Lib. VIIE. zati a tanta grandezza per la ftretta e coftante alleanza coi Re d'Acolhuacan, e di Tlacopan, che s' erano renduti padroni di turta quella rerra: che il loro potere era fmifurato, e la loro tirannia proporzionata alla loro pofianza: che il Re del Meffico s'impadroniva dell' cro dei fuoi fudditi, ed i Rifcotitori dei tributi richiedevano dai tributarj le loro figlie per violarle, ed $\mathrm{i}$ loro figlj per facrificarli, oltre ad altre inaudite veffazioni. Cortès glifi moftrò affai moffo a compaffione delle fue difgrazie, e s' efibì ad ajutarlo in tutto, lafciando per un' altra occafione il trattare fulla maniera di farlo; perchè allo. ra gli premeva d'andare a Chiahuitztla per veder lo ftato dei fuoi vafcelli. In quefta vifita gli fece il Cempoallefe un prefente di lavori d' oro, che importò, per quel che dicono alcuni Storici, mille zecchini in circa.

Il dì vegnente fi prefentarono a Cortés quattrocento Uomini da foma, mandatigli da quel Signore pel trafporto del bagaglio, ed allora feppe da Donna Marina l'ufanza, ch' era preffo quelle Nazioni, di fornire fpontaneamente, e fenza verun intereffe d'uomini da foma ogni qualunque perfona riguardevole, che paffaffe per le loro Città.

Da Cempoalla fi portarono gli Spagnuoli a Chiahuitztla piccola Città fituata fopra un monte fcofcefo e dirupato, poco più di dodici miglia da Cempoalla verfo Tramontana, e tre dal nuovo porto. Quì ebbe Cortès un' altra conferenza col Si. gnore di quella. Città, e con quello di Cempoallan, il quale per quefta cagione cola fi era fatto trafportare. Nel medefimo tempo, in cui difcorrevano full' affare della libertà vi capitarono con un gran feguito cinque Nobili mefficani, Rifcotito. ri dei regj tributi, moftrandofi oltremodo fdegnati contro i Totonachi, perchè aveano avuto l'ardire d'accogliere fenz' alpettare il regio confenfo quegli Atranieri, ed efigendo da loro venti vittime umane da facrificarfi ai lor Dei per la efpia. zione di si fatto delirto. Turboffi tutta la Citrà per tal huova, e maffimamente i dae Signori, che fi riconofcevano più 9. 10. gionamento in Chiadicinque miniftri Regii。 
colpevoli. Cortès, faputa da Donna Marina la cagione della Lib.VIII.loro cofternazione, trovò uno ftraordinario ripiego per tirargli da quell' intrigo. Suggerì a quei due Signori l'ardito configlio di pigliar i rifcotitori e metterli in prigione, e benchè da principio ricufaffero di farlo, parendo loro un attentato troppo temerario e pericolofo, finalmente fi piegarono alle fue iftanze. Furono dunque carcerati nelle loro gabbie quei cinque Nobili, ch' erano entrati in quella Città con tanto orgoglio, e con tal difpregio degli Spagnuoli, che neppur s' erano degnati di guardarli, effendo pafiati innanzi a loro.

Appena ebbero fatto quefto primo paffo i Totonachi; che prefo con ciò coraggio s'avvanzarono infin' all' ecceffo di voler facrificarli quella fteffa notte; ma furono diffuafi da Cortès, il quale effendofi conciliato con quella cattura l'amore, ed il rifpetto dei Totonachi: volle pure colla liberazione dei prigioni guadagnarfi la benevolenza dei Mefficani. Ia fua condotta artifiziofa, e doppia fa fenz' altro palefe il fuo ingegno; ma non può effere lodata, fe non da quei Cortigiani, che non fanno altra Politica, che l'arte d'ingannare gli uomini, e che mettendo in non cale l'onefto, foltanto cercano l'utile nelle loro azioni. Cortès dunque diede ordine alle fue guardie di trarre la notte dalle gabbie due dei Meflicani, e di condurli alla fua prefenza nafcoftamente, e fenza che fe ne poteffero accorgere i Totonachi. Cosi fu fatto, ed i Mefficani reftarono tanto obbligati al Generale Spagnuolo, che gli fecero mille ringraziamenti, e lo configliarono di non fidarfi dei barbari e perfidi Totonachi. Cortès gl' incaricò di efporre al loro Sovrano il fuo gran rincrefcimento per l' attentato di quei montanarj contro i fuoi miniftri ; ma che come egli avea meffi lor due in libertà, cosi farebbe cogli altri tre. Effi partirono immediatamente per la Corte, condotti da alcuni Spagnuoli in una barca infin di là dai confini di quel!a Provincia, e Cortès il di feguente fi finfe adirato contro le guardie, per la cui trafcuraggine erano fcappati quei prigioni; ed acciocchè non avveniffe lo fteffo degli altri diffe, che voleva afficurargli in 
un' altra prigione più ftretta, e per farlo credere, gli fece condurre incatenati fu' vafcelli; $\mathrm{ma}$ indi a poco gli pofe Lib.VIII. nafcoltamente in libertà, come $i$ primi.

Fece colto correr la voce per tutte le montagne de' $\mathrm{To}$ tonachi, ch' erano ormai liberi dal tributo, che pagavano al ConfedeRe di Meffico, e che fe per avventura v'erano altri Rifco-razione titori di tributi, il faceffero fubito fapere per pigliarli. Col de'Totofuon di quefta voce fi rifvegliò in tutta la Nazione la dol. nachicoce fperanza della libertà, e cominciarono a venire a Chia- gnuoli. huitztla parecchj altri Signori non men per ringraziare il loro pretefo liberatore, che per deliberar fopra i mezzi d' affermar la loro libertà. Alcuni, che non aveano ancora cacciato da' loro animi la paura de' Mefficani, pretendevano, che s'addimandaffe perdono al $\mathrm{Re}$ dell' attentato commeffo contro a'fuoi Miniltri; ma prevalfe per le fuggeftioni di Cortès, e de' Signori di Cempoalla, e di Chiahuitztla, l' oppolto fentimento di fottrarfi alla tirannica dominazione de' Mefficani coll'ajuto di que' prodi ttranieri, efibendofi a mettere un efercito formidabile fotto il comando del Generale Spagnuolo.

Cortès dopo d'efferfi abbaftanza afficurato della fincerità de' Totonachi, $e$ informatofi delle loro forze, fe ne prevalfe di quelto momento favorevole per indurquella numerofa $\mathrm{Na-}$ zione a dare ubbidienza al $\operatorname{Re}$ Cattolico. Celebroff queft' atto coll' intervento del Notajo dell' armata, e con tutte le altre folennità legali.

Conchiufo felicemente quefto grand' affare, prefe Cortès congedo da quei Signori per andare a mettere in opera un Fondazio. altro progetto di grandiffima importanza, e poco innanzi da ne della lui formato, cioè quello di piantar in quella colta una Co. Veraçolonia forte, che folfe per loro ricovero nelle difgrazie, fortezza per mantenere i Totonachi nella fedeltà già giurata agli Spagnuoli, fcala per le nuove truppe, che lor veniffero in ajuto da Spagna, o dalle Ifole Antille, e magazzino da riporvi gli effetti, che a loro foffero mandati dai loro Nazionali, o che effi voleffero mandare in Europa. Fondof: 
Ii dunque quefta Colonia nel paefe medefimo de' Totonachi; L.ib.VIII. in una pianura, che v'è appiè della montagna di Chiahuitzla, dodici miglia da Cempoalla verfo Tramontana, e preffo. al nuovo porto. $(p)$ Appellaronla Villarica ( cioè Cittá ric* ca ) della Veracroce per le moftre di ricchezza, che $v$ ' aveano vedute, e perchè vi sbarcarono il Venerdi Santo,e que. Ita fu la prima colonia degli Spagnuoli nel continente dell ${ }^{2}$ America Settentrionale. Cortès fu il primo nel metter ma. no alla fabbrica per incoraggire col fuo efempio la fua gente, la quale coll' ajuto de' Totonachi 'coftruffe in fra poco tempo un numero fufficiente di cafe, ed una piccola fortezza capace di far qualche refiftenza all' armi de' Mefficani.

Frattanto erano arrivati a Meffico que' due primi Rif-

Nuova cotitori, che avea già mefli Cortès in libertà, ed aveano in. ambafcia-formato il $R e$ di tutto ciò, ch' era accaduto confingolari lo. rente di di del Generale Spagnuolo. Motezuma, il quale era già in Motezu- procinto di mandare un efercito per gafligar l'infolente tema. merità di quegli Stranieri, e per difcacciarli da' fuoi domini fi rafferend con quella nuova; ed obbligato da' fervizj fatti da quel Generale a' fuoi Miniftri, gli mandò due Principi fuoi nipoti ( figliuoli forfe del fuo fratello Cuitlahuazin, ) accompagnati da numerofa Nobiltà, e fervitù con un regalo: di lavori d' oro, che importava più di mille zecchini. Ringraziarono Cortès a nome del $\mathrm{Re}$, ed infieme fi lagnarono di lui, perchè s' era inoltrato tanto nell' amicizia de' ribelli Totonachi: onde quefta Nazione avea avuta l' infolenza di

ne.

(p) Quafi tutti gli Storici hanno prefo abbaglio intorno alla fondazione della Veracroce, mentre dicono, che la prima colonia degli Spagnuoli fia fata l'Antigua, o fia antica fondata ful fiume del medefimo nome, e credono non effere Atati più di due luoghi appellati Veracroce, cioè la Veracroce antica, e la nuova piantata fullo fteffo renajo, dove sbarcò Cortès; ma non $v$ 'è dubbio, che tre fono ftate le Città del medefimo nome di Veracroce. La prima fondata nel isi9. preffo al porto di Chiahuitztla, alla quale refto poi folamente il nome di Villarica: la feconda $l$ Antica Veracroce fondata nel 1523., o 1524 ., e la terza la Nuova Veracroce, la quale oggiconferva il nome di Veracroce,e fu fondata per ordine del Conte diMonterey Vicerè del Meffico ful fine del fecolo XVI. , o ful principio del XVII, ed ebbe da Filippo III. il titolo di Città nel 2615 . 
negare il tributo, che dovea pagare al fuo Sovrano: Sog. giunfero, che foltanto per riguardo a tali Ofpiti non era ve.Lib.VIII. nuto un efercito per punire la ribellion di que Popoli; ma che alla fine non refterebbono impuniti. Cortès, dopo aver fignificato con le più convenevoli elpreflioni la fua gratitudine procurò purgarfi dall' accufa dell' amicizia co' Totonachi colla neceffità, in cui s'era trovato, di cercar de'viveri per le fue truppe per cagione d'effere ftato abbandonato da' Mefficani. Diffe poi, che per ciò che riguardava il tributo, non era poffibile, che quella Nazione ferviffe infieme a due Signori : ch' egli fperava portarfi fra poco alla Corte per foddisfare più compitamente al $\mathrm{Re}$, e fargli vedere la fincerità della fua condotta.

1 due Principi dopo d'aver veduto con gran piacere e maraviglia l' efercizio militare della cavalleria Spagnuola, fe ne tornarono alla Corte. Il Signor di Cempoalla, a cui era affai difpiaciuta quell' ambafciata, per vieppiù ftrignere l'alleanza cogli Spàgnuoli, prefentd a Cortès otto Vergini ben veftite, acciocchè fi maritaffero co' capitani, e tra effe v' era una fua nipote, la quale deftinava allo fteffo Generale. Cor. tès, il quale parecchie volte avea difcorfo con lui ful pun. to della Religione, gli diffe, che non poteva accettarle, fe prima non rinunziavano l'idolatria, ed abbracciavano il Criftianefimo; e quindi prefe occafione per efporgli di bel nuovo le pure e fante verità della noftra Religione, e ragionò con quanta forza potè contro il vano culto de'loro bugiardi $\mathrm{Nu}$. mi, e maffimamente contro l'orrenda crudeltà de'loro facrifizj. Ad una sì fervorofa efortazione rifpofe il Cempoallefe, che benchè fommamente pregiaffe l'amicizia. di lui non po. teva pure compiacergli nel lafciare il culto di quegli Dei, mento dalla cui mano ricevevano la falute, l'abbondanza, e tutti degl' Ii beni che avevano, e dalla cui collera provocata colla in. doli di gratitudine temer doveano i più feveri gaftighi. Infiammof- alla. fi più con tal rifpofta il zelo militare di Cortès: onde volto a' fuoi foldati lor diffe "Orsù, Spagnuoli, che afpettia" mo? Come polfiamo foffrire, che coltoro, che fi vantano "d' effe- 


\section{$3 z$}

Lib.VIII.", voli del Demonio quel culto, che fi debbe al noftro uni" co, e vero Dio? Come permettiamo, che gionalmente "fotto i noftri occhj gli fagrifichino si crudelmente delle "vittime umane? Animo, Soldati, animo: ora è tempo di pa. " lefare, che fiamo Spagnuoli, e che abbiamo avuto per " eredità dai noftri antenati il zelo ardente per la noftra fan" ta Religione. Spezziamo gl' Idoli, e tolghiamo dalla vifta " di quefti infedeli quefto reo fomento della loro fuperfi" zione. Se l'ottenghiamo faremo al noftro Dio il più gran" de fervizio, che poffiamo. Se muojamo nell' imprefa, E"gli ci ricompenfarà con una eterna gloria il facrifizio, che "gii faremo della nottra vita."

Il Cempoallefe, il quale nel fembiante di Cortès, e nei movimenti de' Soldati fcorgeva chiaramente il loro intento, fece cenno alla fua gente di apparecchiarfi alla difefa dei loro Dei. Cominciavano già gli Spagnuoli a falir per la fcala del Tempio, allorchè i Ceinpoallefi sbalorditi,e fdegnati, gridarono, che fi guardaffero di fare quell' attentato, fe non volevano, che fubito piombaffe fopra di loro tut: ta la collera degli Dei. Non effendo Cortès capace d'impaurirfi colle loro minacce, lor rifpofe, che gia fpeffe volte gli avea ammoniti di difmettere quella infame fuperftizione: che poichè non avevano mai voluto prendere il fuo configlio tanto a loro vantaggiofo, neppure egli voleva avergli più per amici: che fe $i$ medefimi Totonachi non fi rifolvevano a tor via quegli abbominevoli fimulacri, egli colla fua gente gli fpezzerebbe; e che fi guardaffero bene di far qualche oftilità contro agli Spagnuoli; perchè incontanente fi fcaglierebbero contro loro con tal impeto, che non lafcierebbero vivo nè anche un Totonaca. A cotali minacce ne aggiunfe Donna Marina un' altra più efficace, cioè che fe volevano opporfi all' intento di quegli ftranieri, in ve: ce d'allearfi co' Totonachi contro a' Mefficani, s'alleerebbono co'Mefficani contro a' Totonachi, ed allora farebbe inevitabile la loro rovina. Quefta ragione diftolfe il Cempoal: 
lefe dal primo impeto del fuo zelo", è 'prevalendo nel fuo animo il timor dell' armi Mefficane a quello de' fuoi Dai, Lib. VIII, diffe a Corrès, che faceffe pur ciò, che gli piaceffe; poichè a loro non baftava l'animo di porre facrilegamente la mano ne'loro Dei. Appena ebbero un tal permeffo gli Spagnuoli, che cinquanta de'loro Soldati montando frettolofamente in ful tempio, levarono dagli altari gl' Idoli, e gli getsarono giù per la fcala. I Totonachi frattanto dirottamente piangendo, e coprendofi gli occhj per non vedere sì gran facrilegio, pregavano con voce dolente $i$ loro Dei di non voler gaftigar nella loro Nazione la temerità di quegli ftranieri; poichè non potevano impedirla fenza facrificarfi al furor de Mefficani. Nondimeno alcuni o men codardi, o più gelofi dell' onor de' loro Numi, fi difponevano a prender vendetta degli Spagnuoli, e farebbono infollibilmente venuti alle mani, fe gli Spagnuoli non aveffero prefo il Signor di Cem. poalla, e cinque principali Sacerdoti, e minacciando di dar loro incontanente la morte, non gli aveffero coftretti a trattener l'impeto de' loro Cittadini .

Dopo un' azione si ardita, nella quale non ebbe veruna parte la prudenza, comandò Cortès a' Sacerdoti di levargli dinanzi, e di gettar nel fuoco i frammenti degl' ldoli. Fu prontamente ubbidito,e pieno allora di giubilo, come fe nello Spezzar gl' Idoli aveffe tolto affatto l'idolatria, e cacciato da que' Popoli la luperftizione, diffe al Signor di Cempoalla, che accettava oramai volentieri le otto vergini, che gli offeriva: che d'allora innanzi conterebbe i Totonachi tra i fuoi amici e fratelli, e che in tutti i loro bifogni gli aju. terebbe contro a' loro nemici; che poichè non dovrebbono più adorare quelle deteftabili immagini del Demonio lor nemico, voleva collocare in quel medefimo tempio una immagine della Madre del vero Dio, affinchè 12 riveriffero, ed imploraffero la fua prottezione in tutti i loro bifogni. Indi fi diftefe in un lungo ragionamento fulla fantità della Religione Criftiana, e terminato che fu, ordinò a' muratori Cenzpoallefi di levar dalle pareti del tempio quelle fchifore macStoria del Meffico Tom.III. 
chie di fangue umano, ch' eff vi confervavano come "trofei Lis.VIII.della loro inumana Religione, di pulirle, e d'imbiancarle curiofamente. Fece fare un altare alla ufanza de' Criftiani, e vi collocò la immagine di Maria Santiffima. Commife alla cura di quattro Sacerdoti Cempoallefi quefto nuovo Santuario, purchè andaffero fempre puliti, e veftiti di bianco in vece di quell' abito negro, e malinconico, che portavano per cagione del loro miniftero. Acciocchè poi non mancaffero mai de' lumi d'avanti a quella facra immagine, infegnò loro l'ufo della cera, che l'api lavoravano nelle loro montagne; ed affinchè nel tempo della fua affenza non vi riponeffero gl'Idoli, o in qualche altra maniera profanaffern quel Santuario, vi lafciò uno de' fuoi foldati chiamato Giovanni Torres, che ftante la fua età era poco utile per la guerra, il quale fece a Dio il facrifizio di reftare tra quegl' infedeli per promuover il fuo culto. Le otto Vergini, dappoichè furono fufficientemente inftruite, ricevettero il fanto battefimo, prendendo il nome di Donna Caterina la nipote del Signor di Cempoalla, e quello di Donna Francefca la figlia di Cuexco uno de' principali Signori di quella Nazione.

$\mathrm{Da}$ Cempoalla ritornò Cortès alla nuova Colonia della Veracroce, dov' ebbe la confolazione di rinforzar il fuo piccolo efercito con altri due Capitani, e dieci Soldati, che quivi approdarono da Cuba : a' quali 's' aggiunfero indi a poco altri fei- uomini, che furono per inganno prefi da un vafcello della Giamaica.'

9. 19.

Prima d' intraprendere il viaggio a Meffico, volle Cortès

Lettere dar contezza al fuo Soviano di tutto cid, che fin'allora era dès,e del- avvenuto, ed acciocchè lé fue nuove foffero più gradite manl' armata dò infieme tutto $l$ 'oro che aveano ammaffato, cedendo la al Re fua parte per fuggéttione del medefimo Generale ciafcuno deco. gli Uffiziali, e de' Soldati dell arma ta. Cortès in quefta lettera preveniva il $\operatorname{Re}$ contro i tentativi del Governatore di Cuba. Si fcriffero pure altre due lettere al $\mathrm{Re}$, una fottofcritta da' Magiftrati della nuova Colonia, ed un'altra $\mathrm{da}^{3}$ principali Uffiziali dell' armata, nelle quali il pregavano di voler 
voler gradire ciò che aveano fatto, e di confermare le cariche di Capitano Generale, e di primo Giudice, conferite giăLis.VIIL. per i loro fuffragj a Cortès, la cui perfona raccomandavano co' più magniffici elogj. Quefte lettere infieme col prefente dell' oro furono mandate in Ifpagna per li due Capitani Alonfo Hernandez di Portocarrero, e Francefco di Montejo, i quali fi fecero alla vela addi I 6 . Luglio dell' anno 15 I 9 .

Appena erano partiti i fuddetti Procuratori, che Cortès, il quale andava fempre rivolgendo nella mente de' difegni ${ }^{5.16 .}$ Azione 6. 16.1 grandi, efegui un'imprefa, che da per fe fola bafterebbe a famora di far conofcere la fua magnanimità, e ad immortalare il fuo Cortès. nome. Per togliere a'fuoi Soldati ogni ricorfo, e con effo ancora ogni fperanza di ritornare a Cuba, e per rinforzare la fua piccola armata con tutti i marinai, dopo d' aver gaftigato coll'ultimo fupplizio due foldati, che macchinavano tradimento e fuga in uno de'vafcelli, e con minori pene corpo. rali altri tre complici, induffe con ragioni e preghiere alcuni de'fuoi confidenti, e qualcuno de' Piloti di cui più fi fidava, a bucar di nafcofto uno o due de' vafcelli, a perfuadere a tutti, che s'erano affondati, perch' erano intarlati dalla broma, ed a rapprefentar a lui, che gli altri non erano più atti al fervizio per la fteffa cagione, effendo ftati tre mefí fermi nel porto.Si prevalfe Cortès di sì fatto inganno, perchè non congiuraffe contra lui la fua gente, trovardofi ridotta alla dura neceffita di vincere, o di morire. Tutto fi fece, 'com' egli lo avea ordinato, e col confenfo di tutta l' armata, dopo d'averne cavate le vele, $i$ cordaggj, i ferramenti, e turto ciò che poteva effere di qualche ufo. "Così per ") uno sforzo di magnanimità, dice il Robertfon, a cui nien: " te è paragonabile nella thoria, cinquecento uomini s' accor. " darono di lor volontà di rinferrarfi in : un paefe nemico, ") pieno di potenti e fconofciute Nazioni con ogni ftrada " preclufa allo fcampo, rimanendo col folo rifugio del loro "valore, e della perfeveranza. "Io non dubito, che fe Cortès non aveffe prefo quel configlin, farebbe ftata affatto impoffibile l'ardita imprefa, che meditava; poichè i foldati E 2 alla 


\section{6}

alla vilta de'grandi oftacoli, che ad ogni paffo incontravane LiB.VIII.avrebbono fchivato il pericolo colla fuga, ed il medefimo Generale farebbe fato coftretto a feguirli.

Libero dunque da quefta follecitudine, ratificata l'alle-

5. 17.0 anza co' Totonachi, e dati gli ordini convenevoli per l'a. degli Spa-vanzamento e la ficurtà della nuova Colonia, pensò a fare gnuoli fin'il fuo viaggio a Meffico. Lafciò nella Veracroce cinquanta al paefe womini fotto il comando del Capitano Giovanni d' Efcalante, fcallefí . uno de' migliori Uffiziali dell' armata, incaricd i Cempoallefi di dare ajuto agli Spagnuoli per compiere la fabbrica della fortezza, e di provvederli delle vettovaglie, che bifognaffero, ed egli fi pole in cammino addi 16 . Agofto con quattrocento quindici pedoni Spagnuoli, con fedici cavalli, con dugento Tlamama, of fia uomini da foma pel trafporto delle bagaglie, e dell' artiglierìa, e con alcúne truppe Totonache, nelle quali v' erano quaranta Nobili, condotti da Cortès, e come aufiliarj per la guerra, e come oftaggi di quella Nazio: ne; i più riguardevoli de' quali erano tre appellati, fecondo che dicono gli ftorici, Teuch, Mamexi, e Tamalli:

Incamminoffe per Xalapan, e Texotla, e dopo aver trad verfato con fomma fatica alcune montagne deferte, e di aria alquanto rigida, arrivò a Xocotla $\left(^{*}\right)$ Città confiderabile, e di belli edifizj, tra i quali s' innalzavano tredici tempj, ed il palazzo del Signore, il qual' era fabbricato di pietra e calcina, e fornito d'un gran numero di buone fale e di camere, la più compita fabbrica, che aveano veduta fin' allora nel nuovo Mono do. Aveva il $\operatorname{Re}$ di Meffico in quefto luogo, e ne' cafali da effo dipendenti venti mila vaffalli, e cinque mila Mefficani di guernigione. Olintetl ( quefto era il nome del Signor di Xocotla ) venne ad incontrar gli Spagnuoli, e gli alloggio co. modamente nella fua Gittà; ma nel provvedergli di vettovaglie fa moftrò da principio alquanto fcarfo, finattantochè per

la in.

(*) Bernal Diaz, e Solis appellano quefta Citta Zocotlan, cid che potrebbe cagionar qualch'errore, mentre farebbe facile il confondere quelta Città con quella di Zacatlan, fituata nella diftanza di trenta miglia da Tlafcalola verfo Tramontana 
la informazione de' Totonachi fi formò un' idea più vantaggiofa della loro bravura, e della forza delle loro armi, e de'loroLis.VIII, cavalli. Nell' abboccamento, ch' ebbe col Generale Spagnuolo, l' uno e l'altro vantarono a gara la grandezza, e la poffanza de' loro rípettivi Sovrani. Cortès efigeva inconfideratamente da lui, che tofto deffe ubbidienza al Re Cattolico, e con qualche quantità d'oro rendeffe omaggio alla fua fovranità. Ho ben dell' oro, rifpofe Olintetl, ma non voglio darlo fenza efpreffo ordine del mio Re. Io fra poco farò, diffe Cortès, che vi ordini di dar l'oro, $e$ quanto avete. $S$ ' egli mel comandera, foggiunfe Olintetl, non che l'oro, e tutta la mia roba, ma darò ancor la mia perfona. Ma ciò che non potè ottener Cortès colle fue minacce da quel Signore, l'ebbe per mera liberalità da altri due perfonaggi ragguardevoli di quella valle, i quali portandofi a vifitarlo a Xocotla, gli prefentarono alcune collane d'oro, e fette, ovvero otto fchiave. Trovoffe quì perpleffo Cortès fulla ftrada che doveva prendere per andare a Meffico. Il Signor di Xocotla, ed i Comandanti del prefidio mefficano gli fuggerivano, che s'incamminaffe per Cholulla; ma egli ftimò più ficuro il configlio, che gli diedero i Totonachi d'avviarfi per Tlafcalla: ed in fatti farebbe perito in Cholulla con tutta la fua armata, fe vi foffe andato a dirittura, ficcome fi vedrà da ciò, che appreffo diremo. Per ottenere poi da'. Tlafcallefi il permeffo di paffar pel loro paefe, mandò al Senato quattro meffaggieri di quegli fteffi Cempoallefi, che feco conduceva; ma quefti, come fra poco vedremo, non fecero l' ambalciata a nome degli Spagnuoli, ma a nome de'Totonachi, o perchè cosł foffe ordinato dal Generale Spagnuolo, o perchè a loro medefimi ciò pareffe più conveniente .

Da Xocotla fi portò l' efercito Spaguuoio a Iztacmaxt io tlan, la cui popolazione fi ftendeva per dieci o dodici mi. glia in due file non interrotte di cafe fabbricate full' una, e full' altra riva d' un piccol fiume, che fcorre pel mezzo di quella lunga, e ftretta valle; ma la propria cittia d' Iztac. maxtitlan fornita di buoni edifizi, ed abitata da fei mila 2ai: 
anime in circa; occupava la cima d' un alto e dirupato monL18.VIII.te, il cui Signore fu uno di que' due perfonaggi, che vifitarono e regalarono Cortès in X ocotla. Alla naturale afprezza del luogo erano ftate aggiunte dall' arte buone muraglie coi loro barbacani, e foffi; $\left({ }^{*}\right)$ poichè a cagione d' effer quella piazza frontiera de' Tlafcallefi, era più efpofta alle loro invafioni. Quivi furono affai ben' accolti, e regalati gli Spagnuoli.

5. 18.

Frattanto ventilavafi nel Senato di Tlafcalia la loro preAltera- tenfione. Tutta quella gran Città s' era alterata colle nuove Tiane de' di sì fatti Atranieri, e maffimamente col ragguaglio, che vi Tlafcal- de- diedero $i$ meffaggieri Cempoallefi del loro afpetto, e della liberazio-loro bravura, della grandezza de'loro vafcelli, dell' agilità e Senato della forza de'loro Cavalli, e dello fpaventevole tuono, e full' affa- della violenza diftruttiva della loro artiglieria. Reggevano se degli allora quella Repubblica Xicotencatl, Signor del quartiere di li. Tizatlan, Maxixcatzin Signor d'Ocotelolco, Generale delle armi della Repubblica, Tlebuexolotzin Signor di Tepeticpae, e Citlalpopocatzin Signor di Quiahuiztlan. I meffaggieri Cem. poallefi $(r)$ furono cortefemente accolti, ed alloggiati nella cafa deftinata per albergo degli Ambafciatori, e poichè ripofarono, e pranzarono, furono introdotti nella fala del fenato, per efporre la loro ambafciata. Quivi dopo d'aver fatto un profondiffimo inchino, e tutte le altre ceremonie folite farfi in tali occafioni, parlarono cosi : " Molto grandi e pro" di Signori, gli Dei vi profperino, e vi accordino delle 2) vittorie fopra i voftri nemici. Il Signor di Cempoalla; $e$ »con

$\left({ }^{*}\right)$ Cortès nella fua feconda lettera a Carlo V. paragona là fortezza d'lztacmaxtitlan colle migliori di Spagna .

(r) Bernal Diaz dice, che i Meffaggieri furono foltanto due, e che tofo che arrivarono a Tlafcalla furono meffi in prigione; ma il medefimo Cortès, che gli mandò, afferma, che furono quattro, e dal contefto del fuo ragguaglio fi fcorge, che Bernal Diaz fu male informato di cio che accadde in Tlafcalla. Il racconto di quefto Scrittore contrario a quello d'altri Storici antichi tanto Spagnuoli, quanto indiani, ha indotti in errore molti Autori, e tra effi il Robertion. 
7) cón effo lui tutta la Nazione Totonaca vi falutano, e vi

"fanno fapere, che dalla parte di Levante fono arrivati nel Lis.Vill.

" noftro paefe fopra grandi barche certi Eroi forti, e fom-

" mamente coraggiofi, coll' ajuto de' quali ci veggiamo ora-

" mai liberi dalla tirannica dominazione del Re di Meffico.

"Efi fi proteltano fudditi d'un poffente Monarca, nel cui

\# nome vogliono vifitarvi, efibendofi di darvi notizia del

" vero Dio, e d'ajutarvi contro il voltro antico e capitale

" nemico. La noitra Nazione per quella ftretta amicizia col-

" la voltra Repubblica, che coftantemente ha coltivata, vi

" configlia di ricevere per amici quefti Eroi, i quali quan.

"tunque pochi, valgono in cambio di molti. "Maxixcatzin lor rifpofe a nome del Senato, che ringraziavano i Signori Totonachi della nuova, e del configlio, e que'bravi Atranieri dell' ajuto, che s' efibivano a dar loro, ma che per deliberare fopra un punto di sì gran rilievo, v'abbifognava del tempo: che frattanto ritornaffero al loro albergo, dove farebbono trattati colla diftinzione, che richiedeva e la loro nafcita, ed il loro carattere. Ritiraronfi pure i Meffag. gieri, ed il Senato entrò in confulta.

Maxixcatzin, il quale era in grande tima preffo tutti e per la fua prudenza, e per la fua benignità, diffe, che non era da ributtarfi il configlio dato, da amici si.fedeli, e sì contrarj al più gran nemico della Repubblica: che quegli ftranieri attefo $i$ contraffegni, che ne davano i Cempoallefi, parevano effere quegli Eroi, che giufta la loro tradizione, doveano arrivare in quel paefe: che i tremuoti, che poco innanzi s'erano fentiti, la Cometa, che allora fi lafciava veder nel Cielo, ed altri parecchj avvenimenti di quegli ultimi anni erano indizj, che foffe già arrivato il tempo del compimento di quella tradizione: che s'effi erano immortali, indarno tenterebbe la Repubblica d'opporfi alla loro entrata." Il noftro rifiuto, aggiunfe cagionar ci potreb. " be danni graviffimi, e pel Re di Meffico farebbe foggetto " di maligno piacere il vedere introdotti per forza in Tlaf" calla, quelli, che la Repubblica non volle di buon grado "accet- 


\section{0}

" accettare : " ch̀ egli perô era di parere di ricévergli amiche:

Lis. VIII.volmente. Quefto fentimento fu afcoltato con applaufo, ma gli contradiffe fubito Xicotencatl, $(S)$ vecchio di grand' autorità per la fua lunga pratica negli affari civili e militaxi. "La noftra legge, diffe, ci ordina d' accogliere i fore"ftieri; non già i nemici, che poffano recar danno allo fta"to. Quefti. tomini, che pretendono entrare nella noftra "Città, più mi fembrano moftri rigettaci dal mare, per"chè non poteva foffrirli nelle fue acque, che Dei venuti " dal Cielo, come fcioccamente s' immaginavano alcuni. "Poffibile che fieno Dei, quelli che cercano con tan. "ta avidità l'oro, ed $\mathbf{i}$ piaceri? $E$ che non dovremmo te") mere da loro in un paefe tanto povero, come il noftro, " dove anche del fale fiamo privi? Fa torto alla bravura della "noftra Nazione, chi la ftima fi facile ad effer vinta da po" chi ftranieri. S' eglino fon mortali, l'armi de' Tlafcallefi il 3) faranno palefe al mondo, e fe fono immortali vi farà del tem. " po per placar con offequj il loro fdegno, e per implorar col " pentimento la loro clemenza. Ributtifi dunque la loro do" manda, e fe mai voleffero entrar per forza, fia colle ar" mi rifpinta la loro temerità. "Quefta contrarietà di fentimenti tra due perfonaggi cotanto autorevoli divife gli animi degli altri Senatori. Quelli, ch' erano portati, pel commer. cio, ed avvezzi ad una vita pacifica, aderirono al parere di Maxixcatzin, e gli uomini militari abbracciarono quello di Xicotencatl. Temiloltecatl, uno de'Senatori $(t)$ fuggerì un temperamento per conciliar ambidue $i$ pareri. Propofe, che fi

man-

( $\int$ Solis afcrive al giovane. Xicotencatl il ragionamento del fuo vecchio Padre; ma noi crediamo dover dar maggior fede agli Storici antichi; che $s$ 'informarono dagli fteffi Tlafcallefi.

( $t$ ) Herrera, e Torquemada dicono, che Temiloltecatl era uno de'quattro Signori di Tlafcalla; ma dalle Memorie di Camargo, e d'altri Tla $\mathrm{f}$ : callefi, e anche dallo teffo Torquemada nel lib. 3. e nel lib. 16. confta, che i quattro Signori erano allora quegli da noi fopra mentóvati. Puó crederfi per falvar la verità di ciò, che dicono Herrera, e Torquemada, che Tlehuexolotzin avelfe ancora il nome di Temiloltecatl, ficcome aveva quello di Tezcacalteuctli; mentre fappiamo, che molti erano con due ed anche con tre nomi appellati. 
mandaffe al Capo di quegli ftranieri una rifpofta cortefe ed anichevole accordandogli il permeffo d'entrarvi : ma nel Lis.VIII. medefimo tempo fi deffe $\mathrm{l}$ ' ordine a. Xicotencatl il giovane, figliuolo del vecchio Xicotencatl d' ufcire colle truppe Otomite della Repubblica a contraftar loro il paffaggio, e provar le loro forze. "Se reftiamo, diffe, vincitori, fara im"mortale la gloria delle noftre armi; fe fiamo vinti, incol" peremo gli Otomiti, e daremo ad intendere, che intraprefero fenza ordin noftro la guerra." (u) Ripieghi po. litici troppo frequenti nel mondo, maffimamente preffo le Nazioni colte; ma non però meno contrari alla buona fede, che li debbono reciprocamente gli uomini. Accettò il Senato il configlio di Temiloltecatl; ma prima di fpedire i meffaggieri colla rifpofta, tiede gli ordini convenevoli a Xicotencatl. Quefti era un giovane intrepido, nemico della pa. ce, e troppo portato per la gloria militare: ond' ebbe cara la commeffione, come quella che gli forniva si bella occafione di moftrar la fua bravura.

Cortès dopo d' aver afpettato otto giorni la rifpofta del Senato, credendo che sì fatta tardanza effetto foffe di quella lentezza, che fuole affettare la maeftà de' potentati, e non dubitando per cid, che g!i dicevano i Cempoallef, di dover effere ben' accolto da' Tlafcallefi, ufci da Iztacmaxtitlan con tutto il fuo efercito, il quale oltrea' Totonachi, e agli Spagnuoli, fi componeva d' un competente numero di truppe Mefficane del prefidio di Xocotla, e marcid in buon ordi. ne, come foleva, fin' alla gran muraglia, che da quella parte feparava gli ftati di Tlafcalla da quelli di Meflico; la defcrizione, e le mifure della quale demmo già nel libro antecedente, là dove raggionammo delle fortificazioni de' Mefficani. Era ftata fabbricata da' Tlafcallefi per difen. Storia del MefJico Tom. Ill. F derfi

(u) Abbiamo gia detto altrove, che moltiflimi Otomiti erano rifuggiti in Tlafcalla per fottrarfi alla dominazione de'Mefficani, e che fervirono afrai bene a quella Repubblica. 
Lis. VIII.fatto de' grandi foffi e delle trincee per la medefima cagione dalla parte di Ponente. L' ufcio della muraglia, che ognora era guardato dalle truppe Otomite, ora quando era più bifogno, trovoffl, non fappiamo perchè, fenza veruna guarnigione: onde !' efercito Spagnuolo entrò - fenza alcun impaccio nelle terre della Repubblica : ciò che altrimenti non avrebbe potuto fare fenza fpargere molto fangue.

Quelto dì, che fu il $3 \mathrm{I}$. Agofto, fi lafciarono vedere alcuni Indiani armati, e volendo raggiugnerli la Cavalleria, che batteva la frada, per avere da loro qualche contezza della rifoluzione del Serato, furono uccifi due cavalli, e feriti altritre, e due uomini:perdita in vero grandepersi poca Cavallerìa. Comparve allora una fchiera, che fi ftimò di quattro mila uomini in circa, contra la quale fi fagliarono gli Spagnuoli, e gli Alleati, e in breve la fconffifero colla morte di cinquanta Otomiti. Indi a poco arrivarono due de' Meffaggieri Cempoallefi con alcuni Tlafcallefi, $(y)$ i quali complimentarono Cortès a nome del Senato, e gli fecero fapere il permeffo, che gli fi accordava, d' andare col fuo efercito a Tlafcalla, incolpando gli Otomiti delle oftilita, che aveano fofferte, ed efibendofi a pagargli $i$ cavalli da loro uccifi. Cortès fece fembiante di crederlo, e proteftò la fua gratitudine verfo il Senato. I Tlafcallefi fi congedarono, e ritirarono dal campo i loro morti per bruciarli. Cortès dal. la fua parte fece feppellire $i$ due cavalli uccifi, perchè la loro vifta non incoraggiffe $i$ nemici per fare nuove oftilità.

(x) Da cid, che differo i Mefficani a Cortès intorno alla muraglia fuddetta, potrebbe qualcuno perfuaderfi, ch' eglino furono quegli, che la fabbricarono; ma non v'è dubbio, che fuda'Tlafiallefi fabbricata.

(y) Bernal Diaz dice, che i primi Meffaggieri Cempoallefi tornarono a Cortès prima che foffe entrato nel paefe di Tlafcalla; ma Cortès afferma fchietramente il contrario. Intorno al ragguaglio de'Meffaggieri Cempoallefi, ch' erano reftati in Tlafcalla, benchè fia ftato creduto da quafi tutti gli forici Spagnuoli, egli è affatto incredibile per le ragioni da noi addotte. Il Dott. Robertion rappporta parecchie congetture per renderlo verifimile; ma indarno. 
Il di feguente marciò l' efercito Spagnuolo infin' alla vicinanza. di due monti, tra $\mathrm{i}$ quali $v$ ' erano alcuni burroni. Lis.VIII. Quivi arrivarono gli altri due meffaggieri Cempoallefi, ch' erano reftati in Tlafcalla, bagnati di fudore e di lagrime, e biafimando la perfidia, e la crudeltà de' Tlafcallefi; perchè non curando il diritto delle Genti, gli aveano malmenati, ed imprigionati, e gli aveanodeftinati al facrifizio, dal quale diceano efferfi liberati fciogliendofi l' uno l'altro. Quefto ragguaglio de' Cempoallefi fu certamente falfo: poichè era affatto impoffibile, non che difficile, che fi liberaffero da per fe le vittime cosi per la Atrettezza della gabbia, dove le tenevano, come per la vigilanza delle Guardie, che le cuftodivano: oltrecchè non v' è memoria, chei Tlafcallefi mancaffero mai al rifpetto dovuto al carattere degli Ambafciatori, e molto meno effendo coftoro d' una Nazione tanto ftrettamente congiunta con loro in amicizia, qual' era la Totonaca. Ciò che pare più verifimile è, che il Senato dappoi che rimandò i primi due meffaggieri, trattenne gli altri due per fpedirgli, allorchè aveffero provato le forze degli Spagnuoli, ed eglino impazienti d' alpettare s' affentarono di nafcolto, e cercarono di fcufare con sì fatti pretelti la loro fuga .

Appena aveano terminato i Cempoailefi il loro ragguaglio, che comparve uno Squadrone Tiafcallefe di mille uomini in circa, i quali tofto ches'avviciparono agli Spagnuoli, cominciarono a tirar contra loro $\int_{d} f_{1}$, dardi, e freccis. Cortès a dopo d' aver loro proteftato d' avanti al Notajo Regio dell' armata per mezzo di tre prigionieri, ch' effi non venivano a far loro verun male, e dopo d'avergli pregati di non trattarli da nemici, vedendo che ciò nulla giovava, diede l'ordine di rifpingerli. I Tlafcallefi s' andarono tirando in dietro, finchè menarono gli Spagnuoli a' burroni, dove non poteffero reggere $\mathrm{i}$ cavalli, e dove gli afpettava un groffo efercito; fu! numero del quale v' è una gran varietà preffo gli Au. tori.(z) Quivi fu una terribile zuffa, nella quale ficredet. F 2

9. I9."

Guerra di Tlafcalla.

(z) Bernal Diaz dice, che l'efercito de' Tlafcallefi era di quaranta mi- 
tero perduti gli Spagnuoli, ma rimitifi nel miglior crdine

Lib.VIII.che poterono, ed incoraggiti dalle efortazioni, e dall' efempio del loro Generale, fi ritraffero da quel frto tanto pericolofo, e venuti nella pianura fecero coll' artiglieria, e co" cavalli una si grande ftrage ne' nemici, che gli coftrinfero a tornarfene in dietro. De' Tlafcallefi moltiffini furono i feriti, e non pochi reftarono morti. Degli Spagnuoli avvegnachè vi foffero quindici pericolofamente feriti, uno folo morì il di feguente. In quefta occafione accadde un famofo duello tra un Capitano Tlafcallefe, ed un Nobile Cempoallefe di quelli, che erano andati meffaggieri a Tlafcalla . Combatterono bravamente un buon pezzo a vilta d' ambidue gli eferciti; ma alla fine prevalle il Cempoallefe, il quale avendo meffo in terra il fuo competitore, gli tagliò la tefta, e la portò in trionfo al Campo. Celebroffi la vittoria con acclamazioni, e colla mufica militare. Il luogo, dove fi diede quefta battaglia, s' appellava Teoatzinco, cioè il luogo dell' acqua divina.

Quella notte campeggiò l' efercito Spagnuolo in un colle, dov' era una torre, diciotto miglia in circa dalla Città capitale di Tlafcalla. Fabbricaronvifi delle baracche per la comodità delle truppe, e fi fecero delle trincee per la loro difefa. Quivi Atette il campo degli Spagnuoli fin' alla pace co' Tlafcallefi .

Cortès per obbligare colle fue oftilità i Tlafcallefi, ad accettar la pace e l' amicizia, che lor offeriva, fortì il di 3. Settembre colla fua Cavallerìa, con cento pedoni Spagnuoli, con quattrocento Cempoallefi, e con trecento Mefficani della guarnigione d' Iztacmaxtitlan, bruciò cinque o fei cafali vicini, e fece quattrocento prigionieri, i quali, dopo d'avergli carezzati, e regalati, rimife in libertà, incaricando i principa-

lä uomini in circa. A Cortes parve, che oltrapaffava i cento mila. Altri storici fcriffero, ch'era di trenta mila. E difficile conofcere ad occhio il sumero d'un grand'efercito, maffimamente allorchè non vi fi offerva l'ordine della Milizia Europea. Io per non efpormi ad errare mi contento di. dire, che l'efercito era groffo. 
li di loro d' andare a offeríre dalla fua parte la pace a' Capi della Nazione . Coftoro fi portarono a dirittura a XicotencatlLis.VIII. il giovane, il quale campeggiava con un groffo efercito fei miglia lontano da quel colle. Queft' orgogliofo Tlafcallefe rifpofe, che fe gli Spagnuoli volevano trattar di pace, andaffero alla Capitale, dove farebbero vittime confacrate $a^{\text {' }}$ loro Dei, e le loro carni deftinare per vivande de' Tlafcallefi, che per quanto a lui toccava, il di feguente verrebbe in perfona a dar loro una rifpofta decifiva. Una tal rifoluzione fatta fapere agli Spagnuoli per gli fteffi meffaggieri gli mife in si gran cofternazione, che quella notte fi prepararono per la morte colla confeffione facramentale, fenza però tralafciare le difpofizioni convenevoli per la loro difefa.

Il di vegnente, 5. Settembre, comparve l' efercito Tlafcallefe non meno terribile alla vifta per la fua innumerabile moltitudine, $(\dot{A})$ che bello per la vaga varietà de' loro pennacchj, e d'altri ornamenti militari : Era divifo in cinque fchiere di dieci mila uomini l' una : ciafcuna portava il fuo proprio ftendardo, e nella retroguardia, giufta la ufanza di quella Nazione, veniva lo ftendardo comune della Repubblica, il quale era, ficcome abbiamo detto altrove, un' aquila d' oro colle ali diftefe. L' arrogante Xicotencatl per dare ad intendere il poco conto, che faceva degli Spagnuoli, e che non voleva pigliarli per fame, ma vincerli colle armi, man. dò loro un rinfrefco di trecento gallinacci, e dugento paniere di tamalli, efortandoli a riftorar le loro forze per la battaglia . Indi a poco diftaccò due mila uomini coraggiofi, acciocchè affa.

(A) Cortès dice, che l' efercito de'Tlafcallefi era di più di 149.000 uomini. Bernal. Diaz afferma, come una cola accertata, e da lui ben faputa, ch' effo coftava di foli 50.000 uomini, cioè 10.000 di Maxixcatzin, 10.000 di Xicotencatl, 10.000 di Tlehuexolotzin, 10.000 di Chichimecha" teuctli, uno de'più riglaardevoli Signori di quella Repubblica, e 10.000 di Tecranecatl, Signor di Topoianco, città confiderabile della medefima Repubblica, benchè quefti nomi fiano Atati troppo alterati da quefto Autore. Quefto numero pare piu verifimile; quello che filegge nella letrera di Cor tès fi può credere sbaglio del primo ftampatore della medefima. 
affaliffero il campo degli Spagnuoli . Quefto affalto fu sìvio. L.B.VIII.lento, che forzando le trincee entrarono nel campo,e com. batterono a corpo a corpo cogli Spagnuoli. Potrebbono effere ftati vincitori quefta volta i Tlafcallefi non tanto per cagione del numero affai fuperiore delle loro truppe, quanto per la loro bravura e la qualita delle loro armi, ch' erano picche, lancie, fpade, e dardi di due, e di tre punte, fe la difcordia inforta tra loro non aveffe agevolata a' loro nemici la vittoria. Il figliuolo di Chichimeca-teuctli, il quale era comandante del corpo di truppe di fuo Padre, $\left({ }^{*}\right)$ effendo ftato ingiuriato con parole dall' arrogante Xicotencatl, ebbe un sì grande fdegno, che lo sfidò per un duello, che decideffe e del loro coraggio, e della loro forte; e non po. tendo da lui ottenere quefta foddisfazione, per prender qualche vendetta, ritird dal campo le truppe, ch' erano fotto $\mathbf{i}$ fuoi ordini, ed induffe allo fteffo configlio quelle di Tlehue. xolotzin. Malgrado un sì grande fcemamento dell' efercito, la battaglia fu affai oftinata e fanguinofa. Gli Spagnuolidopo d'a vere coraggiofamente rifpinto le truppe, che aveano affalito il loro campo, marciarono in ordine di battaglia contro il corpo dell' armata Tlafcallefe. La ftrage, che faceva nella moltitudine affollata l'artiglierìa, non baftava per fare a' Tlafcallefi voltar le fpalle, ne per impedir, che empiffero prontamente que' vuoti, che lafciavano i morti; anzi colla loro fermezza, ed intrepidezza aveano meffi in ifcompiglio gli Spagnuoli a difpetto de' clamori, e de' rimproveri di Cortès, e de' fuoi Capitani. Finalmente dopo di quattro ore di combattimento tornarono vittoriofi gli Spagnuoli al loro campo, benchè non ceffarono però d'affalirgli fpeffe volte i Tlafcallefi in quello fteffo giorno. Degli Spagnuoli mancò un fol uomo, e furono feriti feffanta, e turti i Cavalli. De' Tlafcallefi furono moltiffimi uccifi ; ma nè pure un cadave-

ro

${ }^{*}$ ) Solis dice, che Chichimeca-teuctli era Alleato de'Tlafcallefi, ma quefto è un manifefto errore, mentre fappiamo da tutti gli ftorici, ch'egli era 'Tlafcallefe, e uno de' più riguarderoli Signori di quella Repubblica. 
ro fu dagli Spagnuoli veduto per la fomma diligenza e pron tezza, con cui gli ritiravano dal campo di battaglia .

Difguftato Xicotencatl dell' efito infelice di quefta fpedizione fece confultar gl' Indovini di Tlafcalla, e coltoro rifpofero, che quegli Atranieri, come figlj ch' erano del Sole, erano inviacibili durante il di; ma tofto che arrivava la notte infieme col calor vitale di quel Pianeta mancavano pure ad efli le forze per difenderfi. In confeguenza di sì fatto oracolo rifolvette quel Generale di dare in tempo di notte un affalıo al campo degli Spagnuoli. Frattanto Cortès ufci di bel nuovo per far delle oftilità ne' vicini Villaggi, de' quali ne brixiò dieci, e tra effi uno di tre mila fuochi, e le ne tornò con alcuni prigionieri.

Xicorencatl, perchè non falliffe il colpo, che meditava contro gli Spagnuoli, volle prima informarfi della difpofizione e delle forze del loro campo. Mandò però cinquanta uomini a Cortès con un regalo accompagnato da correfi efpreffroni di benevolenza, incaricando coftoro d'offervar tutto; ma non poterono fare con tanta diffimulazione le loro offervazioni, che non fe ne accorgeffe Teuch, uno de'tre principali Cempoallefi, ed avvertiffe fubito Cortès. Quefto Gene. rale avendo chiamate in difparte alcune delle Spie, le co. ftrinfe con minacce a dichiarare, che Xicotencatl s' apparecchiava per dar la notte vegnente l'affalto, e ch'eglino erano ftati mandati a pofta per offervar, per qual parte del campo gli farebbe più agevole l'entrata. Cortès fentita la loro confeffione, fece tagliar le mani (B) a tutti e cinquanta, e gli rimandò a Xicotencatl, incaricandoli di fargli fapere, che o veniffe di giorno al fuo campo, o di notte, 0 gnora gli farebbe conofcere chi erano gli Spagnuoli, e parendogli quefte circoftanze affai opportune per la battaglia, prima che $i$ nemici aveffero forniti tutti i loro preparativi per l'affalto, ufci ful far della notte, con un buon numero di

(B) Alcuni Storici Spagnuoli dicono, che alle fpie Tlafcallefi furono foltanto tagliatele dita; ma lo fteffo Cortès dice, che fece loro tagliar le mani. 
di truppe, e co'fuoi cavalli, a' quali fece metter de' fona: Lis.VIII glj ne'pettorali, e fe $\mathfrak{n}^{\prime}$ andd ad incontrare $i$ nemici, che appunto marciavano verfo il campo Spagnuolo. La vifta del gaftigo efeguito nelle Spie, ed il rumore de' fonagli nel lilenzio e nel bujo della notte fecero tanta paura a' Tlafcalle$f_{i}$, che fubito fi fcompigliarono, e fuggiroro per diverfe ftrade, ed il medefimo Xicotencatl fe ne tornò fvergognato a Tlafcalla. Prefe quindi occafione Maxixcatzin per inculcare il fuo primo fentimento, aggiungendo ora alle ragioni già addotte da lui la fperienza funefta di tante fpedizioni infelicemente riufcite: ciò che moffe tutti gli animi del Senato alla pace.

5. 20.

Mentre che fi ventilava quefto affare in Tlafcalla, in

Nuove Mefico fi confultava fopra ciò, che dovea farfi con quegli ambe pre- Aranieri. Motezuma confapevole delle vittorie degli Spa. fenti di gnuoli, e temendo ormai la loro confederazione co' TlaMotezu- Ccallefí; chiamò il Re di Tezcuco fuo nipote, il Principe ma a Cor- Cuitlahuatzin, ed altri fuoi Configlieri, efpofe loro lo tato delle cofe, palesd i fuoi timori, e dimandò a loro configlio. Il Re di Tezcuco fi mantenne nel fuo primo parere; cioè che quefti ftranieri foffero magnificamente trattati in tutti i luoghi, per dove paffaffero: che foffero benignamente ammeffi nella Corte, e foffero afcoltate le loro propofizioni, come s' afcolterebbero quelle di qualfifia vaffallo, moftrando ognora il Re la fua fuperiorità, e guardando quel decoro, che convienfi alla maefta del trono: che fe effi macchinaffero mai qualche cofa contro alla perfona del $\mathrm{Re}, \mathrm{o}$ contro allo ftato, s'adoperaffero contra loro la forza, e la feverità. Il Principe Cuitlahuatzin ridiffe ancora ciò, che aveva detto nell' altra conferenza, cioè, che non era fpediente l'accogliere nella Corte quegli ftranieri : che fi mandaffe al loro Capo un buon regalo, glifi addimandaffe che cofa gli piaceva di quel paefe pel gran Signore, a nome del quale veniva, e glifi offeriffe l'amicizia e la buona corrifpondenza de'Mefficani; ma infieme gli li faceffero delle nuove iftan. $z e$, acciocchè fe ne tornaffe alla fua patria. Tra gli altri 
Configlieri alcuni abbraeciarono il parere del Re di Tezcuco, ed altri quello del Signor d' Iztapalapan, al quale aderílis.Vill. ancora Motezuma. Quefto fventurato $\mathrm{Re}$ non trovava da per tutto, fe non oggetti, e motivi, onde temere. 'L' im. minente confederazione de' Tlafcallefi cogli Spagnuoli lo te. neva in una fomma inquietudine. Da un altro canto inquietavalo l'alleanza di Cortès col Principe Ixtlilxochitl fuo nipote e nemico giurato, il quale dacchè congiurò contro il Re di Tezcuco fuo fratello, non avea mai lafciate l'armi, ed allora appunto fi trovava in Otompan alla tefta $d$ ' un efercito formidabile. Accref́ceva quefti timori la ribellione di parecchie Provincie, che aveano feguito l'efempio de Totonachi .

Mandò dunque fei Ambafciatori a Cortès con mille ve: fit curiofe di bambagia, ed una buona quantita d'oro, e di vaghe penne, e gl' incaricò di eongratularfi con lui delle fue vittorie, d'offerirgli altri più grandi regali, e di diffua. derlo del viaggio a Meffico rapprefentandogli la malagevolezza della ftrada ed altri oftacoli difficili da fuperarfi. Par. tirono prontamente quefti Ambafciatori con un feguito di più di dugento uomini, ed arrivati al campo degli Spagnuo. li efeguirono appunto quanto era ftato loro ordinato. Cortès gli ricevè con tutto l'onore al loro carattere dovuto, e fi protefto fommamente obbligato alla bontà di sì gran Mo. narca; ma trattenne a bella pofta gli Ambafciatori, fperando che frattanto occorreffe qualche zuffa co'Tlafcallefi, la quale accreditaffe d'avanti a' Mefficani la bravura delle fue truppe, e la fuperiorità delle armi Europee; o pure fatta la pace colla Repubblica foffero teftimonj della feverità, colla quale voleva rimproverare a'Tlafcallefi la loro oftinazione. Non iftette guari, che gli capitò l'occafione, che tanto defiderava. Tre fquadroni nemici piombarono ful campo degli Spagnuoli con urii fpaventevoli, e con una tempefta di frecce, e di dardi.Cortès, con tuttochè aveffe tol to quel. lo fteffo giorno un purgante, montò a cavallo, ed ufci intrepidamente contra i Tlafcallef, i quali fenza troppa fatiStoria del Meffico Tomo III. 
ca furono fconfitti a vifta degli Ambafciatori Mefficani.

Lis.VIII. Perfuafi alla fine i partigiani del vecchio Xicotencatl;

5. 28. che non era fpediente alla Repubblica la guerra cogli SpaPace, e gnuoli ; e temendo inoltre, che quefti 's alleaffero $\mathrm{co}^{\circ}$ confede- Mefficani, rifolvettero di comun confenfo di far la pace, de'Tla- e prefero per mezzano d'effa colui medefimo, ch' era ftato fcallefi Generale in quella guerra. Xicotencatl, benchè da principio coglispa-rifiutaffe di farlo per lo roffore che avea dell' efito infaufto gnuoli. della guerra, fu alla fine obbligato a incaricarfi della commeffione. Portoffi al campo degli Spagnuoli con una nobile e numerofa comitiva, falutò Cortès a nome di tutta la Repubblica, fi fcusò delle già fatte oftilità col pretefto d'averlo creduto Alleato di Motezuma, tanto a cagione de' fuperbi prefenti mandatigli da Meffico, quanto per la gran truppa mefficana, che conduceva feco, promife una ferma pace ed una eterna alleanza tra gli Spagnuoli ed i Tlafcallefi, e gli prefentò un foco d'oro, ed alcune balle di vefti di bam. bagia, fcufando la fcarfezza dell' offerta colla povertà del loro paefe cagionata dalla perpetua guerra co' Mefficani, i quali impedivano loro il counmercio con altre Provincie. Cortès non tralafciò veruna dimoftrazione di rifpetto verfo Xicotencatl: fece fembiante d'effere foddisfatto delle fue fcufe; ma richiefe, che la pace foffe fincera e durevole; poichè fe giammai la rompevano, prenderebbe una sì terribile vendetta di loro, che foffe per fervir d'efempio agli altri Popoli.

Fatta la pace, e congedato Xicotencatl, fece Cortès celebrare il Santo Sacrifizio della Meffa in rendimento di grazie ali' Altiffimo. Ognuno porrà immaginare il difpiacere, ch' ebbero gli Ambafciatori Mefficani nel veder conchiufo sì fatto accomodamento. Lagnaronfi con Corrès, e gli rinfacciarono la fua troppa facilità nel dar credenza alle promeffe d'uomini si perfidi, come i Tlafcallefi. Dicevangli, che quelle apparenze di pace non ad altro fine 's' indirizzavano, che a quello d'infpirargli della confidenza per tirarlo alla loro Capitale, e far quivi fenza loro pericolo ciò, che non 
aveano potuto ottenere colle armi in campagna: che para. gonaffe la condotta di quel Senato con quella della CorreLibivill, di Meffico. I Tlafcallefi, dopo d'aver accordato loro con moftre di pace il permeffo d'entrare nel loro paefe, non ceffarono di fare ad eff la guerra, finchè non riconobbero affatto inutili tutti i loro sforzi. Da' Mefficani pel contrario yon aveano fofferta niuna oftilità, anzi aveano avuto la più diftinta accoglienza, ed i più grandi offequj, e fervizj in tutti $i$ luoghi de'loro domini, dov' erano capitati, e dal loro Sovrano, le più rilevanti prove di benevolenza, e d'amicizia. Cortès rifpofe, che non credette mai di far verun torto con quell' accomodamento alla Corte di Meffico, a cui fi proteftava fommamente obbligato; poichè egli voleva aver pace con tutti: che del refto e non temeva i Tlafcallef in cafo che effer voleffero fuoi nemici: che per lui, e per gli altri Spagnuoli tanto valeva l'efter affaliti nella Citrà, quanto nella campagna; tanto di di, quanto di notte, mentre fa. pevano vincere in qualunque luogo e tempo; che anzi per quel medefimo, che coloro dicevano de' Tlafcallefi, egli voleva portarfi alla loro Città per aver occafione di far quivi una firepitofa vendetta della loro perfidia.

Troppo lontani erano i Tlafcallefi da quella dislealtà j che loro imputavario i Mefficani; imperciocchè infin da quel momento, in cui decretò la pace il Senato, furono fempre mai i più fedeli alleati degli Spagnuoli, ficcome s'andrà vedendo nel decorfo di quefta ftoria. Bramava il Senato d'avere a Tlafcalla Cortès con tutte le fue truppe per vieppiù Atrignere la fcambievole amicizia, e per trattare feriamente delia confederazione contro i Meficani, ed aveano già per mezzo de' loro meffuggieri invitato quel Generale a prendere alloggio nelle loro cafe; proteftando il difpiacere, che avevano nel vedere si illuftri amici della Repubblica in tan. ta fcomodità .

Non fu l'alleanzz de' Tlafcallefi l'unico frutto, cho raccolfero gli Spagnuoli dalle loro vittorie. Nel medefimo campo, nel quale avea afcoltato l'Ambafciator di TlafcalG 2 la, 
la, ricevè Cortès indi a poco quei della Repubblica di Hue: Lis.VIII.xotzinco, e quelli ancora del Principe Ixtlilxochitl. Gli Huexotzinchi, ch' erano ftati già vaffalli della Corona di Meff5. 22. co, e nemici de'Tlafcallefi, s' erano fottratti dalla dominafciata del zione de' Mefficani, e confederatifi co'Tlafcallefi lor vicini, Principe ed ora feguirono il loro efempio nella confederazione cogli chitl, e Spagnuoli. Il Principe Ixtlilxochitl mando degli Ambafciaconfede- tori a Cortès per congratularfi con effo lui delle vittorie ride' Huexotzin- per Teotlalpan, dove voleva unire le fue forze con quelle chi. degli Spagnuoli per far la guerra al Re di Meffico. Cortès poichè fi fu abbaftanza informato della qualità, delle pretenfioni, e delle forze di quel Principe, accettò volencieri, la fua alleanza, e s'efibi a porlo nel trono d'Acolhuacan.

Allo ftefío tempo ritornò dalla Corte l' Ambafciator Mefficano, che fi afpettaya, con un prefente di giojel: li d'oro, che importava mille e cinquecento zecchini, e di dugento preziofi abiti di penna, e con nuove inftanze di Motezuma per diftornare quel Generale dal viaggio a Meffico, e dall' amicizia co' Tlafcallefi: sforzi inutili della pufillanimità di quel Monarca, mentre lo fpendere tant' oro nel far de' prefenti a quegli ftranieri altro in vero non era, che comperar più caro le catene, che ftrigner doveano fra poco. Ia. fua libertà.

5. 23. Sei giorni erano fcorfi dopo la pace fatta co' Tlafcallefi; Sottomef-allorchè i quattro Signori di quella Repubblica per obbligar fione del-gli Spagnuoli ad andare a Tlafcalla fi fecero trafportar in blica di fedia portatile al loro campo con un grand' accompagnaTlafcalla mento. Le dimofrazioni di giubilo, e di rifpetto furono al Re ftraordinarie e dall' una, e dall' altra parte. Quell' illuftre Cattoli- Senato non contento di ratificar l'alleanza, diede fpontanea-
co. mente obbedienza al Re Cattolico: ciò che fu tanto più accetto agli Spagnuoli, quanto era ftata più cara a la libertà, che goduta aveano da tempo immemorabile. Lagnaronfi amorevolmente della diffidenza di Cortès, e colle loro preghiere l'induffero a rifolvere pel di vegnente la fua partenza a Tlafcalla.

Man- 
Mancavano oramai cinquanta cínque Spagnuoli di quel numero, che s' era arrolato in Cuba, ed i rimafti erano perLis.VIII. lo più feriti, o malconci, e ciò tale e tanto sbigottimento cagionato avea ne'Soldati, che non folo fparlavano privatamente del lor Generale; ma inoltre lo fcongiurarono ditornarfene alla Veracroce; ma Cortès gli rincorò, e con efficaci ragioni d'onore, e col fuo proprio efempio di coraggio e di coftanza ne'pericoli, e nelle fatiche gli riaccefe a quell' ardita imprefa. Finalmente prefero di bel nuovo coraggio colla fperanza di felice riufcita, fondata nelle confederazio. ni allora fatte.

Gli Ambafciatori Mefficani, che ancor teneva feco Cor- 5. 24: tès, ricufarono d'accompagnarlo a Tlafcalla ; ma egli li per- Entrata fuafe d'andar feco promettendo loro, che a lato di lui fa. Spagnuo. rebbero affatto ficuri. Superato quefto impaccio marciò l'e- li in Tiafercito ben' ordinato, ed apparecchiato per qualfiffia novità. fcalla. Nelle Città di Tecompantzinco, e d'Atlihuetzian, fu accolto con tutta la magnificenza poffibile, benchè non paragosabile con quella della Capitale, da cui ufcirono ad incontrar gli Spagnuoli i quattro Signori della Repubblica con una bella e numerofa danza della Nobiltà, e con sì gran folla di Popolo, che da qualcuno fu ftimata di più di cento mila perfone: numero non inverofimile attefo la popolazione di Tlafcalla, e la forprendente novità di quegli uomini Atraordinarj, che rifvegliava la curiofità di tutti que Popoli. In tutte le ftrade della Città v'erano formati, gìfta l'ufanza di quelle Nazioni, archi di rami e di fori, e da per tutto rifonava una mufica confufa di ftrumenti $e$ d'acclamazioni con sì gran dimoftrazioni di giubilo, che più pareva celebrarfi il trionfo della Repubblica, che quello de' fuoi nemici. Quefto di tanto memorabile negli annali di Tlafcalla fu il 23 . Settembre 1519.

Era allora quefta Città una delle più ragguardevoli del paefe d'Anahuac. Cortès nelle fue lettere a Carlo V. afferma, che nella grandezza, nella popolazione, nella qualità degli edifizj, e nell' abbondanza delle cofe neceffarie alla 
vita, deffa fuperava Gragata, allorchè fu tolta a' Mori ; e $L_{13}$ V VIII.che nel fuo mercato, di cui egli fa la defcrizione, concorrevano giornalmente fino a trenta mila mercatanti. Il me. defimo Conquiftatore teftifica, che avendo egli ottenuto dal Senato, che fi numeraffero le cafe, e gli abitatori, ch'erano nelle Città, ne'Villaggi, e ne' Cafali della Repubblica, fi trovarono più di cencinquanta mila cafe, e più di cinqueeento mila abitanti.

Aveano apparecchiato i Tlafcallefi per gli Spagnuoli, e rutti $i$ loro Alleati un bello e comodo alloggio. Cortès volle, che gli Ambafciatori meflicani alloggiaffero in un'appartamento vicino al fuo, tanto per far loro onore, quanto per levar da'loro animi la diffidenza de' Tlafcallefi. I Capi della Repubblica per dare agli. Spagnuoli una nuova pruova della loro fincera amicizia, prefentaroso a Cortès fecondo l'ufanza di que' Popoli, trecento belle giovani . Cortès le rifutd da principio, adducendo che dalla Legge Criftiana divietavafi la poligamia; ma poi per non difguftarli ne accettò alcune, come damigelle di Donna Marina. Maigrado un tal rifiuto tofto gli prefentarono cinque vergini della prima Nobiltà, le quali pure accettò Cortès per iftrignere vieppiù l'a. micizia con quella Repubblica. Quefte vergini infieme coll altre furono prontamente inftruite, e rinunziando alla fuperftizione de'loro genitori furono folennemente battezzate in un tempio, che fi fece ripulire ed apparecchiare per celebrarvi i facrofanti inifteri della nofira Religione. Una delle cirque Signore era figlia del Principe Maxixcatzin , la quale prefe nel batrefimo il nome di Donna Elvira, e l'ebbe il Capitano Gio: Velalquez di Leon. Un'altra, figlia del vecchio Xicotencatl, chiamoffi Donna Luigia Techquibuatzin, - fu data al Capitano Pietro d'Alvarado, (C) e l'altre tre

(C) Ebbe Alvarado da Donna Luigia due figli Don Pietro, e Donna Elleonora Alvarado. Coftei fir maritó con $\mathrm{D}$. Francefco della Cueva, $\mathrm{Ca}$ valiere dell' Ordine di S. Giacomo, Governatore di Guatemala, e cugino del Duca di Alburquerque, da quefto maritaggio nacquero parecchj fo glinoli. 
If diedero a' Capitani Criftofano d' Olid, Gonzalo di Sando. val, e Alonfo d' Avila.

Incoraggito da sì felice cominciamento, volle Cortès perfuadere i Capi della Repubblica e la Nobiltà a deteftare la loro fuperftizione, e riconofcere l'unica vera Divinità; ma eglino, benchè perfuafi dalle ragioni di lui, confeffaffero la bontà, ed il porere del Dio, che adoravano gli Spa. gnuoli, non però s'induffero a rinunziar a' loro pretefi Dei, perchè gli ftimavano neceffari per l' umana felicità. "Il no" Ptro Dio Camaxtle, dicevano, ci accorda la vittoria fopra "i noltri nemici: la noftra Dea Matlalcueje ci manda la - pioggia a' noltri campi neceffaria, e ci difeude dalle inon", dazioni del Zahuapan. (") A ciafcuno de' noftri Dei fiamo " debitori d'una parte della felicita della noftra vita, e la lor " collera provocata colla noftra ingratitudine potrebbe tirare fop:a lo ftato i più terribili galtighi. "Cortès, animato da un zelo troppo ardente e violento, voleva fare negl'ido. li di Tlafcalla, ciò che fatto avea con buona riufcita in quelli di Cempoallan; ma il P. Olmedo ed altre perfone riguardevoli lo diftolfero da un sì temerario attentato, rapprefentandogli, che sì fatta violenza, oltrechè non era convenevole alla promulgazione pacifica del Vangelo, cagionar potrebbe la total rovina degli Spagnuoli in una Città tanto popolofa, e tanto attaccata alla fuperfizione. Nulladimeno non cefsò mai ne' venti giorni, che quivi dimorò di rimpro. verar ad effi l'abbominevole crudeltà de' loro facrifizj, e d' in culcare la purità e la fantità della Religione Criftiana, la falfità de' Numi, che adoravano,e la efiftenza d' un Effere Su. premo, il qual regge tutte le cagioni naturali, e veglia con ammirabile provvidenza fulla confervazione delle fue creatu. re. Quefte efortazioni fatte da un uomo tanto autorevole, e di cui aveano formato i Tlafcallefi una idea troppo fublime, avvegnachè non produceffero turto il frutto, che fi defiderava, furono pure affai utili; poichè da effe moffo il Se:

nato

(*) Fiume di Tlafcalla. 
nato alla fine acconfenti, che fi rompeffero le gabbie; e fi

Lis.VIII. rimetteffero in libertà tutti i prigionieri, e gli fchiavi, che v'erano cuftoditi per effere facrificati a'loro Dei nelle fefte folenni, ovvero ne' pubblici bifogni dello ftato.

Così ogni giorno fi ftabiliva vieppiù con nuove dimo. Atrazioni l'alleanza de' Tlafcallefi a difpetto delle replicate fuggeftioni degli Ambafciatori Mefficani per romperla. Cortès quantunque ben perfuafo della fincerita de' Tlafiallefi, avea dato ordine alle fue truppe di mantenerfi ognora in arme per ciò che avvenir poteffe. S' offefe di ciò il Senato, e fi lagnò amaramente della diffídenza di Cortès dopo tante e sì chiare pruove della lor buona fede; ma Cortès fi fcusò proteftando, che ciò noa fi faceva per diffidenza de' Tlafcallefi; ma bensì perchè quella era l' ufanza degli Spagnuoli: Acchetoff il Senato colla rifpoita e gli piacque tanto cotal difciplina ne' Soldati, che Maxixcatzin volle introdurla nelle truppe della Repubblica.

Finalmente Cortès, poichè fi procacciò nel tempo, che Atette in Tlafcalla, una notizia più diftinta della fituazione della Città di Meflico, delle forze di quel Regno, e di tutto ciò che giovar poteva al fuo intento, determinò continuare il fuo viaggio; ma prima di partire regalò a' Tlafcallefi un gran numero de' più belli abiti, che gli a vea mandati il Re di Meffico. Era egli dubbiofo della ftrada, che doveva prendere per andare a quella Corte. Gli Ambafciatori Mefficani volevano, che andaffe per Chololla, dove s'era apparecchiato un buon alloggio per tutta la fua gente. I Tlafcallefi lo diffuadevano da un tal configlio rapprefentando la perfidia de' Cholullefi, e lo configliavano d'incamminarfi per Huexotzinco, Stato confederato del pari co' Tlafcallefi, e cogli Spagnuoli; ma Cortès fi rifolvette d'andar per Chololla cosi per compiacere agli Ambafciatori, come per moftrare a' Tlafcallefi il poco conto, che faceva degli sforzi de' fuoi nemici.

I Cholollefi erano ftati già alleati de' Tlafcallefi; ma full' arrivo degli Spagnuoli erano confederati co' Mefficani, a nemi: 
semici giurati di quella Repubblica. La cagione di si grand' inimicizia era ftata la perfidia dei Cholollefi. Coftoro in unaLis.VIII. battaglia, che come alleati dei Tlafcallefi diedero ai Mefficani, effendo nella vanguardia dell' efercito, fi mifero con un' improvvifa evoluzione nella retroguardia, e attaccando i zia tra Thafcallefi alle fpalle, mentre che i Mefficani gli battevano alla fronte, fecero in loro una grande Atrage. L'odio acce. fo nei cuori dei Tlafcallefi per sì deteftabile tradimento cer lefí cava qualche occafone di vendicarlo, e niun'altra parve loro più opportuna di quefta, allorchè fi trovavano confederati cogli Spagnuoli. Or per infpirar lo fteffo odio a Cortès, e muoveilo alla guerra contro quello Stato, il fecero avvertito della condotta dei Cholullefi verfo di lui; poichè non aveano mandato $\mathrm{i}$ loro meffaggieri a fargli dei complimen. ti, laddove gli aveano mandati gli Huexotzinchi, con tutto che foffero alquanto più difcofti di loro. Raccontavangli altres' il meffaggio, che dicevano aver ricevuto dai Cholollefi, rimproverando loro l' alleanza fatta cogli Spagnuoli, chiamandogli codardi e vili, e minacciandoli, che qualora attentaffero qualche cofa contra quella Santa Città, morrebbono turti annegati; imperciocchè infra gli altri loro errori s' erano perfuafi, che ogni volta che lor piacefle, potrebbono foltanto col radere le muraglie del Santuario di Quetzalcoatl, far indi nafcere dei groffi fiumi, che in un momen. to allagaffero la città, e quantunque i Tlafcallefí temeffero sì fatto infortunio, il defiderio della vendetta fuperava la loro paura.

Moffo Cortès da si fatte fuggeftioni mandò quattro nobili Tlafcallefi a Cholollan per faper da quei Signori la cagione di non avere avuto verfo di lui quel riguardo, che aveano avuto gli Huexotzinchi. I Cholollefi fi fcufarono coll' inimicizia dei Tlafcallefi, dei quali non potevano mai fidar. fi. (D) Quefta rifpolta fu portata da quattro plebei, ciò ch' Storia Antica del NefJico Tom.III. era

(D) Torquemada aggiunge, che i Cholollef ritennero il principale dei mefraggieri Tlafcallefr appellato Patlabuatzin, e con inaudita crudeltà gli 
era tuna manifefta dimoltrazione di difprezzo: Avvertito Cof: LIB.VIII.tès dai Tlafcallefi fece dire a quei Signori per quattro Cemipoallefi da lui colà mandati, che l' ambáciata d' un Monarca si grande, come il Re di Spagna, non era da confidarfi a si vili meffaggieri, mentre neppure eglino fteffi erano degni di fentirla:che fapeffero, che il Re Cattolico era vero Signore di tutto quel paefe, e che a nome di lui veniva ad efigere l'omaggio di quei popoli: che coloro, che a lui fi fottometteffero, farebbero onorati, ed i ribelli farebbero pu. niti giufta il lor merito: che però compariffero infra tre giorni in Tlafcalla, per dare obbedienza al loro Sovrano; altrimenti farebbero trattati come nemici. I Cholollefi quantunque tra loro fi burlaffero, єom'è da crederfi, d'un'ambafcia. ta cotanto arrogante, tuttavia per diffimular il loro maligno intento fi prefentarono il di feguente a Cortès, pregandolo di fcufar la loro mancanza cagionata dall' inimicizia dei Tla. fcallefi, e proteftandofi non che amici degli :Spagnuoli, ma vaffalli ancora del $\operatorname{Re}$ di Spagna.

5. 26.

Entrata degli di Tlafcalla con tutta la fua gente, e con un gran numero Rifoluto dunque il viaggio per Cholollan, ufcì Cortès Spagnuo-di truppe Tlafcallefi (E), le quali :tofto licenziò, fuorchè lolla . Iei mila uomini, che feco conduffe. Poco prima 'd'arrivare a Chololla, vennero ad incontrarlo i principali Signori, ed i Sacerdoti cogl'incenfieri in mano, e con mufica di frumenti, e dopo d'aver fatte le folite ceremonie di rifpetto, differo al Generale, ch'entraffe pure con tutti i fuoi Spa. gnuo-

fcorticarono la faccia e le braccia, e gli tagliarono le mani; ma quefto racconto è fenz'altro falfo; perciocchè sì fatta atrocità non poteva effere ignorata dagli Spagnuoli; ma nè Bernal Diaz, nè Cortes, nè verun altro dei primi Storici ne fa menzione. Cortès non l'avrebbe tralafciata nella fua lettera a : Carlo $V$. per giuftificar la feverità del gaftigo :efeguito nei Cholollefi: neppure è verifimile, che dopo un tale attentato fatto contra uno dej fuoi meffaggieri avelfe alpettato per punirlo altri indizj della perfidia dei Cholollefi.

(E) Cortès dice, che i Tlafcallefi, che l'accompagnarono fin' a fei mit glia avanti d'arrivar a Chololla, erano cento mila guerrieri in circa. Bernal Diaz mette foltanto due mila dei dieci mila, che gli efbi il Senato; $\mathrm{ma}$ quefto Auture pare elferfi dimenticato. 
gnuoli e coi Totonachi; ma non permetteffe ch'entraffero i Tlafcallefi lor nemici. Acconfentì Cortès per compiacer lo. Lis.VIII. ro, ed i Tlafcallefí reftarono accampati fuor della Citrà, imitando nella difpolizione dell'accampamento, nell' ordine delle: fentinelle, e in altre cofe la dif́ciplina militare degli Spagnuoli. Nell' entrata dell' efercito Spagnuolo in Chololla vi fu. una fimile folla di popolo, e le fteffe ceremonie, acclamazioni, ed offequi, ch' erano già Atati in Tlafcalla; ma. non cosł: finceri.

Era allora Chololla una Città popolofa diftante diciototo miglia da. Tlafcalla ad Oftro, e feffanta incirca da Mefo fico: a Levante, e non meno celebre pel commercio dei fuci abitatori, che per la! fua Religione.. Era fituata, ficcom' è prefentemente, in una: bella pianura, ed in poca lontananza. da: quel groppo d'alte montagne', che circondano la valle di Meffico: dalla parte di Levante. La fua popolazione in quel tempo era, fecondo che: afferma. Cortès, di quaranta mila cafe in circa, e' quafi altrettante: ve n' erano nei circonvicini: Villaggi, che le fervivano in vece: di fobborghi. Il fuo: commercio confifteva in manifatture di bambagia, in gem. me; ed in vaffellame di creta, ed erano affai famofi i giojellieri ed i pentolai di Chololla. Per ciò che' riguarda la Religione pud dirli, che Chololla era la: Roma d'Anahuac。 E'efferfi trattenuto tanti: anni: in quelia: Citta is celebre Quetzalcoatl, e: l'aver tanto favorito i fuoi: Cittadini fecero che: dopo lis apoteofi di quefto nume, gli fi confecraffe con ifpezial culto. La forprendente moltitudine di tempj, che vi: aveva, e maffimamente: il tempio maggiore eretto fopra un monte fatto a mano" che finora: fuffifte, tirava a quel luogo riputato fanto infiniti pellegrini non folo dalle Citto vicine, ma eziandia dalle: Provincie: più difcofte

Fu alloggiato Cortès con tutte le fue truppe in certe cafe grandi; dove i due primi: giorni furono: abtiondantemente provveduti di vettovaglie; ma tofto coninciarono a fcarfeggiare fin' a non fornir loro: altro; che acqua e legne. Non fu quefto l' unico argomento del loro animo maligno,

$$
\mathrm{H} 2 \text { poi- }
$$


poichè ad ogni momento fi fcorgevano nuovi indizj del tra: L.s.VIII.dimento, che macchinavano. Gli alleati Cempoallef offervarono, che nelle frade della Citta v'erano alcune buche, dove aveano ficcato delle Aanghe acute, e le aveano coperte con terra, le quali non parevano fatte per altro, che per inabilitare i cavalii. Otto vomini poi venuti dal campo dei Tlafcallefi l'avvertirono, ch' effi aveano veduto ufcir della Citta folla di donne, e di fanciulli : fegno non dubbio prefo fo quelle Nazioni di qualche guerra imminente. Oltracciò fo fapeva, che in alcune ftrade aveano formato delle trincee, $e$ aveano fatto dei grandi ammaffamenti di faff fopra i terrazzi delle cafe. Finalmente una Dama Cholollefe, che s'era innamorata della bellezza, dello fpirito, e della difcrezione di Donna Marina, la prego di falvarfi nella fua cafa dal pericolo, che fopraftava agli Spagnuoli : ond' ebbe occafione Donna Marina d'informarli di tutta l'orditura della confpirazio. ne, e avvisò incontanente Cor:ès . Co ftui feppe a bocca dalla medefima Dama Cholollefe, che i fuoi Concittadini avea. no concertato di far man baffa fugli Spagnuoli coll' ajuto di venti mila Mefficani, $\left(^{*}\right)$ che campeggiavano vicino alla Città. Non contento di quelti rifcontri ircaricò Donna Marinas d'adoperare tutte le fue arti per far venire al fuo alloggio due Sacerdoti, i quali confermarono tutto ciò, che avea dichia. rato la Dama.

Vedendofi Cortès in si grave rifchio di perire, determinò adoperare $i$ mezzi più opportuni per falvarfi . Fece venire alla fua prefenza le perfone principali della Gittà, e diffe loro, che fe aveano qualche querela contro gli Spagnuoli, l'efponeffero fchiettamente, liccome fi conveniva ad uomini onorati, e los darebbe una convenevole foddisfazione. Rifpofero coloro, $\mathrm{ch}^{\prime}$ erano ben foddisfatti della fua condotta, e pronti a fervirlo: che qualora determinaffe la fua partenza, farebbe, abbondan-

temen-

$\left(^{*}\right)$ Bernal Diaz dice, che l'efercito Meflicano, per quel che fi feppe, cra di venti mila uomini. Cortès afferma, che gli ftelli Signori di Chololla gli confeffarono, che tal efercito non era meno di cinquanta mila uo mini. 
temente provveduto di tutto ciò, che bifognafle pel viaggio, ed ancor di gente da guerra per la fua ficurezza. Accettò Cor.Liz. VIII. tès la loro offerta, e prefiffe la fua partenza pel giorno feguente. I Cholollefi fe n' andarono contenti, perchè lor pareva, che tutto andaffe bene per la felice riufcita del loro tradimento; e per afficurarlo vieppiu, facrificarono ai loro Dei, per quel che dicono, dieci fanciulli, cinque di ciafcun feffo. Cortès ragunò i fuoi Capitani, lor palesò i perverfi difegni dei Cholollefi, e loro impole di dire il lor fentimento. Alcuni volevano, che fi fchivaffe il pericolo ritirandofi alla Cirtà di Huexotzinco, appena nove miglia da Chololla difcolta, o pu. re a Tlafcalla; ma per la maggior parte fi compromifero nella determinazione del loro Generale. Cortès diede gli ordini, che gli parvero più confacenti al fuo intento, proteftando, che non fi crederebbe mai ficuro in Meffico fenza lafciar ben punita quella perfida Città. Ordinò alle truppe aufiliarie di Tlafcalla, che il di vegnente fullo fpuntar del Sole piombaffero fopra la Città facendo man baffa fopra tutti i Cittadini, fenza perdo. nar a niuno, fe non che alle donne ed ai fanciulli.

Venne finalmente quel giorno tanto infaulto per Cholol- \$. 27: la. Apparecchiarono gli Spagnuoli i loro cavalli, la loro arti- Strage glieria, e le loro armi, e fi mifero in ordine in un granchololla. cortile del loro albergo, che doveva effere il teatro principale di quella tragedia. Arrivaronvi i Cholollefi fullo fpuntar del di. I Signori con infin' a quaranta Nobili e con gli uomini da foma entrarono nelle fale e nelle camere per levar l' equipaggio, e fubico vi furono polte delle guardie, acciocchè non poteffero fcappare. Le truppe Cholollefi entrarono (almeno in gran parte) nel cortile infieme col principal Signore di quella Città a richiefta, com' è da crederfi, del medefimo Cortès, il quale montando a cavallo parlò a loro in quefta maniera. "Io, Signori, mi fono adoperato per farvi miei amici; en"trai pacificamente nella voltra Città, e quì non avete avu. "to verun torto nè da me, nè da alcuno dei miei; anzi " perchè non avelte a lagnarvi, non permifi, che v'entraf"Cero le truppe aufiliarie dei Ilafcallefi. Oltracciò vi bo.

2) pris: 


\section{2.}

L.IB.VII 2. pregato di dirmi fchiettamente, fe vi abbiamo fatto quale 22. che aggravio per foddisfarvi, ma voi con deteftabile per" fidia avete ordito fotto fembianza d'amicizia, il più cru. "del tradimento per farmi perire con tutta la mia gente: "nulla ignoro dei voltri maligni difegni a, E chiamando in difparte: quattro, 0 cinque Cholollefi, loro addimandò, che ragione: aveano avuta per rifolverfi ad un sì efecrabile attentato . Eglino rifpofero, che gli Ambafciatori: Mefficanis per. far piacere al loro. Sovrano, gli aveano indotti a macchinar loro la morte. Cortès allora con un fembiante pieno dis fuoco parlo così agli Ambafciatori, che fi trovavano prefen ti :, Quefti, malvagi per ifcufar il lora delitto incolpano. „2 del tradimento e voi, ed il voftro Re; ma nè io vi cre. " do capaci di si fatta fcelieratezza, nè poffo perfuadermi " che il gran Monarca Motezuma voleffe far meco da ne.. " mico crudele nel tempo fteffo, che ni dà le più rilevan"ti prove della fua amicizia, e che potendo con aperta „2. forza opporfi alle mie pretenfioni, fr prevaleffe dei tradi. 22. tori per fraftornarle- State put ficuri , che farò rifpettar "le: voftre- perfone nella: Atrage, che fiamo per fare. Oggi " periranao quelti traditori, e farà meffa in conquaffo la "Loro Città . Chiamo il Cielo, e la Terra in teftimonio, gp. che la loro perfidia è quella, che arma le noftre braccia. 32. Per una: vendetta tanto contraria: al noftro genio.

Ciò detto e fatto il fegno d'affalire collo fparo d'uno fchioppo, fi fcagliarono con tal furore gli Spagnuoli contro quelle mifere vittime, che di: tutti quelli, che fi trovavano nel cortile, con tutto che foffero molti, non reft vivo neppure: uno.. I rivi di fangue, che fcorreyano per quel cortile, ed i lagrimevoli lamenti des moribondi bafterebbera a. far ribrezzo, e pieta a qualunque: cuore, che non foffe fpinto dal furor dellia vendetta o. Non avendo più a fare den tro della cafa, ufcirono nelle ftrade, infanguinando col medefimo rigore le fpade in quanti Cholollefi a loro fi prefentavano . I Tlafcallefi dalla lor parte-entrarono nella: Città, come leoni affamati, ravvivando, la loro ferocia coll'odio dei. 
dei loro nemici, e colla brama di compiacere ai loro nuovi alleati. Un colpo si fiero, e sì inopinato mife fubito in $\mathrm{L}_{18 .}$ VIIL. difordine i Cittadini; ma effendofi ragunati in parecchj fqua. droni fecero per qualche tempo una vigorofa refiftenza, finchè fcorgendo la Atrage, che in loro faceva l'artiglierìa, e riconofcendo la fuperiorità dell' armi europee, di nuovo fi fcompigliarono. La maggior parte di loro cercò lo scampo nella fuga : alcuni ricorfero alla fuperfizione di rader le muraglie del tempio per allagar la Città; ma trovando affatto inutile tal diligenza, procurarono fortificarfi nelle cafe e nei tempi. Neppur ciò fu giovevole; perchè ecco che $\mathrm{i}$ lor nemici appicciano il fuoco a rutti gli edifizj, dove trovano qualche refiftenza. Ardono le cafe, e le torri dei tempj: non altro fi vede nelle ftrade, che cadaveri infanguinati o mezzo bruciati, nè altro fi fente, che clamori infultanti e minacciofi dei confederati, folpiri deboli dei moribondi, imprecazioni contro ai vincitori, e lamenti dei loro Dei, perchè gli hanno abbandonati in sì gran calamità . Tra molti, che fi ricoverarono melle torri dei tempj, non vi fu altro che uno, che $\dot{s}$ arrendeffe ai vincitori: tutti gli altri 0 perirono inceneriti, o pure fi procacciarono una morte men dolorofa precipitandofi da quell' altezza.

Con quefta orribile ftrage, (F) nella quale perirono più

(F) Negli fcritti di Monfignor de las Cafas fi legge affai sfigurato quefto avvenimento di Chololla. E' vero, che fu troppo rigorofa la vendetta, ed orribile la "frage, che vi fr fece; ma nè mancatono agli Spagnuoli per punire i Cholollefi quelle ragioni, che noi abbiamo addotte, quefto Prelato non ne fa alcuna menzione, nè $v$ 'intervennero quelle odiofe circoftanze, che egli rammemora, e che non fi trovano preffo veruno Storico antico. Per farci credere, che gli Spagnuoli fecero quella ftrage foltanto per capriccio, e che frattanto che i Soldati faceano fcorrere rivi di rangue, il Generale cantava allegramente non fo che verfetti, farebbe d'unpo almeno, che lo 'ftefro Prelato il deponeffe, come teftimonio oculato, "o che allegaffe tali documenti, che valeffero a f́ncellare quella idea, che ci danna di Cortès quanti il conobbero, 'e faceffero verifimile ciò, che è affatto incredibile. Ma nè Monfignor de las Cafas fi trovò prefente, nè adduce prove fufficienti a meritar la noftra fede. Egli fenza il debito accorgimento fi prevalfe di qualche informazione datagli da alcúno dei molti nemici di Cortès. Io non fono panegirifta di quefto Conquifatore per ifculare 
più di fei mila Cholollefi, reftò allora fpopolata la Città : Lis.VIII.I tempi, e le cafe furono faccheggiate, impadronendofi gli Spagnuoli delle gemme, dell' oro, e dell' argento, ed i Tlafcallefi della robba da veftirfi, delle penne, e del fale. Appena terminata la tragedia di Chololla, comparve colà un efercito di venti mila uomini mandati in foccorfo dalla Re. pubblica di Tlafcalla fotto il comando del Generale Xicotencatl. E' da crederfi, che ciò foffe ftato effetto di qualche avvifo fpedito ta notte innanzi a quel Senato dai Capi delle truppe Tlafcallefi, che campeggiavano fuor della Cíttà. Cortés gradi il foccorfo, regalò a Xicotencatl, ed ai fuoi Capitani una parte del bottino, e lo pregò di tornarfí col fuo efercito a Tlafcalla, perchè non era più neceffario, e ritenne feco quei fai mila uomini, che l'aveano ajutato nel gaftigo di Chololla, acciocchè l'accompagnaffero nel viag. gio a Meffico. Così fi rafsodò pì̀ l'alleanza degli Spagnuoli coi Tlafcallefi.

S. 28. Ritornato Cortès al fuo alloggio dov' erano rimafti, meflione come prigionieri, quaranta Cholollefi della prima Nobiltà, dei Cho- quefti lo pregarono di far luogo fra tanto rigore alla cledeiTe- menza, e ai permettere, che uno o due di loro andaffero pejachefi a richiamar le donne, i fanciulli, e gli altri fuggitivi, che alla coro- andavano marriti; e pieni di fpavento per le montagne. gna. Moffo Cortès a pietà fece ceffar il furor dell' armi , e pubblicare un indulto generale. All' intimarfi un tal bando fo videro fubbito alzarfi tra gli uccifi alcuni, che aveano contraffatto la morte per ifchivarla, e venire verlo la Citta truppe di fuggitivi, piangendo chi la morte del fuo figliuo1o, o del fuo fratello, chi quella del fuo marito. Fece Cortès levar dai tempj, e dalle Itrade $i$ cadaveri, che oramai cominciavano a putire, e rimile $\mathrm{i}$ Nobili prigionieri in liber. tà.

ruoi falli; ma fono Atorico, fon uomo, e fon Crifiano per non affermare ciò, che non credo, e per non credere si gran malvagità d'un individuo della mia fpezie fenza gravi fondamenti. Defcrivo il fatto di Chololla, qual lo trovo preffo gli Storici più finceri, che fi trovarno prefenti, o s informarono tanto dagli antichi Spagnuoli, quanto dagl' Indiani. 
tà. Refto fra pochi giorni quella Città sì ben popolata, che non pareva mancar veruno dei fuoi abitanti. Quivi ricevetteLis.VIII, Cortès i complimenti degli Huexotzinchi, e dei Tlafcallefi, ed il giuramento di fedelà alla Corona di Spagna dei medefimi Cholollefi, e dei Tepejachefi : aggiuftò le differenze, che v'erano tra le due Repubbliche di Tlafcalla, e di Cho. lolla; e riftabill la loro antica alleanza ed amicizia, la qua. le d'allora innanzi non mai mancò. Finalmente per foddisfare ai doveri della umanità e della Religione fece rompere le gabbie dei tempj, e mife in libertà tutti $\mathrm{i}$ prigionieri $\mathrm{e}$ gli fchiavi, che erano deftinati al facrifizio. Fece inoltre nettare il tempio maggiore, e v'inalberò lo fendardo della Croce, dopo d'aver dato ai Cholollefi, ficcome faceva a tutti gli altri popoli, preffo cui fi fermava, qualche idea della Religione Crittiana.

Orgogliofo it Generale Spagnuolo per s̀ felici fucceffi, $\mathbf{5} 29$. - pur bramofo di far paura a Motezuma, incaricò gli Am-ambafciabafciatori Mefficani di dire al loro Signore, che fe già avea ta eprevoluto entrar pacificamente in Meffico, ora vedendo ciò, che fente del gli era accaduto in Chololla, s'era determinato d'entrar co- Meffico. me nemico a fargli quanto male poteffe. Gli Ambalciatori rifpofero, che prima di prendere una tal rifoluzione, faceffe più diligenti ricerche ful fatto dei Cholollefi per certificarfi delle buone intenzioni del loro Sovrano: che fe gli pareva bene, fi porterebbe un di loro alla Corte a prefentar al Re le querele di lui. Acconfentì Cortès, e dopo fei giorni ritornò l'Ambafciatore, portando ad effo Generale un gran regalo confitente in dieci piatti d'oro, che importavano cinque mila zecchini, in mille cinquecento abiti, ed in una gran copia di vettovaglie, ringraziandolo a nome del fuo Sovrano del gaftigo efeguito ne'perfidi Cholollefi, e proteftan. do, che l' efercito gia levato per foprapprendere gli Spagnuoli nella Arada, era degli Acatzinchefi, e degl'Itzocanefi, alleatı di Chololla, $\mathrm{i}$ quali benchè fudditi della Corona, aveano pure prefe l'armi fenza verun ordine del loro Sovrano. Ciò fu confermato col giuramento degli Ambafciatori, Storia Anticadel Meffico Tom. 111. I e Cor- 


\section{6}

e Cortès fece fembiante di reftar pienamente foddisfatto.

Lis.VHII. Non è facile chiarire il vero in quefto particolare, nè poffiamo aftenerci di biafimar la leggerezza de' noftri Autori nel affermare si francamente ciò, che affatto ignoravano. Perchè fi vorrà dar credenza a' Chollolefi, uomini doppj e fin. ti, come tutti confeffano, e non a'Mefficani, ed allo fteffo Motezuma, il quale per l'eminenza del fuo carattere era più degno di fede? La condotta coftantemente pacifica di quel Monarca verfo gli Spagnuoli, non avendo loro fatto verun male in tante e si belle occafioni, che ebbe di fopraffargli, e la moderatezza, con cui fempre mai parlò di loro ( cid che non negano gli fteffi Storici) fanno inverifimile la fcufa de'Cholollefi; ma per altro le danno qualche apparenza di verità certi indizj, benchè ofcuri, dello fdegno di Motezuma, e fopra tutto le oftilità fatte in quefto medefimo tempo fulla guernigione della Veracroce da un poffente Feudatario della Corona di Meffico.

5. 30.

Rivoluzioni in TotonaQuaubpopoca,

$\left.{ }^{*}\right)$ Signor di Nauhtlan (appellata dagli Spagnuoli Almeria ) Citta fituata fulla Cofta del feno Mefo ficano trenta fei miglia a Tramontana dalla Veracroce, e capan. preffo a' confini dell' Imperio Mefficano da quella parte, ebbe ordine da Motezuma di ridurre i Totonachi alla dovuta ubbidiestza, tofto che Cortès fi ritiraffe da quella Cofta . Egli per fare il fuo dovere richiefe con minacce da que' Popoli il tributo che pagar dovevano al loro Sovrano. I Totonachi renduti infolenti col favor de' loro nuovi alleati, rifpofero con arroganza, che non doveano verun omaggio a chi non era più lor $\mathrm{Re}$. Or vedendo Quauhpopoca, che nulla giovavano le fue richiefte per rimettere nella fubordinazione quegli uomini, she aveano troppa confidenza ne' loro alleati, e niun rifpetto al loro Sovrano, mettendofi alla tefta delle truppe Mefficane, che v' erano nella guarnigione di quelle frontiere, cominciò a far delle fcorrerie per

(*) Bernal Diaz l'appella Quetzalpòposa, il guale è ancora nome Melficano. 
li luoghi di Totonacapan, caftigando coll' armi la loro ribellione. I Totonachi portarono i loro lamenti a GiovanniLis.VIIL, d'Efcalante, Governatore del prefidio della Veracroce, e lo pregarono d'opporfi alla crudeltà de' Mefficani, efibendofiad ajutarlo con un buon numero di truppe. Elcalante mandò al Capo de' Meficani un'ambafciata cortefe per diftorlo da quelle oftilità, le quali non potevano, fecondo che egli credeva, effer gradice dal Re di Meffico, che tanto s' era im. pegnato nel favorir gli Spagnuoli protettori de' Totonachi . Qua uhpopoca rifpofe, ch' egli meglio di lui fapeva, fe era - no, 'fiacevole al fuo $\mathrm{Re}$ il gaftigo di que' ribelli : che fe gli Spagnuoli volevano foftenergli, egli colle fue truppe gli afpetterebbe nelle pianure di Nauhtlan, acciocchè l'armi decideffero della loro forte. Non poté comportare una tal rifpofta il Governatore: onde marciò incontanente verfo illuogo prefiffo con due cavalli, e due piccoli cannoni, zon cinquanta pedoni Spagnuoli, e con dieci mila Totonachi in circa. Nel primo attacco de' Mefficani fubito fi fcompigliarono $i$ Totonachi, e per la maggior parte fi mifero a fuggire; $\mathbf{m a}$ ad onta della loro codardia gli Spagnuoli continuarono coraggiofamente la zuffa, facendo non poco male ai Mefficani . Coftoro, come quelli che non aveano mai fperimentata la violenza dell' artiglieria, e la maniera di combattere degli Europei, fi traffero impauriti alla vicina città di Nauhtlan. Gli Spagnuoli gl' incalzarono con furia, e attaccarono il fuoco ad alcuni edifizj; ma quefta vittoria lor coftò la vita del Governatore, il quale infra tre giorni morì delle ferite, di fei o fette foldati, e di molti Totonachi. Un di quefti foldati, il quale aveva la tefta groffa e l'afpetto fiero, fu fatto prigione, e mandato a Meffíco da Quauhpopoca; ma effendo morto per le ferite nella ftrada, non portarono altro a Motezuma, che la tefta, la cui fembianza fece tanto ribrezzo a quel $\mathrm{Re}$, che non volle che foffe a fuoi Dei offerta in alcun tempio della Corte.

Ebbe Cortès la nuova di quefte rivoluzioni prima di

$$
\text { I } 2 \text { par- }
$$


partir da Chololla; (G) ma non volle dire allora niente; Lis.VIII.nè palefar l'inquietudine, che gli recò per non ifcoraggire i fuoi foldati.

5. 3r. Or non avendo più a fare in Chololla, riprefe il viag-

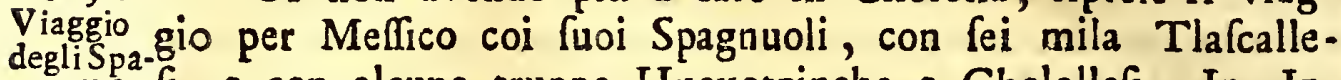
gnuolifi- $\mathfrak{f}$, e con alcune truppe Huexotzinche e Cholollefi. In Izn'a Tlal-calpan, Villaggio di Huexotzinco, quindici miglia lontano manalco. da Chololla, vennero di nuovo a fargli i loro complimenti i Signori Huexotzinchi, e l'avvifarono, che da quel luogo $v$ 'erano due frade per andare a Meffico; l'una aperta, e ben acconciata, la quale conduceva a certi burroni, dov'era da temerfi qualche imbolcata de' nemici, e l'altra di frefco ingombrata ed impacciata con alberi a bella potta tagliati, la quale era pur la più corta, e la più ficura. Cortès $s$ approfittò dell' avvifo, ed a difpetto dei Mefficani fece levar via gl'impaccj dalla ftrada fotto colore d'effere la malagevolezza piuttofto allettamento pel coraggio degli Spagnuoli ; e continuò il fuo viaggio per quel gran bofco di pini, e di quercie fin'a falire fulla cima d'un alto monte appellato Ithualco tra i due Vulcani Popocatepec, e Iztaccihuatl, dove trovarono delle cafe grandi deftinate per alloggio de' mercatanti Mefficani, Quivi s' avvidero dell' ardita imprefa del Capitano Didaco d'Ordaz, il quale pochi giorni innanzi per dare a conofcere a que' Popoli il coraggio della fua. Nazione fali infieme con altri nove Soldati fin quafi all'altiffima cima del Popocatepec, benchè non potè offervar la bocca, o fpiraglio di quel gran Vulcano a cagione dell'alta neve, che $\checkmark$ 'era, e delle nubi di fumo, e di cenere, che lanciava dal. le fue vifcere. $(H)$

Dalla

(G) Tutti o quafi tutti gli Storici dicono, che la nuova di quelle rivoIuzioni arrivo a Cortès, allorchè era in Meffico; ma lo fteffo Cortès afferma, che l'ebbe in Chololla.

(H) Bernal Diaz, e quafi tutti gli Storici dicono, che Ordaz fall in fulla cima del Popocatepec, ed offervo la bocca di quel famofo monte; ma Cortès, il quale il rapeva meglio, dice l'oppofto. Nondimeno ottenne Ordaz dal Re Cattolico di metter un Vulcano nel fuo fcudo d'armi. Quefta 
Dalla cima d'Ithualco offervarono gli Spagnuoli la belliffima Valle di Meffico, ma con fentimenti affai diverfi ; Liв.VLIL. poichè alcuni fi dilettarono nella veduta de' fúoi laghi, delle fue amene pianure, delle verdeggianti montagne, e delle belle, e molte città, che v' erano dentro ed attorno agli fteffi laghi : in altri fi ravvivò la fperanza d'arricchirfi colla preda di tanta grandezza di paefe, quanta indi fo fcorge$\mathrm{va}$; ma alcuni più prudenti in riguardando una si grande popolazione, riputaronc temerità l'affrontare sì gravi rifchj: ficchè furono da una tale paura foprapprefi, che fi farebbono quindi ritornati alla Veracroce, fe Cortès prevalendofi $e$ della fua autorita, e delle ragioni fuggeritegli dal fuo buon ingegno, non gli aveffe incoraggiti all' imprefa .

Frattanto Motezuma, cofternato per l'avvenimento di Chololla, fi ritirò al palazzo tlillancalmecatl, deftinato pel tempo di duolo: ed ivi ftette otto giorni digiunando, ed efercitandofi nelle folite aufterità per procacciarfi la protezione de' fuoi Dei. Da quefto medefimo ritiro inviò a Cortès quattro perfonaggi della fua Corte con un prefente, e con nuove preghiere e pretelti per diftornarlo dal viaggio, efibendofi a pagare annualmente certo tributo al $\mathrm{Re}$ di Spagna, ed a dare al Generale Spagnuolo quattro fome d'oro, $\left({ }^{*}\right)$ ed una a ciafcuno dé fuoi Capitani e Soldati, fe daqual luogo, dove fi trovavano, fi ripatriaffero. Tanto grande era il ribrezzo, che faceano gli Spagnuoli a quel fuperftiziofo Principe! Non avrebbe fatto più premurofe diligenze per ifchivar la loro vifta, s'aveffe prefentito tutto il male, che doveano fargli. Gli Ambalciatori giunfero Cortès in Ithual.

co :

grand'imprefa era rifervata per Montagno, ed altri Spagnuoli, i quali dopo la conquifta di Meffico non folamente offervarono la fpaventevol bocca di quel Vulcano, ma ancora v'entrarono non fenza evidente pericolo della vita, ed indi cavarono una gran quantita di zolfo per far della polvere per l'armi da fuoco.

$\left({ }^{*}\right)$ Elrendo la foma ordinaria d'un Meflicano di cinquanta libbre Spagnuole, o fia d'ottocento oncie, poffiamo congetturare, attelo il numero degli Spagnuoli, che ciò che voleva dar loro Motezuma per diftornargli dal viaggio alla Corte, era più di tre milioni di Zecchini. 
co: il regalo da loro portato era di parecchj lavori d'oro; Lrs.VIII.che importarono mille e cinquecento zecchini. Cortès fece a loro turti gli offequi, poffibili, e rifpofe ringraziando il Re dal fuo prefente, e delle fue magnifiche promeffe, alle quali fperava corrifpondere con buoni fervizj; $\mathrm{ma}$ infieme proteltaado, che non poteva tornare indietro fenza renderfi col pevole di difubbidienza al fuo Sovrano, e promettendo di non recar colla fua venuta il più leggiere pregiudizio allo Stato: che fe dopod'aver efpofta a bocca a fua Maefta l'ambafciata, che portava, e che non poteva ad altra perfona fidare, ella giudicaffe non convenire al bene del fuo regno la dimora degli Spagnuoli nella Corte, egli fenza indugio riprenderebbe la ftrada per ritornare alla fua patria.

Accrefcevafi l'inquietudine di Motezuma per le ruggeftioni de'Sacerdoti, e maffimamente pel ragguaglio, che gli fecero di certi oracoli de'loro bugiardi numi, e di non fo che fpaventevoli vifioni, che dicevano avere avute in que' gionni. Ciò mife il fuo animo in tal cofternazione, che fenz' afpettar l' efito dell' ultima ambafciata mandata agli Spagnuoli, tenne di nuovo configlio col $\operatorname{Re}$ di Tezcuco, col fuo fratello Cuitlahuatzin, e cogli altri perfonaggj., cui confultar foleva, tutti i quali fi mantennero nel primo lorfentimento; Cuitlahuatzin in quello di non permettere agli Spagnuoli l'entrata nella Corte, e di fargli o di grado o per forza, ufcir del regno, e Cacamatzin in quello d'accoglierli come Ambafciatori, poichè non mancavano forze al Re di Meffico per reprimerli, cafo che macchinaffero qualche cofa contro la Real perfona, o contra lo Stato. Motezuma, il quale fin'allora aveva fempre aderito al parere del fuo fratello, ora abbracciò quello del Re di Tezcuco, ma in. fieme incaricò quefto medefimo Re d' andare ad incontrare gli Spagnuoli, e d'adoperarfi per diffuadere il Generale dal viaggio alla Corte. Allora Cuitlahuatzin volto al $\operatorname{Re}$ fuo fratello gli diffe. "Gli Dei vogliano, Signore, che non ac" cogliate in cafa voltra chi da effa vi fcaccj, e che allorchè "vorrete rimediar al male, abbiate tempo, e mezzi per 
"farlo. "Che abbiamo a fare, rilpole il $\mathrm{Re}$, mentre ino" di favorirci, profperano i noftri nemici. Io fono ormai "rifoluto, e vorrei, che tutti fi rifolveffero di non fuggire, " nè moftrar alcuna codardia, avvenga che che fia; ma mi "fanno pietà $i$ vecchj, ed $i$ fanciulli, che non hanno for"za per difenderfi. "

Cortès, congedatifi gli Ambafciatori Mefficani, fi mo:fe colle fue truppe da Ithualco, e s'incammind per Amaquemecan, e Tlalınanalco, due Città fra loro diltanti nove miglia in circa, e fituate fulle pendici di quelle gran montagne. A maquemecan infieme co'vicini cafali, conteneva ven. ti mila abitanti. (I) In quefti luoghi furono ben accolti gli Spagnuoli, e parecchj Signori di quella Provincia vifitarono Cortès, e gli prefentarono certa quantità d'oro, ed alcune fchiave: $i$ quali fi lagnasono amaramente delle veffa zioni, che foffrivano dal $\operatorname{Re}$ di Meflico, e da' fuoi Miniftri negli fteffi termini, che l'aveano già fatto quelli di Cem. poalla, e di Chiahuitztla, e per fuggeftione de'.Cempoallefi, e de'Tlafcallefi, che accompagnavano Cortès, fi confedera. rono cogli Spagnuoli per l'intereffe della liberrà. Sicchè quanto più s'innoltravano gli Spagnuoli in quel paefe, tanto più s'andavano aumentando le loro forze a guifa d'un rufcello, che coll' acque, che va ricevendo nel fuo corfo fi va ingroffando fin'a divenir un gran fiume.

Da Tlalmanalco marcid l'efercito a Ajotzinco, villaggio fituato fulla riva meridionale del lago di Chalco, (L) dov'era il porto per le barche mercantili, che commercia-

vano

(I) Amaquemecan dagli Spagnuoli detta Mecameca è prefentemente un villaggio non per altro rinomato, che per effere ivi nata la celebratiffima Monaca Giovanna Agnère della Croce, donna di prodigiofo ingegno, e di Don volgar letteratura.

(L) Solis confonde Amaquemecan con Ajotzinco, Amaquemecan non è ftata mai fituata, com'egli dice, nella riva del lago; ma da queftodiftante più di dodici miglia nella falda d'un monte. La vifita del $\mathrm{Re}$ di Tezcuco fu renza dubbio in Ajotzinco, come affermano gli Storici bene inAtruiti, 
vano co'paefi, che fono a Mezzogionno di Meffico. La cu. Lis. VIII.riofità d'offervare il quartiere degli Spagnuoli cofto cara ad alcuni Mefficani; perchè le fentinelle fpagnuole, ftimandogli fpie per la paura, che tuttora aveano di qualche tradimen.

5ifita to, ne uccifero quindici incirca quella notte. Il di feguente del Redi allorchè erano pronti a marciare, v'arrivarono quattro noTezcuco bili Mefficani colla nuova, che veniva il $\mathrm{Re}$ di Tezcuco a a Cortès. vifitare il Generale Spagnuolo a nome del Re di Meffico fuo Zio. Non iftette guari, che giunfe lo fteffo $\mathrm{Re}$ portato in una lettiga fregiata di vaghe penne fugli omeri di quattro fuoi dimeftici, e feguito da una numerofa e brillante comitiva di Nobiltà Mefficana, e Tezcucana. Tofto che ven. ne a vifta del Generale Spagnuolo, fmontò dalla lettiga, cominciò a camminare a piedi preceduto da alcuni fuoi fervitori, che andavano levando dalla ftrada tutto cid, che po. trebbe offendere i fuoi occhj, ovvero i fuoi piedi. Gli Spagnuoli reftarono maravigliati di tanta grandezza, e quindi congetturarono quanta farebbe quella del Re di Meffico. Cortès ufci ad incontrarlo alla porta dell' albergo, e lo prevenne con un profondo inchino, al quale corrifpofe il $\mathrm{Re}$ toccando colla deftra la terra, ed indi portandola alla boc. ca . Entrd con un' aria fignorile e maeftofa in una delle fale, e meffofi a federe, fi congratulò con quel Generale, e co' fuoi Capitani del loro felice arrivo, e proteftò la gran volontà, che aveva il Re di Meffico fuo Zio di ftringere amicizia, d'avere una buona corrifpondenza col Monarca di Levante, da cui erano fati in quel paefe mandati, ma infieme efagesò le difficoltà, che bifognava fuperare per andare alla Corte, e pregò Cortès di mutar propolito, fe voleva compiacer al Re. Cortès rifpofe, che fe mai voleffe tornar indietro fenza far la fua ambafciata, mancherebbe al fuo dovere, e darebbe un gran difgulto al fuo Sovrano, che l'avea mandato,

ftruiti, e fi fcorge chiaramente dalla defcrizione di Cortès, e neffuno fin" a Solis ha detto, che fia ftata in Amaquemecan. Bernal Diaz dice, che quefta vifita fi fece in Izpalatenco; ma quefto è un manifefto sbaglio cagionato da mancanza di memoria. 
dato, maffimamente trovandofi oramai cosł vicino alla Corte dopo d'averfuperati i pericoli di un viaggio si lungo. Liz. VIII. $S_{e}$ d dunque così, diffe allora il $\mathrm{Re}$, nella Corte ci vedremo. e congedandofi cortefemente dopo d' effere ftato regalato d'alcune bagattelle d'Europa, vi lafciò una parte della Nobiltà, acciocchè accompagnaffe Cortès nel viaggio.

Da Ajotzinco marciarono gli Spagnuoli a Cuitlahuac, Citra fondata in una ifoletta del lago di Chalco, la quale, benchè piccola, era pure, a detta di Cortès, la più bella, che fin' allora avea veduta. Comunicava quefta Città col continente per due ftrade larghe e comode tabbricate ful lago: l'una a Mezzogiorno lunga due miglia, e l'altra a ferten. trione, che aveva più di due miglia di lunghezza. Cammi* navano gli Spagnuoli molto allegri di veder la moltitudine e la bellezza delle Città, che v'erano nel lago, i tempj e le torri, che s'innalzavano fopra gli altri edifizj, l'alberete che abbellivano i luoghi abitati, gli orti e giardini galleg. gianti nell' acque, e le innumerabili barche, che vi folca. vano, ma non men paurofi dal vederfi attorniati da una immenfa folla di Popolo, che da tutti que'luoghi vi concorreva ad offervarli : per lo che comandò Cortès a' fuoi, che andaffero bene ordinati, ed alleftiti, ed avverti gl' Indiani di non ingombrare la ftrada, nè accoftarí alle file, fe non volevano effer trattati da nemici. In Cuitlahuac furono be. ne alloggiati, e regalati. Il Signor di quella Città fi lagnò fegretamente con Cortès della tirannìa del $\mathrm{Re}$ di Meffico, fi confederò con lui, e gli fece fapere la comodità della Arada per andare alla Corte, e la cofternazione, nella quale aveano meffo Motezuma gli oracoli degli Dei, i fenomeni del Cielo, e la felicità dell' armi fpagnuole.

§. 33 .

$\mathrm{Da}$ 'Cuitlahuac s' incamminarono per l'altra ftrada ver. Vifita de' fo Iztapalapan; ma nell' andare fu Cortès da una nuova fe- Principi licità trattenuto. Il Principe Ixtlilxochitl vedendo, che Cor-co,ed entès non aveva voluto fare il viaggio per Calpolalpan, dove trata del'afpettava, rifolvette di venir ad incontrarlo nella Arada gnuoli in d' Iztapalapan. Marciò però con un buon numero di trappe quefta Storia Antica del Meffico Tom.III. K K e pal- Corte. 
e pafso preffo a Tezcuco. Confapevole di ciò il Principe Lir.VIII. Coanacotzin fuo fratello, il quale dopo que difgutti avvenuti tre anni addietro, di cui abbiamo fatto menzione, vivea tuttora da lui alienato, o fpinto dall' amore fraterno, o moffo dalla fperanza di maggiori vantaggi, che avrebbe u. nendo i fuoi intereffi a quelli del fuo fracello, venne ad in. contrarlo nella ftrada. Quivi fi ccmunicarono fcambievolmente i loro fentimenti, fi rappacificarono, e s'unirono per confederarfi cogli Spagnuoli. Camminarono infieme fin' ad Iztapalatenco, dove raggiunfero quegli ftranieri. Cortès vedendo venir tante truppe armate, ebbe qualche inquietudine; ma poichè fu informato della qualità de' perfonagg;, che venivano a trovarlo, e del motivo della loro venuta, ufcì ad incontrarli, e fattifi dall'una, e dall' altra parte i dovusi complimenti, invitarono amendue i Principi Cortès a portarfi alla Corte di Tezcuco, ed egli li lafcio facilmente perfuadere per la grand' utilità, che fperava trarre dal 'rincipe Ixtlilxochitl il cui attaccamento agli Spagnuoli s'era già renduto abbaftanza palefe.

Era allora Tezcuco, benchè alquanto inferiore a Merfico nella magnificenza, e nello fplendore, la più grande e popolata Città del paefe d'Anahuac. La fúa popolazione, comprefa quella delle Città d'Huexotla, di Coatlichan, d'Atenco ( le quali per effere a quella contigue fi potevano ftimare fuoi fobborghi ) era per quel che dice Torquemada, di cento quaranta mila cafe. Agli Spagnuoli parve più grande al doppio di Siviglia. La grandezza de' tempj, e de' palazzj Reali, la bellezza delle Atrade, le fontane, ed i giardini fornirono abbondante materia alla loro ammirazione.

Entrò Cortès in quefta gran Città $(M)$ accompagnato. da

(M) Dell' entrata degli Spagnuoli in Tezcuco non fa menzione Cortès, nè Bernal Diaz, nè Acofta, nè Gomara, nè Torquemada; ma fi fcorge chiaramente da un luogo della lettera fcritta da Cortès a Carlo V. l'anno 1522. Herrera, e Solis ne fanno pur menzione, ma frammitchiano alcune circoftanze oppofte al vero. Dicono, che prima andarono gli Spagnuoli a Tezcuco, e poi a Cuitlahuac, nel che moftrano aver ignorato la fituazio- 
da $i$ due Principi, e da molta Nobiltà Acolhua tra un im. menfo concorfo di popolo. Fu alloggiato con tutto il fuoLie.VII. Efercito nel palazzo principale del $\mathrm{Re}$, dove il trattamento della fua perfona fu corrif́pondente all' albergo. Quivi gli efpofe il Principe Ixtlilxochitl i fuoi pretef diritti al regno d'Acolhuacan, e le fue querele contro il fuc fratello Cacamatzin, e contro il $\mathrm{Re}$ di Meffico fuo Zio. Cortès gli promife di metterlo in poffeffo della corona, fubiso che aveffe conchiufe le fue negoźiazioni in Meffico, e fenza trattenerfi in quella Corte, marciò verfo Iztapalapan. (N)

Era Iztapalapan una grande e bella Città fituata verfo la punta di quella piccola penifola, che v'è tra i due la- 5. $34^{\circ}$ ghi, quello di Chalco a Mezzogiorno, e quello di Tezcuco degli a Tramontana. Andavafi da quefta penifola alla ifoletta di SpagnuoM ffico per una itrada felciata lunga più di fette miglia, e palapan., fatta ful lago molti anni addietro. La popolazione d'Iztapalapan era allora di più di dodici mila cafe fabbricate per la maggior parte in parecchie ifolette affai vicine tra loro, e contigue alla fteffa penifola, preffo alle quali $v^{\prime}$ erano innumerabili orti e giardini galleggianti. Reggeva allora quefta Città il Principe Cuitlahuatzin fratello di Motezuma, ed.immediato fucceffor di lui nella corona di Meffico, il quale infieme coll' altro fuo fratello Matlatzincarzin, Signor della Citrà di Cojohuacan, accolfe Cortès colle-medefime dimoltrazioni adoperate già dagli altri Signori, per li luoghi de' quali era paffato. Complimentollo con un' aringa beK 2

ne

ge di que'luoghi. Affermano altresì, che Cacamatzin accompagnò Cortès - Tezcuco; ma ci confta non effer ciò vero e per la relazione di Cortès, e per ti manofcritti antichi cirati da D. Ferdinando d'Alba Ixtlilxochitl. Niente dicono della riconciliazione di que'due Principi?', nè efprimono il motivo, che ebbe Cortès per andare a Tezcuco fviandof dalla frada, che conduceva a Meffico. Io adotto in cid che riguarda le circoftanze del viag. gio a Tezcuco il ragguaglio di Betancurt, il quale foriffe fulle memorie d'Alba, e di Siguenza.

(N) Uno ftorico Indiano citato de D. Ferdinando d'Alba Ixtlilxochit dice, che in quefta uccafione fu battezzato il Principe Ixtlilxochitl con altri dugento Nobili di quella Corte; ma quefta una favola tanto inverifimile, che non ha bifogno d'impugnazione. 


\section{6}

ne intefa, ed alloggio lui e tutte le truppe, che l'accompagnava-

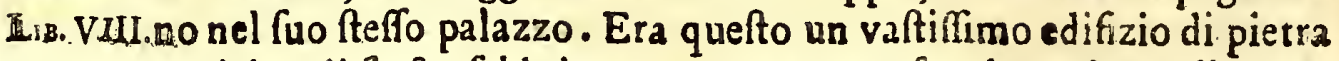
e calcina di frefco fabbricato, e non ancora fornito. Oltre alle mol. te fale, e Itanze comode per l'abitazione, il cui tetto era di cedro, e le mura coperte di vaghi tappeti di bambagia, ed oltre a' gran: di cortili, dov' ebbero il loro quartiere le truppe alleate degli Spagnuoli, aveva un giardino di forprendente grandezza ed amenità, defcritto già da noi , là dove ragionammo dell' Agricoltura de' Mefficani. Dopo definare conduffe il Principe i fuoi Ofpiti a quefto giardino, nel quale fi ricrearono affai, e vi for: marono una grande idea della magnificenza meficana. In quefta. Città offervarono gli Spagnuoli, che in vece delle: querele, e delle mormorazioni, che altrove aveano fentito, altro non fentivafi che lodi del governo, perchè la vicinanza della Corte gli rendeva più cauti nel parlare.

Il di vegnente ben prefto marciarono gli Spagnuolipes quella grande frada, che univa, come abbiamo detto, Iztapalapan con Meffico, la quale era tagliata con fette piccoli canali pel paffaggio delle barche dall' un lago all' altro. fopra effi v' erano de'ponti di travi per la comoditz̀ de' paf: feggeri, le quali levavano facilmente ogni volta che volevano impedire il paffo a' nemici. Dopo effere paffati per Mexicalszinco, ed aver vedure Colhuacan, Huitzilopochco, Cojohuacan, e Mixcoac, Città tutte fondate fulla riva del lago, arrivarono tra una folla incredibile: di. Popolo ad un luogo ap pellato Xoloc, dove s'univa a quefta ftrada quella di. Cojohuacan. Nell'angolo, che formavano quefte due ftrade non: più d' una mezza lega difcofto dalla Capitale, v' era un buon: baluardo con due torricelle circondato da una muraglia alta più di dieci piedi col fuo parapetto merlato, con due ufcj, e con un ponte levatojo:- luogo memorabile nella toria del Meffico per cagione d' effervs ftato il campo del Generale. Spagnuolo nell' affedio di quella gran Citta. Quivi fece alto: l'efercito per ricevere i complimenti di più di mille Nobili mefficani, turti uniformemente veftiti, $i$ quali nel paffar diranzi al Generale Spagnuolo gli facevano un inchino colo 
la folita ceremonia di toccar la terra, e baciarfi la mano.

Terminati quefti complimenti, ne' quali fi trattenneroLis.VHI. più d'un'ora, continuarono gli Spagnuoli il loro cammino cosł bene ordinati, come s' andaffero a dare una battaglia. \$. 35. Poco prima d'arrivar alla Cirta ebbe Cortès l'avvilo, che degliSpaveniva ad incontrarlo il Re di Meffico, ed indi a poco fi gnuoli in lafcid vedere con un ragguardevole e numerolo accompagna. Mefico, mento. Precedevano tre Nobili portando in mano bacchet te frazioni d'oro innalzate, come infegne della Maeftà, colle quali era del Re, avvertito il Popolo della prefenza del Sovrano. Veniva Mo. Nobiltà, tezuma riccamente veftito fopra una letriga coperta di lamine d'oro, che portavano quatro Nobili fulle loro fpalle, e fotto un'ombrella o parafole di piume verdi fregiate di parecchj lavori d'oro. Portava pendente daøli omeri un man. tello ornato di ricchiffimi giojelli d'oro, e di gemme, nella tefta una corona fottile dello fteffo metallo, e ne' piedi delle fuole ancor d'oro legate con iftrifcie di cuojo tempeftate d' a ro e di gemme. Accompagnavanlo dugento Signori affai meglio veftiti degli altri Nobili, ma tutti fcalzi, a due a due, ed accoltati dall' una e dall' altra parte alle muraglie delle cafe per moltrar il rifpetto, che portavano al loro Sovrano. Tofto che fi videro il $\mathrm{Re}$, ed il Generale Spagnuolo, fmontarono tutti e due, quefti dal cavallo, e quegli dalla lettiga, e cominciò a camminar appoggiato nelle braccia del $\mathrm{Re}$ di Tezcuco, e del Signor d' Iztapalapan. Cortès dopo aver fatro al $\mathrm{Re}$ un profondo inchino, s'accolto a lui per mettergli al collo un cordonetto d"oro, nel quale erano infilza. te delle pallottoline di vetro, che fembravano gemme, ed il Re chinò il capo per riceverlo; ma volendo Cortès abbracciarlo, non gli fu permeffo da que' due Signeri, che faceano da braccieri. (O) Dichiarogli il Generale in una bre.

ve

(O) Solis nel ragguaglio di quell'incontro ha quattro sbagli. r. dice, che il prefente di Cortès fu una banda, ovvero un catena di vetro. 2. che que' due Signori che accompagnavano Motezuma, non permifero a Cortès il. mettergli al collo la banda. 3. che ciò fecero con qualche fdegno. 4. che però furono riprefi daì Re. Tutto ciò è falfo, inventato a capriccio, ed oppotto al ragguaglio del medefimo Cortès. 
ve aringa, come il richiedevano le circoftanze, la fua beneLis.VIII.volenza, il fuo rifpetto, ed il piacere che aveva d'effere arrivato a conofcere sì gran Monarca. Motezuma gli rifpofe in poche parole, e fatta la folita ceremonia di toccar la terra e baciarfi la mano, gli ricompensò il prefente delle pallottoline di vetro con due collane di belle nacchere, dalle quali pendevano alcuni gamberi grandi $d^{2}$ oro fatti al naturale. Incaricò il Principe Cuitlahuatzin di condurre Cortès all' alloggio, ed egli ritornò col Re di Tezcuco.

Tanto la Nobiltà, quanto l'immenfo Popolo, che da" terrazzi, dalle porte, e dalle finettre delle cafe offervava tutto cid che avveniva, erano maravigliati e sbalorditi non meno per la novità di tanti oggetti ftraordinarj a' lor occhj prefentati, che per la inaudita degnazione del loro Re, la qua. le contribul affai ad ingrandire la riputazione degli Spagnuoli. Quelti marciavano pieni ancor di maraviglia dal vedere la grandezza della Città, la magnificenza degli edifizj, e la moltitudine degli abitanti, per quella grande e larga ftrada, che fenza fcoftarfi punto dalla rettitudine, continuava quella d'Iztapalapan fabbricata ful lago fin'alla porta meridio. nale del tempio maggiore, alternandofi nei loro animi coll ${ }^{2}$ ammirazione il timore della loro forte, vedendofi cosi pochi nel centro d' un Regno ftraniero. Cosir camminarono un miglio e mezzo dentro la Città infin'al palazzo ch' era già ftato del $\operatorname{Re}$ Axajacatl, non guari difcofto dalla porta occidentale del fuddetto tempio deftinato per loro albergo. Quivi gli alpettava Motezuma, il quale però fe n'era andato innanzi .. Allorchè arrivò Cortès alla porta di quel palazzo, prefolo il Re per la mano l' introduffe in una gran fala, lo, fece federe in una predella fimile nella forma a quelle de' noltri altari, e coperta d'un vagó tappeto di bambagia, ed appreffo ad una muraglia, coperta ancor effa d'un tappeto fregiato d'oro e di gemme, e congedandofi cortefemente gli diffe: "Voi co' voftri compagni liete adeffo nella voftra propria ca"fa: pranzate e ripolatevi, ch'io frappoco ritornerò." Andoffene il $\operatorname{Re}$ al fuo palazzo, e Cortès fece fubito fa. 
re una falva di tutta l'artiglieria per impaurire con sì fatto ftrepito i Mefficani. Frattanto andò a vedere tutte le ftanzeLis.VIII. del palazzo per allogar la fua gente. Era sì grande quefto edifizio, che vi s'alloggiarono comodamente e gli Spagnuoli, ed $i$ loro Alleati, $i$ quali infieme colle donne e colla fervitù, che quelli feco loro conducevano, trapaffavano i fette mila. Da per tutto v'era una gran pulizia: quafi tutte le ftanze aveano letti di ftuoje di giunco, e di palma, giufta la loro ufanza, con altre ftuoje arrotolate per guanciali, e fotto cieli di bambagia, e fcranne fatte d'un folo pezzo. Alcune ftanze aveano il pavimento coperto di ftuoje, e le muraglie parimente coperte di tappeti di cotone di varj colori. Le mura erano affai groffe, ed in certe diftanze v'erano alcune torri. Sicchè gli Spagnuoli vi trovarono quan. to ne potevano bramare per la loro ficurezza. Il diligente e cauto Generale diftribuil fubito le guardie, formò una batterìa de' fuoi cannoni dirimpetto alla porta del palazzo, ed adoperò tanta diligenza per fortificarfi, come fe quelio fteffo giorno afpettaffe qualche affalto da' fuoi nemici. Imbandifft quel giorno a Cortès ed a' fuoi Capitani un magnifico pranzo fervito dalla Nobilì̀, e pel refto dell' efercito fi portarono diverfe e copiofe vivande, benchè d'inferior qualità. Quefto di tanto memorabile non meno per gli Spagnuoli, che per li Mefficani fu li 8. Nov. I519. dopo fette mefich' erano arrivati gli Spagnuoli nel paefe d'Anahuac.

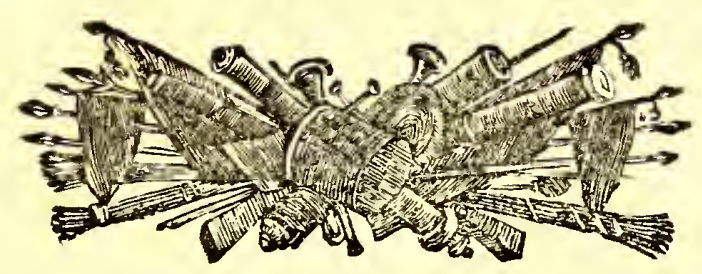


Bo

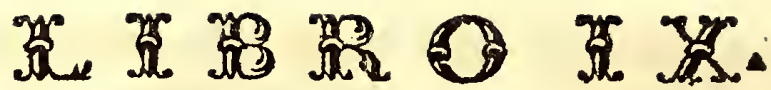

Conferenze del Re Motezumacol Generale Spagnuolo. Imprigio. namento del Re di Meffico, e d' Acolbuacan, e d'altri Signori. Supplizio atroce di Quaubpopoca. Tentativi del Governatore di Cuba contro Cortès, e fconfitta di Panfilo Narvaez. Uccifrone di molti Nobili, e follevazione del Popolo mefjicano contro gli Spagnuoli. Morte del Re Motezuma. Combattiz menti, pericoli, e fronfitta degli Spagnuoli. Battagliad'O. tompan, e ritirata degli Spagnuoli in Tlafcalla. Elezione del $R e$ Cuitlabuatzin. Vittoria degli Spagnuoli in Tepejacac, in Xallatzinco, in Tecamacbalco, ed in Quaubquechollan. Strage fattavi dal vajuolo. Morte del Re Cuitla. buatzin, e de' Principi Maxizcatzin, e Cuicuitzcatzin. Elezione in Mefjico del Re Quaubtemotzin.

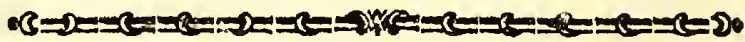

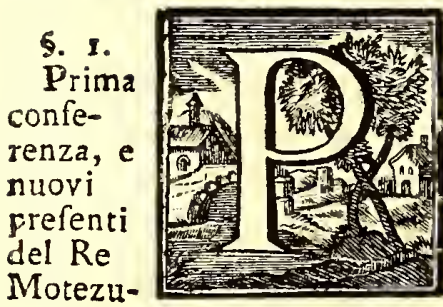

Oichè gli Spagnuoli ebbero pranzato, ed or: dinato tutto cid, che alla ficurezza loro conveniva, tornò a vifitargli il $\operatorname{Re}$ accompagna. to da molta Nobiltà. Cortès venne ad incontrarlo co' fuoi Capitani, e tutti e due accoppiati entrarono nella fala principale, dove prontamente apparecchiarono un' altra prema.

della accanto di quella del Generale Spagnuolo. Il $\operatorname{Re}$ gli prelentò molti lavori curiofi d'oro, d'argento, e di penne, e pì̀ di cinque mila vefti finiffme di bambagia. Mefofi poi a federe fece anche federe Cortès, reftando tutti gli altri in piedi. Cortès gli proteftò con grandi efpreffioni la fua gratitudine, e volendo continuare il fuo difcorfo, l'interrup: pe Morezuma con quefte parole.

"Prode Generale, e voi fuoi compagni, tatti i miei ") dimeftici e cortigiani fono teftimonj del piacere, che ho " avuto del voftro felice arrivo a queita Corte: e fe finora " avea fatto fembianza di ripugnare, ciò è ftato folamente 》) per 
per condifcendere ai miei fudditi. La voltra fama ha aggranditi gli oggetti, e turbati gli animi. Dicevafi, che Liz. LX. eravate Dei immortali, che venivate montati fopra fiere di portentofa grandezza e ferocia, e che vibravate dei fulmini, col quali facevate tremar tutta la terra. Altri fpacciavano, che eravate moftri rigettati dal mare: che la fame in la- $^{-}$ ziabile dell'oro v'avea fatto abbandonar la voltra patria : che eravate troppo portati per le delizie, e si ghiotti, che uno di voi mangiava tanto, quanto dieci dei noftrali. Ma tutti quefti errori fi fon dileguati colla pratica, che hanno avuto con voi $i$ miei fudditi. Già fi fa, che fiete uomini mortali come noi, benchè alquanto differenti nel colore e nella barba. Ábbiamo già veduto coi noftri occhj, che que. fte fiere tanto rinomate altro non fono, che cervi più corpulenti dei noftri, e che i voltri pretefi fulmini non fono altro, che una fpezie più bene intefa di cerbottane, le cui palle fi fpingono con maggiore itrepito, e fanno maggior male. Quanto poi alle voltre qualità perfonali fiàno bene informati da coloro, che vi hanno praticato, che fiete benigni, e generofi, che tollerate pazientemente $i$ difagi, che non adoperate il rigore; fe non contra coloro, che provocano il veltro fdegno colle loro oftilità, nè vi fervite dell'armi, fe nun per la giufta diféa delle voftre perfone. "

"Io non dubito, che voi fimilmente avrete già cacciate dalla voftra mente, ovvero tolto caccierete quelle falfe idee, che vi ha fatto concepire l'adulazione dei miei vaf. falli, o la malevolenza dei miei nemici. Vi farà pure chi vi abbia detto, che io fon uno degli Dei, e che prendo a mio fenno la forma di Leone, o di Tigre, o di qualche altro animale; ma già vedete (in dicendo quefto pighò colle dita la pelle del fuo braccio) che fono di carne ed offa, come gli altri mortali, benchè più nobile per la mia na. fcita, e più poffente per l'altezza della mia dignità . I Cempoallefi, i quali colla voftra protezione fi fon fottratti dalla mia ubbidienza ( $m a$ non refterà impunita la loro ribellione) vi avranno fatto credere, che le mura ed i tet; Storia Antica del Meffico Tom. III. L L ti, 
" ti de miei palazzi fon d' oro, ma i voftri propri occhj polLIs. IX. " fono fmentirli. Quefto è uno de'miei palazzj, e già ve") dete, che le mura fono di pietra e calcina, ed i tetti di " legno. Io non negherd, che fieno grandi le mie ricchez"ze ; ma le fanno maggiori l'efagerazioni de' miei fudditi. "A Alcuni fi faranno lagnati preffo voi della mia crudelta $e$ ") tirannìa ; ma egli chiamano tirannia l'ufo legitrimo della " fuprema autorità, ed appellano crudeltà la neceffaria fe: 9) verità della giuftizia.."

"Depofto dunque e da noi, e da voi qualunque fvana 9) taggiofo concetto cagionato dalle falfe informazioni, io ac", cetto l' ambafciata di quel gran Re, che vi manda, ho ca"ra la fua amicizia, ed offerifco a fua ubbidienza tutto il 9 mio Regro; poichè attefo i fegni, che abbiamo affervati "nel Cielo, e ciò che veggiamo in voi, ci pare ormai ar"rivato il tempo, che fi compiano gli oracoli de' noftrian. "'tenati, cioè, che doveffero venire dalla parte di Levante " certi uomini diverfi da noi negli abiti, e ne' coltumi, i qua"2 li dovrebbono efser Signori di tutti quefti paefi ; imperoc-) chè noi non fiamo originarj di quefta terra: non fono moly) ti anni, che $i$ noftri antenati vennero quì dalle regioni fet, tentrionali, e noi non abbiamo finora retti quefti popoli, fe "non come Juogotenenti di Quetzalcoatl noftro Dio e legit: "timo :Signore. "

Cortès rifpofe ringraziandolo affai de'fingolari benefizj fin' allora da lui ricevuti, e per l'onorevole concetto, che s'e. ra formato degli Spagnuoli. 'Dilsegli, ch' era inviato dal mag. gior Monarca dell' Europa, il quale avvegnachè potelse afpirare a qualche cofa di più, come quegli che era difcendente di Quetzalcoatl, tuttavia fi contentava di ftabilire una confederazione, ed amicizia perpetua con fua Maeftà, e con i fuoi fuccefsori : che il fine della fua ambafciata non era quel. lo di torre altrui ciò che pofsedeva, ma quello bensì d'annunziarli la vera Religione, e di communicargli alcuni avvifi importanti per migliorare il governo, e rendere felici i fuoi vafsalli: ciò che farebbe in un' altra occafione, fe fua 
Maeftà degnaffe afcoltarlo. Accettollo il Re, ed effendofi in. formato del grado e della condizione di ciafcheduno degli Lis. IX. Spagnuoli, fi congedò, ed indi a poco mandò loro un gran regalo, cioè per ciafcuno dei Capitani cerri lavori d'oro, e tre balle di veftr preziofe di penne, e per ciafcun Soldato due balle di vefti di bambagia. Quefto felice principio avrebbe potuto afficurar agli Spagnuoli la poffeffione pacifica di quella vafta Monarchia, fe fi foffero condotti più dalla prudenza, che dal coraggio. (a)

Il dì vegnente volendo Cortès rendere la vilita al Re, s. z. gli mandò a dimandar udienza, e l'ottenne sì prontanente, Vifitadì che coloro che gli portarono la rifpofta, furono gli fteffi in. Cortès troduttori degli Ambafciatori, che doveano condurlo, ed in. al Re. fruirlo nel ceremoniale di quella Corte. Veftiffl Corrès del. la più sfarzofa gala, che avea, e conduffe feco i. Capitani Alvarado, Sanduval, Velafquez di Leon, ed Ordaz, ed in. fieme cinque Soldati a Portaror fi. al Real palazzo tra un'im. menfa folla di popolo, e tolto che giunfero alla prima porta, coloro che gli accompagnavano s'ordinarono in due file, una da ogni banda; poichè l'entrarvi affollati. fi ftimava man. canza di rifpetto alla Maefià o. Dopo effer paffati per tre cortili ed alcune fale all'ultima anticamera per arrivare: alla fala d'udienza, furona quivi cortefemente accolti da parecchj Signori, che facevano la guardia, e coftretti a fcalzarfi, ed a coprirfi gli abiti sfarzofi con velti groffolane. Allorchè entrarcno nella fala d' udienza, il Re fece alcuni paffi verfo Cortès, e prefolo per la mano, e volgendo amorevolmente lo fguardo agli altri, gli fece tutti federe. Il ragionamento

L. 2.

fus

(a) Il dottu, e giudiziofo P. Acona ragionando di quefta prima conferenza di Motezuma nel lib. VII. cap. 25 . della fua Storia dice cosi., Mol$\Rightarrow$ ti fon di parere, che attefo lo ftato delle cofe in quelprimo giomo, fareb"be Aato agerole- agli. Spagnuoli il fare del Re e del Regno tutto ciò che "aveffero voluro, e cominicar loro. la legge di Gesì. Crifto con gran pa"ce, e contento di tutti; ma i gindizi di Dio fono profondi, e molti "erano i peccati di amendue le parti : onde avvenne altrimenti, benchè " alla fine compi il Signore il fuo. difegno di far mifericordia a quella "Nazione dopo arere giudicato e punito coloro, che preffo lui lo meri, tarano. ", 
fu lungo e fopra diverfi foggetti. Il Re fece parecchie diEab. IX mande, tanto ful governo politico, quanto fulle cole naturali di Spagna, e Cortès dopo avergli in tutto foddisfatto $s^{\prime}$ introduffe fcaltramente in materie di Religione. Gli efpofe. l' unità di Dio, l'Incarnazione del Verbo divino, la creazione del Mondo, la feverità del giudizio di Dio, la gloria, con cui premia i giufti, e le pene eterne, a cui condanna i peccatori. Indi ragionò de' riti del Criftianefimo, e particolarmente dell' incruento facrifizio della Meffa per farne il paragone cogl' inumani facrifizi- de' Mefficani, declamando fortemente contra la barbara crudeltà d'immolar vittime uma. ne, e di cibarfi della loro carne . Motezuma rifpofe, che intorno alla creazion del Mondo erano d'accordo; poichè quello fteffo, che avea detto. Cortès, l'aveano già intefo da' loro maggiori : che del refto s'era già informato da' fuoi Ambafciatori della Religione degli Spagnuoli. \# Io per ali) tro, foggiunfe, non dubito della bontà del Dio, che ado" rate; ma s' egli è buono per la Spagna, i noftri Dei foפ) no parimente buoni pel Meffico, ficcome ce l' ha fatto " vedere la fperienza di tanti fecoli. Voi pertanto rifparmia. ") tevi la fatica di volermi indurre a lafciare il loro culto. "Quanto poi a' facrifizj io non fo perchè fia da biafimarfi il "facrificar agli Dei quegli uomini, che o da' proprj lor de. " litti, o dalla loro forte nella guerra fono già deftinati al"la morte." Ma benchè non riufciffe Cortès "nel perfuadergli la verità della Religione Criftiana, ottenne pure, per quel che dicono, che non s'imbandiffe più nella real menfa della carne umana, o perchè colle ragioni di Cortès fi deftaffe nella fua mente il ribrezzo naturale di si fatta vivanda, o pure perchè voleffe almeno in ciò compiacer gli Spagnuoli. Fece altresi fpiccar in quefta occafione la fua regia beneficenza verfo loro, regalando a Cortès ed ai quat. tro Capitani parecchj lavori d' oro, e dieci balle di vefti fine di bambagia, ed a ciafcun Soldato una collana d'oro.

Ritornato Cortès al fuo quartiere (cos̀ chiameremo da ora innanzi il palazzo del Re Axajacatl, dove s'alloge 
giarono gli Spagnuoli) comincid a riflettere al pericolo, in cui fi trovava nel centro d'una Città tanto forte e popolo. Lis. IX, fa; e rifolvette di conciliarli gli animi della Nobiltà con una buona condotta, e con maniere offequiofe, ed amorevoli, ed ordinò alla fua gente, che in tal maniera fi regolaffe, che non poteffero lagnarfi di loro i Mefficani; ma mentre che pareva badare colla maggior diligenza alla pace, rivolgeva nella fua mente de' penfieri arditi e temerarj, alla pace aftatto contrarj, ed effendogli d'uopo per maturarli l' informarfi prima co'fuoi occhj delle fortificazioni di Mef́fico, e delle forze de' Mefficani, addimandò al $\operatorname{Re}$ il permeffo di vedere i palazzi Reali, il tempio maggiore, e la piazza del mercato. Accordollo benignamente il Re, come quegli che nulla fofpettava dello fcaltro Generale, nè previde le confe. guenze della fua troppa indulgenza. Videro pure gli Spagnuoli tutto ciò che vollero, trovando da per tutto abbondante materia d'ammirazione.

Era allora la Città di Meffico fituata, come abbiamo già detto, in un'ifoletta del lago di Tezcuco quindici mi. zione glia a Ponente da quella Corte, e quattro a Levante da della Citquella di Tlacopan. $\left(^{*}\right)$ Si paffava dal continente all'ifolet-Miafico. ta per tre grandi ftrade di pietra e terra fabbricate a bella pofta full'ifteffo lago, quella d'Iztapalapan a Mezzogiorno lunga più di fette miglia, quella di Tlacopana Ponente due miglia in circa, e quella di Tepejacac a Tramontana di tre miglia (b): e tutte tre cosi larghe, che vi potevano andare accoppiati dieci uomini a cavallo. Oltre a quefte tre ftrade

ve

$\left(^{*}\right)$ Nella carta geografica fi rapprefentano per isbaglio più vicine fraloro quefte Città.

(b) Il Dott. Robertfon mette in vece della Atrada di Tepejacac quella di Tezcuco, la quale nel luogo dove defcrive Meffico, fitua verfo Maeftro, e dove parla de'pofti dell' armata Spagnuola nell'affedio di quella Capitale, verfo I evante, avendo già detto che verfo Levante non v'era frada veruna ful lago; ma non vi fu, nè potè mai efsere ftrada veruna ful lago da Mefíco a Tezcuco a cagione della fomma profondità del fuoletto in quella parte, e cafo che vi forse ftata, non farebbe di tre fole miglia, come afferma quefto Autore, ma di quindici, quanto è lo fpazio del lago frapposto. 
ve n'era un'altra alquanto più ftretta per li due acquidotti Lis. IX di Chapoltepec. L'ambito della Città, non comprefi i fobborghi, era di più di nove miglia, ed il numero delle cafe almeno di feffanta mila. (c) Era divifa la Citta in quattro quartieri, e ciafcun quartiere in parecchie contrade, $i$ nomi mefficani delle quali fi confervano finora preffo gl' Indiani. Le linee diviforie dei quattro quartieri, erano le quattro larghe Atrade corrifpandenti alle quattro porte dell'atrio del tempio. maggiore. Il primo quartiere appellato Tecpan (og. gidi S. Paolo. ) comprendeva tutta queila parte, che v'era tra le due ftrade corrifpondenti alle porte meridionale, ed orientale. Il fecondo Mojotla (oggidi. S. Giovanni.). tra le Itrade meridionale ed occidentale. Il serzo Tlaquechiubcan (oggidi Santa Maria) tra le ftrade occidentale e fettentrionale, ed il quarto Atzacualco (oggidis S. Sebaltiano) tra le ftrade fettentrionale ed orientale. A quefte quattro parti nelle quali fu divifa quella Città infino dalla lua fondazione s'aggiunfe, come una quinta parte, la. Città di Tlatelolco fituáta a Maeftro, reftando dopo la conquifta del Re Axajacatl unita a quella di Tenochtitlan, e compofta di tutte due quella di Meffico.

V'era.

(c) Torquemada afferma, che la popolazione della Capitale era di r 20.000. cafe; ma il Conquiftatore anonimo, Gomara, Herrera, ed altri Storiciconvengono nel numero di 60: 000 . cafe, non già di, 60. 000. abitanti, coine dice Roberton; poichè non vi fu Autore antico, che la Atimaffe sì piccola. E' vero che nella traduzione italiana della relazione del conquifta-

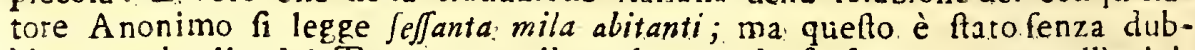
bio uno sbaglio del Traduttore, il quale avendo forfe, trovato nell'originale fefanta mil Vecinss tradufie. 60. 000 . abitanti, dovendo dire. 60. 000. fuocbi; poichè altrimenti fi direbbe, che Cholollan, Xochiınilco, Iztapalapan, ed altre si fatte Citta erano più grandi di quella di Meffico. Ma nel fuddetto numero non fi comprendono le cafe de'fobborghi. Ci confta rer la tefimonianza d'Herrera e di. Bernal Diaz, che verfo Ponente fi continuavano le cafe all' una, ed all' altra parte della frada di Tlacopan infin' al Continente, cioè per uno fpazio di 2. miglia. Verfo. Libeccio. v'era il fobborgo di Aztacalco, verfo Mezzogiorno quelli d'Acatlan,, di, Malcuitlapilco, d'Atenco, e d'Iztacalco, e verfo Maefto. Zancopinca, Huitznahuac, Xocotitlan, Coltonco, ed altri. E'da crederfi, che Torquemada voleffe contar anche le caf́e de' fobborghi; ma con tutta cio mi pare eccéhivo il numero di 120.000. cale. 
V'erano attorno alla Città molti argini, e chiule per trattener l'acque, quando era d'uopo, e dentro d'effa tanti La r. IX. canali, che appena v'era contrada, dove non fi poteffe andar in barca: cid che non meno contribuiva ad abbellir la Città, ed a render più agevole il trafporto delle vettova. glie, e di tucte l'altre derrate del loro commercio, che ad afficurar vieppiù i Cittadini contro a' tentativi de' loro ne. mici. Le ftrade principali erano larghe e dritte. Quanto all' altre alcune erano meri canali, dove non fi poteva andar altrimenti che in barche, altre erano felciate, e fenza verun' ac. qua, ed altre aveano un canaletto tra due terrapieni, i quali fervivano alla comodità de'viandanti, ed allo fcarico delle barche, o pur erano giardinetti, con alberi piantativi, e fiori. Per ciò che riguarda gli edifizj, oltre a' molti tempj ed a' magnifici palazzi Reali, di cui abbiamo altrove ragionato, v' erano pure altri palazzi o cafe grandi, che aveano fabbricato i Signori Feudatarj per loro abitazione in quel tempo, nel quale erano obbligati di rifedere nella Corte. Sopra tutte le cafe, fuorchè fopra quelle de' poveri, v' erano de' terrazzi co' loro parapetti, ed in alcune ancora de' merli, e delle torri, benchè affai più piccole di quelle de'tempj: ficchè i Mefficani provvidero alla loro difefá non meno nelle ftra. de, e nelle cale, che ne' tempj.

Oltre alla grande e rinomata piazza di Tlatelolco, do. ve fi faceva il principal mercato, v' eranc altre piazzette di. ftribuite per tutta la Città, dove fi vendevano $i$ viveri ordi. narj. V'erano altresi in parecchj luoghi delle fontane, e de' vivaj, maffimamente preffo a' tempj, e molti giardini parte piantati al livello della terra, e parte in alti terrazzi.

1 molti, e grandi edifizj pulitamente imbiancati e forbiti , l'alte torri de' tempj fparti per li quartieri della Città, i canali, l'alberece, ed i giardini formavano un compleffo tanto bello, che gli Spagnuoli ron parevano mai paghi di vederlo, maffimamente allorchè l'offervarono dall'atrio fuperiore del tempio maggiore, il quale, non che la popolazione di quella Corte, dominava ancora $i$ laghi, e le belle e gran- 
di Città che v' erano all' intorno. Non meno maravigliati reftarono nel vedere i palazzi Reali, e la forprendente varieı̀ di piante e d'animali, che vi s'allevavano; ma niun'. altra cofa empl di maggior ftupore i loro animi, che la gran piazza del mercato. Non vi fa Spagnuolo, che non la celebraffe con fingolari lodi, ed alcuni di loro, che aveano viaggiato per quafi tutta la Europa, proteftarono, ficcome ne fa fede Bernal Diaz, di non aver mai veduta in veruna piazza del Mondo nè un sì gran numero di negozianti, nè tanta varietà di mercanzì, nè un sì bell' ordine e difpofizione in tutto.

9. '4.

Allorchè gli Spagnuoli falirono in ful tempio maggio:

Sfoghi re, vi trovarono il Re, il quale s'era anticipatanente pordel zelo tato colà per impedire coll!a fua prefenza qualuaque attendicortès tato, che far fi voleffe contra $i$ fuoi $D$ i. Dopo aver offer. ligione. vata ftimolatine dal Re medefimo da quella alrezza la Città, Corrès gli domandò permaffo di vedere i Santuarj, ed egli l'accordò dopo aver confultati i Sacerdoti. Entraronvi gli Spagnuoli, e contemplarono non fenza compaffione e ribrezzo la cecità di quei popoli, e l'orrenda ftrage, che in loro faceva la crudele fuperftizione dei loro facrifizj. Cortès allora volgendofi al $\mathrm{Re}$ gli diffe:" Mi maraviglio, Signo" re, che un Monarca tanto favio come fiete voi, adori " come Dei quelte abominevoli figure del Demonio.." "Se " io aveffi faputo, rilpofe il $\mathrm{Re}$, che dovevate fparlar con "si fatto difpregio dei noftri Dei , nun mi farei mai pie"gato alle voltre domande." Cortès vedendolo tanto fde. gnato gli addimandò $\int_{c u f a}$ e fi licenzio per riportarfi al quartiere., Andate pur in pace, diffe il $\mathrm{Re}$, che io refte" rò quì per placar la collera dei noftri Dei provocata colle is voftre beftemmie.",

Malgrado quelto difgufto ottenne Cortès dal Re non folamente il permeffo di fabbricare dentro il recinto del quartiere una cappella ad onor del vero Dio, ma ancor gli operaj ed i materiali per la fabbrica, nella quale fi celebro la Santa Meffa, finchè vi fu del vino, e giornalmente vi con.

corre- 
revano i Soldati a far le loro divozioni. Piantò altresi nel principal cortile una gran Croce, acciocchè vedeffero i Mef- Liz. IX. ficani la fomma venerazione, in cui era preffo gli Spagnuo. li quel fanto ftrumento della noftra redenzione. Voleva inoltre confacrare al culto del vero Dio lo fteffo fantuario d' Huitzilopochtii, ed allora fu trattenuto dal rifpetto del $\mathrm{Re}$ e de' Sacerdoti; ma l' efegul alcuni mefi dopo, effendofi ac: quiltata una maggior autorità coll' imprigionamento del $\mathrm{Re}_{3}$ e con altre azioni non meno temerarie, che oramai vedremo. Spezzò gl'Idoli, she vi fi veneravano, fece ripulire ed acconciare il fantuario, vi collocò un crocif́ffo, ed una immagine della Madre di Dio, (d) e meflo inginocchione innanzi a quefte facre immagini ringraziò l' Altiffimo d' aver loro accordato di adorarlo in quel luogo gia per tanto tem. po deftinato alla più abominevule, e crudele idolatria. Quefto medefimo zelo per la Religione l' induffe a replicar fpeffo al $\mathrm{Re} i$ fuoi ragionamenti fulle fantiffime verita del Criftanefimo; ma quantunque Motezuma non folfe difpolto ad ab. bracciarle, nondimeno moffo dalle ragioni di lui comandò, che d'allora innanzi non fi facrificaffero più vittime umane; e benchè non compiaceffe al Generale Spagnuolo nel rinunziar alla idolatria, lo carezzava ognora, e non v' era giorno, nel quale non faceffe nuove finezze e regali agli $S p a-$ gnuoli. L'ordine, che diede il Re a'Sacerdoti intorno a' facrifizj non fu coftantemeate offervato; e quella grand'armo. nì, che v'era ftata fin'allora, fi turbò per l'inaudito atten. tato del Generale Spagnuolo.

Non erano fcorfi più di fei giorni dopo l'entrata degli Spagnuoli in Meffico, allorchè Cortès trovandofi ifolato in Storia del Mefjico Tom. III.

$\mathrm{M}$

mez-

(d) L' immagine della Madre di Dio, che collocò Cortès nel fantuario d' Huitzilopochtli credefi effere fata quella medefima, che prefentemente fi venera col titolo della Madonna de los Remedios, o fia del Soccorfo, in un magnifico tempio difcolto otto miglia in circa dalla Capitale verfo Ponente. Si dice, che la portò feco al Mefico un Soldato di Cortès appellato Villafuerte, e che il di apprefro quella terribil notte, neila qua!e furono fconfitti gli Spagnuoli, ei la nafcofe nel luogo, dove alcuni anni dogo fu ritrovata, cioè in quello fteffo, dove oggidì è venerata: 
mezzo ad un immenfo popolo, e conof́cendo quanto perico:
LAB. IX. lo correrebbe la loro vita, fe mai fi cangiaffe, come potreb5. 5. be avvenire, l'animo del $\mathrm{Re}$, fi perfuafe, che non v'era Imprigio-altro configlio da prenderfi per la loro ficurezza, che quello namento d'impadronirfi della fteffa perfona del $\mathrm{Re}$; ma effendo un Motezu. tal configlio tanto ripugnante alla ragione, la quale da lui ma. efigeva ed il rifpetto alla Maeltà di quel Monarca, e la gratitudine alla fua beneficenza, cercò de' pretelti per acchetar la fua colcienza, e per mettere il fuo onore al coperto, (e) e non ne trovò altro più confacente di quello delle rivoluzioni della Veracroce, la nuova delle quali avuta già in Chololla riferbata avea nel petto fin' allora; ma volendo ora fervirfene, la palesò a' fuoi Capitani, acciocchè feriamente penfaffero a ciò che far converrebbe per fottrarfi da sì gravi pericoli : e per giuftificar vieppiù il fuo attentato, e muo. vere i fuoi Spagnuoli ad efeguirlo, fece chiamar parecchie perfone principali de' fuoi alleati, ( la cui informazione dovreb.

(e) Che l' intento di Cortès forfe quello d' impadronirfi di Motezuma in qualunque maniera fi folfe, e che le rivoluzioni della Veracroce non forfero altro che un mero pretefto per mettere in opera il fuo difegno, confta evidentemente dalla fua lettera a Carlo V.de' 30. Ottobre I 520. ", Paf" fados, invictiflimo Principe ( dice nel cap. 23. di quelta lettera, o fia , ragguaglio) feis dias defpues que en la gran Ciudad de Temiftitan en" trè ( dovea dire: Tenocbtitlan ) e habiendo vifto algunas cofas de ella, " aunque pocas, fegun lo que hai que ver y notar: por aquellas me pare", cio, y a un por In que de la tiera habia vifto, que convenia al Real , Servicio, y à nueftra feguridad, que aquel Seńor ( Motezuma) eftuviera ", en mi poder, y no en toda fu libertad; porque no mudaffe el propofi" to, que moftraba en fervir à V. Alteza, mayormente que los Efpañoles "fomos algo incomportables e importunos, e porque enojandofe nos podia "hacer mucho daño, y tanto, que no hubieffe memoria de nofotros fegun ¿fu gran poder; e tambien porque teniendole conmigo, todas las otras ", tierras, que à èl eran fubditas, vendrian mas aina al conocimento y fer"vicio de V. Mageftad, como defpues fucediò. "A Ancor più Ichiettamente il palefa nel cap. 2. della fteffa lettera citando un' altra da lui foritta al medefimo $\operatorname{Re}$ dalla Veracroce. ", Certifiquè, dice, à V. Alteza, que lo " habria ( a Motezuma ) o prefo, o muerto, o fubdito à la Coron a Real ") de V. Mageftad, y con efte propofito y demanda me parti de la Ciudad " de Cempoal. "Ora allorchè Cortès fi partì da Cempoalla non erano ancor accadute le rivoluzioni della Veracroce, nè avea avuto dal Re vesun torto, ma piuttofto delle finezze angolari, e de' più magnifici prefenti. 
vrebbe fempre mai effergli folpettola a cagione della capitale loro nimifà co' Meflicani, ) e addimando loro, fe avea. Lib. IX. no offervato qualche novità negli abitatori di quella Corte. Eglino rifpofero, che la plebe meflicana era allora diverrita nelle pubbliche allegrezze, che avea ordinato il $\mathrm{Re}$ per ce. lebrare l'arrivo di sì nobili ftranieri; ma che nella Nobilta fcorgevano certo contegno fofpettofo, e tra l'altre cole aveano fentito dir da loro, che farebbe facile il levare $i$ ponti da' canali, ciò che pareva indicare qualche fegreta colpirazio. ne contro gli Spagnuoli.

Non potè Cortès dormire quella notte per l'inquietudine, e paffolla tucta girando penfierofo pel quartiere. Una fentinella gli fece fapere allora, che in una ftanza v' era un ufcio di frefco murato. Cortès lo fece aprire, ed entrandovi trovò molte Atanze dov' era depofitato il teforo del defunto Re Axajacatl. Vide quivi molti idoli, una gran quantita di lavori d'argentó, d'oro, di gemme, di penne, e di cotone, e parecchie altre cole, che pagavano alla Corona le Provincie tributarie, o pure prefentavano i Signori Feudatarj al loro Sovrano: Dopo avere offervato con iftupore si gran ricchezza, fece Cortès murare un' altra volta l'ufcio, lafciando allora tut to nello ftato di prima.

La mattina feguente ragunò i fuoi Capitani, rapprefentò loro le oftilita fatte dal Signor di Nauhtlan contro il prefidio della Veracroce, e contra i Totonachi loro alleati, le quali fecondo che dicevano gli fteff Alleati, non fi fareb. bono fatte fenza l'ordine, o il permeffo del Re di Mefico. Efpofe colla maggior energia il graviffimo pericolo, in cui fi trovavano, e dichiarò loro il fuo difegno, efagerando i vantaggi, che doveanfi afpettare dalla efecuzione, e diminuendo i mali, che potrebbono cagionarli. I lor fentimenti furono varj. Alcuni rigettavano il configlio del Generale, come temerario ed impraticabile; e dicevano, che farebbe meglio il domandar permeffo al $\mathrm{Re}$ di ritirarfi dalla Corte; poichè chi con tanta premura, e con sì gran prefenti avea cercato di diftornargli dalla rifoluzione di portarfi a Meffico 
facilmente accorderebbe loro la partenza. Altri fimavano Lip. IX. bensi neceffaria la partenza, ma credevano, che fi dovea far di nafcolto, per non dare occafione a' Meflicani di qualche tradimento; ma la maggior parte di loro tirati anticipatamente dal medefimo Generale al fuo partito, ficcome è da crederfi, acconfentì al configlio di lui, ributtando gli altri, come ignominioli, e più pericolofi.," Che fi dirà di noi 2) dicevano, vedendoci ufcire fubito d'una Corte, dove fiamo " Atati colmi d'onore? Chi farà che non fi perfuada, che la ") paura è quella, che ci caccia? Or fe mai perdiamo la ri\%) putazione di coraggiofi, che ficurezza poffamo prometter"ci $o$ in que'luoghi de' Mefficani, per li quali dovremo: \#neceffariamente paffare, o tra i noftri Alleari, che non fa. "ranno più trattenuti dal rípetto delle nottre armi ? "Si prefe finalmente la rifoluzione di pigliare Motezuma nel. fuo. palazzo, e condurlo prigione al loro quartiere : rifoluzionebarbara e ftravagante fino all' ecceffo fuggerita dal timore. della loro forte, e dalla fperienza della loro propria felicità, la quale più d'ogni altra cofa incoraggifce gli uomini ad avanzarfa alle più ardue imprefe, e gli manda fovente in precipizio.

Per l' efecuzione d' un sì pericololo attentato mife Cortès in arme tutte le fue truppe, e le diftribù ne' luoghi convenienti. Comandò a cinque de' fuoi Capitani, ed a venti-: cinque foldati, di cui più fi fidava, che fi portaffero a due: a due a palazzo, ma in tal maniera, che vi concorreffero. tutti ad un tempo, come fe ciò foffe a cafo, ed egli vi andò: colla interprete Donna Marina ottenuto avanti il benepla. cito del Re in quella ora, nella quale foleva vifitarlo. Fu: introdotto cogli altri Spagnuoli nella. fala d'udienza, dove: il Re affai lontano da prefentire ciò ch' era per accadergli, gli accolfe colla folita amorevolezza. Gli fece federe, lor: regalò alcune cofe d'oro, e davvantaggio prefentò a Cortès una delle fue figlie. Cortès dopo avergli fignificata colle piùr cortei efpreffioni la fua gratitudine, fi fcusò d'accertarla, al-. legando ch' egli era ammogliato in Cubz, e fecondo. la leg:- 
ge divina de' Criftiani non gli era permeffo d'aver due mo. gli; ma alla fine l'ammife nella fua compagnia pernon dar Labi IX. difgulto al $\mathrm{Re}$, e per avere occalione di farla Criftiana, fic. come in fatti l'ottenne. Agli altri Capitani diede parimente il Re alcune figlie di Signori Meflicani di quelle', che avea nel fuo ferraglio. Si trattennero poi un pezzo fopra varj foggetri; ma vedendo Cortès, che que' difcorfi lo difraevano dal fuo intento, diffe al $\mathrm{Re}$, che quella vifita s'indirizzava a dargli parte dell' andamento del Signor di Nauhtlan fuo vaffallo: lagnoffi delle oftilita fatte da quel Signore contro i Totonachi per la loro amicizia cogli Spagnuoli, della guerra fatta agli Spagnuoli della Veracroce, e della morte del Governatore Efcalante, e di fei Soldati di quella. guernigione. "10, fnggiunfe, debbo render conto al mio. "Savrano della morre di quelti Spagnuoli, e per poter dar"gli una convenevole foddisfazione, bo fatto ricerca intor" no ad una condotta tanto irregolare. Tutti v' incolpano ", come il principale Autore di si fatte rivoluzioni; ma io "fon lontano da immaginare una tal perfidia in sì gran. "Monarca, come quella di perfeguitarci da. nemico in quel"la provincia nello fteffo tempo, in cui ci ricolma di gra "zie nella Corte. Io non dubito, rifpofe il Re, che quelli, "che $m$ 'incolpano della guerra di Nauhelan Sono i Tlafcal"lefi miei giurati nemici; ma vi protelto, che non vi ho. "avuto verun influffo. Quauhpopoca s'è avanzato a farla. "fenza mio ordine, anzi contra la mia propria volontà : ed. "affinchè vi confti della verità, io il farò fubito venire. " alla Corte, e ve lo darò in mano. "Chiamò immediatamente due fuoi Cortigiani, e confegnando a loro certa gemma, in eui era fcolpita l'immagine del Dio della Guerra, la quale portava ognora pendente dal braccio, e fervi$v a$ in vece di figillo per contraffegno de'fuoi comandi, ordinò loro d'avviarfi colla maggior celerità poffibile verfo Nauhtlan; ed indi condurre alla Corte Quaubpopoca, e l'altre perfone principali, ch'erano intervenute nella morte degli Spagnuoli, e: lor diede autorità di. levar delle truppe, $c$ 


\section{4}

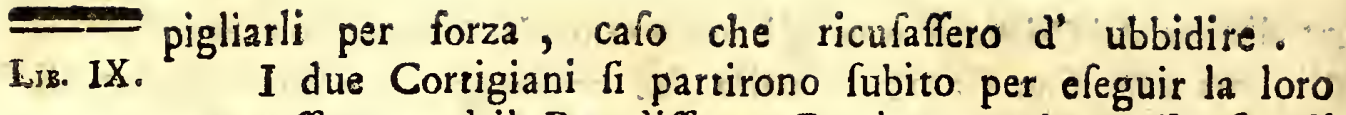
commeffione, ed il Re diffe a Cortès: "Che poffo far di " più per afficurarvi della mia fincerità ? Io non dubito d' ef"f fa, rifpofe Cortès; ma per diffipar l' errore, in cui fono an" che i voftri fteffi vaffalli, che fia ltato efeguito per ordin " voftro l'attentato di Nauhilan, vi vuole una dimoftrazione "Atraordinaria, la qual faccia palefe la voftra betrevolenza "verfo di noi; e niun'alrra mi pare più confacevole a que. " fto fine, che quella di degnarvi di venire a convivere con "noi finattantochè fiano condotti $\mathrm{i}$ rei, e per la loro confef"fione Gi renda manifefta la voftra innocenza . Cid fervirà " per foddisfare al mio Sovrano, per giuftificar la voltra con" dotta, e per onorarci, e metterci al coperto fotto l' ombra: " di voftra Maeftà. "Malgrado le artifiziofe parole, colle quali procurờ Cortès di orpellare la fua ardita ed ingiuriofa pretenfione, il $\operatorname{Re}$ fe ne accorfe fubito, e fi turbd. " Dove " mai s'è veduto, diffe, che i $\operatorname{Re}$ fi lafcino condur prigioni? " ed ancorchè io voleffi avvilire in sì fatta maniera la mia " perfona, e la mia dignità, non fi metrerebbono incontanen" te in armi tutti $i$ miei Vaffalli per liberarmi? Io non fo. " no uomo da potermi nafcondere o fuggirmene a' monti. "Senza fottopormi ad una tal infamia, for quì tuttora pron" to a foddisfare alle voftre querele.," La cafa , Signore, difse "' allora Cortès, alla quale v' invitiamo, è uno de' voftri palazzis nè farà maraviglia a'voftri fudditi avvezzi già a vedervi fpeffo cangiar d'abitazione, il vedervi ora venire ad abitare il palazzo del voftro defunto Padre Axajacatl fotto il pretefto di palefarci la voftra benevolenza. Cafo poi che i voftri fudditi ardifcano fare qualche cola contra voi o contra noi, abbiamo pure affai di coraggio, forti braccia, e buone armi per reprimere la loro temerita. Del refto io v'impegno la mia fede, che da noi farete tanto onorato,e cos̀̀ ben fervito, come da'voftri proprj fudditi Il Re perfeverd nella fua ripugnanza e Cortès nelle fue iftanze finattantockè uno de' Capitani Spagnuoli troppo ardito, ed incon- 
fiderato, avendo a male, che s'indugiaffe l' efecuzione del loro difegno, diffe con collera, che lafciaffero i difcorfi, e fi rifol- Lis. IX. veffero a trarlo per forza, o ammazzarlo. Il $R e$, il quale nel fembiante dello Spagnuclo fcorfe il propofito di lui, addiman. dò a Donna Marina, che diceva quel furiofo ftraniero. "Io "Signore, rifpofe difcretamente coftei, colne fuddita voftra " defidero la voftra felicità, e come confidente di quefti uo. " mini fo i loro fegreti, e conofco il loro genio. Se voi de" gnate far ciò, che richiedono, farete da loro trattato con " tutto quell'onore, e diftinzione, che fi debbe alla voftra "Real perfona; ma fe perfiftete riella voftra renitenza cor"re pericolo la voltra vita. "Quell' infelice Re, il quale dacchè ebbe la prima nuova dell'arrivo degli Spagnuoli,era ftato fopraffatto da un timor fuperftiziofo, e più pufillanime di giorno in giorno diveniva, vedendofi ora in tale ftretto, e perfuadendofi che prima che giugnefsero le fue guardie a foccorrerlo, potrebbe efser già perito per le mani di quegli uomini tanto arditi e rifoluti, cedette finalmente alle loro iftanze. "Io, diffe, mi voglio fidar di voi: andiamo pure, "andiamo; poichè così il vogliono gli Dei, e fubito ordinò, che gli appreftaffero la letriga, e fi mife in effa per trafportarfi al quartiere degli Spagnuoli.

Io non dubito, che i Leggitori fentiranno nel leggere, e nel confiderare le circoftanze di quefto ftraordinario avvenimento quel medefimo difpiacere, ch' io fento nello fcriverlo; ma è d'uopo non meno in quefto, che in altri avvenimenti di quefta ftoria levar la mente al Cielo, e riverire col più profondo rifpetto gl'altifimi configlj della Divina Provvidenza, la quale prefe gli Spagnuoli per iftromenti della fua giuftizia, e della fua mifericordia, caftigando in alcuni la fuperftizione e la crudeltà, ed illuminando gli altri colla luce del Vangelo. Non cefferemo mai d' inculcar quelta verità, e di far conofcere anche nelle azioni più fregolate delle creature la bontà, la fapienza, e l'onnipotenza del Creatore.

Ufci finalmente Motezuma del fuo palazzo per non ritor- 


\section{6}

tornarvi piut: Ufci proteftando a' fuoi Cortigiani, che peE

LiB. IX. certe cagioni confultate già cogli Dii fe n'andava di fuo buon grado a vivere per alcuni giorni con quegli ftranieri, comandando loro, che così lo pubblicaffero per tutta la Corte: Bortofi con tutto quel treno e magnificenza, che portar foleva ogni volta che fi lafciava vedere in pubblico, e gli Spagnuoli andavano preffo a lui guardandolo fotto colore d'onorarlo. Divolgoffi incontanente per tutta la Città la nuova di quello ftraordinario avvenimento, e vi concorfe in folla il popolo: alcuni inteneriti piangevano, ed altri fi mettevano per terra come difperati. Il $\mathrm{Re}$ procurava acchetargli, fignificando loro il piacere, col quale andava a ftare tra que' fuoi amici; ma temendo qualche grande fcompiglio, diede ordine a' fuoi Miniftri di fcacciar dalla ftrada la ciur: maglia, ed impole pena di morte a chiunque cagionaffe qualche inquietudine. Giunto al quartiere carezzò gli Spagnuoli, che vennero ad incontrarlo, e prefe per la fua abitazione l'appartamento, che più gli piacque, il quale addobbarono prontamente i fuoi dimetici co' più fini tappeti di cotone; e di penne e co'migliori mobili del Real palazzo. Cortès pole guardia nella entrata di quell' appartamento, e raddoppiò quelle, che ordinariamente $v^{3}$ erano per la ficurezza del quartiere. Intimò a tutti gli Spagnuoli ed Alleati, che lo trattaffero, e gli fervifiero con tutto quel rifpetto, che fi conveniva alla fua Maeta, e permife, che entraffero de' Meffica: ni a vifitarlo, quanti voleffero, purchè foffero pochi per volta: ficchè nulla di quanto avea nel fuo palazzo gli mancava; fuorchè la libertà.

6. 6.

Dava quivi Motezuma liberamente udienza a'fuoi VafVita del falli, udiva le loro queftioni, pronunziava delle fentenze, e Re nella reggeva il Regno coll' ajuto de' fuoi Miniltri e Configlieri. Serprigione. vivangli i fuoi dimeftici colla fteffa diligenza e puntualità, che da loro foleva adoperarli. Servivagli alla tavola una fchiera di Nobili ordinati a quattro a quattro, e portando in alto i piatti per maggior oftentazione. Dopo avere fcelto quello che gli piaceva, partiva il refto tra gli Spagnuoli, che gli af: 
fiftevano, e tra i Nobili Mefficani, che lo fervivano. Non contenta di ciò la fua generofità faceva frequenti e magnifici Lis. IX. regali agli Spagnuoli.

Cortès dalla fua banda moftrava tal zelo, perchè la fus gente gli portaffe il rifpetto, che gli fi doveva, che fece fruftare un Soldato Spagnuolo, perchè gli rifpofe brufcamente, - l' avrebbe fatto impiccare, fecondo che affermano alcuni Storici, fe lo Ateffo $\mathrm{Re}$ non fi foffe interpofto in favor del reo. Ma fe quefti era degno d'un tal gaftigo per aver mancato con sì fatta rifpofta al rifpetto dovuto alla Maeftà di quel $R e$, che pena meritava chi l'avea privato temerariamente della fua libertà? Ogni volta che Cortèsentrava a vifitarlo, gli faceva la fteffa riverenza, e gli fteffi complimenti, che far foleva quando andava a palazzo. Per follazzarlo nella prigione faceva fare a' foldati l' efercizio dell' armi, o pure facevagli giocare innanzi a lui, ed il medefimo Re fi degnava talora giocare con Cortès, e col Capitano Alvarado ad un giuoco, che gli Spagnuoli chiamavano il bodoque, e moftrava piacere di perdere per aver occafioni di efercitare la fua liberalita. Una volta perdette dopo il pranzo quaránta pezzi d'oro non lavorato, cioè, per quan. to poffiamo congetturare, cento e feffanta oncie almeno. Co. si facilmente fcialacquano le loro ricchezze coloro, che l'han. no acquiftate fenza propria fatica.

Vedendo Cortès la liberalità, o piuttolto prodigalità di lui, gli diffe un giorno, che alcuni foldati furfanti aveano tolto dal teforo del fuo defunto Padre Axajacatl certi pezzi d'oro; $\mathrm{ma}$ che gli farebbe fubito riporre, dov' erano.," Pur" chè non tocchino diffe il Re, le immagini degli Dei, nè " ciò che è deftinato al loro culto, prendano quanto vo. "gliono. "Avutone un tal permeffo traffero quindi gli Spa. gnuoli più di mille abiti fini di cotone. Cortès comandò, che vi fi riponeffero; ma Motezuma s'oppofe dicendo, ch'e gli non riprendeva giammai ciò, che una volra avea dato Fece altresi Cortès imprigionare alcuni Soldati, perchè avea. no pigliato dal medefimo teforo certa quantità di fliquidam.

Storia Antica del Meffico Tom. III. 


\section{8}

bra; ma a richiefta del Re furono tofto meffi in liberta:

LIs. IX. Nè contento Motezuma di concedere le fue ricchezze agli Spagnuoli, prefentò a Cortès un' altra fua figlia, la quale accettò quefto Generale per maritarla con Criftofano d'Olid Maeftro di campo delle truppe fpagnuole. Quefta Principeffa ficcome l'altra già prefentatagli, furono prontamente inftruite e battezzate fenza veruna contraddizione del lor Padre .

Non dubitando più Cortès della buona volontà del Re, renduta già palefe non meno nelle ftraordinarie dimoftrazioni di liberalità, che nel piacere che moftrava d'effere tra gli Spagnuoli, dopo alcuni giorni di prigione gli accordò l'ufcire del quartiere, e l'efortò ad andare ogni volta che voleffe a divertirfi nella caccia, alla quale era oltremodo affezionato. Non rifiutò quell' avvilito Monarca queft' ufo miferabile della fua libertà, che gli fi accordava : ufciva fpeffo, a fi portava ora a' tempj a far le fue divozioni, cra al lago a caccia d'ucceli $i$ acquatici, ora al bofco di Chapoltepec, od a qualche altro luogo di diporto guardato fempre da un buon numero di Soldati Spagnuoli. Quando andava pel lago, lo fcortavano moltiffime barche, o pure due brigantini fatti far da Cortès, tolto che entrò in quella Corte. $\left(^{*}\right)$ Quando fi portava a qualche bofco, l'accompagnavano due mila Tlafcallefi oltre alla numerofa comitiva di Mefficani, che andava ognora per fervigio di lui ; ma non pernottava mai fuor del quartiere.

Più di quindici giorni erano già fcorfi dopo l' imprigioSupplizio namento di Motezuma, allorchè ritornarono que' due Cortidel Sig. giani mandati a Nauhtlan, conducendo feco loro Quauhdian, e popoca, un figliuolo di lui, ed altri quindici Nobili compli。 nuovo ci della morte del Capitano Efcalante. Quauhpopoca veniinfulto va riccamente veftito fopra una lettiga. Allorchè arrivò al âtà del Requartiere, fí fcalzò giufta il ceremoniale di palagio, e fi coprì

(*) Per efporre tutta in una volta la vita di Motęuma nella prigione rammemoriamo quui alcuni arvenimenti di molto pofteriori a quelli, che fiamo per raccontare. 
pri d'un abito groffolano: fu introdotto all' udienza del Re, e fattevi le folite ceremonie di rifpetto gli diffe: „Eccovi, Lis. IX. " molto grande e poffente Signore, il voltro fervo ubbidien" te a' voltri ordini, e pronto a compire in tutto la voftra "volontà." "Affai male vi fiete condotto quefta volta, "gli rifpofe con ifdegno il $\mathrm{Re}$, nel trattar come nemici "quegli ftranieri, ch' io ho accolto amichevolmente nella " mia Corte, ed è ftata affai grande la voftra temerità nell" ", incolparmi, come autore di si fatto attentato: farete per"tanto punito, come traditore al voftro Sovrano, "e volendo Quauhpopoca fcularfi, non volle afcoltarlo, e lo fece fubito confegnare a Cortès infieme coi complici acciocchè dopo difaminato il delitto gli caftigaffe colla peria, che fi conveniva. Cortès lor fece le dovure interrogazioni, ed eglino confeffarono fchiettamente il farto fenza incolpar da principio il $R e$, frnchè vedendofr minacciati di tormenti, e credendo inevitabile il loro fupplizio, dichiararono, che quanto aveano fatto era loro ftato comandato dal $R e$, fenza $i$ cui ordini non aurebbono mai avuto ardire d' intentar che che fia contro agli Spagnuoli.

Cortès fentira la loro confeffione; e facendo fembianza di non credere la loro fcufa, gli condannò ad effere bruciati vivi avanti il Real palagio come rei di lefa maeftà. Portoffi immediatamente all' appartamento del Re con tre o quat* tro de' fuoi Capitani ed un Soldato, che portava in mano de' ferri e fenza tralafciare neppur quefta volta le folite ceremonie e complimenti, diffe al $\mathrm{Re}$ : "Sono già ftati, Si"gnore, efaminati i rei, e tutti hanno confeffato il loro "delitto, ed incolpano voi, come autore della morte de' miei "Spagnuoli. Io gli ho condannati al fupplizio, che meri"tano, e che meritate anche voi attefo la loroconfeffione; " ma a vendo per altro riguardo a' grandi benefizj, che fino" ra ci avete fatto, ed all' affezione che avete manifeftata " verfo il mio Sovrano e verfo la mia Nazione, voglio ac" cordarvi la grazia della vita; ma non poffo fchivare il "farvi fentire una parte della pena, che meritate pel vo. $\mathbf{N} \approx$ 》 Atro 
"ftro delitto. ", Ciò detto comandò adiratamente al SoldaLis. IX to, che gli metteffe i ferri a' piedi, e fenza voler fentir nulla, volto le fpalle, e fe $n$ ' andò. Fu si grande lo ftupore del Re nel veder fottopofta a tanto oltraggio la fua perfona, che nè gli lafciò verun moto per la refiftenza, nè veruna parola per efprimere il fuo dolore. Stette un buon pezzo quafi privo di fenfo. I dimeftici, che gli affiftevano dichiaravano con mute lagrime il lor dolore, e gittandofi a' fuoi piedi, gli alleggerivano colle loro mani il pefo de' ferri, e fi ftudiavano di rifparmiargli il contatto d'effi frapponen. dovi delle fafcie di cotone. Ritornato a fe dalloftupore proruppe in alcune azioni d'impazienza, ma prontamente fi rafferenò, afcrivendo la fua difavventura alla fovrana difpofizion degli Dei.

Appena fatta quefta azione tanto ardita, pafsò Cortès ad efeguire un'altra imprefa non meno temeraria. Dopo aver dato ordine alle guardie del quartiere di non permettere, che v'entrafse verun Mefficano a veder il $\mathrm{Re}$, comandò, che fofse condotto al fupplizio Quauhpopoca col fuo figliuolo, e cogli altri rei. Gli conduffero gli fteffi Spagnuoli armati, e pofti in ordine di battaglia per far retta al popolo, fe forfe volefse impedir la efecuzione; ma che potrebbe far quella piccola truppa contra all' immenfa moltitudine de' Mefficani, che doveano efsere fpettatori di quel gran fuccefso, fe Iddio, il quale tutto difponeva per l' efecuzione de' fuoi altifimi difegni, non avefse impedito gli effetti, che doveano cagionarfi dall' inaudito attentat's di que' pochi uomini? Il fuoco s'accefe davanti al palagio principal del Re. Le legna, che, vi s'a. doperarono furono una gran quantità d'archi, di frecce, di dardi, di lance, di fpade, e di fcudi, ch' erano in un'armerìa perchè il richiefe dal $\mathrm{Re}$ Cortès per liberarfi dalla inquietudine, che gli cagionava la vifta di tante armi. Quauh popoca legatigli i piedi e le mani e pofto fulle legna nelle quali doveva abbruciarfi, proteft di nuovo la fua innocen. za, e ridifse, che quanto avea fatto, era itato per efprefso comando del fuo $\mathrm{Re}$ : fece poi delle preghiere a'fuoi Dei, 
- fece coraggio a'fuoi compagni per foffrir la morte. S'accefe il fuoco, ed in pochi minuri furono confunti $(f)$ a vilta Lis. IX. d'un immenfo popolo, il quale non fi mofse, perchè fi perfuafe, come è da crederfi, che quel fupplizio s'efeguiva per ordine del Re; ed è afsai verifimile, che a nome di lui folse pubblicata la fentenza.

Non fi può giuftificare in verun modo la condotta di Cortès, poichè oltre all' arrogarfi un' autorità, che non gli conveniva, fe egli credecte daddovero, che il $\mathrm{Re}$ era ftato au. tore delle rivoluzioni della Veracroce, perchè condannare a morte, e ad una morte tanto acerba quegli nomini, che non aveano altro delitto, che quello d'efeguire puntualmente gli ordini del loro Sovrano? Se non credeva colpevole il $\mathrm{Re}$, perché fottoporlo a tanta ignominia, mettendo in non cale il rifpetto dovuto al fuo carattere, la gratitudine, che fi conveniva alla fua beneficenza, e la indennita, che fi doveva alla fua innocenza? Io congetturo, che Quauhpopoca ebbe dal Re l'urdine precifo di rimettere i Totonachi fotto l'ubbidienza di quella corona, e per non poter compiere un-tal ordine fenza imbrogliarf cogli Spagnuoli, come quelli che proteggevano $i$ ribelli, portd le cofe agli eftremi, che abbiamo veduti .

Tolto che furono giuftiziati i rei, fi portò Corrès all'ap. partamento di Motezuma, e falutandolo amorevolmente, e vantando la grazia che gli faceva nell' accordargli la vita, gli fece levare i ferri. Il giubilo, che ebbe allora Motezuma, fu proporzionato al cruccio, che gli avea cagionata l'i.

gno.

(f) Solis, dove fa menzione della fentenza di Cortès contro Quauhpopoca, dice cosi: "Juzgofe militarmente la caura,y fe les dio fentencia de " muerte con la circunftancia de que fueffen quemados publicamente fus "cuerpos., , Nel che fenza efprimere la fnezie di fupplizio, a cui furono. condannati, dà ad intendere, che non furono $i$ rei bruciati, ma tolo tanto i loro cadaveri : ciò che non conviene alla fincerita, che fi richiede da uno ftorico. Egli fi ftudiò di diffinulare cid, che non fi confaceva col panegirico del fuo Eroe; ma poco giova la fua diffimulazione, mentre non folamente gli altri Atorici, ma anche il medefimo Cortès l' afferma schietramente nella fua lettera a Carlo V. Vedafi Spezialmente il Cronichifta Herrera nella fua Decada 2. lib. 8. cap. 9. 
gnominia. Dileguoffi affatto il timore, che avea di perder Lis. IX. la vita, e ricevette la libertà, come un benefizio impareggiabile. Tanto s'era avvilito l' animo di quel Re! Abbracciò con fomma amorevolezza Cortès, frgnificogli con fingolari dimoltrazioni la fua gratitudine, e quel giorno fece itraordinarie finezze agli Spagnuoli, ed a' fuoi proprj vaffalli. Cortès levò le guardie, che v' erano, e diffe al $\mathrm{Re}$, che qualora voleffe, poteva reftituirfi al fuo palagio, ben ficuro che il $\operatorname{Re}$ nol farebbe; poichè avea fpeffo fentito da lui, che non gli conveniva ritornare al fuo palagio, mentre foffero nella Corte gli Spagnuoli. In fatti non volle lafciare il quartiere, proteftandn il pericolo, che correvano gli Spagnuoli, qualora gli abbandonaffe; ma può crederfi, che ancora il difornaffe dal riprendere la fua liberc̀ il fuo proprio pericolo; poichè non ignorava, quanto avevano a male i fuoi vaffalli l'avvilimento del fuo animo, e la troppa fua condifcendenza verfo gli Spagauoli.

E' affai verifimile, che il fupplizio di Quauhpopoca ca: 5. 8. gionaffe qualche fermentazione infra la Nobiltà; poichè inTentari- di a pochi giorni Cacamatzin $\mathrm{Re}$ d'Acolhuacan, non potenvidel Re do più comportare l'autorità, che andavano acquiftando gli d'Acol- Spagnuoli nella Corte di Meffico, e vergognandofi di vedecontro a- re in sì miferabile ftato il Re Motezuma fuo Zio, gli mangli Spa- dò a dire, che fi ricordaffe d'effere Signore, e non voleffe gnuoli. farfi fchiavo di quegli ftranieri; ma vedendo, che Motezu. ma rifiutava d'arrenderfi al fuo configlio, fi rifolvette di fas egli medefimo la guerra agli Spagnuoli. La rovina di coltoro farebbe tata inevitabile, fe la ftima in cui era Cacamatzin preffo i Meflicani, ed i Tezcocani, foffe ftata uguale alla fua intrepidezza, e rifoluzione; ma i Mefficani fofpettarono, che fotto color di zelo per l'onor di fuo Zio, nafcondeffe qualche fina ambizione, ed il difegno d'ufurpar la corona di Meffico. Appo i Tezcocani fuoi fudditi non era molto accetto a cagione del fuo orgoglio, e del male da Iui fatto a fuo fratello il Principe Cuicuitzcatzin, il quale per ifchivar la perfecuzione era rifuggito in Meffico, ed era pir accetto per la fua indole piacevole e popolare. An- 
Andoffene dunque Cacamatzin a Tezcuco, e convocati i fuoi Configlieri, ed i più riguardevoli perfonaggi della fua Lis. IX. Corte, lor rapprefentò il lagrimevole ftato, in cui frova. va la Corte di Meffico pel foverchio ardire degli Spagauoli, e per la pufillanimità del Re fuo Zio: l'autorità, Góe que' pochi ftranieri s'andavano arrogando, le graviffime ingiurie da loro fatte alla perfona del $\mathrm{Re}$ imprigionandolo, come $\mathrm{fe}$ foffe un vile fchiavo, ed anche agli fteffi Dei, introducendo in quel Regno il culto d'altri Numi Atranieri: efagerò i ma. li, che da tali principj potrebbono rifultare contra la Corte - contra il Regno d'Acolhuacan. "E' tempo ormai, dice"va, di combattere per la noftra Religione, per la noftra " patria, per la nottra libertà, e pel noftro onore, prima " che s'accrefca la poffanza di quefti uomini o con nuovi "rinforzi venuti dal loro paefe, o con nuove alleanze nel " noftro contratte. "Finalmente impofe loro di palefare liberamente il loro fentimento. I Configlieri per la maggior parte fi dichiararono pel partito della guerra, o per compiacer al $\mathrm{Re}$, o perchè in fatti erano dello fteffo fentimento, ma certi anziani affai autorevoli differo al $\mathrm{Re}$ con libertà, che non fi lafciaffe cosi facilmente portare dall'ardore giovanile : che avanti di prender alcuna rifoluzione avvifaffe, che gli Spagnuoli erano nomini guerrieri e rifoluti, e pugnavano con arme affai fuperiori, che non poneffe mente tanto al fuo parentado con Motezuma, quanto all' alleanza ed amicizia d'effo lui cogli Spagnuoli : che si fatta amicizia, della quale v' erano certi e chiari argomenti gli farebbe facrificar coll' ambizione di quegli ftranieri tutti gl' intereffi del fangue, e della patria.

Malgrado quefte rapprefentazioni fu rifoluta la guerra, ed immediatamente fi cominciarono a fare $i$ preparativi col maggior fegreto; non però con tanto, che non arrivafe la nuova ed a Motezuma, ed a Cortès. Quefto Generale entrò in una graviffima inquietudine, ma confiderando peral. tro, che tutte le imprefe temerarie gli riufcivano, deliberò di prevenire il colpo, marciando colle fue truppe a dare un 
affalto a Tezcuco: Diftornollo Motezuma da un sí ardito

EIB. IX. configlio, informandolo delle forze di quella Corte, e della immenfa moltitudine de' fuoi abitatori. Determinoffi dunque Cortès di mandare un' ambafciata a quel $\mathrm{Re}$, ricordandogli l'amiquia contratta già in Ajotzinco, allorchè $v$ 'andò ad incontrarlo a nome del Re fuo Zio, e dicendogli che ponderaffe che quanto è facile l'intraprendere la guerra, tanto è difficile il riufcirne: che a lui tornerebbe più a conto il mantenerfi in una buona corrifpondenza col Re di Caftiglia, e colla Nazione Spagnuola. Cacamatzin rifpofe, ch' egli non poteva aver per amici coloro, che gli toglievano l'onore, che opprimevano la fua patria, che oltraggiavano il fuo fangue, e che fpregiavano la fua Religione: che nè fapeva chi foffe il Re di Caftiglia, nè gl' importava il faperlo: che fe volevano fchivare il colpo, ch' era per venire fulle loro tefte, ufciffero incontanente da Meflico, e ritornaffero alla loro patria.

A difpetto d' una rifpofta sì forte, replicò Cortès l'am: bafciata; ma effendogli tato rifpofto in fullo fteffo tuono, fi lagnò con Motezuma, e per impegnarlo in quell' affare finfe di fofpettar d'effo lui, che aveffe qualche influffo negli oftili difegni del fuo nipote. Motezuma fi purgò del fofpetto colle più fincere protefte, e s'efibì d'interporvi la fua autorità. Mandò pertanto a dire a Cacamatzin, che veniffe a Meffico a vifitarlo: ch' eg!i troverebbe maniera d'accomodar quelle differenze. Cacamatzin fdegaato di veder Motezuma più impegnato in favor di coloro, che opprimevano la fua libertà, che di colui, che s'adoperava per reftituirgliela, gli rifpofe, che fe dopo tanta infamia foffe reftato in lui qualche fentimento d'onore, fi vergognerebbe di vederfi fatto fchiavo di quattro furfanti, i quali mentre l'adefcavano con buone parole, l' oltraggiavano co' fatti: che poichè non baftava a muoverlo nè il zelo della Religione Mefficana, e degli Dei Acolhui difpregiati da quegli ftranieri, nè la gloria de'fuoi Antenati ofcurata ed avvilita perla fua codardia, egli voleva dare ajuto alla Religione, vendicar 
gli Dei, confervare il Regno, ricuperar l'onore e la liberta di lui, e di tutta la Nazione: che però egli verrebbe inLir. IX. fatti, ficcome lo pregava, a Meffico non gia colle mani nel feno, ma impugnando la fpada per cancellar col fangue degli Spagnuoli l'obbrobrio de' Mefficani.

Colternoffi il Re Motezuma per una tal rifpofta, temendo d'effer vittima in quella tempefta o deila vendetta Imprigiodegli Spagnuoli, o del furore del Re Cacamatzin: onde fi namento rifolvette di adoperarvi un rimedio eltremo per impedirla, e del Re di falvar la fua vita con un tradimento. Diede però fegre- huacan, tamente ordine a certi Uffiziali Mefficani, che fervivano nel- e d'altri la guardia del Re fuo nipote, di procurare con ogni loro ed efaldiligenza e fenza incugio di prendere la Real perfona di tazione lui, e condurla colla maggior cautela a Meffico; perchè ciò del Prinimportava affai al ben pubblico del Regno. Suggeri loro la cipe Cuimaniera di farlo, e forfe anche lor fece qualche dono, e tzin. lor promife qualche premio par incoraggirli all' imprefa. Coftoro poi follecitarono altri Uffiziali o dimettici del $\mathrm{Re} C a$. camatzin, che riconobbero più difpolti a quella fazione, e col loro ajuto ottennero tutto cio, che voleva Motezuma. Tra gli altri palazzi il Re d'Acolhuacan uno ne aveva fabbricato fulla fponda del lago in tal maniera, che per un canale, che fcorreva fotto, potevano entrare ed ufcire le barche. Quivi, dove allora rifedeva il $\mathrm{Re}$ Cacamatzin, difpofero un buon numero di barche con gente armata, e nel bujo della notte, col quale fi coprono i più gran delitti, diedero addoffo improvvifamente al $\mathrm{Re}$, e prima che venir poteffe qualche gente a difenderlo, lo pofero in una barca, e colla maggior preftezza lo portarono a Meffico. Motezuma fenza verun riguardo al carattere di Sovrano, nè al parentado di Cacamatzin, lo diede fubito in mano a Cortès. Quelto Ge. nerale, il quale per quanto appare dalla fua condotta, non aveva veruna idea di quel rifperto, che fi debbe alla maeftà Reale anche nella perfona d' un barbaro, lo mife in ferri, e lo rinchiufe fotto la cultodia di buona guardia. Le riflefioni da farfi lopra quefto, ficcome fopra altri ftraordi: Storia Antica del MefJico Tom. 111.

O nari 
narj avvenimenti della noftra Storia, fono troppo facili : LIB. IX. triviali per doverci trattenere nel corío della narrazione.

Cacamatzin, il quale avea cominciato il fuo infaufto Regno colle diffenzioni del fuo fratello Ixtlilxochitl, e collo fmembramento dello ftato, lo fini colla perdita della corona della libertà, e della vita. Determinò Motezuma, acconfentendovi Cortès, che la corona d'Acolhuacan fi deffe al Principe Cuicuitzcatzin, il quale era ftato da Motezuma tratte. nuto nel fuo palagio, dacchè per ifchivare le perfecuzioni del fuo fratello Cacamatzin, $(g)$ s'era ricoverato in Meffico e meffofi lotto la fua protezione. In quefta elezione fi fece torto a' Principi Coanacotzin, ed Ixtlilxochitl, i quali per effere nati dalla Regina Xocotzin, aveano maggior diritto alla corona. Non fi può fapere-il morivo, ch' ebbe il Re di Meffico per rifiutare Coanacotzin, giacchè per ciò che riguarda Ixtlilxochitl apparife, che non volle accrefcere la poffanza d'un nemico tanto terribile. Checcheffia, Motezuma fece riconofcere Re Cuicuitzcatzin dalla Nobiltà Tezcu. cana, e l'accompagnò infieme con Cortès infino alla barca fulla quale dovea valicar il lago, e gli raccomandò l'amicizia coltante de' Mefficani, e degli Spagnuoli; poichè agli uni ed agli altri era debitore della corona.

Portoffl Cuicuitzcatzin a Tezcuco accompagnato da mol. ti Nobili dell' una e dell' altra Corte, ed ivi fu ricevuto con acclamazioni, con archi trionfali, e con balli, trafportandolo la Nobiltà in una lettiga dalla barca al real palagio, dove il Nobile più anziano gli fece un lungo ragionamento a nome di tutta la Nazione congratulandofi con lui, efortandolo

(g) Cortès nella fua lettera a Carlo V. dice, che Cuicuitzcatzin era figliuolo di Cacamatzin : ma quefto fu uno sbaglio del copifta di quella lettera, o pure del medefimo Cortès, poichè ci confta, ch' erano fratelli, benchè foltanto dalla parte del Padre: nè poteva effere ciò che dice. Cortès; poichè egli medefimo afferma in un' altro luogo, che Cacamatzin era un giovane di venticinque anni, e peraltro rapprefenta Cuicuitzcatzin in età da poter reggere il Regno. Oltracciò lo fteffo Cortès in un' altra lettera fcritta addi Is. Maggio 1 522. dice chiaramente, che que' due Signori erano fratelli. 
dolo all' amore de' fuoi vaffalli, ed efibendofi ad amarlo come Padre, ed a riverirlo come lor Signore. Neffuno è ca. Lis. IX. pace d'efprimere il dolore, che si fatte nuove cagionarono a Cacamatzin vedendofi nella più frefca età (poichè non aveva più di venticinque anni ) privato della corona, che tre anni innanzi avea ereditata da fuo Padre, e ridotto alla ftrettezza ed alla folitudine d' una carcere dallo fteffo $\mathrm{Re}$, cui voleva liberare, e dagli fteffi Itranieri, che avea voluto fcacciar dal Regno.

Aveva oramai Cortès nel fuo potere $\mathbf{i}$ due più poffenti $\operatorname{Re}$ d'Anahuac, e non iftette guari, che pigliò ancora il Re di Tlacopan, i Signori d'Iztapalapan, e di Cojohuacan, fratelli tutti e due del $\operatorname{Re}$ Motezuma, due figliuoli di quefto medefimo $\mathrm{Re}$, Itzquauhtzin Signor di Tlatelolco, un Sommo Sacerdote di Meffico, e parecchj altri de' più rag. guardevoli perfonaggi, benchè ignoriamo le circoftanze di quefti imprigionamenti; ma è da crederfi, che gli andaffe pigliando ad uno ad uno, allorchè entravano a far vifita al Re Motezuma.

Incoraggito quel Generale con sì profperi fucceffi, $e$ vedendo il Re di Meffico tutto facrificato alle vogiie dilui, Sottomergli diffe, ch'era ormai tempo di far che i fuoi fudditi ri- fione di conofceffero il Re di Spagna lor legittimo Sovrano, come $\underset{\mathrm{ma}}{\mathrm{M} \text {, }} \mathrm{e}$ quello che difcendeva dal $\operatorname{Re}$ e Dio Quetzalcoatl. Mote-della Nozuma, al quale non baftava l'animo di contraddirgli, con-biltà mefvocd la principal Nobiltà della Corte, e delle Città circon- ficana $\mathrm{Re}$ vicine. Vennero tutti prontamente a ricevere i fuoi ordini, Spagna. e ragunati in una gran fala del quartiere, ed intervenendovi Cortès con alcuni altri Spagnuoli, fece loro il $\mathrm{Re}$ un lungo difcorfo, nel quale proteftò l'amore, che a loro portava come Padre, da cui non doveano temere, che lor proponeffe veruna cofa, che non foffe giufta e vantaggiofa. Ricordò loro l'antica tradizione fulla devoluzione dell' Imperio Mefficano ne'difcendenti di Quetzalcoatl, i cui luogotenenti erano ftati, ed egli, ed i fuoi anteceffori, ed i fenomeni offervati negli elementi, i quali giufta l' interpretazione de' Sacer-

$$
\mathrm{O}_{2}
$$
doti , 
doti, e degl' Indovini fignificavano, ch' era oramai arrivato L18. IX.il tempo in cui fi compieffer gli oracoli. Io non dubito, che faceffe anche menzione del memorabile avvenimento e vaticinio della fua Sorella Papantzin, da noi nel libro V. ramıemorato, il quale verifimilmente fara ftata la principal cagione dell' avvilimento del fuo animo. Indi pafsò a paragonare i contraffegni offervati negli Spagnuoli, con quelli della tradizione: onde conchiufe che il Re di Spagna era appunto il legittimo difcendente di Quetzalcoatl, cui però cedeva il Regno, e dava l'ubbidienza, ed efortava tutti a far lofteffo. (b) Nel dirfi fuddito d'un altro Re fentì così gran pe:

na,

(h) Le circoftanze della fuddetta affemblea, dell' omaggio renduto al $\mathrm{Re}$ di Spagna, e dell' ordine intimato da Motezuma a Cortès d' ufcire dalla Corte fi raccontano dagli Storici con tal varietà, che neppure due di loro fitroveranno, che fieno perfettamented'accordo. Io nella narrazione di quefti avvenimenti tengo dietro principalmente ai racconti di Cortès, e di Bernal Diaz tutti e due teftimonj oculati. Il Solis afferma, che il riconofcimento di Motezuma fu un mero artifizio: che non ebbe mai intenzione d' adempiere ciò, che prometteva: cbe l'intento di lui exa quello di sbrigars dagli Spagnuoli, $e$ di temporeggiare per intendersela poi colla fua ambizione Senza curarfí della fua parola. Ma fe l'at to di Motezuma fu un mero artifizio, e non penfava ad :effettuare cio che prometteva, perchè nel dirfi vaffallo d' un altro Monarca ebbe tanto dolore, che gli troncó la voce, e gli cavò le lagrime, ficcome dice lo fteffo Autore ? Se non penfava ad altro, che a sbrizarfi dagli Spagnuoli, non era d'uopo di si fatta finzione. Quante volte potè con fare un fol cenno a' fuoi vaffalli, fe aveffe voluto, o facrificartutti gli Spagnuoli a' fuoi Dei, o pure lafciando loro la vita fargli condur legati al porto, acciocciè quindi riprendefero la frada per Cuba? Tutta la condotta di Motezuma fu affattooppofta alle intenzioni, che in lui fuppone il Solis; ma nulla fmentifce tanto la fua accura, quanto la chiara teftimonianza data dalla Corte di Spagna, la quale in parecchj refcritti fpediti in favor della Real difcendenza di Motezuma, accordandole delle efenzioni e de' privilegj ftraordinarj, dichiara, che tali privilegj non poffono fervir d'efempio a verun' altra ca$\mathrm{fa}_{2}$; perchè niun' altra, foggiunge, ha fatto a Spagna sì gran fervizio, come quello che le fece l'Imperatore Motezuma nell' incorporar colla fua volontaria ceffione in quella corona, un Regno tanto ricco e tanto grande, quanto quello del Meffico. Se l' ubbidienza data da Motezuma al Re Cattolico folfe ftata tale, quale cela rapprefenta il Solis, fi direbbe, che la Corte di Spagna credeva incorporato il Regno del Meffico nelia Corona di Caftiglia mercè d' una celfione finta ed ingannevole, e d'un mero artifizio di Motezuma: ciò che farebbe un graviffimo torto alla Criftiana dirittura de' Re Cattolici.Il Betancurt nella part. 2. tratt. 3. del fuo Teatro Me/ficano cita i luddetti refcritti, i cui originali faranno renz' altro nell' archivio de 'Signori Conti di Motezuma e di Tula . 
na, che gli troncò il difcorfo e gli fece fultituir le lagrime alle voci. Il pianto del $R e$ fu feguito da si amari finghioz. Lis. IX. zi di tutta l'affemblea, che intenerirono, e moffero a pieta gli Spagnuoli. A' finghiozzi poi fuccedette un malinconico filenzio, il quale fu rotto da uno de' più autorevoli Signori Mefficani con quefte parole. "Poichè, Signore, diffe al Re, "è arrivato il tempo, che fi compiano gli oracoli antichi, "e gli Dei vogliono, e voi cel comandate, che framo fud. " diti d'un altro Signore, che abbiamo noi a fare, fe non "che fottometterci alle fovrane difpofizioni del Cielo inti" mateci per la voltra bocca?

Cortès allora ringraziò il $\mathrm{Re}$, e turti i Signori, che v'erano prefenti della pronta e fincera loro fommeffione, e proteftò, che il fuo Sovrano non pretendeva toglier la corona al $\mathrm{Re}$ di Meffico, ma foltanto far riconofcere il fuo alto dominio fovra quel Regno, che Motezuma non folamente feguiterebbe a comandare a' fuoi fudditi, ma eziandio eferciterebbe la Iteffa autorita fopra tutti que'popoli, che foffero per fottometterfi agli Spagnuoli. Congedata l'affemblea fece fare Cortès un pubblico Atrumento di quell' atto con tutte le folennita, che gli parvero neceffarie, per mandarlo alla Corte di Spagna.

Or'avendo fatto cosi felicemente quelto paffo, rapprefentò a Motezuma, che poichè aveva gia riconolciuto il do. minio del $\mathrm{Re}$ di Caltiglia fopra que' paefi, era d' uopo pale. fare la fua fubordinazione con qualche contribuzione d'oro, 5. II. Primo e d'argento, allegando per ciò il diritto, che aveano i So- la corovrani d' efigere si fatto omaggio da' loro vaffalli per mante- na di Canere lo fplendore della corona, per futtentare i loro Miniftri, per le fpefe della guerra, e per gli altri bifogni dello Stato. Motezunra con regia magnificenza gli diede il teforo del Padre fuo Axajacail, che fi confervava, come abbiam già detto, in quel medefimo palagio, del quale niente avea tolto finora Cortès, contuttochè gli foffe ftato efpreffamente permeffo dal Re il prenderne tutto ciò, che voleffe. Tutto quelto teforo venne nelle mani degli Spagnuoli inlieme con 
tutto quello che contribuirono i Signori Feudatarj di quella Lib. IX corona: il che fu tanto, che con ciò, dopo aver feparata la quinta parte pel Re di Spagna, ebbe Cortès quanto abbifognava per pagare $i$ debiti da lui fatti in Cuba nell alleftimento dell armata, e per rimunerare i fuoi Uffiziali e Soldati, reftando ancora affai per le fpefe da fare nell' avvenire. Pel Re fi deftinarono, oltre alla quinta parte dell'o. ro e dell' argento, che fuffero, certi lavori, che confervaro. no interi per riguardo al loro maravigliofo artifizio, $i$ quali giufta il computo di Cortès importavano più di cento mila ducati; ma la maggior parte di quefta ricchezza fi perdette, ficcome apprefto vedremo.

5. 12

Trionfavano gli Spagnuoli nel vederfi padroni a sì poInquie- ca fpefa di tante ricchezze, e nell' aver fottomeffo fenza vetudine No-runa fatica al loro Sovrano un Regno cosi grande ed opubiltà Mef-lento; ma la loro felicità era omai foverchia, ed era d' uoficana, e po fecondo la condizione delle cofe umane, che andaffero a nuovi di vicenda i fucceffi profperi cogli avverfi. La Nobiltà Meffica. Motezu- na, la quale fin' allora s' era tenuta in un rifpettofo filenzio ma. per la fomma loro deferenza alla volontà del lor Signore, vedendolo ora in tanto avvilimento, il $\mathrm{Re}$ d'Acolhuacan, ed altri riguardevoli perfonaggj meffi in ferri, e la Nazione fottopofta al dominio d' un Monarca Itraniero, che non conofcevano, cominciò prima a bisbigliare, e poi a fparlarcon maggior libertà, a biafimare la loro tolleranza, a far ragunanze, ed anche, per quel che fi dice, a levar delle truppe per liberar da quella ignominiofa oppreffione il lor $\mathrm{Re}$, e la loro Nazione. Parlarono a Motezuma alcuni de' fuoi favoriti, rapprefentandogli il dolore, che della fua difgrazia aveano i fuoi vaffalli, confiderando feemato il fuo potere, ed ofcurato lo fplendore della fua dignità, e la fermentazione che oramai cominciava a fentirfi non meno tra la Nobiltà, che tra il baffo popolo, impazienti di vederfi fottopolti ad un $\operatorname{Re}$ Atraniero, e condannati a facrificargli il frutto delle loro fatiche. Efortaronlo a fcuotere il timore, che s'era impadronito di lui, ed a ricuperar la fua autorità: poi- 
chè fe egli nol faceva, il farebbono i fuoi vaffalli, i quali s'erano determinati di fcacciar dalla Corte, e dal Regno LiB. IX. quegli ofpiti tanto infolenti, e perniciofi. Da un'altro canto i Sacerdoti gli efageravano il detrimento, che foffriva la Religione, e l'impaurivano colle minacce, che dicevano farfi da' loro Dei fdegnati, e rifoluti di negar la pioggia a'cam. pi, e la loro protezione a' Mefficani, fe non mandava via quegli uomini tanto contrarj al loro culto. Parecchj Storici troppo facili nel credere le apparizioni degli ppiriti aggiun. gono, che il medefimo Demonio fi fece vifibile al Re, minacciandolo di molti mali, che fulla fua perfona, e ful Regno piombar farebbe, fe fofferiva più a lungo gli Spagnuo. li, e promettendogli, fe gli cacciava, di perpetuare nella fua famiglia la corona di Meffico, e di rendere fommamen. te felici i fuoi vaffalli.

Moffo Motezuma da tante rapprefentazioni e minac. ce, vergognandofi, che gli foffe rinfacciata la fua codardia, ed intenerito per la difgrazia del fuo nipote Cacamatzin, cui feinpre avea amato con fingolar tenerezza, e per quella del fuo fratello Cuitlahuatzin, e di altri perfonaggi della prima Nobiltà, benchè non acconfentiffe al partito di toglier la vita agli Spagnuoli, ficcome alcuni gli configliavano, fi rifolvette pure a dir loro apertamente, che fe n'andaffero da quel Regno. Fece però un di chiamar Cortès, il quale confapevo. le delle conferenze fegrete, che ne' giorni precedenti avea avuto il $\operatorname{Re}$ co' fuoi Miniftri, co' Nobili,' e co' Sacerdoti, fentì una gran turbazione nell' animo fuo; ma diffimulando quanto gli fu poffibile, fi porto incontanente al $\mathrm{Re}$ accompagnato da dodici Spagnuoli. Motezuma l'accolle con minore amorevolezza di quella che foleva moltrargli, e gli palesò fchiettamente la fua rifoluzione. "Non potrete met"tere in dubbio, gli diffe, il grand'amore che v' ho portato " dopo tante, e si chiare dimoftrazioni. Finora v' ho tenuto 》) volentieri nella mia Corte, anzi ho voluto rimanervi qui a " convivere con voi pel fingolare piacere, che ho della voftra "famigliarità e converfazione. Per quanto a me appartiene, 
LIB. IX." vi riterrei tuttora fenza veruna novita, ogni giorno fommi: "niftrandovi fempre maggiori ripruove della mia benevolenza; " ma non fi può ; perciocchè nè i miei Dii lo permettono, " nè lo tollerano i miei vaffalli. Mi trovo minacciato de' più " terribili gaftighi del Cielo, fe vi lafcio ftare più tempo nel " mio Regno, e ne' miei vaffalli è cominciata a fentirfi tale " inquietudine, che fe non ne levo prontamente la cagione, " mi farà poi affatto impoffibile l'acchetarli : onde bifogna " tanto pel mio, quanto pel voftro bene, e per quello di tut" to quefto Stato, che voi v' apparecchiate per ritornare alla "voftra patria. „ Cortès quantunque trafficto dal piì acerbo dolore, nondimeno affettando una gran ferenirà nel fuo fem. biante rifpofe, che il fuo animo era pronto ad ubbidirgli; ma che mancandogli vafcelli pel trafporto a cagione d'efferfi rovinati quelli, fu'quali era da Cuba venuto, vi bifognava del tempo, de'lavoratori, e de' materiali per farne altri. Motezuma allora pieno di giubilo per la prontezza, con cui s' efibiva ad ubbidire, ed abbracciandolo gli diffe, che non faceva meftiere di precipitar la partenza : che fabbricaffe pure i fuoi vafcelli : ch' egli gli fomminiftrerebbe il legname neceffario, c la gente per tagliarlo, e per trafiportarlo al porto. In fate ti diede fubito l'ordine ad un buon numero di legnajuoli, acciocchè tagliaffero il legnanae necelsario da un pineto, che v'era poco difcofto dal porto di Chiahuitztlan, e Cortès dal fuo canto vi mandò alcuni Spagnuoli, affinchè foprantencieffero al taglio, afpettando che frattanto fi cangiaffe lo ftaro delle cofe in Meffico, o pur gli capitaffero de nuovi foccorfa di Spagnuoli mandatigli dalle Irole, o da Spagna. (i):

Otto

(i) Cuafi tutti gli forici, Spagnuoli dicono, che allorchè il Re fece chiamar Cortès per intimargli l'ordine di partire, avea alleftito un efercito per farfi ubbidir per forza, fe mai vi foffe qualche refiftenza, ma vi è una gran varietà fra loro, poichè alcuni affermano, ch' erano in arme cento mila uomini, altri fcemano quefto numero della metà, ed altri finalmente il riducono a cinque mila. Io mi perfuado che vi fia ftata in fatti qualche truppa alleftita, non pero per ordine del Re, ma foltanto d'alcuni Nobili di quelli, che aveano prefo un più grasld'impegno in quefto affare. 
Ottó giorni dappoichè fu prola quefta riloluzione fece Motezuma di bel nuovo chiamare Cortès, e quefto Gene.Lib. IX: rale entrò in nuova inquietudine. Il $\mathrm{Re}$ gli difle, che non era più d'uopo di fabbricar de' vafcelli, perchè poco innanzi erano approdati al porto di Chalchiuhcuecan diciotto baftimenti frmili a' fuoi già diftrutti, ne' quali potea imbarcarfi colla fua gente: che affretraffe per tanțo la fua ipartenza, perciocchè ciò conveniva al bene del Regno. Cortès diflimuio il giubilo, che ebbe per una tal nuova, e ringraziando Dio nel fuo cuore d'avergli mandato un sì opportuno foccorfo, rifpole al $\mathrm{Re}$, che fe que'vafcelli dovevano far viaggio verío Cuba, egli era pronto a partire; ma che altrimente farebbe d'uopo di continuare la fabbrica de'fuoi va. fcelli. Vide pure, e difaminò le pitture di quell' armata mandate al Re da'Governatori della Colta, e non dubitò ch'ef. fa foffe di Spagnuoli; ma affai lontano da penfare, che deffa folfe mandata contro lui, fi perfuafe piuttolto, ch' erano ritornati i fuoi Procuratori inviati l'anno fcorfo alla Corte di Spagna, e che portavano feco loro i regj difpaccj, e con: ducevano un buon numero di truppe per la conquilta.

Quefta gran confolazione gli duró, finattantochè gli arrivarono le lettere di Gonzalo di Sandoval Governatore del- §. 13. la Colonia della Veracroce, nelle quali gli faceva fapere, del Goche quell' armata compolta d'undici valcelli e fette brigan. vernatotini, d'ottanta cinque cavalli, d'ottocento pedoni, e più di redicacinquecento uomini da mare, con dodici pezzi d'artiglierìa Cortès. ed abbodanti munizioni da guerra fotto il comando del Generale Panfilo Narvaez, era mandata da Didaco Velafquez. Governatcre di Cuba contro lo fteffo Cortès, come valfallo ribello a traditore al fuo Sovrano. Ricevè quelto forte col. po alla prefenza del $\operatorname{Re}$ Motezuma; ma fenza moftrar nel fuo fembiante veruna turbazione, diede ad intendere al $\mathrm{Re}$, che coloro, i quali erano approdati a Chalchiuhcuecan, era. no nuovi compagni mandarigli da Cuba. Dolla fteffa diff. mulazione usò verfo i fuoi Spagnuoli, finchè non ebbe i loro animi preparari.

Storia del Meffico Tom. III. 


\section{14}

E' fuor di dubbio, che quefta occalione fin unz di quel: Lis. IX le, nelle quali fece fpiccar Cortès la fua invitta coltanza e magnanimità. Trovavafi da una parte minacciato di tutta la potenza de' Mefficani, fe reftava nella Corte; e da un'altra parte vedeva alleftita contro fe fteffo un' armata de' fuoi medefimi Nazionali fuperiore di lunga mano alla fua; ma la fua accortezza, la fua induftria fingolare, ed il fuo maravigliofo coraggio cangiarono in bene tutto il male, che gli fopraftava. Procurò così per lettere, come per alcuni mediatori, di cui più fi fidava, conciliarfi l' animo di Narvaez, e metterlo alla ragione, facendogli varj partiti, e rapprefentandogli i vantaggi, che avrebbero gti Spagnuoli, fe s' uniffero ambedue l' armate, ed operaffero d'accordo; e per l'oppofto i mali, che dovrebbe cagionar agli uni, ed agli altri la difcordia. Narvaez per configlio di tre difertori di Cortès era già sbarcato con rutia la fua armata nella Cofta di Cempoalla, e s'era meffo in quartieri in quella Città il Signor della quale, conofcendo che i nuovi fuoi Ofpiti erano ancora Spagnuoli, e credendo che venivano ad unirfi col fuo amico Cortès, o pur temendo la loro poffan$\mathrm{za}$, gli accolfe con fommo onore, e gli provvide di tutto cio che abbifognavano. Motezuma credendo da principio lo fteffo, mandò a Narvaez de' ricchi prefenti, e diede ordine a' fuoi Governatori di fargli gli fteffi offequj, che aveano già fatto a Cortès; ma indi a pochi giorni s'accorfe della difcordia, che v'era fra loro a difperto della gran diffimula. zione di Cortès, e de'luoi sforzi per impedire, che una tal nuova arrivaffe al $\mathrm{Re}$, od a' fuoi vaffalli.

Ebbe allora Motezuma la più bella occafione del Mondo per diftuggere e gli uni, e gli altri, fe egli aveffe covati nel cuore que' fanguinofi configli, che parecchj Storici vollero imputargli. Narvaez s'adoperò per alienarlo da Cortès, e da quelli del fuo partito, incolpando tutti di tradimento, e promettendo di caftigar l'inaudita loro temerità nell' im. prigionar si gran $R e$, e di liberar tanto lo fteffo $R e$, quanto tutta la Nazione dalla loro oppreffione; ma Motezuma 
fu si lontano da macchinar qualihe cola per sl fatte fugge. ftioni contro Cortès, che anzi quando quelto Generale gli Lis. IX. fece fapere la fua fpedizione contro Narvaez, moftrò Motezuma un gran difpiacere del rifchio, a cui s'efponeva con truppe si inferiori, e s' efibi a levar prontamente un buon efercito per mandarlo in ajuto di lui.

Avea già Cortès adoperate tutte le diligenze poffibili per venire ad un accomodamento pacifico, e ad ambedue l'armate vantaggiofo fenz' altro effetto, che quello di ricevere nuovi difpregi e minacce dall arrogante e fiero Narvaez. Vedendofi dunque coftretro a far la guerra a' fuoi Nazionali, e non volendo per la fua diffidenza prevalerfi del foccorfo, che gli efibiva il $\mathrm{Re}$ di Meffico, pregò il Senato di. Tlafalla d'alleftire quattro mila uomini di guerra per condurli feco, e mandò a Chinantla un fuo Soldato appellato $T_{0}$. billa uomo affai pratico del meftier della guerra, acciocchè domandaffe due mila ucmini a quella bellicofa Nazione, e fi procacciaffe trecen:o picche di quelle, che ufavano gli fteffi Chinantechi per far refittenza alla cavalleria di Narvaez, poichè erano più lunghe e più forti di quelle degli Spagnuoli . Lafciò in Meffico cento quaranta (l) Spagnuoli con tutti i loro alleati fotto il comando del Capitano Pietro d'Alvarado, raccomandando a loro, che guardafsero, e trattafsero bene il $R_{\text {, }}$ e procurafsero mantenerí in buon'armonia co' Meflicani, malfimamente colla famiglia Reale, e colla Nobiltà. Nel pren. der congedo dal $\mathrm{Re}$ gli diffe, che vi lafeiava nel fuo luogo il Capitano Tonatiub (con quefto nome del Sole era appellato da Meflicani Alvarado, perch' era biondo ) incarica. to di fervire in tutto a fua Maefta: che la pregava di continuar la fua protezione fugli Spagnuoli: ch'egli andava a P. 2

tro-

(I) Bernal Diaz dice, che gli Spagnuoli che reftarono in Meflico furono ottanta tre. Nell' edizioni moderne delle lettere di Cortès fi dice, che tilrono cinquecento, $\mathrm{ma}$ in un'edizione antica fi mette il numero di 140 , il quale mi pare il vero attefo il numero totale delle truppe fpagnuole. Il numero di 500. è evidentemente falro, e contraddice al ragguaglio del medefimo Cortès. 
trovar quel comandante di frefco venuto, ed a far quanto LiB. IX.gli foffe poffibile per mettere in efecuzione i fuoi regj ordini. Metezuma dopo avergli fatto nuove protefte della fua benevolenza, lo fece provvedere abbondantemente di vettovaglie, e d'uomini da foma pel trafporto del bagaglio, e lo licenziò con fomma amorevolezza.

Parti Cortès da Méffico ful principio di Maggio dell' an no 1520 . dopo effere tato fei mefi in quella Corte, con fettanta Spagnuoli, ed alquanta Nobiltà Mefficana, che volle accompagnarlo per qualche tratto di ftrada. Parecchj Storici fi fon perfuais, che i Mefficani andavano per far le fpie, e dar contezza al Re di quanto vi avveniffe; ma Cortès non gli credette tali, benchè peraltro non fi fidaffe di loro. Fece il viaggio per Chololla, dove 's' unì con lui il Capitano Velafquez, il quale ritornava da Coatzacualco, effendovi ftato mandato con qualche truppa da Cortès per cercare un porto più comodo pe' vafcelli. Quivi ancora ricevette Cortès una buona provvifione di viveri mandatagli dal Senato di Tlafcalla; ma non ebbe i quattro mila uomini, che avea domandato: o perchè non ardiffero entrar in nuove zuffe cogli Spaguuoli, come afferma Bernal Diaz; o perchè non voleffero allontanarfi tanto dalla loro patria, come dicono altri Storici; o perchè vedendo Cortès con forze si inferiori a quelle del fuo nemico, temeffero di reftar vinti in quella fpedizione. Alcune giornate prima d'arrivare a Cempoalla fu Cortès raggiunto dal Soldato Tobilla colle trecento picche di Chinantla, ed in Tapanacuetla, villaggio difcofto trenta miglia in circa da quella Citta, gli fi unì il famofo Capitano Sandoval con felfanta Soldati del prefidio della Veracroce.

5. 14. Finalmente dopo aver fatte nuove richielte al Narvaez,

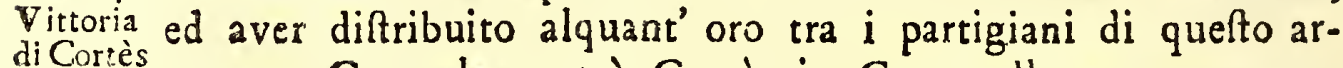
contro rogante Generale, entrò Cortès in Cempoalla a mezza notNarvaez. te con dugento cinquanta uomini, $(m)$ fenza cavalli, necon altre

(m) Bernal Diaz dice, che Cortès andò a Cempoalla con 206. uomini. Torquemada numera 266. oltre a cinque Capitani; ma Cortès, che il feppe meglio di loro, afferma, che furono 250. 
aitre arme, che picche, fpade, rotelle, e pugnali, ed incamminandofi pian piano, e fenza verun rumore al tempio Lr. IY. maggiore di quella Citta, dove aveano $\mathrm{i}$ 'nemici il loro quartiere, vi diede un si furiofo affalto, che prima di fpuntar la luce del di s'era già renduto padrone del quartiere, di tutti i fuoi nemici, dell' artiglierìa, dell' arme, e de'cavalli, reftando morti quattro foltanto de' fuoi Soldati, e quindici de' nemici, e molti dell' una, e dell' altra parte feriti. $(n)$ Si fece riconofcere da tutti Capitano Generale, e fupremo Magiltrato, mife in ferri nelta fortezza della Veracroce e Narvaez, e Salvatierra perfona riguardevole, e nemico giurato di Cortès, e fece levar da'valcelli le vele, i timoni, e le buffole. Appena cominciava la luce di quel dì (il quale fu la Domenica di Pentecofte 2\%. Maggio) che arrivarono $i$ due mila Chinantechi in buon ordine, e bene armati, (o) i quali vennero foltanto ad effer teftimon; del trionfo di Cortès, e del roffore de' partigiani di Narvaez, vedendofi vinti da sì pochi nemici, e men armati di loro. La felicita di quefta fpedizione fi dovette in gran parte all'imparêg. giabile bravura di Sandoval, il quale con ottanta uomini fa. Ii fal tempio in mezzo ad una tempelta di faette e di palle, affall il Santuario, dove s'era fortificato Narvaez, e s'impa. dronì della perfona di lui.

Or trovandofi Cortès con diciorto vafcelli, con preffochè due mila uomini di truppa Spagnuola, con cento cavalli in circa, e con fufficiente copia di munizioni da guerra, penso a fare nuove fpedizioni nelle Cofte del Golfo Mefricano, ed avea già deltinati i Capi, che doveano comandarle, e la gente

che

(n) Evyi ancora della varietà preflo gli Autori intorno al numero de morti in quell' affalto; io metto quello, che mi pare più verifimile attefo il ragguaglio degli fteffi Autori.

(o) Alcuni Autori dicono, che $\mathbf{i}$ Chinantechi intervennero nell' affalto del quartiere di Narvaez; ma Bernal Diaz, il quale vi fu prefente, afferma l"oppofto. Cortès non fa menzion di loro. Chi volefre informarfi di turte le circcftanze di quefta gloriofa fpedizione di Cortès, potrà confultare gli Storici della corfuifta; poiche noi le tralafciamo, come non confacenti alla noftra Storia. 
che dovea andar fotto $\mathrm{i}$ loro ordini, allorchè gli vennero da Lis. IX. Meffico delle infaufte nuove, che fraftornarono i fuoi pro. getti, e lo coltrinfero a ritornare in frett alla Capitale.

In quel tempo, in cui Cortès era affente da Meflico,

S. 15. venne la fefta dell' incenfamento d' Huitzilopochtli, che $f_{1} f_{d}$ della No-ceva nel mefe Toxcath, il quale in quell' anno cominciò adfiltà Mef- di I 3. del noftro Maggio. Celebravafi quefta fefta, la più folleva- folenne di quelle che fi facevano ogni anno, con balli del zono det $\mathrm{Re}_{\text {z }}$ e della iNobiltà, de'Sacerdoti, e del Popolo. Pregd la controgli Nobiltà il Capitano Alvarado d'acconfentire, che il Re fi Spagnuo-portaffe al tempio a fare il fuo dovere. Scufoff Alvarado, o li. per gli ordini datigli da Cortès, o perchè temette, che $i$ Mefficani macchinaffero qualche novità avendo feco loro it $\mathrm{Re}$, ben confapevole quanto fieno facili a cangiarfi in tumulti le pubbliche allegrezze. Si prefe pertanto il partito di far quel ballo religiofo nel cortile del palazzo, $(p)$ o quartiere degli Spagnuoli o per difpofizione di quel Capitano, o per ordine dello fteffo Re per poter intervenirvi fecondo il loro coftume. Venuto il giorno della fefta concorfero in quel cortile molti uomini della prima Nobiltà (del cui numero (q) non contta ) ben fregiati di parecchj ornamenti d'oro, di gemme, e di penne. Cominciarono a cantare e bal. lare in ful fuon degli ftrumenti, e frattanto fece Alvarado occupar da alcuni Soldati le porte. Quando poi vide i Mefficani

(p) Gli Storici della conquifta dicono comunemente, che il ballo fi fece nell' atrio del tempio maggiore, ma non è verifimile, che l'immenfa folla di Popolo, che vi concorreva, permettefre di fare si orrenda ftrage nella Nobilta, maffimamente effendo quivi l'armerie, onde potevano prender quante arme voleffero per opporfi alla temerità di que' pochi Atranieri, nemmeno pud crederfi, che gli Spagnuoli voleffero merterf in un si evidente rifchio di perire. Cortès e Bernal Diaz non fanno menzion del luogo cel ballo. Il P. Acofta dice, ch' effo fi fece in palazzo, né potè effere altro, fe non quello, dove allora abitava il Re. Li'inverifimiglianza, che fi fcorge nel ragguaglio di quegli Storici, ed il giusdizio e l'antichità del P. Acofa ci fanno anteporre la teftimonianza di quefto, folo fcrittore a quella di tutri gli altri.

(q) Prefro Gomara furono feicento i Nobili, che intervennero nel ballo, prefo altri Storici futono più di mille, e prefro Monfignor de las Cafas più di sue mila. 
ficani più rifcaldati, e forfe arche ftancati dal ballo, diede cenno a' fuoi d'affalirli, e coftoro fi fcagliarono tofto con fu-L.s. IX. ria contro a quegli fventurati, i quali nè poterono far refiftenza, perch' erano difarmati, e ftracchi, nè fu a loro poffibile lo fcampar colla fuga, perchè erano occupate le porte. Fu terribile la trage, lagrimevoli i gridi, che mandavano al Cielo i moribondi, e copiofo il fangue, che vi fi fparfe. Quefto fatal colpo fu uno de' più fenfibili a' Mefficani; im. perciocchè vi perdettero il fiore della lor Nobiltà, e per perpetuarne preffo i loro difcendenti la memoria, compofero fopra un tale argomento de' cantici flebili, o fia elegie, le quali fi confervarono molti anni dopo la conquifta. Terminata quella tragica ed orrenda funzione, fpogliarono gli Spagnuoli i cadaveri di tutta la ricchezza, di cui erano fregiati .

Non $f_{1} F_{2}$ il motivo che induffe Alvarado ad un fatto sì temerario ed inumano. Alcuni dicono, che altro non $\mathrm{fu}$, fe non quello della maledetta fame dell' oro. $(r)$ Altri affermano, e ciò pare più verifimile, ch' effendogli ftato detto, che $i$.Mefficani volevano in quella fefta dare un colpo maeftro agli Spagnuoli, per fottrarfi dalla loro opprellione, $e$ rimettere in libertà il loro $\mathrm{Re}$ e Signore, che gli Spagnuoli aveano in prigione, egli gli prevenne ftimando vero quel detto volgare, chi affalifce vince. $(S)$ Checcheffia, la fua condotta non può fcufarfi d'imprudenza, e di crudeltà.

La

(r) Gli Sorici Mefficani, il P. Sabagun nella fua Storia m. ro Mnnfignor de las Caras nel fuo formidabile fcritto della difruzione delle Indie, e Gomara nella fua Cronaca della $N$. Spagna affermano, che l'avarizia d'Alvarado fu la cagione della ftrage fatta nella Nobiltà mefficana; ma io non poffo crederlo fenza pruove più efficaci. Gomara e Monfignor de las Ca a fas tennero dietro fenz' altro al Sahagun,e coftui all' informazione de'Merficani,'i quali come quelli ch'erano nemici degli Spagnuoli, non fono in cio degni di fede.

(f) E' affatto incredibile, che i Mefficani macchinaffero nell occafione del ballo contra gli Spagnuoli guel tradimento che fuppongono parecchj Storici, e molto più che aveffero già apparecchiate le pignatte per cuocervi la carne degli Spagnuoli, ficcome dice il Torquemada. Quefte form favole inventate per giuftificare Alvarado. Ciò che mi pare più verifumile è 
La Plebe irritata con un colpo tanto fenfibile, tráttd d'al: Liz. IX lora innanzi gli Spagnuoli, come nemici capitali della patria. Affalirono alcune truppe mefficane con tal impeto il quartiere, che rovinarono una parte del muro, minarono per diverfe parti il palazzo, e bruciarono le munizioni; ma furo: no rifpinti col fuoco dell' artiglieria e degli fchioppi, ond' ebbero agio gli Spagnuoli di rifare il muro. Quella notte ripofarono pure dalla fatica della giornata, ma il giorno appreffo fu si furiofo l'affalto, che gli Spagnuoli fi credettero di dover perire, ed in fatti non farebbe reltato nè anche uno di loro in vita, ficcome erano già frati uccifi fei o fette, fe il Re moftratofi alla ciurma degli affalitori non aveffe colla fua autorità raffrenato il loro furore. Il rifpetto alla Maeftà del loro Sovrano ritenne la Plebe, che non affaliffe coll' arme il quartiere; ma non la diltolfe da altre forti d'oftilità : bruciò pure i quattro brigantini, che avea fatto fare Cortès per falvarfi in effi, cafo che non poteffe fcampare per le ftrade fatte ful lago, e fi rifolvette di diftruggere colla fame gli Spagnuoli, negando a loro i viveri, e procurando impedire l' intro: duzione d'effi con un foffo fcavato lattorno al quartiere.

In tale ftato fi trovavano gli Spagnuoli in Meffico, allorchè Alvarado avvisò Cortès, pregandolo per due meffaggi Thafcallefi d'affrettare il fuo ritorno, fe non voleva trovar tutti morti. Lo fteffo gli addimandò il Re Motezuma, facendogli fapere, quanto rincrécevole gli era ftata quella follevazione de'fuoi vaffalli cagionata dal temerario e fangui: nofo attentato del Capitano Tonatiuh.

Cortès, dopo aver dato i fuoi ordini di trasferire la Colonia della Veracroce ad un altro fito più vicino al porto di Chalchiuhcuecan (benchè non fi mife allora in efecuziose quefto difegno) marciò colla fua gente a gran giornate verfo la Capitale. In Tlafcalla fu magnificamente albergato

nel

che i Tlafcallefi per quel gran odio, che portavano a'Mefficani, mifero in telta ad Alvarado il pretefo tradimento. Nella Storia della Conquifta abbiamo parecchj efempj di si fatte fuggeftioni de'Tlafcallefi contra i loro nemici. 
nel palazzo del Principe Maxixcatzin . Quivi fece la raffe. gna delle fue truppe, e trovò novanta fei cavalli, e milleLis. IX. trecento pedoni Spagnuoli, a' quali s' aggiunfero due mila Tlafcallefi datigli da quella Repubblica. Con quefto efercito entrò in Meffico a' 24. Giugno. Non vi trovò verun contrafto nell' entrata; ma fubito s' accorfe della fermentazione popolare, che v'era tanto per la poca gente, che fi vedeva. nelle ftrade, quanto per alcuni ponti, ch' erano ftati levati da' canali. Allorchè entrò nel quartiere con quel giubilo degli uni e degli altri, che è facile ad intenderfi, Motezuma venue ad incontrarlo nel cortile colle più offequiofe dimotrazioni d'amicizia; ma Cortès o infolentito per la vittoria ottenuta contro Narvaez, e per la gran gente, che avea fotto i fuoi ordini, o perfuafo ch' era d' uopo il far fembianza di credere il $\mathrm{Re}$ colpevole dell' inquietudine de' fuoi vafíalli, pafsò a dilungo e fenza badar a lui. Il Re traffitto dal più vivo dolore in vedendofi si indegnamente difpregiato, fe n'andd al fuo appartamento, dove gli fi accrebbe il cordoglio colla nuova, che immediatamente gli portarono i fuoi fervitori, delle parole ingiuriole, che avea detto contra fua Mae. ftà il Generale Spagnuolo. ( $t$ )

Riprefe Cortès colla maggior feverità il Capirano Alva. rado, e gli avrebbe dato fenz' altro il gaftigo, che meritava, fe il permetceffero le circoftanze del tempo e del reo. Prefentiva la gran tempefta, ch'era ormai per piombare fulle Storiadel Mefjico Tom. $I I I$.

(t) Lo Storico Solis non vuol credere il difpregio di Motezuma fatto da Cortès, e per difender quefto Generale fa torto a Bernal Diaz, che afferma ciò, come teftimonio oculato, ed al Cronichifta Herrera, che il racconta dietro a buoni docamenti. Accufa immeritevolmente Bernal Diaz di parzialità contro Cortès, e d'Herrera dice, che può fofpettarfi, che voleffe adottar il ragguaglio di Diaz per poter adoperarvi una fentenza di Tacito: Ambizione, foggiunge, pericolofa megli Storiti; ma in niun altro più che nel medefimo Solis; poich ò ogni perfona imparziale e bene infiruita nella Stotia di Meffico vedrà in leggendo quella del Solis, che quefto Autore in vece d'aggiuftar le fentenze alla narrazione, aggiulta al contrario la narrazione alle fentenze. Finalmente qualora egli non alleghi delle ragioni migliori di quelle che adopera contro Bernal Diaz, dobbiamo dar fede alla teftimonianza di quefto sutore, che fu prefente alfatto. 
loro tefte, e non gli parve favio configlio il farfi nemico in LIB IX occafione di tanto pericolo uno de' più prodi Capitani, che aveva nella fua armata.

Colle nuove truppe condotte a Meffico da Cortès v'avea un efercito di nove mila uomini, e non potendo ftar tutti nel quartiere, occuparono ancora alcuni edifizj di quelli, ch' erano dentro il recinto del tempio maggiore nella parte più vicina al quartiere. Colla moltitudine s'accrebbe parimente la penuria de' viveri già cagionata dalla mancanza di mercato; poichè per l'odio degli Spagnuoli non volevano farlo i Mefficani. Mandò però Cortès a dire con grandi minacce a Motezuma, che deffe ordine di farfi il mercato, acciocchè eglino vi fi provvedeffero di tutto il bifognevole. Rifpore Motezuma, che i perfonaggi più autorevoli, di cui potrebbe fidarfi, per far efeguire un tal ordine, fi trovava. no, ficcome lui, in prigione: che metteffe qualcuno di loro in libertà, affinchè a ciò poteffe badar. Cortès traffe dalla prigione il Principe Cuitlahuatzin fratello di Motezuma, affai lontano da prevedere, che la libertà di quel Principe do. vrebbe cagionar la rovina degli Spagnuoli, Cuitlahuatzin non ritornò mai al quartiere, nè rimife il mercato, o perchè non volle favorire gli Spagnuoli, o perchè non vi aceonfentirono i Mefficani, anzi lo coftrinfero ad efercitare la fua carica di Generale. In fatti egli fu colui, che d'allora innanzi comandò le truppe, e foprantefe alle oftilità contra gli Spagnuoli, finattantochè per la morte del fuo fratello fu eletto $\mathrm{Re}$ di Meffico.

5. 16. Quel giorno, nel quale entrò Cortès in Meffico, non Zuffe tra fece verun moto la Plebe; ma nel di appreffo cominciarono caniegli a tirar con frombole tanti faffi contra gli Spagnuoli, che Spagnuo- fembrava, fecondo che diffe Cortès, una tempelta, e tante
Ii nella Capitale.frecce, che coprirono tutto il pavimento del corcile, ed i terrazzi del palagio, ed erano tanti gli affalitori, che non fi vedeva il fuolo delle ftrade. Non parve bene a Cortès di ftar fulla difefa; perchè non s'afcriveffe a codardia, onde maggior coraggio prendeffero $i$ nemici. Fece per tanto 
una fortita contro loro con quattrocento uomini parte Spagnuoli, e parte Tlafcallefi. I Mefficani s'andarono ritirando Liz. IX. con poco loro danno, e Cortès dopo aver fatto appiccar il fuoco ad alcune cafe, ritornò al quartiere; ma vedendo, che i nemici continuavano le lor oftilità, tece forrire il Capitano Ordaz con dugento Soldati. I Mefficani fecero vifta di fcompigliarfi e di fuggire per allontanarli dal quartiere, ficcome in fatti l'ottennero; ma improvvifamente fi videro gli Spagnuoli da ogni parte circondati da'Nemici, ed attaccati da un corpo di truppe alla fronte, e da un altro alle fpalle, benchè tanto tumultuariamente, e con tal difordine, che gli uni davano impaccio agli altri. Allo fteflo tempo fi lafciò vedere fopra $\mathrm{i}$ terrazzi una gran ciurmaglia, la quale inceffantemente tirava de' faffi e delle frecce. 'Trovaronfi allora gli Spagnuoli in gran pericolo, e quefta occafione fu fenz' altro una di quelle, nelle quali fece fpiccar più la fua bravura il prode Ordaz. Il combattimento fu molto fanguinofo, ma fenza gran danno degli Spagnuoli, i quali cogli fchioppi, e le baleftre fgomberarono i terrazzi, e colle picche e le fpade rifpinfero la moltitudine, che inondava la Atrada, e cosi poterono finalmente ritirarfi al quartiere, la. fciando morti molti Mefficani, e de'fuoi non più d'otto; ma ufcirono quafi tuiti feriti ed anche lo ftefio Ordaz. Tra i mali, che fecero in quefto di i Mefficani agli Spagnuoli, fu quello d'attaccar fuoco a diverfe parti del quartiere, ed in una d'effe fu tal l'incendio, che gli Spagnuoli furono coftretti a gitrar giù la muraglia, ed a difender quella breccia coll'artiglieria, e con molta gente, che vi pofero fin'alla notte nella quale i nemici diedero loro luogo di rifar la muraglia, e di curare i feriti.

Il dì vegnenre ( 26 . Giugno ) fu più terribile l'affalto e più grande la furia de' Mefficani. Gli Spaguuoli fi difende. vano con dodici pezzi d'artiglieria, i quali faceano una grande Atrage nella folla degli affalizori; ma ficcome quefti erano in si gran numero, s'occultavano i morti fotto i piedi di quelli, che fottentravano nel loro luogo. Cortès in vedendo la Q 2 loro 
Horo oftinazione, forti colla maggior parte delle fue truppe, Lis. IX.e s' incamminò combattendo per una delle tre principali ftrade della Città; efpugnò alcuni ponti, appiccò il fuoco ad alcune cale, e dopo aver combattuto quafi tutto il di, ritorno al quartiere con più di cinquanta Spagnuoli feriti, lafciando morti innumerabili Mefficani.

La fperienza fece conofcere a Cortès, che il maggior danno veniva alle fue truppe da' terrazzi, onde per ifchivarlo fece fare tre macchine da guerra (dagli Spagnuoli appellate Mantas ) sì grandi che ognuna poteffe portar venti Soldati armati, coperte con un forte tavolato per difendere i Soldati da' faffi de'terrazzi, fornite di ruote per agevolare il moto, e colle loro fineftrelle o cannoniere per lo fparo degli fchioppi. Mentre fi confruivano quefte macchine, avvennero delle novità grandi in quella Corte. Motezuma falito fopra una torre di quel palazzo, avea indi offervato uno do' fuddetti combatrimenti, ed avea veduto tra la folla del popolo il fuo fratello Cuitlahuatzin comandando le truppe Mefficane. A vifta di tanti oggetri lagrimevoli fu affalito l'animo di lui da una turba di penfieri malinconici. Vedeva da una parte il pericolo che correva di perder la corona e la vita, e da un'altra gli fi prefentavano la diftruzione degli edifizj della Capitale, l'uccifione de' fuoi vaffalli, e la felicita de' fuoi nemici, e non trovava altro rimedio a tanti mali, fe non quello della prorsta ulcita degli Spagnuoli. In si fatti penfieri palsò quella notte, ed il dì feguente a buon ora chiamò Cortès, e gli parlò fullo fteffo propofito, pregandolo inftantemente di non differir più la fua partenza da quella Corte. Non avea d'uopo Cortès di sì fatte preghiere per rifolverfi al partito del viaggio. Trovavafi oltremodo bifognofo di viveri: il cibo fi dava già per milura a'Soldati, ed era sì poco, che baftava foltanto a mantener la vita, non già la forza neceffaria per opporfi a tanti nemici, da' quali erano in. ceffantemente travagliati. Finalmente vedeva, ch' era tanto impoffibile per lui il renderfi padrone di quella Città, come vorrebbe, che nè anche vi potrebbe fuffiftere. $D_{2}$ un altro 
eanto gli rincrefceva affai l'abbandonar l'imprefa cominciata, perdendo in un momento colla fua partenza, tutti que'van- Lis. IX. taggi, che s' era procacciati col fuo coraggio, colla fua induAtria, e colla fua felicità; ma cedendo al tempo, rifpofe al $\mathrm{Re}$, ch'egli era pronto a partire per la pace del Regno, purchè i fuoi vaffalli deponeffero l' arme.

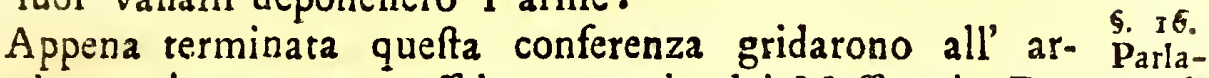
me nel quartiere per un affalto generale dei Mefficani. Da mento del per tutto procuravano di falir fulla muraglia per entrarvi, Real Po. mentre che alcune truppe d'arcieri polte in convenevole di- polo, e ftanza tiravano una immenfa moltitudine di frecce per fupera- fetti. re l'oppofizione degli affediati, ed alcuni affalitori s' innoltra. rono tanto, che malgrado il fuoco dell'artiglieria, e degli fchioppi mifero piede dentro il quartiere, e combatterono a corpo a corpo cogli Spagnuoli . Coftoro ftimandofi oramai quafi vinti e fopraffatti dalla moltitudine, pugnavano da difperati. Motezuma in vedendo il loro conflitto, ed il fuo proprio rifchio, fi rifolvetta di farfi vedere per trattener colla luz prefenza e colla fua voce il furor dei fuoi vaffalli. Meffefi però l'infegne regie, e fcortato da alcuni fuoi Miniftri, e da dugento Spagnuoli fali ful terrazzo, e fi prefentò al Popolo, intimando filenzio i Miniftri, acciocchè poteffe fentirfi la voce del Sovrano. Sul comparir di lui cefsò l'affalto, ammutolirono tutti, ed alcuni s' inginocchiarono per riverirlo. Parlò ad alta voce, e fece loro in fuftanza quefto brieve difcorfo: "Se il " motivo, che v'induce a prender l'arme contro quefti Stra"nieri, è il zelo per la mia libertà, ho pur caro l'amore " e la fedeltá, che mi moltrate; ma v'ingannate nel cre" dermi prigioniere di loro, mentre è in mia balia il lafciar " quelto palazzo del mio defunto Padre, ed andarmene al " mio, qualora mi piaccia. Se poi il voltro fdegno è cagio" nato dalla lor dimora in quefta Corte, vi fo fapere, ch" " eglino m'hanno dato parola di partire, ed io v'afficuro, " che fenz'altro partiranno tofto che voi ponghiate giù $l^{\prime}$ " arme. Ceffi dunque la voltra inquietudine: fatemi in ciò "palefe la voltra fedeltà, fe già non è vero quello, che ho ") fen: 
, fentito dire, cioè che avete ad un altro Signore giurata Lı. IX., quella ubbidienza, che a me dovete: il che nè io poffo " perfuadermi, nè voi far potrete fenza tirarvi addoffo tut"ta la collera del Cielo.,"

Reftò il Popolo per un poco muto, finchè un uomo più ardito (u) degli altri alzò la voce, chiamando il Re codardo ed effeminato, e più atto a maneggiare il fulo e la fpola, che a reggere una si coraggiofa Nazione, come la Mefficana, e rinfacciandogli, che per la fua codardia fi foffe, farto con tanta viltà prigioniere dei fuoi nemici, e non contento d'ingiuriarlo con parole prendendo in mano l'arco gli tirò una freçia. La plebe, come quella ch'è tanto facile a muoverfi col primo impulfo, che le fi dà, feguì prontamente il fuo efempio: cominciaronfi a fentir da per tutto degl' improperj, ed a piover dei faffi e delle frecce verfo quella parte, dov'era il Re. Gli Storici Spagnuoli dicono, che con tutto che la perfona Reale coperta foffe con due rotelle, fu pur ferita da una faffata nel capo, da un'altra in una gam. ba, e da una frecciata in un braccio. Quindi fu dai MiniAtri alla fua camera pórtato, travagliato affai più dallo sdegno, e dalla rabbia, che dalle ferite.

Frattanto i Mefficani perfiftevano nel loro affalto, e gli Spagnuoli nella loro difefa, infinattantochè alcuni Nobili chiamarono Cortès a quel medefimo luogo, dov'era ftato ferito il $\mathrm{Re}$ per difcorrere con lui intorno a parecchj articoli, che non troviamo dichiarati preffo gli Storici. Cortès addimandò loro, perchè voleano trattarlo da nemico, non aven. do ricevuto verun torto da lui., Se volete, gli differo, fchi. "var le noftre oftilità, ufcite fubito di quelta Città; fe nò, "noi framo rifoluti di morire, o pur di farvi morir tutti.", Cortès foggiunfe, ch'egli non fi lagnava di loro, perchè a. veffe paura delle loro arme, ma perchè gli rincrefceva d' ef. fere

(a) Il P. Acofta dice, che il Mefficano, che diffe quelle villanie al Re, fu Quauhtemotzin nipote di lui, e poi ultimo Re di Meffico; ma io not credo. 
fere da loro fteffi coltretto a dar loro la morte, ed a diftrug. gere una s̀̀ bella Città. I Nobili fe n' andarono replicandoLis. IX. le loro minacce.

Compite finalmente le tre macchine da guerra, ufci con effe a buon'ora Cortès ai 28 ., o 29. Giugno $(x)$, e s'incamminò per una delle tre ftrade principali della Citta con tre mila Tlafcallef, ed altre truppe aufliarie, colla maggior parte degli Spagnuoli, e con dieci pezzi d'artiglieria. Arrivati che furono al ponte, ch'era ful primo canale, appofero alle cafe le macchine e le fcale per ifcacciare la ciurma, ch'era fopra i terrazzi; ma furono tante e sì grofre le faffate quindi tirate contra le macchine, che le fracaffarono. Gli Spagnuoli combatterono coraggiofamenre infin'al mezzo di fenza poter mai efpugnar il ponte: per lo che ritornaro. no vergognati al quartiere, lafciando un di loro morto, e conducendo feco molti feriti.

Incoraggiti per tanto i Melficani, fi fortificarono cinquecento Nobili nell' atrio fuperiore del tempio maggiore ben forniti d'arme e di vettovaglie, e quindi cominciarono a far gran danni agli Spagnuoli con faffate e frecciate, mentre che altre truppe mefficane gli affalivano per la ftrada. nio.

Mandovvi Cortès un Capitano con cento Soldati per ifracciare i Nobili da quel luogo, il quale, perchè troppo alto e vicino, dominava il quartiere; ma avendo tentato per ben tre volte la falita, furono vigorofamente rifpinti. Determinoffi però quel Generale a dar lui medefimo l'affalto, con tutrochè aveffe infin dal primo combattimento una buo. na ferita nella mano finiftra: legoff la rotella nel braccio, ed avendo fatto cerchiar il tempio da un numero competen. te di Spagnuoli e di Tlafcallefi, cominciò a falir per le fcale con una gran parte della fua gente. I Nobili affediati contraltavano loro con gran coraggio la falita, e rovefcia.

rono

(x) E indicibile la varietà degli Autori intorno all'ordine, ed alle circofanze de'combattimenti, che vi furon in que'giorni. Io tengo dietro al ragguaglio di Cortès, che mi pare in ciò il più autorevole. 
rono alcuni Spagnuoli; frattantochè altre truppe meflicane Lis. IX. entrate nell'atrio pugnavano furiofamente con quelli, che cerchiavano il tempio. Cortès, febbene con fomma fatica e difficoltà, mife pur piede colla fua gente nell'atrio fuperiore. Quivi fu la maggior forza, ed il maggior pericolo della zuffa, la quale duro più di tre ore. I Mefficani parte vi morirono a fil di fpada, e parte fi gittarono ai piani inferiori del tempio, dove continuarono: la pugna finchè tutti furono uccifi. Cortès fece attaccar fuoco ai Santuarj, e fi reftitu' in buon ordine al quartiere. Vi perirono quarantafei Spagnuoli, e tutti gli altri ufcirono feriti e bagnati di fangue. Quefto famofo combattimento fu uno di quelli, nei quali fi pugnò con maggior coraggio dall' una e dall' altra parte; e per ciò il rapprefentarono dopo la conquifta tanto i Tlafcallefi, quanto i Mefficani nelle loro pitture.

Alcuni Storici aggiungono a ciò la circoftanza del gran rifchio, in cui fi trovò fecondo che effi dicono, Cortès d' effere precipitato da due Mefficani, i quali rifolutifi di facrificare la propria lor vita pel bene della patria, afferrarono Cortès aell' orlo dell' atrio fuperiore per trarlo feco loro nella caduta credendofi di por fine alla guerra colla morte di quel Generale; ma quefto fatro, del quale non fanno menzione nè Cortès, nè Bernal Diaz, nè Gomara, nè verun altro degli Storici più antichi, s'è renduto più inverifimile per le circoltanze aggiuntevi da alcuni Scrittori moderni. $(y)$

(y) Solis dice, che $\mathrm{i}$ due Mefficani s'accoftarono inginoccbione a Cortès in atto d'implorar la fua clemenza, e fenza indugiare fi buttarono git colla preda nelle mani aumentando la violenza dell' impulfo colla forza naturale del lor proprio pefo; che Cortès fa sbroglio da loro, e gli ributto con qualche diffcoltaे. Io pure la trovo affai grande per credere una forza si corprendente in Cortès. Gli umaniffimi Signori Rainal, e Robertfon moffi a pietà, per quanto appare, pel pericolo di Cortès lo provvidero quefti di hon fo che merli, e quegli d'una inferrata, dove s'appigliaffe per isbrogliarfi da'Meficani; ma nè i Meflicani fecero mai dell' inferrate, nè in quel tempio erano de'merli. E' da maravigliare, che quefti Autori tanto increduli rapporto a cid, che viene teftificato e dagli Spagnuoli, e dagl' Indiani, credano poi cid, che nè fi trova preffo gli Autori antichi, _e neppur è verifimile. 
Ritornato Cortès al quartiere, s'abboccò di bel nuovo con certi Mefficani ragguardevoli, rapprefentando loro ilLis. IX. danno che ricevevano dall' armi Spagnuole. Coloro rifpolero, che ciò nulla importava, purchè foffero rovinati gli Spagnuoli, che cáo che non foffero da' Mefficani ammazzaci, dovrebbono infallibilmente peire della fame rinchiufi nel quartiere. Cortès avendo offervato quella notte qualche trafcuraggine ne' Cittadini, forti con alcune compagnie di Soldati, ed incamminandofi per una delle tre frade principali, appiccò il fuoco a più di trecento cafe. $\left({ }^{*}\right)$

Il di vegnente poichè furono racconciate le macchine, forti con effe, e colla maggior parte delle fue truppe, e s' avviò per la grande ftrada d' lztapalapan con miglior riufcita della prima volta; imperocchè a difpetto della vigo. rofa refiftenza, che faceano i nemici nelle trinciere da loro fatte contro il fuoco degli Spagnuoli, efpugnò i quattro primi ponti, ed attaccò fuoco ad alcune cale di quella ftrada, e fi approfittò de' materiali d'effe per empierne i folf o canali, acciocchè non vi foffe difficoltà nel paffaggio, calo che i Meflicani levaffero i ponti. Lafciò ne' pofti efpugnati una fufficiente guernigione, e ritornò al quartiere con molti Sol. dati feriti, lafciando dieci o dodici morti .

Nel d’̀ appreffo continuò i fuoi attacchi per la medélio ma ftrada, efpugnò i tre ponti, che gli mancavano, ed incalzàndo i nemici, che gli difendevano, arrivò infin' a terra ferma. Mentre che badava a far empiere gli ultimi foffi per agevolare, com'è da crederfi, la fua ritirata dalla Corte per quella fteffa ftrada, per dov'era già entrato fetre mefi innanzi, gli fu detto che $\mathrm{i}$ Mefficani volevano capitolare, e per fentir le loro propolizioni ricornò in fretta al quartiere con la cavalleria, lafciando tutta l'infanteria per guardare i

Storia Antica del Meffico Tom. III. $\mathrm{R}$ pon.

$\left({ }^{*}\right)$ Cortès dice, che bruciava le cale, ma ciò non vuol dire, che ardevano rutte, e reftavano incenerite; ina foltanto, che appiccava ad efre il fuoco, il quale in alcune faceva molto male, in altre poco, ed in altre ninno. Bernal diaz dice, che fi durava fatica per farle ardere, perchè erano coperte di terrazzi, e Separate l'una dall' altra. 
ponti efpugati. Gli propofero i Mefficani, ch'érano pronti LiB. IX.a far ceffare tutte l'oftilità; ma che per far la capitolazione aveano d'uopo della perfona d'un Sommo Sacerdote, ch' era ftato fatto prigione dagli Spagnuoli, allorchè diedero l'affalto al tempio. Cortès lo mife fubito in liberta, e fi capitolò l'armiftizio. Ciò pare effere ttato un mero ftratagemma degli Elettori per ricuperare quel capo della Religione, della cui perfona aveano bifogno per l'anzione del nuovo $\operatorname{Re}$, che aveano gia eletto, o erano omai per eleggere; perchè appena ebbe Cortès il piacere della fofpenfion dell' armi, che varrivarono alcuni Tlafcallefi portando la nuova, che $\mathbf{i}$ Mefficani aveano riprefi i ponti, ed uccifi alcuni Spagnuoli, e che veniva una gran folla di guerrieri contro il quartiere. Cortès andò ad incontrarli colla cavallerìa, e rompendogli con fomma fatica e pericolo per farfi ftrada, ricuperò i ponti ; ma nel tempo, in cui efpugnava gli ultimi, aveano già tolto i Mefficani agli Spagnuoli i quattro primi, ed aveano cominciato a trar fuori $\mathrm{i}$ materiali, con cui aveano coloro empito i foffi. Tornò finalmente Cortès ad efpugnarli, e fi ritirò al quartiere con sutta la fua gente Aanca, malconcia, e ferita.

Cortès nella fua lettera a Carlo V. gli rapprefenta il gran pericolo, che in quel giorno corfe di perder la vita, ed afcrive ad una particolar provvidenza de! Signore l'averla fcam. pata fra una s̀̀ gran moltitudine di nemici. Egli è certo, che da quel momento, nel quale i Mefficani fi follevarono contro gli Spagnuoli, avrebbono potuto rovinarli tutti infieme co'loro alleati, fe aveffero offervato un miglior ordine nel combattere, e fe vi foffe ftata una maggior cencordia tra i Capi fubalterni, che foprantendevano agli attacchi; ma i capi non erano d'accordo, come poi vedremo, ed il popolaccio era portato foltanto dall' impeto del fuo furore tumultuario. Da un' altra parte non può dubitarfi, the gli Spagnuoli parevano effer di ferro, mentre nè cedevano al rigor della fame, nè alla neceffità del fonno, nè alla continua fatica, nè alle ferite. Dopo aver impiegato tutto il di 
nel combattere co'loro nemici paflavano la notte feppellendo i morti, curando i feriti, e riparando a' mali cagionati da' Lis. IX. Mefficani durante il di nel quartiere, ed anche in quel poco terripo, che davano al neceffario ripolo, non lafciavano mai l'arme, pronti tuttora a prefentarfi a' nemici.Ma ancor più palefe fi farà la durezza di quegli uomini né terribili combattimenti, che frappoco efporremo.

In uno di quefti giorni, verifimilmente il 30. Giugno, morì dentro il quartiere degli Spagnuoli il $\mathrm{Re}$ Motezuma ${ }^{\text {5. } 19 .}$ nel cinquantefimo quarto anno della fua età, nel decimot- Re Motavo del fuo Regno, e nel fettimo mefe della lua prigione zuma II. Intorno alla cagione, ed alle circottanze della fua morte vi Signori. c̀ tal varietà e contraddizione preffo gli Storici, ch'è affat. to impoffibile di rintracciare il vero. Gli Storici Mefficani n'incolpano gli Spagnuoli, e gli Spagnuoli incolpano i Merficani. (z) Io non poffo perfuadermi, che gli Spagnuoli fi rifolveffero a toglier la vita ad un $R e$, a cui doveano tan. ti beni, e dalla cui morte non potevano afpettarfi, fe non molti mali. La perdita di lui fu compianta, fe diamo fede a Bernal Diaz autore oculato e finceriflimo, non meno da Cortès, che da ciafcuno de' Capitani e de' Soldati, come farebbe quella del lor proprio Padre. Egli infinitamente li favor ò folfe in tutto per la fua propria inclinazione, 0 in parte ancora per paura; fempre: mai fi moltro verfo loro di buon cuore; almeno non $v$ 'è ragione di creder l'oppofto, nè fi fa, che mai fparlaffe contro agli Spagnuoli, com' eglino. fteffi il proteltarono.

\section{R 2}

Le.

(z) Cortès, e Gomara affermano, che Motezuma morì della faffata, che gli diedero nel capo i fuoi vaffalli. Solis dice, che la morte gli fi cagio no da non averfi voluto curar la ferita. Bernal Diaz aggiunge a si fatta ommiffione la volontaria inedia. Il Cronichifta Herrera dice, che la ferita non era mortale, ma che mori del crepacuore e della rabbia. Il P. Sahagun, e gli Storici Mefficani, e Tezcucani affermano, che gli Spagnuoli l'ammazzarono, ed un di loro efprime la circolanza d'avergli un Soldato traffitto colla fpada l'anguinaglia. Tra quefti ultimi Storici alcuni dicono, che la morte di lui accadde la notte della fconfitta degli Spagnuoli, altri affermano, che avrenne innanzi. Acofa, Torquemada, e betancurt. la rifervano al Divino giudizio. 
Le fue buone e cattive qualito poffono intenderfi dal racIzy. IX. conto delle fue azioni. Egli fu circofpetto, magnifico, liberale, zelante della giuftizia, e grato a' fervizj de' fuoi fudditi; ma la fua ritrofa circofpezíone rendeva inacceffibile il trono a'lamenti de' vaffalli. La fua magnificenza, e la fua liberalità s'appoggiavano alle gravezze del Popolo, e la fua giuftizia degenerava in crudeltà. $F u$ efatto e puntuale in ciò , che apparteneva alla Religione, ed affai zelante del culto de' fuoi Dei, e dell' offervanza de' riti. (A) Nella fua giovinezza fu portato per la guerra, e coraggiofo, e reitò vincitore, fecon. do che $f i$ dice, in nove battaglie; ma negli ultimi anni del fuo Regno le delizie domeltiche, la fama delle prime vittorie degli Spagnuoli, e fopratutto la fuperftizione avvilirono a tal fegno il fuo animo, che pareva aver cangiato di feffo, ficcome dicevano i fuoi fudditi. Dilettavafi affai della mufica, e della caccia, ed era dettro tanto nell' efercizio dell' arco e delle frecce, quanto in quello della cerbottana. Era di buona ftatura, e di poco buona carnagione, di faccia lunghetra, e d'occhi vivi.

Lafciò in morendo parecchj figliuoli, de' quali tre pe: rirono nella infaufta rotte della fconfitta degli Spagnuoli, o per le mani degli fteffi Spagnuoli, come affermano i Mefficani, o per le mani de' Mefficani fecondo che dicono gli Spagnuoli. Di quelli, che fopravviffero, il più grande fu Johua: licahuatzin, il quale nel battefimo s' appellò Don Pietro $M o$. tezuma, e da coftui difcefero i Conti di Motezuma, e di Tula. Ebbe Motezbma quefto figliuolo da Miahuaxochitl $\left({ }^{*}\right)$ figlia

(A) Solis dice, che Motezuma appena piegava la cervice, cioè chinava il capo, ai fuoi Dei, che avea ura più grande idea di fe, chè degli Dei \&c. Ma quefte, ed altre fimili cole, che afferma quefto Storico, fono affatto contratie alla verità, ed alla teltimonianza degli Autori Indiani, e Spagnuoli, che corobbero quel Re. Il medefimo Solis roggiunge, che il Demonio lo favoriva con frequenti vijite. Ma come favorir tanto colui, che il difpregiava? Si fatta credulita non 1 conviene ad un Cronichifta maggior del!' Indie.

(*). Solis alterando, come fuole, il nome di quefta Regina l' appella Niagua Sucbil. Coftei foppravviffe alla conquifta, e prefe nel battefimo i! zome di Donna Maria Miabuaxochitl. 
Figlia d' Ixtlilcuechahuac, Signor di Tollan. Da un altra mo. glie ebbe Tecuichpotzin Principeffa bella, dalla quale difcen-Lie. IX. dono le due nobilifime cafate di Cano Motezuma, e d'Andrada Motezuma. Oltre a quefti fappiamo, che avea un altro figliuolo ch' era Signor di Tenajoccan, il quale effondo fcam. pato, e ricoveratofi in Tepozotlan, allorchè gli Spagnuoli ufirono fconfitti di Meffico, fu poi folennemente battezzato, effendo vicino a morte, ful fine dell' anno I524., o ful principio del 1525 . (B) I Re Cattolici accordarono ingolari pri. vileg; alla polterita di Motezuma per riguardo all' impareg. giabile fervizio loro fatto da quel Monaria nell' incorporare nella Corona di Caftiglia colia fua volontaria ceffione un Regno si grande, e si ricco, come quello del Meffico. Felice lui, fe dopo aver ceduto il fuo Regno al Re di Spagna, fi foffe procacciato il Regno del Cielo; ma nè le replicate in. ftanze fattegli da Corrès in tutto il tempo della fua prigio. ne, nè le continue efortazioni impiegate dal P. Olmedo, maffimamente negli ultimi giorni della vita di lui, baftaro. no per indurlo ad abbracciar la fede di Gesù Crifto, (C) la quale indi a poco fu si facilmente abbracciata da' fuoi valo falli. Configlj altiffimi della predeftinazione, che non poffono indagarfi da'martali.

Tolto

(B) Quefto figlivolo di Motezuma, Signor di Tenajoccan prefe nel bat: tefino il nome del fuo patrino Rodrigo di Paz, cugino del Conquiftatore Cortès. Intervennero al batterimo i Magiftrati Spagnuoii di quella Corte, e fuferpellito il cadavero del fuddetro Principe con la pompa, che fi conyeniva, nella Chiefa di S. Giufeppe dei PP. Francelcani, prima parrucchia della Nusova Spagna.

(C) Didaco Mugnòz Camargo, nobile Tlafcallefe dice nei fuoi manofcritti, che Motezuma poco prima di morire fu battezzato, ed anche nomina coloro, che io tennero a battefimo, cioè Cortès, Alvarado, ed $\mathrm{O}$ lid; ma ciò è fenz' altro falfo; poichè non può crederfi, che Cortès nom ne faceffe menzione nella fua lettera a Carlo V. mentre tanto importava per la fua giuffificazione. Bernal Draz teltimonio oculato rarumemora it rammarico del $\mathrm{P}$. Olmedo per non aver potuto ridur quel- Re al Criftia nefimo. Gumara dice, che Motezuma addimandd il battefimo nel Carnovale di quell'anno: che fi differi fin'alla pafqua acciocchè forfe più folenriz, ed allora fi fraftornò per. l'arrivo di Panfilo. Narvaez; ma è fuor di dubbio, che per la pafqua non era ancor arrivata a Meflico la nuova dell' armata di Narvaez: non potè dunque frattornarfi per effa il battefimo. 
Tofto che morì il Re, il fece Cortès fapere al PrinciLis. IX pe Cuitiahuatzin per mezzo di due illuftri prigioni, ch' erano ftati prelenti alla morte di lui, ed indi a poco fece metter fuori il Real cadavero da fei Nobili Mefficani, accom. pagnati da parecchj Sacerdoti, ch' erano rimilmente in prigione. $(D)$ La vifta d'effo eccitò un gran pianto nel Popolo ( ultimo omaggio, che faceano al lor Sovrano ) inalzando con le lodi le fue virtù infin" alle ttelle que' medefimi, che poco innanzi non trovavano in lui, fe non vizj da biafimare. La Nobiltà, poichè ebbe fparfe copiofe lagrime ful freddo corpo del fuo fventurato Re, il portò ad un luogo della Città appellato da loro Copalco (E) dove lo bruciaro. no colle folite ceremonie, e feppellirono con fomma riveren. za le ceneri, febbene non mancarono alcuni uomini indegni, c ribaldi, che l'infultarono con villanie.

In quefta medefima occafione, fe mai è vero ciò che raccontano parecchj. Storici, fece Cortès gettar dal quartiere in un luogo chiamato Tebuajoc i cadaveri d'Itzquauhtzin, Signor di Tlatelolco, e d'altri Signori prigionieri, i cui no. mi ignoriamo, uccifi tutti, fecondo che coloro affermano per ordine di Cortès, benchè niuno efprima il motivo di sì fatta rifoluzione, la quale cafo che foffe ftata giufta, non però potè fcufarfi d'imprudenza; poichè la vilta di quella ftrage dovea neceffariamente irritar lo fdegno de' Mefficani, ed indurgli nel fofpetto d'effere ttato fimilmente uccifo ddgli Spagnuo.

(D) Torquemada, ed altri Autori dicono, che il Cadavero di Motezuma fu gettato nel Tehuajoc infieme cogli altri cadaveri; ma dâi ragguaglio di Cortès e da quello ancora di Bernal Diaz, confa, che fu mefro fuor del quartiere fulle fpalle dei Nobili.

(E) Herrera congettura, che Motezuma fia ftato: feppellito in Chapoltepec, perchè gli Spagnuoli fentirono un gran pianto verfo quella parte.So. lis afferma pofitivamente, ch'effo fu feppellito in Chapoltepec, e che ivi era il fepolcro dei Re; ma tutto ciò è a ffatto, contrario al vero, perchè Chapoltepec non era diftante meno. di tre miglia dal quartiere : ficchè era impofibile, che gli Spagnuoli fentiffero il pianto, che vi fi faceva, maffimamente trovandofi nel centro d'una Città tanto, popolofa, ed in tempo. di tanta turbolenza e rumore. I Re poi non aveano, un luogo fiffo per la loro fepoltura, e particolarmente ci confta per la depofizione dei Mefficani, che le ceneri di Motezuma furono, reppellite in Copalco. 
gnuoli il lor Sovrano. (F) Checcheffia di tal fatto, i Tla. telolchi porrarono fopra una barca il cadavero del loro Si-Lis. IX. gnore, e celebrarono con gran pianto di quel popolo le fue effequie.

Frattanto continuavano i Mefticani con maggior ardo. re i loro attacchi. Cortès contuttochè faceffe una grande ftrage di loro, e foffe quafi fempre reftato vincitore, pure vedeva bene, ch' era più il fangue fparfo da'fuoi Soldati, che $i$ vantaggi provegnenti dalle fue vittorie, e che alla fi. ne la mancanza di viveri e di munizioni, e la moltitudine de'nemici doveano neceffariamente prevalere alla bravura delle fue truppe, e alla fuperiorita delle fue arme. Credendo per tanto affolutamente neceffaria la pronta partenza degli Spagnuoli, chiamò a corfiglio i fuoi Capitani per deliberar ful tempo ed il modo d'efeguirla. I loro pareri furono diverfi. Chi diceva, che doveano partir di giorno facendofi ftrada coll'armi, fe i Mefficani vi s'opponeffero. Chi voleva, che cio fi faceffe di notre. Quefto appunto fu il parere d' un Soldato appellato Botello, che fi vantava d'Aftrologia, a cui deferiva Cortès più di quello, che fi conveniva, ingannato dall' aver vedute certe fue predizioni cafualmente avve. rate. $\mathrm{Si}$ rifolvette dunque, anteponendo le vane offervazio. ni di quel mefchino Soldato alla luce della prudenza militare 'd'ufcir di notte tempo col maggior fecreto poffibile, come fe baftar poteffero tutte le loro diligenze per occultare alla vigilanza d'un sì gran numero di nemici la marcia di nove mila uomini colle loro arme, co'loro cavalli, coll' aptiglieria, e col bagaglio. Fu pur effa prefifa per la notte del I. Luglio $(G)$ notte infaufa e menorabile per gli Spa* gnuo.

(F) Intorno alla morte di quei Signori nulla dicono Cortès, Bernal Diaz, Gomara, Herrera e Solìs; ma la rapportano, come certa, Sahagun, Torquemada, Betancurt, e gli Storici Mefficani. Io per riguarda a que fti, e per la fedeltà, che debbo al Pubblico la racconto pure; ma con qualche diffidenza a cagione dell' inverifimiglianza, che vi trovo.

$(G)$ Bernal Diaz dice, che la f́confitta degli Spagnuoli accadde la notte del di ro. Luglio; ma io credo, che ciò fia ftato unosbaglio dello Stampa- 
gnuoli a cagione della grande Atrage, che foffrirono, per lo Lrs. IX.che le diedero il nome di noche trifte, col quale è fin' ad ora conofciuta nelle loro ftorie. Fece Cortès far un ponte di legno, che fi poreffe portare da quaranta uomini per fervir fi d'effo nel paffaggio de'foff. Fece poi metter fuori tutto l' oro, l'argento, e le gemme, che fin' allora aveano ammaffato: ne cavò la quinta parte, che apparteneva al loro Re, e la confegnò agli Uffiziali di fua Maeftà, proteftando l'im. poffibilita, in cui fi trovava, di confervarla e falvarla . Lafciò il refto a'fuoi Uffiziali e Soldati, acciocchè ognuno pi : gliaffe ciò che voleffe; ma infieme gli avvertì, quanto meglio farebbe l'abbandonar tutto a' nemici; poichê liberi da quel pefo potrebbero con minor difficoltà falvar le loro vite. Molti di loro, non volendo reftar privi del principale oggetto delle loro brame, e dell' unico frutto delle loro fa. tiche, s'addoffarono quella preziofa foma, forto il cui pefo perirono vittime non meno della loro avarizia, che della vendetta de'lor nemici.

9. 20 .

Ordind Cortès la fua marcia nel maggior filenzio della Terribilenotte, la quale rendevafi più ofcura per le nuvole, che $v^{\prime} e$. 'confíta rano, e più molefta e pericolofa per cagione d'una piccola degli Spa- pioggia, che non ceffava. Diede la vanguardia all invitto nella lor Sandoval con altri Capitani, e con dugento pedoni, e venritirata! ti cavalli, la retroguırdia a Pietro Alvarado colla maggior parte delle truppe Spagnuale. Nel corpo dell' efercito fi con: ducevano i prigionieri, la gente di fervizio, i! bagaglio, e Cortès con cinque cavalli e cento pedoni per dar pronto ajuto, dovunque foffe maggior bifogno. Le truppe aufiliarie dî Tlafcalla, di Cempoalla, e di Cholulla, nelle quali erano allora piu di fette mila uomini, fi compartiruno nelle tre parti dell' efercito. Avendo poi implorata la protezione del Cielo, cominciarono a marciare per la ftrada di Tlacopan. La maggior

tore; perchè Cortès afferma, ch'eglino nella lor ritirata arrivarono a Tlafcall a il di 10. Luglio, e dal diario della loro marcia fcritto da quefto Conquiftatore fi fcorge, che la fconfitta non potè accadere, fe non nel 1. Luglio. 
gior parte di loro pafsò felicemente il primo foffo, o fia canale coll' ajuto del ponte, che feco portavano, fenza trovareLis. IX. altra refiltenza, che quella poca, che far poterono le fentinelle, dalle quali era guardato quel polto; ma accortifi i Sacerdoti, che vegliavano ne'tempj, gridarono all' armi, e colle loro cornette eccitarono il popolo. In un momento fi videro gli Spagnuoli affaliti e per acqua, e per terra da un numero infinito di nemici, i quali colla ftefia loro moltitudine e difordine s'impacciavano nell' attacco. Fu affai terri. bile e fanguinofo il combattimento nel fecondo foffo, eftremo il pericolo, e traordinarj gli sforzi degli Spagnuoli per ifcampare. Il bujo della notte, lo Arepito dell'arme, i clamori minacciof de' combatrenti, i lagrimevoli lamenti de? prigionieri, ed i languidi fofpiri de'moribondi formavano un compleffo non men compaffionevole, che orribile. Quì la voce fentivafi d'un Soldato, che domandava ajuto a' fuoi compagni, e là quella d'un altro, che chiedeva a Dio mifericordia. Tutto era confufione, clamori, ferite, ed uccifione. Cortès da buon Generale fcorreva intrepidamente quà, e là, paffando fpeffo e ripaffando a nuoto i folli, incoraggendo gli uni, ajutando gli altrı, e dando agli zvanzi del fuo fcompigliato efercito tutto quell' ordine che potevafi non fenza gran rifchio d' effere uccifo, o fatto prigione. Il fecondo foffo s'empi a tal fegno di cadaveri, che fopra effi paffarono quegli della retroguardia. Alvarado, che la comandava, trovori nel terzo foffo cosi furiofamente incalzato da'nemici, che non potendo a loro far fronte, nè paffarvi a nuoto feñza evidente pericolo di perire per le lor mani, ficcò la lancia nel fondo del foffo, ed afferrò il piede d'effa colle braccia, e dando uno ftraordinario impulfo al fuo corpo, fi lanciò d'un falto di là dal folfo. Quefto falto ftimato da turti un prodigio d'agilit' diede a quel luogo il nome, che fin' ad ora conferva, del Salto d'Alvarado. (I)

Storia del Meffico Tom. III.

$S$

$\mathrm{La}$

(I) Bernal Diaz fi beffa di coloro, che credevano si fatto falto d'Alvarado; e dice, chiera affatto impofibile artefo la larghezza e la profondità 
La perdita de' Mefficani in quefta infaufta notte fu fen: Lis. IX.z'altro grande. Di quella degli spagnusli parlano, ficcome in altri computi, con fomma varietà gli Autori . $(L)$ Io ftimo vero il computo, che rapporta Gomara, come quegli, che moltra averne fatto diligenti ricerche ed efferfi informato tanto dal medefimo Cortès, quanto da altri Conquiftatori, cioè, che perirono oltre a quattrocento e cinquanta Spagnuoli, più di quattro mila uomini di truppe aufiliarie, e tra effl, fecondochè dice Cortès, tutti i Cholullefi. Furo. ro ancora ammazzati tutti, $(M)$ o quafi tutti i prigionieri, tutti gli uomini e le donne, ch' erano al fervizio degli Spagnuoli, e quaranta fei cavalli, e fi perdettero tutte le ricchezze da loro ammaffate, tutta l'artiglierìa, e tutti i ma. nofcritti di Cortès contenenti il ragguaglio di ciò, ch' era avvenuto fin'allora agli Spagnuoli. Tra gli Spagnuoli, che mancarono, i più riguardevoli furono i Capitani Giovanni Velafquez di Leon, intimo amico di Cortès, Amador di Lariz, Francefco Morla, e Francefco di Saucedo, uomini tutti e tre di gran coraggio, e merito. Fra i prigionieri perirono lo fventurato $\mathrm{Re}$ Cacamatzin, ed un fratello, un figliuolo, e due figlie del Re Motezuma. (N) Accompagnò que-

di quel foffo; ma gli altri Autori il rapportano come certo, e noi lo troviamo confermato da una coltante tradizione.

(L) Cortès dice, che perirono r 50. Spagnuoli; ma o egli rminul a bella pofta il numero per particolari interefli, o vi fu sbaglio dei copifti, o del primo ftampatore di quella letiera. Bernal Diaz numera 870 Spagnuoli morti; ma in quefto numero comprende, come egli ftelfo afferma, non folamente quelli, che furono uccifi in quella infaufta notte, ma eziandio quelli, che perirono nei giomi feguenti fin'al loro arrivo a Tlafcalla. Solis non ne numera più di 200 , e Torquemada 290 . Nel numero delle truppe aufiliarie, che vi perirono, Cono d'accordo Gomara, Herrera, Torquemada, e Betancurt. Solis dice folamente, che mancarono più di mille Tlafcallefi ; ma ciò non s'accorda cul computo di Cortès, nè con quello d'altri Autori.

(IM) Cortès afferma, che furono uccif tutti i prigionieri; ma fi debbe eccetruare Cuicuitzcatzin mefto già da Cortès ful trono d'Acolhuacan; poichè fappiamo pel ragguaglio dello fteffo Cortès, che colui era prigione, benchè ignoriamo la cagione, e da un'altra parte ci confta, ch'egli fú poi uccifu in Tezcuco, ficcome apprefo vedremo.

(N) Torquemada afferma, come una cofa bene accertata, che pochi gior- 
quefte Principeffe nella lor difgrazia Donna Elvira, figlia del Principe Tlafcallefe Maxixcatzin.

Non potè Cortès, malgrado la grandezza del fuo cuoLis. IX. re, trattener le lagrime a vilta di tal calamità. Si mife a federe fopra una pietra in Popotla, villaggio vicino a Tlacopan, non già per ripofar dalle fue fatiche, ma per pian. ger la perdita de' fuoi amici e compagni. In mezzo a tan. ti difattri ebbe almeno il conforto di fentire, ch' erano in falvo i fuoi più bravi Capitani Sandoval, Alvarado, Olid, Ordaz, Avila, e Lugo, $i$ fuoi interpreti Aguilar, e Donna Marina, ed il fuo ingegniere Martino Lopez, ne'quali principalmente confidava di poter riparare il fuo onore, e conquiftar Meffico.

Trovavanfi gli Spagnuoli cosi malconcj ed indeboliti per la fatica e le ferite, che fe i Mefficani gli aveffero infeguiti, non ne farebbe reftato neppur uno in vita; ma ap. s. 2r. pena arrivati all' ultimo foffo, ch' era in quella ftrada ful la- Marcio go, ritornarono alla Citta, o perchè fi contentarono della degli Atrage gia fatta, o perchè avendo trovato $i$ cadaveri del Re Spagnio. d'Acolhuacan, de' Principi Reali di Meffico, e d'altri Signori, s'occuparono nel pianger la loro morte, e nel fare le loro effequie. Lo fteffo fenz' alcro avranno fatro co' loro parenti, ed amici morti, lafciando in quel giorno nette le ftrade ed $\mathrm{i}$ foff, e bruciando i cadaveri, prima che infettaffero l'aria colla loro corruzione.

Allo fpuntar del di fi trovarono gli Spagnuoli in Popotla fparfi, ftanchi, ed angultiati, ed avendoli raccolti e riordinati Cortès, marciarono per la Città di Tlacopan travagliati inceffantemente $\mathrm{da}$ alcune truppe della medefima Città, e di quella ancora d' Azcapozalco infin' ad Otoncalpolco, tempio fituato fulla cima d'un piccolo monte nove miglia a Ponente dalla Capitale, dore prefentemente è il

$$
\mathrm{S}_{2} \text { cele- }
$$

ni dappoichè Cortês pigliò Cacamatzin, lo fece ftrangolar nella prigione. Cortès, Bernal Diaz, Betancurt, ed altri dicono, che fu uccifo infieme cogli altri prigionieri in quella memorabil notte. 
celebre Santuario e magnifico tempio della Madonna detta Lis. IX. de los Remedios, o fia del Soccorfo. Qui fi fortificarono fecondo la loro poffibilita per difenderfi con minor fatica dalle truppe nemiche, che li travagliarono tutto il di. La notte ripofarono un poco, ed ebbero qualche rinfrefo fomminiftrato loro dagli Otomiti di due vicini cafali, che vivevano impazienti fotto il giogo de'Mefficani. Da quelto luogo cominciarono ad incamminarfi verfo Tlafcalla, unico loro ricovero in tanta calamità, per Quauhtitlan, Citlaltepec, Xoloc, e Zacamolco, incalzati per tutta la Itrada da parecchie truppe volanti de' nemici. In Zacamolco fi trovarono si affamati, ed a tal miferia ridotti, che mangiarono a cena un cavallo, quello ftefo giomo ammazzato da' nemici, ed ebbe la fua parte it medefimo Generale. I Tlafcallefi fi gettavano a terra per mangiar l'erba, che vi trovavano, implorando l'ajuto de'lor Dei.

5. 22.

Il giorno feguente appena meffifi in ifrada pel monte Battaglia d'Aztaquemecan videro da lontano nella pianura di Tonan famola poco difcolta dalla Città d' Otompan un numerolo e brillan-
d'Otom- te efercito, o di Mefficani, come dicono comunemente gli
pan. to Scrittori, o pur compolto, come io credo, delle truppe d' $\mathrm{O}$. tompan, di Calpolalpan, di Teotihuacan, e d'altri luoghi circonvicini ragunatevi per fuggeftione de' Mefficani. Alcuni Storici fanno afcendere quefto efercito a dugento mila uomini, numero giudicato dagli Spagnuoli folamente ad occhio, ed aggrandito forfe dalla loro paura. Coltoro fi perfuafero, ficcome ne fa fede il medelimo Cortès, che quel giorno doveffe effere l'ultimo della loro vita. Ordinò quefto Generale le fue languenti truppe, slargando la fronte di quel miferabile efercito, acciocchè reltaffero in qualche maniera coperti $i$ fianchi d'effo colle piccole ale della poca cavalleria, che gli era rimatta, e col fembiante pieno di fuoco diffe loro: "In s) tale Atretto, ci troviamo, che è neceffario vincere, o mo. "rire. Fate animo, Caftigliani, e confidate, che colui, che "ci ha fin'ad ora liberati da tanti pericoli, ci fcamperà an. "che da quefto. "Diedeŕ finalmente la battaglia, la qua- 
le fu affai fanguinora, e durò più di quattio ore. Cortès ve. dendo le fue truppe diminuirfi, ed in gran parte fcoraggirfi, Lis. IX. ed i fuoi nemici venir più orgogliofi non oftante il danno che ricevevano dall' armi fpagnuole, prefe un'ardita e pericolo. fiffma rifoluzione, colla quale ottenne la vittoria, e pofe in falvo gli avanzi del furo mefchino efercito. Gli venne in men. te ciò che fovente avea fentito dire, cioè, che i Meflicani fi fcompigliavano e fuggivano, qualora vedevano morto il loro Generale, o perduto lo ftendardo. Cihuacatzin Generai di quell' efercito veftito d' un ricco abito milicare con un vago pennacchio nel capo, e con uno fcudo indorato nel braccio era portato in una lettiga fulle fpalle d'alcuni Soldati. Lo ltendardo, che giufta la loro ufanza portava, era una rete d'oro filfa nella punta d'un' afta, la quale avea forte. mente legata fulla fchiena, e s'innalzava dieci palmi in circa fulla telta di lui. $\left({ }^{*}\right)$ Offervolla pur Cortès nel centro di quella gian moltitudine di nemici, e rifoluto di dare un col. po decifivo, comandò a'fuoi prodi Capitani Sandoval, Alvarado, Olid, ed Avila, che gli teneffero dietro per guardargli le fpalle, e con altri, che l'accompagnavano, s'avventò per quella parte, dove gli parve men malagevole l'imprefa con tal impeto, che alcuni gettava in terra colla lancia, ed altri colle ftatfe. Così s'andò inoltrando per le fchiere nemiche fin' a raggiugnere il Generale, ch' era accompagnato da alcuni Uffiziali, e con un colpo di lancia lo diftele al fuolo. Giovanni di Salamanca bravo Soldato, che accompagnaya Cortès, fmontando preftamente dal cavallo, pofe fine alla vita di lui, levandogli il pennacchio, lo prefentò im. mediatamente a Coriès. (O) L'efercito nemico, appena che vide il fuo Generale morio, e prefo lo ftendardo, fi fcom. pi-

(*) Quefta forte di ftendardo era appellato dai Mefficani Tlabuizmatlaxopilli.

(O) Carlo V. accordò alcuni privilegi a Gio: di Salamanca, e fra gli al tri quello d'uno fcudo d'armi per la fua cara dov'era un pennacchio per memoria di quello, che avea tolto al Generale Ciluacatzin, allorchè l'uscife. 
pigliò, e fi mife a fuggire. Gli Spagnuoli ineoraggiti col Lis. IX. gloriofo fatto del loro capo gl' incalzarono, facendo di loro una grande ftrage.

Quefta vittoria fu una delle più famofe, ch' ebbero l'armi Spagnuole nel Nuovo Mondo. Segnalofí in effa fopra tutri il Generale Spagnuolo, di cui dicevano poi i fuoi Capitani e Soldati, che non aveano veduto mai un si gran coraggio, ed una sì grande attività, come quella, che fece fpiccar in quella giornata; ma ebbe una grave ferita nel capo, la quale peggiorando vieppiù ogni giorno riduffe ad eftremo pericolo la fua vita. Bernal Diaz loda giuftamente la bravura di Sandoval, e $f_{a}$ vedere quanta parte ebbe quefto famofo Uffiziale nella vittoria, facendo animo a tutti non meno coll' efempio, che coll' efortazioni. E' ftata parimenta celebrata dagli Storici Spagnuoli Maria d' Eftrada, moglie d'un Soldato Spagnuolo, la quale armata di lancia e rotella correva tra l'ofte nemica ferendo ed uccidendo con una intrepidezza molto ftrana nel fuo feffo. De' Tlafcallefi dice Bernal Diaz, che combatterono come Leoni, tra' quali fi diftinfe Calmecahua, Capitano delle truppe di Maxixcatzin. Quefti prefe nel battefimo il nome di Don Antonio, e fi rendette celebre ancor più che per la fua bravura, per la fua lunghiffima vita di cento trenta anni.

La perdita de'nemici fu fenz'altro grande, ma affai minore di quella, the rapportano parecchj Autori, i quali la fanno afcendere a venti mila uomini: numero affatto incredibile attefo il miferabile ftato, a cui erano riaotti gli $\mathrm{Spa}$ gnuoli, e la mancanza d'artiglieria, e d'altre armi da fuoco. Per l'oppofto la perdita degli Spagnuoli non fu sì poca, come la rapprefenta Solis; $(P)$ poichè vi perirono quafi tut ti

(P) Solis per efagerare la vittoria d'Otompan dice, che tra quei di Cortès furono alcuni feriti, dei quali morirono due, o tre Spagnuoli in Tlafcalla; ma quefte Autore, attento folamente alla pulitezza del linguaggio, alle lodi, ed alle fentenze, fi curò poco dei conti. Egli afferma, che Cortès conduffe feco a Meffico dopo la fconfitta di Narvaez I roo Uomini i quali con altri 80, che fecondo che egli dice, reftarono con Alvarado, fan- 
ti i Tlafcellefi, e molti Spagnuoli a proporzion del numero delle loro truppe, ed ufcirono tutti feriti.

Stanchi finalmente gli Spagnuoli d'infeguire i fuggitivi, riprefero la ftrada verfo Tlafcalla per la parte Orientale di quella pianura, dove ftettero quella notte allo fcoperto, ed il medefimo Generale dopo la fatica e le ferite avute in quella giornata, fece perfonalmente la guardia per maggiore lor ficurezza. Non erano già gli Spagnuoli più di quattrocento e quaranta. Oltre a quegli ch' erano ftati uccifi ne' combattimenti precedenti la notte della infaufta loro ufcita da Meffico, perirono in effa e ne' fei giorni feguenti ottocento fettanta, ficcome afferma fpeffo Bernal Diaz teftimonio oculato, molri de' quali effendo ftati fatti prigionieri da' Mef. ficani, furono inumanamente facrificati nel tempio maggiore della Capitale.

Il di feguente ( 8 . Luglio $\left({ }^{*}\right)$ I520. ) entrarono, al. 5. 23. zando le mani al Cielo, e ringraziando l'Altiffimo, ne' do Ritirata minj di Tlafcalla, ed arrivarono a Huejotlipan $\left.{ }^{\star \star *}\right)$ luogo degli,Spaconfiderabile di quella Repubblica. Temevano pure di tro. Tlafcalla. var qualche novità nella fedeltà de' Tlafcallefi, fapendo bene quanto fia comune negli uomini il vederfi abbandonati nelle loro

no r18o. Nei combattimenti precedenti alia ronfitta degli Spagnuoli in Meffico, appena fa menzione di qualcuno morto. Nella fconfitta numera foltanto 200 , e nel ragguaglio del loro viaggio fin'a Tlafcalla non rapporta altri, che quei due o tre, che morirono in Tlafcalla delle ferite avute in Otompan. Dove dunque fono, o come fparirono gli altri cinquecento e prù uomini, che vi mancano per compiere il numero Ir8o? Un' altra idea affai diverfa ci danno della battaglia d'Utompan coloro, che vi fi trovarono, ficcome può fcorgerfi nelle lettere di Cortès, e nella Storia di Bernal Diaz., O quanto era, dice quefto Autore, furiofa e fpavente"vole da vederfi quefta battaglia! come combattevano a corpo a corpo, o, e con qual furia ci attaccavano i cani (cosi chiama per ingiuria i ne"mici!) Che ferite ed uccifione facevano in noi colle loro lancie e fpade "Sxc., e dopo poche righe dice cosl: ", Torno a dire, che ci ferivano ed " ammazzavano molti dei noftri Soldati.,

$\left(^{*}\right)$ Bernal Dia'z dice, che la battaglia d'Otompan $f u$ il dì 14. Luglio; ma quefto fu uno sbaglio di memoria; poichè Cortès afierma, ch' entrarono nei domini di Tlafcalla il di 8. un giorno dopo quella battaglia .

$\left.{ }^{* *}\right)$ Huejotlipan è appellato da Cortès, e da Herrera Gualipan, da Bernal Diaz Gualiopar, e da Solis Gualipàr. 
loro calamità da' più cari amici; ma tolto fi difingannarono;

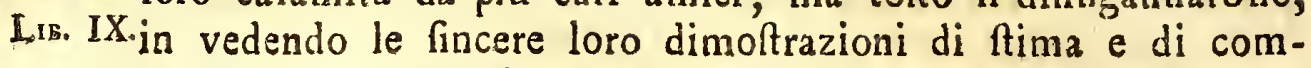
paffione per le paffate difgrazie. Appena ebbero la nuova del loro arrivo i quattro Capi di quella Repubblica, che fi. portarono ad Huejotlipan per complimentarli accompagnati da un Signore de' più principali d' Huexotzinco e da molta Nobiltà. Il Principe Maxixcatzin, quantunque afflitto per la morte della fua cara figlia Donna Elvira, procurò confolar Cortès colla fperanza della vendetta, afficurandofi di prenderla nel coraggio degli Spagnuoli, e nelle forze della Repubblica, che infin d'allora gli promile, e lo fteffo s'efibiro. no a far gli altri Signori. Cortès gli ringraziò della fingola. re lor benevolenza, e prendendo lo fendardo tolto il giorno innanzi al General Mefficano, lo regalò a Maxixcatzin, ed agli altri Signori prefentò alcune pregevoli fpoglie. Le donne Tlafcallefi fcongiurarono Cortès di vendicar la morte de'lor figliuoli, e parenti, e sfogarono la lor pena in mille imprecazioni contro la perfidia de' Mefficani.

Poichè ebbero ripolato tre giorni in quefto luogo, paffarono alla Capitale della Repubblica, quindi diftante quindici miglia per curar le loro ferite, delle quali morirono otto Soldati. Il concorfo di Popolo nella loro entrata in Tla. fcallan fu uguale, e forfe anche più grande di quello, che vi $\mathrm{fu}$ allora, quando la prima volta v'entrarono. L' accoglienza, che lor fece Maxixcarzin, e la cura, che prefe di lozo, furono degne del fuo animo generolo, e della fua fincera amicizia. Gli Spagnuoli fi riconofevano ogni giorno più obbligati a quella Nazione, la cui amicizia coftantemente coltivata fu il mezzo più efficace non folo per la conquifta della Capitale dell'Imperio Mefficano, ma eziandia per quella di turte guante le Provincie, che s'oppofero ai progreffi dell' armi Spagnuole, e per la foggiogazione dei barbari Cicimechi ed Oromiti, da cui furono tanto travagliati i Conquiftatori .

Mentre che gli Spagnuoli ripofavano in Tlafcalla dalle loro fatiche, e curavano le loro ferite, i Meflicani s'adope- 
ravano per rimediare $a^{\circ}$ mali della Corte, del Regno Erano pur grandi e lamentevoli i danni fatti loro da un an. Lis. IX. no in quà; poichè oltre alle gran fomme d'oro, d'argento, di gemme, e d'altre cole preziole da loro lpele parte in prefenti fatti agli Spagnuoli, e parte in omaggio al Re di Spagna, delle quali alquanto fenza dubbio recuperarono, s'era ofcurata la fama delle loro armi, e fcemato il rifpet to alla Corona di Meffico.: s'erano fottratri dalla loro ubbidienza i Totonachi, ed altri Popoli : s'erano renduri più infolenti $i$ lor nemici: fi trovavano danneggiati $i$ tempj, e 5. 24. Elezione in Me Mico, e di= fpofizionide! Re Cuitlatzin. rovinate molte cafe della Capitale, e foprattutto lor man. cava il $\mathrm{Re}$, molte perfone Reali, ed una gran parte della Nobiltà. A quefti danni ricevuri dagli Spagnuoli s'aggiunfero quelli, ch' eglino fteffi fi. cagionarono colla guerra civile, la notizia della quale dobbiamo a'manofcritti d' uno Storico Mefficano, che fi trovava allora in quella Corte, e fopravviffe alcuni anni alla ruina dell' Imperio.

Allorchè: gli Spagnuoli erano nella Corte travagliati dalla fame per l'oftilità de'Mefficani, parecchj Signori. della prima Nobiltà o per favorire il partito degli Spagnuoli, o ciò che fembra più verifimile, per foccorrere il loro $\mathrm{Re}$, il qual' effendo tra gli Spagnuoli, doveva patir la medefima lor necelfità, gli provvedevano nalcolamente di viveri, e forfe anche $f$ dichiararono apertamente in favor di loro, confiaando nella propria loro autorità. Per quefta cagione s'accele tra' Meflicani una sì funelta diffenfione, che non porè fpegnert: fe non colla morte di molti chiari perfonaggi, e particolarmente di Cibuacoatl, di Tzibuacpopoca, di Cipocatli, e di Tencuecuenotzin, gli uni figliuoli, e gli altri fratelli del $\operatorname{Re}$ Motezuma, fecondo che afferma il fuddetto Storico.

Aveano d' uopo i Meflicani di mertere alla telta della loro Nazione un uomo capace di riftabilir l'onor d'effa, e di riparar alle perdite avute nell' ultimo anno del Regno di Motezuma. Fu eletro Re di Meffico il Principe Cuitlahuatzin o poco innanzi, o poco dopo la fconfitta degli Spaguuoli. Era egli, come abbiam già detto, Signor d'Iztapalapan, Storia del Mefjeco Tom. III.

Con- 
Configliere intimo del Re Motezuma Suo fratello, e Tlach-

Lis. IX.cocalcatl, o fia General d'efercito. Era uomo favio, e di gran talento, ficcome ne fa fede il fuo nemico Cortès, liberale, e magnifico, come il fuo fratello. Dilettavali affai dell' Architettura, e della coltura de' giardini, come fi vide nel magnifico palazzo, che fabbricò in Iztapalapan, e nel celebre giardino, che vi piantò, de' quali fanno menzione con gran lode quafi tuti gli Storici antichi. La fua bravu: ra, e la fua perizia militar gli acquiftarono una grande ftima preffo i fuoi Nazionali, alcuni Spagnuoli bene informati del carattere di lui affermarono, che fe egli non foffe ftato fraftornato dalla morte, non farebbe tata mai efpugnata dagli Spagnuoli la Capitale. (2) E' da crederfi, che i facrifizj, che fi fecero mella felta della fua incoronazione furono di quegli Spagnuoli, ch' egli fteffo fece prigioni nella not: se della loro confitta.

Tofto che furono terminate le fefte della fua incoronazione, s'applicò a rimediar a' mali della Corte', 'e dell' Im. perio. Diede ordine di riparar i tempj danneggiati, e di rifar le cafe rovinate, aumentò e migliorò le fortificazioni delIa Capitale, mancò de' meffi alle Provincie dell' Imperio, incoraggendole per la comun difefa dello Stato contro a quei nemici Atranieri, e promife di rilevar da ogni tributo coloro, che prendeffero l'armi in favor della Corona. Mandò altresi degli Ambafciatori alla Repubblica di Tlafcallan con un

(Q) Solis dà a Cuitlahuatzin il nome di Quetlabaca, e dice di lui, che wiffe nel trono pocbi giorni, ma quelli che baftavano per lafciar preffo ai fuoi nazionali quafi scancellata dalla fua dappocaggine la memoria del fuo nome. Ma quefto è falfo, ed oppofto al ragguaglio di Cortès, di Bernal Diaz, di Gomara, e di Torquemada, Autori affai meglic informati del Solis. Come potea fcancellarfi la memoria del fuo nome preffo i Mefficani, confervandofindelebile prefro gli 'Spagnuoli, come quelli che il confíderavano Autore della terribile loro fconfitta del r. Luglio, ficcome, coftoro medefimi lo teftificano? Cortès fi ricordava tanto di Cuitlahuatzin, e conferva. va tale rdegno contro lui per quei difaftri, che quando fi trovo con forze fufticienti per intraprendere l'affedio di Meflico volendo vendicarfi di quel $\mathrm{Re}$, e non potendo prender vendetta della fua perfona, perch' era già trapaffato, la prefe della Città favorita da lui. Quefto fu il motivo, come dice lo fteffo Cortès, della fua Ipedizione contra Lztapalapan. 
un buon prefente di vaghe penne, d'abiti di cotone, e di fale, li quali furono accolti con onore giufta le leggi Atabi- Lib. IX. lite fra le Nazioni dirozzate di quel paefe. Il propofito di tale ambafciata fu per rapprefentare a quel Senato, che ben- 5. 25. che fin'allora foffero ftati tra loro nemici capitali i Meffica- Ambani, ed i Tlafcallefi, era omai d'uopo d'unirfi infieme, co-Re Cuime originarj d'un medefimo paefe, come Popoli d'una me- tlahuadefima lingua, e come adoratori de' medefimi Numi, contro Tlacali nemici: comuni, della patria, e della Religione: che aveano lefi. già veduto la fanguinofa ftrage, che aveano fatto in Meffio. co, ed in, altri. luoghi quegli inumani ed: orgogliofi: Itranieri, i facrileghi loro attentati contro i fantuarj, e contro le ve: nerabili immagini degli Dii, la. loro ingratitudine e perfidia contra il fuo fratello ed anteceffore, e contro i più: rifpetta. bili perfonaggi degli Anahuatlachi, e la infaziabile lor fame dell' oro, che gl' induceva: a mancare alle più fante leggi dell'amicizia: che fe la Repubblica continuava a fecondare i perverfi difegni di que' moltri, avrebbono da. loro alla fine quella ricompenfa, ch' ebbe il Re Motezuma dell' umanita, con cui gli accolfe nella fua Corte, e della liberalita, con cui gli favorì per tanto tempo: i Tlafcallefi farebbono de. teftati da tutte le Nazioni per aver dato ajuto a si iniqui ufurpatori, e gli Dei farebbono piombar fulla Repubblica tutto il furor della lor collera per. efferfi, confederata. co' nemici del loro culto. Se per contrario fi dichiaravano, come gli pregava, nemici di quegli uomini odiati dal Cielo, e dalla Terra, la Corte di Meflico farebbe un'alleanza perperua, ed avrebbe d'allora innanzi un commercio libero colla Repuba blica, onde quefta fchivar potrebbe la miferia, a cui era ftata fin'allorz ridotta: tutte le Nazioni d' Anahuac le farebbono obbligate, per un si importante fervizio, e gli Dei col fangue di, si fatte vitume placati, manderebbono ne' loro campi la pioggia neceffaria, farebbono felici le loro ar$\mathrm{mi}$, e renderebbono celebre per, tutta la terra il nome de. Tlafcallefi.

Il Senato, dopo aver afcoltato l' ambalciata e congedato. T 2 . dalla. 
-dalla fala d'udienza gli Ambafciatori fecondo la loro ufanza; LIR. LX.vi rimale per confultar fopra quel grande affare. Non vi mancarono alcuni, a' quali parvero giulte le propofizioni della Corte di Meffico, e confacenti alla felicità della Repubblica; efagerando i vantaggi, che s'offerivano a loro, e da un'al. tra parte l' efito infaufo dell' imprefa deg!i Spagnuoli in Meffico, e la ftrage fatta nelle truppe Tlafcallefi, che v' erano ftate fotto i loro ordini: Alzò la voce tra gli altri il giovane Xicotencatl, il qual era ftato fempre mai nemico capitale degli Spagnuoli, e procurd di perfuadere con quante ragioni potè l'alleanza co' Mefficani, aggiungendo, che farebbe molto meglio il confervar gli antichi coltumi della lor Nazione, che non il fortometterí alle nuove e Atravaganti ufanze di quella gente indomita ed imperiofa: che non poteva immaginarf un'altra occafione più opportuna per liberarfi affatto dagli Spagnuoli, che állora quando fi trovavano fcemati, indeboliti; ed abbattuti. Maxixcatzin, il quale per l'oppofto era finceramente affezionato agli Spagnuoli, ed era fornito d' un miglior ingegno per conofcere il dritto delle Genti, e d' una volontà più ben difpofta per offervarlo, ributtò il fentimento di Xicorencatl, biafimando come un' abbominovele perfidia it configlio di facrifficare alla vendetta de' Mefricani' quegli nomini travagliati dalla fortuna, che aveano cercato il loro aft: lo in Tlafcalla, confidati nelle protefte, e nelle dimoftrazio. ni del Senato, e della Nizione. Soggiunfe, che fe fi lufinga. vano de'vantaggi, che offerivano i Mefficani, egli gli fperava più grandi dalla bravura degli Spagnuoli, che fe poi v' era ragione di non frdarfi degli Spagnuoli, men fidar fi doveano de' Mefficani, della cui perfidia aveano tanti efempj: finalmente che niun altro delitto farebbe capace d'irritar tan. to la collera degli Dei, e d'ofcurar la gloria della Nazione, come sì fatta malvagità contra quegli ofpiti innocenti. Xi. cotencatl inculcava il fuo configlio rapprefentando a' Senatori uno tchifofo ritratto dell' indole, e de' coftumi degli Spagnuoli. I' altercazione fu s̀̀ grande, e rifcaldò a tal fegno gli animi; che Maxixcatzin trafportato dalla collera diede una fpinta a $\mathrm{Xi}$. 
Xicotencatl, e lo precipitò per alcuni gradini, che v'erano, chiamandolo fediziofo, e traditore alla patria . Cotal dimo. LiB. IX. Atrazione, fatta da un uomo tanto circolpetto, e tanto rifpettato, ed amato dalla Nazione, obbligò il Senato a far imprigionar Xicotencatl.

La rifoluzione, che vi fi prefe fu quella di rifpondere ali' ambafciata, che la Repubblica era pronta ad accettar la pace e l'amicizia della Corte di Meffico', qualora non foffe per farfi con un'azione si indegna; e con un delitto sł enorme, come quello di facrificar i fuoi ofpiti ed amici; nua quando fi cercarono gli Ambafciatori per intimar loro la rif́pofta del Senato, erano già nafcoftamente partiti da Tla. fcalla, perciocchè avendo offervato quel popolaccio alquanto inquieto nel loro arrivo, temettero, che voleffe far qualche attentato contro il rifpetto dovuto al lor carattere. E' però da crederfi, che il Senato mandaffe degli Aunbafciatori Tlao fallefi per portar la rifpofta a quella Corte. I Senatori procurarono occultar agli Spagnuoli il motivo dell'ambafciata, e tutto cio ch' era avvenuto nel Senato; ma a difpetto del. le loro diligenze il feppe Corrès, il quale ringraziò, com'e. ra giufto, Maxixcatzin de'fuoi bucni ufficj, e s' efibi a corrifpondere alla idea vantaggiofa, che aveva della bravura e dell'amicizia degli Spagnuoli .

Non contento il Senato di si fatte pruove della fua gran fedelta, diede di bel nuovo l'ubbidienza al Re Cattolico, e ciò ch'è più, moffi i quattro Capi della Repubblica dalla grazia dello Spirito Santo rinunziarono all' Idolatria, e 5. 26 . Battefimo de' dopo bene inftruiti furono battezzati dal P. Giovanni Didz, di Tla Cappellano dell' armata Spagnuola, effendo lor patrini Cortès, ed i fuoi principali Capitani. Celebroffi quefta funzione colle più gran dimoftrazioni di giubilo, tanto degli Spa. gnuoli, quanto de'Tlafcallefr. Chiamoffi Maxixcatzin nel battefimo D.Lorenzo, Xicotencatl il vecchio Don Vincenzio, Tlehuexolotzin Don Gonzalo, e Citlalpopoca D. Bartolommeo. (R)

Se-

(R) Nè Cortès, nè Bernal Diaz fanno motto di tal battefimo. Herrera 
Seguirono il loro efempio alcuni Tlafcallef, ma di coftoro Lis. IX. non tutti perfeverarono nella fede; perchè non erano ficco: me era d'uopo, intimamente perfuafi della verità del Crifianefrmo.

Erafi già Cortès liberato dal pericolo, a cui efpofe la Sbigotti- fua vita il colpo ricevuto nel capo. nell'ultima battaglia, e mento gli altri Spagnuoli, fuorchè alcuni, che ne morirono erano d'alcuni guariti delle terite coll'ajuto de' Cerufici. Tlafcallei. Nel temli. po della fua malattia non avea Cortès penfato ad altro, che a' mezzi da adoperarfi per condurre a buon fine la grand'im: prefa della conquifta di Meffico, ed a ciò ottenere a vea fatto tagliar una gran quantità di legni per la confruzione di tredici brigantini; ma allorchè formava quelti grandi, progetti, molti de' fuoi Soldati rivolgevano de'penfieri affai diverfi. Vedevanfi fminuiti, poveri, e mal concj, e sforniti d'armi e di cavalli. Non potevano dimenticarf: del terribile con. flitto e della tragica notte del I. Luglio, nè voleano di nuovo efporfi a si fatte fventure. Fomentavanfi fcambievolmente le loro idee, ed i loro timori, e biafimavano l'oftinazione del loro Generale in una imprefa si temeraria. Dal. le mormorazioni private s'avanzarono a fargli una richiefta legale, volendo, obbligarlo con molte ragioni a ritornare al: la. Veracroce, laddove potrebbono procacciarfi foccorfi di trup. pe, d'armi, e di munizioni per intraprendere con magoiori forze $l_{a}$ conquifta, che allora ftimavano affatto impoffibile. Turboft Cortès con quefta novita, la quale fraftornava tur:i i fuoi difegni, ma con quel talento, che avea di perfuadere

\footnotetext{
$f_{a}$ foltanto menzione di quellor di Maxixcatzin, e Solìs aggiunge quello di Xicotencatl. Qualche Autore fa Miniftro del battefrmo il P. Olmedo, ed alcuni dicono, che Maxixcatzin lo ricevette. nell' ultima fua infermita. Ma egli è certo, che tutti i quattro Capi di quella Repubblica furono battezzati avanti la conquifta, benchè Torquemąa, e. Betancurt non fiano d'accordo ful tempo. Si fa ancora, che Maxixcatzin non differi il battefimo fin'all'ultima infermità, e che i quattro Signori furono battezzati non già dal' $\mathbf{p}$. Olmedo, ma dal P. Diaz. Oltre ad altre pruove ciò confta dalle pitture artiche, ch' erano in parecchi Conventi dei PP. Francefcani fatte dai Tlafcallefi, e vedute dallo Storico Torquemada.
} 
dere a' fuoi Soldati quanto voleva, fece loro un efficace difcorfo, col quale gl' induffe a defítere dalla loro pretenfione. Lis. I X. Rimproverò loro quella codardia, rifvegliò ne' loro cuori i fentimenti d'onore, facendo loro un ricordo lufinghevole de' loro gloriofi fatti, e delle protefte piene d'ardore, e di coraggio, che fovente gli aveano fatto : fece loro chiaro quanto più pericolofo riufcir dovrebbe il loro ritorno alla Veracroce, che non la dimora in Tlafcalla, afficurolli della fedeltà di quella Repubblica, della quale fi moltravano ancor dubbiofi: finalmente gli pregò di fofpendere la lor rifoluzione fin'a veder l' efito della 'guerra; che voleva fare contro la Provincia di Tepejacac, nella quale f́erava aver nuove ripruove della fincerita de' Tlafcallefi:

I Signon della Provincia di Tepejacac confinante colla Repubblica di Tlafcalla; s'erano dichiarati àmici di Cortès, Guegri e fudditi del Re di Spagna infin da quell'orrendo macello, Spagnuoche gli Spagnuoli fecero in Cholollan; ma vedendo poi ab- li contro battuti gli Spagnuoli, e vitroriofi i Mefficani; fi rinifero jachefi. Cotto l'übbidienza del Re di Meffico, e per procàcciarfi la grazia di lui, ammazzarono alcuni Spagnuoli, che dalla Ve: racroce faceano viaggio a Meflico non confapevoli della tragedia de' loro compagni : ammifero ae' loro luoghi della guernigione mefficana, ed occuparono la ftrada che conduceva dalla Veracroce a Tlafcalla, e non contenti di ciò fecero alcune fcorrerìe nelle terrè di quella Repubblica. Deliberd Cortès di far loro la guerra non meno per caftigar la loro perfidia, che per afficurar la trada di quel porto per li foccorfi, che indi afpettava. Spingevalo áltresi a tale fpedizione il giovane Xicotencatl; il qual era ftato gia rimeffo in libertà per la mediażione del medefimo Generale Spagnuolo; e per levar via ogni qualunque fofpetto, che vi foffe contro lui per quello, ch' era avvenuro nel Senato, s'efibi ad ajutarlo in quella guerrà con un buon efercito. Cortès accettò l'offerta ; ma prima di prender l'armi, richiefe amichevolmente qualche foddisfazione da' Tepejachefi, e gli efortò à lafciare il partito de' Mefficani, promettendo di perdonar lo: 
ro il delitto commeffo nell' ammazzamento di quegli SpaLIB. IX.gnuoli; ma effendo ftate da loro ributtate le fue propofizioni, marciò contrd quella Provincia con quattrocento venti Spagnuali, e con fei mila arcieri Tlafcallefi, frattanto che Xicotencatl ragunava un efercito di cinquanta mila uomini. In Tzimpantzinco Città di Tlafcallan gli fi aggregarono tante truppe di quella Repubblica, d' Huexotzinco, e di Chololla, che fi credette vi foffero fin' a cencinquanta mila uomini.

La prima loro Spedizione fu contro Zacatepec, luogo della confederazione de' Tepejachefi. Gli abitatori d'effo fecero un'imbofcata agli Spagnuoli. Si combattè dall' una, e dall'altra parte con gran coraggio ed oftinazione; ma furono alla fne vinti i Zacatepechefi, reftando moltiffimi di lo. ro morti nel csmpo. (S) Indi marciò l'efercito contro A: catzinco, Città dieci miglia a Scirocco da Tepejacac, nella qual entrarono trionfanti gli Spagnuoli dopo aver guadagnata una battaglia poco men difficile di quella di Zacatepec: Da Acatzinco mandò Cortés parecchj diftaccamenti a bruciar alcuni luoghi di qué contorni, ed a fot tomettere altri alla fua ubbidienza, ed allorchè gli parve effer tempo d' attaccare 12. Città principale, s'incamminò con tutto il fuo efercito verfo Tepejacac, dov' entrò fenza veruna refiftenza de' Citfadini. Quivi dichiard fchiavi molti prigionieri prefi in quella Provincia, e gli fece bollare con un ferro infocato lecon: do la barbara ufanza di quel fecolo, applicandone la quinta parte al Re di Spagna, ficcome fi faceva di tutto quanto acquiftavano, e compartendo il refto tra gli Spagnuoli, e gli Alleati. Vi fondò giufta la maniera di parlar degli Spagnuoli di quel tempo, una Città, che appellò Segura della

Fron-

(S) Parecchi Storici dicono, che la notte feguente la battaglia di Zaca. tepec ebbero gli Alleati degli Spagnuoli una gran cena di carne umana, parte arroftita in un forprendente numero di Spiedi di legno, e parte leffa in cinquanta mila pignatte; ma quefo racconto mi pare una favola. Non è verifimile, che tralafciaffero nel loro ragguaglio un avverimento si notabile nè Cortès, nè Bernal Diaz, il qual'è troppo prolifo e nojofo nella narrazione di si fatte crude!tà. 
Frontera, la qual fondazione fi riduffe a ftabilirvi de' MagiArati Spagnuoli, e farvi una piccola fortificazione. $\left({ }^{*}\right)$.

LIB. IX.

Le truppe Melficane, ch' erano nelle guernigioni di quella Provincia, fi ritirarono da effa, perchè non aveano forze G. $^{29}$. fufficienti per refiftere alla porenza de' loro nemici; ma nel- di Qualo Iteffo tempo fi lafcio vedere Sopra la Città di Quauhque- uhquechollan (**) difcofta da quella di Tepejacac più di quaranta miglia verfo Libaccio, un efercito di Mefficani maudatovi dal Re Cuitlahuatzin per impedire agli Spagnuoli il paffo da quella banda alla Corte, fe mai l' intentaffero. Era Quauhquechollan una Città confiderabile, dov' erano da cinque in fei mila famiglie, molto amena, e forte non meno per fua natura, che per arte. Era naturalmente difefa da una banda da una montagna alta e dirupata, e da un'altra da due fiumi poco fra loro difcofti. Tutta la Città era circondata $d a$ una forte muraglia di pietra e calcina, alta venti piedi in circa, e larga dodici, con un buon parapetto da per tutto, che avea quafi tre piedi d'altezza. Non v' erano per entrarvi più di quattro porte in quelle parti, dove l'eftremità della muraglia fi raddoppiavano, formando due femicircoli, ficcome fi vedono rapprefentati nella figura da noi data nel libro VII. Accrefcevafi la malagevolezza dell' entrata per l'elevazione del fuolo della Cittá; la qual' era tanta, quanta l'altezza della fteffa muraglia: ficchè per entrarvi era d'uopo di falire per alcuni gradi affai alti.

Il Signor di quella Citta, il qual era parziale degli Spagnuoli, mandò un' ambafciata a Cortès, proteftando it fuo vaffallaggio al $\mathrm{Re}$ di Spagna riconofciuto gia Signore di quella terra nella celebre affemblea, che tenne il Re Motezuma colla Nobiltà mefficana alla prefenza di Cortès: ch'e-

Storia Antica del Meffeco Tom. III. $\mathrm{V}$ gli

(*) Suffifte fir' ad ora la Citta di Tepejacac, o fia Tepeaca come l'ap. pellano gli Spagnuoli; ma il nome di Segura della Frontera fu tofo mefro in obblio. Carlo V. le diede il titolo e l'onore di Citta Spagnuola nel I 545 . Oggidi appartiene al marchefato del Valle.

( $\left.{ }^{* *}\right)$ Quauhquechollan è appellata dagli Spagnuoli Guaquecbula, o Huacacbula. Oggidi è un amenifimo villaggio d'Indiani abbondante di buone frutta. 
gli bramava di moftrar la fua fedelta; ma non gli era perIrs. IX.meffo da' Melficani: che prefentemente era in Quauhquechollan un buon numero d'Uffiziali Mefficani, e fin'a trenta mila uomini da guerra parte in quella Citta, e parte ne". luoghi circonvicini per impedire ogai qualunque confederazione cogli Spagnuoli: pertanto lo pregava di venire a foccorrerli, e liberarli dalle veffazioni, che foffrivano da quelle truppe. Gradì Cortès l'avvifo, e mandd fubito co'medefimi meflaggieri un foecorfo di iredici cavalli, di dugento pedoni Spagnuoli, e di trenta mila uomini di truppe aufiliarie fotto il comando del Capitano Olid. I meffaggi giulta l'ordine del loro Signore 's efibirono a condur l' efercito per una ftrada poco battuta, ed avvertirono il Comandante Olid, che allorchè l'efercito folfe oramai vicino alla Città, i Quauhquechollefi dovrebbono affalir di mano armata gli alberghi degli Uffiziali Mefficani, e procurar di pigliarli, o d'ucciderli, acciocchè entrandovi poi l'efercito degli Spagnuoli foffe loro più agevole lo rconfiggere i nemici deftituiti già $\mathrm{da}$ loro condottieri. Ma dodici miglia prima di giugnere a Quauhquechollan il comandante Spagnuolo entrò in folpetto, che gli Huexotzinchi non fi foffero fegretamente confederati co Quauhquechollefi e co' Mefficani per rovinare affat to gli Spagnuoli. Quefto folpetro cagionato da finiftre informazioni, e renduto più verifimile dalla moltitudine d' Huexotzinchi, che fpontaneamente s'aggregarono all' elercito, lo coftrinfe a ritornare a Chololla, dove fece pigliar alcuni Hue. xotzinchi de'più ragguardevoli, ed i Mellaggj di Quaukquechollan, e gli mandò con buona guardia a Cortès, acciocchè faceffe le fue ricerche, intorno al pretefo tradimentrs.

Rincrebbe affai a Cortès sì fatta condotta contro amici si fedeli come gli Huexotzinchi. Nulladimeno gli difaminò diligentemente, fcoprì l'innocenza e la buona fede degli uni e degli altri, e s'avvide, che le paffate difgrazie aveano fatti gli Spagnuoli più paurofi, e la paura gl'induceva, come fuole, ad inlofpertire più che non fi conveniva. Carezzò è regalò quanto potè gli Huexotzinchi, ed i Quauhque. 
uhquechollefi, ed accompagnato da effo loro marciò incon-, tanente per Chololla con cento pedoni, e dieci cavalli, de- Lis. IX. terminato d'efeguir perfonalmente quefta imprefa. Trovò in Chololla gli Spagnuoli impauriti, fece loro coraggio, ed in di marciò per Quauhquechollan con tutto l'efercito, il qua. le allora conftava di più di trecento Spagnuoli, é di più di cento mila Alleati. Tanta era la prontezza di que' Popoli nell' armarfi contro i Mefficani per fottrarfi dalla loro dominazione. Prima d'arrivare a Quauhquechollan fu avvifato Cortès da quel Signore, ch' erano già ftate prefe tutte le mifure: che i Mefficani confidavano nelle loro fentinelle po. fte fulle torri delle Citta, e nelle ftrade; ma che effe erano già ttate fegreramente pigliate da' Cittadini.

Appena videro i Quauhquechollefi l'efercito, che veniva in foccorfo loro, che affalirono mano armata gli alberghi de' Mefficani con tal violenza, che prima d'entrar Cortès nella Città, gli prefentarono quaranta prigionieri. Quando v'entrò quel Generale, combattevano tre mila Cittadini il principal albergo degli Uffiziali Mefficani, i quali quantunque molto inferiori in numero, fi difendevano pure con tal coraggio, che i Quauhquechollefi non potevano efpugnar la cafa, con tutto che fi foffero renduti padroni de' terrazzi. Vi diede Cortès l'affalto, e l'efpugnò; ma a difpetto delle diligenze da lui adoperate per prender qualcuno, da cui informarfi dello ftato prefente della Corte, pugnarono con tal oftinazione i Mefficani, che tutti furono uccifi, ed appena potè prender qualche lume da un Uffiziale moribondo. Gli altri Mefficani, ch' erano fparfi per la Citrà, n' ufcirono precipitofamente per incorporarfi col groffo dell' efercito, accampato in un luogo al o, che dominava tutti $\mathrm{i}$ contorni, il qua. le in un momento fi mife in ordine di battaglia, ed entrò nella Citrà appiccando il fuoco alle cafe. Cortès afferma che non avea mai veduto un altro elercito di più bella comparfa per cagione dell' oro, e de pennacckj, di cui vedeváfi fregiato. Gli Spagnuoli corfero alla difefa colla loro cavalleria, e con molte migliaja d'Alleati, e gli coßtripfero a ritirarfi ad 
un luogo alto e malagevole, ma effendo i Meffrcani ancor la

Lis. IX. da' lor nemici incalzati, fi ricoverarono nella cima d' un' altiffima montagna, lafciando molti di loro morti nel campo. I vincitori dopo aver faccheggiato il campo nemico, ritornarono alla Città pieni di gloria, e carichi'di fpoglie. $(T)$

'5 30. Tre giorni riposò l' efercito in Quauhquechollan, e nel Guera quarto marciò verfo Itzocan $\left({ }^{*}\right)$ Citta di tre in quattro mila d'Itzo- famiglie, fituata nella falda d' un monte, dieci miglia in cir-

ca da Quauhquechollan, circondata da un fiume profondo, e da una piccola muraglia. Le fus itrade erano bene ordinate, e tanti i fuoi tempj, che tra grandi, e piccoli parvero a Cortès intorno a cento: il fuo clima è caldo per effere fituata in una valle profonda chiufa da alte montagne, ed il fuo terreno, come quello di Quauhquechollan fertilifimo, ed adombrato da alberi di bei fiori, e d'eccellenti frutte. Signoreggiava allora quello ftato un Perfonaggio del Real fangue di Meffico, al quale il diede in feudo Motezuma dopo aver fatto morire non fo per qual cagione il legittimo Signore, che il poffedeva, e prefentemente v' era una guernigione di cinque in fei mila uomini di truppe Mefficane. Tutto ciò intefo dal Signor di Quauhquechollan moffe Cortès alla fpedizione contro Itzocan. Il fuo efercito s'era tanto accrefciuto, che afcendeva, per quel ch' egli afferma, a cento venti mila uomini in circa. Diede l'affálto alla Città per quella parte, dov' era men difficile l'entrata. Gl' Itzocanefi fecondati dalle truppe regie fecero da principio qualche refiftenza; ma effendo ftati alla fine fuperati dalle forze tanto fuperiori degli affalitori, fi fcompigliarono, e fuggirono per la parte oppolta della Città, ed avendo paffato il fiu. me,

(T) Bernal Diaz nega, che Cortès andaffe in perfona alle fpedizioni di Quaubquechollan e d'Itzocan; ma lo fteffo Cortès l'afferma efpreffamente, e parla in tal maniera di quefte due Città, che ancorchè egli non l'affermaffe, dovremmo credere, che interveniffe a quella guerra. Bernal Diaz forfe 's'era dimenticato dopo quarant'anni fcorfi. Cortès fcrifle la fua feconda lettera a Carlo V. nella quale ne parla, pochi giorni dopo quelle fpedizioni.

(*) Itzocan è chiamata Izucar dagli Spagnooli, 
me, levarono $i$ ponti per non effere infeguiti da'loro nemici. Gli Spagnuoli ed i loro Alleati a difpetto delle dificol.Lib. IX. tà, ch'ebbero nel valicare il frume, gl'incalzarono per più di quattro miglia uccidendo alcuni, facendo altri prigioni, e lafciando tutti impauriti. Ritornato Cortès alla Città fece attaccar fuoco a tutti i Santuarj, e per mezzo d'alcuni prigionieri chiamò i Cittadini, che andavano fparfi per le montagne, dando loro falvo condoito, acciocchè tornaffero fenza verun timore ad abitar le loro cale.

Il Signor d'Itzocan s' era affentato dalla Città, e meffo in viaggio per la Corte, dacehè fu veduto l'efercito nemico. Ciò baliò alla Nobiltà, a cui forfe non era accetto quel Signore, per dichiarar vacante lo Stato: per lo che $s$ accorarono coll' autorità e la protezione di Cortes di darlo ad un figliuolo del Signor di Quauhquechollan, e d'una figlia di quel Signore, cui fece morir Motezuma, e perché era ancor ragazzo di pochi anni, gli furono affegnati Tutori il fuo proprio Padre, un fuo Zio, e due Nobili. Quefto ragazzo fu in brieve inftruito nella dotrrina Criftiana, e battezzato,

La fama delle vitrorie degli Spagnuoli volò fubito per tutta la terra, e tirò parecchj popoli all'ubbidienza del Re Cattolico. Oltre a Quauhquechollan, Itzocan, ed Ocopetlajoccan, Città grande poco difcolta da quelle due, $(V)$ vennero alcuni Signori a far omaggio alla Corona di Caftiglia da otto luoghi di Coaixtlahuacan $\left({ }^{\pi}\right)$ parte della gran provincia di Mixtecapan, diftante pit di cento venti miglia a Mezzod’ da Quauhquechollan, cercando turti 2 gara l'alleanza, e l'amicizia d'uomini sì prodi.

Ri-

(V) Ocopetlajoccan è detto da Cortès Ocupatujo per ignoranza della lingua meflicana, e l'Autore delle Note fulle lettere di Cortès ftampate in Meffico nel i 770. credette, che foffe Ocuituco; ma quefto luogo non è cosł vicino a Quauhquechollan, come era preffo Cortès il fuo Ocupatujo. Torquemada, benchè peraltro molto efatro nello fcrivere i nomi meflicani, chiama quel Luogo ora Acapetlajoccan, ora Acapetlabuacan.

${ }^{*}$ ) Coaixtlahuacan è appellata da Cortès Coaftoaca, e dicefi da lui vicina 
Ritornato Cortès a Tepejacac fece la guerra per mezzo Lis. IX.de' fuoi Capitani ad alcune Città, che aveano fatto dell' 0 . filità contro gli Spagnuoli. Gli abitatori di Xalatzinco, Cit6. 3r. tà poco difcolta dalla ftrada della Veracroce, furono vinti di Xala- dal famolo Sandoval, ed i principali di loro condotti prigiotzinco,di nierri a Cortès, il quale vedendoli umiliati e pentiti, gli richalco, e míre in libertà. Quei di Tecamachalco, Città confiderabile di Toch- della Nazione Popoloca fecero una gran refiftenza; ma alla tepec. fine s'arrendettero, e due mila di loro furono fatti fchiavi. Contra Tochtepec Città grande ful fiume di Papaloapan, do. v'era guernigione mefficana, mandò un Capicano appellato Salcedo con ottanta Spagnuoli, de' quali noa reftò neppure uno vivo, per recare a Cortès la nuova delia loro fconfitta. Rincrebbe affai a quel Generale quefta perdira, la quale rapporto alle poche truppe Spagnuole, che allora aveva, era molto grande, e per vendicarla mandò $\mathrm{i}$ due bravi Capitani Ordaz ed Avila con alcuni cavalli, e venti mila Alleati, i quali ad onta del gran coraggio, con cui fi difefero i Mer. ficani, efpugnarono quella Città coll' uccifione di molti nemici.

Non fu la perdita di que' Soldati quella che più rin. crebbe a Cortès. Quegli fteffi che poco innanzi l'aveano fcongiurato di ritornare alla Veracroce, perfiftettero sì oftinatamente nella loro richiefta, che fu coltretto d'accordar loro il permeffo di tornare non già alla Veracroce per afpettare ivi nuovi foccosfi, ma a Cuba per effer più lontani da' pericoli della guerra, parendo manco male a quell' accorto Condottiere, lo fminuir le fue truppe, che il tener de' malcontenti, che col loro difpiacere rallentaffero il coraggio, $e$

raf-

\footnotetext{
a Tamazolla dove alcuni mefi innanzi avea mandato alcuni Spagnuoli a cercar delle miniere. Il fuddetto Autore delle Note fulle lettere di Cortès, dice, che quefta Tamazolla è in Cinaloa; ma quefto è uno dei grandi fpropofiti che fi leggono in quelle note, Lo fteffo Cortès afferma, che Tamazolla diftava quaranta leghe da Itzocan, laddove Cinaloa diftà più di. 400. Nemmeno parla Cortès di Huaxjacac, o fia O Oxaca dove dice Coaftoaca, ficcome vuol quell'Autore, ma di Coaixtlahuacan, detta dagli Spagnioli Juflabuasa.
} 
raffreddaffero gli animi degli altri; ma quelta perdita fu prontamente e vantaggiofamente riparata con un buon numero Lis. IX. di Soldati, che con cavalli, armi, e munizioni arrivarono al porto della Veracroce, mandati gli uni dal Governatore di Cuba in ajuto di Narvaez, e gli altri dal Governatore di Giamaica alla fpedizione di Panuco: i quali tutti s'aggregarono volentieri al partito di Cortés, cangiandofi in iftrumenti di felicità quegli fteffi mezzi, che da'fuvi nemici per la fua rovina s'adoperavano.

Le vittorie degli Spagnuoli, e la moltitudine de' loro Alleati aggrandirono talmente il loro nome, e conciliarono fatta dal tal autoritá a Cortès preffo que' popoli, ch' egli era l'arbi-Vajuolo. tro delle loro differenze, ed a lui, come fe foffe fupremo Siguor di quella terra fi portavano per ottenere la conferma Cuitladell'inveltitura degli Stati vacanti, ficcome accadde in quel- buatzin, li di Cholollan, e d'Ocotelolco in Tlafcalla, vacanti e l'u. Principe no, e l'altro per morte cagionata dal vajuolo. Quelto fla. Maxixgello del genere umano affatto fconolciuto fin'allora nel Nuo. carzin, vo Mondo, vi fu portato da un Moro fchiavo di Narvaez. zione del Coltui l'attaccó a' Cempoallefi, e quindi fi propagò il contagio per tutto l' Imperio Mefficano con indicibil danno di quelle Nazioni. Vi perirono molte migliaja d'uornini, ed alcuni luoghi reltarono fpopolati. Quelli, la cui compleffione prevalfe alla violenza del male, reftarono sì sfigurati, e fegnati con si profonde tracce del veleno ful viro, che faceano orrore a chiunque li guardava. Tra gli altri mali cagionati da quefta nuova malatía fu troppo fenfibile a' Mefficani la morte del lor $R e$ Cuitlahuatzin, dopo tre o quattro mefi di Regno, ed a' Tlafcallefi ed agli Spagnuoli quella del Principe Maxixcatzin.

I Mefficani eleffero $\operatorname{Re}$ Quauhtemotzin nipote del defunto Cuitlahuatzin, mentre non reftava verun fratello de' due ultimi Re. Era coltui giovane di venticinque anni, e di grande fpirito, e benchè per la fua età non molto pratico nel meltier della guerra, continuò pure le difpolizioni militari del fuo anteceffore. Sposò fua cugina Tecurchpotzin, 
figlia del Re Motezuma, e moglie gì del fuo Zio Cuitla? Lis. IX.huatzin.

La morte di Maxixcatzin fu affai compianta da Cortès così per l'amicizia, che avea ftretta con lui, come pure perchè ad effo lui fi dovea principalmente quell' armonia, ch'era fra $\mathrm{i}$ Tlafcallefi e gli Spagauoli. Per tanto dopo aver renduta ficura la trada della Veracroce, e d'aver mandato alla Corte di Spagna il Capitano Ordaz con un diftinto rag: guaglio fcritto all' Imperatore Carlo V. di tutto ciò , che gli era fin'allora avvenuto, ed il Capitano Avila alla ifola di $S$. Domenico a follecitar nuovi foccorfi per $!_{a}$ conquifta di Meffico, egli fi partì da Tepejacac per Tlaícalla, e v'entrò veftito di bruno, e facendo altre dimoftrazioni di dolore per $\mathbf{l}$ morte del Principe fuo amico. Conferì a richiefta degli fteffi Tlafcallefi, ed a nome del Re Cattolico lo Stato vacante d'Ocotelolco, uno de' quattro principali di quella Repubbli $c a$, ad un figliuolo del defunto Principe, ragazzo di dodici anni, il quale nel battefimo prefe il nome di Don Giovanni Maxixcatzin, (") effendo da allora innanzi il nome del Padre foprannome del figliuolo, e di tutta la fua chiara difcendenza, e per fargli qualche particolar onore per riguardo a' meriti del Padre fuo, l' armò da Cavaliere all' ufanza di Caftiglia .

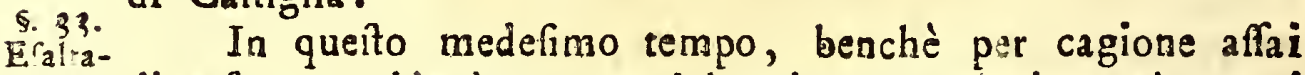
zione del diverfa, accadde la morte del Principe Cuicuitzcarzin, cui Principe aveano meffo Motezuma e Cortès ful trono d'Acolhuacan in tzin, e luogo del fuo fventurato fratello Cacamatzin. Non gli fu permuic di meffo di godere della fua pofticcia dignità, perchè tofto gli Cuicuitz-
catzin. tolfe la libertà colui medefimo, che gli avea dato la corona. Ufcì di Meffico tra gli altri prigioni quella notte della fconfitta degli Spagnuoli; ma ebbe allora la fortuna, o piuttofto difgrazia di fcampare, mentre dovea frappoco perder più igno:

minio.

${ }^{*}$ ) Solis dice, che $s^{\prime}$ appellava Lorerzo; ma quefto fu il nome del $\mathrm{Pa}-$ dre; il figliuolo s' appello Giovanni, ficcome ne fa fede Torquernada، il quale to feppe dagli itefr Tlafcallef. 
miniofamente la vita: Accompagnó gli Spagnuoli ne loro conflitti fin'a Tlafcalla, dove ftette finattantochè o impazien.Lir. IX. te dell' oppreffione, o bramofo di ricuperare il trono, fe ne fuggi legietamente a Tezcuco. Regnava allora in quetta Corte il fratello di lui Coanacotzin, a cui morto Cacamatzin toccava giufta la legge di quel Regno la Corona. Appená vi fi prefento Cuicuitzcatzin, che fu fatto prigione da' Miniftri Regi, i quali ne diedero pronto avvifo al $R e$, affente allora in Meffico. Coltui lo fece fapere al $R e$ Quauhte. motzin fuo cucino, il quale, Aimando fpia degli Spagnuoli quel fuggitivo Principe, fu di lentimento di farlo morire. Coanacorzin o per compiacere a quel Monarca, o piurtolto per levare a Cuicuitzcarzin l'occafione di voler ricuperare il trono con pregiudizio del fuo dritto, e della pace del Regno, gli fece dar fenza indugio la morte. Cosi fini quello fventurato Principe, la cui efaltazione non fervi ad altro, che a far più grande e più Itrepitofa la fua caduta. (Z)

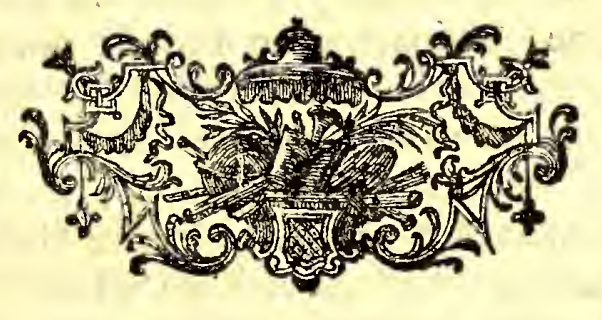

Storia Antica del Meffico Tom. III. X II.

(Z) Non v' è veruno Storico Spagnuolo, fuorchè Cortès, che faccia menzione della fuga, dell' imprigionamento, e della morte di Cuicuitzcatzin. Gomara accenna foltanto la fua morte. Quefto Autore l'appella Cocuzca, Herrera Quifquizca, e Cortès Cucazcacin. Aggiunge, che avea ancora il nome d'Ipaljucbil, cioc̀ Icpalxecbitl. 
162

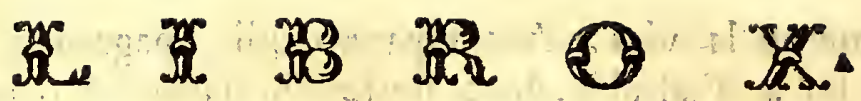

Marcia degli Spagnuoli a Tezcuco: le loro negoziazioni có. Meffccani, le loro fcorrerie e battaglie ne' contorni de' lagbi meficani: le loro Spedizioni contro Jacapichtlan, Quaubnabuac, ed altre Citta. Fabbrica de' brigantini.

Congiura d'alcuni Spagnuoli contro Cortès. Rafsegna, divifione, e pofti dell' efercito Spagnuolo. AfJedio di Meffico, imprigionamento del $R e$ Quaubtemotzin, e rovina dell' Imperio Mefficano.

9. I.

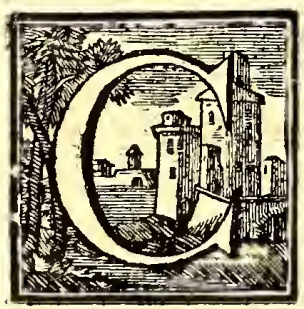

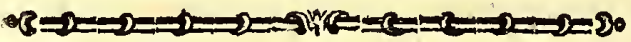

R. Io il altri materiali de' vafcelli da ho fa dis e marcia antecedente, per fervirfene ne'brigancini, per impegolarli dell'efer- fece cavar una fufficiente quantità di pece da' pini della gran gnuolo a montagna Matlalcueje. (a) Avvisò gli Huexotzinchi, i ChoTezcuco. lullefi, i Tepejachefi, ed altri alleati, acciocchè alleftiffero le loro truppe, e fece ammaffare una gran copia di munizioni da bocca e da guerra pel numerofo efercito, che do: vea

(a) Solis aggiunge, che allora cavarono zolfo dal celebre Vulcano Popocatepec per far della polvere; che colui che il cavò s'appellava Montáno, ed a cio perfuadere allega la teftimonianza di Laet; ma egli è certo, che hon fi cavó zolfo da quel Vulcano prima della conquilta di Meffico, e che colui che lo cavò nel ' 522 . 's' appellava Montaño, o fia Montagno, non Montano, come dice Solis, nè per dimoltrar la verità di queftu fatto bifognava prevalerfi dello rcritto d'un Olandefe; poichè ci contta per la teftimonianza degli Autori 'Spagnuoli Herrera, Torquemada, \&c. e per gli Aeffi privilegj accordati dal Re Cattolico alla pofterità di Montagno. 
yea impiegarfi nell' affedio di $\mathrm{M} *$ ffico; ed allorchè gli parve, che foffe tempo di marciare, fece la raffegna della fua trup. Lis. X. pa, la quale conftava di quaranta cavalli, e di cinquecento cinquanta pedoni. Divife quella poca cavalleria in quattro parti, e l'infanteria in nove compagnie, armate l'une di fchioppi, l'altre di balifte, l'altre di fpada, e rotella, e l'altre di picche. Dal cavallo, ful quale ftava ordinando le fue truppe, fece loro quefto parlamento: "A Amici e compagni "miei, qualunque difcorfo io voglia fare per animar la vo. "Ara bravura, è affatto fuperfluo, mentre tutti ci ricono" fciamo obbligati a riparar l'onore delle noftre armi, ed "a vendicar la morte de' noftri Spagnuoli ed alleati. An" diamo alla conquifta di Meffico, imprefa la più gloriofa " di quante mai a noi fe ne poffono offerire in tutta la no" Atra vita. Andiamo a caftigare con un colpo la perfidia, " l'orgoglio, e la crudeltà de' nottri nemici, ad ampliare $\mathbf{i}$ " dominj del noftro Sovrano aggiungendovi un Regno si gran" de, e si ricco, a fpianar la ftrada al Vangelo, ad aprir "la porta del Cielo a tanti milioni d'anime, ad afficurar " colla fatica di pochi giorni l'agiatezza delle noftre fami" glie, ed a rendere immortale il noftro nome: fimoli tut"ti capaci d'incoraggire anche gli animi più codardi, non "che de"cuori si nobili e generofi, come i voftri. Io non "vi veggo veruna difficolta, che non poffa dalla voltra bra"vura fuperarfi. Sono in vero molti i noftri nemici; ma " noi fiamo a loro fuperiori nel coraggio, nella difciplina, " e nell'armi.Oltrechè abbiamo fotto $i$ noftri ordini un nu" mero si grande di truppe aufiliarie, che da effe ajutati ", conquiftar potremmo non folo una, ma molte Città egua"li a quella di Meffico. Sia pur effa forte quanto fi voglia; " ma non l'è tanto, che poffa refiftere agli attacchi, che " dobbiamo dargli per terra e per acqua. Finalmente Iddio, " per la cui gloria pugniamo, s'è dichiarato di voler fe" condare i noftri difegni. La fua provvidenza ci ha confer. "vato in mezzo a tanti periglj e difaftri, ci ha mandato "de' nuovi compagni in luogo di quelli, che abbiamo per$\mathrm{X}_{2}$ " duti, 


\section{4}

"duti, ed ha cangiato in ben noftro que' mezzi, che da' Eia. X." noftri nemici s'adoperavano per la noftra rovina. Che non "dovremo fperare per l'avvenire dalla fua mifericordia? E"gli è noftro Condotriere in quefta grande fpedizione: teniamogli pur dietro, e non ci rendiamo indegni della fua s) protezione colla noftra diffidenza e pufillanimita.

I Tlafcallefi, i quali procuravano contraffare la dicciplina degli Spagnuoli, vollero anch' eff far la raffegna delle loro truppe alla prefenza di Cortès. Cominciava l' éfercito dalla mufica militare di cornette, lumaconi, ed altri si fatti ftumenti da fiato, dietro alla quale venivano i quattro Capi della Repubblica armati di fcudo e fpada, e fregiati di vaghifimi penaacchj, che fopravanzavano più di due piedi fopra i loro capi. Portavano i capelli legati con bendelle d'oro, nelle labbra e nell' orecchie de' pendenti di gemme, e ne' piedi delle farpe di gran pregio. Ad effi tenevano dietro quatero loro fcudieri armati d'arco e frecce. Venivano dopo i quattro ftendardi principali della Repubblica, ciafcheduno colla fua propria infegna fatta di penne. Indi cominciarono a paffare in file ben ordinate le truppe d'arcieri a venti a venti, lafciandofi vedere in certe diftanze gli fendardi particolari delle 10 . ro compagnie, compolta ognuna di arecento o quattrocento uomini, le quali erano feguite dalle truppe armate di fpada e di fcudo, ed alla fine da quelle de' picchieri. Herrera e Torquemada affermano, che gli arcieri erano feffanta mila, i pic. chieri dieci mila, e gli altri armati di fpada quaranta mila.(b)

Xico-

(b) Solìs tenendo dietro, per quel ch'egli dice, a Bernal Diaz, non numera nella raffegna dei Tlafcallef più di 10.000 uomini, e riprende Herrera, perchè difcottandofi dal ragguaglio di Bernal Diaz ne conta 80. $000 ; \mathrm{ma}$ in cid, ficcome in altri punti, fi reorge bene la trafcuraggine di" Solis nel confultar gli Autori. Bernal Dia non fa menzione della raffegna dei Tlafcallefr; foltanto dice, che Cortès domando al Senato dieci mila uomini, ed il Senato rifpofe, ch'e ra pronto ad alleftire un numero a frai maggiote di truppe. Herrera non conta 80.000 uomini, come dice il Solis, ma i 0.000 , ficcome pud vederfi nella fua Decada 2. lib. 2. cap. 20. Herrera è ftato feguito in tal computo da Torquemada e da Betancurt. Ojeda, il quale vi fu prefente, ed era Condottiere delle truppe alleate afferma, ch' effe erano I 50.000 uomini; ma in quefto computo comprefe gli Hizexozzinchi, i Cholollefi, ed i Tepejachefi'。 
Xicotencatl il giovane fece ancora il fuso parlamento ad e. fempio di Cortès, nel quale diffe alle fue truppe, che il Liв. $\mathbf{X}$. di appreffo, ficcome era a loro ben noto, doveano marcia. se co prodi Spagnuoli contra i Mefficani lor capitali nemi. ci: che benchè il fol nome de'Tlafcallefi baftaffe per impaurire tutte le Nazioni della terra, s'adoperaffero pure per ac. quiftarfi nuova gloria colle loro azioni.

Cortès dal fuo canto convocd i principali Signori degli Stati alleati, e gli efortò ad una coltante fedeltà verfo gli Spagnuoli, efagerando loro i vantaggj che doveano fperare dalla rovina de' Mefficani, ed i mali, che doveano temere, Se mai per fuggeftione de' Mefficani, o per paura della guerra, o per incoftanza d'animo mancaffero alla fede data. Indi pubblicò un bando militare per la regola delle fue truppe, il quale conteneva i feguenti arricoli.

I. Niuno beftemmi contro Dio, ne contro la B. Vergine, nè contro i Santi.

II. Niuno abbia lite cons altri, nè metta maro alla $\int p a b$ da, a ad altra arma per ferislo. ferramenti.

IV. Niuno sforzi veruna donna fotto pena della morte.

V. Niuno pigli la roba altrui, nè punifca verun Indias: no fe non è fuo fcbiavo. me Jo.

VI. Niuno vada a far delle fcorrerie fenzia il noftro per.

VII. Niuno faccia prigione verun Indiano, nè faccbegg fenza il noftro perme fjo la cafa di lui.

VIII. Niwno malmeni gli Alleati; anzi per tutti i ver. $f_{i} f_{i}$ procusi mantener la loro amicizia.

E perchè nuila giova il pubblicar delle leggi, qualora. non fi zela la loro offervanza, e non fi punifcono i delinquenti, fece impiccar due mori fuoi fchiavi, perchè rubaro. no un gallinaccio, e due mantelli di banbagia. Con quelti ed alrri si fatti gaftighi, fece rifpettar i fuoi ordini cotanto neceffarj per la confervazione delle fue sruppe. 
Poichè ebbe date tutte le difpofizioni, che gli parvero LIs. X. confacenti al buon efito della fua imprefa, marciò finalmen. te con tutti i fuoi Spagnuoli, e con un buon numerod' Al. leati il di 28. Decernbre 1520. dopo aver fentito la Santa Meffa, ed invocato lo Spirito Santo. Non volle condurre allora feco tutto l'efercito d'Alleati ch' era ftato il giorno innanzi raffegnato, così per cagione della difficoltà, che vi farebbe nel mantenere un si gran numero di truppe in Tezcuco, come perchè ftimò più neceffario il lafciarne la maggior parte in Tlafcalla per la ficurtà de' brigantini, quando foffe tempo di trafportarli. (c) Delle tre Atrade, che $v^{3}$ erano per andare a Tezcuco prefe Corrès la più malagevole, perfuadendofi prudentemente, che non dovendo in effa afpettarlo i Mefficani, riufcirebbe più ficura la fua marcia. $S^{\prime}$ avvid dunque per Tetzmellocan, villaggio appartenente allo Stato di Huexotzinco. Addì 30 . offervarono dalla cima più alta di quelle montagne la bella valle di Meffico, parte con giubilo, perchè quivi era l'oggetto delle loro brame, e parte con qualche difpiacere per la memoria de'loro difaftri. Nel cominciare a fcendere verfo la valle riconobbero la ftrada impacciata con molti tronchi e rami dxalberi traverfativi a bella pofta, ed ebbero d'uopo d'impiegar mille Tlafcallefi nello fgombrarla. Tofto che giunfero alla pianura, furono affaliti da alcune truppe volanti de' nemici; ma effendo ftati alcuni d'effi dagli Spagnuoli uccifi, furono meffi in fuga gli altri. Quella notte alloggiarono in Coatepec, Luogo otto miglia difcofto da Tezcuco, ed il dì vegnente incamminandofi a quella Corte folleciti della difpofizione de' Tezcucani, ma parimente rifoluti di non tornare in dietro fenza aver prefa vendetta de'loro nemici, videro venir verfo loro quattro Perfonaggi autorevoli difarmati, e uno di loro con una banderuola d'oro in mano, e riconofcendo Cortès, che ciò

era

(c) Non fi dubita, dice Solls, cbe ufcì Cortès da Tiafcalla con più di feffanta mila uomini; ma egli è certo, che non fi fa quanti conduffe feco; roichè nè Cortès, nè Bernal Diaz efprimono il numero. Gomara dice, ch' erano più di 80. 000 . 
era un contraffegno di pace, $s^{\prime}$ avanzd per abboccarfi con loro. Erano quefti quattro meflaggieri mandati dal Re Coa- Lis. $X$. nacotzin per complimentare il Generale Spagnuolo: per invitarlo a portarfi alla fua Corte, e per pregarlo di non fare veruna oftilità ne'fuoi Stati, i quali gli prefentarono la banderuola, che avez trentadue oncie d'oro. Cortès malgrado quefti contraffegni d'amicizia rinfacciò loro la morte data pochi mefi innanzi dagli abitatori del villaggio di Zoltepec a quaranta cinque Spagnucli, cinque cavalli,e trecento Tlafcallefi, che gli acccompagnavano carichi d'oro, d' argento, e d'armi per gli Spagnuoli, ch' erano allora in Meftico, ed efeguita con tal inumanità, che aveano fofpefo, come tro. fei ne' tempj di Tezcuco le pelli degli Spagnuoli colle loro armi ed abiti, e quelle de' cavalli co'loro ferri. Soggiunfe, che giacchè non era poffibile a loro il compenfar la perdita di quella gente, doveano almeno pagargli l'oro e l'argento rubato: che fe non gli davano la dovuta foddisfazione, per ciafcuno Spagnuolo uccifo farebbe egli morire mille Tezcu. càni. I meffaggieri rifpofero, che di ciò non erano colpevo. li i Tezcucani, ma i Mefficani, pe' cui ordini il fecero i Zoltepechefi, che con tutto ciò eglino s' efibivano ad adoperare ogni diligenza poffibile, acciocchè tutto foffe loro reftituito, e prefo cortefemente congedo dal Generale Spagnuolo, ritornarono in fretta a Tezcuco colla nuova del pronto arrivo degli Spagnuoli a quella Corte.

Entrò Cortès col fuo efercito in Tezcuco l'ultimo di di quell' anno. Vennero ad incontrarlo alcuni Nobili, e lo conduffero ad uno de'palagj del defunto $\operatorname{Re}$ Nezahualpilli, il qual' era così grande, che non folamente vi s'alloggiarono que' feicento Spagnuoli, ma vi potevano ancora ftare co- $\begin{gathered}\text { degli } \\ \text { Spagnio- }\end{gathered}$ modamente, per quel che dice Cortès, altri feicento. Tofto li inTezriconobbe quel Generale notabilmente diminuito il concorfo $\underset{\text { rivoln- }}{\text { cuco }}$ e di Cittadini nelle ftrade, parendogli che non vi vedeva nep tioni di pur la terza parte di Popolo, ehe avea gia veduto in altre quella ocaafioni, e particolarmente offervò, che vi mancavano le Corte. donne, ed i fanciulli, indizio manifelto della cattiva difpofi. 
zione di quella Corte. Per non accrelcere la diffidenza de? Lrs. X. Cittadini, e per non efporre a qualche difaftro la fua gente pubblicd un bando, nel quale fotto pena di morte vietava a'fuoi Soldati l'ufcire fenza il fuo permeffo dal quartiere: Dopo definare offervarono da' terrazzi del palagio, che gran gente abbandonava la Città, incamminandofi gli uni a'bo. fchi vicini, gli altri a diverfi luoghi del lago. La notte vegnente s'aflente lo tetio Re Coanacorzin, trafportandofi a Meflico in una barca a difperto di Cortès, che voleva pigliarlo, ficcome avea gia fatto co' tre fuoi fratelli Cacamatzin, Cuicuitzcatzin, ed Ixtlilxochitl. Non poteva prender Coanacotzin verun altro partito; perchè com' era poffibile, che fi ftimaffe ficuro fra gli Spaguuoli dopo aver veduto ció che aveano fatto co' fuoi fratelli, e col Re Motezuma fuo Zio, e mafimamente temendo, che molti de' fuoi medefimi fudditi prendeffero quindi occafione per dichiararfi fuoi nemici, gli uni per paura degli Spagnuoli, o per l' intereffe particolare delle loro famiglie, gli altri per vendicar la morte di Cuicuitzcatzin, ed altri aucora per metter ful trono Ixtlilxochit! .

Le rivoluzioni, che immediatamente accaddero in quel: Ia Corte, giultificarono abbaftanza la determinazione da lui prefa. Appena v'era fato tre giorni Cortès, che gli fi prefentarono i Signori d'Huexotla, di Coatlichan, e d'Atenco, tre Città cosìvicine, come abbiam già detto, a Tezcuco, che parevano altrettanti fobborghi di quella gran Corte, pregan:dolo di volere accettar la loro alleanza ed amicizia. Cortès; come quegli che nulla più bramava, che d'ingroffar il fuo partito, gli accolle benignamente, ed efibi loro la fua protezione. La Corte di Meffico, tolto che feppe tal novita, mandò a que' Signori una fevera riprenfione, facendo lor dire, che fe la cagione d'efferfi appigliari ad un si vil partito era la paura che aveano della poffanza de' loro nemici, fapeffero pure, che $i$ Meflicani fi trovavano con forze affai più grandi, colle quali vedrebbono frappoco mefli in conquaffo gli Spagnuoli, infieme co' loro favoriti alleati i Tlafcallefí che fe s'erano a ciò ridotsi 
dotti dall' intereffe degli Stati, e delle poffeflioni; che avea no in. Tezcuco paffaffero a Meffico, ne' cui dominj fa rebbono loro Liz. X. affegnate delle terre affai migliori, ma que' Signori in vece d'impaurirfi colla riprenfione, o d'arrenderfi alle promeffe, pigliarono i meffaggieri, e gli mandarono a Cortès. Coftui gli addimandò del motivo della loro ambafciata, ed eglino rifpofero, che fapendo che quei Signori erano nella fua grazia, venivano a pregarli di voler elfer mediatori per la pace tra $i$ Meflicani e gli Spagnuoli. Cortès, facendo Sembianza di creder ciò che dicevano, gli rimife in liberta, e gl'incaricò di dire al loro Sovrano, ch'egli non voleva la guerra, né la farebbe mai, fe non foffe coftretto dall' oftilita de'Mefficani; che per tanto s'avvedeffe, e fi guardaffe di far verun male agli Spagnuoli, o a'loro Alleati, perchè altrimenti eglino farebbono da nemici, e rovinerebbono infallibilmente le 10ro Città.

Molto invero importava a Cortés l'alleanza di quelle tre. Città; ma più d'ogni cola gli era d'uopo di conciliarfi la feffa Corte di. Tezcuco tanto per la gran Nobilià, che v'era, quanto per la fua influenza nell' altre Città del Re. gno. Dacchè entro in quella Corte procurò ognora guada. gnarfi gli animi colla civilta, e colle buone maniere, e lo fteffo avea raccomandato a'fuoi, vietando feveriflimamente ogni forta d'oftilità verfo i Cittadini. Riconobbe da principio fra Ja Nobilta un partito favorevole al Principe Ixtlilxochitl, cui teneva, non fo per che cagione in Tlafcalla. Lo fece condur alla Corte da un buon numero di Spagnuoli, e di Tlafcallefi, prefentollo alla Nobilta, ed ottenne, che foffe da loro riconofiuto per Re, ed incoronato colle medefime ceremonie e dimoftrazioni d'allegrezza, che far folevano verfo i loro legittimi Sovrani. (d) Promore Cortès l' elaltazio. Storia Antica del Mefjico Tom. III.

(d) Solis nel racconto dell' efaltazione del Principe Ixtlilxochitl oltre alle immaginarie aringhe, che pone in bocca di Cortès, e de'Tezcucani, incorfe almeno in fette errori fuftanziali. I. Suppone viro in quefto tempo Cacamatzin, laddove pel ragguaglio di Cortès, e di tutti gli Storici cicon- 
ne di quefto Principe cosł per vendicarfi del legittimo Re

Lis. X. Coanacotzin, come perchè il Regno foffe da lui dipendente. Il Popolo l'accettò o perchè non ebbe ardire d'opporli agli Spagnuoli o forfe perchè erano infaftiditi del governo di Coanacotzin. Era Ixtlilxochitl giovane di ventitre anni in circa. Infia dalla prima entrata di Cortès in Tiafcalla s' era dichiarato apertamente per gli Spagnuoli, $s^{3}$ era efibito a loro col fuo efercito, e gli avea invitati a fare il viaggio a Meffico per Otompan, dov' egli allora fi trovava; ma a difpetto della fua buona volonta, e de'fuoi offequj era pure prigioniere degli Spagnuoli, allorchè coltoro ufcirono fconfitti da Meffico, e fu ritenuto da loro in Tlafcalla finchè fu chiamato al trono. Le circoftanze di quefto avvenimento ci fanno credere, che la fua prigionia fia ftata una decorofa oppreffione della fua libertà, colorata con qualche bel pretefto di quelli, che fuol inventar la politicadegli uomini, qualora a cagione di qualche diffidenza voglion metterfi al ficu-

ro.

fta, che fu uccifo nella notte della fconfitta degli Spagnuoli, o poco innanzi. 2. Dubita prima, e poi afferma pofitivamente, che in quefto medefimo tempo regrava in Tezcuco Cacamatzin, laddove fappiamo per la teftimonianza di quafi tutti gli Storici, che regnava Coanacotzin - 3. Fa Cacamatzin :fratello di Nezahualpilli ( cui appella Nezabal) effendo ftato fuo figlio, ficcome fanno tutti quelli che hanno fudiato la ftoria di que' PopoR. 4. Suppone Nezahualpilli ucciro da Cacamatzin : favola nun mai udita nella Storia di Tezcuco. 5. Grede morto Nezahiualpilli, allorchè regnava l'antecelfore di Motezuma. Or l'anteceffore di Motezuma nori nel 1502 . : dunque Nezahualpilli 'fu uccifo, al più tardi, quelto medefimo anno da $\mathrm{Ca}$ camatzin, fecondo che dice Solis. Cuando ebbe l'ardire d'ammazzar il fuo Re fi debbe credere, che aveffe almeno quindici anni: dunque nel igrg. allorchè il fuddetto Cacamatzin vifito Cortès in Ajotzinco, aveva almeno 32. anni; eppure lo fteffo Solis dice in altro luogo, che era allora giovane di 2 g. anni,; ma egli è certo, che Nezahualpilli mori nelıgr6. 6. Suppone.Cacamatzin ufurpators della corona, quando n'era legittimo erede, ficcome confa per la Storia. 7. Finge, che il nuovo Re fi trovava in Tezcuco, quando yi arrivò Cortès, che coftui non l'area mai veduto, che la prima volta, che il Principe gli fi prefento, fi compiacque tanto della fua eloquenza, e gentilezza, che fenza poter trattenerfi l'abbracciò, ma tu tro ciò è fallo; poichè ci confta per le lettere del medefimo Cortès, e per la Storia di Herrera di Torquemada, ed altri, che quel Principe ( il cui nome ignorò Solis ) era più d'un anno, ch'era ftato veduto daCortès, e più di fei mefi era fato fuo prigioniere, e che per incoronarlo io fece venir da. Tlafcalla. 
ro. Colla lunga pratica degli Spagnuoli s'avvezzo alle loro ufanze e maniere. Fu inftruito nella Criftiana Religione, e Lis. X. battezzato col nome di Don Ferdinando Cortes Ixtlilxochitl per riguardo al Generale Spagnuolo, che fu il fuo patrino. Non ebbe nel trono fe non fe qualche apparenza di Maefta; mentre più che Signor de' fuoi fudditi, fu miniftro della volontà degli Spagnuoli, cui rendette de'gran fervizj non folamente nella conquilta di Meffico, nella quale fervì colla fua perfona e colle fue truppe, ma eziandio nalla reedificazione di quella Capitale, per la quale forni alcune migliaja d'Architetti, di Muratori, e d'Operaj. Mori affai giovane nel I $523^{\circ}$, e gli fuccedette nella fignoria $\mathrm{d}$ : Tezcuco il fuo fratello Don Carlo, di cuí poi faremo onorevole ricordanza: Coll' efaltazione d'Ixtlilxochitl, e cogli offequj, che Cortès gli faceva, s'ingrolsò confiderabilmente il partito degli Spagnuoli, e tutte quelle famiglie Tezcucane, che s'erano alfentate dalla Corte per paura delle loro oftilità, rendute oramai ficure ritornarono liete alle loro cafe.

$S$ 'era rifoluto Cortès di tener il fuo quartiare in Tez. cuco, e però s' era adoperato per fortificar quel Real palagio dove alloggiavano le fue truppe. Non potea pigliar configlio. più confacevole al fuo intento. Tezcuco, come Capitale del Regno d'Acolhuacan, e Città tanto grande, abbondava d' $\theta$ gni forta di vettovaglie pel fuftentamento dell' efercito: avea buoni edifizj per la loro abitazione, buone fortificazioni per la loro difefa e copia di ogni fpezie d'artefici per tutti $i$ lavori, di cui aveano bilogno. I dominj poi di Tezcuco, confinanti con que' di Tlafcalla, rendevano agli Spagnuolí più agevole la neceffaria loro comunicazione con quella $\mathrm{Re}$ pubblica: la vicinanza del lago importava affai per la coAtruzione de' brigantini, e la vantaggiofa fituazione di quella Corte rendeva gli Spagnuoli confapevoli di tutti i movimen. ti de'loro nemici fenza efporli alle loro oftilità .

Dopo aver ben ordinate le cofe di Tezcuco rifolvette Cortès di dare un affalto alla Cirtà d'Iztapalapan per vendicarfi contro effa, ed $\mathrm{i}$ fuoi Citradini delle offefe ricevute Y 2 
da Cuidlahuatzin loro antico Signore, cui riconofceva AutoLrs. X. re della memorabile fconfitta del I. Luglio: Lafciò in Tezcuco una guertsgione di più di trecento Spagnuoli e molti

5. 3. Alleati fotto il comando di Sandoval, ed egli marciò con ne peri- più di dugento Spagnuoli, più di tre mila Tlafcällefi, (b) colofa e molta Nobiltà Tezcucana. Prima d'arrivare ad IztapalaIztapala. pan, vennero ad incontrarli alcune truppe di nemici, finpan. gendo d"opporfi alla loro entrata, e pugnando parte in ter$r a$, e parte in acqua, ma nel combattere s'andavano ritirando alla Città, facendo fembianza di non poter far retta al loro attacco. Cosi impegnati gli Spagnuoli ed i Tlafcal: lefi nell' incalzarli entrarono nella Città, le cui cafe trova. rono in gran parte fpopolate, perchè i Cittadini s'erano ricoverati colle loro mogli, co' loro figlj, e colla maggior parte della loro roba, nelle cafe che aveano nell' ifolette del la. go; ma furono ancor quivi da' loro nemici perfeguitati pugnando anche nell' acqua. Era omai ben avanzata la notte, allorchè gli Spagnuoli allegri per la vittoria, che credevano aver ottenuto, s' occupavano nel faccheggiar le cafe, ed $i$ Tlafcallefi nell'appicciarvi il fuoco, ma tofto fi cangiò il loro giubilo in ifpavento, imperciocchè colla fteffa luce dell' incendio offervarono, che fgorgava l'acqua de' canali, e comincíava ad inondar le cafe. Riconofciuto il periglio fi fuonò alla ritirata, e s'abbandonò precipitofamente la Città per riprender la frada per Tezcuco, ma a difpetro della loro diligenza arrivarono ad un luogo, dov' era tanta acqua, che gli Spagnuoli vi paffarono a ftento, e de' Tlafcallefr s' annegarono alcuni, e fi perdetre la maggior parte del botrino. Non farebbe rimalto neppur uno di loro vivo, fecondo che afferma Cortès, fe fi foffero trattenuti tre ore più nella Città, perciocchè i Cittadini, volendo annegar tutti i loro nemici, ruppe. so l'argine del lago, ed allagarono la Città. Il di vegnente

con-

(e) Gomara dice, che andarono a quella fpedizione fei mila Tlafcallef, Solis ne numera dieci mila; ma Cortes afferma, che furono da tre, in quattro mils. 
continuarono il loro viaggio lungo il lago, travagliati ognora, e beffaci da' nemici. Non riulci gradevole agli Spagnuoli Lis. X: quefta fpedizione; ma benchè vi perdeffero !e fpoglie, e molti foffero feriti, non morirono piì di due Spagnuoli, ed un ca. vallo. La perdica degl' Iztapalapanefi fo affai maggiore; poichè oltre al difcapito, ch'ebbero nelle loro cafe, reltarono, per quel che dice Cortès, più di fei mila morti.

Il difgufo, ch' ebbe Cortès per quelta fpedizione, fi compensò tolto col contento che ricevette per l'ubbidienza, che gli diedero per mezzo de'loro ambafciatori Mizquic, Otompan, ed altre tre o quattro Citia di que'contorni, allegando per ottener la fua grazia, ch'effendo ftati follecitari da'Mefficani a prender l'armi contro gli Spagnuoli, non vi acconfentirono mai. Cortès, come quegli che s'andava ognora procacciando maggior autorita, quanto più s'ingroffava il fuo partito, richiefe da loro come condizione neceffaria per ottenere la fua alleanza, che pigliaffero tutci i meffaggieri, che foffero lor mandati da Meffico, e tutti i Meflicani che capitaffero nelle loro Città. Eglino s' efibirono a farlo, benchè non fenza gran difficolra, e d'allora innanzi furono coltantemente fedeli agli Spagnuoli.

Quefta confederazione fu immediatamente feguita da quella di Chalco, Cittá, e Stato confiderabile della riva orien. tale del lago dolce; imperocchè fapendo Cortès, che i Chalchefi volevano aderixe al fuo partiro, ma non of vano dichiararfi pel timore delle guernigioni mefficane, ch' erano nel loro Stato, vi mandò Sandoval con venti cavalli, dugento pedoni Spagnuoli, ed un buon numero d'Alleati, e gli diede l'ordine d'incanminar prima certe truppe Tlalcallefi, che voleano portare alla loro patria quella parte, che aveano fcam. pato del bottino d'Iztapalapan, ed indi rivolgerfi fopra Chalco per ifcacciar da quello Srato i Mefficani. Diede Sandoval la vanguardia a' Tlafcallefi; alcune truppe Mefficane, che s'e. rano mefle in aguato, fi fcagliarono improvvifamente contra loro, gli fcompigliarono, uccifero loro alcuna gente, e tolfero loro il botrino; ma fopravvenendo gli Spagnuoli fcon-

fiffe:

§. 4.

Confea derazione d'Otompan, e d'altre Città cogli Spa. gnuoli: 
fiffero, e fugarono i Mefficani. Recuperato il bottino contiE18. X. nuarono i Tlafcallefi fenza pericolo il loro viaggio, e San. doval marciò verfo Chalco, ma molto prima d'arrivare alla Città gli venne all' incontro il groffo della guernigione mefficana, il quale, fecondo che affermano alcuni Storici, fi componeva di dodici mila uomini. Si diede la battaglia, la quale durò due ore, e finì coll' uccifione di molti Mefficani, e colla fuga degli altri. I Chalchefi, confapevoli della vittoria, vennero con gran giubilo ad incontrar gli Spagnuoli, e gl' introduffero trionfanti nella Città. $(f)$ Il Signor di quello Stato, morto del vajuolo poco innanzi, avea negli ultimi momenti della fua vita raccomandato caldamente a' due figli, che lafciava, che fi confederaffero cogli Spagnuoli, che coltivaffero la loro amicizia, e che aveffero Cortés per $\mathrm{Pa}$ dre. In confeguenza della fua ultima volontà fi portarono que'due giovani a Tezcuco, accompagnati dall' efercito Spa gnuolo, e da molta Nobilt't Chalchefe: prefentarono a Cortès in oro il valfente di cencinquanta zecchini, e ftabilirono 1 alleanza, nella quale fi mantennero coftantemente fedeli. La cagione di ribellarfi si facilmente tanti popoli di quell' Imperio era negli uni la paura dell' armi Spagnuole, e della poffanza de' loro alleati, e negli altri l'odio della dominazione mefficana. Non è proffibile, che lia coftante la fedeltà de'fudditi, qualora nella fubordinazione influifce più il terrore, che la beneficenza. Non v'è trono più vacillante che quallo che fi foftiene più colla forza dell' armi, che coll'amor de'Popoli. Cortès, dopo avere accarezzato i due giovani Chalchefi, divife fra loro quello Stato, o a richiefta di loro medefimi, a per fuggeftione della Nobiltà. Diede al più

(f) Solis nel ragguaglio di quefto avvenimento incorre in due errori geografici. I. Suppone la Città di Chalco contigua a quella d'Otompan, non fapendo, che fra effe $v$ ' era la Corte di Tezcuco, ed altre Citta confiderabili del Regno d'Acolhuacan, ficcome fi vede nella noftra carta geografica de' laghi Mefficani.'2. Dice, che gli ftati di Chalco, e di Tlafcalla erano confinanti, laddove v' era fra loro un gran bofco di più di quindici miglia, ed una parte de' dominj di Huexotzinco, e da un' altra banda era frappofta la parte più popolata del Regno d'Acolbuacan. 
piè grande l'inveftitura della Citta prineipale con altri luoghi, ed al più piccolo aggiudicò Tlalmanalco, Chimalhua-Lis. IX. can, A jotzinco, ed altri.

Non ceffavano frattanto i Meffeani di far delle fcorrerie negli Stati, che s' erano confederati cogli Spagnuoli, ma la diligenza di Cortès nel mandarvi de' foccorfi rendeva inutili per lo più i loro sforzi. Tra gli altri vennero in fra pochi giorni a Tezcuco gli fteffi Chalchefi ad implorare l'ajuto degli Spagnuoli; poichè avevano faputo, che i Mefficaai s' apparecchiavano a far un gran colpo in quello Stato di frefco fottratto alla loro dominazione. Non potè Cortès fervir quefta volta alle loro pretenfioni; perché avendo omai finito il lavoro degli alberi, de' panconi, e di tutto il fornimento de'brigantini, avea d'uopo delle fue truppe per farlo trafportar con ficurezza a Tezcuco. Diede per tanto loro il configlio di confedetarfi cogli Huexotzinchi, co' Cholollefi, e co' Quauhquechollefi. Rifiutavano una tal confederazione i Chalchefi per l'antica loro inimicizia con que' popoli; ma alla fine l'accettarono, moffi dall' inftanze di Cortès, e coAtretti dalla neceffita. Appena erano partiti i Chalchefr, che arrivarono opportunamente a Tezcuco tre mefraggieri di Huexotzinco e di Quauhquechollan, mandati da que' Signori a Cortès per fignificargli la loro follecitudine per cagione di certi fummi, indizj non ofcuri di guerra, ofervati dalle fentinelle, ch'eglino avęano fulle cime delle montagne, e per offerirgli le loro truppe pronte a'fuoi ordini, qualora fe ne voleffe fervire. Profitoffi Cortès di si bella occafione per confederar quegli Stati cor. quello di Chalco, obbligandoli a rinunziare pel bene comune ai particolari loro rifentimenti. Fu sı̀ ferma quefta alleanza, che d'allora innanzi s'ajusa. rono fcambievolmente contro i Mefficani.

Effendo omai tempo di trafportare a Tezcuco il legna- Trafsorme, le vele, il cordaggio, ed i ferramenti de' brigantini, to de' mandò Cortès a tal fine Sandoval con dugento pedoni Spa- materiagnuoli, e quindici cavalli, incaricandols d' andar prima a Zol- gantinitepec-e far contro que' Cittadini una rigorofa vendetta per 
l'uccifione di que quaranta cinque Spagnuoli, e trecento Lis. X. Tlafcallefi, di cui abbiam fatto fopra menzione. I Zoltepechefi, allorchè videro venir contro loro quella tempefta, $a b$. bandonarono le loro cafe per ifcampar la vita colla fuga; ma furono incalzati dagli Spagnuoli, molti di loro uccifi, ed al: tri fatti fchiavi. Quindi marciô Sandoval a Tlafcalla, dove trovò tutto alleftito pel trafporto de' materiali la vorati de' brigantini. Il primo brigantino fu fatto da Martino Lopez Soldato Spagnuolo, che faceva da ingegniere nell' armata di Cortès, e fu meffo a pruova nel fume Zahuapan. Sul mo: dello d'effo furono fabbricati da' Tlafcallefi gli aliri codici. $S$ ' efeguì il trafporto col maggior apparato e giubilo de' Tlafcallefi parendo loro poco pefante quella carica, che contribuir doveva alla rovina de' loro nemici. Otto mila Tlafcallefi portavano addoffo le travi, le vele, e gli altri arnefi, che fi richiedevano per la fabbrica de'brigantini, due mila venivano carichi delle vettovaglie, e trenta mila uomini armati per la difefa fotto gli ordini di tre capi principali Chichimecatl, o fia Chichimeca-teuctli, $\left(^{*}\right)$ Ajotecatl, e Teotepil, o Teotlipil. Quefto convoglio occupava, per quel che dice Bernal Diaz, più di fei miglia. Quando ufcirono di Tlafcalla, comandava la vanguardia Chichimecatl; ma tofto che mifero il piede fuori de' dominj di quella Repubblica, Sandoval gli diede la retroguardia; perchè temeva qualche attacco da'nemici. Ciò cagionò un grave difgufto al Tlafcallefe, come quegli che fi vantava della fua bravura, allegando che in tutte le battaglie nelle quali fin'allora s'era

tro-

$\left(^{*}\right)$ Quefto Chichimecatl, che fa una figura luminofa nella noftia Storia, non pare, che fia ftato il Padre, il quale era già molto vecchio, ma il figlio così ancora appellato, cioè colui medefimo, che nella guerra de'Tlaf-

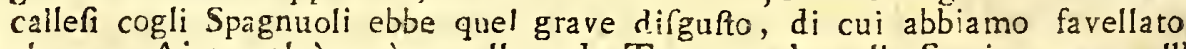
altrove. A iotecatl è cosi appellato da Torquemada nella Storia, ma nell' indice lo chiama Ajutecatl. All'altro Capo dà nella Storia il nome di Teotepil, e nell' indice quello di Térlypil. Io forpetto, che quel Nobile Tlafcallefe fia ftato Acxotecat, Signor d'Atlibuetzian, cioè quel 'Padre inumano, che in odio della Fede Criftiana uccife poi due de' fuoi figli. Cortès appella que'Capi Tutecatl e Teutiplb. 
trovato, avea fempre occupato ad efempio de' fuoi Antenati il polto piû rifchiofo, ed ebbe bifogno Sandoval d'adoperarLis. X. delle ragioni e delle preghiere per contentarlo. Cortès veftito della più sfarzofa gala, ed accompagnato da turti $i$ fusi Uffiziali venne ad incontrarli ed abbracciò e ringraziò que'Signori Tlafcallefi de'buoni loro uffizj. Sei ore fpefero nell' entrare in Tezcuco col miglior ordine, e gridando $\mathrm{Ca}$. figlia, Caftiglia, Tlafcalla, Tlafcalla in mezzo allo Itrepito degli ftrumenti militari.

Appena arrivato il General Chichimecatl, fenza voler Spedizioprender ripofo della fatica del viaggio, pregò Cortès d'impie- ni contra gar lui e la fua truppa contro i nemici. Cortès, il quale le Città non altro afpettava, che l'arrivo delle truppe aufiliarie di can,edi Tlafcalla per efeguire una fpedizione, che tempo fa medita- Tlacova, lafciando in Tezcuco una buona guernigione, e dati pan. gli ordini opportuni intorno al compimento de'brigantini, $f$ mife in marcia ful principio della Primavera del 1521. con venticinque cavalli, e fei piccoli cannoni d'artiglieria, con trecento cinquanta pedoni Spagnuoli, trenta mila Tlafcallef, ed una parte della Nobilta Tezcucana:e perchè temeva, che i Tezcucani, di cui non fi fidava ancora, non deffero qualche avvifo fegreto a' nemici, e fraftornaffero i fuoi difegni, ufè di Tezcuco fenza palefare il termine della fua fpedizione. Cammino l'efercito dodici miglia verfo Tramontana, e ftette quella notte allo fcoperto. 11 dì vegnente andò ad attaccar Xaltocan, Citta forte fituata in mezzo ad un piccolo lago con una frada, che vi conduceva tagliata, come quelle di Meffico con alcuni foffi. L'Infanteria Spagnuola, ajutata da un buon numero d'Alleati, pafsò i foffi fra un denfo nembo di dardi, frecce, e faff, con cui furono molti feriti; ma non potendo i Cittadini foffrir più la ftrage, che in loro facevano l'armi Spagnuole, abbandonarono la Città, e fi falvarono colla fuga. I vincitori faccheggiarono le cafe c ne abbruciarono alcune.

- 1l giorno dappreffo s'incamminarono alla grande e bella Citta di Quaubritlan, ficcome Cortès l'appella a ragione; Storia Antica del Mefjeco Tom. III. 
ma la trovarono fpopolata, perchè i Cittadini impauriti per Lis. X. ciò. ch' era avvenuto a Xaltocan, procurarono poifi in ficuro. Quindi paffarono a. Tenajoccan, e ad Azcapozalco, e perchè non trovarono refiftenza in quelle tre Città, non vi fecero verun male. Finalmente arrivarono alla Curte di Tlacopan, termine che s'era propolto Cortès per follecitarvi qualche accomodamento colla Corte di Meffico, e fè mai non riufciffe, per informarí più da vicino de'loro difegni e preparativi. Trovò gli abitanti di quella Città difpolti a contraftar loro l'entrata. Affalirono coltoro colla folita furia gli Spagnuoli, e combatterono coraggiofamente un buon pezzo; ma alla fine non potendo foltenere il fuoco degli fchioppi, e l'impeto de' cavalli, fi ritraftero alla Città Gli Spagnuoli, perchè era tardi, s'alloggiarono in una gran cafa del fobborgo. Il giorno feguente appicciarono fuoco: i Tlafcallefi a molte cafe della Città, e ne fei giorni, che vi ftettero gli Spagnuoli, ebbero continue zuffe, e vi furono alcuni duelli famofi tra i Tlalcallefi, ed i Tlacopanefi; ma combatterono gli uni e gli altri con fingolar bravura, e sfogarono: l'odio, che fcambievolmente $f$ portavano in mille obbrobrj. I Tlacopanefi appellavano. i Tlafcallefi damigelle degli Spagnuoli, fenza la protezione de'quali non avrebbono mai avuto ardire d'innoltrarfi fino a quella Città. I Tlafcalleti: a vicenda lor rifpondevano, che a' Mefficani piutrolto ed a tutti i lor partigiani fi doveva il titolo di donne, poichè effendo tanto fuperiori di numero, a' Tlafcallefi non aveano potuto mai foggiogarli . Non furono pure efenti gli Spagnuoli da sì fatti infulti . Gl' invitarono per burla ad entrare: in Meffico per comandare ivi da Signori, e per goder di tutti i piaceri: della vita . ". Vi pare, Criftiano, di"cevano a Cortès, che andranno adeffo le cofe, come l'al3. tra volta?: Penfate forle, che regni in Meffico un altro 9. Motezuma, facrificato alle voltre voglie?. Entrate pure, 9. entrate nella Corte, dove fi farà di tutti voi un gran fa". crifizio a' nottri Dei. "Nelle zuffe, ch"ebbero in quelti giorni gli Spagnuoli, entrarono in quella fatale ftrada, e 
s' accortarono a que' memorabili fofi, ne' quali nove mefi innanzi erano ftati fconfitti. Vi trovarono una terribile re- Lri: X. fítenza, e tutti in una volta furono per perire; perchè impegnati nel perfeguitare certe truppe mefficane, ch'eran venuce a bella potta ad infultarli per tirarli al pericolo fi trovarono improvvifamente affaliti dall' una e dall'altra banda della ftrada da un sì gran numero di nemici, che a ftento retrocedettero, combattendo furiofamente fin' a terra ferma. In quefto conflitto furono uccifi cinque Spagnuoli, e molti feriti. De' Meflicani reftarono molti morti in quelta, e nell'altre pugne. Cortès, difgultato per la mala riufcita della fua fpedizione, ritornò col fuo efercito per la medefima ftra- da a Tezcuco, ricevendo nella fua marcia nuovi infulti da' nemiei, i quali afcrivevano la fua ritirata a paura, e codardia. (g) I Tlafcallefi, che accompagnarono gli Spagnuoli in quella fpedizione, avendo ammaifato una gran fomma di fpoglie domandarono permeffo a Cortès di portarle alla loro patria, e Cortès l'accordò volentieri. (b)

Z 2

San-

(g) Solis volendo fmentir Bernal Diaz dice così "Checchè dica il noftro ;. Storico per ifcreditare quefta fpedizione (di Tlacopan) fu defla si im" portante al fine principale, che appena ritornato Cortès a Tezcuco, vi "vennero fupplichevoli a dar l'ubbidienza i Cazicchi di Tucapan, di Ma", rcalzingn, e d'Auhtlan ( cosi appella Tuzapan, Mexcaltzinco e Nauh, tlan) ed altri Popoli della riva fettentrionale: ciò che da a conofcere, ," che gli Spagnuoli ritornarono con riputazione \&c. ", Ma diffimulando I'efpreffione ambigua di riva fettentrionale, la quale i "Lettori non pratici della geografia di quiel Regno intenderanno forre della riva del lago, dovendo intenderla di quella del mare,e l'errore che evvi nel direchevennero Tezcuco i Signori di que'luoghi, laddove fappiamo per la teftimonianza di Cortès, che vi mandarono i loro ambarciatori, egli '̀ certo, che que' Signori non poterono indurfi a mandarvi tal ambafciata dalla nuova di ciè ch'era avvenuto a Tlacopan, perchè i loro Ambalciatori arrivarono a'Tezcuco quattro giorni dopo quella rpedizione, ficcome afferma Bernal Diaz, teftimonio oculato, e le loro Città erano diftanti da quella Corte più d’ soo. miglia.

(h) Herrera Torquemada dicono, che Cortès fece togliere violente. mente a' Tlafcallefi gli ornamenti d'oro, di cui andavano fregiati dopo la fpedizione di Tlacopan, e che $i$ Tlarcallefi furono talmento fdegnati di una tal indegnità, che in due giorni ne difertarono più di venti mila. Se vio folfe tato vero, Cortès farebbe ftato l'uomo più rciocco ed impruden. 


\section{0}

Sandoval, il quale nell' affenza di Cortès avea avuto LiB. X.cura di quella piazza, ufci da effa due giorni dopo l'arrivo di quel Generale con venti cavalli, trecento pedoni Spa5. 7. gnuoli, ed un gran numero d'Alleati per andare a foccors Spedizio-rere 'i Chalchefi, i quali temevano un grande affalto da' MefnidiSan-ficani; ma avendo trovato in Chalco un gran numero di tra Huax-truppe di Huexotzinco, e di Quauhquechollan, ch' erano vetepec, e nute in loro ajuto, e fapendo, che il maggior danno fi faJacapich- ceva a quella Città da' Mefficani, ch' erano nel prefidio di Huaxtepec, Citsà fituata fu'monti quindici miglia a Mezzodi da Chalco, s'incamminò colà. Nella lor marcia furono affaliti da due groffi corpi di nemici, ma prontameote gli fconfiffero, ciò che in gran parte fi dovette all' innumerabile moltitudine d'Alleati, che feco loro conducevano gli Spagnuoli. Entrarono coftoro in Huaxtepec, ed alloggiarono in certe cafe grandi per ripolare, e curare i feriti; ma immodiatamente ebbero un nuovo affalto da' Meflicani, che lico. ftrinfe a riprendere l'armi per rifpingerli, ficcome fecero, perfeguitandoli per pì̀ di tre miglia fin' a lafciarli affatto fconfitti. Indi ritornarono alla Cirtà, dove ripofarono due giorni. Era allora Huaxtepec Città celebre non meno per le fue eccellenti manifatture di cotone, che pel fuo mara. vigliofo giardino, di cusi abbiam fatto già menzione.

$D_{a}$ Huaxtepec mandò Sandoval de' meffaggieri ad offerir la pace agl'abitatori di Jacapichrla, luogo fortiffimo diftante fei miglia, fituato nella cima d'un monte quafi inacceffibile alla cavalleria, e difefo da una competente guernigione di Meflicani; ma effendo ftate le fue propofizioni da loro ributtate, marcid verfo quella Città determinato di darvi un colpo, che rintuzzaffe il loro orgoglio, e liberaffe

per-:

te, e quella medefima avarizia, che fece perir tanti Spagnuoli nella loro urcita da Meffico, avrebbe fraftornato l'imprefa della Conquilta; ma il racconto di quegli Storici è affatto contrario a cid, che dicono Cortès e Bernal Diaz teftimonj oculati, e Gomara Autore antico. Tutti e tre concordemente affermano, che iTlafcallefi domandarono licenza d'andare a Tlafcalla, che Cortès l' accordò volentieri, e ch'eglino portarono moito allegri il bottino da loro fatto in quella feedizione. 
perpetuamente i Chalchefi dal male, che lor veniva da quella parte. I Tlafcallefi e gli altri Alleati s'impaurirono a vi- Lrz. $X$. fta della difficclià e del pericolo: ma Sandoval, animato da quel gran coraggio, che fpiccava in tutte le fue azioni, fo rifolvette a morire o vincere. Cominciò a falire colla fua infanteria, dovendo nello fteffo tempo fuperare e l' afprezza del monte, e la moltitudine di nemici, che lo difendevano con un nembo di dardi, di fafli, ed anche di pietre fmifurate, le quali benchè fi rompeffero nell' urtar che facevano nelle rupi frappofte, i loro frammenti ferivano gli Spagnuoli; ma nulla fu capace di trattenerli fin'ad entrar nella Cittá, bagnati di fudore, e di fangue, ed a loro efempio altrettanto fecero gli Alleati. La fatica e le ferite infiammarono talmente il loro fdegno, e con tal furia fi fagliarono contro i nemici, che molti fuggendo dalle fpade fi precipitarono per le balze del monte. Tanto fu il fangue, che vi fi fparfe, che tinfe un rufcello, che vi correva, ed imbrattò talmente le fue acque, che in più d' un'ora non fe ne poterono fervire i vincitori per appagar la gran fete, che gli travagliava. (i) , Fu quelta, dice Cortès, una delle più fegnalate vittorie, ") nella quale diedero gli Spagnuoli le maggiori pruove del "loro coraggio, e della loro coftanza . "Quefta giornata coftd la vita a Gonzalo Dominguez, uno de' più bravi Sol: dati di Cortès, la cui perdita fu affai fenfibile a tutti . Irritati i Mefficani colla ftrage di Jacapichtla (*) armarono

(i) Bernal Diaz fi beffa di Gomara, per quefta narrazione dell' acque intorbidate dal fangue, e foggiunge, che non avevano bifognodi bere quell" acqua, mentre $v^{\prime}$ erano alcune forgenti di buon acqua; ma le quefe forgenti fi trovavano nello fteffo luogo della zuffa, è da crederfi", che reftarfero ancor effe tinte di fangue; s'erano diftanti da quel luogo, non erano gli Spagnuoli in iftato di cercarle. Bernal Diaz non fi trovo in quella fpedizione, e noi dobbiamo dar maggior fede al ragguaglio di Cortès : ", $F u$ " sì grande, dice coftui, il macello, che i noftri Spagnuoli fecero de' ne" mici, e tal la ftrage che $\mathbf{i}$ nemici fecero di loro medefimi, precipitando"fi da quella cima, che tutti coloro, che vi fi trovarono, affermano, che " un ruf́cello, che circondava quáfi tutto quel luogo, reftò tinto di fangue ", per più d'un ora: ficchè non ne poterono bere.,

(*) Herrera, e Solìs alterando il nome di quella C̈ittà, l'appellano Capiflan. 


\section{2}

rono prontamente venti mila uomini, e gli mandarono in LIB. X.due mila barche contra Chalco. I Chalchefi implorarono, come altre volte, l' ajuto degli Spagnuoli, ed i loro meffaggieri giunfero, allorchè rizornava da Jacapichtla col fuo efercito Sandoval, fiacco, malconcio, e ferito. Cortès, afcrivendo con troppa leggerezza le replicate oftilità de' Mefficani contro $i$ Chalchefi a trafcuraggine di quell' impareggiabile Comandante, fenza voler prima informarfi della condotta di lui, nè fentirlo, nè permettergli un momento di ripolo, gli comandò di marciar fubito a Chalco con que' Soldati, ch' erano men feriti, per dare ajuto a quegli Alleati. Rincrebbe fommamente a Sandoval quello fmacco fattogli dal fuo Generale, allora quando da lui avrebbe dovuto aifettarfi le più gran lodi; ma fu tanta la fua prudenza nel diffimular l' ingiuria, e tanta la fua prontezza nell' ubbidire, quanto era tato il fuo coraggio in quell ardua fpedizione. Parti fenza indugio a Chalco; ma quando $v$ arrivò, trovo già finita la battaglia, nella quale reftarono vittoriofi i Chal. chefi coll'ajuto de'loro nuovi Alleati gli Huexotzinchi, ed i Quauhquechollefi; e febbene ebbero una perdita confiderabile, uccilero pure molti nemici, e fecero quaranta prigionieri, e fra effi un General d'efercito, e due Perfonaggj della prima Nobiltà, $i$ quali furono da' Chalchefi confegnati a Sandoval, e da quefti a Cortès. Quefto Generale ravvedutofi del fuo fallo, e bene informato dell' irreprenfibil condotta di Sandoval, procurò placare il giufto di lui rifentimento con fingolari dimoftrazioni di ftima, e d'onore.

Volendo poi Cortes far qualche accomodamento co Mefs. 8. ficani, così per ifchivar le fatiche ed i difagj della guerra, Negozia-come per poter impadronirfi di sì belle Cirtà fenza rovinarzione in- le, rifolvette di mandare a Meffico que' due Perfonaggj pridi Cortès gionieri con una lettera al Re Quauhtemotzin, la quale nella Cor benchè non poteffe effere intefa in quella Corte, perchè ne te di Mef-ignoravano affatto $i$ caratteri, era pur credenziale, ed un
fico. contraffegno della loro ambafciata. Efpofe a que' meffaggieri il contenuto della lettera, e gl'incaricò di rapprefentare al 
loro Sovrano, ch' egli non altro pretendeva, fe non che il Re di Spagna foffe riconofciuto. Signor di quella terra, giu-Lis. Xfta ciò che era: Atato accordato dalla Nobiltà Mefficana in quella rifpettabile affemblea, che fi tenne in Meffico innanzi al $\operatorname{Re}$ Motezuma: che fi ricordaffero dell' omaggio, che allora. fecero tutti i Signori Mefficani al gran: Monarca dell' Oriente: che voleva ftabilire una pace ferma, ed una eterna alleanza con loro, e non faceva la guerra, fe non cofretto dalle loro oftilità: che gli rincrefceva di dover fpargere tanto fangue mefficano, e diftruggere sì grandi e belle Cirsà: ch' eglino: medefimi erano teftimonj della bravura degli Spagnuoli, della fuperiorità delle loro armi, della molo titudine de' loro Alleati, e della felicità de loro progreffi: che fi ravvedeffero finalmente, e non l'obbligaffero colla loro oftinazione a continuar la guerra fin'alla total rovina della Corte, e dell' Imperio.

Il frutto di quefta ambafciata fi riconobbe fubito ne"lamenti de"Chalchefi, i quali confapevoli delle grandi forze, che fi radunavano contra quello Stato, vennero ad implorar l'ajuto degli Spagnuoli, prefentando a Cortès dipinte in una tela le Cista, che s'armavano per ordine del Re di Meffico contra loro, e la ftrada, che doveano fare. Frat-. tanto che Cortès alleftiva le fue truppe per quella fpedizione, arrivarono a Tezcuco i Meffaggieri di Tuzapan, di Mexcaltzinco, e di Nauhtlan, Città fituate nella cofta del feno Mefficano di là dalla Colonia della Veracroce, a dare a no me de'lor Signori l'ubbidienza: al Re di Spagna.

A' 5. Aprile ufcì Cortès da Tezcuco con trenta cavalli: trecento pedoni. Spagnuoli, e venti mila Alleati, lafciando gnuolo Sandoval il comando di quella piazza, e la cura de'brigantini montaAndò a dirittura a Tlalmanalco, ed indi a Chimalhuacan, $\left({ }^{* *}\right)$ ridionalia. do.

$\left(^{* *}\right)$ Erano, e vi fono ancora due luoghi di quefto nome $: 1$ ' uno, fituato fulla fponda del lago Tezcucano nel principio della penifola d'Iztapalapan è appellato femplicemente. Chimalbuacan; l' altro pofto nelle montagne che fono a mezzodi della Valle Méficana s'appella Cbimalbuacan-Cbalco, e quefo fu quello, ore andò Cortès. 


\section{4}

dove s'ingrofsò il fuo efercito con altri venti mila e più uo-

LIB,X. mini, (l) i quali o per vendicarfi de'Mefficani, o per l' intereffe delle fpoglie, o com'io credo per l'uno e per l'altro venivano da parecch; luoghi per fervire in quella guerra. Quindi tenendo ognora, com'è da crederfi, quella ftrada, che rapprefentarono nella loro pittura i Chalchef, s'incamminarono per le montagne meridionali verfo Huaxtepec, videro preffo alla frada un monte affai fcofcelo, la cui cima era occupata da una gran moltitudine di donne $e$ di fan. ciulli, e la falda da moltifimi guerrieri, i quali confidati nella natural fortezza di quel luogo, fi beffarono con urli e fifchj degli Spagnuoli. Cortès, non potendo comportar quella beffa, fece dar un affalto da tre bande al monte; ma appena erano cominciati a falire a grande ftento fra una tempefta di dardi e di faffi, che chiamò alla ritirata; perchè oltrechè s'avvide, che l'imprefa era temeraria, e pià difficile, che fruttuofa, fi lafciò veder un efercito di nemici, che marciava verfo quella parte coll' intento d' affalire alle fpalle gli Spagnuoli, allorchè foffero più impegnati nell' affalto. Cortès venne loro incontro colle fue truppe bene ordinate. La battaglia durò poco, perchè i nemici riconofcendofi inferiori di forze, abbandonarono tolto il campo. Gli Spagnuoli gl'infeguirono per più d'un'ora e mezza fin' a fconfiggerli del tutto. La perdita degli Spagnuoli in quefta battaglia fu quafi un nulla; ma nell' affalto del monte furono otto di loro uccifi, e molti feriti- $(m)$

La fete, che travagliava quivi l'efercito, e l'avvifo, ch'ebbe Cortès d'un altro monte tre miglia diltante, e fimilmente occupato da'nemici, lo coltrinfero a marciare verfo

(I) Cortès dice, che in Chimalhuacan gli s'aggiunfero più di 40.000. nomini, e Bernal Diaz afferma, che furono più di venti mila; ma queftinumera foltanto quelli, che vi fi aggregarono di nuovo, e Cortès ef́prime la fomma totale rifultante da quelli, che feco conduffe da Tezcuco, é da quelli, che gli s'aggregarono in Chimalhuacan .

(m) Cortès nelle fue lettere non numera più di due Spagnuoli uccifi nell: afralto di quel monte; ma Bernal Diaz fa menzione d'otto, sapporta $i$ sero nomi. 
fo quella parte. Offervd in una colta del monte due cava. lieri o fra rupi dominanti, difefe da molti guerrieri; ma Lis. X. coftoro credendo, che gli Spagnuoli intentafiero l'affilto dalla banda oppofta, abbandonarono le rupi, e la accorfero, .dove maggiore pareva il pericolo. Cortès, come quegli che fapeva ben approfittarfi di tutte le congiunture, che gli prefentava la forte, o l'inavvedulezza de' fuoi nemici, ordinò ad uno de'fuoi Capitani di procurar d'occupare con un competente numero di foldati qualcuna di quelle due supi, mentre che egli tratteneva per la banda oppofta gli aflediati. Cominció dunque a falire con fomma fatica; ma quando arrivò ad un pofto tanco alto, quanto era quello, dov' erano i nemici, vide inalberata la bandiera Spagnuola in una delle rupi o cavalieri. I nemici, trovandoli da due parti affaliti, ed avendo gia cominciato a fentire il danno, che lor facevano l'armi da fuoco, s'arrendettero. Cortès gli accolfe con fingolare umanità; ma richiefe da loro, come condizione neceffaria per ottenere il perdono, che induceffero ad arrenderfi parimente coloro, che occupavano il primo monte, ficcome in fatti avvenne.

Libero omai Cortès da quelti impaccj, s' incamminò per. Huaxtepec, Jauhtepec, e Xiuhtepec alla grande ed amena Città di Quauhnahuac, ( ${ }^{*}$ ) Capitale della Nazion Tlahuica, diftante più di trenta miglia da Meffico verlo mez. zogiorno. Era quelta Città affai forte per la fua fituazione; poichè da una banda circondata da montague dirupare, e dall'altra da una cava protoada fette pertiche in circa, dove fcorreva un rufcello. Non vi potea entrar la cavalleria, fe non per due Atrade ignorate allora dagli Spagnuoli, o per li ponti, fe non folfero ftati levati, allorchè effi vi comparStoria Antica del Meffico Tom. III. A a vero.

(*) Il no:ne Quatihnahuac è uno de' più alterati dagli Spagnuoli. Cortès appella quefta Città Coadnabaced, Bernal Diaz Coadalbaca, Solis Quatlabaca \&c. Prevalfe poi quello di Cuernabaca, col quale è prefentemente conofciuta dagli Spagnuoli; ma gl' Indiani ritengono l'antico Quauhnahuac. E.' deffa uno de' irenta luoghi, che diede Carlo V. a Corrès, ed oggidi è parte degli Stati del Signor Duca di Monteleon, come Marchefe della Valle d'Oaxaca, 
vero. Mentre coftoro cercavano un luogo opportuno, dove L18. Xo ifar l'affalto, i Quauhnahuachefi tiravano contra loro un indicibile quantità di frecce, di dardi, e di faffi. $\mathrm{Ma}$ avendo offervato un coraggiofo Tlalcallefe, che due alberi grandi, che prendevano l'origine dalle due bande oppofte della cava, piegandofi l'uno verfo l'altro, aveano incrocicchiati ed intrecciati fcambievolmente i loro rami, fece d'effi un ponte per paffare all'altra banda, $l$ ' efempio del quale fu tolto imitato, quantunque a grande ftento, e con gran pericolo, da fei foldati Spagnuoli, e poi da molti altri tanto Spagnuoli quanto Tlafcallefi $(n)$ una sì fatta intrepidezza impauri talmente coloro, che da quella parte difendevano l'entrata nella Città, che fubito fi ritirarono, ed andarono ad unirfi agli altri Cittadini, che per l'altra parte della Citta s'opponevano alle truppe condotte da Cortès, ma allorchè erano più impegnati nella difefa, fi trovarono improvvifamente affaliti da quelle truppe, che dietro a quel coraggiofo Tlafcallef́e erano già entrate per la parte indifefa nella cirtà. Lo fpavento ritraffe i Cittadini dalla difefa, e gli mife in fuga pre. cipitofa verfo le montagne: ficchè gli Alleati abbruciarono fenza verun contrafto una buona parte della Città. Il Signor d'effa il qual era fuggito cogli altri, temendo d'effer rag. giunto nelle montagne dagli Spagnuoli, prefe il configlio d'arrenderfi, proteltando che non l'avea fatto innanzi, per. chè alpettava, che lo fdegno degli Spagnuoli it foffe sfogato nella Città; e foddisfatto con altre oftilità, s'afteneffe d'incrudelire contra la fua perfona.

Poichè ebbe ripofato l'efercito parì da Quauhnahuac carico di fpoglie verfo Tramontana per un gran bofco di pini,

(n) Solis fenza far menzione di quel Tlafcallefe, attribuifce tutta la gloria di quell'azione a Bernal Diaz, nel che contraddice a Cortès, ed agli altri Storici. Lo fteffo Bernal Diaz, il quale nella narrazione di quefto avvenimento fi fa tutto l'onore, che può, fi vanta bensì d' effere ftato uno di coloro, che non curando il pericolo della vita, paflarono fugli alberi della cava; ma non fi dà la gloria d'effere ftato il primo nel paffaré, nè d'aver fuggerito il configlio. Vedafi ciò, che ne dicono Cortès, Gomara, Herrera \& $c_{0}$ 
pini, deve pati una gran fete, ed il di feguente fi trovarono preffo alla Città di Xochimilco. Quefta bella Città, la Liz. X. più grande dopo le Corti di tutte quelle della Valle mefficana, era fondara fulla faonda del lago di Chalco, poco più di dodici miglia dalla Capitale. Il fuo popolo era affai numerolo, i fuoi tempj erano molti, i fuoi edifizj magnifrci, Xochie fingolarmente belli $\mathrm{i}$ Cuoi giardini galleggianti nel lago, ond'ebbe il nome di Xochimilco. $\left(^{*}\right)$ Avea a guifa della Capitale molti canali, o foffi, ed ora per paura degli Spagnuoli aveano fatte molte trinciere. Tofto che videro venir l'efercito, levarono i ponti da' canali per rendergli più difficile l'entrata. Gli Spagnuoli divifero l'efercito in tre fquadroni per affalire da altrettance bande la Città; ma da per tutto trovarono una gran refiftenza, e non poterono efpugnare il primo fofo, fe non dopo un terribile combattimento di più di mezz'ora, nel quale furono uccifi due Spagnuo. li, e molti feriti; ma fuperati alla fioe quefti oftacolis, en. trarono nella Città, incalzando i Xochimilchefi: i quali dalle barche in cui s'erano ricoverati, perfeverarono combattendo infin'alla notte. Sentivanfi alle volte in fra i combattenti alcune voci, che domandavano la pace, ma accortifi gli Spagnuoli, che si fatte voci non ad altro s'indirizzavano, che a guadagnar tempo per mettere in ficuro le loro famiglie, e la loro roba, e per ricevere il foccorfo, che afpertavano da Meffico, gli ftrinfero più, finattantochè non trovando: refiftenza, fi ritirarono per ripofare, e curare $i$ riti; ma appena cominciavano a refpirare, che fi videro affaliti da un gran numero di nemici, che vennero formati in ordine di battaglia per quella iteffa ftrada per dov' erano en. trati gli Spagnuoli. Coftoro furono ridotti al maggiore ftretto, e lo fteffo Cortès corfe gran pericolo di reftar prigione de'nemici; imperocchè effendo caduto di ftanchezza il fuo cavallo, ficcom' egli dice, o meffo in terra a colpi de' Xochimilcheli, come affermano altri Storici, continuò combarten.

$$
\text { A } a_{2} \text { do }
$$

ง. Ir. Conquifta di milco. 


\section{8}

- do a piedi colla fua lancia; ma fopraffatto da' nemici non LiB. X. avrebbe potuto evitar la fua rovina, fe un prode Tlafcallefe, $(0)$ e dietro a lui due fervitori del medefimo Cortès, ed altri Soldati Spagnuoli non foffero veruti opportunamente in fuo ajuto. Sconfitti finalmente i Xochimilchsti, ebbero gli Spagnuoli l'agio di ripofare alquanco dalle fatiche della giornata, nella quale furono uccifi alcuni de'lor Soldati, e quafi tutti feriti, ed il medefimo lor Generale, ed i principali Capi, Alvarado, ed Olid. Quattro Spagnuoli fatti prigionieri furono condotti alla Capitale, e fenza indugio facrifcati, e le loro braccia e le gambe mandate in parecchj luo. ghi per incoraggire i fudditi contro i nemici dello Stato. E' fuor di dubbio, che tanio in quelta, quanto in altre molte occafioni potè Cortès effere facilmente ammazzato da' fuoi nemici, fe coltoro non aveffero avuto quella fciocca premura di pigliarlo vivo per facrificarlo a' loro Dei.

La nuova della prefa di Xochimilco mife in gran cofternazione la Corte di Meffico. Il Re Quauhtemotzin convocò alcuni Capi militari, e lor rapprefentò il danno ed il pericolo cagionati a Meffico dalla perdita d'una piazza tanto riguardevale, il fervizio che farebbono agli Dei, ed alla Nazione nel recuperarla, ed il coraggio, e le forze, che vi bifognavano per vincere quegli arditi e perniziofi ftranieri. Diedefi però immediatamente l'ordine d'armare un efercito di dodici mila uornini da mandar per terra, ed un altro da mandar per acqua, e s'efeguì con tâl prontezza, che appena aveano ripofato gli Spagnuoli dalla fatica del giorno innanzi, quando fu avvifato Cortès dalle fue fentinelle della marcia

(o) Herrera, e Torquemada dicono, che il di vegnente dopo il pericolo, in cui fi trovò Cortès, avendo cercato il Tlafcallefe, che gli avea dato ajuto, non fi potè trovare nè vivo, nè morto: onde per la divozione, che quel Generale portava a S. Pietro, fi perfuadette, che quefto Santo A poftolo forfe ftato colui, che l'a peva fcampato. Non fo, donde abbiano prefo quefti Autori sì fatro aneddoto; poichè Berna! Diaz, e Gomara, e quel ch'è più, il medefino Cortès affermano, che colui che venne a favorirlo fu un Tlafcallefe fenza far veruna menzione di quel Santo, nè dell' erése poi fparito quell' uomo. 
cia de' Mefficani verfo quella Città. Divife quefto Generale il fuo efercito in tre fchiere, e diede a' fuoi Capitani gli ordini Lis. $X$. più opportuni: lafciò qualche truppa di guernigione nel quartiere, e comando, che venti cavalli con cinquecento Tlafcallefi paffaffero a traverfo i nemici ad occupare un vicino monticeilo, e quivi afpertaffero i fuoi ulteriori ordini per affalire. I Comandanti Mefficani venivano pieni d'orgoglio, ed oftentando certe fpade europee prefe gia agli Spagnuoli nella fconfitta del I. Luglio. La battaglia fi diede fuor della Città, ed allorchè parve bene a Coriès ordinò, che la truppa polta ful monticelio affaliffe alle fpalle i Mefficani. Coftoro, vedendofi da per tutto attaccati, fi fcompigliarono, e fuggirono, lafciando nel campo cinquecento morti. Gli Spagnuoli ritornati al quartiere feppero, che la truppa ivi rimalta era ftata in gran pericolo a cagione della moltitudine de' Xochimilchefi che l'a veano combattuta. Cortès, dopo d'effere ftato tre giorni in Xochimilco in frequenti combattimenti co' nemici, fece appicciar fuoco a' tempj ed alle cafe, ed andò al mercato, il qual' era fuori della Città, per ordinar la fua gente per la marcia . I Xnchimilchefi, perfuadendofi, che la loro partenza forfe effetto della paura, attaccarono con gran clamori la retroguardia; ma furono talmente battuti dagli Spagnuoli, che non ofarono più affalirli.

Avanzoffi Cortès col fuo efercito infin' a Cojohuacan, s. $\mathbf{x}$. Città grande fituata nella riva del lago, fei miglia cliftante Marcia da Meffico verfo Mezzogiorno, coll' intenzione d'offervar degli tutti que' polti per meglio difporre l'affedio della Capitale. li attorTrovò la Città fpopolata, ed il giorno feguente n'ufci per no a'lariconofcere la ftrada, che conduceva da quella Città infin' al- ghi fin'a la Itrada d'Iztapalapan. Vi trovò una trincea fatta da' Mefficani, e diede ordine all' infanteria d'attaccarla, e malgrado della rerribile refiftenza de'nemici, che la difendevano, l'efpugnarono pure, reftando feriti dieci Spagnuoli, e morti alcuni Mefficani. Salito Cortès fulla trincea, vide la Arada d'Iztapalapan ingombrata da una moltitudine innumerabile di nemici, ed il lago da alcune migliaja di barche, e dopo aver offer- 
Offervato tutto ciò, che al fuo difegno fi confaceva, ritornò L18. X. alla Citta, a' cui tempj e cafe fece appicciare il fuoco. Da Cojohuacan, marciò l'eferciro a Tlacopan, effendo travagliato nel cammino da alcune trupps volanti di nemici, che affalirono il bagaglio. In una di quefte zuffe, in cui corfe gran pericolo Cortès, gli fecero prigionieri due fuoi fervitori, i quali condotti a Meffico furono incontanente facrificati. Arrivò Cortès a Tlacopan affitto per tal difgrazia; ma gli fi accrebbe il difpiacere allorchè dall' atrio fuperiore del maggior tempio di quella Corte contemplò infieme con altri Spagnuoli quella fatale ftrada, nella quale alcuri mefí innanzi avea perduti tanti fuoi amici e foldati, e conliderò attentamente le gran difficoltà, ch' era d' uopo fuperare per renderfi padrone della Capitale. Alcuni gli fuggerivano, che mandafie per quella itrada le fue truppe a far qualche oftilità a' Mefficani; ma egli non: volle efporle a sì gran rif́chio, onde fenza trattenerfi più in quella Città ritornò per Tenajoccan, Quauhticlan, Citlaltepec, ed Acolman a Tezcuco, dopo aver girato in quelto viaggio attorno a tutti i laghi della valle mefficana, ed offervato quanto facea di meftieri per efeguire felicemente la grand'imprefa, che meditava.

5. $1 \xi_{0}$. In Tezcuco continuò Cortès tutti i preparativi per l'afracontra fedio. Erano già accomodati i brigantini, terminato un caCortès. nale lungo un miglio e mezzo, fufficientemente: profondo, e dall' una e da!l' altra banda fornito di ftecche per ricevere l'acqua del lago, nella quale dovevano gittarfi i brigantini, e fabbricara una macchina per gettarlio.(p) Le truppe, che avea Cortès fotto i fuoi ordini erano innumerabili, ed anche il numero degli Spagnuoli s' era confiderabilmente ac-

cre-

(p) Gomara dice, che in quel canale lavorarono quattrocento mila uomini della Corte e del Regno di Tezcuco; prichè ne' cinquanta giorni, che fi lavorò in quel canale, ogni giorno s'impiegavano otto mila Operajnuovi. Aggiunge, che il fuddetto canale avea mezza lega di lunghezza I2. piedi di larghezza, e dove meno, quattro braccia fpagnuole di profondità; ma io credo, che vi fia qualche sbaglio intorno alla larghezza, e non dubito, che fra frata di piu di r 2 . piedi. 
crefciuto con quelli, che pochi giorni innanzi erano venuti di Spagna in un vafcello che approdò al porto della Vera Lis. X. croce carico di cavalli, d'armi, e di munizioni da guerra. Tutto pareva tendere ad un efito felice, allorchè fu l'imprefa nel maggior pericolo di rovinarfi. Certi Soldati Spagnuoli, partigiani del Governator di Cuba, moffi dall' odio di Cortès, o dalla invidia della fua gloria, o ciò che pare più verifimile, dalla paura de' periglj, che lor fopraftavano nell' afiedio della Capitale, s' accordarono fegretamente di tor la vita a Cortès, a' fuoi Capitani Alvarado, Sandoval, e Tapia, ed a tutti quelli, che vedevano più attaccati al partito di quel Generale. Aveano già i congiurati non che determinato il tempo e la maniera d'efeguir con ficurezza il colpo, ma eletto anche coloro, a'quali doveano conferirfi le cariche vacanti di Generale, di Giudice, e di Capitani; ma uno de' complici, pentito del fuo misfatto, rivelò opportunamente a Cortès il tradimento. Quefto Generale fece immediatamente pigliare Antonio di Villafaña, capo della congiura, commile ad un giudice l'efame del reo, ed avendo coftui confeffato Ichiettamente il cuo delitto, fu per giuftizia impiccato ad una finettra del quarriere. Intorno a' complici diffimulò prudentemente Cortès, facendo fembianza di ron crederli colpevoli, ed attribuendo alla malignità di Villafaña l' infamia, che dalla fua confeffione rifultava contra loro; ma acciocchè nell' avvenire non foffe tanto efpolta a si fatti pericoli la fua vita, creò una guardia del corpo compofta di parecchj foldati, della cui fedeltà, e del cui coraggio era ben ficuro, i quali l'accompagnavano di di, e di notre, e badavano ognora alla confervazione della fua perfona.

Impedita dusque col gaftigo del principal reo quella perniziofa congiura, s'applicò Cortès con maggiore attività a dar l'ultima mano alla fua grande imprefa. Addi 28. A. preparaprile, poichè fu celebrata la Meffa dello Spiritofanco, nel- l'afredio la quale fi comunicarono tutti gli Spagnuoli, e che furono di Merda un Sacerdote benedetti i brigantini, fi gettarono quetti all'acqua, e fpiegando immediatamente le vele, comincia- 
rono a folcar pel lago collo fparo dell' artiglieria e degli Lis. X. Cchioppi, il quale fu feguito dal canto del $T_{e}$ Deum colla mufica degli ftrumenti militari. Turte quefte dimoltrazioni fi dovettero alla gran confidenza, che avea Cortès ne'brigantini, per la felicita della fua imprefa, fenza i quali forfe non avrebbe mai potuto condurla a buon fine. Fece poi la raffegna del fuo efercito, e vi trovò ottanta fei cavalli, e più d'ottocento pedoni Spagnuoli, tre grandi cannoni di ferro, quindici minori di rame, mille libbre caltigliane di pislvere da Chioppo, ed una gran quantità di palle, e di faette, effendof raddoppiato il numero e le forze del fuo piccolo elercito co' loccorfi venutigli quell' anno da Spagna, e dall'ifole Antille. Fece loro per incoraggirli un parlamento fimile a quello che avea gia fatto, allorchè ufc̀ di Tlaicalla. Mandò de'meffaggieri a quefta Repubblica, a Chololla, ad Huexotzinco, e ad altre Città, facendo loro fape: re, ch'era già terminata l'opera de'brigantini, e pregandole di mandargli infra dieci giorni quante truppe fcelte poteffero, per effere omai giunto il tempo di por l'affedio a quella fuperba Corte, che avea per tanti anni oppreffo la loro libertà. Cinque giorni avanti la fefta Ji Pentecolte arrivò a Tezcuco l'efercito di Tlafcalla, il quale conftava, fecondo che afferma lo fteffo Cortès, di più di cinquanta mila uomini forto il comando di parecchj capi famofi tra $i$

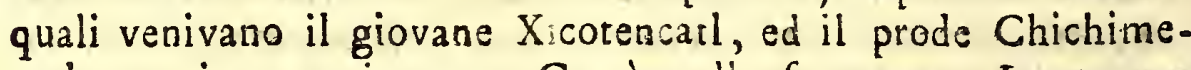
catl, a cui venne incontro Cortès colla fua gente. Le truppe d'Huexotzinco, e di Chololla paffarono colà per le montagne diChalco, giulta l'ordine loro dato. Ne' due giorni feguenti vennero altre truppe di Tlafcallan, e d'altri luoghi circonvicini, le quali infreme colle fuddette faceano più di dugento mila uomini, fecondo cho ne teftifica il loro Condotriere Alfonto d'Ojeda.

Il Lunedi di Pentecofte (20. Maggio) ragunò Cortès la fua gente nella piazza maggiore di qualla Corte per far la divifione dell' efercito, per nominare i Comandanii, per aflegnare ad ognuno di loro il luogo, dove formar dovea il 
fuo campo, e le truppe che doveano effere fotto lui, e per pubblicar di nuovo il bando militare pubblicato già in Tla. Lis. $\mathbf{X}$. fcalla. Ordinò a Pietro d'Alvarado di ftare a campo nella Città di Tlacopan, per impedire che entraffe da quella parte ${ }_{\text {:Dirpori. }}^{\text {rs. }}$ 5. Is. qualche foccorfo a' Mefficani, e gli affegno trenta cavalli, cen-zione delto feffantotto pedoni Spagnuoli, diftribuiti in tre compagnie forto altrettanti Capitani, e venticinque mila Tlafcallefi con due 'éercito nell' cannoni d' artiglieria. Criftofano d' Olid fu creato Maeftro di campo, e capo della divifione deftinata per la Città di Cojoaffedio huacan, e gli furono affegnati trentatre cavalli, cento feffantotto pedoni Spagnuoli, fotto altri tre Capitani con due cannoni, e più di venticinque mila Alleati. A Gonzalo di Sandoval furono dati ventiquattro cavalli, cento feffantatre pedoni Spagruoli fotto due Capitani con due cannoni, e gli Alleati di Chalco, d'Huexotzinco, e di Cholollan, i quali erano più di trenta mila, e gli ordinò Cortès d'andar prima a rovinare la Città d'Iztapalapan, ed indi metterí a campo in quel luogo, che a lui pareffe più confacevole al fine di Itrignere $\mathbf{i}$ Meflicani. Cortès, malgrado le rimoltranze fattegli da' fuoi Capitani e Soldati, prefe il comando de' brigantini, mentre ftimava più necaffaria in effi la fua affiftenza. Compartì ne' tredici brigantini trecento venti cinque Spagnuoli, e tredici falconetti, affegnando a ciafcun brigantino un Capitano, dodici Soldati, ed altrettanti rematori: ficchè tutto l'efercito deftinato a cominciar l'affedio della Capitale conftava di novecento diciaffette Spagnuoli, e più di fettantacinque mila uomini di truppe aufiliarie $(q)$ il cui numero indi a poco Storia Antica del Mefjeco Tom. III. B b s'ac-

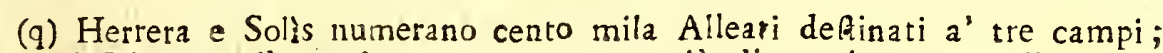
Bernal Diaz per l'oppolto non ne numera più di ventiquattro mila, otto mila per ciafcun campo. Noi diamo maggior fede a Cortès, come quegli che meglio d'ogni altro fapeva il numero di truppeaffegnate a ciafcun comandante. Solis dice, che Bernal Diaz fi lagna. fpeffe volte, che gli Alleati lor davano più impaccio, che ajuto; ma ciò è falfo, anzi fpeffe volte vanta il fuddetto Diaz il grand'ajuto, che avevano dagli Alleati, ed il loro coraggio nel combattere contro i Meflicani. I Tlafcallefi noftri amici, dice nel cap. 15I. ci ajutarono affai bene in tutta la guerra come nomini coraggiof. Tutta la fua Storia è piena di si fatte efprellioni, ficcome pu- 
s'accrebbe, come vedremo, infino a dugento mila e pià. TutLis. X. te l'altre truppe, ch' erano venute a Tezcuco, o rimafero là per impiegarfi, qualora foffe d'uopo, o ritornarono a' loro luoghi, meatre non erano tanto diftanti dalla Capitale, che non poteffero venire prontamente all'affedio, ogni volta che foffero chiamate.

5. 16. Partirono infieme da Tezcuco Olid, ed Alvarado colle Suppli- loro truppe per andare a' pofti loro affegnati dal Generale. zio di Xi- Tra i più riguardevoli Tlafcallefi, che accompagnavano Alcotencatl. varado, v' erano il giovane Xicotencatl, ed il fuo cugino pilteuctli. Quefti in una contefa, che avvenne, fu ferito da uno Spagnuolo, il quale non curando imprudentemente ǵli ordini intimati loro dal Generale, nè il rifpetto dovuto a quel Perfonaggio potè cagionare col fuo attentato la diferzione de' Tlafcallefi. Rincrebbe affai a coftoro l'oltraggio, e fecero palefe in alcune dimoftrazioni il loro fdegno. Procurò acchetargli Ojeda lor condottiere, e diede perneffo 2 Pilceucti d'andare a curarfi alla fua patria. Xicotencatl, a cui non meno pel fuo impiego, che pel fuo parentado era più che a verun altro fenfibile tal ingiuria, non trovando ailora altro modo di vendicarfi, abbandonò di nafcolto l'efercito, e prêfe infieme con altri Tlafcallefi la ftrada per la fua patria. Alvarado ne diede prontamente avvifo a Cortès, e coitui diede ordine ad Ojeda di raggiungerlo e prenderlo, e prefo che fu, lo fece impiccare pubblicamente o nella fteffa Città di Tezcuco, (r) ficcome dicono Herrera, e Tor: que-

re le lettere di Cortès, e la narrazione d' altri Storici. Bernal Diaz foltanto dice, che nelle lor ritirate per la Atrada di Tlacopan erano impacciati dalle truppe aufiliarie; ma qualunque truppa numerofa, che vuol far la fua ritirata per una ftrada ftretta, ha dell' impaccio nella felfa moltitudine .

(r) Cortès non fa menzione di quefto avvenimento di Xicotencatl: può crederfi, che aveffe qualche particolar ragione di tacerlo. Bernal Diaz afferma, che Xicotencatl andava a Tlafcalla per impadronirfi dello Stato di Chichimecatl, mentre che coftui era nella guerra; ma ciò è affatto inverifimile. Vi fono degli Autori, che dicono, ch' egli era portato a Tlafcalla dall' amor d'una Dama. Lo nel ragguaglio di quefto fucceffo tengo dietro a Torquemada, ed Herrera, perchè fcriffero fopra i manofcritti di Ojeda, e di Camargo, ch' erano affai bene informati. A Solis pare incre- 
quemada, 0 in un luogo ad effo vicino, come afferma Bernal Diaz, pubblicata prima da un banditore la cagione della Lis. $X$. fua condanna, ch'era quella d'aver difertato e d'aver follecitati i Tlafcallefi contro gli Spagnuoli. E'da crederfi, che Cortès non s'arrifchiaffe all' efecuzione d'un tal fupplizio feuz' aver prima otrenuto, ficcome efpreffamente afferma Herrera, il confenfo del Senato di Tlafcalla: ciò che non era difficile attefo la loro Severità nel punire $i$ delitti anche nelle perlone più colpicue, e l'odio particolare, che portavano a quel Principe, il cui orgoglio non potevano più foffrire. Un sì Atrepitolo gaftigo, che avrebbe dovuto naturalmente eccitar gli animi de Tlafcallefi contro gli Spagnuoli, impauri a tal legno ed effi, e gli altri Alleati, che da allora innanzi offervarono più puntualmente le leggi della milizia, e fi mantennero più fubordinati a que' Capi ltranieri traendo frutto gli Spagnuoli anche da'loro Iteffi falli; ma non temettero i Tlafcallefi di fare alcune dimoftrazioni di ftima e di vene. razione verfo il loro Principe, piangendo la fua morte, diftribuendo fra loro come pregevoli reliquie le fue vefti, e ce. lebrando, com'è da crederfa, colla dovuta magnificenza le fue efequie. La famiglia e la roba di Xicotencatl furono aggiudicate al Re di Spagna, e trafportate a Tezcuco. Nella famiglia erano trenta mogli, e tra la roba una gran quantita d' oro.

Alvarado ed Olid continuarono la loro marcia verfo Tlacopan, là onde paffarono a romper l'acquidotto di Cha. $B$ b 2

polte.

dibile, che Xicotencatl fia fato giuftiziato in Tezcuco: "perchè farebbe " ftato un tropp arrifchiarfi il rifolverfi Cortès ad una sì violenta efecu"zione fotto gli occhi d'un numero si grande di Tlafcailefi, a'quali do", veva neceflariamente rincrefcere quell' ignominiofo gaftigo fatto in uno "de' primi uomini della loro Nazione; "ma affai più s'arrifchiò Cortès nell'imprigionare il Re Motezuma nella fua fteffa Corte, e fotto gli occhj d'un numero inccinparabilmente più grande de'Mefficani, a'quali doveva fenz'altro rincrefcere quel grand' affronto fatto al primo uomo della lor Nazione. Se nella Conquifa di Meffico non foffero intervenuti altri fatti parimente temerarj, farebbe forfe efficace la ragione del Solis: oltre che Cortès non diede, per quel che dice Fierrera, la fentenza contro Xicotencatl fenza il confenfo del Senato di Tlafcalla, ed io non dubito, che a nome dello feffo Senato deffa fia ftata pubblicata. 
poliepec per levar l" acqua a' Mefficani ; ma non poterono efe-

Lis. $X$. guire una si importante oftilita fenza una gran refiftenza de nemici, $i$ quali, ancivedendo quelto colpo, aveano facto e

5. I7. per terra, e per acqua i loro preparativi per la difefa. Cofto-

Prime ro furono fconfitri, ed i Tlalcallefi nell'incalzarli uccifero oftitita venti di loro, e fecero fette ovvero otto prigionieri. Fatto degliSpa-s' felicemente quefto primo paffo, rifolvettero que' Comanprincipio danti d'andar per la Arada di Tlacopan ad efpugnare qualche dell'affe-foffo; ma fu sì grande la moltitudine de' Meffrcani, che Capitale. vennero contra loro, e sì folto il nembo di frecce, di dardi, e di faff, che contra loro tirarono, che uccifero otto Spagnuoli, e ne ferirono più di cinquanta, ed a ftento poterono coftoro ritira?fi vergognati a Tlacopan, dove $s^{\prime}$ accampò Alvarado giulta l'ordine del Generale, ed Olid marciò a Cojo. huacan nel di 30. Maggio confacrato quell' anno alla folennità del Corpus Domini, nel quale cominciò, fecondo il com. puto di Cortès, Y'affedio della Capirale.

Frattanto che Alvarado ed Olid s'occupavano nel riem: piere alcuni foffi, ch'erano nella riva del lago, ed acconciavano alcuni paffi per la comodita della cavalleria, il Comandante $\left(^{\star}\right)$ Sandoval col numero fopraddetto di Spagnuali, e con pi ù di trentacinque mila Alleati parti da Tezcuco il di 3 r. Maggio col propofito di prender per affalto la Città d'Iztapalapan, contra la quale era particolarmente impegnato Corrès. Entrovvi pure Sandoval, facendo una rerribile ftrage col fuoco nelle cafe, e coll armi negli abitanti, i quali impauriti procu. rarono fcampar la vira nelle barche. Cortes, per affalire nel medefimo tempo quella parte della Città, ch' era nell'acqua, dopo aver fatto fcandagliare tutto il lago, s' imbarcò colla fua gente ne'brigantini, e navigò a vela, ed a remo verfo Iztapajapan. Diede fondo preffo ad un monticello ifolato, poco difante da quella Città, la cui cima era occupata da molti ne-

$\left({ }^{*}\right)$ Solis dice, che marciarono infreme da Tezcuco Criftofano d'Olid, e Gonzalo di Sandioval; ma quefto è fato uno sbaglio di quell' Aurore, derendo dire Pietro d'Alvarado in rece di Gonzalo di Sandoval. 
nemici rifoluti a difenderf, e ad offendere gli Spagnuoli quatito lor fofle poffibile. $(\rho) \mathrm{Vi}$ sbarcò Cortès, e fuperando Lie. $\mathrm{X}$. con cencinquanta uomini l'afprezza, e la difficoltz della fa. lita, e la refiltenza de'nemici, efpugnò il monte coll' uccifione di quanti lo difendevano. ( $t$ ) Ma appena l'aveano efpugato, che videro vesir contra loro una gran flotta di barche (u), chiamate cogl' indizj del fumo che nel primo comparir de'brigantini li diedero tanto in que! monte, quan. to in alcuni tempj di que' contorni. Imbarcaronfi fubito gli Spagnuoli, e ftestero fenza muoverf fulla difefa, finattantochè fecondari da un vento gagliardo, che opportunamente fi levò, ed aumentando la velocità de' brigantini coll'impulfo de' remi s'avventarono contro le barche, rompendone alcune, ed altre ribaltandone coll'urto. Alcuni de' nemici perirono feriti dalle palle, e molti reltarono annegati. Tutte l'altre barche fuggirono perfeguitate per più d'otto miglia da' bri. gantini infin'alla Capitale .

Il Comandante Olid, tolto che vide da un tempio di Cojohuacan la zuffa de'brigantini, marcid colle fue truppe in ordine di battaglia per la trada, che conduceva a Meffico, efpugnò alcuni foffi, e trincee, ed uccife molti nemici. Cortès dalla fua parte raccolfe quella fera i fuoi brigantini,

(f) Nella cima di quel monticello fabbricò lo Storico Solis ura fortezza ben capace. Nico, ch'egli la fabbricò, perchè non v'è memoria prefo vesun altro Storico, che vi fra tata mai veruna fortezza nè grande, nè pico cola. Lo ftefto Cortès, il quale vanta la fua vittoria, non fa menzione, fe non delle trinciere, che vi erano.

(t) Solìs dice, che Cortès accordò la vita alla maggior parte di coloro, che difendevano if monticello del lago; ma lo fteffo Cortès afferma, che giuno di loro fcampd ta vita. Quefto monte per memoria della vittoria ottenuta da Cortès fu appellato d'allora in quà il Peñon del Marqués, cioè la rupe del Marchefe.

(u) Bernal Diaz dice, che la florta, che venne contra Cortès, era compofta di tutte le barche, che fi trovavano in Mefsico, ed in tutti i luoghi pofti ful lago, ma quefta è un'iperbole fgangherata. Solis afferma, chequella flotta confava di quattro mila cánoe; ma Cortès, il quale comandava i brigantini, ed avea maggior intereffe, che non Rernal Diaz, nè Solis nell' efagurare il numero delle barche nemiche per render piu famofa la fua vittoria foltanto dice, ch'elfe furono piu di cinquecento. 


\section{8}

ni, ed andò con effi a combattere il baluardo, che era fic. Li5. X. come abbiam già detto in quell'angolo, che formava la ftrada di Cojohuacan con quella d'Iztapalapan. Il combatiè per acqua e per terra, e malgrado della bravura, colla quale fi difele la guernigione mefficana che v'era, pure l'efpugnò, e con due grandi cannoni d'artiglieria fece una orrenda ftrage nella moltitudine, che occupava il lago e la ftrada. Quel luo. go appellato da' Mefficani Xoloc, parve affai vantaggiofo a Cortès $(x)$ per iftabilirvi il fuo campo, ed in fatti non era facile il trovarne un altro più confacevole $a^{\prime}$ fuoi difegni; poichè in effo fi rendeva padrone della principale ftrada, e di quella parte del lago, aove poteano entrare maggiori foc. corfi alla Città, ed oltracciò della ftrada di Cojohuacan per la comunicazione col campo d' Olid. La poca diftanza di quel luogo da' campi di Cojohuacan, e di Tlacopan importava affai a Cortès per dar prontamente i fuoi ordini, e per porgere ajuto, dovunque abbifognaffe. Finalmente la vicinanza a Meffico contribuiva ad agevolar gli affalti. ( $y$ )

Quivi ragunò $i$ brigantini, ed abbandonando la fpedizione contra Iztapalapan prefe la rifoluzione di dar tolto principio agli affalti. Fece però venire al fuo campo la metà celle truppe di Cojohuacan, e cinquanta pedoni fcelti delle truppe di Sandoval. Quella notte fi fenti venir verfo qual campo una gran folla di nemici. Gli Spagnuoli fapendo, che i Mefficani non folevano combattere la notte, fe non quando erano ficuri della vittoria, da principio s'impaurirono; ma febbene qualche danno ricevettero dai nemi-

$\mathrm{Ci}_{3}$

(x) $I$ P. Sahagun nella fua Storia M. S. della conquifta dice, che Cortès per mezzo di certi Perfonaggi fuoi prigionieri chiamó il Re e la Nobilta di Meffico, ad un luogo appella to Acacbinanco, e mette l'aringa, che fece efponendo loro i motivi della guerra; ma un tal abboccamento nè mi par vero, nè verifimile. Cortès, il quale racconta minutamente quanto egli diceva ai Mefficani, e quanto i Mefficani dicevano a lui, non avrebbe certamente tralafciato una cofa tanto notabile.

(y) Betaricurt dà ad intendere, che Cortès s'accampó dentro la Città ; ma ciò è affitto contrario al vero, ed al ragguaglio del medefimo Cortès, il quale afferma, che il cuo campo era lontano meza lega dalla Città. 
ci, pure gli costrinfero eol fuoco dell'artiglieria, e degli fchioppi a ritirarî alla Citra. Il di vegnente fi videro a liti da una prodigiola moltitudine di guerrieri, i quali con urli Spaventevoli aggrandivano il numero nell' immaginativa degli Spagnuoli. Cortès, effendogli opportunamente arrivato il foccorfo, che alpettava da Cojohuacan, fece una fortita colla fua gente meffa in ordine di battaglia. Si combattè dall' una e dall'altra parte con gran coraggio ed oftinazione; ma gli Spagnuoli, ed $\mathrm{i}$ loro Alleati efpugnarono un foffo, ed una trinciera, e coll' artiglierìa, e coi cavalli fecero tanto male ai Mefficani, che gli obbligarono a ricoverarfi nella Città; e perchè dalla parte del lago, che v'era a ponente della ftrada, erano incomodati dalle barche Meflicane, fece Cortès slargare un foffo della trada, acciocchè vi poteffero paffare i brigantini, i quali fi fcagliarono impetuofamente contra effe, le perfeguitarono infin'alla Città, ed attaccarono il fuoco ad alcune cale dei fobborghi .

Frattanto Sandoval terminata felicemente, benchè non fenza gran rifchio, la fpedizione d'Iztapalapan, marciò colle fue truppe verfo Cojohuacan. Nella ftrada fu affalito dalle truppe di Mexicaltzinco; ma le rconfiffe, e fece appicciar fuoco alla Città. Cortès, confapevole della fua marcia, e d'un gran foffo, ch'era ttato di frefco fatto nella Itrada, gli mandò due brigantini per agevolare il paffaggio dell'efercito. Quefto marciò verfo Cojohuacan, e Sandoval andò con dieci cavalli al campo di Cortès. Quando v'arrivò, trovò quegli Spagnuoli in combattimento coi Mefficani. La fatica del viaggio, e della battaglia di Mexicaltzinco non bafto a diftoglierlo dalla zuffa. Combattè pure col folito coraggio, ma nel combattere gli fu traffitta la gamba cun un dardo, e con effo lui furono anche feriti molti altri Spagnuoli. Quefti vantaggj dai Mefficani ottenuti non fono da paragonarfi colla perdita, ch'ebbero quel giorno, nè colla paura, che lor fece il fuoco dell'artiglieria, la quale fu si grande, che in molti giorni non ofarono accoftarfi al campo degli Spagnuoli. Coftoro paffarono fei giorni in continue zuffe: 
zuffe: $\mathrm{i}$ brigantini girando attơrno alla Città attaccavano il Lis. X.; fuoco a molte cafe dei fobborghi, e nelle loro fcorrerie fcoprirono un canale grande e profondo, pel quale potevano agiatamente entrar nella Città: ciò che fu nell' avvenire di gran vantaggio agli Spagnuoli.

Alvarado dalla fua parte ftringeva, quanto poteva, i Mefticani, prendendo in frequenti pugne alcuni folf e trinciere della ftrada di Tlacopan; na furono uccifi alcuni de' fuoi, e molti feriti. Offervò egli, che per la ftrada di Tepejacac, fituata verfo Tramontana, s' introdacevano continuamente dei foccorfi nella Città, e s'accorfe, che per quella ftrada farebbe facile la fcappata agli affediati, qualora fi trovaffero in iitato di non poter più refiftere agli Affediatori. Il fece prontamente fapere a Cortès, e coftui comandò a Sandoval, che andaffe con cento diciotto pedoni Spagnuoli, e con un grandiffimo numero d'Alleati ad occupar quel luogo, e quindi impediffe i foccorfi, che venivano ai nemici. Ubbidì Sandoval, quantunque travagliato ancora dalla ferita della gamba, e s'impadroni fenzd contrafto di quel luogo, reftando d'allora innanzi impedita ai Mefficani ogai comunicazione

$5,28$. per terra colle altre Città. $\left({ }^{*}\right)$

Prima

Fatto ciò, determinò Cortès di fare il giorno fe: entrata guente un'entrata nella Città con più di cinquecento Spafediatori gnuoli, e più d' ottanta mila Alleati da Tezcuco, da Tlafcalnella.Cit-la, da Chalco, e da Huexotzinco, lafciando in guardia del sà. Campo qualche Cavalleria con dieci mila Alleati; ed ordinando a Sandoval, e ad Alvarado d'entrarvi ciafcuno per la fua ftrada nello fteffo tempo colle loro truppe, che non era-

(*) Il Dott. Robertfon dice: ", che Cortès volle affalir la Città da tre " parti differenti: da Tezcuco dal lato orientale del lago, da Tacuba a po", nente, e da Cuyocan ( cioè Coyohuacan ) verfo mezzo giorno. Quefe . Città, foggiunge, erano pofte fulle principali felciate che conduconoal" la Capitale, e che fono fatte per la fua difefa. Diede a Sandoval il co"mando della prima \&c. "Ma quefto è un errore; poichè a Levante non era nè poteva effere veruna felciata a cagione delia profondita del lago. Sandoval s' accampó non già in Tezcuco, donde era impoflibile l' affalir Meffico, ma in Tepejacac verfo Tramontana. 
erano meno d'ottantá mila uomini. Marciò Cortès per la fua Arada col fuo numerofo efercito bene ordinato e fian- $L_{i z_{c}} \mathbf{X}$. cheggiato da'brigantini, ed appena fatto un poco di Itrada s'imbattè in un largo e profondo foffo, ed in una trinciera alta più di dieci piedi. S'oppofero coraggiofamente i Mefricani al loro paffaggio, ma rilpinti coll'artiglieria de'brigantini, paffarono gli Spagnuoli incalzandogli fin' alla Città, dove trovaroso un altro gran forfo, ed una forte ed alta triaciera. L'impeto dell' acqua in quetto follo, la folla de' nemici, che vi concorfero alla difefa, i loro gridi fpaventevoli e minacciof e la folta pioggia di frecce, di dardi, e di faff, che tiravano, tratiennero qualche tempo la rifoluzione degli Spagnuoli; ma avendo finalmente collo fparo di tutta l'artiglieria e di tutte l'armi da fuoco fcacciati dalla trinciera coloro, che la difendevano vi pafsò l'efercito, e s'avanzd efpugnando altri folfi, e trinciere fin'ad una piazza principale della Cictà, ch'era piena di popolo . Malgrado la ttrage, che vedevano farfi nella moltitudine da un gran cannone piantato nell' ingreffo della piazza, non ardivanogli Spagnuolid' entrarvi, finchè il medefimo Generale, rinfacciando loro quella ignominiofa paura, e fcagliariof intrepidamente contro i nemici, fece coraggio a' fuoi Soldati. I Mefficani impauriti da sì grand'intrepidezza rifuggirono dentro il recinto del tempio maggiore, e vedendofi ancor là affaliti, fi ricoverarono negli atrj fuperiori de'tempj, dove parimente furono perfeguitati; ma all'improvvifo fi trovarono gli Spagnuoli affaliti alle fpalle da altre truppe mefficane, e meffi in tale Itretto, che non potendo foltener la furia de' nemici nè dentro il recinto del tempio, nè fuori nella vicina piazza, furono coltretti a ritirarfi nella ftrada, per la quale erano entrati nella Città, fafciando in potere de'nemici il cannone d'artiglieria. Indi a poco fopravvennero opportunamente nella piazza tre o quattro cavalli, e perfuadendofi i Mefficani, che veniva contra loro tutra la cavalleria, fi fcompigliarono per la paura, che aveano di quelle grandi e focofe beftie, ed abbandonarono ignominiora.

Storia Antica del Meffico Tom. III. C c men. 
mente il tempio, e la piazza, le quali furono fenza induLis. X. gio occupate dagli Spagnuoli. Dieci o dodici Nobili mefficani s' erano fortificati nell' atrio fuperiore del tempio maggiore; ma a difpetto della loro oftinata refiftenza furono vinti ed uccifi dagli Affalitori. L' efercito Spagnuolo nella fua ritirata attaccò il fuoco alle più grandi e più belle cafe della ftrada d'Iztapalapan, benchè non fenza graviffimo pericolo per l'impeto, con cui davano addoffo i Meflicani alla retroguardia, e pelimale che le facevano da terrazzi. Alva: rado, e Sandoval fecero colle loro truppe una grande frage de' Mefficani, e gli Alleati meritarono in quefta giornata grandi encomj dal Generale Spagnuolo.

6. I9. Aumentavanfi ogni giorno talmente le forze degli SpaAumen- gnuoli con nuovi foccorfi, e con nuove alleanze di Città, to delle e di Provincie intere, che non effendo Ptati da principio aufiliarie ne'tre loro campi più di novanta mila ucmini, in fra podegli Spa-chi giorni arrivarono a dugento quaranta mila. Il nuovo gnuoli. $\operatorname{Re}$ di Tezcuco per manifeitare a Cortès la fua gratitudine procurava conciliargli tutta la Nobiltà del fuo Regno, ed armo in quefta occafione un efercito di cinquanta mila uo: mini, che mando in ajuto agli Spagnuoli fotto gli ordini $d^{\prime}$ un Principe fuo fratello, ch' ebbe nel battefimo il nome di Don Carlo Ixtlilxochitl, $(z)$ giovane, della cui bravura fanno chiara teftimoniazza gli Storici antichi, e tra gli alsri l'iffeffo Cortès, il quale vanta l'opportunità, e l' im. portanza di tal foccorfo. Rimafe quel Principe con trenta mila

(z) Cortès l' appella Ifrifucbil : Bernal Diaz, e Sollis, alterando più il nome, il chiamano Sucbil. Torquemada contraddicendo a fe ferfo dice, che quefto giovane era Coanacotzin fratello maggiore di Don Ferdinando Ixtlilxochitl, e dopo poche pagine fa quefto medefi mo Coanacotz in Configliere principale del Re Cuauhtemotzin, duránte l'affedio della Capitale; ma egli è certo, che il giovane condottiere dell' efercito Tezcucano fu Don Carlo Ixtlilxochitl, al quale, morto il fuo fratello Don Ferdinando Cortès Ixtlilxochitl, dopo la conquifta, diede il Conquiftatore Cortès il'invefticura dello Stato di Tezcuco. Coanacotzin fi trattenne nella Corte di Meffico dal principio di quefto anno 'fin' alla conquifta. Fu fatto prigioniere infieme col Re Quauhtemotzin, e fu fimilmente con lui giuftiziato treannidopo in Izancanac, allorchè faceano turti e due viaggio con Cortès verfo Comajahua. 
mila uomini nel campo di Cortès, e gli altri venti mila fi compartirono ne' campi di Sandoval, e d'Alvarado o. QueftoLas. X. foccorfo de' Tezcucani fu tolto feguito dalla confederazione de'Xochimilchefi, e degli Otomiti montanari cogli Spagnuo. li, le quali nuove truppe accrebbero di venti mila uomini l'efercito di Cortès.

Non mancava altro a quefto Generale pel compimento dell' affedio, fe non l'impedire i foccorfi, che s' introducevano per acqua nella Città. Ritenendo perciò fette brigantini, mandò gli altri fei verfo quella parte del lago, che v' era fra Tlacopan e Tepejacac, acciocchè quindi agevolmente poteffero dare ajuto a' campi di Sandoval, e d'Alvarado, qualora quefti Comandanti il richiedeffero; e mentre non foffero da effi impiegati, andaffero a due a due corfeg. giando, e procuraffero di pigliar tutte le barche, che por: taffero viveri, o truppe alla Città.

Or trovandofi Cortè̀s con un numero sì grande di trup. pe alleate, determinò di fare in fra tre giorni un"entrata nella: Città. Diede perciò gli ordini opportuni, e' nel giorno prefiffo marciò colla maggior parte della fua cavalleria, con trecento pedoni Spagnuoli, con fette brigantini, e con una moltitudine innumerabile d'Alleati. Trovarono i foff aperti, le trinciere rifatte, ed $\dot{i}$ nemici bene alleftiti per refíftere; con tutto ciò efpugnarono coll' ajuto de'brigantini tutti $i$ foff e le trinciere, che v' erano infin'alla piazza principale di Tenochtitlan. Quivi fece alto l'efercito, non permettendo Corrès, che s' inoltraffero più fenza lafciare fpianati tutti i paffi difficili, che avea efpugnato, ma frattanto che dieci mila Alleati s'occupavano nel riempiere i foff, gli altri: s'applicarono a bruciare e rovinare alcuni tempj, cafe, e paldzzi, e tra gli altri quello del Re Axajacatl, dove aveano gia avuto gli Spagnuoli il loro quartiere, ed il celebre palazzo degli uccelli del Re Motezuma. Dopo fatte quefte oftilità a grande ftento, e con grave pericolo a cagione degli sforzi, che faceano i Mefficani per impedirle, fuond Cortès la ritirata, la quale s'efegui felice- 
mente, quantunque inceffantemente travagliata foffe la reLis. X. troguardia dalle truppe nemiche. Lo fteflo fecero dal loro canto Alvarado, e Sandoval. Quelta giornata fu fenz' altro di gran fatica per gli Spagnuoli, ed i loro Alleati, ma eziandio d'indicibila cordoglio per li Meflicani tanto per la perdita di tanti belli edifizj, quanto per gli fcherni, coi quali erano infultati dagli fteff loro vaffalli confederati cogli Spagnuoli, e da'Tlafcallefi lor nemici capitali, che nel combattere lor moftravano le braccia, e le gambe de' Mefficani, che aveano uccifo, e minacciavano di voler mangiar: le quella notte a cena, ficcome in fatri lo fecero.

5. 2o. cani di fcavare i folf ricolmati, e di riparar le trinciere

Il di vegnente a buon'ora per non dar tempo a' Meffientrace nella Città. abbattute, fortì Cortès dal fuo campo nello fteffo modo del giorno precedente; ma ad onta della fua diligenza i Meffi. cani aveano già rifatto la maggior parte delle fortificazioni diftrutre, e le difefero con tale oftinazione, che non potè l'efercito degli Affediatori efpugnarle, fe non dopo un fu. riofo combattimento di cinque ore. Inoltroffi l' efercito, ed efpugnò due foffi della ftrada di Tlacopan; ma effendo ormai per terminare il di, fi ritirò al fuo campo, pugnando sutrora colle truppe nemiche, che davano addoffo alla re. troguardia. Gli fteffi combattimenti dell' efercito di Cortès ebbero quelli di Sandoval, e d'Alvarado, dovendo gli Affediati combattere in un medefimo tempo con tre numero. fiffrmi eferciti fuperiori a loro nell' armi, ne' ca valli, ne' brigantini, e nella difciplina militare. Alvarado dal fuo canto avea già rovirate tutte le cafe, che v' erano dall' una e dall' altra banda della ftrada di Tlacopann; $(A)$ poichè la popolazione della Capitale fi continuava per quella parte in.

(A) Quefte cale non erano fabbricate nella fteffa ftrada, ma preffo ad effa nelle ifolette, che v'erano dall' una, e dall' altra banda. Non fappiamo che forfe nelia Arada altro edifizio, che un tempio, il qual era in quella parte, dove slargandofi la ftrada formava una piazzetta. Quefto tempio fu prefo da Alvarado, e vi mantenne ura guernigione quafi tutto il tem po dell' afredio. 
infin' al continente, ficcome ne fanno fede Cortès, e Bernal Diaz.

Cortès avrebbe voluto rifparmiar alle fue truppe la Liв. $X$. gran fatica di replicare ogni gionno i combattimenti per efpugnare gli fteff foff e le fteffe trinciere; ma nè potea lalciarvi della guernigione per confervare gli acquifi fenza facrificarla al furor de'nemici, nè volea accamparfi dentro la Città, ficcome lo confgliavano alcuni de' fuoi Capitani; poichè oltre a'continui affalti, che di e notte dovrebbono tollerare da' nemici, non potrebbono quindi così facilmente, impedire i foccorfi, che venivano alla Città, come dal po: fto di Xoloc.

Mentre quefti foccorfi andavano mancando agli Affedia. ti, s'aumentavano quelli degli Affediatori, i quali in que-Confedefto medefimo tempo ne ricevettero uno ianto a lor vantaggio- razione fo, quanto a' nemici nuocevole. Gli abitanti delle Citrà fi- chieCittuate nella riva, e nell' ifolette del lago di Chalco, erano tà del lic* fin' allora ftati nemici degli Spagnuoli, e poteano recar mol-go cogli to danno al campo di Cortès, fe le loro truppe l'aveftero da li. una parte della ftrada affalito nel medefimo tempo, nel qua le da un'altra parte l' affalivano i Mefficani; ma eglino non aveano intentata veruna oftilita contra gli Spagnuoli, forfe perchè la rifervavano ad occafione più opportuna. I Chal. chefi, ed altri Alleati, cui non tornava a conto la vicinan: za di tanti nemici, procuravano tirarii al loro partito ora con promeffe, ed ora con minacce, e con veffazioni, e tanto potè la loro importunita, e forfe anche la paura della ven. detta degli Spagnuoli, che vennero al campo di Cortès per confederarfi, con lui i Nobili d'Iztapalan, di Mexicaltzinco, di Colhuacan, d'Huitzilopochco, di Mizquiz, e di Cuitiahuac, le quali Città erano una parte confiderabile della val. le Mefficana. Rallegroff infinitamente Cortès di queft' alleanza, e richiefe da loro, che non folamente l'ajutaffero colle lor truppe, e colle lor barche, ma eziandio, che trafportaffero de'materiali per far delle capanne lungo quella ftrada, perchè effendo quella la fagione delle pioggie, pa: tiva troppo la fua gente per mancanza d'abitazione. 
Tutto ciò fu sì bene da loro efeguito, che immediata Lis. $X_{0 .}$ mente mifero fotto gli ordini di Cortès un corpo confidera. bile di truppe, il cui: numero non fi dice, e tre mila: barche per ajutare i brigantini nel loro corfo, nelle quali trafportarono prontamente: i materiali neceffarj, e fabbricarono tantebaracche, che vi poterono agiatamente ftare tutti gli Spagnuoli con due mila Indiani impiegati nel: loro fervizio; poi. chè il groffo delle truppe Alleate: era accampato in Cojohua. can, quattro miglia diftante da Xoloc: e non contenti' di sì grandi foccorfi apportarono al campo di Cortès molte vettova. glie, e principalmente: del pefce: e delle ciriegie in gran quantità o.

Trovandofi dunque Cortès colle fue forze tanto accre: fciute, entrò con effe due o tre giorni di feguito nella: Cittă , facendo una confiderabile Atrage de'Cittadini . Egli: fi pero. fuadeva, che doveffero arrenderfi gli Affediati vedendofi: contra: un si ecceffivo numero di truppe, e Cperimentando i perniziofi. effetti dell' oftinata lor refiftenza; ma s'ingannò, poichè i Mefficani erano rifoluti di perder prima le: loro vite, che 12. lor libertă. Rifolverte però di far continue entrate nella. Città per coltringerli con inceffanti oftilità a domandar la pace, che rifiutavano. Formò de' fuoi baltimenti due arma te, compofta ciafcuna di tre brigantini, e di mille: e cinquecento barche', ordinando loro d'accoftarfi alla Cirtà, d'appicciare il fuoco alle cafe, e di fare a' Meflicani quanto male lor fofle poffibile. Diede ordine a Sandoval, e ad: Alvarado di far lo fteffo dal loro: canto, ed egli con tutti $i$ fuoi Spagnuoli, e per que! che pare, con ottanta mila Alleati $(B)$ marciò come folea, per la trada d'Iztapalapan verfo la Città fenza poter ottenere nè in quefta nè in altre entrate di quefti giorni altri vantaggi, che quelli d'andar a poco a poco fminuendo il numero de'nemici, rovinando alcuni edifizj,

(B) Io congetturo, che le truppe alleate, che accompagrarono: Cortès in quefta entrata, fieno ftate ottanta mila uomini, perchè Cortès afferina, che quel giorno erauo più di cento mila nel fùs campo, de' quali ventio ventidue mila faranno ftatj impiegati nelle due flotte di barche. 
fizj, ed inoltrandofi ognigiorno alquanto più col fine d'aprirfi comunicazione col campo d'Alvarado, febbene allora Lis. $x$. non gli fu polfibile d'ottenerlo.

Alvarado colle fue truppe fecondate da'brigantini avea già efpugnato un tempio, che v' era in una piazzetta della Itrada di Tlacopan, nel quale mantenne d'allora innanzi una guernigione a difpetto de' violenti affalti de' Mefficani . Avea parimente prefo alcuni foff e trinciere, e lapendo che S. 22. Operazioni $\mathrm{d}^{\prime}$ Alvarado, e prola maggior forza de nemici era in Tlatelolco, dove rifiede- tzin. va il Re Quauhtemotzin, e dove s'era ricoverata infinita gente di Tenochtitlan, indirizzò verfo quella parte le fue operazioni; ma quantunque combatteffe con tutte le fue forze per terra, e per acqua, non potè inoltrarfi fin dove voleva per la gagliarda oppofizione degli Affediati, ne' quali combattimenti perirono molti e dall' una, e dall'altra parte. In una delle prime zuffe fi lafciò vedere un membruto $e$ coraggiofo Tlatelolchefe traveftito da Otomito con un Ichcahuepilli, o fia corazza di cotone, e fenz' altre armi, che uno fcudo e tre faff, e correndo velociffimamente verfo gli Affediatori tiro fucceffivamente i tre faffi con tal deftrezza, e con tal forza, che con ciafcheduno abbattè uno Spagnuolo, cagionando non minore fdegno agli Spagnuoli, che paura e meraviglia $a^{\prime}$ loro confederati. S' adoperarono con ogni loro diligenza per averlo tra le mani; ma non poterono mai pigliarlo, perchè in turti i combartimenti compariva diverfamente veftito, ed in tutti faceva gran danns agli Affediatori, avendo peraltro tanta velocità ne' piedi per falvarfi, quanta forza nelle braccia per offendere. Il nome dique. fo celebre Tlatelolchefe era Tzilacatzin.

Alvarado, infuperbito per alcuni vanraggi ottenuti fopra i Mefficani, volle un giorno inoltrarfi fin' alla piazza del mercato: avea già efpugnate alcune trinciere ed alcuni foffi, e tra gli altri uno, ch' era largo più di cinquanta pie: di, e profondo più di fette, e dimenticato per la felicità di farlo turare, ficcome gli avea ordinato il fuo Generale, s'inoltrò con quaranta o cinquanta Spagnuoli; ed alcuni Alleati. 
leati. Accortifi i Mefficani della fua trafcaraggine piombaz Lib. X. rono tofto fopra loro, gli fconfiffero, e fugarono, e nel ripaffare il foffo uccifero alcuni Alleati, e fecero prigioni quattro Spagnuoli, i quali furono incontanente facrificati a vifta d'Alvarado, e della fla gente nel tempio maggior di Tlatelolco. Rincrebbe affai a Cortès quefta difgrazia' come quella che dovrebbe accrefcer l'animo e l'orgoglio de' nemici, e fi portò fubito a Tlácopan per riprendere feveramente Alvarado della fua difubbiadienza, e della fua temerita; ma informato del coraggio, con cui 's'era condotto in quella giornata, e s'era impadronito de' pofti più difficili, fi contentò d'una benigna ammonizione, inculcando i fuoi ordini fulla maniera di far l'entrate.

5. 23. Le truppe di Xochimilco, di Cuitlahuac, e d'altre Tradi- Città del lago, ch' erano nel campo di Cortès, volendofi mento approfittare dell' occafione, che lor fi prefentava 'nell' entrate chimil- degli Spagnuoli, di faccheggiar le cafe de' Mefficani, adopechefi e rarono la più abbominevole perfidia. Inviarono una fegreta Dopoli. ambafciata al Re Quauhtemotzin, proteftando th inviolabil loro fedelta alla corona, e lagnandofi degli Spagnuoli, perchè gli forzavano a prender l'armi contra il natural to ro Signore, e foggiungendo, che volevano nella prima loro entrata unirfi co'Mefficani contra que' neenici della patria per ammazzarli tutti, e por così fine a tante calamità. Lodò i) $R e$ il loro propofito, affegnò loro i polti, che doveano occupare, ed anche rimandò loro de' doni per rimunerar la pretefa lor fedeltà. Entrarono quefti traditori, come folea no, nella Cittz, e fingendo prima di rivolgere le loro armi contro gli Spagnuoli, cominciarono poi a laccheggiar le cafe de' Mefficani, uccidendo coloro, che lor refiftevanó, ed imprigionando le donne, ed i fanciulli; ma accortifi tofto i Mefficani del tradimento, fi fcagliarono contra loro con tal furia, che quafi tutti i colpevoli pagarono colla vita la lor perfidia. Moltiffimi furono uccifi nella zuffa, e gli altri fatii prigioni furono per ordine del $\mathrm{Re}$ incontanente facrificatio Quefto tradimento pare non effere ftato configliato, nè efe: guito, 
guito, fe non da una parte del popolaccio di quiclle Città, gente malnata, ed ognora pronta a si fatti delitri.

Erano omai venti giorni, che gli Spagnuoli faceano continue entrate nella Citia: onde alcuni Capitani e Soldati Atanchi per tanti combattimenti, il cui frutto vedevano ancor lontano, fi lagnavano col Generale, e lo fcongiuravano d'avventurare tutte le gran forze, che aveva, ad un colpo decifivo, che gli tiraffe finalmente da tasti periglj e fatiche. Il difegno di coltoro era quello d'inoltrarfi fin' al centro di Tlatelolco, dove aveano radunato le loro forze $i$ Mefficani per rovinarli affatto in una fola gionnata, o almeno indurli ad arrenderfi. Cortès, il quale conolceva affai bene, quanto pericolofa foffe una tal imprefa, procurava dittornarli da effa colle più efficaci ragioni; ma nulla giovando, nè potendo peraltro ripugnare ad un configlio, ch' era divenuto quafi generale, fi piegò alla fine alle loro importune iltarze. Ordinò al Comandante Sandoval, che con cen. to quindici pedoni, e dieci cavalli andafe ad unirfi ad Alvarado, che metteffe in un'imbofcata la cavalleria, e levaffe il bagaglio, fingendo di far partenza, e d'abbandonare l'affedio della Città, acciocchè allettati i Mefficani ad in-feguirli folsero afsaliti alle fpalle dalla cavalleria: che co' fei brigantini procuraffe impadronirfi di quel gran foffo, dove fu fconfitro Alvarado, facendolo riempiere ed appianare: che non faceffe un paffo avanti fenza lafciar bene accomodata la ftrada per la ritirata, e che s'adoperaffe per entrare a mano armata nella piazza del mercato.

Il di preffio per l'affalto generale marciò Cortès con venticinque cavalli, con tutta la fua infanteria, e con più di cento mila Alleati. Formavano l'ali del fuo efercito dall'una, e dall'altra banda della ftrada i fuoi brigantini con più di tre mila barche aufiliarie. Entrò fenza verun contralto nella Città, e divife tolto il fuo efercito in tre parti, acciocchè per altrettante ftrade arrivar poteffero ad un ora alla piazza del mercato. Il comando della prima divifione fi diede a Giuliano Alderere, Teforiere del Re, il

Storia Antica del Meffico Tom. III.

D d

qua.

ङ. 24. Vittoria de'Merficani. 
quale era ftato colui, the con maggior impegno avea fconj Lis. X. giurato Cortès d'intraprender quefta fpedizione, e gli fu ordinato d'incamminarfi per la ftrada principale e più larga con fettanta pedoni Spagnuoli, fette cavalli, e venti mila Alleati. Delle altre due ftrade, che conducevano dalla ftrada maeftra di Tlacopan alla piazza del mercato, la meno Atretta fu affegnata a' Capitani Andrea di 'Tapia, e Giorg io Alvarado ( fratello di Pietro d'Alvarado ) con ottanta pedoni Spagnuoli, e più di dieci mila Alleati, e dell' altra più ftretta e malagevole s' incaricò il medefimo Cortès con cento pedoni Spagnuoli, e col groffo delle truppe aufiliarie, lafciando nell' entrata di ciafcuna Arada la cavalleria e l'artiglierìa. Entrarono tutti ad un tratto combattendo coraggiofamente. I Mefficani fecero da principio qualche refiftenza ma poi fimulando codardia fi ritirarono, abbandonando i folfi agli Spagnuoli, affinchè coftoro allettati dalla fperanxa della vittoria s'avanzaffero a maggiori pericoli. Alcuni Spagnuoli s'inoltraron' fin'alle ftrade più vicine alla piazza del mercato, lafciando incautamente addietro un largo foffo mal ricolmato, ed allorchè più incoraggiti cercavano a gara d'entrare $i$ primi in quella piazza, fentirono il formidabil fuono della cornetta del Dio Painalton, la quale fi fonava da' Sacerdoti ne'cafi di pubblico e preffante bifogno per eccitare il popolo a prender l'armi. Vi accorfe fubico una si gran moltitudine di Mefficani, e piombò con tal furia fopra gli Spagnuoli e gli Alleati, che gli fcompiglio, e gli coltrinfe a tornare precipitofamente indietro infin' al foffo $(C)$ in apparenza ricolmato con fafcine, ed altri materiali leggieri, e volendo paffarvi fopra, s'affondavano pel pefo e per la violenza della moltitudine. Quì fu il maggior conflitto e pericolo

(C) Solls mette quefto foffo fuor della Città, e dice, che nell' ufcir da efto furono affaliti gli Spagnuoli da' Mefficani; ma quefto è un manifefto errore; poichè ci con $\mathrm{Aa}$ da' ragguagli di Cortès, e d'altri Storici, che il fuddetto foffo era fra la Atrada maefta di Tlacopan, e la piazza del mercato, e che per andar gli Spagnuoli al luro campo dovettero traverfare la maggior parte della Città. 
ricolo de'fuggitivi; poichè non potendo ad un' ora 'e paffare a nuoto, e difenderfi, erano feriti e prefi da' Mefficani. Liв. X. Cortès, il quale colla diligenza propria d'un buon Generale era venuio al foffo, allorchè vi arrivarono le truppefconfitte, procurò fermarle co'fuoi clamori, acciocchè noz agevolaffero col lor difordine la frage cominciata a far da' nemici; ma che voci fon capaci di trattener la fuga d'una moltitudine fcompigliata, alla quale dà fretta la paura? Trafitco dal più vivo dolore per la perdita de' fuoi, e non curando il fuo proprio pericolo, s'accoftò al folto per ifcampar quelli che poteffe. Alcuni ufcivano difarmati, altri feriti, ed altri quafi annegati. Procurò mettergli in ordine, ed incamminarli verfo il campo, reftando egli in dietro con dodici o venti uomini per guardar loro le fpalle; ma ap. pena fi cominciò a marciare, ch'egli fi trovó in un paffo fretto circondato da' nemici. Quel giorno farebbe ftato l' ultimo per lui, a difperto dello fraordinario coraggio, con cui fi difefe, efi farebbe perduta infieme colla fua vita tutta la fperanza della conquifta di Meffico, fe $i$ Mefficani in vece di dargli, come facilmente poterono, la morte, non fi foffero impegnati in pigliarlo vivo per onorar con sì illuftre vittima $\mathrm{i}$ loro Dei. Aveanlo già prefo, e conducevanlo fenz' altro al facrifizio, fe la fua gente avvifata della fua prigionia non foffe prontamente venuta a liberarlo. Dovette Cortès principalmente la fua vita e la fua liberta ad un foldato della fua guardia appeliato Criftofano d'Olea, uomo di gran coraggio, e di fingolar deftrezza nell' armi, ( $\left.{ }^{*}\right)$ il quale in un'altra occafione l'avea liberato da un imil pericolo, ed in quefta lo falvò a colto della fua propria vita, tagliando con un colpo di fpada il braccio di quel Mefficano, che l'a. vea pigliato. Fu altresì debitore Cortès della fua libertà al Principe Don Carlo Ixtlilxochitl, $e$ ad un bravo Tlafcallefe appellato Temacatzin.

$$
\text { D. d } 2
$$

Ufci-

$\left.{ }^{*}\right)$ Bernal Diaz in molti luoghi della fua Aoria loda affai il coraggiod'Olea, la cui morte fu oltremodo fenfibile ed al fuo Generale, ed a'fuoi compagni, 
Ufcirono finalmente gli Spagnuoli, benchè a grande Lis. X. Atento, e con non poche ferite nella ftrada larga di Tlacopan, dove potè Cortès ordinarli, prendendo egli la retroguardia colla cavalleria; ma l'ardire ed il furore, con cui li perleguitavano i Mefficani, erano tali, che pareva loro im. polibile lo fcampar la vita. Coloro, che erano entrati per le altre due Atrade, aveano avuti de' terribili combattimenti; ma perchè furono più diligenti nel turare $i$ foffi, fu loro men difticile la ritirata, allorchè Cortès diede loro l'ordine di marciare alla piazza maggiore di Tenochritlan, dove fi radunarono. Quindi videro con graviffimo loro fpiacere elevarfi da' caldani del tempio maggiore il fumo del copal, che bruciarono i Mefficani in rendimento di grazie per la vittoria ottenuta; ma s'accrebbe davvantaggio la lor pena, quan. do videro alcune tefte di Spagnuoli gettate verfo di loro da' Mefficani per ifcoraggirli, e fentirono dirî, che aveano uccifo i Comandanti Alvarado e Sandoval. Dalla piazza s'incamminarono per la ftrada d' Iztapalapan al loro campo, incalzati ognora da una gran moltitudine di nemici.

Alvarado, e Sandoval s'erano sforzati d'entrar nella piazza del mercato per una frada, che conduceva da quella di Tlacopan a Tlatelolco, ed aveano felicemente avanzate le loro operazioni infia' ad un polto poco lontano da quella piazza, ma avendo veduti i facrifizj d'alcuni Spagnuoli, e fentito dirfi da' Mefficani, che erano ftati uccifi e Cortès, ed i fuoi Capitani, fi ritirarono con fomma difficoltà; imperciocchè a que'nemici, che innanzi gli combattevano, s'aggiunfero coloro, che aveano fconfitte le truppe di Cortès..

La perdita, ch'ebbero in quella giornata gli Affediatoji f fu di fette cavalli, di molte armi, e barche e d'un cannone diartiglieria, di più di mille Alleati, e di pià di feffanta Spagnuoli, parte uccifi nella battaglia; e parte fatti prigioni, ed immediataménte facrificati nel tempio maggior di Tlatelolco a vifta della gente d'Alvarado. Fu eziandio uccifo il Capitano d' un brigantina. Cortès ebbe una ferita 
in una gamba, ed appena vi fu tra gli Affediatori chi non reftafle ferito o malconcio. $(D)$

Celebrarono i Mefficani otto giorni continui la vittoria con illuminazione e mufica ne'tempj: fecero volar la fama per tutto il Regno, e poriar per le provincie le tefte degli Spagnuoli uccifi per impaurir quelle, che s'erano ribellate alla corona, e rivocarle alla Joro ubbidienza, liccome in fatti l'ottennero da alcune. Scavarono di nuovo i folfi, ripararono le trinciere, e rimifero la Città fuorchè i tempje le cale rovinate da nemici nello tato, in clii era prima di cominciarfi l'affedio.

Frattanto gli Spagnuoli fi tenevano fulla difefa ne'loro campi, curandofi le ferite, e riltorandofi per i futuri combattimenti; ma affinchè non fi prevaleffero della loro dap. pocaggine i Mefficani per introdur de'viveri nella Citta gantini, poctind Cories che i brigantini andaffero a due a dutaordinò Cortès, che $\mathrm{i}$ brigantini andaffero a due a due cor-gemmi feggiando pel lago. I Mefficani, riconofcendo la fuperiorità de' Méde'baftinenti e dell' armi Spagnuole, e non potendo adopeficani. rare armi uguali, procurarono almeno in qualche maniera pareggiare $i$ brigantini. Aveano per cio fabbricate trenta barche grandi dagli Spagnuoli dette Piraguas, ben fornite, a coperte di grofl tavolati per poter da effe combattere fenza tanto rifchio d'effer danneggiati. Determinarono far con effe un'imbofcata a' brigantini tra i bofchetti di canne, ch' erano ne' cefpuglj galleggianti nel lago, e ficcarono in parec. chj luoghi delle itanghe groffe nafcolte fotto acqua, accioc. chè urtando in effe $\mathrm{i}$ brigantini fi rompeffero, o almeno $s$ 'imbarazzaffero per la difefa. Difpolta dunque l' imbofcata, fecero ufcir da' canneti del lago tre o quattro barche ordinarie, acciocchè provocando due brigantini, che vi corfeg-

$$
\text { gia- }
$$

(D) Cortès non numera più di trenta cinque, o quaranta Snagnuoli uccifi, nè più di venti feriti, ma egli feguendo l'orme d'altri Generali di'minuifce le fue perdice, ficcome fece nel ragguagliar la fconfitra del r. Luglio dell' anno antecedente: onde ci parve meglio di tener dietro in quefto punto a Bernal Diăz, il quale moftra avere avuto una particolar cura di contar gli Spagnuoli, che andavano mascando. 
giavano, gli menaffero poi fuggendo at luogo dell' imbolcaL. X. ta. Gli Spagnuoli, tofto che le videro, corfero dietro a lo: ro, ma allorchè erano più impegnati nell'incalzarle, urtasono i brigantini nelle ftanghe, ed al tempo fteffo vennero fuori le trenta barche grandi, e gli affalirono da ogni parte. Corfera gli Spagnuoli gran pericolo di perder infieme co' loro baftimenti le loro vite; ma frattanto che col fuoco degli fchioppi trattenevano i nemici, ebhero agio alcuni deftri nuotatori di levar le Itanghe, onde liberati da quell' impaccio poterono prevalerfi dell'artiglierìa per fugar le barche . I brigantini reftarono affai malconcj, gli Spagnuoli fériti, e de' due Capitani, che gli reggevano, $l^{2}$ uno fu uccifo nella zuffa, e l'altro morì infra tre giorni delle ferite. I Meflicani rac. conciarono le loro barche per replicar lo tratagemma; ma avvifato fegretamente Cortès del luogo, dove fi mettevano in aguato, difpofe anch' egli un'imbufcata di fei briganrini, ed approfittandofi dell' efempio de' nemici, ordinò, che un brigantino s' accoftaffe al luogo dov'erano in aguato le barche, e quando le fcopriffe, fi metteffe a fuggire verfo quella parte, dov' erano imborcati gli Spagnuoli. Avvenne tutto, come Cortès l'avea penfato; perchè i Mefficani, vedendo il brigantino, ufcirono prontamenie dall' imbofcata, ed allorchè fi credevano più ficuri della preda, ufcirono gli altri cinque brigantini contra loxo, e cominciarono fubito a giocar dell' artiglierìa, col cui primo fparo ribaltarono alcune barche, e fcompigliarono le altre. Perì la maggior parte de' Mefficani, ed alcuni furono fatti prigioni, e tra gli altri alcuni Nobili, de' quali volle fervirfene Cortès per foliecitar qualche acsomodamento colla Corte di Meffico.

5. 26 .

Mandò dunque a dire al $\operatorname{Re}$ Quauhremotzin per mezzo Amba- dì quefti nobili prigionieri, che confideraffe :, quanto s' andafruttuofa vano fcemando le forze di Meffico nello teffo tempo, in cui al Re di s'andavano aumentando quelle degli Spagnuoli: che alla fine Meffico. dovrebbono arrenderfi alla maggior poffanza: che ancorchè gli Spagnuoli non entraffero in quella Corte a far delle oftilità, bafterebbe foltanto l'impedir loro i foccorti per farli mo 
rir di fame: che potevano ancora evitare $i$ difaftri, che lor fopraftavano: che fe acconfentivano alle propofizioni di pace, Lis. $X$. egli farebbe incontanente ceffar tutte le oftilith, il Re refterebbe nella pacifica poffeffione della corona con tutta ta grandezza, con tutto il potere, e con tutta l'autorita, di cui avea fin'allora goduto, ed i fuoi fudditi rimarrebbono liberi e padroni di tutti $i$ loro beni fenza richiedere altro da fua MaeItà, nè da'fuoi fudditi, fe non l'omaggio, dovuto al Re di Spagna, come a fupremo Signore di quell' Imperio, i dritti del quale erano già ftati riconofciuri dagli fteffi Mefficani, come appoggiati full' antica tradizione de'loro antenati : che fe per l'oppofto s'oftinava nella guerra, egli farebbe privato della corona, la maggior parte de' fuoi vaffalli perderebbe la vita, e quella bella e gran Città farebbe affatto rovinata . Il $R e$ confultò co' funi Configlieri, co' Generali d'efercito, e co' Capi della Religione : efpofe loro il foggetro dell' ambafciata, lo ftato di quella Corte, la Ccaríezza di viveri, l'affizione del popolo, ed i mali ancor maggiori, che lor minacciavano, e comandd loro, che diceffero liberamente il lor parere. Alcuni antivedendo l' efito della guerra,-inclinavano alla pace; altri Spinti dall' odio degli Spagnuoli, o dallo ttimolo dell' onore configliavano la guerra. I Sacerdoti, l'autorità de' quali tanto in quefta materia, quanto in altre, era fommamente rifpettata, $s^{\prime}$ opporero fortemente alla pace, allegando parecchj pretefi oracoli de'loro Dii, il cui fdegno dovea temerfi, fe fi piegavano alle pretenfioni di que' crudeli nemici det lor culto, e la cui protezione dovea follecitarfi con preghiere e facrifizj. Prevalfe alla fine quefto pa. rere pel timor fuperfiziofo, che s' era impadronito de' loro fpiriti, e fecondo effo fi ripole al Generale Spagnuolo, che contiruaffe pur la guerra, poichè eglino aveano rífoluto di difenderfi infin'all' ultimo refpiro. Se ad una tal rifoluzione foffero ftati ridotti non già dalla fuperftizione, ma dall' onore, e dall'amor della patria, e della propria libertà, non farebbono ftati tanto biafimevoli, perchè quantunque inevitabile prevedeffero la loro rovina, continuando la guerra, 
-non aveano fperanza di migliorar la loro fortuna colla pace: LIB. X La fperienza de' paffaii avvenimenti non permetteva loro di fidarfi delle promeffe, che lor in facevano, onde dovea loro rapprefentarfi più confacevole all' idee dell'onore il morir coll' armi nelle mani per la difefa della parria, e della libertà, che non l'abbandonar la medefiına patria all' ambizione di quegli Atranieri, e ridurfi colla loro refa, ad una trifta e miferabile fervitù.

Due giorni dopo la fconfitta degli Spagnuoli arrivarono Spedi- al campo di Cortès alcuai Meffaggieri mandati dalla Città zioni di Quauhnahuac a lagnarfi de'gran mali, che lor facevano Malinal- i Malinalchefi lor vicini, i quali, fecondo che coloro afferchefi, e mavano, volevano confederarfi co' Cohuizchi, Nazione affai contro i numerofa per diftruggere Quauhnahuac, perchè s' era alleata Matla- cogli Spagnuoli, ed indi paffare i monti per venire ad affalire con un grand' efercito il campo di Cortès. Quefto Generale, avvegnachè fi trovaffe in sitato di domandar piutto. fto ajuto, che di darlo, contuttociò per la riputazione dell' armi Spagnuole, e per prevenire il colpo, che gli fi minacciava, mandò il Capitano Andrea di Tapia co' meffaggieri, con dugento pedoni Spagnuoli, con dieci cavalli, e con un buon numero d'Alleati dandogli l'ordine d' unirli colle truppe Quauhnahuachefi, e di far tutto ciò, che credeffe convenire al fervizio del fuo $\mathrm{Re}$, ed alla ficurà degliSpagnuo. li. Tapia efeguì ciò, cha gli fu impofto dal fuo Generale, ed in un piccolo luogo fituzto fra Quauhnahuac e Malinalco'; ebbe una gran battaglia co' nemicr, gli fconfirte, e gl' incalzò infin'alla falda dell' alto monte, ful quale era la Città di Malinalco. Non potè dare ad effa, coms avrebbe voluto, un affalto, perchè il monte era inacceffibile alla cavalleria; ma diede il gualto alla campagna, e perchè era omai per compierfi il termine di dieci giorni, che gli era ftato prefcritto, ritornò al campo di Cortès.

Indi a due giorni arrivarono allo fteffo campo i meffaggieri degli Oromiti della Valle di Talloc can, domandando ajuto contro i Matlatzinchi, Nazione potente e guerriera del- 
Ja medefina valle, $i$ quali lor faceano continua guerra, aveano abbruciato uno de' lor luoghi, ed aveano fatto loro mol. Liz. $X_{\text {. }}$ ti prigionieri, ed oltre a ciò s' erano accordati co' Mefficani d'affalire con tutte le loro forze il campo di Cortès dalla banda di terra ferma nel medefimo tempo, in cui i Mefficani l'affaliffero dalla banda della Citta. Nell' entrare, che aveano fatto gli Spagnuoli in Meffico aveano qualche volta fentito i Mefficani minacciarli del poter de' Matlatzinchi ; ed ora s'accorfe Cortès pel ragguaglio degli Oromiti del gran pericolo, che correrebbe, fe defle tempo a' nemici di porre in efecuzione il loro difegno. Non volle confidar al altro quefta importante fpedizione, fe non al prode ed invitto San. doval. Queft' uomo infaticabile, contuttochè foffe ftato ferito nel giorno della fconfitta di Cortès, avea fatto que'giorni dopo da Generale, girando inceflancemente per tutti e tre i campi, dando le più opportune difpofizioni per la lor $f i$. curezza. Appena fcorfi quattordici di dopo. la fconfitta, marcio verfo la Valle di Toloccan con diciotto cavalli, cen cẹnto pedoni Spagauoli, e con feffanta nuila Alleati. Nella ftrada videro alcuni contraffegni della ftrage fattavi da' Matlatzinchi, e quando entrarono nella valie, trovarono un luogo di frefco rovinato, e videro le truppe nemiche, che anda. vano cariche di fpoglie, le quali furono da loro abbandonate, tofto che comparvero gli Spagnuoli volendo reftar pitz sorigati per la battaglia. Paffarono un fume, che traverfa la valle, e rimafero nella riva ad afpettar gli Spagnuoli. Sandoval lo valico intrepidamente col fuo efercito, affalt i ne. mici, li mife in fuga, e gl' inleguì per ben nove miglia, fin' ad una Città, dove coloro rifuggirono, lafciando morti nel campo più di mille Matlatzinchi. Sandoval affediò la Città, e coltrinfe $i$ nemici ad abbandonarla, ed a ricoverar. $f($ in una fortezza fabbricata nella cima d'un monte fcofcefo. Entrò l' efercito vittoriofo nella Citrà, e dopo averla faccheggiata, appicciarono fuoco agli edifizj, e perchè era tardi, e le truppe erano troppo ftanche, fi rifervò l'affalto della fortezza pel giorno feguente, nel quale credendo d'avere Storia Antica del Mejfico Tom. III. E e. un 
un gran contrafto, trovarono la fortezza vuota. Determinó La. X.Sandoval paffar nel fuo ritorno per alcuni luoghi, che s'erano dichiarati parimente contrarj; ma non ebbe d' uopo d'adoperar contra effi l'armi, perchè impauriti dal veder contra loro un si grand' efercito, ingroffato con numerofe truppe d'Otomiti, tofto s' arrendettero. Sandoval gli accolfe con fomma benignita, e richiée da loro, che induceffero la Nazione Matlatzinca a fare amicizia cogli Spagnuoli, rapprefentando loro $i$ vantaggi, che da effi fperar potevano, e per I' oppoito i mali, che loro recar dovrebbe l' inimicizia.. Quete fpedizioni furono di grandiffima importanza; poichè quattro giorni, dappoichè era ritornato Sandoval, arrivarono al campo di Cortes parecchj Signori Matlatzinchi, MalinalcheII, e Cohuixchi (E) a dimandare fcula delle loro oltilità, ed a tabilire una confederazione, che fu tanto utile agli Spagnuoli, quanto nocevole a'Mefficani.

Non aveano più gli Spagnuoli nemici da temere dalla parte del continente, ed avea Cortès in fua difpofizione un sì ecceffivo numero di truppe, che avrebbe potuto impiegar nell' affedio di Meflico affai più gente di quella, che mandó Serfe contra la Grecia, fe per cagione della fituazione di quella Capitale non foffe ltata piuttofto d'impaccio una s' gran moltitudine d'Affediatori. I Mefficani per contrario fi trovavano abbandonati da'loro amici, e da' loro fudditi, circondati da nemici, ed afflitti dalla fame. Avea quelia fventurata Corte contro te gli Spagnuoli, il Regno d' Acolhuacan, le Repubbliche di Tlafcalla, di Huexotzinco, e di Cholollan, quafi turte le Citrà della Valle Mefficana, e le numerofillime Nazioni de' Totonachi, de' Mixtechi, degli Otomiti, de' Tlahuichi, de' Cohuixchi, de' Matlatzinchi, ed altre: ficchè

(E) Cortès fcrive Cuifco, in vece di Cohuixco. L'autore delle note fulle lettere di quel conquiftatore credette, che parlaffe d'Huifuco, perchè non reppe, che v'era una gran Propincia appellata Cohuixco. Huifuco ( preffo i Mefficani Huitzoco) era, ed è anche oggi un luogo ofcuro, non già Provincia grande, ficcom' era, fecondo che afferma il medefimo Cortès, quella di Cuifco. 
chè oltre a'nemici efterni più della metà dell' Imperio avea cofpirato alla fua rovina, e l'altra metà almeno non la favoriva . Lis. X.

Mentre il Comandante Sandoval impiegava il fuo coraggio contro i Matlatzinchi, diede anche prova del fuo contro i Mefficani il Tlafcallefe Chichimecarl. Quelto famofo Gene- memorale, come vide, che gli Spagnuoli dopo la fcoufita fi man-rabile tenevano fulla difefa, determinò di fare un'entrata in Meffico nerale con foli i fuoi Tlafcallefí. Ufci dunque del campo d'Alvarado, dov'era fempre fato infin dal principio dell' affedio ac. Chichimecatl. compagnando gli Spagnuoli in tutti i combattimenti, e fa. cendo ognora fpiccar la fua bravura. Efpugnò quefta volta tutti i folfi, cherano nella ftrada di Tlacopan, e lafciando in guardia del più pericolofo quattrocento arcieri, acciocchè gli afficuraffero il paffo nella ritirata, entrò col groffo delle truppe nella Città, dov'ebbe una terribile zuffa co' Mefficani, nella quale furono uccifi e feriti molti e dell'una,e dell' al. tra parte. Lufingavanifi Mefficani di potere fconfiggerlo nel paffo del foffo, incalzaronlo fortemente nella ritirata; ma mercé gli arcieri polti full'altra riva del foffo, lo ripafso felicemente co' fuoi Tlafcallefi, e ritornò pieno di gloria al loro campo. ( $F)$

E. e 2

I

(F) Bernal Diaz dice, che gli Spagnuoli reftarono dopo la fconfitta abbandonati dagli alleati, i quali: per la paura, che mifero loro i Meflicani con certe minacce, che lor fecero a nome de'lor Dii, fe n' andarono tutti alle loro cafe: che nel campo di Cortès non reftò altri, che il Principe Don Carlo con quaranta Tezcurani: in quello di Sandoval un Signor di Huexotzinco con cinquanta Uumini, ed in quello di Alvarado il General Chichimecatl con 80. Tlafcallefi. Ma ciò. non può effere, perchè due giorni dopo quella fconfitta partì dal campo di Cortès il Capitano. Tapia a far la guerra a' Malinalchefi, e conduffe feco molti Alleati, ficcome afferma lo fteffo: Bernal Diaz. Dodici giorni dopo. Tapia partì dal medefimo Campo il Comandante Sandoval con feffanta mila Alleati, come dice Coriès. Nel tempo in cui Sandoval faceva l'a guerra a'Matlatzinchi, cioè fedici o diciotto giorni dopo la fconfitta, fece la fua famofa entrata il General Chichimecatl, e- non potè farla fenza molre migliaja di Tlafcallefi. Egli è certo, che non fe n'andarono tutti gli Alleati,e che re alcuni fe n'andarono, fubito ritornarono, perchè indi a pochi gioni era ne' tre cam$\mathrm{pi}$, e maffimamente in quello di Cortès un nummero aftai maggiore di quello, che vi era innanzi la fconfitta. Cortès nulla dice di si fatto difertamento; e non è verifimile, che l'aveffe tralafciato nel raccontare al Re. i. fuoi difagj. 
I Mefficani per vendicare l'ardire de' Tlafcallefi affahLis. X. rono una notte il campo d'Alvarado; ma effendo ftati a tempo fentiti dalle fentinelle, corfero all' armi gli Spagnuoli, e gli Alleati. Durò la zuffa tre ore, nel qual tempo avendo Cortès dal fuo campo fenti to una cannogata, $e$ fo. fpettando ciò che in fatti era, gli parve opportuna quefta occafione per fare un'entrata nella Città colla fua gente, ch' era già guarita delle ferite. I Mefficani, ch' erano andati a Tlacopan, non potendo fuperar la refiftenza degli Spagnuoli, ritornarono alla Città, nella quale trovarono l'efercito di Cortès: fi combattè con ardore, ma fenza verun confi. derabile vantaggio nè dell' una, nè dall' altra parte.

In quefto medefimo tempo, quando $v$ 'era maggior bifogno d'armi e di munizioni, approdo un vafcello alla Veracroce, che portò agli $S$ pagnuoli un nuovo foccorfo, col quale fi mifero in iftato di continuar le loro operazioni. Il Principe Don Carlo Ixtlilxochitl avea configliato il Genera le Spagnuolo di non impegnarfi in nuovi affalti, ne' quali dovea patir troppo il fuo efercito: che fenza efporfi a tanti mali, e fenza rovinare i belli edifizj di quella Capitale potrebbe d'effa impadronirfi foltanto coll' impedire l'introda. zione de'viveri; poichè quanto più grande foffe il numero degli affediati, tanto più prefto confumar dovrebbono i pochi viveri, che aveano. Quefto favio configlio tanto più pregevole, quanto men fi dovea afpertar da un Principe, che per la fua età, e per la fua intrepidezza bramava occafroni di far fpiccare il fuo coraggio, piacque tanto a Cortès, che fenza poterfi trattenere corfe ad abbracciarlo, fignificandogli con fingolari efpreffioni la fua graticudine. 'S' appigliò per alcuni giorai ad un tal configlio; ma poi ftanco della lunghezza dell' affedio tornd alle primiere oftilità, ma prima di continuarle offerì di nuovo la pace a' Mefficani, facendo Joro il paragone delle forze degli uni e degli altri, e replicando le ragioni, che a loro a vea già altra volta propofte. I Mefficani rifpofero, che non lafcierebbono mai l'armi, infinattantochè gli Spagnuoli non fe n'andaffero alla lor patria. 
Or vedendo Cortès la rifoluzione de' Mefficani, dopo quaranta cinque giorni d'affedio, e che quanto più gl' invi. Lis. $X$. tava alla pace, tanto più oftinatamente la rigettavano, determinò di non dare un paffo avanti nella Città fenza diftruggere tutti gli edifizj, che v'erano dall' una e dall' altra banda della itrada, così per ifchivare il danno, che da' ter- Meffico, azi faceano alle fue truppe, come per coltrignere oli affe e bravadiati con sł fatte olilità ad accettar le fue propolizioni cune Sollecitò però, ed ottenne da' Signori Alleati alcune migliaja di villani gualtatori forniti degli Atrumenti neceffarj per rovinar gli edifizj, e riempere $i$ folfi. Fece ne' giorni feguenti alcune entrate nella Città co' fuoi Spagnuoli, e brigantini, con più di cencinquanta mila Alleati, diftruggendo edifizj, riempiendo folfi, e fcemando coll'uccifione il numero de' fuoi nemici, benchè non fenza grave pericolo della fua perfona, e della fua gente; poichè poco mancò, ch'egli non foffe fatto prigione, fe non foffe ftato da' fuoi foldati ajutato, e le fure truppe furono talvolta coltrette a fottrarfi con la fuga dal furor de'Mefficani: vi perirono alcuni Spagnuoli ed Alleati, e due brigantini erano già quafi vinti da una flotta di canoe; ma ua altro brigantino, che vi fopravvenne, gli traffe dal pericolo.

Si rendette famofa in quefte entrate la bravura d' alcune donne Spagnuole, $\left(^{*}\right)$ che accompagnarono volontariamente $\mathrm{i}$ lor mariti alla guerra, e co continui difagi, che fotfrivano, e cogli efempj di valore, che aveano ognora d'avanti agli occhj, erano divenute foldati. Faceano la guardia, marciavano co'lor mariti armate di corazza di cotone, di rotella, e di Spada, e fi gettavano intrepidamente in mezzo a'nemici, aumentando malgrado il loro feffo il numero degli Affediatori.

A' 24. Luglio fi fece una nuova entrata nella Citta con

(") Quefte donne s'appellavano Maria d'Efrada, del cui coraggio al bia* mo fatto menzione altrove, Beatrice Bermudez de Velafco, Giovanna Martin, Elifabetta Rodriguez, e Beatrice Palacios. 
con un numero più grande di truppe di quello delle altre

L a. X. volte, $(G)$ e combattendo vigorofamente s'. impadronirono. gli Spagnuoli di quella trada, per la quale comunicava la ftrada larga d' Iztapalapan con qualla di 'Tlacopan: ciò che tanto avea bramato. Cortès per la libera comunitazione del fuo campo con quello d'Alvarado. ESpuznaronó, e riempiro. no parecchj folfi, ed abbruciarono e rovinarono molri edifizj, e tra gli altri un palagio del Re Quauhtemotzin, il qual'era un vafto e forte edifizio circondaro di foff. Delle: quattro parti della Città reltarono quel giorno tre in poter degli Spagnuoli, effendo ftati ridotti gli Affediati alla parte di Tiatelolco, la quale a cagione d'effervi più acqua, era più forte e più ficura.

Da una Dama Mefficana prefa nell' ultimo affalto inte. fe il Generale Spagnuolo il miferabile ftato della. Città per la penuria di vettovaglie, e la difcordia, che era fra gli Affediati; perchè il $R e$, i. fuoi parenti, e molti Nobili s' erano. determinati di morir piutrofto, che arrenderfi; ma il popolo era fcoraggito ed infaltidito dell' affedio. Ciò fu conferma to da due difertori di mediocre condizione, che fpinti, dalla. fame vennero al campo di Cortès.

Per quefte nuove fi rifolvette Cortès: di non: lafciar paffar niun giorno fenza entrar nella Città fin' ad efpugnarla, o, rovinarla; onde vi ritornd a' 25 . col fuo efercito, ed efpugnò una. larga frada, nella qual'era un foflo si: grande, che in tutta la giornata non í potè riempiere o turare. Demolirono o bruciarono tutte le cale, ch'erano in quella parte: a difpetto della refiftenza de nemici. I Mefficani, in vedendo gli Alleati impiegati nell'atterrar gli edifizj: ", Rovinate. 3, pur traditori, lor dicevano, rovinate cotelte cafe, che po" fcia avrete la fatica di rifarle. "Noi, rifpondevano gli 3. Alleati, noi fenz'altro le rifaremo, fe voi folte vincitori;

э), ma.

(G) Dice Cortes, che gli Alleati come videro la felicità delle armi Spagnuole, vennero in si gran copia a fervir nell' affedio, che, non fi potevano numerare. 
j) ma fe folte vinti, voi medefimi le rifarete, acciocchè vi "2abitino i voltri nemici. "Or non potendo i Mefficani Lis. X. riparar gli edifizj, facevano nelle itrade alcune piccole fortificazioni di legno per danneggiare da effe gli Affediatori, ficcome li danneggiavano innanzi da'terrazzi, e per dare impaccio alla cavalleria, empirono la piazza di pietre; ma gli Affediatori fe ne fervirono per turare $i$ folli.

Nell' entrata, che vi fi fece a' 26. ', 's' efpugnarono due grandi folfi, che avevano fatto di nuovo i Meflicani. Alvarado per la fua parte 's andava avanzando ogni giorno più nella Città, ed addi 27. s'inolrrò tanto, efpugnando parecchj folf e trinciere, che giunfe ad occupar due torri vicine al palagio, dove rifiedeva il $\mathrm{Re}$ Quauhremorzin; ma non potè quindi paffare avanti, ficcome voleva, a cagione della fomma difficolià, che vi trovò ne' folfi, e della gagliarda re. fiftenza de nemici, i quali lo coltrinfero a retrocedere incalzando furiofamente la fua retroguardia. Cortès, avendo offervato una ftraordinaria fumata, che s'inalzava da quelle torri, e fofpettando ciò che in fatti era avvenuto, entrò come. foleva nella Città, ed impiegò tutta la giornata nell' accomodare tutti i paffi cattivi. Non gli mancava già più d'un canale e d'una trinciera per entrar nella piazza del mercato. Determinoffi d'inoltrarfi infin lá, ficcome in fatti l'ottenne, e quella fu la prima volta dopo cominciato l'affe. dio, che convennero le truppe di lui con quelle di Alvarado con indicibil contento degli uni, e degli altri. Entrò Cortès con qualche Cavalleria in quella piazza, e vi trovò, innumerabil popolo alloggiato ne'portici, perchè non poteva. no capir nelle cafe di quella contrada. Sali ful tempio, donde offervò la Città, e riconobbe, che delle otto parti d'effa una foltanto reftava da efpugnare. Fece attaccar il fuoco all' alte e belle torri di quel tempio, nel quale, ficcome in quello maggior di Tenoctitlan era adorato l'idolo del Dio della guerra. La Plebe Mefficana in vedendo quella gran famma, che pareva elevarfi infin al Cielo, fece un gran pianto. Cortès mollo a pietà dal vedere un si numerofo po. polo 
polo a tale tretto ridotto, fece ceffar quel giorno tutte l 0 : Lis. X.ftilità, e parlar di nuovo agli Affediati, acciocchè s'arrendeffero; ma eglino rifpofero, che nol farebbero mai, che cafo che un fol Mefficano reftaffe vivo, quegli continuerebbe la difefa fin'a morire.

Paffati poi quattro giorni fenza combattimento entrò di

5. 30 . nuovo Cortès nella Città, e vi fi abbattè in una gran folStatola- la d'uomini miferabili, di donne, e di fanciullideboli, magrimero- cilenti, e quafi moribondi dalla fame, la quale era tanMeffica- to grande, che molti viveano foltanto d'erbe e di radici ni. paluitri, d'infetti, ed anche di fcorze d'alberi. Moffo quel Generale a pietà comandò alle fue truppe, che non faceffe. ro loro verun male: palsò alla piazza del mercato, e vi trovò i portici ripieni di gente difarmata : indizio manifefto dello fcoraggimento del popolo, e del difpiacere, con cui tollerava l'oftinazione del Re, e della Nobilià. La maggior parte di quella giornata fi pafsd in negoziazioni della pace; ma vedendo Cortes, che ciò nulla giovava, diede ordine al Comandante Alvarado d'inoltrarfi armata mano per una gran. de ftrada, dov' erano pir di mille cale, ed egli con tutto il fuo efercito andd ad affalire da un' altra banda. Fu sł grande la Atrage, che fecero quel giorno negli Affediati, che tra morti e prigionieri furono più di dodici mila. Gli Alleati incrudelivano a tal fegno contra quelle infelici vittime, che non la perdonavano nè ad età, nè a feffo, non baftando a trattener la lor crudeltà gli ordini feveri del Go: nerale.

Il di vegnente ritornò Cortès con tutte le fue forze; ma comandd, che non fi faceffe verun male agli Affediati, cosi per la compaffione, che gli cagionava ta vilta delle loro miferie, come per la fperanza, che avea d'indurli ad arrenderfi. I Mefficani, vedendo venir contra loro un si gran numero di truppe, e tra effe gli fteff lor fudditi che già lor fervivano, ed ora gli minacciavano della morte, trovandofi ormai a tanta miferia e calamità ridotti, ed avendo d'avanti agli occhj tanti oggetti lagrimevoli ; poi- 
chè neppare aveano dove mettere il piede, fe $n$ on fopra cadaveri de' loro Cittadini, sfogarono in orrendi clamori la $L_{18} . X$. lor rabbia, ed addimandavano la morte come l'unico rimedio a' loro mali. Pregarono Cortés alcuni Plebei d'abboccarfi con certi Nobili, che difendevano una trincie ra per trattar di qualche accomodamento. Cortès fi poriò ad effi, benchè poca fperanza aveffe di riúcire. Erano appunto coftoro di quelli, che non potevano pir̀ comportare i difagj dell' affedio: onde allorchè videro accoftarfi a loro il Generale Spagnuolo, moft dalla difperazione gli differo: "Se voi fiete " frgliuolo del Sole, fiecome alcuni fi vanuo immaginando, ") perchè effendo voftro Padre si veloce, che nel breve Ipa"zio d'un gierno termina il fuo corfo, voi ftate tanto a "liberarci da tanti mali colla morte. Vegliamo pur morire " per andare al Cielo, dove ci alpetta il noftro Dio Hui" tzilopochtli per darci il ripofo delle noftre fatiche, ed il (3) premio de' noftri fervizj. "Cortès propofe loro varie ragioni per muovergli ad arrenderf; ma rifpondendo coloro, che nè ciò era in lor podeftà, nè aveano fperanza di poter perfuaderne it $\mathrm{Re}$, fi ritird per follecitarlo per mezzo d'un illuftre Perfonaggio, cui tre giorni innanzi avea fatto pri. gioniere, ed era un Zio del Re di Tezcuco. Fu colui, ben. chè ferito, incaricato di portarfi a Tlatelolco a trattar quell' affare col Re; ma non fi vide altro fiutto della fua ambafciata, che gl' iterati clamori, co'quali addimandava il popolo la morte. $(H)$ Alcune truppe Mefficane fi fcagliavano difperatamente contro gli Spagnuoli; ma erano così indebo. lite dalla fame, ch' era poco il male, che faceano, e troppo grande quelio, che aveano da'loro nemici.

Ritornd Cortès il giorno feguente alla Città, afpettan. do ad ogni momento, che s'arrendeffero i Mefficani, e fen. za permettere, che fi faceffe loro verun danno s'indirizzò a Storia Antica del Meffeco Tom. III. F f cer-

(H) Si diffe, fecondochè fcrive Cortès, che tofto che quel Signore fi prefentò a Quauhtemotzin per efporgli le propofizioni di pace, fu facrificato fer ordine di quel Re; ma ficcome queftu fatto non ha altro fondamento, che quel rumore vago, non è degno della noßra fede. 
certi uomini principali, ch' erano in una trinciera, già noti LIB. X.a lui infin dalla prima volta, che fu a quella Corte, e loro addimandò perchè volevano sì oftinatamente difenderfi,

Diligen- non potendo più refiftere, e trovandof in tale ftato, che ze inuti-con un fol colpo potrebbe toglier la vita a tutti. Coloro li per la rifpofero, che vedevano beniffino, ch' era inevitabile la: lo-s Meflica- ro rovina, ed avrebbon voluto impedirla; ma non potevano, mi. perchè non toccava a loro il deliberare. Nondimeno fi efi-: birono di pregare il Re di voler fentire le propofizioni di pace. In farti fi portarono incontanente a palagio, ed indi a poco tornarono dicendo, che perchè era tardi non poteva il $\mathrm{Re}$ venire, e che non dubitavano, che il di dappreffo farebbe con Cortès in quella medefima piazza. Era nel centro d'effa un gran terrapieno quadro, dove facevano i Mefficani le loro rapprefertazioni teatrali, ficcome abbiamo detto altrove. In quefto teatro fece metter Cortès de' tappeti, e delle predelle per tenervi la bramata conferenza, e fece apparecchiare un buon pranzo pel $\mathrm{Re}$, e per la Nobiltà che dovea accompagnarlo. Venuto il giorno, fece avvifare il $R e$, che l'afpettava in quel luogo; ina il Re gli mandd cinque. Perfonaggi affai autorevoli, fcufandofi di venire in perfona per certa indifpofizione che avea, e perchè non fi fidava degli Spagnuoli. Cortès gli accolle con fomma benignità; diede loro un decorofo banchetto, e gli rimandd al $\mathrm{Re}$, affinchè lo pregaffero a nome luo di voler venire in quel luogo fenza paura; poichè egli impegnava la fua fede di rifpettar, come fi conveniva la fua Real perfona: che la. fua prefenza era afiolutamente neceffaria, e fenza effa non fi poteva conchiuder nulla, ed accompagnò queft a mbafciata con un prefente di vetrovaglie, ch' era allora it più pregevole. Gli Ambafciatori, dopo aver fatto conofcere nel. pranzo la gran neceffita, che pativano fe n'andarono, ed indi a due ore ritornarono, portando a Cortès un regalo di vefti finiffime, che gli mandava il $\mathrm{Re}$, e replicando le fuddette fule. Tre giorni vi paffarono in quefte negoziazioni fenza verun frutto. 
Cortès avea dato ordine agli alleati di ftarfene fuori - della Città, perchè i Mefficani l' aveano pregato di non per. Lis. X. mettere; che v' interveniffero, allorchè fi teneffe. la con. ferenza col Re; ma ora avendo perduta ogni fperanza d'ac- T.32. comodamento; richiamò tutte le truppe del fuo Campo, nel- conflito, le quali erano più di cencinquanta mila uomini, e quel. edorrenle ancora del campo d'Alvarado, e con turte quefte forze de' Mefo infieme fi:mife a combattere certi foff e trinciere, ch' erano ficani. 'le maggiori fortificazioni, che reftavano a'Mefficani, e ad un ora Sandoval col fuo efercito affali la Città dalla banda di Tramontana. Quefta fu la giornata più infautta per quella Citis, nella quale fi fparfe più copiofamente che mai il fangue Mefficano, non avendo ormai quegli infelici Cittadini nè armi per rifpignere la moltitudine e la furia de' lor nemici, nè forze per difenderfi, nè anche terra per combattervi. Il fuolo della Città era coperto di cadaveri, e l'acqua de'foffi e dé canali era tinta di fangue. Non fi vedeva altro, che rovina e ftrage, nè altro sì fentiva, che pianti lagrimevoli, e clamori di difperazione. Gli Alleati incrudelirono talmente contra quella gente miferabile, che più s'affaticavano gli Spagnuoli nel contener la lor crudelta, che nel combattere co' loro nemici. La Arage, che in quella giornata fi fece ne' Mefficani fu si grande, che tra morrie prigionieri, per quel che dice lo fteffo Cortès, olırepafsò le quáranta mila perfone.

L'intollerabil fetore di tanti cadaveri infepolti coftrinfe allora gli Affediatori a ritirarfi dalla Citià ma il giono feguente ( 13 . Agofto) vi ritornarono per dar l'ultimo alfalto a quel cantone di. Tlatelolco, che ancor reftava in po. ter de' Mefficani . Conduffe feco Cortès tre cannoni d'arriglieria, tà, e de' e tutte le fue truppe. Affegnò a ciafcheduno de' Capitani il Re. luogo, d'onde dovea dar l' affalto, e comandò loro, che $s$ adoperaffero quanto lor foffe poffibile per coltrignere gli Affediati a gittarfi all' acqua verfo quel luogo, dove afpetrava Sandoval con tutti i brigantini, il qual' era una 'fpezie di porto circondato dapertutto di cafe, dove approdavano le Ff 2 bar. 
barche mercantili de' Negozianti, the venivano al Mercato E18. X. di Tlatelolco: e foprattutto che procuraffero pigliare il Re Quauhtemotzin; poichè ciò. baftava per rendergli padroni della Città, e por fine alla guerra; ma prima di venipe a quefto: colpo decifivo, fece nuovi tentativi per ridur gli Affediati a qualche accomodamento. Fu pure a cio indotto non folamente dalla compaffione di tanti miferabili, ma eziandio dal defiderio d'impadronirfi de' tefori del $\operatorname{Re}$, e della Nobiltà; imperciocchè fe a prendeva per affalto quell? ultima parte della Citta, i Meflicani privi d'ogni Iperanza di fcampar le loro ricchezze, potrebbero gettarle nel lago, affinchè non le godeffero i vincitori, e cafo che nol faceffero, farebbono effe pigliate dagli Alleati, i quali come quelli ch' erano innumerabili, e più pratici delle cafe, poco.o niente lafcierebbono agli Spagnuoli nello fcompiglio e nelia confufion dell' affalto. Tornè perciò a parlar da un luogo eminente a certi Mefficani ragguardevoli da lui ben concofciuti, rapprefentando loso l' eftremo lor pericolo, e pregar. doli di far nuove iftanze al: $\mathrm{Re}$, acciocchè fi piegafe a quell' abboccamento tanto da lui bramato pel bene del Re medefimo, e di tutti i fuoi fudditi; poichè fe fi mantene. va ancora nel propofito di difenderfi, egli era determinato di non lafciar quel giorno un Meficano vivo. Due di quei Nobili s' incaricarono di perfuadere il Re, ma appena partiti ritornarono, accompagnando il Cibuacaatl, o fupremo $\mathrm{Ma}$ giftrato della Corte. Fu accolto da Cortes. con fingolari dimoftrazioni d'onore e di cordialita, ma egli con un' aria fignorile, colla quale pareva, che moftrar voleffe il fuo animo fuperiore a tutte le calamità, parłò così a Cortès.: "Ri„S Sparmiatevi, - Generale, la fatica di follecitar l' abboc" camento col mio Re e Signore Quauhtemotzin. Egli \&े "rifoluto di morir piuttofto, che comparire innanzi a "voi. Non faprei dirvi, quanto mi rincrefca una tal rifo. "luzione; ma non v'è rimedio. Voi per tanto prendete " quel configlio che più vi piace, ed operate giufta i vo. "Ari difegni. "Cortès gli diffe, che andaffe pure a difpos- 
re gli animi de'fuoi Cittadini alla morte, che tolto dovrebbono foffirire.

Erano frattanto venute a renderfi agli Spagnuoli truppe numerofe di donne, di fanciulli, e di gente melchina, affrettandofi a gara per fottrarfi da quell' eftremo periglio, ed annegandofi alcuni di quelli, che fi sforzavano di paffar a nuoto $\mathrm{i}$ folfi, per la fomma loro debolezza. Cortès comandd, che non fi facefle male a coloro che s'arrendevano; anzi comparti in parecchj pafti alcuni Spagnuoli, acciocchè collo loro autorità raffrenaffero la barbara crudelià degli Alleati; ma a difpetto de'fuoi ordini perirono tra le mani di quelle inumane e furiofe truppe più di quindici mila tra uo. mini, donne, e fanciulli.

I Nobili e gli uomini mrlitari che s'erano oftinati nella rifoluzione, di difenderfi infin' all' ultimo refpiro, occupavano i terrazzi delle cafe, ed alcune felciate. Cortès veden. do, che era tardi, e non volevano arrenderfi, fece giocar l' artiglieria contro loro, e ciò non baftando, fece con un' archibutata fegno per l'affalto. Affallirono tutti ad un'ora gli Affediatori, e ftrinfero a tal fegno i deboli ed afflitti Cittadini, che non reftando a loro nella Gitta verun luogo, dove poteflero difenderfi dalla furia d' un si gran numero di nemici, fr gettarono molti nell' acqua, ed altri venivano a darfi a'vincitori. Aveano i Meficani alleftite delle barche per fottrarfi colla fuga dal poter degli Spagnuoli; ma Cortès accortofi di un tal futterfugio avea dato b'ordine a Saudoval d'impoffeffarfi co'brigantini del porto di Thatelolco, e di pigliar le barche. Malgrado della fomma diligenza di Sandoval molte barche fcapparono, e tra l'altre quella appunto, che portava le Perfone Reali. Effendofene avveduto quelL'attivo Comandante, ordind a Garcia d'Holguin, Capitano del più veloce brigantino, di dare ad effa la caccia; il quale fiede tal fretta, che in breve le raggiunfe, ed apparecchian. dofi gli Spagnuoli, a far fuoco contro i fuggitivi, coftora fermarono i romi, e gettarono l'armi in contrafegno d'arrenderfi. Erana fogra quella gran barca o piragua . Il Redi 
Meffico Quauhtemotzin, la Regina Tecuichpotzin fua fpofa

Lis. X. il Re d'Acolhuacan Coanacotzin, ed il Re di Tlacopan' Terlepan. quetzaltzin con altri Perfonaggj. Abbordò il brigantino, ed il Re di Meffico, avanzandofi verfo gli Spagnuoli, diffe a quel Capitano: "Io fono, o Capitano, voltro prigioniere, nćal. " tra grazia da voi richiedo, fe non che abbiare alla ke. " gina mia Spofa ed alle fue damigelle quel riguardo, che "fi dee al lor feffo, ed alla lor condizione: "e prendéndo per la mano la Regina, pafso con effo lei al brigantino. Offervando poi, che il Capitano Spagniolo era follecito delle altre barche, gli diffe, che non dubitaffe, che turti tofto che fapeffero, il lor Sovrano effer prigione, verrebbono a mo. rir con lui.

Conduffe il Capitano Holguin quegl' illuftri prigionieri a Cortès, il quale trovavafi allora nel terrazzo d' una cafa di Tlatelolco. Gli accolfe con tutte le dimoftrazioni d'ono. re e d'umanità, e gli fece federe. Quauhtemotzin con gran. d'animo gli diffe: "Ho fatto, o prode Generale, e per la " miá difefa, e per quella de' miei fudditi tuttociò, che da " me richiedevano l'onor della mia Corona, e lo zelo pel "mio popolo; ma per effermi ftati contrarj gli Di mi " veggo ora privato della corona e della libertà. Io fono "voftro prigioniere; difponete a voftro piacere della mia "perfona: " e ponendo la fua deftra fopra un pugnale chie portava Cortès alla cintola, foggiunfe :, toglieremi con " quefto pugnale una vita, che non perdei nella difefa del "mio Regno. "Cortès fi sforzò di confolarlo con buone ragioni, proteftandogli che nol confiderava come fuo prigioniere, ma come prigioniere del più gran Monarca dell' ELropa, nella cui clemenza confidar doveva, che non che la liberrà, che per difgrazia avea perduta, ma gli farebbe an. che reftituito il trono de' fuo chiariffimi anteati, ch' egli avea si degnamente occupato, e difefo. Ma che conforto poteva avere da si fatte protefte, o che fede poteva dare alle parole di Cortès colui, ch' era ftato fempre fuo nemico, avendo veduto, che al Re Motezuma non baftò l' effere fuo 
amico e protettore per non perder la corona, la libertà, e la vita? Egli richiefe da Cortès, che non fi faceffe verun Liz. $X$. male a fuoi fudditi, $e$ Cortès richiefe vicendevolmente da lui, che comandaffe, che turti $i$ fuoi fudditi s'arrendeffero. L'uno e l'altro diedero i loro ordini, e tutti e due furono prontamente ubbiditi. Fu ordinato altresh, che tutti i Mefficani ufciffero dalla Citrà fenz armi, e fenza carica, e fecondochè afferma un reltimonio oculato e finceriffimo ${ }^{*},\left(^{*}\right)$ tre giorni e tre notti fi videro turte le tre ftrade piene d'uomini, di donne, e di fanciulli aeboli, macilenti, e lordi, che ne utcivano per andare a ricoverarfi in altri luoghi. Il fetore, che rendevano tante migliaja di corpi putrefatti ed infepolti era, si intolerabile, che cagiono qualche indifpofzione al Generale de'Conquiftatori. Le cale, le itrade, ed i. canali crano pieni di cadaveri sfigurati: (I) il fuolo della Città fr trovò in alcune parti fcavato da' Cittadini, che cercavano fotto terra delle radici, di cui cibarfi, e molii albesi fi videro fcorricati per provvedere alla eftremità della fame. Fece il Generale feppellire $i$ cadaveri, e bruciar per tutta la Città un'immenfa quantità di legne, così per purgar quell' aria infetta, come per celebrar la fua vittoria.

Sparfafi fubito per tutta la terra la nuova della prefa della Capitale, diedero ubbidienza a Cortès. le provincie dell' Imperio, benchè non vi mancarono alcune, che anche due anni dopo travagliarono colla guerra gli Spagnuoli. Gli

Allea -

(") Bernal- Diaz del Caftillo.

(I) "Es verdad, y Ju:o amen, que toda la laguna, y cafas, y barba" coas eftaban llenas de cuerpos y cabezas de hombres muertos: que yo " no sè de que manera lo efcriba: pues en las calles, y en los mifmos "patios de Tlatelulco no habia otras colas, y no pudiamos andar, fina " entre cuernos y cabezas de Indios muertus. Yo he leido la deftruicion "; de Jerufalen; mas fi en ella hubo tanta mortandad como efta yo no lo " sè \&c. "Bernal Diaz nel cap. is6. della fua ftoria. Taliefpreffionid'un teftimonio oculato tanto fincero, e che non fapeva efagerate, danno qualche idea di quell'ortenda ftrage. Io forpetto, che da' Mefficani lafciatiforfern a bella poffa infepolti i cadaveri, per ifcacciar colla puzza gli Affediatori: nè poffo alrro perfuadermi, attefo la fomma premura di quelle Nazioni nell' efequie de' lor defunti. 
Alieati ritornarono a loro paefi oltremodo lieti della preda; Lis. X. e contenti d'aver meffo in conquaffo quella Corte, la cui dominazione non potevano comportare, o de cui armi ti tenevano in una perpetua inquietudine, fenz accorgerf, che colle proprie lorn armi fi fabbricavano le catene, che doveano ftrignere la loro libertà, e che rovinato quell' Imperio, doveano reftare avvilite tutte le altre Nazioni.

Il bottino fu affai minore di quello, che fperavano i vincitori. La roba da veftire, che vi fi trovo fu divifa per la maggior parte tra gli Alleati. Quei lavori d'oro; d'argento, e di penne, che pel fingolate loro artifizio fi conlervarono interi, fi mandarono in prefente all' Imperator Carlo V. Tutto il relto dell' oro, che fi fece fondere, appena arrivo a 19. 200. oncie, $(K)$ cosi perchè i Mefficani ne gettarono la maggior parte nel lago, $(L)$ come perchè i particolari tanto Spagnuoli, quanto i loro Alleati procurarono nel faccheg: giar la Città ricompenfarfí nafcofameate delle loro fatiche.

La préa di quella gran Corte accadde a' 13. Agofto I521. cento novanta fei anai, dappoichè fu fondata dagli Aztechi, e cento feffanta nove anni dappoichè fu eretta in Monarchì, la quale reffero undici Re. L'affedio di Meffico paragonabile neile difgrazie e nella itrage con quallo di Ge. rufalemme, duro fettanta cinque giorni, nel qual tempo di dugento mila e più Alleati ne perirono alcune migliaja, e di novecento Spagnuoli più di cento. Il numero de' Mefficani morti non fi fa; ma attefo il ragguaglio di Cortès e di Ber. nal

(K) Corrès dice , che l'oro che fi fece fondere pefava 130. 000 Caftellanos, che fanno 19. 200 oncie. Bernal Diaz dice, che quell oro importó 380. 000 pefos, it che pare effer più. Tra le fpoglie, che fi mandarono 2 Carlo V. v'erano perie d'enorme grandezza, gemme pregevoliffime, e lavori maravigliofi d'oro. La nave, fulla quale fi mandarono, fu predata da Giovanni Florin famofo Corfale francele, ed il teforo fu mandato alla Corte di Francia, la quale autorizzava si fatte ruberie fotto il ron men celebre che frivolo preferto d'effere it $\operatorname{Re}$ Criftianiflimo figliuolo d'Adamo al pari del Re Cattolico.

(L) Bernal Diaz dice, che egli vide cavar dal lago alcune cole d'oro, e tra le altre un fole fimile a quello, che mando il Re Motezuma a Cortès, allorchè coftui fi trovava nella fpiaggia di Chalchiuhcuecan. 
nal Diaz, e ciò che ne dicono altri Storici pare, che gli uccifi freno ftati più di cerro mila. Intorno poi a quelli, Lis. X. che morirono di fame, o d' infermita cagionata dall' acqua falmaltra, cho beevano, o dall' infezion dell'aria, il mede. fimo Cortès afferma, che furono più di cinquanta mila. La Città reltò quafí tutta rovinata. Il $\mathrm{Re}$ di Meffico, malgra. do le magnifiche promeffe del Generale Spagnuclo, fu dopo pochi giorni meffo ignominiofamente alla tortura ( la qual tollerò con invitra coftanza) acciocchè dichiaraffe, dov'erano le immenle ricchezze della Corte, e de' tempj: (M) ed indi a tre anni fu per certi fofpetti impiccato infieme col Re di Tezcuco, e di Clacopan. (N) I Melficani con tutte Storia Antica del Mefjeco Tom. III. $\mathrm{G} g$ le

(M) La tortura, che fiede al $\operatorname{Re}$ Quauhtemotzin, fu quella di bruciargli a poco a poco i piedi dopo avergli unti d'olıo. Accompagnollo, e mori ne'tormenti un intimo di lui privato. Bernal Diaz aggiunge, che fu ancora tormentato con efro lui il $\mathrm{Re}$ di Tlacopan. Cortès fuo malgrado s'appigliò a si indegna e barbara rifoluzione per condifcendere alle iftanze d alcuni ingordi Spagnuoli, i quali infolpettirono, ch egli non volefemetere il Re alla tortura per approfittarfi fegretamente di tutto il teforo Reale.

(N) Quauhremotzin Re di Meffico, Coanacotzin Re d'Acolhuacan, Tetlepanquetzaltzin Re di Tlacopan furono appiccati ad un albero per fentenza di Cortès in Izancanac, Città capitale della Provincia di Acallan in uno de' tre giorni precedenti la quarefima dell'anno I525. La cagione della loro morte fu certo difcorfo, ch'ebbero fra loro fulle loro difgrazie, infinuando quanto agevole lor farebbe, fe voleffero, ammazzare Cortès, e tutti gli Spagnuoli, ed il ricuperar la loro libertà, e le loro corone. Un traditor Mefficano per procacciarfi la grazia del Generale Spagnuolo, gli diede contezza di tutto, alterando il fenfo delle parole, e rapprefentando come una congiura ordinata ciò che non era ftato altro che un mero difcorfo in aria. Cortès, il quale era allora in viaggio verfo la provincia di Coma abua con pochi Spagnooli indeboliti dalla fatica, e con piu di tre mila Meflicani che conduceva feco, fi perfuafe, clie non v'era altro rimedio per ifchivate il pericolo, da cui fi credeva minacciato, fe non quello di-far morire i tre Re. Qucha efecuzione, dice Bernal Diaz, fu troppo inginfta e liafomata da tutti noi, che con lus viaggiavamo in quella gionata. Cagionò a Cortès una gran malinconia, ed alcune vegghie. Il medefimo Autore foggiunge, che il P. Gio: di Varillas Frate delld Peligione della Madonna della Mercede li confeso e confortò nel fupplicio: ch' eglino erano buoni Criftiani, e che morirono ben difpofti: ond'è manifefto, ch'erano Rati battezzati, ma fra tanti ftorici del Meflico, non ve n'è uno she fáccia menzione d'un avvenimento sì noiabile, e si gloriofo, come quello del battefimo di quefti tre Re, empiendo peraltro tante pagine e tanti 
le Nazioni, che contribuirono alla loro rovina, reftarono a Lis. X. difpetto delle criftiane ed umaniffime difpofizioni de' Re Cattolici, abbandonati alla miferia, all'oppreffione, ed al dif. prezzo non che degli Spagnuoli, ma anche de' più vili fchiavi Africani, e degl' infami lor dircendenti, caftigando Dio nella miferabil pofterità di quelle Nazioni 'l' ingiuftizia, la crudeltà, e la fuperftizione dé loro antenati: orrendo efempio della giuftizia divina, dell' inftabilità de' Regni della terra.

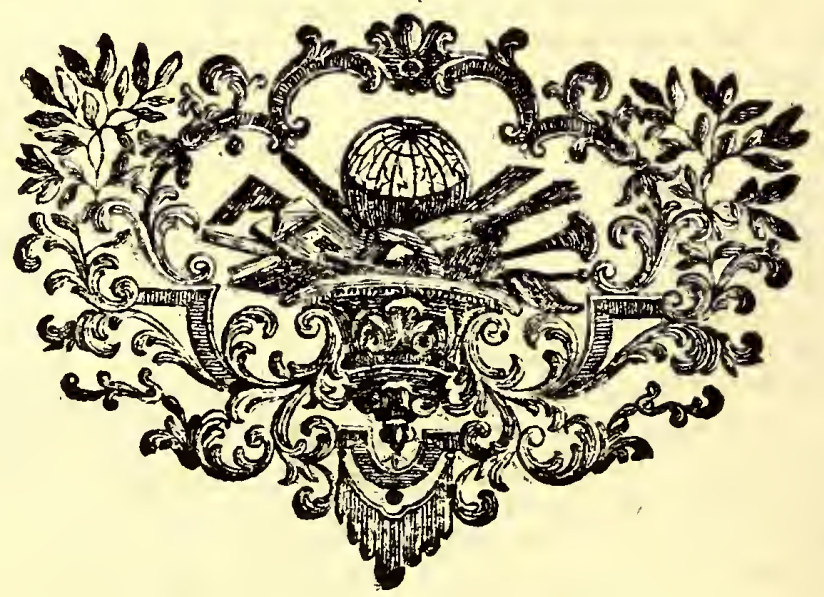

DI.

fogli di bagattelle : e quel ch' è peggio, Torquemada il quale faticò venti anni nella ftoria del Mefrico, ed ingrofsd i fuoi tre ftupendi volumi co' ragguagli della fcoperta delle Ifole di Salomone, delle rivoluzioni delle Filiprine, della perfecuzione del Giappone, e di mille altre cofe fuor di propofito, non fa neppur cenmo della converfion di que' Re. 


\section{I S C E N D E N Z A}

\section{DEL RE MOTEZUMA}

MOTEZUMA Re IX. di Meffico, ammogliato con Miabuaxocbitl fua nipote

Don Pietro Jobualicabuatzin Motezuma, ammogliato con Donna Caterina Quauxocbitl fua nipote

D. Diego Luigi Ibuitemotzin Motezuma, ammogliato in Irpagna con Donna Francefca della Cueva.

D. Pietro Tefifon Motezuma della Cueva, I. Conte di Motezuma,

di Tula , e Vifconte d'Iluca, a mmogliato con Donna Girola ma Porras.

D. Diego Luigi Motezuma e Porras II. Conte di Motezuma \&cc., ammogliato con Donna Luigia Jofre Loaifa e Carrillo, figlia del Conte dell' Arco.

Donna Maria Girolama Motezuma Jofre de Loaifa III. Conteffa di Motezuma \&c. maritata con D. Giufeppe Sarmiento de Valladares, il quale fu Vicerè del Meflico, e I. Dica d' Atrifco.

Donna Faufta Du- Donna Melchiorra menica Sarmien- Sarmiento Moteto Motezuma, IV. zuma, V. ContefConteffa dí Mo- fa di Motezuma, tezuma, morta morta fenza fucfanciulla in Mer- ceffone nel 1717. fico nel 1697 . onde ricaddero gli Stati di Motezu$\mathrm{ma}$ in Donna Terefa Nieto \&c. figlia del I. Matchere di Tenebron

Donna Terefa Francefca Motezuma e Porras maritata con D. Diego Cifneros de Guzman.

Donna Girolama de Cifneros Motezuma, maritata con D. Felice Nieto de Silva, 1. Marchefe di Tenebron.

Donna Terefa Nieto de Silva e Motezuma, II. Marchefa di Tenebron, e VI. Conteffa di Motezuma \&ce. maritata con D. Gafparo d'Oca Sarmiento e Zuńiga .

D. Girolamo d'Óca Motezuma \&c. III. Marchefe di Tenebron, e VII. Conte di Motezuma, ammogliato con Donna Maria Giufeppa de Mendoza.

D. Gioacchino d'Oca Motezuma e Mendoza, VIII. Conte di Motezuma, IV. Marchefe di Tenebron e Grande di Spagna, oggi vivente.

Vi fono tanto in Ifpagna, quanto in Meffico altri sami di quefta nobilifima firpe. 


\section{I S C E N D E N Z A DI FERDINANDO CORTE'S}

D. FERDINANDO CORTE'S, Conquiftatore, Governatore, e Capitano Generale del Meffico, I. Marchefe della Valle d'Oixaca, ammogliato in feconde nozze con Donna Giovanna Ramirez d'Arellano e Zuñiga, figlia di D. Carlo Ramirez d'Arellano, II. Conte d' Aguilar, e di Donna Giovanna de Zuniga, figlia del Conte di Bañares, primogenito di D. Alvaro de Zuñiga, I. Duca di Bejar. Fu loro figlio ${ }^{(*)}$ I.

D. Martino Cortès Ramirez d'Arellano, II. Marchele della Valle, ammogliato colla fua nipote Donna Anna Ramirez d'Arellano. Furono lor figli I 1 .

I D. Ferdinando Cortès Ramirez d'Arellano, III. Marchele della Valle, ammogliato con Donna Mencia Fernandez de Cabrera e Mendoza, figlia di D. Pierro Fernandez Cabreta e Bobadilla, II. Conte diChinchon, e di Donna Maria de Mendoza, e della Cerda, forella del Principe di Melito. Non ebbe D. Ferdinando altro, che un figliuolo, il quale mor fanciullo: onde gli fuccedette il fuo frateHo

2 D. Pierro Cortès Ramirez d'Arellano, IV. Marcheie della Valle, anmogliato con Donna Anna Pacheco della Cerda, forella del II. Conte di Montalban. Morì fenza figli, e però gli fuccederte la fua Sorella

3 Donna Giovanna Cortès Ramirez d'Arellano, V. Marchefa della Valt le, maritata con Don Pietro Carrillo de Mendoza, IX. Conte di Priego, Affiftente e Capitano Generale di Siviglia, Maggiordomo maggior della Regina Donna Margherita' d'Auftria. Fu lor figlia

I I I.

Donna Stefani.a Carrillo de Mendoza e Cortès, VI, Marchefa della Valle, moglie di D. Diego d'Aragon, IV. Duca di Terranova, Principe di Caftel Vetrano, e del S. R. I., Marchefe d'Avola, e della Favara, Coneftabile ed Almirante di Sicilia, Commendatore di Villafranca, Vicerề di Sardegna, Cavaliere dell infigne Ordine del Toron d'Oro. Fu lor figlia, unica

\section{I $V$.}

Donna Giovanna d'Aragon Carrillo de Mendoza e Cortès, $V$ V Da chefra di Terunova, e VIl. Marchefa della Valle, Cameriera, maggior della Regina Donna Luigia d'Orleans, e poi della Regina Donna Marianna d'Auftra, maritata con D. Ettore Pignatelli, V. Duca di Montelione, Principe di Noia, Marchele di Cerchiara, Conte di Borello, di Caronia, e di Santangelo, Vicerè di Catalogna, Grande di Spagna \&c. Fu lor figlio unico

(*) Oltre all' erede del Marchefato ebbe il Conquiftatore Cortès parecchj altri figli, parte legitimi, e parte battardi . I légittini furons r. Douna Maria Cortès \&c. Inaritata con D. Luigi de Quignones V. Conte di Lina. 2. Donna Caterina motta in Sivit glia. 3. Donna Giovanna moglie di D. Ferdinando Enriquez de Ribera, II. Duca d'AlEalà, Marchele di Tarifa, Conte de los Molares'. 4. Donna Eleonora anınogliata in Meficó con. Giovanni Tolofa, Bifcaglino. I baftardi furono. I- D. Martino Cortes natogli dalla fanofa Donna Marina, Cavaliere dell' ordine di S. Giaconn. 2. D. Luigi antogli da.

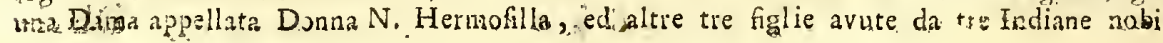


$\mathrm{V}$.

D. Andrea Fabrizio Pignatelli d'Atagon Cartillo de Mendoza a Cortès.VI. Duca di Montelione, VI. Duca di Terranova, VIII. Marchẹe della Valle, Grande di Spagna, Gran Camerlingo del Regno di Napoli, Cavaliere del Toron d'Oro \&c., ammogliato con Donna. Terefa Pimentel e Benavides, figlia di D. Antonio Alfońfo Pimentel de Quinones, XI. Conte di Benavente, di Euna, e di Mayorga, Gr. di Spazna \&c., e di Donna Elifabetta Francefca de Bẹnàides; Ill. Marclíefa di Javalquinco, e di Villareal. Fu lor figlia $=$ V I.

Donna Gicranna Pignatelli d'Aragon, Pimentel, Carrilto de Mendo' za e Cortès, VIL. Duchefra di Montelione, VI. Duchefa di Terranova, IX. March. della Valle, Gr. di Spagna-\&xo moglie dit D Niccold Pigna. telli de'Principi di Noia, e di Cerchiara, Principe del S. R. 1., Viçerè di Sardegna, e di Sicilia, Cavaliere del Tolon d'Ora \&c. Fu lor figlio $\mathrm{V} I \mathrm{I}$.

D. Diego Pignatelli d'Aragon \&c. VIII? Duca disMontelione, VIII. Duca di Terranova, X. March. della Valle, Grabd' Almiratite, Coneftabile del Regno di Sicilia, Gr. di Spagna, Principe del S. R. I. Cavaliere del Toron d'oro \&c., ammogliato con Donna Margherita Pignatelli 'de' Duchi de Bellofguardo. Fil lor figlio

V I I I.

D. Fabrizio Pignatelli d'A Aragon \&c. IX. Duca di Montelione, IX. Duca di Terranova, XI, March. della Valle, Gr. di Spagna, Principe del S. R. 1. \&c., ammogliato con Donna Coftanza Medici dei Principi d'Ottajano. Fu lor figlio

\section{X.}

D. Ettore Pignatelli d'Aragon \&c., X. 'Dúca di Montelione, X. Duca di Terranova, XII. Marchefe della Valle d'Oaxaca, Grande di Spagna, Principe del S. K. I. Vive prefentemente in Napoli ammogliato con Donna N. P'iccolomini de' Duchi d'Amalfi .

Da quella nobilifuma coppia, che abbiam mefra fotto il num. VI., nacquero quatro figli', Diego, Ferdinando, Antonio, e Fabrizio, ed altrettante figlie, Rofa, Maria Terefa, Stefania, e Catetina .: 1. D. Diego fu l'erede del Marchefato della Valle, o fia del. Vaglio, come dicono in Napoli, e de' Duati di Montelione, e di Terranova 2. D. Ferdinando fposo Donna Lucrezia Pignatelli, Principefa di Strongoli, il cui figlio D. Salvatore tclfe Donna Giulia Mattrgli de Duchi di Marigliano.3.D. Anronio s'aminoglio in Ifrágna colla figlia unica del C. di Fuentes. Da tal maritaggio nacque D. Gioacchino Pignatelli d Aragon, Moncayo \&c. C. dì Fuentes, Marchidi Cofcojuela, Gr. di Spagna, Principe del S. R. I. Cayaliere del Tofon d'oro, di S. Giacomo \&c. Ambarciatore del Re di Spagna nelle Corti d'Inghilterra, di Francia, P Pefidente del Real Configlio degli Ordini militari, il cul figlio D. Luigi, oggi vivente, ha frorato la figlia unica ed erede di Cafimiro Pignatelli, Conte a Egmont, Duca di Bifaccia \&c. Cavaliere del Tofon, e Tenente Gènerale delle Atmi del $\operatorname{Re}$ Criftianilumo 4: D. Fabrizio prefe per moglie Virginia Pignatelli, Corella della Principeffa di Strongoli, il cui figlio D. Michele è Marchefe di Salice, e Guagmano sa Rofa fu data in ifpola al Principe di scalea 6. Maria Terefa al Marchefe di Wefterlo, Signor Boemo 7. Stefania al Principe di Bifignano. 8. Caterina al Conte d"Acerra." 
I
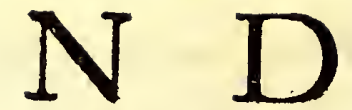

I
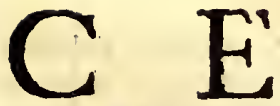

D E L T O MO I I I.

L I B R O V I I I.

I Rimi viaggi degli Spagnuoli alle cofte d'Anahuac. Pag. 3
Carattere de'principali Conquiftatori del Meffico.

3 Armata e viaggio di Cortès.

4 Vittoria degli Spagnuoli in Tabafco

5 Notizia della famofa Indiana Donna Marina.

6 Arrivo dell' armata al porto di Chalchiucuecan.

7 Inquietudine di Motezuma: prima ambafciata e prefente mandato da lui a Cortes.

8 Prefente di Motezuma pel Re Cattolico.

9 Ambafciata del Signor di Cempoalla, e fue confeguenze. 23

Io Imprigionamento in Chiahuitztla de'Miniltri Regj. 27

II Confederazione de'Totonachi cogli Spagnuoli .

12 Fondazione di Veracroce.

13 Nuove ambafciate e prefenti di Motezuma.

I4 Spezzamento degl' idoli di Cempoalla .

Is Lettere di Cortès, e dell' armata al Re Cattolico.

I6 Azione famofa di Cortès.

17 Viaggio degli Spagnuoli fin' al paefe de'Tlafcallefi .

18 Alterazione de'Tlafcallefi, e rifoluzione di quel Senato fu. gli affari degli Spagnuoli.

19 Guerra di Tlafcalla.

20 Nuove ambafciate e prefenti di Motezuma a Cortes.

21 Pace e confederazione de'Tlafcallefi cogli Spagnuoli

22. Ambafciata del Principe Ixtlilxochitl, e confederazione dea gli Huexotzinchi.

Sofl Hexotzing 52

23 Sattomeffione della Repubblica di Tiafcalla al Re Cattolico. $5^{2}$

24 Entrata degli Spagnuoli in Tlafcalla.

25 Inimicizia tra' Thafcallefi, ed i Cholullefi.

26 Entrata degli Spagnuoli in Cholulla.

27 Strage fatta in Cholulla.

28 Sottomeffone de' Cholutlefi, e de'Tepejachefi alla Corona di Spagna .

29 Nuova ambafciata e prefente del Re di Meffico.

30 Rivoluzioni in Totonacapan. 
3 I Viaggio degli Spagnuoli fin'a Tlalmanalco.

32 Vifita del Re di Tezcuco a Cortès.

33 Vilita de' Principi di Tezcuco, ed entrata degli Spagnuoli in quella Corte.

34 Entrata degli Spagnuoli in Iztapalapan.

35 Entrata degli Spagnuoli in Meflico, e dimoftrazioni del Re, e della Nobiltà.

\section{I B R O I X.}

I Prima conferenza, e nuovi prefenti del Re Motezuma. 80

2 Vifita di Cortès al Re. 83

3 Defcrizione della Città di Meffico.

4 Sfoghi dello zelo di Cortès per la Religione. 88

5 Imprigionamento del Re Motezuma. 90

6 Vita del Re nella prigione.

7 Supplicio del Signor di Nauhtlan, e nuovo infulto alla Maeftà del ke.

96

8 Tentativi del Re d'Acolhuacan contro agli Spagnuoli.

98

9 Imprigionamento del Re d'Acolhuacan, e d'altri Signori, ed elaltazione del Principe Cuicuitzcatzin.

io Sottomeflione di Motezuma, e della Nobiltà Mefficana al Re di Spagna.

I I Primo omaggio de'Mefficani alla Corona di Spagna.

12 Inquietudine della Nobiltà mefficana, e nuovi timori di Motezuma.

102

105

I3 Armata del Governatore di Cuba contro Cortès.

I4 Vittoria di Cortès contra Narvaez.

is Strage della Nobilta mefficana, e follevazione del Popolo contro agli Spagnuoli.

16 Zuffe tra'Mefficani, e gli Spagnuoli nella Capitale.

17 Parlamento del $\operatorname{Re}$ al Popolo, e fuo effetto.

18 Combattimento terribile nel tempio.

19 Morte del Re Motezuma, e d'altri Signori.

110

113

116

20 Terribile fconfitta degli Spagnuoli nella lor ritirata .

21 Marcia faticofa degli Spagnuoli.

22 Battaglia famofa d'Otompan.

23 Ritirata degli Spagnuoli in Tlafcalla .

24 Elezione in Meffico, e difpofizioni del Re Cuitlahuatzin.

25 Ambafciata del Re Cuitlahuatzin a'Tlafcallefi .

145

26 Battefinio de' quattro Signori di Tlafcalla .

27 'Sbigottimento d'alcuni Spagnuoli .

28 Guerra degli Spagnuoli contro i Tepejachefi.

29. Guerra di Quauhquechollan. 


\section{0}

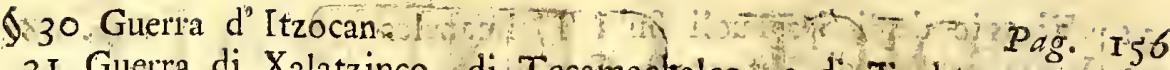

$3^{I}$ Guerra di Xalatzinco, di Tecamachalco, di Tochtepec. Th 8

32 Strage fatta dal Vajuolo. Morte Lel Re Cuitlahuatzin, e del

Principe Maxixcatzin, ed elezione del ReQuauhtemotzin. I 59

33 Efaltazione del Principe Coanacotzin, e morte di Cuicui. tzcatzin.

\section{I B R O. X.}

$\$$ I Raffegna e marcia dell' Efercito Spagnuolo a Tezcuco. 162

2 Entrata degli Spagnuoli in Tezcuco; e rivoluzioni di quellaCorte I 68

3 Spedizione pericolofa contra Iztapalapan.

4 Confederazione d'Otompan, e. d'altre Città cogti Spagnuoli. 172

3 Trafprto de' materiali de' brigantini .

6 Spedizioni contra Xaltocan, e Tlacopan.

7 Spedizioni contra Jauhtepec, e Jacapichtla.

175

8 Negoziazione infruttuofa di Cortès nella Corte di Meffico. I 82

9 Murcia dell'Efercito Spagnuolo per le montagne meridionali. I 83

Io Conquifta di Quäuhnahuas.

I I Conquinta di Xochimilco.

I2 Marcia degli Spagnuoli attomo a' laghi fin' a Tezcuco.

I 3 Congiura contira Cortès.

14. Ultimi preparativi per l'affedio di Meffico.

I Difpofizione dell' efercito nell'affédio della Capitale.

I6 Supplizio di Xicotencatl.

17. Prime oftilità degli Spagnuoli, e principio dell'affedio della Capitale.

I 8 Prima entrata degli Affediatori nélla Città .

Ig Aumento delle truppe aúfiliarie degli Spaginuoli:

20: Nuove entrate nella Città .

2I Confederazione di parecchie Città del lago cogli Spagnuoli. .

22 Cperazioni d'Alvarado, e prodezza di Tzilacatzin:

23. Tradimento de' Xochimilchefi, e d'altri Popoli.

24 Vittoria de' Mefficani.

25 Combattimenti de' brigantini, e ftratagemmi de' Mefficani.

26. Ambafciata infruttuofa al Re di Meflico.

627. Spedizioni contra i Malinalchef, e contro i Matlatzinchi.

29 Strage fatta in Meffico, e bravura d'alcune donne.

30 Stato lagrimevole de' Mefficani .

3 I Diligenze inutili fer la refa de'Mefficani.

- 32 Terribil conflitto, ed orrenda Arage de' Mefficami. 227.

33 Ultimo af alto e prefa della Città ,'e de'Re. 227

- Difcendenza del Re Motezuma.

- Difcendenza di Ferdinando Cortès. 


\section{INDICE GENERALE}

\section{DELLE COSE NOTABILI}

\section{CONTENUTE NELLA STORIA ANTICA}

\section{DEL MESSICO.}

$\times C=2$

Il numero romano addita il tomo, l'arabico denota la pagina, la N. vuol dire che la cola fi trova nelle note, e la M. fignifica Meffico.

A

A Beto di forprendente grandezza nella Valle d'Atrifco. I. 6 I

Abiti comuni de' Meflicani. II. 223. Abiti Reali . I15. 241.

Sacerdotali 38. 39. 46. Militari. 140. 142. Funebri. 93. Acacia. Grand'Abbondanza di vera Acacia nel M. I. 66

Acamapitzin eletto $\operatorname{Re}$ di Meffico. I. 173. morto 177

Acbiote, o rocou, frutto d' un albero del M. Che ufo ne faceano i Mefincani? 1. 5.6.

Acolbuacan, regno. La fua eftenfione I. 28. Le ventinove Città d'effo che contribuivano alle rpere del Real palazzo, e del tempio. 245 .

Acolbuatzin, Principe. Spora una figlia del Re Xoiotl. 1. 137. Si rende padrone di Tepotzotlan. I42. Muore 143.

Acolbui, Nazione celebre. La fua origine \&c. I. 136

Acolman, Città prefa dal Pr. Nezahualcojotl. I. 209.

Acolnabuacatl, Principino Mefficano uccifo da'Tepanechi. I. 183.

Acqua. Perchè è falmåtra quella del lago di Tezcuco. 1. 37. Acque petrificanti, e minerali. 38 . Acqua benedetra. II. 2x. 80 .

Acquidotti di Chapoltepec. II. 203. di Cempoalla . 204

Adulterio. Come fi puniva? Il. 130.

Agricoltura. Era in gran pregio preffo i Meflic. II. 152. maniera di coltivar la terra. I53. I lavori della campagna compartiti tra gli uomini, e le donne. 155

Aguilar, Diacono Spagnuolo, interprete di Cortès. Le fue avventure. III. so Abuaubtli, uova commentibili di mofche paluftri. 1. 108.

Abuejactli, Serpe affai velenofa. I. 97.

Abuitzotl, eletto $\operatorname{Re}$ di Meffico. I. 2s6. Fabbrica, e dedica il tempio maggior di Melfico. 257. le fue conqúifte. 258. Ia fua morte, ed II fio elogio. 262 
Abuitzotl. quadrupade anfibio 1. 80

$\mathcal{A} i$. V'erano per li figliuoli de' Signori. II. In

Ajacaxtli, fonaglio de Mefficani. '11. 179

Ajotocbtli, Armadillo, o quadrupede teftaceo. I. 72

Alabaftro in M. I. 44. Lo lávöravano i Meffićani. II. 205

Alberi. La lor varietà, grandezză, ed eccellenza. I. "6r."

Albergbi. V' erano nelle ftrade per li viandanti. II. 168

Alimenti. Quali erano quelli de'Mefficani?'II: 21 7

Alleanza famofa de'Re di M., d'Acolhuacan, e di Tlacopan. I. 224. Altare, com' era quello de' (acrifizj? 1I. 46.

Alvarado ( Pietro d') Conguiftatore. Il fúo ritratto."111. 8. il fuo crudele attentato contro la Nobiltà mefficana. '118. il fuo falto ftupendo. 137. Le fue operazioni nell' affedio della Capitale . I95. 200. 204.207 Alvarado, fiume grofo. 1. 36 .

Ambafciata. Si mandava ora al Re, o Signore, ora alla Nobiltà, ed ora al Popolo di qualche paefe. II. Irg. Maniera di farla. Ir8. Quella d'Itzcoatl a Nezahualcójotl. I. 208., ed al Tiranno Maxclaton. 213. Quelle di Motezuma al Conquiftatore Cortès . III r 19: 22. 30.48.65. Quella di Cuitlahuatzin a' Tlafcallefi. I46. Quella di Cortès al Senato di Tlafcalla. 37 .

Ambafciatori. La loro ufanza, il loro abito, e la loro immunità. II. I 18 Amecameca, offia Amaquemecan, regno de' Cicimechi. I. 132. Città preffo al Vulcano Popocatepec. III. 7 I

Amimitl. Dio della pefca. II. 20

Amiztli, quadrupede anfibio. I. 74

Amolli, radice adoprata in vece di fapone. II. 228

Anfifibena del M. I. 98.

Anima. Che penfavano i Meflicani, e gli Otomiti dell'anima umana, e di quella de'Bruti? HI: 4 . T'́ 'luoghi per le anime feparate da' corpi. Ivi .

Anmali. Che Spezie n' erano allevate da'Mefficani. II. 158. Tutti quelli, che vi furono trafportati da Europa, fon ben riufciti. I. 69.

Anno mefficano. Com'era?'II. 58. Anno divino che cofa foffe? 84. Anni mefficani, rifcontrati co' crintiani. 230. Figure "rapprefentative dell'Anno. 64. Efpofizione di tali figure. 248 .

Api. Deferizione di fei fpezie d'Api del M. I. 107.

squile del M. I. 8i

Aquile, Ordine militare. II. 140

Arcbi negli edifizj mefficani. II. 201. Archi famofi di Cempoalla . 204

Arcbitettura dómeftica de 'Mefficani. II. 199. militare. I49. Religiofa. 27. e regu.

Argine fatto far dal Re Nezahualcojotl nel lago meffic. I. 234 .

Armi difenfive de' Mefficáni. II. 14I. Armi offenfive. I43.

A femblea famora del Re Motezuma', è della Nobiltà mefficana full'affare degli Spagnuoli. III, 107.

Afronomia. Si ftudiava in Tezcuco. I. 225. cognizioni aftronomiche de'

Toltechi. I 28. del Re Nezahualcojotl. 246. de'Mefficani. II. 62. Offervazioni che ne faceva il Re Nezahualpilli. I. 294.

Atolli, farinata di frumentone molto ufuale prefo i Mefficani. II. 219 Auferità de' Mefficani. II. 52. de' Miztechi . e de' Teohuacanefi. 54. de' Tlarcallefi. 5s. 
Axajacatl; Re di Meffico. Vince i Tehuantepechefi. I. 241. Conquifta Tlatelolco. 248., ed il paefe de' Matlatzinchi. 252. Muore 353. Axajacatl, Mofche palufri. V. Mofca. Axolotl, I ucertone acquatico affai fingolare. I. Iog.... Aztechi. V. Meficani.

Aztlan, patria de'Mefficani, e delle altre tribù di Nabuatlachi. 1. 156

B

$\mathrm{B}$ Agni troppo urati da' Meflicani, II. 2 I 4

Balli. Quelli de' Mefficani vari, e belli. II. 179. V. Fefte.

Balla, Macchina per valicare i fiumi. II. 168

Balfamo mefficano: I. 63. Quanto $\mathrm{fi}$ vendette in Roma il primo balfamo mefficano? Ivi N. s'aduprava nelle ferite da' cerufici mefficani. II.213 Banditore, impiego onorevole preffo i-Melficani. II. 128

Bando pubblicato da'Mefficani, in. Tezcuco. I. I9r. da Cortès in Tlafcalla. III. 1.65

Barcbe. La lor forma, e grandezza, ed il lor numero ecceffivo ne' laghi mefficani. II. 168

Bitume giudaico, offia Asfalto. Abbondanza d'effo nelle cofte del M. ed ufo che ne faceano i Mefficani. I. 43

Bobo, pefce delicato. I. I०3

Boja, impiego onorevole prefro i Mefficani. II. 128

Bofchi grandi di mufe, di melaranci, e di limoni. I. 50. di chicozapoti. 52. di cedri, d'ebani, di caobe, e d'altri legni affai preziofi. 60. Bofchi reali. II. is 7 .

Botetto, pefce il cui fegato è troppo velenofo. 1. 103.

Brina. 'V'è della brina in qualche luogo del $M$. tutti i giorni dell' anno. I. 39.

Bugia. Che pena areva in M? II. 134. in Acolhuacan. 137.

$\mathrm{C}$

Acabuate, frutto d'una pianta isngolare. I. : 53.

Cacamatzin, Re d'A cclhuacan..1. 298 . Fa un accordo co' fuoi fratelli.3or Il fuo parere fulle pretenficni' degli Spagnuoli. III. 48. 70. Vifita Cortès in Ajotzinco. 72. I fuoi tentativi contra gli Spagnuoli. 102. Perde la corona, la libertà, e la vita. 105. 138.

caccao. Quattro fpezie. I. 54. Quanto ne confumava ogni anno il Re Nezahualcojotl? 244. S'impiegava per moneta. II. I65. Ne facevano parecchie bevande. 219

Caccia generale. Come fi faceva? II. 160. Modo di cacciare gli fcimiotti,e le anitre. 161. e le ferpi . 162. Caccia per li facrifizj che fi facevano nella fefta di Mixcoatl. 79

Cacomiztle, quadrupede. Si defcrive. I. 80.

Calendario mefficano del primo anno del fecolo. II. 234. Efpofizione del Calendario. 57.

Calmecabua; Capitano Tlafcallefe, che ajutò gli Spagnuoli nella conquifta di M. e viffe anni cento trenta. III. I42

Camaleonte mefficano diverfo dal volgare. 1. 94 


\section{4}

Cammote, radice pregiata. I.' 57

Candele. Non erano in ufo prefro i Mefficani. II. 225

Capelli. Quelli de'Mefficani neri, lifci, e grofli. I. I18. come gli portan vano? II. 140.224.

Caragna . ragia medicinale. I. 65

Caratteri, o fegni convenzionali delle cofe ufati da' Mefficani per rapprefentar quelle, che non hanno figura propria. II.: Igr. Caratteri numerali 192.

Carceri de' Melficani . Com'erano? IH. 138.

Cardinale, uccello affai bello e canoro 1.89 .

Caricbe onorevoli nélla Corte di Meffico. H. 117. in quella di Tezcuco. I. $18 \mathrm{r} .225$

Carne. Non ne mangiavano tanto Mefficani, quanto gli Europei. II. 221 S'imbandiva nelle lor tavole la carne degli uomini facrificati. 47

Carte: Facévanla i Melsicani di feta, di cotone, di maguei, e d'altre piante. II. I89. I Quauhnahuachef, ed altri Popoli pagavano ogni anno alla Corona di M. fedici mila pezze di carta. 126

Cafa. Cöm'erano 'le cafe de' Mefsicani? II. 200

Calampulga, ragno velenofo. I. I 10

Cedro. Abbondanza, e grandezza de' Cedri nel M. I. 60

Ceiba, albero grandifsimo, il cui frutto contiene una fpezie di cotone alfai fino. I. 62

Cempoalla, grande e bella Città nella cofta del feno Mefficano, dove furono bene accolti gli Spagnuoli. III. 25. Vi Spezzano gl'idoli,' 3 I

Centeotl, Dea della terra, e delle biade. 11. 16. Monaci ad effo lei confa. crati. 44. Fefte, che le facevano. 68.75

Centzontli, uccello affai rinomato pel fuo canto. I. 89

cera. Non fe ne fervivano i Mefficani. II. 1225. Imparano da Cortès a farne delle candele. 111. 34 .

Cbacbalaca, uccello che fa un gran rumor nei borchi 2 I. 86

Cbajote, frutto comune nel M. I. 52

Chalchef̂, tribù dei Nahuatlachi. I. isr." La lor crudeltà. 230. Vogliono alzar $\mathrm{Re}$ un fratello del Re Motezuma Ilhuicamina: 238. foggiogati dal medefimo Re. ivi. Confederati cogli' Spagnuoli. III. I 73

Cbalco, Cirtà confiderabile. I. 30 , conquiftata dal Re Motezuma 238. travagliata dai Mefficani per la fua alleanza cogli Spagnuoli. III.I 75

Chalco (Lago di ) I. 30, 37

Cbapopotii. V. Bitume giudaico.

Cbia, pianta La faa defcrizione I. 54 . Facevano certe bevande della femenza. If. 220 , e ne cavavano un eccellente olio per la pittura.1. 44

Cbiapanef. La lor tradizione intorno al diluvio univerfale, ed alla popolazione dell' Armerica I. 15o. I lor anní e mefi. II. 6s

Chichimecatl, bravo Generale Tlafcallefe : La fua contefa con Xicotencatl. III. 46. Vuol per la fua bravura il potto più rifchiolo nell' efercito. 176. Fa un' entrata in Meffico coi "fuoi Tlafcallefi, e combatte gloriofamente coi Mefficani. zrg

Cbilli. V. Perverone.

Cbimalpopoca, eletto $\operatorname{Re}$ di Meffico. I. 186. Fatto Signor di Tezcuco dal Tiranno Tezozomoc. I90. Affifte al funeral del Tiranno. 195. Il furo difcorfo col Principe Tajatzin."Ig6. Ingiurie fattegli dal Tiranno 
Maxtlaton. 199. Vuol morir facrificato al Dio della guerta, è prefo dai Tepanechi, e meffo in prigione in Azcapozalco. 200. e s'appicca nella carcere. 202

Chinampa. V. Orti galleggianti.

Cbirurgia dei Mefficani. II. 216

Cbololian; offia Cbollula, Città grande. La fua defcrizione. III. 59. Strage fattavi dagli Spagnuoli. 62. Piramide, e tempj di Cholollan. 11. 33. Teatro che v'era. 177

Cbolollefz più pratici del commercio, che della guerra. I. 284. La lor guerra coi Tlafcallefi. 278. Cagione della loro nimiftà. III. 57. La lor condotta yerfo gli Spagnuoli. Ivi. Danno ubbidienza al Re Cattolico. 64. Uccifi in M. allorchè vi furono fconfitti gli Spagnuoli. 138

Cicimechi, nazione antica. La lor origine, ed i lor coftumi. I. 132. I lor Re. 133, e fegu. La lor lingua. I. 153. Dai Cicimechi, e dagli Acolhui ti fa per li maritaggi una fola nazione, 137. I Cicimechi barbari confufi dagli Spagnuoli cogli Otomiti. 148

Cibuacoatl,' Dea. II. 9

Cibuacoatl fupremo Magiftrato. Non fi poteva appellar dalle fue fentenze. II. 127. Chi ufurpava la fua autorita, o le fue infegne era reo di morte. Ivi .

Cibuacuecuenotzin. Fatto memorabile d'effo lui. I. 187

Cioccolata inventata dai Mefficani, II. 219

Circuncifione. Non fu in ufo preffo i Mefficani. II. 73

Clima del Meffico. I. 39. Divario tra' paefi di clima freddo, e quelli di clima caldo. 116

Goanacotzin Re d'Acolhuacan fa morire il fuo fratello Cuicuitzcatzin. 111. 16r. Per paura degli Spagnuoli fi ricovera in Meffico. 168. Prefo dagli Spagnuoli infieme col Re di Meffico 230. e poi impiccato. 233

Coatl, ftrumento d'agricoltura. II. I 53

Coatlicue, Dea dei fiori. Il. 2x. Fefta che le faceano. 67

Coatlicue, creduta Madre del Dio della guerra. II. I8.

Coatzontecoxocbitl, fiore, affai bello, I. 46

Cościniglia, infetto celebre per la tintura. La fua defcrizione. I. I12. Maniera d'allevarla. 11. is 9

Cocco frutto d' una palma, che noi crediamo trafportato dälle Ifole Filippine. I. 48

Cocco d'olio, frutto d'un' altra palma. I. 59

Coccodrillo, Il Mefficano è come quello del Nilo. I. 96. Modo d'ammazzare i Coccodrilli. II. 162. I Zopiloti diftruggono le loso uova 1. 83

Cojametl, quadrupede alquanto fimile al Cinghiale. Non ha l'ombellico fulla fchiena. I. $7 \mathrm{I}$.

Cojobuacan, Città riguardevole della valle Mefficana, conquiftata dal Ré Itzcoatl. I. 223. danneggiata dagli Spagnuoli. III. 190. Vi s'accampa il Comandante Olid nell affedio della Capitale. 198

Cojote, Fiera del Meffico. La fua defcrizione. 1. 76

colezione. Con che la faceano i Mefficani? 11. 226

Colbuacan, Città confiderabile. I. 30. N. Sacrifizio inumano d'una Principeffa di Colhuacan $\cdot 1.7 \mathrm{I}$

Colbui tribù dei Nahuatlachi. I. 151. Fanno fchiavi i Mefficani. 164. La lor guerra coi Xochimilchefi. 165 
Collegj di Sacerdoti', di giovani, e di putte. II. 42. 43. ron

Colombella, perce delicatifimo. I. 103

Colonna. Le colonne dei Mefficani non aveano bafi, nè capitelli . II. 202. Colonne di fmifurata grandezza in Mictlan..204

Comalli tefto da cuocere il pane, ed il caccao. II. 225

Commercio dei Meflicani. II. 163. V' era vera compra e vendita . 165. Commercio nel lago di M. 169. Giudici di commercio . 166

Conquifatori Spagnuoli. Il lor carattere. III. 6. Furono ftrumenti della giuftizia, e della mifericordia di Dio. 95. Vollero fare fchiavi gl' Indiani, e non fu loro permeffo. Il. 136

Consiglio. Configli Reali in Meffico. II.' ri 7. In Tezcuco. I. 225

Cortis (Ferdinando) famofo Conquiftatore. Il fuo ritratto. III. 6. La fua armata. ro La fua condotta verfo i Tabafchefi. 12. I fuoi primi abboccamenti coi Mefficani. 15. e feg. Fa pigliar cinque Miniftri del Re di M. 27. e fpezzar gl'idoli di Cempoalla. 31. Diftrigge la fua flotta. 35. fa tagliar le mani a cinquanta fpie dei Tlafcallefi. 47. Libera in Tlafcalla $\mathrm{i}$ prigionieri deftinati al facrifizio. 55. Difefo ful fatto di Cholulla contro le accure di Monfig. de las Cafas. 63. I fuoi primi abboccamenti col Re Motezuma . 77. e fegu. Sfoghi del fuo zelo per la Religione. 88. Conduce prigioniere Motezuma al fuo quartiere. 89. Brucia vivo Quauhpopoca e mette in ferri Motezuma.99. $s^{\prime}$ intriga col Re d'Acolhuacan. s02. e lo mette in ferri. 105. Vince il General Narvaez, ed aumenta il fuo efercito. 116. I fuoi conflitti in Meffico. 122. Affalifce coraggiofamente il tempio. 127. Le fue fortite contro i Mefficani. 329. Fa morire alcuni Signori. 134. Piange la morte dei fuoi amici, e foldati. (139. Uccide il General dei nemici nella battaglia d'Otompan. 14r. Vefte bruno per la morte di Maxixcatzin. I60. Il fuo parlamento alle truppe per incoraggirle all' affedio della Capitale. 162. Fa Ixtlilxochit! Re d'Acolhuacan. 169.s' accampa in Xoloc. 198. Prefo dai Mefficani, e meffo in libertà dai fuoi Soldati. 187. 211. Procura abboccarfi col Re Quauhtemotzin, e non può ottenerlo. 228. Trattiene il furor delle truppe aufiliarie 229. Mette alla tortura, il $\operatorname{Re}$ di Meffico, e poi l' impicca infieme coi Re $d^{2} A$ coltruacan, e di Tlacopan. 233

Cotone, affai comune nel Meffico. I. 55. Ne facevano carta i Mefficani. II. 189. e tele di molte forti. 207

Coxolitli, fpezie di fagiano. I. 85

Cozcaquaubtli, o Re dei Zopiloti, uccello. I. 82

Cucaraccia , infetto fchifofo. I. 109

Cucujo, fcarafaggio luminofo. La fua defcrizione. I. Io6

Cueitl, gonna mefficana. II. 223

Cuicuitzcatzin, fatto Re d'Acolhuacan da Motezuma III. s06. uccifo per ordine del fuo fratello Coanacotzin. 160

Cuitlabuac, Città nel lago di.Chalco, prefa dal General Motezuma. I. 227. v' entrano gli Spagnuoli. III. 75

Cuitlabuatzin, Signor d' Iztapalapan. Le fue qualità III. 145. II fuo famofo giardino. II. 156. Il fuo palazzo. III. 76. Il fuo parere fugli $S p a-$ gnuoli. 48. 70. Gli accoglie in Iztapalapan. 76. Prefo da Cortès. 107. Rimeffo in libertà . I22. Fatto Re di Meffico dopo il fuo fratello Mo. tezuma. I45. Muore del Vajuolo.I 59. fdegno di Cortès contra lui. I $7 x$ 
$\mathrm{D}$ Anta, o Tapir, quadrupede grande. I.' 75

Deftatori, uccelli curiofi. I. 9 I

Diafpro. di diverfe fpezie nel M. I. 44

Digiuno. Diverfe forti di digiuni preffo i Mefficani. II. 53. Digiuno di quattro anni in Teohuacan. 54. Digiuno Atraordinario del Sommo Sacerdote. 55. Quello dei Tlafcallefi nell' anno divino. ivi.

Diluvio univerfale. Tradizione d'effo preffo i Chiapanefr. I. 150. preffo : Mefficani. II. 6. Come lo rapprefentavano? V. la figura dopo la pagina 192 . del tomo 2. e la \{piegazione d'effa nella pag. 257

Dio, come appellato dai Mefficani? Il. 4. che idea aveano della Divinità? ivi. Come la riverivano? 24. DEI MESSICANI. Il Dio della providenza . 7. Gli Dei del Cielo. 8. dell' aria . Ir. dell' acqua . I4. del fuoco, della terra, e delle biade. I6. dell' inferno, e della notte. I 7 . della guerra. 17. e feg. Del commercio, della caccia, della pefca, e della medicina. 20. del vino, e dei fiori. 21. degli Orefici, e degli artefici di ftuoje. 22. delle allegrezze. ivi. della vecchiaja. 23. I Dei Penati. ivi. La Madre degli Dei. 22

Bonna. Abiti delle donne mefficane. I1. 223. I lor impieghi domeltici 216 I lor lavori nella campagna. Is 5

E

Difrcio. Avvanzi d'edificj antichi in Mictlan. II. 203. preffo al fiume

E Gila, e nella Pimeria. I. 159.. Preffo a Zacatecas. 16r. In Tezcuco. 226. In Cholulla. II. 33. in Teotihuacan. 34. nel difretto di Tlafcalla, in Guatulco, e preffo a Molcaxac. I50. in Coatlichan.*15I. in Tezcutzinco, ed in Meffico. 203. in Cempoalla.204

Educazione fingolare della gioventù mefficana. II. 100

Elettori del Re di Meffico. Chi erano? II. I I

Elezione del $\operatorname{Re}$ di M. Come fi faceva? II. II 2

Eredi. Chi erano preffo i Mefficani, ed i-Tlafcallefi? II. I23

Efortaziont dei Mefficani ai loro figli. II. IO4

Efrada (Maria d') donna (pagnuola. Combatte coraggiofamente coi Mefficani. III. I42. 22 I

Etd. Quattro ne diftinguevano i Mellicani. II. 57

\section{F}

$\mathrm{F}$

Agiano. Tre rpezie di Fagiani nel M. I. 85

Fame nel M. I. 234. 282. dei Mefficani nell affedio di M. III. 224

Farfalla. Varietà e vaghezza delle Farfalle del M. I. 109

Ferro. Ve ne fono multe miniere nel M. I. 43

Fefte fecolari. II. 84. Fefte del Dio dell' acqua. 66. 67. 74. 79. 81. Del Dio degli Orefici. 6r. del Dio della guerra. 70. 76. 80. di Tezcatilipoca. 68. della Dea delle biade . 75. del Dio del fuoco. 76. 83. della Dea della caccia. 79. della Madre degli Dei. 77. 83. dell' arriyo de- 


\section{8}

gli Dei. 78. dei Defunti. 77. Felta gtande della Dedicazione del tempio maggiore. I. 257

Feudatario. I Signori feudatari della Corona di Meflico doveano rifedere una gran parte dell' anno nella Corte. I. 266

Feudo. Quali erano i Feudi nel M? II. 124

Ficaja fingolare nel M. 1. 62

Figliuoli. La loro educazione. II. xoo, e fegu. Efortazioni, che lor facezno i lor Genitori. ro4. Imparavano per lo piü il meftier dei lor Genitori.11r. Com'ereditarano? 123. Che pena aveano coloro, che fcialacquavano i beni paterni? 134 .

Figura. Efpofizione delle figure del fecolo, dell' Anno, del Mefe, delle Città, dei Re, e del Diluvio. Il. 348. Quali erano lề figure che adopravano i Mefficani nelle lor pitture? agr

Fiori notabili del M. I. 46

Fiumi groffi del M. I. 36

Fonti del M. I. 38

Formiche del M. 1. Ir Madre delle Formiche, Terpe beila cosł apcellata, perchè abita ordinariamente nei formicaj,ed accompagna le formiche. 1. 97

Fortificazioni. Ufate dai Mefficani. Iİ. I49. quelle di Tlafcalla . I. 156.III. 150. Quelle di Quauhquechollan. III. I53. Quelle di Meffico. II. Is I.

Frecce. Deitrezza nel tirarle. II. 43.

Frumentone, offia Maix. Diverfe fpezie. I. 5.6. Fu trafportato da America in Europa. ivi. Ne facerano i Mefficani il lor pane. Il. 2r8. e parecchie bevande. 219. Modo di feminarlo, e di coltivarlo. 194.

Fratte proprie del Melfico, o trafportatevi da Europa. I. 48. e fegu.

Fulmini frequenti nel M. I. .40.

Fwnerali. Quelli del Re Xolotl. I. I40. del Re Quinatzin. I45. del Tiránno Tezozomoc. 195. ceremonie funerali. 11. 93.

Fuoco. Come lo cavavano? II. 226. Felte grandi della rinnovazion del fuoco. 84

\section{G}

$\mathrm{G}$

Arrapata, intetto perniciofo de' prefi caldi. I. I2.

Garze nel M. affai belle. 1. 84 .

Gemme. Che fpezie ne fono nel M.? 1. 43. come le lavoravano gli artefci mefficani? II. 206.

Geroglifici, e figure fimboliche ufate da'Mefficani. II. I9r.

Getto. Lavori eccellenti che ne faceano i Melticani. 11. 195.

Giardini di Motezuma. 1. 273. d'Iztapalapan. II. 1 56. d' Huaxtepec. 157

Giganti. Crani, offa, e carcami interi di veri Giganti ritrovati in parecchi luoghi del M. 1. 1zs.

Giorni intercalari preffo i Mefsicani.. II. 62

Giudici mefsicani. Quali erano, e come giudicavano? II. 127. Giudici del

Commercio. 166. d'Acolhuacan. 128. 1 Giudici che mancavano al lor obbligo erano rei di morte. 130

Giuoco de' Volatori. II. 182. del Pallone I84. a Itri giuochi. 185.

Giuramento, com'era quello de' Mefsicani, e quanta fede faceva? II. 24.

Gomme, e ragie del Mefsico. I. 63. Gomma arabica a frai abbondante nel

M. 
M. 66. Gomma copal 65. Gomma lacka V. Lacca.

Granai. Com' erano quelli de' Mefficani? II. I55

Grijoiva (Gio: de) Comandante Spagnuolo. Il fuo viaggio alle cofte del

M. III. 4. Creduto Quetzalcoatl da' Mefficani. 5. Ritorna in Cuba.6 Gerra. Maniera di dichiarare, è 'di far la guerra. II. I46. Guerra tra' Colhui, ed i Xochimilchi. I. 165. di Pojaubtlan. I53. Tra'Tepanechi, e gli Acolhui. i 85. Tra' Mefficani, ed i Tepanechi. 215. Guetra di Coaixtlahuacan . 235. di Cotafta 237. di Chalco 239. di Tlatelolco 248. di Xiquipilco 252. di Huexotzinco 254.258. Guerra de'Mefficani co' Tlafcallefi 275 , e fegu. Guerra degli Spagnuoli. V. Spagnuoli.

H

$\mathrm{H}$

Amaca, o ponte penfile. Com'era? II. 169

Huaxjacac, Città antica nel paefe de'Zapotechi. I. 33. N.

Huematzin, celebre Aftronomo de'Toltechi. I. 128

Huexotzincatzis, Principe tezcucano condannato a morte dal fuo Padre Nezabual pilli. 1. 295

Huexotzinco, Città grande, e Repubblica. I. 33

Huexotzinchi. Fanno la guerra a' Tlafcallefi. 1. 155. 277. e fegu. Si confederano co' Re d'Acolhuacan contra il Tiranno Maxtlaton. 206. La lor guerra cogli Acolhui 254., e co' Cholullefi 28. Si confederano co' Miztechi 236., e poi co' Cotaftefi contra i Mefficani 237. Si confedetano cogli Spagnuoli. III. 52

Huitzilibuitl, eletto Re di Meffico. I. I78. morto I86.il fúo figliuolo ucciro da' Tepanechi. I 83

Huitzilopocbco, Città della Valle mefficana. I. 30. N.

Huitzilopocbtli Dio della guerra, II. 17 . Fefte che fi facevano ad onor di lui. 70. 76. 8o.

Huitzitzilin. V. Succiafore.

Huixtocibuatl, Dea del Sale. II. 20

IDoli. La lor materia, e la lor moltitudine. II. 24 . Idolo di Tezcatlipoo

ca 7. di Tlaloc is. d'Huitzilopochtli 19. 24. Idoli degli Dei Penati.23 Impiegbi. V. Cariche.

Interjazione. Chi la facevano? II. 39. 5I. Era anche un ofequio meramente civile. Ivi. Incensazione $d^{\prime}$ Huitzilopocbrli, fefta grande, che ff facera nel mefe quinto. 70

Incenfo. Che cofe adoprayano i Mefficani in cambio d'incenfo? Il. 39

Incefo. Come fi puniva? II. i $3 \mathrm{I}$

Incontinenza de' Sacerdoti come fi puniva? II. 4I. r32. quella delle vergini confacrate al fervizio degli Dei.42

Injetti del M. 1. 10s. e fegu.

Jolloxocbitl, offia Fior del cuore affai odorofo. I. 46

Ipocaufto mefficano. La fua ftruttura, ed il fuo ufo. II. 214

Ifrice mefficano. I. 80

Itzcoatl, eletto Re di Meffico. J. 206. fa la guerra a' Tepanechi 215. Conquifta Azcapozalco 218. Cojohuacan 222. Xochimilco, Cuitlahuac, e 
Quauhnahuac 226. Mette Nezahualcojoti ful trono d'Acolbuacan 22r. Fa la famofa alleanza co' Re d'Acolhuacan, e di Tlacopan 224. La fua morte, e il fuo elogio: 229

Itzcuintepotzotli. Quadrupede curiofo del M. I. 77

1tzocas, Citta bella prefa dagli Spagnuoli. I. I 56

Itztli, pietra minerale affai pregiata. Che faceano d'efra i Mefficani? I. 45. II. 205

Ixtlilxocbitl, Re d'Acolhuacan, I. 184. La fua guerra contra i ribelli Tepanechi. 185. Le fue difavventure 187. La fua morte tragica. 189

Ixtlilzocbitl, Principe inquieto. Le rivoluzioni da lui cagionate 297. e fegu.

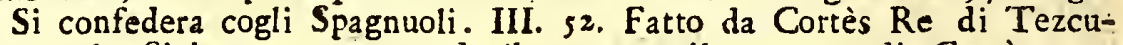
co 169. Si battezza, e prende il nome, e il cognome di Cortès 17r. Ajuta gli $S$ pagnuoli nell' affedio di Meffico. 202

Ixtlilxocbitl (D. Carlo) Principe Tezcucano affai bravo. Ajuta gli Spagnuol 1 nell' affedio di Meffico. III. 202. Libera Cortès da' Mefficani: 21 r. Savio configlio dato da effo lui a Cortès. 220

Iztacmaxtitlan, Città, e fortezza. La fua defcrizione. Ill. 37 J

Iztapalapan, gran Città. La fua defcrizione. III. 75. I fuoi giardini. II. 156. V' entrano gli Spagnuoli mano armata, e trovanfi in gran pericolo d'effere annegati. III. 172

Iztapalapan, penifola tra' due laghi meflicani. I. 36

\section{L}

L Acca. La Lacca non è opera delle Formiche, ma vera gomma . I. 66. N. Ladro. Che pena aveano i Ladri in M? II. 133. in Tezcuco. 137

Lagbi del M. 1. 37. Moto ftraordinario nel lago di Tezcuco. 292

Lana. Non $n^{\prime}$ aveano i Mefficani. II. 207.

Latte. Non ne prendevano i Mefficani. II. $22 \mathrm{I}$

Leggi. Chi le faceano? II. 129. Leggi penali contra varj delitti.130. Leggi fugli fchiavi. 134. Leggi d'altri paefi. 137. Ottanta ne pubblice Nezahualcojotl. I. 242

Legni pregevoli del M. 1. 60

Leone del M. I. 70

Letto. Qual era quello de' Mefficani? II. 224

Lingua. Quella de'Mefficani copiofa, efpreffiva, e cortefe. II. 172. Quella degh Otomiti difficile e piena d'afpirazioni. I. 148. Cuella de' $\mathrm{Ta}$ rafchi dolce, e fonora. 49. Che lingua parlavano i Toltechi, i Cicimechi, ed i Tlafcallefi? 153 . N.

Lino. Se ne troyò in Michuacan, ed in altri paefi. I. 5 s. N.

Zuiquidambra, ragia preziora del M. I. 64

Locufa. Quanto male fanno in alcuni paefi del M. le Locufte? I. 109

Lucertole, e Lucertoni del M. I. 94.

Lume. Con che cola fi faceano lume i Mefficani? II. 225.

Iuna, adorata da' Mefficani. II. 9. La fua apoteofi. 10 
Maguei, offia Alod americano. Ve ne fono diverfe fpezie. 1. 58. Quanto utile ricavavano i Mefficani da tali piante? II. 158. e quanto ne ricavano gli Spagnuoli? 222. Quanto vino fi puó cavar da una pian. ta? ivi.

Malattie del M. I. 117.

Manati, beftia marina affai groffa. I. 100

Manta, beftia marina affai perniciofa. I. rox. Offervazione intorno ad un pafo di Plinio. Ivi N.

Marina, ( Donna) Indiana molto celebre. Il fuo elogio. III. I3. Rivela a Cortès la corpirazione de' Cholullefí 60

Marmo nel M. I. 44

MaJferizie de' Mefficani. II. 224

Matrimomo. Erano legittimi i matrimonj de' Mefficani. II. 93. Come fi celebravano? 89. Come fi facevano in Ichcatlan? 92. Non era permeffo il matrimonio fra perfone congiunte nel primo grado. 89. I3I

Maxixcatzin, Signor di Tlafcalla. Il fuo difcorfo nel Senato full' affare degli Spagnuoli. III.38. Induce il Senato alla pace.48. Si battezza. I49 Muore. 159

Maxtlatl, cintura, colla quale coprivano i Mefficani le loro vergogne. II. 223.

Maxtlaton, Principe Tepaneco. La fua nimifà co' Mefficani. I. 18r. Uccide il fuo fratello Tajatzin, ed ufurpa il regno d'Acolhuacan. 197. Ingiuria il Re Chimalpopoca. 199., e l'imprigiona 200. Perfeguita il Principe Nezahualcojotl. 203. Perde la corona, e la vita . 218

Medicina de' Mefficani.,Il. 2I I. Semplici in effa adoprati 2I2. Olj, infufroni, \&c. 213.

Mercanti. Le loro ufanze ne'viaggi. II. 167

Mercato. Grandezza, varietà, difpofizione, ed ordine maravigliofo de'mercati. II. 163. 566. Chi rubava nel Mercato era quivi incontanente uecifo. 133 .

Mefe. Numero e nomi de'mefi mefficani. II. 59. Figure de'mefi 65., e la loro fpiegazione 248. Mefi Chiapanefi 65.

Meficani. Il lor carattere. I. I18. la loro origine, e la lor pellegrinazione 156. e fegu. La loro fchiavitù in Colhuacan 164. La lor miferia ne' principj $170 \mathrm{La}$ lor Monarchìa 173 . Gli aggravj loro impofti 775 . La lor inquietudine cagionata dall arrivo degli Spagnuoli. III. Iro. Sollevanfi contra loro 120 , e gli fconfiggono 136 . I lor patimenti nell' afredio 224.227

Mefico, regno. La fua eftenfione, e le fue provincie. I. 29.

Mefisio, Città Etimologìa di tal nome. I. 168. Come, e quando fondata? 166. La fua fituazione. 30. La fua defcrizione. III. 85. Inondata. I. 233. 260. Affediata dagli Spagnuoli. III. 196. e prefa 229. e regu.

Metalli più ufuali preffo i Mefficani. I. 4z.

Metate, o Metlatl. pietra da macinare il frumentcne, ed il caccao. II. 224 Mexicotechuatzin, dignità facerdotale. Il fuo impiego. II. 38.

Michuacan, regno. La fua fituazione, ed eftenfione. I. 28

Milizia. Uffiziali della milizia mefficana. Il. 139. Ordini militari. 140

Minerali, e miniere del M. I. 42 .

Miztecapan, Provincia grasde del M. L. 32. Commercio di cocciniglia in efla. IIs 
Miztecbi, Nazione numerofa, ed induftriofa. I. 150 . Le lor rozze. II. 23 Monaci confacrati alla Dea Centeotl. La lor vita, e la lor riputazione. II. 44

Moneta. Ve n'erano cinque forti preffo i Mefficani. II. Its

Monifterj, o Collegj di Sacerdoti. Erano varj. II. 43

Monti del M. I. 4I. Quello d'Orizaba più alto del Pico di Teneriffa . ivi N. Moquibuix. ultimo Re di Tlatelolco. Vince i Cotaftefi. I. 237. E' vinto
ed uccifo da'Mefficani 248

Mofca. Mofche luminofe. I. I08. Mofche paluftri, che fi mangiavano da' Melficani. Iri.

Motezuma llhuicamina. La fua prodezza, e le fue arventure. I. 209., e fegu. Eletto Re di Meffico. 229. Caftiga i Chalchefi 23 I. 239." Fa morir Quauhtlatoa Re di Tlatelolco. 233 . Le fue conquifte $233.23 \%$. La fua motte 239

Motezuma Xocujotzin. Re di Meffico. La fua elezione. I. 264 . Le fue buone e cattive qualità 265. 274. Il fuc portamento, e ceremoniale. 267. Magnificenza de' fuoi palazzi, giardini \&c. 271 .' La fua inquietudine per l'arrivo degli Spagnuoli. III, s. 18. Le fue ambafciate, e prefenti a Cortès 19. 22. 30. 4.8. 65. 69. Riceve gli Spagnuoli nellia Corte. 77. Il fuo difcorfo nel primo abboccamento con Cortès 80. Regala gli Spagnuoli 83. il fuo difcorfo fulla Religione. Ivi. E' prefo da Cortès 8.9., e mefro in ferri 100 . La fua vita nella prigione 96. Fa pigliare il Re d'Acolhuacan fuo nipote, e lo dà in mano a Cortès. ro5. Fa omaggio al Re di Spagna ro7. Ordina a Cortès di partirf dalla Corte. I ro. Parla al Popolo, ed es vilipefo e ferito. I25. Muore. 131. Opinioni fulla fua morte. Ivi. N. Le fue elequie 134. La fua afcendenza I. 303. La fua difcendenza. IIL. 235

IAuraglia celebre di Tlafcalla. II. I 50

Musa, frutto. Ve ne fono quattro fpezie afrai abbondanti nel Meffico. I. 49. Offervazione fopra un paffo di Plinio intorno a tal frutto.ivi.N.

Mufaici eccellenti di penne. II. 197. di conchiglie fpezzate. 199

Mufica. Quella de' Mefficani cattiva. Il. 178. Mufica militare. 146

$\mathrm{N}$ Abuatlacbi, Sette Nazioni o tribù venute dal fettentrione a popolare Meffico. I. Is to

Narvatez ( Panfilo ) Conduce un'armata contra it Conquiftatore Cortès. IH. II:3. E' vinto e prefo da Cortès. II6.

Nezabualcojotl, Principe famolo, perfeguitato dal Tiranno Maxtlaton. I. 203. Le fue diligenze per ottenere la corona d'Acolhuacan 205. L'ottiene 22r. Le fue difpofzion nel governo 23: Pubblica ottanta leggi $2_{42}^{2}$. Severo nell' amminiftrazione della giultizia 243 . I fuoi progreffi melle fcienze 245. Il fuo fentimento full' idolatria 246 . L'ultime fue difpofizioni, e la fua morte 247. Le fue fpefe annuali 244

Nezabualpilli, nominato Re d'Acolhuacan. I. 247. Le fue nozze 253. Punifce colla morte il fuo figliuolo 295. La fua clemenza verfo i biragnofi 297. Come interpreta i fenomeni offervati nel regno di Motezuma 287. Il fuo ingegno 297. La fua morte 294.

Nigua, infetto pernicioSo. I. In

Nobiltà. Diverfi gradi di Nobiltà preffo i Mefficani confuft dagli Spagnuo1i. If. 120., e prefentemente ayviliti \$2x. Accordo fra la Nobiltà, e 
la Plebe meflicana $a^{\prime}$ tempi del Re Itzeoatl. I. 215.220

Nopaltzin, Re de' Cicimechi. Il fuo governo. I. I40. La fua morte 143

Nozze de'tre Principi Acolhui. I. 137. di Nezahualcojotl 23\%. di Nezabualpilli 253. de'Miztechi. 11. 93. Ceremonie delle Nozze. V. Riti Nuziali.

\section{O}

Bblazioni ne'tempj, e nelle cafe de'Meflicani. II. so

Occbione, perce fingolare. I. I03

Oceloxocbitl, offia Fior della Tigre affai bello. 1. 47

Olintetl, Signor di Xocotla . Come tratto gli Spagnuoli ? III. 36

Olmecbi, Nazione antica d'Anahuac, cacciati da'Tlafcallefi. I, I47

Omacail, Dio delle allegrezze. II. 22

Ometeuctli, ed Omecibuatl, Dei del Cielo. II. 8

Omicidio. Come fi puniva in M? II. 130. In Acolhuacan. 137

Onobualco, nome antico di Jucatan, e di Tabafco. I. 35

Oratoria pregiata da' Mefficani. Il. 74

Ordaz ( Didaco) bravo Capitano Spagnuolo. Sale ful Vulcano Popocate. pec. III. 68. Combatte coraggiofamente co' Meflicani. 123. Efpugna la Città di Tochtepec. 158

Ornamenti de' Mefficani. II. 224.

Orti galleggianti nel lago Mefficano.II. Is2. Fatti da' Meflicani per ordine del Re d'Azcapozalco, 1. I 76

ofpizio. V'erano Orpizj in Meflico per alloggiare iSignori foreftieri. II. 3 I

Otomiti. Notizie di queft'antica Nazione.1. 147. Servono bene a'Tlafcallefi contro i Mefficani 277. Si confederano cogli Spagnuoli. III. 203. Che fentivano intorno all'anima? II. 4. I lor facrifizj 47. Le loro nozze. 92

Otompan, offia Otumba, Città confiderabile del regno d'Acolhuacan. I. 29. Si ribellano i fuoi abitatori contra il Re Ixtlilxochitl 187 . ed ammazzano un Ambalciatore di lui 188. Battaglia famofa d'Otompan. III. 140

P Alazzi del Re di Meffico. I. 27I. II. 202. del Re d'Acolhuacan. I. 232. del Signor d'Iztapalapan. III. 76. del Signor di Xocotla. III. 36 Palma. Diverfe Cpezie di Palme nel M. I. 58

Pallone. V. Giuoco.

Pane de' Mefficani. Com' era, e come fi faceva? II. 218

Papantzin, Principeffa. Succeflo mernorabile d'efto lei. I. 289

Papagalli. La lor varietà ed abbondanza nel M. I. 90

Pafferi mefficani eccellenti nel canto. I. 90.

Pellicano. Ve ne fono due spezie nel M. I. 84

Pena. Che pene erano in ufo preffo. $\mathrm{i}$ Mefficani? II. I30. 138

Penne. Lavori eccellenti, che ne facevano i Mefficani. II. 197. 20\%. 209

Pepe di Tabafco. Defcrizione dell'albero, che lo porta. I. 55.

Pefca de'Mefficani nel lago. II. 162:

Pefci de' Mari d'Anahuac. I. 99.

Piante del Meffico. I. 45. e regu. Quali erano le più coltivate? II. 158.

Piantanimali, o Zoofiti del M. I. II 6

Pietre prezevoli del M. I. 43

pioggie copiofiffime nella ftate. I. 40 
Piftrice di due fpezie. I. roz.

Pittura. Diverfe pitture ufate da'Mefficani. II 187. Tela e colori di cuí fi fervivano. 189 . Carattere della lor pittura, e modo di rapprefentar gli oggettirgr. Efpofizione di fette pitture full' educazione de figliuoli. I02. e d'altre efprimenti le città tributarie, ed i tributi. 124. Pitture dell' armata Spagnuola mandate al Re Motezuma. III. 5. I 8

Poesia. Carattere della Poesia mefficana. II. 175. coltivata più che altrove in Tezcuco. I76. Componimenti poetici del Re Nezahualcojot I. 245 .

Poeta. Come foampd la Vita un poeta condannato alla morte? II. 176

Poliglotto, uccello famoro. V. Centzontli.

Ponti. Com' erano quelli de' Mefficani. Il. 169

Poffefroni della corona, della Nobiltà, del Comune de' luoghi, e de'Tempj. II. I 22

Pofte e Corrieri, preffo i Mefficani. La lor velocità. II. IIy.

Prefagj dell' arrivo degli Spagnuoli. I. 266, e 'egu.

Provincie dell' Imperio Meflicano. I. 31. Tributi che pagarano. II. I 24.

Pucuaro. Pietruzze di Pucuaro I. 38.

Uadrupedi del M. I. 69. e fegu.

Q

Quaglie. Quanto abbondanti nel M. I. 85

Quaquavppitzabuac, Re di Tlatelolco. I. I 74

Quateotzin, Chalchefe. La fua umanità verfo i prigionieri meflicani, e la fua difgrazia. I. 2 so

Quaubrabuac, grande, bella, e forte Città de'Tlahuichi prefa da' Meflicani. I. 227 , e poi dagli Spagnuoli III. 185

Quaubpopoca, Signor di Naubtlaa. S'intriga cogli Spagnuoli. Ill. 66. Lo bruciano vivo. 98

Quaubquecbollan, Città . Defcrizione d'effa, delle fue fortificazioni. III. 153

Quaubtitlan, Città grande e bella della valle Mefficana..I. 30.1I.177.conquiftata dal Re Itzcoarl I. 228. Sacrifizj inumani che vi fi facevano. II. $5 \mathrm{r}$

Quaubtlatoa Re di Tlatelolco uccifo da' Mefficani. I. 232

Quaubtemotzin. Eletto Re di Meffico. III. I59. le fue diligenze per riprender Xochimilcho. I 88. Ributta le propofizioni di Cortès 215.2225 . Non vuole abbuecarfi con Cortès. 226. 228. prefo dagli Spagnuoli 229. meflo alla tortura, ed impiccato. 233

Quetzalcoatl, Dio dell' aria, già fommo Sacerdote di Tula. Che raccontavano della fua vita, del fuo governo e de' fuoi prodigi? II 11. Opinione del Dr. Siguenza intorno alla perfona di Quetzalcoatl. 13. Tempj confacrati ad onor di lui. 12. 30. 33. Ordine di Religiofi confacrati al fuo culto 43 .

Quinatzin Re d'Acolhuacan. Ribellioni accadute nel fuo, regno. I. I44 Onori fattigli nella fua morte. 145

R. Adici ufuali nel M.I. 47 $\mathrm{R}$

Ragie utili del M. I. 63. Ragia elaftica, offia ule. 67

Ragni del M. I. I10.

Rame di due forti in Zacatolan. I. 43 
Ra ne di quattro fpezie I. 99.

Re Toltechi. I. 117. Cicimechi. 146. Elezione, unzione, ed incoronazio. ne de' Re di Meffico. II. I12. e fegu. Abiti, ed infegne Reali. IIs

Rettili del M. I. 93. e regu.

Riti-fuperftiziofi nella nafcita de'Figli, II. 86. nelle Nozze. 89. ne' Funerali. 93. Riti fingolari nella fefta di Tezcatlipoca 68., in quelle d' Huitzilopochtli. 70. 80. ed in quella dell' arrivo degli Dei . 78

Roballo, pelce delicato. I. I02

Rospo. Grandezza ftroordinaria de' Rofpi in alcuni paefi caldi. I. 95

Rofpo, pefce fingolare. La fua defcrizione. I. I02

Ruffiana. Che pena aveva in Meflico? II. 132

SAcerdote. Numero, e gradi de' Sacerdoti Mefficani. II. 36. I loro im pieghi, il lor abito, e la lor vita. 39. Aufterità de' Sacerdoti di Teohuachan, ${ }^{2}$

Sacerdoteffe. Il lor impiego. II. $4 \mathrm{I}$

Sacrifizio. Come fi faceva il facrifizio comune di vittime umane? II. 45. e il facrifizio gladiatorio? 47. Non v'è certezza intorno al numero de' facrifizi, che annualmente fi facevano 49. Sacrifizio inumano d'una Principeffa di Colhuacan. I. I 7I. Sacrifizj orrendi in Quaubtitlan. II. 5x. Sacrifizj d'a nimali . 5o. 5s. Numero eccellivo di facrifizj nella dedicazione del tempio maggiore. I. 257

Salaffo. Come l'uravano i Melficani? II. 214

Sandoval ( Gonzalo de ) famofo Conquiftatore Spagnuolo. Il fuo elogio.. III. 8. Afralifce coraggiofamente il tempio di Cempoalla,e piglia il General Narvaez. II 7. Sconfigge i Meflicani preffo a Chalco, 174. ed in Huaxtepec. I8o. conduce le Truppe 'Tlafcallefi a Tezcuco co' materiali deterigantini dopo aver caftigati i Zoltepechefi . I75. Dà un affalto terribile a Jacapichtlan. I80: La fua pazienza, ed ubbidienza. I82. Piglia per affalto Iztapalapan. 199. S'accampa con un buon numero di truppe in Tepejacac per affediar la capitale. 200. Vince i Matlatzinchi. 2r7. Fa pigliare i Re di Meffico, d'Acolhuacan, e.di Tlacopan, che fuggivano. 229.

Sapone, Che uravano i Mefficani in vece di fapone? II. 227

Scalá. Com' erano le icale de'tempi ? II. 28

Scarpe. Com' erano quelle de'Mefficani? II. 223

Scbiavo. Quante forti di fchiavi erano preffo i Meflicani? II. 134. Potevano aver peculio, ed anche altri fehiavi, che lor ferviffero,e la loro fchiavitù non era ereditaria 135. Si compravano fchiavi da facrificare. 49. 135. Leggi fugli fchiavi 134. La fchiavitù degl' Indiani fu affatto tolta da' Re Cattolici. 136

Scimie. Ve ne fono parecchie fpezie nel M. I, 76

Scorpioni. Ofervazioni intorno ad effi. I. no

Scudi di diverfe fatte adoprati da'Mefficani. II. I4I

Scultura de'Mefficani. II. I 94

Scuole pubbliche, e Seminarj per la gioventù. Il. 109. Scuole di Pittura, di Mufica \&c. fondate in Tezcuco dal Re Nezahualcoiotl. I. 326

Secolo mefsicano. II. 229. Efpofizione del fecolo. 57 . Figure del fecolo.64.

Dichiarazione d'effe. 248. Gran fefta nel cominciare il fecolo. 84.

Sepolcrı de'Mefsicani, de'Cicimechi, e de' Miztechi. 97. 
Serpi di diverfe fpezie. I. 25. Offervazioni fu'lor denti, e fulla maniera di comunicare il veleno. 96. Serpe de' fonagli. Ivi. Serpe luminofa. 97. Serpi allevate da' Mefsicani. 98

Seta del M. 1. Iro.

Smeraldi. La lor abbondanza nel M. I1. 206. 209. Smeraldi di gran valo, re portati da Cortès in Ifpagna. 206

Sodomia. Come fi puniva in M. 1I. 132., e in Acolhuacan? 137

Sole, divinizzato da' Mefsicani. II. 9. Tempj in Teotihuacan confacrati al Sole, e alla Luna. 34. Ogni giorno facevano nove incenfazioni ad onor del Sole. 39. e gli facrificavano delle quaglie. 50. Digiuno ad onor del Sole. 53. L'anno Mefsicano aggiuftato preflo i Mefsicani al corfo folate. 58.62

Spada mefsicana. Com'era? II. 144

spada, pefce diverfo da quello del mar di Groenlandia. I. roz

Spagnuoli. Prefagj ful lor arrivo. I. 288. i primi lor viaggi alle cofte del M. III. 3. e fegu. La lor vittoria in Tabafco ro. Sbarcano in Chalchiuhcuecan. I4. Entrano in Cempoalla 25. Fondano la Colonia della Veracroce 29. Si confederano co'Totonachi. Ivi. La lor guerra co' Tlafcallefi. 4I, e regu. Fanno la pace. 5ó. Strage fatta da loto in Chololla.6x. La folenne lor entyata in Mefsico. 77. S'alloggiano nel palazzo del Re Axajacatl. 78. Son regalati dal Re Motezurna. 83. La lor maraviglia nel veder quella Città . 87. I lo" conflitti in effa . I22. L' orribile loro fconfitta. I33. Mangiano nella lor ritirata un Cavallo. I40. La famofa lor battaglia in Otompan. Ivi. Si ricoverano in Tlafcal. 1a. 143. La lor guerra in Tepejacac. 15 1 . in Quauhquechollan. 153. In Itzocan. 156. In Xallatzinco, ed in altri luoghi, 158. La lor entrata in Tezcuco. I67. La loro fpedizione contra Iztapalapan. 172. Le loro fcorrerie ne' contorni del lago Mefsicano. 177. Pigliano Quauhnahuac. 185 , e Xochimilco. 187. Congiurano alcuni di loro contra Cortès. I go. Sono fconfitti, el alcuni di loro facrificati da' Mefsicani. 2ro. Trattengono nell' affedio della Capitale la crudel tà de' loro Alleati. 227. Si rendono padroni di Mersico. 23I

Spedali per li poveri. II. 36. Per li Soldati invalidi fondati da Motezuma II. in Colhuacan I. 275., e da Nezahualpilli in Tezcuco, 297

Spie da' Mefsicani appellate Quimichtin, o Sorci. II. 147. Come furono trattate da Cortès le Spie de' Tlafcallefi? III. 47

Stendardi de' Mefsicani, e de' Tlafcallefi. II. I45. Com'era quello che pigliò Cortès nella battaglia d'Otompan. III. I $4 \mathrm{I}$

Strada. Le Strade pubbliche fi racconciavano ogni anno. II. 168. Stradeful lago Mefsicano. III. 85

Strumenti muficali. II. I 78. Strumenti d'agricoltura. 153

Succiafore, uccellino maravigliofo ed affai bello. I. 86

$\mathrm{T}$ $T$

Abacco. L'ufo d'effo prefto i Mersicani. II. 227. Ve ne fono due 'pezie. Ivi N.

Tabafcheß vinti dagli Spagnuoli. III., 10

Tajatzin, Principe erede della Corona d'Azcapozalco. I. 194. La fuaconferenza col Re di Mefsico. ig6. uccifo dal fuo fratello Maxtlaton. I98 Tapajaxin, lucertola orbicolare. La defcrizione d'effa. I. 94

Tapir. V. Danta. 
Tarantola, ragno grande e pelofo. I. I1 1

Tarafchi, o Michuacanefi, nazione, Notizie d'effa. I. 148

Teatro Mefficano. Com'era? II. I76

Tecalli. V. Alabaftro.

Tecamaca, ragia medicinale. I. 65

Tecbicbi, quadrupede del M. I. 73. che ne facevano i Mefficani ne' funerali? II. 94

Tecbotlala, Re d'Acolhuacan. Succede a Quinatzia. 1. 146. Il fuo governo. 180 . La fua morte. 184

Tecomatl. V. Vafi.

Tetle o fiaccole nuziali. II. 9 I

Tele di varie fatte e materie, che facevano i Meflicani. II. 207

Temazcalli, o Ipocaufto meflicano. Struttura d'eflo, e modo di fervirfene. II. 2 I 4

Temolin, due Tpezie di Scarafagi. I. 107

Tempio famofo del Re Nezahualcojotl. I. 246. Tempio maggior di Meffco edificato dal $\operatorname{Re}$ Ahuitzotl. 257. Defcrizione e mifure d'effo. II. 25. Edifizj anneffi al tempio maggiore. 30. Tempj di Tezcuco, di Chololla, e di Teotihuacan. 33. Moltitudine di tenupj, e lor rendite. 35

Tenajoccan, Corte antica de'Re Cicimechi. 1. 134. prefa per affalto dagli Huexotzinchi confederati del Re Itzcoatl. 219

Teokuacan, o Tebuaian Città e Stato confiderabile del regno di M. Aufterità che viefeguivano i Sacerdoti. II. 54. Che pena vi avea il Sacerdote reod' in continenza.4I. I Teohuacanefi deftriffimi nel frecciare. I 43 Teopixqui, nome generale de'Sacerdoti. II. 39

Teotetl, offia pietra divina fimile al marmo nero. II. 7 .

Teotibuacan. Piramidi e tempi preßso a quefta Città. II. 34

Tectl, nome di Dio, che vale lo fteffo, che il Tbeos de Greci. II. 172

Tepanecbi, tribù di Nahuatlacbi. I. 151. Sconfitti da'Tlafcallefi. I 54. vinti e loggiogati da'Mefficani. 2 I 8

Tepejacac, Città, e Stato confiderabile del regno di M. I. 37. che tributo pagava alla corona? 1I. I 26. Guerra degli Spagnuoli in Tepejacac. III. IsI

Tepejacac, Vilaggio tre miglia da. M. a tramontana, dove s'accampò il Comandante Sandoval nell' affedio della Capitale. III. 200. V'è prefentemente il più famofo fantuario del nuovo Mondo. II. 22

Teponaztli, firumento muficale de' Meflicani. II. I 79

Terra del M. in gran parte montuofa. 1. 36. Divifione delle Terre prefto i Mefficani. II. 122 .

Teucti, primo grado di nobiltà in Tlafcalla. Come s'acquiftava? II. I 20

Teucli. Giudice di contrada in M., luogotenente del Tlacatecatl. II. I28

Tentilie, Governatore Mefficano. Fa buona accoglienza agli Spagnuoli. III. I6

Tezcuco, Gran Città. La fua fituazione- I. 28. Fondata da'Cicimechi 137 Corte de'lor $\mathrm{Re}$, e capitale d'Acolbuacan. 144. La pirt culta di tutto il paefe d'Anahuac 247., e la più grande. 11I. 74. Dalla in feudo il Tiranno Tezozomoc al $\operatorname{Re}$ di M. I. 190. La recupera Nezahualcojo:l 208. V'entrano gli Spagnunli la prima volta III. 74. vi fanno la lor piazza d'armi. I $7 \mathrm{I}$

Trzozomor, Regolo d'Azcapozalco. Si ribella contra il Re Ixtlilxochitl. I. 185. lo fa morire, ed ufurpa la corona d'Acolbuacan. I89. Impone nuovi aggravj a' fuoi fudditisgl. Muore. I94 
Tiburone, beftia marina troppo vorace. I. 100

Tigre Mefficana. I. 70

Tigri, Ordine militare appo i Mefsicani. II. $\$ 40$

Tizatlalli, terra bianca minerale. II. 189

Tizoc, eletto Re di Meffico. I. 253. morto 256

Tlacatecall, Magiftrato, e Tribunale di grand'autorità. II. 127

Tlacatecatl, carica di General d'efercito. II. 147

Tlacatecco, luogo nel tempio, dove fi ritiraya il Re dopo la fua elezione. II. II4

Tlacateotl, Re di Tlatelolco. I. 184

Tlacocbcalcatl, fupremo grado nella Milizia. Il. 139

Tlacocbtli, o Dardo mefficano. Com' era? II. I 44

Tlacopan, oflia Tacuba, Città, e Corte.I.28. Vi fi fonda una piccola Monarchìa 224. Vi s'accampa il Comandante Alvarado nell' affedio di Meflico. III. 196

Tlacuatzin, Quadrupede affai fingolare. I. 72

Tlabuichi. Tribù di Nahuatlachi. I. I gI. Il lor paefe 3I

Tlabuicole, celebre Generale Tlafcallefe fatto prigioniere dagli "Huexotzinchi. 1. 28I. Sacrifica to in Meffico. 282

Tlaloc, Dio dell' acqua, e de' Monti. II. 14. Fefte di lui. V. Fefte.

Tlescalla, Repubblica. La fua origine. I. 154. La fua eftenfione e fituazione 29. Il fuo governo I55. Le fue leggi. II. 138. Chi reggevamo la Repubblica allorchè vi arrivarono gli Spagnuoli? III. 38. Numero delle cafe, e de' fudditi della Repubblica. 54

Tlafcalla, Città capitale della Rep. del medefimo nome. La fua fituazione. I. 29. Divifione de' fuoi quartieri. I55. La fua grandezza. III. 53

Tlascallefi. La lor origine. I. $15 \mathrm{r}$. La famola lor vittoria in Pojauhtlan. 354. Fondano Tlafcalla, ed altri luoghi 55. Il lor carattere 156. La lor guerra co' Mefficani 275. Danno ajuto a' Miztechi, ed a' Cotaftefi contra i Mefficani, e fono fconfitti 236. Troppo gelofi di confervar le loro cafe e famiglie. II. I23. I lor diverfi pareri fulle pretenfioni degli Spagnuoli. III. 38. La lor guerra cogli $S_{\text {pagnuoli }}$ II. La lor pace e confederazione 50. Danno ubbidieniza al Re di Spagna 52. Áccompagnano gli Spagnuoli nel lor viaggio 56. fconfitti con effo loro in Meffico 138. uccifi nella battaglia d'Otompan 143. Fanno buona accoglienza agli Spagnuoli nella lor ritirata . 144. La lor rifpofta all'ambafciata del Re di M. I49. Rivifta delle lor truppe, che doveano ajutar gli Spagnuoli nell'affedio di Mefficb. 164

Tlatelolco, Città fondata nel lago mefficano. I. i 70 Conquiftata da Meflicani, ed unita a quella di M. 248

Tlatelolcbi. Cagione della lor difcordia co' Meflicani. I. 16r. La lor divifione 170.1 lor $\operatorname{Re} 174$. e regu.

Tilizapotl, othia Zapote nero, frutto deliziofo. I. 5I

Tlotzin, Re de' Cicimechi. I. I 43

Tollan, offia Tula, Città antica e celebre. I. 3I. Fondata da' Toltechi, e divenuta metropoli di quella Nazione 126. Vi arrivano i Mefficani 161

Toltecbi, Nazione antica, e famora. La lor origine, ed i primi loro ftabilimenti in Anahuac. I. 126. I lor $\operatorname{Re} 127$. I lor coftumi. Ivi. Le for difgrazie I 30 . Le lor alleanze co' Cicimechi 135. Fabbricarono l' alta piramide, o tempio di Cholulla 129. Aneddoti del Cav. Boturini intorno a' Toltechi 128.330 
Toteotzin, Signor di Chalco troppo crudele. Vuol ractificare il prode Mo tezuma. I. 210

Totonacapan, Provincia grande del Meffico. I. 34

Totonacbi. Si confederano cogli Spagnuoli. III. 29

Tributo. Che tributo pagavano le Provincie del M. IL. 24 . Tribu to di ptdocchj impofto dal Re Motezuma a'mendici. I. 275

Tuza, quadrupede curioro del genere delle Talpe. I. 79

Tzacua, uccello fingolare. I. 92

Tzilacatzin, famofo Tlatelolchefe. La fua forza ed agilità. III 207

$\mathrm{V}$

Ajuolo. Strage fatta da efro nel M. III. 159

$V a f i$ da bere preffo i Mefficani. II. 225

Ubbriacbezza punita rigorofamente in M. II. 134. In Acolhuacan. I37

Uccelli di rapina. I. 8 r. notturni 83 . ácquatici 84 . da mangiare 85. di vaghe penne 86. cantori 88. parlatori 90

Veracroce, prima colonia degli Spagnuoli. III. 29. Tre Città del medefimo nome 30. N.

Vermi particolari del M. I. rog

Vespe di varie fpezie. I. 108

Vino di frumentone, e di maguei. 1I. 228

Viti troyate nel M. I. 48

Ulùa ( S. G'o: d') iroletta, e porto. Perchè cosł appellati? III. 6

Unzione. Come fi faceva quella de' Re di M ? II. I Is. Unzione de' fommi

Sacerdoti 37. Altre unzioni laperftiziofe 40

Volte negli edifizi mefficani. II

Vulcani del M. I. 40

X

Icalli, vafo da bere. II. 22 ;

$\mathrm{X}$

Xicotencatl, Signor di Tlafcalla: Il fuo parere full'affare degli Spagnuoli. III. 40 . Si battezza 149

Xicotencatl il giovane, General d'efercito in Tlafcalla. Il fuo carattere. III. 4I. Fa la guerra agli Spagnuoli 45. Confulta gl' Indovini di Tlafalla 47. Si porta a nome del Senato a Cortès per far la pace so. Il fuo parere nel Senato contra gli Spagnuoli 148 . Lu fa impiccare Cor tès. 194

Xiloxochitl, o Tiata, fior fingolare. I. 47

Xiquipilli. Che cola era? II. 147. 165

Xiubteuctli, Dio del fuoco. 11. 16. Fefte ad onor di lui. 76. 83. 51,

Xocbimilco, gran Città della Valle mefficana. 1. 30. Conquiftata dal Re

ltzcoatl 226. combattuta, e danneggiata dagli Spagnuoli. III. 187

Xocbimilchefi, tribù di Nahuatlacbi. I. Is 1 . La lor guerra co' Colluui 16s

Vinti e foggiogati da' Mefficani 226

Xocotla, Città confiderabile del M. III. 37

Xolotl, Re. Conduce in Anabuac una numerola colonia di Cicimechi. I.

133. Si ftabilifce in Tenajoccan 134. Favorifce i Toltechi 135. Fa

buon' accoglienza agli Acolhui, e ad altre Colonie. Ivi. Fa la divi-

fione degli Stati 138. La. fua morte, ed efequie. 139

Moloilzcuintli, quadrupede del M. I. 77

2 Apatz, o Tzapotl, nome di parecchj frutti del M. I. s2

Zopilate, Uccello utiliffimo nel M. I, 82. Legno del Zopilote affai bello. 60 
$E R \mathcal{A T} \mathcal{A}$

Pag. 7. lin. I 8 intraprefe
$C \oplus R R I G E$

imprefe.

ananàs.

forbito.

vi numera.

ricompenferà.

di venti mila cale.

nell' affermare.

all' a mbizione.

furero.

agli 8. Luglio.

e levandogli.

Cugino.

darle.

e da quefto.

fi perfuafe.

fi diedero.

Iztapalapan .

Mizquic.

deferzione.

riempiere.

Oltre a quefti, o ad altri errori fcufabili mella pog. I5. manca ffotto la pofilla feguente: 5. 6. Arrivo dell' asmata al porto di Chalchiuhcuecian.

SBAGLI SFUGGITI NE' DUE PRIMI TOMI.

Nel tomo I. pag. I26. dore fi dice parlando della pellegrinazione de' Toltechi, ch'efra cominciò l'anno I Tecpatl, cioè il 596. detl' era volgare, debbe dir così l'anno I. Tecpath, cioè il 544. dell' ere volgare, come fi legge nel mio originale Spagnuolo, e nelle mie differtazioni: quell' altro fu uno sbaglio nella traduzione. Nella medefima pagina dove fi dice, che la Monarchìa dei Toltechi cominciò nell' anno VIII. Acatl, cioè nel 667 dell' era volgare debbe dire nell' anno VII. Acatl. $\mathcal{O}^{\circ}$, poichè l'anno 667. non fix VIXI, ma VII. Aiatl. Nel tomo 2. pag. 59. dove fi dice, che ogni qrattro anni s'anticipava un giorno l'anno meficano per cagione del giono intercalare del nobro anno bifeftile; onde negli ultimi anni del fecolo meficano cominciava aili 14. Febbrajo \&5c. debbe dire, cominciave alli 13. Febbrajo.

Nella pag. 16. del medefimo tomo 2. dove fi dice che alla Dea Centeotl

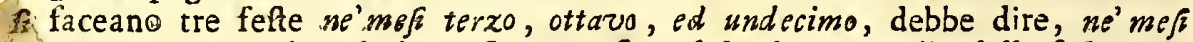
uruarto, ottavo, ed undecimo, ficcome fi vede nel ragguaglio delle fefte pag. 68 , e nel Calendario pag. 236.

Nel fudiletto Calendario pag. 239, dove fi legge Fefta di Maculitocbili, debbe dire, Fefta di Macuiltocbtli, e nel fine del Calendario, dove fi dice, L'anno Seguente II. Acatl comiacia da IL. Mizquili, debbe dire, da II, Miquiztli.

AVVISO DELLO STAMPATORE.

Il tomo delle differtazioni non fi pubblicherà probabilmente infin'al mefe d'Agofto di queft'anno ( $178 I_{\circ}$ ) ma $\mathrm{fi}$ faranno tucti gli sforzi poffibili acciocchè venga più prefto in luce. 


\section{STORIA ANTICA \\ DEL MESS I C O}

CAVATA DA' MIGLIORI STORICI SPAGNUOLI,

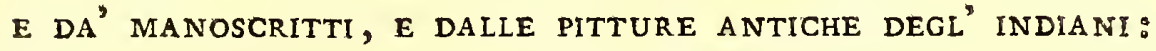

$$
\text { DIVISA IN DIECI LIBRI, }
$$

E CORREDATA DI CARTE GEOGRAFICHE,

E DI VARIE FIGURE:

E

D I S E R T A Z I O N I

Sulla Terra, fugli Animali, e fugli abitatori del Meffico.

O P E R A

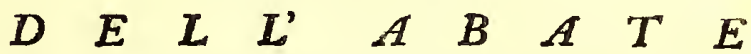

D. FRANCESCO SAVERIO

C L A V I G E R O

$T$ O MO IV.

CONTENENTE LE DISSERTAZIONI。

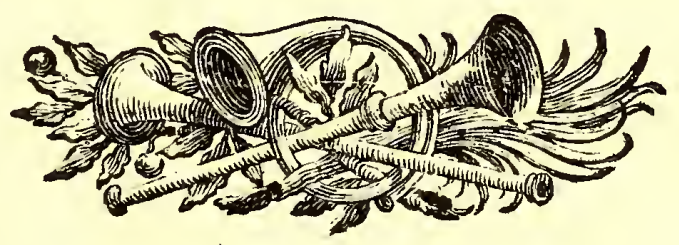

IN CESENA MDCCLXXXI.

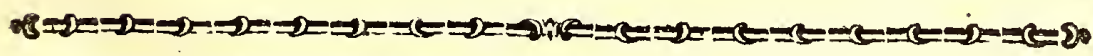

Per Gregorio Biasini ali' Insegna di Pallade Con Licenza de' Superiori. 



\title{
A SUA ECCELLENZA
}

\author{
$1 \mathrm{~L} S I \mathrm{G} N O R$
}

\section{DON GIOVANNI RINALDO CONTE CAR LI}

Cavaliere e Commendatore del Sacro Ordine de'SS. Maurizio

e Lazaro, Configliere Intimo attuale di Sua Maeftà limp.

R. Cef. ed Ap., e Prefidente già del Regio Ducale Magiftrato Camerale nella Lombardìa Auftriaca \& $c_{\text {. }}$

Francesco Saverio Clavigero.

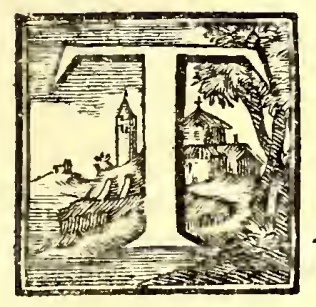

Anto per far palefe l'alta ftima, che ho; della voftra rifpettabil Perfona, quanto per ringraziarvi a nome degli Americani, io vi dedico la prefente opera: la quale, benchè non degna del voftro fingolar merito, è nondimeno la cofa, che ho piu cara.

Egli è a me rincrefcevole d'effere ttato creduto una volta contrario, foltanto per qualche diverfità A 2 di 


\section{4}

di fentimenti, a un sì celebre Autore, che per tanti capi fi è conciliata la ftima di tutti i Letterati. Gli Americani poi vi fono oltremodo tenuti, avendo efli avuto in Voi un Difenfore non meno illuftre per la fua nafcita, che ragguardevole per le fue rilevanti cariche, e foprattutto chiariffimo per li fuoi luminofi fcritti: (*) il qual in mezzo a più fpinofi affari di Stato ha faputo trovar tempo per iftudiare accuratamente la Storia dell' America, ed ha aruto coraggio per difender quelle difpregiate Nazioni contra tanti rinomati Europei dichiarati lor nemici e perfecutori.

Spero, che quefta opera mia, compofta anch'effa per dileguar gli errori pubblicati in Europa contra l'America, farà da Voi gradita, come un contraffegno del mio rifpetto, e della gratitudine degli Americani verfo di Voi.

DIS-

(*) Le opere pubblicate dal Sig. C. Carli fono I. Della Spedizione degli Argonauti. 2. della Teogonia d Efiodo. 3. Delle Monete, e della Inftituzione delle Zecche in Italia. 4. L'Uomo Libera. 5. Le Lettere Americane, delle quali fonfi pubblicati due tomi, e non iftarà guari a pubblicarfi il terzo. 6. Una Differtazione pubblicata fin dal 1745. contro i volgari pregiudizj della Stregonerìa, delle Larve domeftiche, degl' incan . tefimi \&c. Opere tutte piene di fquifita erudizione. 


\section{5 \\ DISSERTAZIONI \\ SU LA TERRA, SU GLI ANIMALI, E SU GLI ABITATORI DEL MESSICO}

Nelle quali $f_{i}$ conferma in parte la Storia antica di queb paefe, s'illuftrano molti articoli di Storia naturale, e fi confutano moltiffimi errori Spacciati intorno all' America da alcuni celebri Autori moderni.

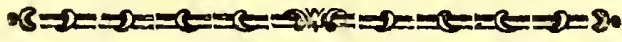

\section{A CHI LEGGE.}

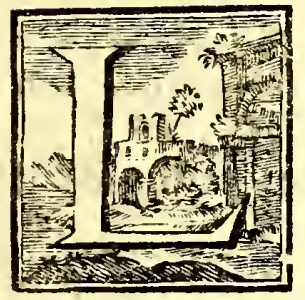

E Differtazioni, che diamo ora in luce, fon neceffarie, non che utili, per illuftrar la Storia antica del Meffico, e per confermar la verita di molte cofe in effa contenute. La prima differtazione è neceffaria per fupplire alla mancanza di notizie intorno alla prima popolazione di quel nuovo Mondo. La feconda, quantunque nojofa, non fi vuole ommettere, perchè fi fappiano i fondamenti della noftra Cronologia, e fara utile per chiunque vorrà nell'avvenire fcriver la ftoria del Meffico. Tutte l'altre parimente fon neceffarie per diftornar gl' incauti lettori da quegli inganni, ne'quali faranno ftati involti da quella gran turba d'Autori moderni, che fenza averne fufficiente cognizione, fi fon meffi a fcrivere della terra, degli animali, e degli nomini dell' America .

Imperocchè quanti in leggendo, per efempio l'opera del Ricercatore $\left({ }^{*}\right)$, non $s^{\prime}$ empieranno le tefte di mille idee fconvenevoli, e contrarie alla verità della mia Storia? Egli è Filo.

(a) Il Sig. de Pavv. nell'opera intitolata: Recherches pbilolopbiques fur les Amerisains. 
lofofo alla moda, ed erudito, maffmamente in certe materie; nelle quali farebbe meglio che foffe ignorante, 0 almeno che non ne parlaffe. Egli condifce i fuoi difcorfi colla buffonerìa, e colla maldicenza, mettendo in ridicolo, quanto v'è rifpettabile nella Chiefa di Dio, e mordendo quanti gli fi parano avanti nelle fue ricerche fenza verun riguardo alla verità, nè all' innocenza. Egli decide francamente, ed in un tuono magiftrale, cita ad ogni tre parole gli Scrittori dell' America, e protefta, che la fua opera è frutto della fatica di dieci anni. Tutto ciò rende affai commendabile l'Autore preffo molti lettori del noftro fecolo filofofico. La fua maldicenza, il difpregio, con cui fparla de' più venerabili- Padri della Chiefa, lo Icherno, che fa de' Romani Pontefici, de' Sovrani, e degli Ordini Religiofi, e il poco conto, che moltra fare de' Sacri libri, in vece di fcemar la fua autorità, potranno accrefcerla in un fecolo, nel quale fi fon pubblicati più errori, che in tutti $i$ fecoli paffati, fi forive con libertà, e fi mentifce con isfacciataggine: non è pregiato chi non è Filofofo, nè fi re. puta Filolofo chi non fi beffa della Religione, e prende il linguaggio dell' empietà.

L'argomento dell'opera del Sig. de Paw è quello di perfuadere al Mondo, che in America la Natura ha degenerato affatto negli elementi, nelle piante, negli animali, e negli uomini. La terra ingombrata da alti monti, e rupi, e nelle pianure allagata da acque morte e guafte, o coperta di valti bofchi, e si folti, che non vi poffono penetrare $i$ raggi folari; $\grave{e}$, dice egli, generalmente affai fterile, e più abbondante di piante velenole, che tutto il refto del Mondo. L'aria mallana, e più fredda affai di quella dell' altro Continente. Il clima contrario alla generazion degli animali . Tutti gli anima!i propri di que' paefi erano più piccoli, più deformi, più déboli, più codardi, e più itupidi di quelli del Mondo ancico, e quelli, che vi fi trafportarono altronde, solto tralignarono; ficcome pure tutte le piante d'Europa trafpiantatevi. Gli uomini appena differivano, dalle beftie, ie non nella figura: ma ancoxa in quefta fi f corgono molte tracce della lor degenerazione: 
il colore olivaftro, la tefta troppo dura, ed armata di groffe capelli, e tutto il corpo privo affatto di pelo. Eglino fon brutti, e deboli, e foggiacciono a molte malattie ftravaganti, cagionate dal clima infalubre. Ma comecchè tali fieno i loro corpi, fono anche più imperfette le loro anime. Eglino fono a tal fegno di memoria sforniti, che oggi non fi ricordano di ciò, che fecero jeri. Non fanno far rifleffioni, nè ordinar le loro idee, nè fon capaci di migliorarle, neppur di penfare, perchè per $i$ lor cervelli foltanto rigirano umori groffi e vifcofi. La loro volontà è infenfibile agli ftimoli dell' amore, e d'ogni altra paffione. La loro pigrizia gli tiene inmerfi nella vita felvaggia. La loro codardia fi fece palefe nella conquifta. I loro vizj morali erano corrifpondenti a quefti difetti fifici . L' ubbriachezza, la 'bugia, e la pederaftia erano comuni nelle ifole, nel Meffico, nel Perù, ed in tutto il nuovo Continente. Vivevano fenza leggi. L.e poche arti da loro conofciute erano troppo groffolane. L'agricoltura era preffo loro del tutto abbandonata, la loro architettura affai mefchina, e più im. perfetti ancora $i$ loro ftrumenti. In tutto il nuovo Mondo non vi erano pì̀ di due Città, Cuzco nell' America Meridionale, e Meffico nella Settentrionale, e queite due altro non erano, che due miferabili Cafali \&c.

Quefto è un leggiero abbozzo del moftruofo ritratto, che il Sig. de Paw fa dell' America. Non l'efpongo interamente, e lafcio ancor quello, che ne hanno fatto altri Autori male informati, o al pari di lui prevenuti, perchè non ho pazienza per copiare tanti fpropofiti. Neppur pretendo fare l'apologìa dell' America, e degli Americani ; perchè vi bifognerebbe un' opera affai voluminofa. Per ifcrivere un errore, o una bugia baftano due linee, e per impugnarla non baftano talora due pagine, e nè anche due fogli: di quanto dunque farebbe meftieri per confutare tante centinaja d'errori? Io però quelli foltanto voglio confutare, che fono oppofti alla verita della mia Storia. Ho fcelto l'opera del Sig. de P., perchè in effa, come in una fentina, o fogna, fi fon raccolte tutte le immondizie, cioè, gli errori di tutti gli altri. Se talvolta parranno 
un pò trioppo forti le mie efpreffioni, ciò è Atato, perchè giu: dico, nob effere convenevole adoperar la dolcezza verfo un uo. mo che ingiuria tutco il nuovo Mondo, e le perfone pì̀ rispettabili del Mondo antico.

Ma avvegnachè l'opera del Sig. de P. fa il principal berEaglio, a cui dirizzo i miei colpi, avrò pur a fare con altri Autori, e trá effi col Sig. de Buffon. Io ho una grande ftima di quelto celebre Autore, e il reputo il più diligente, il più accorto, ed il più eloquente Naturalitta del noftro fecolo; anzi credo, che non vi fia ltato finora al Mondo un altro, che meglio di lui abbia dato a conofcere gli animali; ma ficome l'argomento della fua opera è tanco valto, cos̀ non è da maravigliare, che talora sbagliaffe, of dimenticaffe di ciò, che avea già fcritto, maffimamente rapporto all' America, dove la Natura è sì varia: per lo che nè tali sbagli, nè le ragioni, che contra effi addurremo, potranno in verun modo pregiudicare alla gran riputazione, della quale egli gode preffo tutci i Letterati del Mondo.

Nel paragonare che fo, l'un Continente coll' altro, non pretendo di far comparir l'America fuperiore al Mondo antico; ma foltanto di moftrar le confeguenze che poffono naturalmente dedurfi da' principj di quegli Autori, che impugno. Si fatti paralelli fon troppo odiofi, ed il vantare appaffionatamente il proprio paele fopra tutti gli altr, pare più proprio dı fanciulli che pugnano, che d'uomini lettiatl che difputano.

Nelle citazioni della Storia de' Quadrupedi del Conte de Buffon mi fon prevaluto dell' edizione fatra in Parigi nella. regia Aamperia in tometri trentuno in 12. , e terminata l'anno I768. In quelle delle Ricercbe del Sig. de PaW mi fon fervito dell' edizione di Londra del $177 \mathrm{I}$. in tre tomi colla impugnazione fartagli da Don Pernery, e colla rifpofta del Sig. de $P$. 


\section{DISSERTAZIONE I.}

SU LA POPOLAZIONE DELL' AMERICA, E PARTI. COLARMENTE SOPRA QUELLA DEL MESSICO.

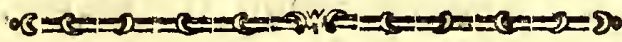

A Ppena troveraffi nella Storia un problema più difficile a A fcioglierf, che quello de!la popolazione dell' America, nè intorno al quale vi fia una più gran varietà d' opinioni. Può dirfi, ch' effe fono tante, quante fono ttate quelle degli antichi Filofofi intorno al Sommo Bene. Ora io non voglio difaminarle tutte, perchè ciò farebbe una fatica infruttuofa: nemmeno pretendo di ftabilire un nuovo fiftema, mentre non vi fono fondamenti da appoggiarlo. Voglio foltanto efporre, e fottomettere al giudizio degli uomini dotti le mie congetture, perchè mi pare, che non faranno affatto inutili; ma per difcorrere con quella chiarezza e precifione, che fi conviene, dividerò il punto generale in parecchj articoli, e dichiarerò in diverfe conclufioni i miei fentimenti

\section{\$. I.}

In qual tempo fi comincio a popolar l' America?

Betancurt $\left({ }^{*}\right)$, ed altri Autori fi perfuafero, che il nuovo Mondo cominciaffe a popolarfi avanti il Diluvio. Ciò potè certamente avvenire, perchè lo fpazio di 1656 . anni fcorfi dalla creazion de' primi uomini infino al Diluvio, giufta la Cronologia del tefto ebraico del Genefr, e della noftra Volgata, e molto più quello di 2242 ., o pur di 22626 anni, fecondo il computo de'Settanta, fu fenz' altro fufficiente per popolar tutto il Mondo, ficcome è fato già da alcuni dimoltrato: almeno

Storia Antica del Meffico Tom. IV. B B dopo

(*) Nel Ieatro Mefficano part. 2. tratt. 3. cap. I. 
dopo dieci, o dodici fecoli poterono alcune famiglie di quelle; che s'andarono fpargendo verfo le parti più Orientali dell' $\mathbf{A}$. fia, paffare a quella parte del Mondo, che oggidi appelliamo America, o foffe, come io credo, a quell' altra unita, o folle da un piccolo tretio di mare feparata. Ma come provare, che in farti fi popolò l'America avanti il Diluvio, come vogliono quegli Autori? Perchè nell' America, dicono, vi furono già de'Giganti, e l'epoca de' Giganti fu antidiluviana. (a) Perche Iddio, diranno altri, non creò la terra, fe non perchè foffe abitata, (b) e non è verifimile, che avendo a tal fine creata l'America, voleffe lafciarla tanto tempo fenza abitatori, e maffimamente avendo egli ordinato a' primi uomini, che fi moltiplicaffero, ed empiffero la terra . (c) Ma ancorchè concediamo a quegli Autori, che il facro telto, nel quale fi fa menzione de' Giganti, debba intenderfi nel fenfo volgare, cioè d'somini di ftraordinaria altezza e corporatura, e non dubitiamo che tali uomini fieno ftati nel nuovo Mondo, ficcome abbiamo detto altrove, a difpetto de' Signori Sloane, $(d)$ de P. e di altri, che non credono, fe non quello, che fono avvezzi a vedere, ciò per altro nulla gioverebbe a confermare quella opi-

(a) Gigantes erant Juper terram in diebus illis. Gen. 6.

(b) Ipfe Deus formans terram, $\mathcal{G}$ faciens eam.. non in vanum creavit eam, ut babitaretur formavit eam. Ifai. 45.

(c) Crefcite, \& multiplicamini, $\sigma^{\circ}$ replete terram. Gen. 1.

(d) Lo fcritto d'Hans Sloane Inglefe, nel quale fi sforza di provare, che i grandi offami ritrovati nell' America fono Atati d'Elefanti \&c., non già di Giganti, fi può leggere, nelle Memorie dell Accademia Real delle Scienze di Parigi dell' anno 1727. Oltre a quello, che abbiam detto nel lib. I. della Storia contra un tal fentimento, aggiungiamo quil l'atteftato del Dr. Hernandez, tefimonio oculare, fincero, e bene intendente: Permulta Gigantum, dice, non vulgaris magnitudinis off a per bofce diesa invent funt, cum apud Tezcocanos, tum apud Tollocenfes. Hac autem, foggiunge, notiora funt, quam ut fides queat illis ab aliquo denegari: "O tamen non me latet, a multis judicari, multa feri non poffe, antequam faEta fint. Adeò verum eft, atque indubitatum quod Plinius nofter dixit: nature vim atque majeftatem omnibus momentis fidc carere. Tract. 1. de Quadrup. N. Hilp. cap. 32. Se negli fcavamenti fatti nell America loltanto fi foffero ritrovate delle offa diftaccate e fparfe, potrebbe a ragione crederfi, che foffero flate appartenenti ad alcune beftie grandi; ma effendo ftati ritrovati e crani umani, e carcami interi, non $v$ 'è̀ 'più luogo alle congetture di Sloane. Vedafi ciò che racconta l'Acofta nel lib. 7. cap. 3. della-fua Storia 
opinione; poichè gli fteffi facri libri ci danno contezza d'alcuni Giganti pofteriori al Diluvio, come d'Og Re di Bazan, $(e)$ e di que' cinque Getei di cui fi parla ne' libri de' Re. Da quefti Giganti, de' quali fi fa menzione nella Sacra Scrittura pel rapporto, che hanno alla ftoria degli Ebrei, polfiamo congetturare, che ve ne foffero molti altri, tanto nella Paleftina, quanto in altri paefi, la cui notizia non importava a' Sacri Storici. Il tefto d' Ifaia nulla prova in favor di quella opinione; poichè quantunque Iddio creaffe la terra, acciocchè foffe abitata, neffuno poò indovinare il tempo da lui prefffo all' efecuzione de' fuoi divini configli .

Il Viaggiatcre Gemelli dice, allegando certe pitture antiche de'Mefficani, $(f)$ che la Citrà di Meffico fu fondata nell'anno II. Calli, corrifpondente, dice egli, all' anno 1325 . della creazion del Mondo, cioè, più di trecento anni avanti il Diluvio; ma quelto enorme fpropofito non fu già un errore della fua mente, ma uno sbaglio della fua penna, ficcome chiaramente $f i$ fcorge in leggendo tutto il contefto della fua narrazione: onde a torto glielo rinfaccia il maldicente Ricercatore, il quale incolpa anche di tal errore il chiariffimo Siguenza, laddove fiamo ficuri, che quefto dotto Mefficano era d'un fentimento affai diverfo. E' pur vero, che la Città di Meffico fu fondata nell'anno II. Calli, e che tal anno fu quello del I 325. ; non però della creazion del Mondo, ma dell'era volgare del Criftianefimo. Il fuddetto viaggiatore in vece di fcriver quefto, fcriffe quell' altro.

Egli de per altro inutile il ricercare, fe l'America foffe B 2

intorno al carcame gigantefco difotterrato nel 1586. in Gesiu del Monte, Villa dei Gefuiti di Meftico vicina a quefta Capitale, allorchè egli vi dimorava. Vedafi pure ciò che dice il Zarate, dotto e riguardevole Scrittore della Storia del Perú, nel lib. 1. cap. 5. intorno alle offa, e crani umani difotters rati a' tempi fuoi in Porto-Vecchio contrada della Provincia di Guayaquil. Vedafi ciò che racconta il finceriffimo Bernal Diaz delle offa prefentate dai Tlafcallefi a Cortès ec. es.

(e) Torrubia nel fuo Apparatoalla Storia Naturale di Spagna incorfe per ben tre volte nel groffo errore di fare Og antidiluviano, ed afferma efpreffamente chiegli fu annegato nel Diluvio:

(f) Giro del Mondo tom. 6. 
popolata prima del Diluvio: imperciocchè per una parte ciờ non potendofi indovinare, e per l'altra effendo certiffimo, che: nel Diluvio perirono tuti gli vomini, bifogna fempre dopo quella inondazion generale cercar per l' America nunvi popolatori. So bene, che alcuni Autori circofcrivona il Diluvio tra: i confini d' una parte dell'Afia; ma fo ancora, che una tal opinione non fi accorda bene colla verità de' facri libri, $(g)$ nè colla tradizione degli fton Amerisani, (b) nè colle offervazio. ni fifiche .

(g) Operti funt omnes montes excelf fub univerfo calo. Quindecim cubitis alvior fuit aqua super montes quos, operuerat. Gen. 7. Pare, che Iddio fuggeetfe: quefte parole al facto Scrittore, per ifmentire le cavillazioni degl' Increduli; poichè non è facile di trovare alre efpreflioni più proprie a fignificar I" univerfalità del Diluvio. $\mathrm{Ma}$ ancorchè quel facro tefto doveffe intendurfi. folamen: te dei monti della Paleftina, e d'altri paefi non rroppo da efra difcolti, fifa come alcuni pretendono, io in vero non poffo capire, come poffa l'acqua, attefo le leggi della Natura full' equilibrio dei liquidi, alzarfi quindici cubiti fópra gli alti monti di quei paefi fenza allagar tutta. I' Afra, l'Africa, I'Euro$\mathrm{pa}$, ed anche I' America. Se poi non fu univerfale il Diluvio, perchè comandare la coftruzione dell'arca laddove la famiglia di Noè avrebbe potuto agia. camente fotrarf dall'inondazione portandofi ad altri paefi, dove non era per. accadere quella calamità? Perchè fas racchiudere nell' arca alcuni individui, d'ogni fpezie di quadrupedi, d'uccelli, e di rettili affine di confervar le spezie fu la fuperficie della terra? ut falvetur femen Juper faciem univerfa terra. Gen. 7. Reftando le fpezie degli animali in moitiffimi altri pae. $\mathbb{1}$, che non doveano allagarfi, una tal diligenza farebbe ftata foverchia, e ri dicola, fpecialmente per rapporto agli uccelli. Per quefte, e per altre sì fat te. ragioni dobbiamo, conchiudere, che coloro, che ftimando divina l'autorita: dei facri libri, negano ciò non oftante I'univerfalità del Diluvio, debbono per carità condurfi a qualche fpedale.

(h) Volendo Iddio fár rif́pettar la fua gituítizia dalla pofterità di Noè, e. confondere l'incredulità dei Mortali, difpofe, che oltre al teftimonio delle laGre fcritture, a corpi marini in gran copia reftati nei monti per eterni monumenti del Diluvio fr confervaffe pure la memoria di quelto fpaventevole e general gaftigo fra le Nazioni Americane. Quefte in fatti fenza avere alcuna motizi dei fanti libri, nè alcun commercio colle. Nazioni dell' antico. Continenre, aveano turtavia contezza del Diluvio, ficcome ne fanno.fede Gomara, Acofta, Ferrera; Calcia, Martinez, Torquemada, Siguenza, Ixtlilxochitl; e rutti quegli Autori, che fecero intorno a ciò diligenti ricerche. I: Toltechis. gii Peolhui, i Tarafchi, O.Michuacanefi, i. Mefficani, i, Miztechi, i Tlafcallefr, i Ehiapanefi, ed ahre Nazioni confervavano la tradiziono del Diluvio

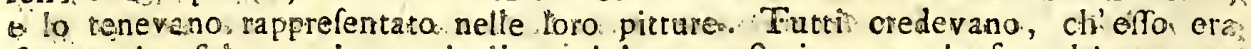
Aato univerfale, e cbe turci gli ucminl erano fati annegati, fuorche ua nom

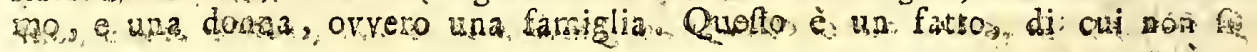


Il Dott. Siguenza credette cominciata la popolazione dell' America non guari dopo la difperfione delle genti. Siccome. non abbiamo i manofcritti di quefto chiariffimo Mefficano, cosi ignoriamo i fondamenti della fua opinione, la quale è peraltro affai conforme alla tradizione de' Chiapanefi, di cui ap. preffe favelleremo. Altri Autori per l'oppofio credono troppo moderna quella popolazione, perchè gli Scrittori della Storia de':Mefricani, e de'Peruani non trovarono preffo quefte Nazioni veruna memoria de'loro particolari avvenimenti, che oltrepaifaffe gli otro fecoli. Ma quefti Autori confondono la popo. lazione del Meffico proprio fatra da' Cicimechi, e dagli Aztechi con quella, che $i$ loro Antenati aveano fatta molti fecoli avanti ne paefi fettentrionali dell' America, nè fanno diftinguere i Mefficani dalle altre Nazioni, che prima di loro occuparono quel paefe. Chi fa, per efempio, quando entrarono nel paefe d'Anahuac gli Otomiti, gli Olmechi, i Cuitlatechi, ed i Michuacanefi? Non é da maravigliare che alcuni Scrittori del M:ffico ron poteffero trovare memoria pit̀ antica d' ctto fecoli; poichè oltre alla perdita della maggior parte de' monu. menti ftorici di quelle Nazioni, di cui abbiamo fatto tante volte menzione, eglino non fapendo per lo pib̀ accordar gli anni Mefícani co'noltrali, traviarono a tal fegno, che incorfero in molti groff anacronifmi; ma coloro, che fi procacciz. rono maggior abbondanza d'antiche, e fcelte pitture, ed' ebbero maggior fagacità per rintracciare la cronologìa di qué popo. li, ficcome Siguenza, ed Ixtlitxochitl, trovarono certamente delle memoric affai più antiche, e fe ne fervirono per li pre. gevoliflimi lo: feritti.

Io in vero non dubito, che la popolazione dell'America fia antichiffrma, ed affai più, che non pare agli Autori Euro pei. I. perchè agli Americani mancavano certe arti, ed invenzioni, come per efempio quella di fervirfi. della cera, o dell' olio

puó dubitare fenza temerità. Vedafi ciò che abbiamo detto intorno: a quefto xella Storia, e ciò ancora che diremo appreffo. Il $\mathrm{P}$. Acofta dice, che tutia gl' Indiani axeano notizia del Diluvio; ma cio debbe intenderfi di quelli, che: vizeano in forieta. 
olio per farfi lume, le quali effendo da un altro canto antichiffime, nell' Afia, e nell' Europa, fono da un altro canto utilifime, per non dir neceffarie, ed una volta imparate, non 1 laf́iano mai. 2. perchè le Nazioni dirozzate del nuovo Mon: do, e particolarmente quelle del Meffico confervavano nelle Joro tradizioni, e nelle lor pitture la memoria della Creazion del Mondo, del Diluvio, della fabbrica della torre di Babel, della confufion delle lingue, e della difperfione delle Genti, come abbiamo detro nella Storia, e ne fanno fede gli Autori predetti, benchè alterata con alcune favole, $e$ non aveano veruna notizia degli avvenimenti accaduti poi in Afia, in Africa, e in Europa, con turto che molti d'effi foffero sì grandi, e sì no. tabili, che non poreva facilmenre fcancellarfi dalla lor memosia. 3. perchè nè preffo gli Americani eravi alcuna notizia de popoli dell' antico Continente, nè preffo quefti s'è trovato al cun rifcontro del paffaggio fatto da quelle Nazioni al nuovo Mondo. Quefte ragioni rendono non già certa, ma affai ve: rifimile la noltra opinione. $\left({ }^{*}\right)$

\section{I I.}

\section{Cbi furono i popolatori dell' America?}

Quegli Spiriti forti, che non riconofcono ne'libri fanti l'impronta della fomma verità, o non ne fanno gran capitale, dicono, che gli Americani non traggono la lor origine da $\mathrm{A}$. damo, e da Noè, e credono, o fingon di credere, che come Iddio creò Adamo, perchè folfe Padre degli Afratici, così fe-

(*) Certo Autore moderno afferma, che la popolazione dell' America è più antica dell' ufo del ferro, perchè tal ufo non fi trovò preffo gli Americani. $\mathrm{Ma}$ queft'opinione è renz'altro falla; poichè l'invenzion del ferro fu anteriore al Diluvio. Di Tubalcain, fefto nipote d'Adamo, fi dice nel capo 4. del Genefi, che fu Fabbro, e lavorò ogni forta d'opere di ferro, e di rame: Sella genuit Tubalcain, qui fuit malleator, $\sigma$ faber in cuncta opera aris, or ferri. Or chi crederà l'A America popolata prima di Tubalcain? Gli Americani non ufavano il ferro, forfe perchè nei paefi fettentrionali dell' America, dove prima fi ftabilirono, non ne trovarono le miniere, e poi fi perdette preffo lore la memoria di quel merallo. 


\section{I S S E R T Z I O N E I.}

ce dopo, o prima di lui altri uomini, acciocchè foffero $\mathbf{P a}$ : triarchi degli Africani, degli Europei, e degli Americani. Ciò non s'oppone, dice un Autor moderno, (i) alla verità de' facri libri ; perciocchè febbene Mosè non fa menzione d'alcun altro primo Patriarca, fuorchè d'Adamo, quefto fu, perchè egli non fr mife a fcrivere la Storia degli altri popoli, ma foltanto quella degl' Ifraeliti. Ma oltrecchè quefto rancido fi. ftema contraddice apertamente alla venerabile tradizione, alle facre fcritture, $(k)$ ed alla comun credenza della Chiefa Cattolica ( il che poco importa per li Filofofi di quella fatta) è ftato pure fmentito dalla tradizione degli fteffi Americani, i quali e nelle loro pitture, e ne'loro cantici fi dicevano difcendenti di quegli uomini, che fcamparono dalla general inondazione. I Toltechi, gli Acolhui, i Mefficani, i Tlafcallefi, i Tarafchi, i Miztechi, i Chiapanefi, ed altri popoli tutti erano d'accordo in quefto punto : tutti dicevano, che $i$ loro antenati erano altronde venuti in que' paefi: additavano la ftrada da coloro tenuta, ed anche confervavano i nomi o veri, - fuppotti, di que' primi loro progenitori, che dopo la confu. fion delle lingue fi fepararono dal refto degli uomini .

Monfig. Francefco Nuñez de la Vega, Vefcovo di Chia. pa, dice nel Proemio delle lue Coftituzioni Sinodali, che nel. la vifita della fua diocefi farta da lui medefimo verfo la fine del fecolo paffato, trovò molti Calendarj antichi de' Chiapanefi, ed un vecchio manofcritto nella lingua di quel paefe fatto dagli fteff Indiani, nel qual fi diceva giufta l'antica loro tradizione, che un certo Votan $\left({ }^{*}\right)$ intervenne alla fabbrica di quel grand'edifizio, che fi fece per ordine del fuo avolo per falire in cielo: che quivi fu dato a ciafcun popolo il fuo lin.

guag-

(i) L'Autore d'una miferabile operetta intitolata, Le Pbilofopbe Douceur it ampata in Berlino l'anno I 775 .

(k) Tres iffi flij funt Noè: ab bis diffeminatum eft omne genus bominum Juper univerfam terram. Gen. 9. Fecit ex uno omne bominum genus inbabitare fuper faciem univerfa terra. Aat. 17. Non può efprimerfi con parole più fignificanti la comune origine di tutti gli uomini tratta da Adamo, e da Noè.

(*) Votan è il principale tra quelli venti uomini chiari, che comunicarono i loro nomi ai venti giorni del mefe Chiapanefe. 
guaggio, e che il medefimo Votan fu da Dio incaricato di far la divifione delle terre d'Anahuac. Soggiunge poi il fuddetto Prelato, che v'era a fuo tempo in Teopixca, luogo grande di quella Diocefi, una famiglia di cognome Votan, che fi credeva difcendente di quell'antico popolatore. Io non pretendo far credere tanto antica la popolazione dell' America fu la fede di quella tradizione de'Chiapanefi, ma folamente dare a divedere che gli Anericani fi riputavano difcendenti di Noè.

Degli antichi Indiani di Cuba raccontano parecchj Storici dell'America, ch' effendo ftati interrogati dagli Spagruoli fu la loro origine, rifpofero ch' eglino aveano intefo da' lor maggiori, che Iddio creò il cielo, la terra, e tutte le cofe: che un vecchio avendo prefentito una grande inondazione, colla quale volle Iddio caftigare i peccati degli uomini, fi fabbrico una gran canoa,e s'imbarcò in effa colla fua famiglia, e con mol. ti animali : ch' effendofi poi fcemata l'inondazione, mandò fuori il corvo, il quale perchè trovò carogne, di cui cibạrfi, non tornò mai alla canoa: che mardò indi a poco la colomba, e quefta tofto ritornò portando nel becco un ramufcello d' Hoba, certo albero fruttifero dell' America: che quando il vecchio vide la terra afciutta, vi sbarco, ed avendo fatto vino d' uve falvatiche, s'imbriacò, e s'addormentò: che allora un de' fuoi figliuoli fi beffò della nudità di lui, ed un altro figliuolo pietofamente il coprì: che deftandofi benediffe quefto, e malediffe quello: finalmente ch'eglino traevano dal figlio maledetto la loro origine, e però andavano quafi ignudi: che gli Spagnuoli, poichè erano ben veftiti, forfe da quell'altro difcendevano.

I Mefficani appellavano Noè Coxcox, e Teocipactli, ed i Michuacanefi Texpi. Quefti dicevano "che vi fu un gran Di"L Luvio, e che Tezpi per non reftare annegato s'imbarco in " un legno lavorato a foggia d' un' arca colla fua moglie, co' "fuoi figliuoli, e con diverfi animali, e parecchie femenze di " frutti, e che effendo fcemata l'acqua mandò fuori quell' uc" cello, che ha il nome d'Aura, il quale rimafe per mangiar 2) de'corpi morti, e poi mandò altri uccelli, che neppor ri- 
s, tornarono, fuorchè quell'uccellino (il fucciafiore) tanto da loro pregiato per la varietà de' colori delle fue penne, il quale gli portò un ramo " $(l)$ e da quefta famiglia tutti credevano di trarre la loro origine. Dunque le abbiam riguar. do o a' facri libri, o alla tradizione degli Americani, dobbiamo cercar nella pofterità di Noè i popolatori del nuovo Mondo.

Ma chi furon effi? Qual de'figliuoli di Noè fu il ceppo delle Nazioni Americane? Il Dotror Siguenza, e la ingegnofiffima Mefficana Suor Giovanna Agnefe della Croce credettero, o pur congetturarono, che i Mefficani ed altre Nazioni d'Anahuac foffero difcendenti di Nephtuim, figliuolo di Mefraim, e nipote di Cham. Il Cav. Boturini fu di parere, che effi difcendeffero non folamente da Nephtuim, ma eziandio dagli altri fuoi cinque fratelli. Il dottiffimo Spagnuolo Arias Montano fi perfuafe, che gli Americani, e particolarmente i Peruani apparteneffero alla pofterità d'Ophir, quarto nipote di Sem. Le ragioni di quefto Autare fon cosi deboli, ed infuffi. ftenti, che non meritano, che fe ne faccia menzione. Di quelle di Siguenza ragioneremo appreffo.

Gli altri Autori che non hanno voluto innoltrarfi nelle loro ricerche fino ad una sì remota antichità, hanno cercato in diverfi paefi del Mondo l'origine degli Americani. Le loro opinioni fono tante, e cosl diverfe, che non è facile di nu. merarle. Chi crede d'aver trovato i Progenitori degli Americani nell' Afia, chi nell' Africa, chi nell' Europa. Tra quelli, che s'immaginano d'averli trovati in Europa, parve ad alcuni che deffi foffero i Greci, ad altri i Romani, ad altri gli Spagnuoli, ad altri gl' Irlandefi, ad altri i Curlandefi, ed a qual cuno ancora $\mathrm{i}$ Ruffs. Tra quelli, che gli reputano originarj

Storia Antica del Meffico Tom. IV. C dell'

(1) Herrera Dec. 3. lib. 3. cap. ro. Vedafi quefto Autore nella Dec. 4. lib. I. Cap. II. intorno a ciò che dicevano della loro origine gl' Indiani di Terraferma. Vedanfi ancora il medefimo Herrera, Torquemada, ed altri intorno alIa tradizione, che era prefro gli Haitini, o fia abitatori dell'ifola Spagnuola. Della tradizione dei Metlicani, degli Acolhur, e dei Tlafcallefi abbiamo ragionato nel lib. II. della noftra Storia. Di quella dei Toltechi fa menzione Boturini, come pure Torquemada, ed altri. Di quella de' Miztechi fcriffe Garcia nel fuo erudito Trattato fulla origine degl' lndiani. 
dell' Africa, chi gli fa difcendere dagli Egizj, chi da' Cartagi:neli, chi da' Numidi. Ma non - v'è maggior varietà di fentimenti, che fra coloro, che credono doverfi all' Afia la popolazione dell'America. Gl' Ifraeliti, i Cananei, gli Affrj, i Fenicj, i Perfani, i Tartari, gl'Indiani orientali, i Chinefi, i Giapponefi, tutti hanno i loro avvocati fra gl Storici; e fra i Filofof di quefti due ultimi fecoli. Alcuni poi non contenti di cercare i fuddetti popolatori ne' paefi conolciuti del Mondo,

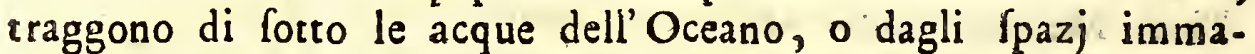
ginarj la famofa ifola Atlantida, per mandar quindi coloni all' America. Ma quelto è poco: poichè vi fono degli Autori, che per non far torto a verun popolo, credono gli Americani difcendenti da tutte le Nazioni del Mondo. (m)

La cagione d'una sì gran varietà, e d'una tale Aravaganza d'opinioni è ttata quella di perfuaderfi, che per credere una Nazione nata da un'altra, non bifognaffe altro, che di trovare qualche affinità in alcune voci delle lor lingue, e qualche fomiglianza ne' loro riti, coltumi, ed ufanze. Táli pur fono i fondamenti di quafi tutte le mentovate opinioni, raccolte già, e con una gran copia d'erudizione illuttrate dal Domenicano Garcia, e da que' dotti Spagnuoli, che con nuove aggiunte riftamparono l'opera di lui, nella quale può vederle chi vo. glia; poichè io crederei di perdere il tempo nel confutarle.

Ma non poffo a meno di non far menzione dell'opinione del Dottor Siguenza, adottata ancora dal chiariffimo Velcovo Francefe Pietro Danielle Huet, perchè mi pare la più ben fondata. Il Siguenza dunque fi perfuafe, che le Nazioni, che popolarono l'Imperio Mefficano appartenevano alla pofterità di Nephtuim, e che i loro progenitori ufciti d'Egitto, non guari dopo la confufione delle lingue, s' incamminarono verfo I'America. Le ragioni, fu le quali appoggiò un tal fentimento, fi trovano foltanto accennate nella Biblioteca Mefficana. Vorrem. mo vederle efpolte con tutta quella forza, e con tutta quella erudizione, con cui faranno ftate fenz' altro feritte da quel dot: tiff.

(m) Quefta opinione improbabile fu guella di Garcia, e di Betancurto 
tiffimo Autore; ma ficcome fiamo privi de'fuoi pregevoliffimi fcritti, così non faremo altro, che accennarle, come fece it Dott. Eguiara nella fuddetta Biblioteca.

Tali ragioni fi riducono, per quanto appare, alla conformità di quelle Nazioni Americane cogli Egizj nell' ufanza d'e. difizj piramidali, e di geroglifici nel modo di computare il tempo, nel veftire, ed in alcuni coltumi, ed a quefto avrà forfe aggiunto la fomiglianza del Teotlde' Mefficani al Theutb degli Egizj, la qual cagionò a Monfig. Huet il medefimo fentimento di Siguenza, benchè per diverfa via. Se quefto penfiere, fi propone come una congettura, io non vi contraddirò ; ma fe fi pretende, ch' effo fia una verità da poterfi affermare, non mi pajono fufficienti quelle ragioni.

Siguenza volle, che i figliuoli di Nephtuim ufciffero d' $\mathrm{E}^{\text {. }}$. gitto verfo l'America non guari dopo la confufion delle lingue: onde dovrebbe fare il paragone de'coftumi degli Ameri. cani con quelli de'primi Egizj, non gia con quelli de' loro difcendenti, che molti anni dopo effi abitarono in Egitto, e de'quali non fi credono difcendenti gli Americani. Ora chi fi perfuaderà, che gli Egizj immediatamente dopo la difperfione delle genti cominciaffero a fabbricar piramidi, ed a fervirfi di geroglifici, e che infin d'allora aveffero già ordinati i loroan. ni, e mefi nella forma, in cui gli ebbero poi? Tutte quefte: cofe furono fenz'altro pofteriori a quell' epoca. Neppur bifognava l'aver vedute le piramidi d' Egitto, acciocchè faltaffe in tefta agli Americani il far sì fatti ediffzj: poichè per ciò baftava il vedere $i$ monti: che fono fu la terra. Chiunque vo. glia fabbricare un alto edifizio per immortalare il fuo nome, facilmente verrà in penfiero di farlo piramidale; perchè niun altro edifizio v'è, che poffa alzarfi a tant' altezza con minori fpefe, perchè quanto più s' innalza tanta minor quantirà di materiali richiede. Oltrechè gli edifizj: Meficani erano affatto diverfi da quelli degli Egizj. Quelti erano vere piramidi; quelli nò; ma erano fabbriche compofte per lo più di tre, di quat sro, o cinque corpi quadri, o quadrilunghi, de"quali i fupe. xiori aveano minor ampiezza degl' inferiori: quelli degli Egio 
zj erano per lo più vuoti; quelli de' Mefficani mafficcj: quefti fervivano di bafi a'loro Santuarj; quelli di fepolcri de' Re. I rempj de'Mefficani, e delle altre Nazioni d'Anahuac erano d' una fpecie sì rara, che non fo, che fieno ftati in ulo ap. preffo alcuna Nazione del Mondo: ficchè debbono confrderarfi, come una invenzione originale de'Tolrechi, o d'altri popola.tori più antichi di loro.

Nel modo di computare il tempo furono i Mefficani più fomiglianti agli Egizj ( parlo degli Egizj pofteriori, non gia de'primi, del cui metodo nulla $f_{1} f a$ ). L'anno Egiziano era folare di 36.5. giorni, come quello de' Mefficani : gli uni, e gli altri contenevano 360. giorni ne' loro mefi, e come gli Egizj aggiu. gnevano cinque giorni all' ultimo lor mefe Mefori, cosi i Mef. ficani al loro mefe lzcalli: nel che convenivano e cogli Egizj, e co' Perfiani; ma del refto v'era un gran divario tra gli. uni, e gli altri. L'anno. Egiziano conftava di mefi dodici, ed i. mefi di giorni trenta; l'anno Mef́ficano $\left({ }^{*}\right)$ componevafi di mefi diciotto, ed $i$ mefi di giorni venti . Gli Egizj, ficcome inoltiflime altre Nazioni dell'antico Continente, contavano per fettimane; i Meffrcani per periodi di giorni cinque nel civile; e di giorni tredici in ciò, che riguarda la Religione.

I Mefficani fi fervivano, come gli Egizj, di geroglifichi; ma quante altre Nazioni non fe ne fono parimente fervite per celare $i$ mifterj della loro Religione?. E fe i Mefficani impararono dagli. Egizj i geroglifichi, perchè non ebbero ancor da loro l'ufo delle lettere? Perchè le lertere, diraffi, furono inventate dopo la loro feparazione, ma come $f \mathfrak{f}$, che prima che coloro fi feparaffero, erano gia ritrovati i geroglifichi?

L'abito de' primi Egizj fara ltato verifrmilmente il mede. fimo degli altri figliuoli, e nipoti di Noè : almeno non $v^{\prime}$ è ragione da farci credere l'oppofto. Intorno poi a"coftumi po. lícici di que'primi uomini nulla fappiamo. I più antichi Egi$3 j$, de'quali abbiamo rifcontri certi, fono fati quelli, cho vi-

( ${ }^{*}$ Parlo dell' anno religiofo dei Mefficani; poichè del loro anno civile 2. c⿻ atronomico nulla rappiamo. 
viveano a' tempi del Patriarca Giufeppe. Ora fe vogliamo fa: re il confronto delle loro ufanze, accennate ne' facri libri, con quelle de' Mefficani, anzichè la pretefa loro medefimezza, troveremo la lor diverfità. Finalmente io non pretendo di dimoftrar falfa l'opinione del Siguenza, ma foltanto di far vedere, ch' effa non è una verità da poterfene afficurare.

Lo ftravagante Ricercatore dice, che i Mefficani traggo. no la lor origine dagli Apalachiti meridionali; ma nè allega, nè può allegare qualche ragione, che renda probabile, o verifimile un tal paradoffo: ed ancorchè cid foffe vero, refterebbe ancora in piedi la difficoltà intorno all' origine degli feffi Apalachiti . E' vero, che per queli' ardito Autore non v' è difficoltà, perchè talvolta dà a divedere, che non gli difpiace lo fpropofitato fiftema del Francefe La Peyrere.

Per ciò poi che riguarda la mia opinione, 'mi par bene d'efporla nelle feguenti conclufioni.

I. Gli Americani difcendono da diverfe Nazioni, ovvero da diverfe famiglie difperfe dopo la confufion delle lingue. Non: potrà dubitar di quefta verita, chiunque abbia qualche contez za della moltitudine, e della fomma diverfità delle lingue americane. Nel Meffico ne ho numerate trenta cinque di quelle che fon finora conofciute. Nell' America meridionale fono affai più . Sul principio del fecolo paffato ne contavano i Portoghefr fino a cencinquanta nel Maragnone. E' vero, che fra alcune di quefte lingue fi fcorge una tale affinità, che dà tofto a divedere, che effe fon nate da una medefima madre, fiecome 'Eudeve, l'Opata, e la Tarabumara nell' America fettentrio. nale, e la Mocobi, la Toba, e l'Abipona nell' America Meridionale; ma ve ne fono molee altre affii pir fra loro differen. zi, che l'Ebraica, e l'Illirica. Poffo affermare fenza pericolo d'ingannarmi, che non fy troveranno nè tra le vive, nè tra le morte lingue dell' Europa, due più fra loro differenti, che la Meflicana, l' Otomiza, la Tarafca, la Miaja, e la Mizteca cinque lingue dominanti in diverfe Provincie del Meffico. Sicchè: farebbe un grande fpropofito il dire, che sì fatte lingue ame. micane fieno ftate diverfi dialetri d'una liagua madre. Come è: pof 
poffibile, che una Nazione alteraffe a tal fegno il fuo primitivo linguaggio, o lo moltiplicaffe in tanti dialetti sì differenti Ira loro, che non avefiero anche dopo molti fecoli, molte voci comuri a tutri, o almeno che non vi foffe qualche affinità, vi reftaffe qualche traccia della lor origine?

Chi potrà mai credere cid che fi legge ( ${ }^{*}$ ) nella Storia del P. Acolta? Cioè, che effendo giunti gli Aztechi, o Mefficani dopo la lunga lor pellegrinazione nel Regno di Michuacan, vollero ftabilirvifi allettati dall'amenità della terra; ma non po. tendo refrarvi tutto il corpo della Nazione, acconfenti il lor Dio Huitzilopochtli, che vi rimaneffero alcuni, ed anche fuggeri agli altri la maniera di farlo, ordinando loro, che allorchè coloro, che doveano rimanere fi bagnaffero nel lago di Pazcuaro, involaffero loro le velti, e tolto fuggiffero per con. tinuare il loro viaggio: che coloro che fi bagnavano vedendofi privi delle lor vefti, e burlati da' loro compagni ebbero un tale 1degno, che rifolvettero non folo di rimanervi, ma eziandio di prendere un nuovo linguaggio, e che quindi ebbe origine la lingua Tarafca. Ancor più incredibile è il racconto adottato da Gomara, e da parecchj Storici; cioè, che da un vecchio appel. lato Iztac Mixcoatl, e dalla fua moglie Itancueitl, nacquero fei figliuoli, tutti di diverfa lingua, chiamati Xolbua, Tenocb, Olmecatl, Xicallancatl, Mixtecatl, ed Otomitl, i quali furono. progenitori d'altrettante Nazioni, che popolarono il paefe d'Anahuac. Queft' allegoria, colla quale volevano fignificare i Mef. ficani, che tutte quelle Nazioni traevano origine da un comun. ceppo, fu da' [uddetti Autori cangiata in favola, perchè mal ansela.

II. Gli Americani non traggono la loro origine da verun popolo oggi efiftente nell' antico Mondo, o almeno non v' de ragione d'affermarlo. Quefta conclufione fi fonda nella medefima ragione dell' antecedente; poichè fe gli Americani difcendeffero da qualcuno di que popoli, potrebbe rintracciarf. la lor origine.

per

(*) Stor. Nat. e Mor. delle Indie lib. 7. Il P. Acofta non moftra d"accon

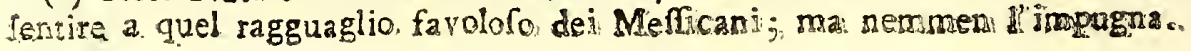




\section{I S E R T A $Z$ I O N E I.}

per qualche traccia rimafa nelle lor lingue, malgrado l'antichirà della lor feparazione; ma tal traccia non s'è potuta fin'ad ora ritrovare, contuttochè molti Autori l'abbiano con grand'impegno ricercata, ficcome può vederfí nell' opera del Domenicano Garcia. Io ho confrontato proliffamente la lingua Mefficana, ed altre americane con parecchie altre cosi vive, come morte dell' antico Continente, e non ho potuto trovar fra le une, e le altre veruna affinirà: La fomiglianza del Teotl de' Meffica. ni col Theos de'Greci $m$ ' ha indotto talvolta a confrontar que fte due lingue; ma fempre vi ho trovato una gran diverfità. Quefto argomento è più efficace rapporto agli Americani per: la loro fermezza e coftanza nel ritener le lor lingue. I Meffi. cani confervano la loro lingua tra gli Spagnuoli, e gli Otomi. ti ritengono la difficile lor favella tra gli Spagnuoli, ed i Meffi. cani, anche dopo due fecoli e mezzo.

Se gli Americani difcendono, com' io credo, da diverte famiglie difperfe dopo la confufion delle lingue, e fin d'allora feparace da quelle altre, che popolarono i paefi dell' antico Continente, indarno 's affaticheranno gli Autori nel ricercar nelle lingue, o ne' coltumi de' popoli afratici l'origine de' popolatori del nuovo Mondo. Io non dubito attefo ciò che ci dicono i fa. cri libri, che poichè fi fu abbaftanza moltiplicata la difendenza di Noè, vi foffe un ordin efpreffo di Dio di fepararfi le famiglie, e di portarfi ciafcuna a popolare il paefe affegnatole. Mosè nel fuo divin cantico parla cos̀ al popolo d'Ifraelle : „Ri" cordati de' giorni antichi, e pon mente a ciafcuna delle paffa. "te generazioni: interroga tuo Padre, ed i tuoi antenati, e ti "diranno, che ailora quando l'Altiffimo divideva le genti, e "feparava i figliuoli d'Adamo, prefcriffe i termini de' popoli "( della Paleftina ) giufta il numero de' figliuoli d' Ifraelle: „(n) nel che fi da a divedere il Signore in atto di compartir le $\mathrm{fa}_{\mathrm{a}}$. miglie, e di prefcrivere i limiti de' paefi, che doveano occupa.

re.

(n) Memento dierum antiquorum, cogita generationes fingulas: interrog $x$ patrem 1uum, $\mathcal{O}$ annunciabit tibi, majorés tuos, ev diccnt tibi. Quando dividebat Altifsmus gentes, quando Separabat flios Adam, confituit terminos populorum iuxtet numerum filiorum Ifrael. Deut. 32. 


\section{D ISSERT A I O N E I.}

re. Quegli uomini, che intraprefero la coftruzione della torre di Babel, s'incoraggivano a lavorare in quella fabbrica con quelte parole: "Venite, diceano, edifichiamci una Città, ed „) una torre, la cui cima tocchi il Cielo, e rendiamo celebre il ") nome noftro, prima che fiam divifi per tutca la terra. "( (0) Sapeano dunque, che doveano effere fparfi per cutti i paefí, e Iddio, perchè con sì fatta fabbrica s'opponevano a' fuoi configlj, - almeno differivano l'efecuzione de' fuoi ordini intorno alla popolazione della terra, confufe il loro linguaggio, e così gli venne fatto di feparargli, e di fpargerli da quel luogo per tutt' i paefi. ( $p$ ) Quindi è verifimile, che Noè, vecchio venerabile, da tutti come Padre riverito, il qual fopravviffe trocento cinquant' anni al diluvio, affegnaffe a ciafcuna famiglia il fuo diItretto fecondo l'inftruzione avuta da Dio, perchè altrimenti non fi farebbe potuto efeguir la divifione fenza guerre fanguinofe, volendo ognuno goder del fuo nativo paefe fenza efporfi a molti periglj, e difaftri in terre fconofciute. Quelto mio fentimento fi rende ancor più verifimile per la tradizione de' Chiapanefi intorno alla popolazione d'Anahuac fatta da Votan, di cui abbiam già parlato. Non però fi dee penfare, che quelle medefime perfone, che dalle altre famiglie fi fepararono in Babel, popolaffero in fatti, ficcome diceano i Chiapanefi, i paefi dell' America; ma i loro difcendenti, mentre quelle prime faniglie faranno andate a poco a poco incamminandofi verfo quella parte, e mol. iplicandofi nel loro viaggio.

$D_{a}$

(o) Venite, faciamus nobis civitatem, or turrim, cujus culmen pertingat ad calum, celebremusque nomen nofrum antequam dividamur in univerfas terras. Gen. II.

(p) Atque ita divifit eos ex illo loco in omnes terras. ib. 


\section{DIS S E R T A Z IO N E I. \\ 23 \\ ๑. III. \\ Da qual parte, e come pafjarono i popolatori, e le beftie
in America.}

Quefto è l'altro punto, ed il più difficile nel problema della popolazion dell' America, nel quale fono, ficcome nell' altro, affai diverfi i fentimenti degli Autori. Alcuni afcrivono la popolazione del nuovo Mondo a certi Mercanti Fenicj, che navigando l'Oceano cafualmente vi capitarono. Altri s' immaginano, che quegli fteffi popoli, che fuppongono effer paffati dall' antico Continente all' Ifola Atlantida, quindi facilmente fi portaffero alla Florida, e da quefto vafto paefe s'andaffero fpargendo per tutta l'America. Altri credono, che vi paffarono dall' Afia per lo ftretto d'Anian, ed altri che vi î trafportarono dalle regioni fettentrionali dell' Europa per non fo che tráccio del mar glaciale.

Il P. Feijoò, Benedettino Spagnuolo, s' efibì pochi anni fa a proporre al Mondo letterario un nuovo fiftema . (q) E qual è quefto fiftema nuovo? Che l'America era già unita dalla parte fettentrionale all' antico Continente, e che per quella vi paffarono gli uomini, e le beftie. Ma quefta opinione è tanto an. tica, quanto il P. Acolta, il quale I44. anni prima del P. Feijod la pubblicò nella fua Storia dell' America : $(r)$ oltrecchè non bafta a fciogliere tutte le difficoltà, che vi fono intorno al paffag. gio delle beftie, ficcome appreffo vedremo.

Il Conte de Buffon malgrado il fuo grand' ingegno, e la fua proliffa efattezza fi contraddice opertamente in quefto punto. Egli fuppone uniti già ambidue i Continenti per la parte della Tartaria Orientale, ed afferma, che per di la paffarono in America $i$ primi Popolatori, e tutte quelle beftie, che vi fi trovarono comuni all' uno, ed all'altro Mondo, come i bifonti (appellati in Meffico Cibolos) i lupi, le volpi, le mar. Storia Antica del Mefjeco TomoIV. D tore,

(q) Teatro critico univerfale tom. s. difc. I 4.

(r) Stor, nat e mor. delle Indie lib, I. cap. $20 ., 2$ I. 
tore, i cervi, i daini, ed altri si fatti quadrupedi, a'quali fi confà il clima freddo, ma che non poteano effere in America leoni, tigri, cammelli, elefanti, giraffe, nè alcuna di quille diciaffette fpezie di fcimie, che fi trovano nell' antico Continen. te, e per dirlo in poche parole, niun quadrupede proprio di clima caldo potè effer comune ad ambidue i Continenti, perchè non era capace di refiltere al freddo de' paefi fettentriona. li, per li quali dovea paffare dall'uno all' altro Mondo. Quefto ridice inceffantemente in tutta la fua Storia Naturale, e con tal fermezza, che per quefta fola ragione bandifce dall'A. merica le gazzelle, le capre, ed $i$ coniglj. Egli non reputa quadrupedi propriamente americani, fe non quelli, che vivono foltanto ne'paefi caldi di quel nuovo Mondo, tra i quali numera le tredici, o quattordici fpezie di fcimie americane, da lui divife nelle due claffi di Sapayirs, e di Sagoini: di quefte foggiunge, niuna era nell'antico Continente, ficcome non v'è nel Continente nuovo alcuna delle diciaffetre fpezie dell' antico. Qual dunque fu l'origine di quefti, e d'altri quadrupedi propriamente americani? Quefto dubbio, il qual occorre fpeffo nella Storia Naturale di quel gran Filofofo refta indecifo fino al penultimo tomo della ftoria de'quadrupedi, nel quale parlando da buon Cattolico ragiona cosi : $(\rho)$, Siccome non può dubitar"fi, che tutti gli animali in generale furono creati nell' an"tico Continente, bifogna ammettere il paffaggio da quefto 2) nell' altro Continente, e fupporre infieme, che quefti anima" li (il daino, il capriuolo, e le muffetre) in vece d'aver ") degenerato, come gli altri, nel nuovo Mondo, vi fi fono per " contrario perfezionati, e che per la convenienza del clima "hanno fuperato la propria lor natura... L'efferfi trovati nel: ") nuovo Mondo tanti animali, che non poffono rapportarfi ad " alcuno del Mondo antico, dà a divedere abbaftanza, che l'o. "rigine di quelti animali propri del nuovo Mondo non debbe , afcriverfi alla femplice degenerazione. Quantunque grandi, ed 2) efficaci fi vogliano fupporre gli effetti, non fi porrà mai con"vin-

(1) Hifoise Nato tom. 29. Difcours fur la degeneration des animomx. 
\# vincere con qualche apparenza di ragione, che quefti animali " fieno Itati originalmente que' medefimi dell' antico Continen" te. E' fenz'altro più conforme alla ragione il credere, che " i due Continenti erano gì contigui, o continui, e che quel" le fpezie, che s'erano ritirate nelle regioni del Nuovo Mon. " do, perchè vi trovarono il Cielo, e la terra più confacevo. "Li alla lor natura, furono quivi racchiufe, e feparate dalle " altre per le irruzioni de' mari, che divifero l'Africa dall' A. "merica. ( $t$ ) Quefta cagione è naturale \&c. "Da quefto difcorfo del Cionte de Buffon fi conchiude I. che non v' è animale propriamente americano; poichè tutri vi andarono dall' antico Continente, dove furono creati . 2. che l' argomento fondato fulla natura degli animali ripugnante al freddo, niente non vale per dimoftrare, che non poterono paffar nel nuovo Continen. te, perchè quelli, che non poteano per la lor natura fare il paffaggio per li paefi fetrentrionali, poterono farlo per quella parte, dov' era già unita l'America all' Africa, ficcome crede quell' Autore. 3. che per dove paffarono nel nuovo Mondo i Sagoini, ed i Sapayùs, vi poterono fimilmente andare gli elefanti, i cammelli, le giraffe, i leoni, le tigri \&c.

Tralafciando poi altre opinioni, che non meritano d' effer commemorate, efporrò in alcune conclufioni il mio fentimento, non già per iftabilire, ficcome ho proteftato, un nuovo fiftema; ma per fomminiftrare de' materiali ad altri migliori ingegni, e per illuftrare alcuni punti della mia Storia.

I. Gli uomini, e gli animali dell' America vi pafjarono dall' antico Continente. Quefta verità è fondata ne' facri libri. Lo fteffo Mosè, che dichiara Noè ceppo comune di tutti gli $\mathrm{D}_{2}$ uomi-

(t) Prego i Lettori di confrontar ciò che dice qui il $\mathrm{Co:}$ de Buffon full' antica unione dell'Africa, e dell' America con quello che fcrive nel tomo 18. laddove ragiona del Leone: "II Leone Americano, dice, non può difcende" re dal Leone dell' antico Continente, perchè non abitando quefto, $\mathrm{fe}$ non "fra i tropici, ed avendogli chiufe la natura, per quel che appare, tutte le " Arade di tramontana, non p tè paffare dalle parti meridionali dell' Afia, e ", dell'Africa in America; mentre quefti due Continenti fon feparati da im" menfi mari: onde dee dirfi, che il Leone americano è un animale proprio, "e particolare del nuovo Mondo." 
uomini dopo il diluvio, dice efpreffamente, che in quella ge: nerale inondazion della terra perirono tutti i fuoi quadrupedi, tutti gli uccelli, e turti i rettili, fuorchè pochi individui, che fi falvarono neil' arca per riftabilire le fpezie. Le replicate efpreffioni, che ufa il Sacro Storico per fignificare l'univerfalita, non permettono di dubitare, che tutti i quadrupedi, e rettili, e gli uccelli, che oggidi fono al Mondo, difcendano da que' pochi individui, che Iddio fottraffe dalla general inondazione; altrimente farebbe ftata foverchia, come abbiamo fopra accennato, ed anche ridicola la diligenza adoperata nel racchiuder quegli animali, e particolarmente gli uccelli nell' arca per confervar le fpezie, ed uno rpropofito non diffimile a quello delle figlie di Lot, le quali, perchè videro arder le Cirtà di Sodoma, e di Gomorra, fi perfuafero, ch'erano periti cutri gli uomini, a che elleno fole folfero rimalte per confervar la fpezie umana fu la terra. II. I primi popolatori dell' America vi poterono paJjare in barcbe per mare, o a piedi per terra, o pel ghiaccio. 1. Potesono paffare in barche o cafualmente rapiti da un veato gagliardo, o a bella polta, fe forfe era piccolo lo ftretto di mare, che feparava l'uno dall'altro Continente. Non v'è dub. bio, che que' popolatori poterono arrivare al nuovo Mondo in quella fteffa maniera, nella quale molti fecoli dopo vi fu poztato quel Marinajo, o Piloto, cui, al dir di molti Autori, dovette Colombo le prime notizie, che lo finfero alla fua glo. riofa e memorabile fcoperta . (u) 2. Vi poterono paffare a piedi per terra fuppolta, come ormai vedremo, l'unione d'ambidue $\mathrm{i}$ Continenti. 3. Poterono anche fur quel paffaggio a piedı per qualche fretto di mare agghiacciato. Neffuno ignona quanto grandi, e quanto durevoli fono i diaccj de' marifettentrianali. Non farebbe dungue da maravigliarf, che uno itrer-

(ui) Alcuni Autori affermano, che quel Marinajo, che diede contezza a Colombo dei nuovi paefi di Ponente, era d'Andaluzia, chi dice ch'egli era Bifcaglino, chi lo fa Portoghefe. Altri poi regano quefto fatto. Checcheffia egli è certo, che nella Storia abbiamo non pochi efeaspi di Vafcelli rapiri dai venti, e porrati molti gradi lontano da quella ftrada, che tenevano. Balta leggere gli efempi recati da Plinio nel lib. 2. cap. 67. e nel lib. 6. cap. 22. della fua Storia Naturale. 
to di mare fra i due Continenti s'agghiacciaffe a tal fegno; che gli teneffe uniti per alcuni mefi, e fopra effo vi paffaflero gli uomini o cercando nuovi paefi, o perfeguitando qualche fiera. Qui non difcorriamo di quello, che in fatti accadde, ma di quello foltanto, che potè accadere.

III. I progenitori delle Nazioni, che popolarono il paefe d' Anabuac ( di cui ora Colamente difcorriamo ) pufarono da' pacfi Settentrionali dell' Europa ne' fettentrionali dell' America, o piuttoflo da' paefs piu orientali dell' Afia, ne' piis occidentali dell' America. Quefta conclufione fi fonda nella coltante, e general tradizione di tutte quelle Nazioni, le quali unanimemente dicevano, effere ftati i loro progenitori gente venuta in Anahuac da' paefi fituati a Tramontana, ed a Maeftro. Una tal tradizione venne confermata dagli avanzi d' alcuni antichiffimi edifizj, da quelle Nazioni fabbricati nella lor pellegrinazione, di cui abbiam fatta menzione nel lib. 2. della Storia, e dalla comun credenza de' popoli fettentrionali. Oltracciò che abbiamo detto altrove, ne abbiamo preffo Torquemada, e Betancurt un chiariffimo documento. In un viaggio, che fecero gli Spagnuoli l'anno i606. dal Nuovo Meffico fino al fiume, che eglino appellarono del Tizon, feicento miglia da quella Provincia verfo Maeftro, vi trovarono alcuni grandi edifizj, e s'abbatierono in alcuni lodiani, che parlavano la lingua mefficana, da' quali feppero, che alcune giornate lontano da qual fiume verfo Tramontana v'era il Regno di Tollan, e molte popolazioni affai grandi, donde ulcirono coloro, che popolarono l'Imperio meft. cano, e che da' medefimi popolatori erano ttate coltratte quelle, ed altre fabbriche. In fatti tutti i popoli d'Arahuac af. fermavano, che verfo Maeftro, e varfo Tramontana v'eraso i Regni, e le Provincie di Tollan, di Teoacolbuacan, d'Amaquemecan, d'Aztlan, di Tebuajo, di Copalla $\sigma_{c}$. ( nomi tutti mefficani ) la cui fcoperta, fe nell'avvenire s'avanza per quelle parti la popolazione degli Spagnuoli, recherà gran lume alla Storia antica del Meffico. Il Cav. Boturini $f_{a}$, fede, che nelle pitture antiche de' Toltechi fi rapprefentava la pellegrinazione de'loro antenati per l'Afia, e per li paefi fectentrionali dell'. 
dell America fino a Stabilirfi nel Regno di Tollan, ed anche fi éfibifce di additare nella fua Storia Generale la ftrada, che tennero nel loro viaggio; ma ficcome egli non ebbe agio di com. porre la Storia, che meditava, cosł non poffiamo dir di più intorno a quefto argomento.

Ora effendo que' paef, ne' quali i progenitori di quelle Nazioni già tempo fi ftabilirono verfo quella parte, dove $l_{2}$ colta più occidentale dell' America tanto 's' avvicina alla cofta più orientale dell' Afia, è probabile, che per quella medefima parte paffaffero dall' uno all' altro Continente, $o$ in barche, fe allora v'era quello ftretto di mare, che v' è oggidi, fecondo che appare dalle moderne fcoperte de' Ruff, 0 a piedi, fe era tutto un Continente, come appreffo vedremo. Le tracce, che andarono lafciando quelle Nazioni, ci conducono fino a quello ftretto, il qual è fenz'altro quel medefimo, che fooperfero i Viaggiatori nel fecolo XVI., ed appellarono Stretto d'Anian. $(x)$

Quanto alle altre Nazioni dell'America, non trovandofi preffo loro veruna tradizione intorno alla parte, per dove paffarono al Nuovo Mondo, nulla poffiamo affermare. Può effere, che tutte paffaffero per dove andarono i progenitori de' Meficani, e può effere ancora, che paffaffero per un altra parte affai diverfa. Io congetturo, che i progenitori delle Nazioni, che popolarono l'America Meridionale, fi portarono là per quella parte, per dove paffarono, come fa appoco diremo, le beftie proprie de'paefi caldi, e che i progenitori di quelle Na. zioni, che abitarono tutti que' paefi, che vi fono dalla Flori. da infino alla parte più fettentrionale dell' America, vi paffarono dal fettentrione dell' Europa. La diverfità di carattere, che fi fcorge nelle tre fuddette claffi d'Americani, e la fituazione de'paefi, che occuparono, mi fanno folpettare, che effe ebbero diverfa origine, e che per diverfe parti fi portarono là i loro progenitori; ma quefto è un mero fofpetto, ed una congettura. Al-

(x) Nelle carte geografiche dell' America pubblicate nel fecolo paffato fi vede comunemente defcritto lo ftretto d'Anian, benchè con non poca varietà. $D_{a}$ pochi anui in qua fi cominciò ad ommettere, perchè fi flimava favolofo; ma dopo le fcoperte dei Rufi hanno cominciato alcuni Geografi a rimetterlo. 


\section{DIS E R T A Z I O N E I.}

Alcuni affegnano un altra parte pel paffaggio de' primi po: polatori, ed è quella dell' Ifola Atlantida, la cui efiltenza im. pugnata dal P. Acofta, fu foftenuta dal Dott. Siguenza, per quanto appare dal ragguaglio del Gemelli, e noviffimamente promoffa con gran copia d'erudizione dal Ch. Autor delle $\mathbf{L}$ ettere Americane. Se nel ragguaglio, che di quell' ifola fa Platone nel Timeo, non vi foffero parecchie favole frammifchia. te, potrebbe forfe l'autorità d' un sì grave Filofofo indurci al. l'affenfo. Lafciando dunque ad altri quefta contefa, venghiamo al punto più difficile del noftro problema.

IV. I quadrupedi, ed $i$ retrili del nuovo Mondo vi pafJaro: no per terra. Quefta verità fi farà palefe dimoftrando l'impro. babilità, o l'inverifimiglianza delle altre opinioni. Il gran Dottor della Chiefa Agoftino fu di parere, che le fiere, e gli animali nocevoli che fono nelle Ifole vi poterono trafportarfi dagli Angioli; ficcome può crederfi che dagli Angioli fteffi fi fece la radunanza degli animali nel luogo, dove era ftata fabbricata l'arca di Noè; imperciocchè non era poffibile, che gli uomini vi radunaffero le fiere erranti per li bofchi, e gli uccelli, che volavano fparfi in cosi diverfe regioni, fe effi medefini non vi foffero thati trafportati dagli Angioli, o pure indotti da una certa inclinazione infufa loro dal Creatore. Ma quefta foluzione, benchè tronchi affatto ogni difficoltà nel paffaggio delle fiere nel nuovo Mondo, non farebbe gradita nel fecolo, in cui viviamo, nè dobbiamo prevalercene, fe non quando aveffimo riconofciuti inutili tutti gli altri ripieghi per falvar la verità dé facri libri.

Lo fteffo Santo Dottore fuggerife $(y)$ altre tre foluzioni per

(y) "Poffunt quidem credi ad infulas natando tranfffe, fed proximas.... "Quod fi homines eas captas fecum adduxerunt, \& eo modo ubi habitabant 2 earum genera inftituerunt, venandi ftudio fieri potuiffe incredibile non eff; "quamvis iuffu Dei five permiffu etiam opera Angelorum negandam non fic " potuiffe transferri. Si vero e terra exortæ fint fecundum originem primam, "qua dixit Deus: producat terra animam viram, clarius apparet non tam "reparandorum animalium caufa, quam figurandarum variarum gentium " propter Ecclefix facramentum in arca fuiffe omnia genera, $f_{i}$ in infulis, "Suo tranfire non poffent, multa animalia terra produxit. "AUGUST. Ib. 16. de Civit, Dei cap. 7. 
per ifpianare quella difficoltà. Poterono, dice, le fiere paffare a nuoto alle ifole : vi poterono effer trafportate dagli uomini, per averne della cacciagione, e poterono anche effer quivi formate dalla terra, ficcome furon fatte nel principio del Mondo. Ma niuna di quefte foluzioni bafta a fpianar le difficolta, che vi fono nel paffaggio delle fiere al nuovo Mondo; poichè quanto alla prima, egli è certo, che quantunque ftretto fi voglia l'intervallo di mare, che feparava $i$ due Continenti, non è credibile, che ardiffero di valicarlo tanti animali, che non fono deftinati a menar la vita nell' acqua, nè fono avvezzi a notare. E' ben vero, che avrebbon poturo alcuni paffare a nuoto, come paffano $i$ cinghiali della Corfica in Francia; ma chi crederebbe altrettanto delle fcimie americane, che fono affatto inabili al nuoto, o del Perico ligero, o fia pigro, che è tanto lento, e tanto ftenta a muoverfi? Oltracciò che cofa potè indur tante beftie a lafciar la terra, e ad abbandonarfi a' pericoli del mare?

Non è meno incredibile, che foffero quegli animali dagli uomini portati fu'vafcelli, malfimamente fe fi fuppone cafuale il loro arrivo alle colte dell' America. Potrebbono pure nel ca. fo d' intraprendere a bella polta quel viaggio portar feco alcuni fcojattoli, ed alcuni fcimiotti curiofi per loro divertimento, alcuni conigli, lepri, e techichi, acciocchè dopo d'efferfi moltiplicati, ferviffero al loro foltentamento, ed alcuni cervi, daini, martore, ed anche tigri per veltirfi delle loro pelli; ma a che fine portar lupi, volpi, faine, cojoti, tlalcojoti, pume, o leoni americani, ed altre si fatte beftie, che in vece di recar loro qualche utilità, lor fono tanto nocevoli? Per la caccia? Ma non potrebbono avere una tal ricreazione fenza verun danno, e con gran vantaggio ne' cervi, ne'daini, nelle capre falvatiche, ne' coniglj, nelle lepri, ed in altri animali men feroci ? E fe mai fi fuppongono tanto fciocchi i primi popolatori dell' America, che voleffero portare a' nuovi loro paefi quelle beftie tanto nocevoli per cacciarle, almeno non faranno ftati sì paz.$z i$, che fi rifolveffero di portar tante fpezie di ferpenti per a: ver poi il piacere d'ammazzarli. 


\section{DISSER T A Z IONE I.}

Per ciò che riguarda la terza foluzione, cioè che Iddio abbia creati gli animali nell' America, come gli avea creati nell' Afia, quella fenz' altro troncherebbe affatto la difficolià, fo non s' opponeffe a' facri libri. Se Iddio dovea creare quelle fpezie dopo il diluvio, perchè diede l'ordine a Noè di guardar nell' arca un certo numero d'individui di tutti i quadrupedi, di tutti i rettili, e di tutti gli uccelli, acciocchè non periffero le fpezie? $U_{t}$ falvetur femen fuper faciem univerfa terre. Se quefto teltodebbe intenderfi foltanto degli animali dell' antico Continente, $e$ non di quelli del nuovo, fimilmente potrebbe quell'altro tefto, nel quale fi dice, che da'tre figliuoli di Noè fu propagato turto il genere umano: ab bis diffeminatum eft omne genus borsinum fuper univerfam terram, foltanto intenderfi de' popolatori dell' Afia, dell'Africa, e dell' Europa, non già di quell dell' America : onde dovremmo appigliarci allo fpropofitato fiftema d'llaacco de la Peyrere, o ad un altro della medefima fatta. Io almeno non poffo diltinguere quel fuper faciem wniverfe terre del primo tefto da quel super univerfam terram del fecondo .

Refta ancora un altro ripiego pel paffaggio delle beftie; ed è quello fteffo, che abbiam fopra accennato in ragionando degli uomini. Potrebbe qualcuno imnaginarf, che le beftie paffaffero per qualche ftretto di mare agghiacciato; ma chi potrà perfuaderfi, che parecchie fpezie di beltie voraciffime fi portaffero a quelle regioni prive di tutto ciò, che potrebbe fervire al loro foftentamento, e che altre, la cui natura ripugna oltremodo al freddo, ofaffero inoltrarfi nel rigor dell' inverno a que' luoghi agghiacciati?

Or non effendo probabile, che le beftie del nuovo Mon: do vi paffaffero a nuoto, o pel mare agghiacciato, nè che vi foftero trafportate dagli uomini, o dagli Angioli, nè che vi foffero nuovamente creati da Dio, dobbiamo credere, che così i quadrupedi, come i rettili, che fi trovarono nell' America, vi paffarono per terra, e per confeguenza ch'erano allora uniti tutti $e$ due $i$ Continenti. Quetto è thato il fentimento d'Acofta, di Grozio, di Buff n, é d'altri grand' uomini. Io Storia Antica del Mefico Tom. IV. 
fon troppo lontano dall' adottare il fiftema del Conte de Buffon in tutta la fua eftenfione. Non potrà mai perfuadermi quelto Filolofo con turta la fua eloquenza, ed erudizione, che tutto ciò, ch'è ora terra fia ftato già letto di mare. Non po. trò mai credere, che l'antico Continente (e lo fteffo dico del nuovo ) foggiaceffe giammai ad una general inondazione diftinta dal diluvio di Noè, e più durevole d'effo. Tutti gli argomenti del Co. di Buffon non baftano a perfuaderci una tal opinione, che pare poco conforme a' facri libri, nella cui ftoria fi dà a divedere, che almeno una parte dell' Afia è ftata popolata dalla creazion de'primi uomini fino al diluvio univerfale, e dacchè s'afciugò la terra fino ad alcuni anni dopo la morte del noltro divin Redentore. Nella ferie de' quaranta fecoli, e più, comprefi nella narrazione della facra fcrittura, non fi trova alcun vuoro, nel qual fi poffa accomodar quella prerefa inondazione. Per ciò poi che riguarda il nuovo Continente, non v'è alcuna ragione capace d' indurci a credere, che vi fra accaduta qualche inondazione generale diverfa da quella di Noè, ficcome dimoftreremo nella noftra III. differtazione.

Ma non v'è dubbio, che il noltro pianeta abbia foggiac. ciuto a grandiffime vicende dopo il diluvio. Le ftorie antiche, e moderne confermano quella verità, che Ovidio cantò a no. me del Filofofo Pitagora:

Vidi ego, quod fuerat quondam folidiffima tellus,

EJje fretum; vidi factas ex aquore terras. (")

Ora fi arano alcune terre laddove un tempo fi navigava; e per contrario oggi folcano le navi per dove già folcava l'aratro. I tremuoti hanno fubbifsate molte terre, ed altre fono Itate elevate da' fuochi fotterranei. (y) I fiumi hanno formato col loro fango nuovi terreni: il mare ritirandofi da alcune colte ha allungata per quella parte la terra, ed inoltrandofi al. trove colle fue irruzioni l' ha raccorciata: ha feparato alcuni

ter-

(") Metamorph. lib. XV.

(y) Nafcuntur $\sigma$ alio modo terre, o repente in aliquo mari emergunt, veluti paria fecum faciente natura, quaque bauferit biatus, alio loco reddente. Plinius Hift. Nat. lib. 2. cap. 86. 


\section{IS S R T A Z I O N E I.}

terreni, ch'erano uniti, ed ha formati nuovi ftretti, e feni. Abbiamo pure efempj di tutte quefte rivoluzioni ne' fecoli paffati. La Sicilia era già unita al Continente di Napoli, ficcome l'Eubea (oggi Negroponte) alla Beozia. Diodoro, Strabone, ed altri Autori antichi dicono lo ftefso della Spagna e dell'A. frica, ed affermano, che per una violenta irruzione fatta dall' Oceano nella terra, che v'era tra' monti Abila, e Calpe, fi ruppe quella comunicazione, e fi formò il mar Mediterraneo. Apprefso i Ceilanefi v' è la tradizione, che una si fatta irruzione del mare feparò la loro ifola dalla penifola dell' India. Lo ftefso credono i Malabari rapporto alle ifole Maldive, ed i Malai rapporto a quella di Sumatra. Egli è certo, dice il Conta di Buffon, che in Ceilano ha perduto la terra trenta, - quaranta leghe di terreno, che le ha tolto il mare, e per l'oppofto in Tongres, !logo de' Paefi baffi, il mare ha ceduto più di trenta leghe alla terra. La parte fettentrionale dell' Egitto debbe la fua efiftenza alle inondazioni del Nilo. (A) La terra, che quefto fiume ha portata da' paefi mediterranei dell' Africa, ed ha depofta nelle fue inondazioni, ha formato ua fuolo di più di venticinque braccia di profondita. Similmente, foggiunge il fuddetto Autore, la Provincia del Fiume Giallo della China, e quella della Luigiana non fi fon formate, fe non del fango de fiumi. Plinio, Seneca, Diodoro, e Strabone rapportano innumerabili efempj di tali rivoluzioni, $(B) \mathrm{i}$ quali tralafcio, perchè non paja troppo proliffa e carica quefta difE 2 ferta-

(A) Faro, o fia Farion ifola d'Egitto, la quale, fecondo che accenna Omero nell'Odifiea, era diftsnte un giorno, ed una notte di navigazione dalla terra fettentrionale d'Egitro, era tanto a quella vicina ai tempi della celebre Cleopatra, che appena diftava fetta Aadi; poichè tanta era la lunghezza del ponte, che quella Regina fece fare ai Rodienfi per dar comunicaz:one a quell' Irola col Continente. Erodoto, Ariftotele, Seneca, Plinio, ed altri Autori antichi fanno menzione di quefto notabile accrefcimento del terreno d'Egitto.

(B) Vedanfi particolarmente Plinio nel lib. 2. della Stor. Nat. Seneca rel lib. 6. delle Queft. Natur. Plinio annovera fra le nuove Ifole comparfe nel mare, e formate per l'innalzamento della terta, quelle di Rodi, di Delo, d' Anafe, di Nea, d'Alone, di Jera, di Tera, di Terafia, ed a' tempi fuoi quella di Tia. Fra le ifole formate coi tremuoti mette Sicilia reparata dal Continente dell'Italia per un inceryallo di dodici miglia, Cipro difaccata dal- 
fertazione, ficcome ancora molte rivoluzioni moderne, che fi leggono nella Teoria della Terra del Co: di Buffon, e preffo altri Autori. Nella noftra America tutti quelli, che hanno offervato con occhj filofofici la penifola di Jucatan, non dubitano, che il fuo terreno fia ltato già letto di mare; e per l'oppofto nel canale di Bahama fi fcorgono parecchj indizj d'effere ftata un tempo unita l'ifola di Cuba al Continente della Florida. Nello ftretto, che fepara l'America dall' Alia, fi trovano moltifime ifole, le quali faranno verifimilmente quelle montagne, che erano in quel tratto di terra, che crediamo fubbiffato da alcuni tremuoti: il che fi rende più verifimile da quella moltitudine di Vulcani, che fappiamo effere nella penifola di Kanfchatka. Congetcuriamo pure, che il fubbiffamento di quella terra, e la feparazione de' due Continenti fieno ftati cagionati da que' grandi, e ftraordinarj tremuoti, di cui fanno menzione le Storie degli Americani, e de' quali fesero un epoca quafi tanto memorabile, quanto quella del Diluvio. Le Storie de' Toltechi fiffano tali tremuoti nell' anno J. Tecpatl; ma ficcome non fappiamo di qual fecolo effo foffe, nemmeno pofiamo indovinare il tempo, in cui fopravvenne quella gran calamità. Se un gran tremuoto fubbiffafse l'iftmo di Suez, e vi fofse allora tanta fcarfezza di Storici, quanta ve n'era ne'primi fecoli dopo il Diluvio, dopo trecento, o quattrocento anni fi dubiterebbe, fe l'Afia fofse Atata un tempo unita per quella parte all'Africa, ed alcuni lo negherebbono arditamente.

V. I quadrupedi, ed i rettiti dell' America paffarono per diverfe parti dall' un Continente all' altro. Fra le beftie americane ve ne fono alcune, la cui natura repugna fommamente al freddo, come le fimie, le dante, i coccodrilli, \&c. Ve ne fono poi altre, il cui naturale le porta a' paefr agghiacciari, co. me le martore, $i$ renni, ed $i$ ghiottoni. Or nè qualte potero.

no

Ia Soria, l'Eubea dalla Beozia, Arflanta, e Nacris dalla Eubea, Berbifco dalla Birinia, e Leucofia dal promontorio delle Sirene. Fra le terre fommerfe nel mare fa menzione dell' ifola Cea, nella grale fo Subbiffarono rrenta anigitia di berreno con una Arage incred bile degli abitatori eca 
no andar nell' America per la zona torrida, nè quelle per le zone fredde; poichè bifognerebbe fare una gran violenza al loro genio, e farebbono fenz'altro morte nella ftrada. Le fcimie, che fono nella Nuova Spagna pafsarono là fenza dubbio dall ${ }^{\circ}$ America meridionale. (C) Il centro della lor popolazione fono i paefi fituati fotto l'equinoziale, e tra efsa, ed il grado XIV. o XV. di latitudine: a proporzione della diftanza dall' equato. re fi va per lo più diminuendo il loro numero, e di là de' tropici non vi fono più, $f e$ non $f e$ in qualche contrada, la quale per alcune circoflanze particolari fia tanto calda, quanto i paefi equinoziali. Chi dunque potrà perfuaderfi, che sì fatte beftie s' incamminafero al nuovo Mondo pel rigido clima del fettentrione? Potrebbe qualcuno dire, che non è inveritimile, che fofsero trafportate dagli uomini, efsendo tanto pregiate per le loro Atravaganze, e per la ridicola loro imitazione dell' Uomo; ma oltrecchè l'argomento, che facciamo fu le fcimie, fi può fare fopra altri quadrupedi, che non hanno verun pregio per efsere cercati, anzi molte cattive qualità perefsere fchivati, non è credibile, che gli uomini volefsero condur feco degl' in. dividui di tante fpezie di fcimie, quante ne fono nell' America, e molto meno d'alcune, che in vece d'efser graziofe, fono per l'oppolto d'un afpetto brutto, e d' un' indole feroce, come quelle che appellano $Z$ ambos: e cafo che gli uomini fi folsero rifoluti di menare al nuovo Mondo due individui almeno d'o. gni fpezie, quefti certamente non vi potevano arrivare nè per i mari, nè per i paefi del lettentrione, quancunque s'adoperafsero $i$ conduttori per difenderli dal freddo. A vrebbono dua. que dovuto trafportarli da' paefi caldi dell' antico Continente a'paefi parimente caldi del nuovo per un mare fottopolto ad un clima non diffimile a quello del paefe proprio di que' guadru

(C) Don Ferdinando d'Alba Ixtlilxochitl, Indiano affai inftruito nelle antichità della fua Nazione, dice nella fua Storia univerfale della Nuora Spagna, che non eranvi fcimie nella terra d Anahuac; che le prime, che vi i lafciarono vedere, vennero dalla banda di mezzogiorno dopo l'epcca dei gran venti. I Tlafcallefi cangiando in $f_{a v o l a}$ queft avyenimento, dicevano, che il Mondo fini una volia con vento, e che quei pochi uomini, che foprayviffeso furusu trasformati in fcimie. 
drupedi, cioè, o da' paefr meridionali dell' Afia a' meridionali dell' America per li mari Indico, e Pacifico, o da' paefi occidentali dell' Africa agli Orientali dell'America per l' Oceano Atlantico. Dunque fe gli uomini trafportarono quelle beftie dall' uno all' altro Mondo, ciò s'efegul per uno di que' mari. Ma quefta navigazione fu cafuale, o fatta a pofta? Se cafuale, come, e perchè conduffero feco tanti animali? Se fu fatta a polta, e con propofito deliberato di paffar dall' uno all' altro Mondo, chi ne diede lor contezza? chi lor moftrò la fituazione di que' paefi? Chi lor additò la ftrada? Come s'arrifchiarono a traggettar si gran mare fenza buffola? Sopra che va. fcelli? Se quefti vi approdarono felicemente, perchè non reftò preffo gli Americani veruna memoria della loro coftruzione?

Oltracciò fono comuni nella zona torrida del nuovo Mondo i Coccodrilli, beftie, le quali richiedono un clima caldo, 0 temperato, e vivono alternativamente nella terra, e nell' acqua dolce. Or per dove paffarono quefte beftie? Non certamente pel fettentrione; perchè la lor natura repugna oltremodo al freddo. Neppur furono trafportati dagli uomini; perchè dove mai fi videro degli uomini così fciocchi, che voleffero imbarcar feco de' coccodrilli, per portarli a que' paefi, dove andavano a popolare? Nemmeno può dirfi, che paffaffero a nuoto; perchè non è poffibile, che s'allontanaffero notando per le ac. que ralmaftre dell' Oceano quafi due mila miglia dalle rive de fiumi, o laghi, ne' quali s' erano allevati, e dove godevano della compagnia degli altri individui della loro fpezie.

Non refta dunque altro ripiego, fe non quello d'ammettere $l^{\prime}$ antica unione de' paefi equinoziali dell' A merica con quelli dell' Africa, e la continuazione de'paefi fettentrionali dell'A. merica con quelli dell' Europa, o dell' Afia : quefto pel paffag. gio delle beftie proprie de'climi freddi, e quella pel paffaggio de' quadrupedi, e de'rettili propri de' climi caldi. Per le ragioni finora addotte ci perfuadiamo, che vi fu già un gran tratto di terra, che univa la parte ora più orientale del Brafile alla parte più occidentale dell' Afriça, e che tutto quefto fpazio di terra farà ftato per avventura fubbiffato da alcuni gran 
tremuoti, reftando foltanto alcuni avanzi d'efso nelle ifole di Capo verde, di Fernando di Noroña, dell' Afcenfione, di S. Matteo, ed altre, e nelle molte fecche riconofciute da parecchj viaggiatori, e particolarmente dal Sig. de Buache, il quale fcandagliò quel tratto di mare con fomma diligenza. (D) Quefte ifole, e fecche faranno ftate verifimilmente le parti più alte di quel Continente fubbifsato. Similmente crediamo, che la parte ora più occidentale dell' America era già unita per mezzo d'un minor Continente alla parte più orientale della Tartaria, e forfe ancora era unita l'America per la Groenlandia con altri paefi fettentrionali dell' Europa .

Il fommo rif́petto, che portiamo a' facri libri, ci coftrigne a credere, che $\mathrm{i}$ quadrupedi, ed $\mathrm{i}$ rettili del nuovo Mondo difcendono da quegl'individui, che fcamparono nell' arca di Noè dal diluvio univerfale, e le ragioni fnora addorte con altre, che tralafciamo per non effere nojofi, ci perfuadono, che tali beftie paffarono per terra, e per diverfe parti nel nuovo Continente. Tutri gli altri filtemi foggiaciono a graviffime difficoltà; nel noftro ve ne fono alcune, ma non affatto infuperabili. La plù grande confifte nell' apparente inverifmilitudine d'un sì gran tremuoto, che fubbiffaffe uno fpazio di terra di più di mille cinquecento miglia, qual era nella noftra fuppofizione quello, che univa l' Africa coll' America, e che l'affundaffe tanto, quanta è la profondità offervata in alcuni fiti di quel mare. Ma oltrechè noi non afcriviamo ad un fol tremuoto quella ftupenda rivoluzione, effendo peraltro nelle vifcere. della terra tanti ammaff di materie combuftibili, l' infammazion delle une potrebbe rapidamente comunicarfi alle altre (nello fteffo modo, con cui fpiega Gaffendo la propagazion del fulmine) e la violenta rarefazione dell' Aria contenuta dentro quelle mine naturali potrebbe ad un ora fcuotere, agitare, e fubbiffare

uno

(D) Il Sig. de Buache prefentò I'anno I737. all' Accademia Real delle Scienze di Parigi le carte idrografiche di quel mare fatte fecondo le fue offervazioni, le quali furono efaminate, ed approvate dall Accademia. Il Chiar. A titor delle Lettere Americane diede nel fuo 2. tomo un tiftretto di quelle carte. 
uno fpazio di terra di due, o di tre mila miglia. Quefto non è impoffibile, nè inverifimile: nè ce ne mancano elempi nella Storia. Il tremuoto, che fi fentì nel Canadà l'anno $1663 . \%$ fubbifsò una catena di montagne di pietra viva, lunga prù dí trecento miglia, reftando tutto quel gran tratto di terra cangiato in una immenfa pianura. Quanto dunque fara ftato il conquaffo cagionato da quegli ftraordinarj e memorabili tremuoti, di cui fanno menzione le ftorie Americane, e co' quali credevano finito il Mondo?

Può anche opporfi al noftro finema, che fe le beftie pafo farono per terra dall' uno nell' altro Continente, non è facile d'indovinar la cagione, per cui vi paffarono alcune fpezie, fenza reftarne ne anche un folo individuo nel Continente antico, e reftarono per l'oppofto alcune Spezie intere nell' antico Continente, fenza che paffafe un individuo d'effe nell' America. Perchè paffarono per efempio le quattordici fpezie di fcimie, che oggi fi trovano nell' America, e non pafrarono le diciaffette Cpezie, che il Conte di Buffon annovera nell'Afia, e nell' Africa, effendo tutte d'un medefimo clima, ed avendo parimente liberta, ed agio per paffare? Perchè paffarono i pigri, e non le gazzelle, che fon tanto veloci? Se poi dall' Armenia, dove fi fermò l'Arca di Noè, s'incamminarono le beftie verfó l'America, dovettero fenz'altro fare un viaggio di fei mila miglia le fpezie deftinate a' paefi equinoziali del nuovo Mondo; portandofi dall' Armenia per la Mefopotamia, e la Siria all'E. gitto: quindi pel centro dell' Africa al fuppolto fpazio di terra, che univa ambidue $i$ Continenti, e da quello finalmente al Brafile: e benchè quanto ad altre bettie non appaja veruna difficolta nel fare quel viaggio in dieci, in venti, od in quaranta anni; nulla di meno per ciò che riguarda i pigri non fi può capire, come poteffero efeguirlo nè anche in fei fecoli, ognora camminando. Se diamo fede al Conte di Buffon, il pigro non può avanzare in un ora più d'una pertica, ovvero fei piedi reali di Parigi: onde per far quel viaggio di fei mila miglia, abbifognerebbe di feicento ottanta anni incirca, ed ancor più, fe crediamo a ciò, che fcriffero Maffei, Herrera, e Pifon, i 
quali affermano, che quel miferabil quadrupede appena può fare in quindici gioni, 0 in dus ftrimane una gittaca di pietra.

Quefto è quello, che può dirfi contro il noftro fiftema; ma alcuni de' fuddetti argomenti hanno maggior forza contro l'altre opinioni, fuorchè contro quella, che impiega gli Angioli nel trafporto delle beftie. Se gli uomini furono coloro, che trafportarono le beftie, perchè in cambio de'lupi, e delle volpi non portarono i cavalli, $\mathrm{i}$ busi, le pecore, e le capre? Come non lafciarono ne anche un individuo di parecchie fpezie nel Continente antico? Se fi vuole, che paffaffero tali beltie 2 nuoto, allora s'aggiunge la difficoltà del viaggio marittimo a quella del terreftre. Se fi fanno paffare tutte le beftie, anche quelle dell' America meridionale pel fettentrione, allora in ve. ce di fare un viaggio di fei mila miglia, avrebbono dovuto fare un altro di più di quindici mila, pel qual viaggio avreb. be bifogno il pigro di 1740 . anni.

Noi dunque rifpondramo alle fuddette obbiezioni I. che non effendo finora conofciuti tutti i quadrupedi della terra, non pofframo fapere quanti fono nell' uno, e nell' altro Continente, e quanti mancano. Il Conte di Buffon numera foltanto du. gento fpezie di quadrupedi. Il Sig. Valmont di Bomare, il quale fcriffe foco tempo dopo quell' Autore, ne numera du. cenro fetantacinque; ma a dire il vero neffuno è capace di contarle tutte; perchè nulla fi fa de' quadrupedı d'alcune regioni mediterranee dell' Africa, d'una gran parte della Tartaria, del paefe delle Amazoni, della Luigiana fettentrionale, delle contrade fituate a tramontana del fiume colorado, del paefe degli Apacci, delle ifole di Salomone, della nuova Olanda \&co i quali paefi fono una parte confiderabile del noltro globo. Non c̀ pur maraviglia, che non s'abjia contezza delle beitie di qué paefi fconolciuti, mentre di quelle de'paefi afldi conofciuti, ed abitati dugento felfanta anni $f_{d}$ dagli Europei, non hanno i Zoologifti quelle notizie, che fi richiedono per ifcrivere una Atoria compita de'quadrupedi. Il Conte di Buffon, conrutcochè fia l'uomo più inftruito in quefta materia, ommette pu. Storia Antica del Mejfico Tom. IV. F 
re alcuni quadrupedi del Meffico, altri fpatiia dal proprio loz paefe, ed altri confonde, ficcome faremo vedere nella differtazione fu gli animali. Ma per ciò che riguarda le beltie, che certa. mente mancavano all' America, come gli Elefanti, i Cammelli, ed i Cavalli, fi poffano addurre parecchie ragioni d'una tal man. canza. Pud effere, che quelle beftie in fatti paffaffero inel nuovo Mondo, e poi vi periffero o ammazzate dalle fiere, o eftinte da qualche epidemia lor fopravvenura.. Può effere ancora, che non paffaffero mai nell'America. Alcune, come gli Elefanti, ed i Rinoceronti, la moltiplicazione de' quali è affai lenta., fi fermarono ne'paefi meridionali dell' Afia, e dell' Africa., perchè vi trovarono un clima convenevole alla lor natura, buoni pafcoli, ed un grande fpazio di terra da poter vivere agiatamente: ficchè non ebbero bifogno d'ufcire di quelle regioni per procacciarfi altrove il lor vitto. E' vero, che molti Autori fi fon perfuafi, che le grandi offa difotterrate preffo al fiume Ohio, ed in altri luoghi dell' America fieno ftate d' Elefanti, ciò che dimoftrerebbe 1 antica loro efiftenza in quel Continente; ma ficcome i Zoologifti moderni non fon d'accordo intorno alla fpezie di quadrupedi, a cui tali offa appartenevano, non può da efse verun argo. mento dedurfi contra noi. (E) Finalmente altre beltie forfe non patsarono nel nuovo Mondo, perchè le tratcennero gli uomini. Io non dubito, che poichè ufcl la famiglia di Noè dell' arca, ritenne nel fuo potere le vacche, le pecore,; e le capre ridotte ad armento, ed a greggia, per prender da efse cosi il veltito ad efempio de'loro antenati, come il foltentamento, giulta il permelso dato da Dio dopo il diluvio. A proporzione che s'anda. vano moltiplicando gli uomini, s'andavano parimente aumensando le loro pofseffioni nell' Armenia, nella Caldea, nella Si ria, nella Perfia, e nell' Egitto, nelle quali regioni reltarono, come

(E) Muller diffe, che quelle Orra erano tate di certi grandiffimi quadrupedi, che egli appella Mammouts. Il Conte di Buffon, fidandofi troppo di lui calcold, che detti quadrupedi erano fei volte più grandi degli Elefanti. Altri credettero, che foffero ofla d'Ippopotami, altri che foffero di beftie marine, ed altri finalmente, che apparteneffero ad altri quadrupedi fconofciuti, ed eftinti. Ma non v'è dubbio, che molte di quelle ofla fono ftate di giganti, come abbiam detro nel lib. 1. della noftra Storia. 
come è da credere, confinate in que' primi tempi gli armenti, e le gregge fotto la cura de' primogeniti delle famiglie; mentre gli: altri quadrupedi, ch' erano in libertà, fuggendo dagli uomi. ni fr portarono a' paefi: non ancor popolati, ed alcuni cercando il clima, ed il cibo confacevole alla lor natura, fi faranno incamminati verfo l' America.. Frattanto molte famiglie deftinate a: popolar diverfi paefi: della terra, prefentendo la lor feparazio. ne, e volendo lafciare: alla pofterita un chiaro monumento della lor magnificenza, intraprefero la coftruzione della. Città , e della torre di Babel .. Iddio confure loro,il linguaggio per coftrignerli 2: portarfi a' paefi loro affegnati, ed eglino coltretti dall' ordine, e dal galtigo del Cielo fi mifero in cammino per diverfe ftra. de: I progenitori di coloro, ch' erano deftinati a popolar l'America, o non conduffero feco gregge, ed armenti, perchè non: poterono averle, o avendole tratte dalla Caldea, le confumarono per mancanza di viveri nella lunga loro pellegrinazione. Egli è certo, che niuna di quelle beftie, che da' primi fecoli fono fate fotto la cura e la direzione degli uomini nel Mondb antico, fi trovò nel nuovo:: il che pare un chiaro indizio, che gli animali paffarono per proprio loro iftinto nel Mondo. nuovo, non gia trafportati dagli uomini . Ciò che diciamo del. le vacche, delle pecore, e delle capre, pofliamo anche congetturarlo degli Afini, e de Cavalli, poichè non dobbiamo dubitare, che anche quefti animali furono ridotri a: fervirù immediatamente dopo ill diluvio. $\mathrm{Ma}$ checcheffia, 1 argomento prefo dal: paffaggio d'alcune beftie, e: non d'altre: nulla prova: contro il noltro fiftema.

Quanto poi al calcolo fopraccennato ful tempo di cui abbifognava. il Pigro per portarfi dall' Armenia fino al Brafile' non vi troviamo verun inconveniente. Ancorchè: aveffe avuto bifogno di mille anni, porè: finalmente arrivare al nuovo Mondo nel cafo, che fi fiano confervati turto quel tempo uniti i due Continenti : la qual ruppofizione non può dimoftrarfi falfa nè dalla ftoria, nè dalla ragione; ma neppur v'è ragione, che ci coitrigna ad ammertere un tal calcolo. Il medefimo Co: di Buffon prosefta, che gli Autori hanno efagerata la lentezza del Pigro, ed il Sigo.

$$
\text { F } 2 \text { : }
$$

diAus. 


\section{DISSERT AZIONE I.}

d'Aubenton riconobbe, che non era effo tanto lento, quanto la teltuzgine. Oltrechè non effendo quefta beftia nocevole, ma piuttofto degna di compaffione, potè effere ajutata dagli uomini, e da un paefe in un altro trafportata.

Tali fono i miei fentimenti intorno alla popolazione dell' America, i quali fottometto al giudizio de' Dottori Criftiani e favj; non però a quello di certi Filofofi milcredenti, e capricciofi, che nè rifpettano l'autorità divina, nè fi curano delle tradizioni umane, nè vogliono afcoltar la ragione.

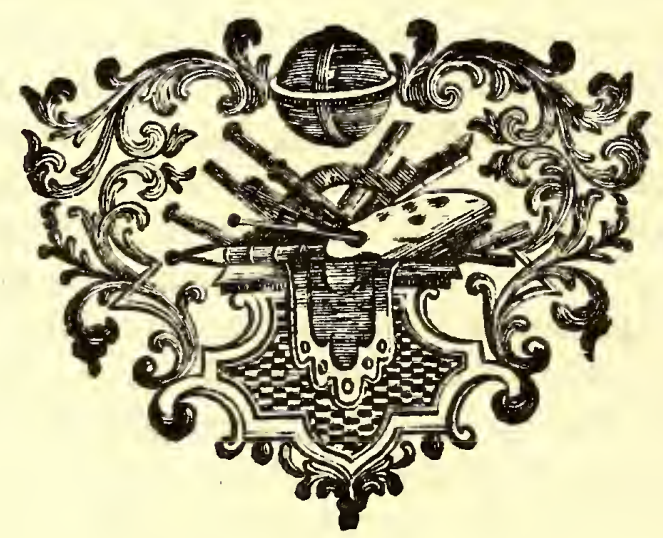




\section{DISSERTAZIONE II.}

\section{SU LE PRINCIPALI EPOCHE DELLA STORIA DEL MESSICO.}

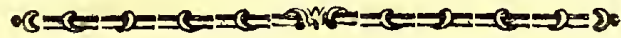

T A fomma varietà, che troviamo preffo gli Autori intorno alla Cronologia della Storia del Meffico, ci coltrigne a difaminare proliffamente l'epoche dei principali avvenimenti. Se aveffimo ciò fatto nel corpo della Storia, farebbe ftato d' uopo interrompere il filo della narrazione con difpute fpinofe. Se I' aveffimo fatto, come volevamo, nelle note, quefte farebbero divenute oltremodo lunghe. La varietà dei fentimenti degli Storici nacque, per quanto appare, dal non avere eglino aggiuftati gli anni Mefficani ai noftrali. Io ho faticato con gran diligenza per rintracciare il vero, e in gran parte mi pare d'effer riufcito, ficcome farò vedere nella prefente differtazione, la quale farà fenza dubbio nojofa per coloro, che non hanno intereffe nel rifchiaramento di quefti punti di cronologia.

\section{I.}

Su l'epoca dell' arrivo dei Toltecbi, e d'altre Nazioni nel paefe d' Anabuac.

Non difcorriamo nella prefente differtazione dei primi po. polatori, dei quali abbiam già ragionato, ma foltanto di quelle Nazioni, che fanno qualche figura nella noftra Storia. Or difcordano primamente gli Autori intorno all'ordine dell'arrivo di tali Nazioni; poichè i Cicimechi per efempio, i quali fecondo Acolta, Gomara, e Siguenza, furono i primi ad al:vare in quel paefe; fecondo Torquemada furono i terzi; e itcondo Boturini i quarti. Non è minore la lor difcordanza intorno al tempo dell'arrivo di ciafcuna Nazione, come andremo vedendo.

Nel: 


\section{D I S:S E R T A Z I O N E: II.}

Neffuno dubita, che $\mathbf{i}$ Toltechi fieno ftati molto antichi: Dalle fteffe ftorie dei Cicimechi confta, che coftoro non giunfero in Anahuac, $\mathrm{fe}$ : non dopo la rovina dei Toltechi, le cui fabbriche riconobbero nel loro, viaggio, ed i cui avanzi trovarono nelle rive dei laghi mefficani, ed in altri luoghi . In quefto punto for d'accordo Torquemada, Betancurt; e Boturini .. Herrera, Acofta, e Gomara non fanno menzione doi Toltechi, forfe: perchè quegli: Autori, di cui effi fervironfi, tralafciarono le:notizie: di quella Nazione: per cagione d'effere: fcarfe, ed ofcure..

Intorno al tempo del loro arrivo in Anahuac, Torque. mada dice nel lib; 3..della. fua Storia, ch' effo avvenne nell'. anno 700. dell' era volgare;: ma da ciò ch' egli fcriffe: nel: lib. I. fi deduce, che accadde verfo, il 648, Il Cavo. Boturini gli fa quafi un fecolo più antichi, mentre: credette regnante in Tula l'anno 660 . Ixtlalcuechahuac. Re fecondo di quella $\mathrm{Na}$ zione. Dalle loro pitture fappiamo, ch'eglino ufcirono da. Hue huetlapallan, llanno I. Téppatl', che dopo aver pellegrinato cen-. to quattro anni fri frabilirono prima، in Töllantzinco, ed indi in Tula, e che la loro monarchía cominciata l'anno V.II. Acatl durò trecento ottanta quattro anni.. Io dopo aver confrontate: quefte epoche dei Toltechi con quelle dei Cicimechi lor fucceffori, mi fon perfuafo, che la loro ufcita da Huehuetlapallan accadde l'anno 544 , e che la: loro monarchì: cominciò l'an-. no 667. Chiunque voglia continuare, retrocedendo verfo quel tempo, la ferie degli anni Mefficani rifcontrati co" criftiani, da noi efpolta nel fine del tomo fecondo, troverà l'anno $544 \%$. dell' era volgare effere tato I: Tecpatl, e l'anno 667. effere fi. mil mente ftato. VII. Acatl. Non v' è per altro ragione di an: ticipare tali epoche, nè poffono pofporfi fenza fconvolgere quel. le delle Nazioni poiteriori.. Ora effendo cominciata quella mo: marchì l' anno 667 , ed effendo durata 384 anni, dee fiffarin : il fine d'effa, ed il conquaffamento de', Toltechi nell'anno, $305 \mathrm{I}$.

Fra la rovina dei Toltechi; e l'arrivo dei Cicimechi non mette Torquemada più idi nove anni; "ma ciò non pud effere?, perchè i. Cicinechi trovarono, come: il medefimo, Aurore di 


\section{I S E R T A Z IO NE II.}

ce, rovinati gli edifizj dei Toltechi, e non è verifimile che fi rovinaffero in foli nove anni. Oltracciò, non può filfarfi in quel fecolo il cominciamento della monarchì Cicimeca fenza aumenrare il numero di quei Re, o fenza prolungare oltremodo la loro vita, come fa Torquemada. Chi fara capace di credere, che Xolosl regnaffe 1 I3. anni, e ne viveife 200.? Che Nopaltzin fuo figliuolo viveffe 170 ., Techotlala fuo terzo nipote regnaffe I04., e Tezozomoc fuo difcendente regnaffe in Azcapozalco anni is 6o., ovvero is 80 .? ' $E$ ' vero, che un uomo di compleffione robulta fecondaro dalla fobrietà nel vitto, e da un clima così mite, come quello del Melico, potrebbe arrivare ad un' età tanro avanzara, e non fon troppo rari nella Sto. ria di quel paefe gli éfempj di quegli uomini, che hanno prolungato la lor vita oltre al termine regolare dei mortali. Calmecahua, uno dei Capitani Tlafcallefi che ajutarono gli Spagnuoli nella conquifta di Meffico, viffe I 30 . anni. Pietro Nie. to, Gefuita, morì nel 1536. d'anni I32. Didaco Ordoñez, Francefcano morl in Sombrerete d'anni : I $7 .,\left(^{*}\right)$ facenda prediche al popolo fino all'ultimo mele della fua vita. Si porreb. be fare un lungo catalogo di quélli, che tanto nei due fecoli paffati, quanto a' noftri dì hanno in quei paefi oltrepafCata l' eta centenaria. Particolarmente tra gl' Indiani non fono cosi rari coloro, che giungono ai novanca ed ai cento anni, confervando fino all'eltrema vecchiaja i capelli neri, la dentatura ferma, e buona la lor vifta; ma effendo ftati sì pochi quelli, che dopo il fecolo XXIII. del Mondo hanno prolungato la lor vita fiao agli anni 150., che fon riguardati, come altrettanti prodigi, non pofiramo acconfentire alla ftravagante cronologì di Torquemada, appoggiata forfe alla fede di qualche pittura o fcritto dei Tezcucani, maffimamente confeffando lo lteffo Aurore, che quelle Nazioni non tennero gran conto degli anni. Noi pertanto crediamo fenza efitazione, che l'arrivo dei

(*) Didaco Ordoñez viffe in religıone anni ro4., e nel facerdozio quafi 95. Nell ultima fua predica prefe congedo dal Popolo di Sombrerete con quells parole di S. Paolo: Bonum certamen certavi, curfum con/umavi \&c. 
dei Cicimechi in Anahuac accadde nel fecolo XII., e verifimilmente verfo l'anno 1170.

Appena erano fcorli otto anni, dappoichè Xolotl, primo Re Cicimeea, s'era ftabilito in Tenajusa, quando vi capitarono delle nuove genti condotte, come abbiam detto nella Storia, da fei Capi. Io non dubito, che quefte nuove genti fieco ftate le fei tribù dei Xochimilchi, dei Tepanechi, deı Colhui, dei Chalchefi, dei Tlahuichi, e dei Tlafcallefi, feparate dai Mefficani in Chicomoztoc, e giunte nella Valle Mefficana non tutte infieme ma con qualche divario di tempo, e coll'ordine da noi accennato. Egli è certo, che allorchè vi arrivarono pochi anni dopo gli Acolhui, trovarono già fondata dai Tepanechi la Città d'Azcapozcalco, e dai Colhui quella di Colhuacan. Si fa peraltro, che quefte tribù arrivarono a quel paefe dopo i Cicimechi : dunque il loro arrivo accadde in quell' intervallo, che vi fu fra l'arrivo dei Cicimechi, e quello degli Acolhui. Or non v' è memoria d'altre genti giunce in quel tempo in Anahuac, fe non di quelle condotte da luddetti fei Capi: quefte dunque furono quelle fei tribù di Nahuatlachi, cioè i Xochimilchi, i Tepanechi, i Colhui, \&c. condotta cia.fcheduna dal fuo Capo. Il P. Acofta fa quefte tribù quafi tre fecoli più antiche, mentre dice, che giunfero alle rive del lago mefficano l'anno 902., dopo una pellegrinazione d'ottanta anni; ma quefta cronologia non s'accorda bene colla Storia, dalia quale confta, che quando Xolotl arrivo alla valle meflicana colla fua colonia di Cicimechi, trovò (popolate le rive di quel lago, e l'arrivo di quefta Colonia non potè avvenire avanti la meta del fecolo XII., giufta quello, che abbiamo detto fopra .

Non fi fa l'anno dell' arrivo degli Acolhui; ma io non dubito, che fia ftato verfo il fine del fecolo XII.; perchè eglino giunfero pochi anni dopo l' arrivo di quelle fei tribù, e per. altro confta dalla medefima Storia, che Xolotl fopravviffe alcuni anni al loro arrivo.

L'ultima Nazione, o tribù giunta in Anahuac fu quella dei Mefficani. Fra tanti Storici da me confultati non ne ho 
trovato neppur uno, che fia di contrario parere, fe non il Be: tancurt, il quale mette gli Otomiti dopo i Meflicani. Il P. Acofta fiffa l'arrivo dei Mefficani alle rive del lago mefficano nell'anno 1208.; perchè afferma, che vi arrivarono 306. anni dopo i Xochimilchi, e l'altre tribù dei Nahuatlachi, le quali crede egli arrivate nel 902. Torquemada, giulta il calcolo fatto dal Betancurt ful ragguaglio di lui, mette l' arrivo dei Melficani in Chapoltepec nell' anno 1269. Una Storia mefficana anonima citata dal Cav. Boturini mette l'arrivo di quella tribù in Tula l'anno I I g6́., ed in quefta epoca pare, che fieno d'accordo parecchj Storici Indiani. Oltracciò quefta cronologìa $s$ ' accorda perfettamente con tutte le alcre epoche: onde noi l'abbiamo adottata, come la più probabile, e quafi certa. Ciò fuppolto bifogna dire, che i Mefficani arrivarono a Tzompan. co l'anno I216., ed a Chapoltepec l'anno 1245.; perchè fis fa, ch' eglino ftettero in Tula nove anni in Tepexic, ed in altri luoghi prima d'arrivare a Tzompanco, undici; in Tzompanco fi trattennero anni fette, ed in altri luoghi prima d'andare a Chapoltepec, ventidue. Dopo effere ftati anni diciaffette in Chapoltepec, paffarono ad Acolco nel 1262., dove ftettero 52. anni, ed indi furono condotti fchiavi a Colhuacan nel I 3 I 4 .

Per ciò che riguarda gli Otomiti, v'è una gran varietà preffo gli Storici. Alcuni gli confondono coi Cicimechi, ficcome l'A Acofta, il Gomara, e la maggior parte degli Autori Spagnuoli. Torquemada nel lib. I. gli diftingue efpreffamente; ma in altri lunghi della fua Storia pare, che gli confonda. Betan. curt, dopo aver copiata la narrazione di Torquemada in tutto ciò, che appartiene ai Toltechi, ai Cicimechi, ed alle altre Nazioni, dice parlando del Regno di Chimalpopoca, Re terzo di Meffico, che in tempo di lui giunfero in Anahuac gli Otomiti, e fi Atabilirono principalmente in Xaltocan. Quefto aned. duto di Berancurt non è da difpregiare; perchè 'enz' altro lo prefe dagli foritri di Siguenza, mentre non fuol difcoftarfi da Torquemida, fe non per tener dietro a quel dotto Mefficano; ma egli falla nella cronologì, mentre fiffa l'arrivo degli Oto: Storia Antica del MeJjico Tom. $1 V$. $\quad G \quad$ miti 


\section{DISSERTAZIONE II.}

miti nell' anno VI. Tecpatl, il quale crede effere ftato l'anne I $38 \mathrm{r}$. S'ingannd certamente; poichè ficcome appare dalla no. Itra tavola cronologica meffa in fine del 2 . tomo, l'anno $138 \mathrm{r}$. non fu VI. Tecpatl, ma VI. Calli, nè allora regnava Chimalpopoca, ma Acamapitzin, come faremo appreffo vedere. Se l'arrivo degli Otomiti nella Valle Mefficana (non già nel paefe d'Anahuac, nel quale molti fecoli prima s' erano tabiliti ) accadde nell anno VI. Tecpatl, e fotto il regno di Chimalpopoca, ciò farà ftato fenz' altro nell' anno 1420. Il non farfi menzione degli Otomiti prima di quefta epoca, e l'effere ftati effi trovati dagli Spagnuoli men civili delle altre Nazioni, fparfi in parecchie provincie, ed in alcuni luoghi ifolati, e circondati da altse Nazioni di diverfo linguaggio ci fa credere, che appunto a quel tempo cominciarono a vivere in focietá fotto la dominazione de'Tepanechi, e pofcia fotto quella de' Mefficani, e de' Tlafcallefi. Io mi perfuado, che a cagione d'aver eglino trovata la terra occupata dalle altre Nazioni, non poterono, come gli altri Atabilirvifi tutti in un fol paefe, benchè la maggior parte di quella Nazione popolaffe quel tratto di terra, che v'è a Maeftro, ed a Tramontana della Capitale, come più vicino al paefe, dove prima viveano sparfi a guifa di fiere.

La cagione d'effere ftati gli Otomiti confufi da molti Storici co' Cicimechi può prenderfi dalla medefima ftoria. Allorchè gli antichi Cicimechi furono renduti civili da' Toltechi, e da' Nahuatlachi, molte famiglie di quella Nazione s' abbandonarono alla vita felvaggia nel paefe degli Otomiti, pregiando più l' efercizio della caccia, che le fatiche dell' Agricoltura. Coftoro ritennero il nome di Cicimechi, e gli altri ridotti a civiltà cominciarono ad appellarfi Acolhui, onorandofi col nome d' una Nazione, ch' era ftimata la più colta. Degli Otomiti poi coloro, che adottarono la vita civile, reftarono col nome d' Otomiti, col quale fon conofciuti nella Storia; ma gli altri, che fparfi ne' bofchi, e frammifchiati co' Cicimechi non volleró difmettere la barbara loro libertà, furono da molti chiamati Cicimechi dal nome di quella celebre Nazione: per lo che alcuni

Scrit: 
Scrittori ragionando di quefti barbari, i quali per più di un fecolo dopo la conquifta di Meffico travagliarono affai gli Spagnuoli, diftinguono i Cicimechi Mefficani da' Cicimechi Otomiti; perchè gli uni parlavano la lingua mefficana, e gli altri l'otomita giufta la Nazione, onde traevano la lor origine.

$D_{d}$ tutto ciò, che frora abbiamo detto, poffiamo conchiudere colla maggior verifimiglianza, the fi può in un argomento cosi ofcuro, che l'ordine, ed il tempo dell' arrivo di quelle Nazioni in Anahuac, fu quefto:

I Toltechi l'anno 648.

I Cicimechi verfo l'anno II 70 .

I primi Nahuatlachi verfo il 1178 .

Gli Acolhui verfo il fine del fecolo XII.

I Mefficani giunfero a Tula l' anno I I96., a Tzompanco l'anno 1216 ., ed a Chapoltepec l'anno 1245.

Gli Oromiti entrarono nella valle mefficana, e cominciarono a ridurfi a vita civile l'anno 1420.

So bene, che i Tepanechi vantavano tanto antica la loso Citrà d'Azcapozalco, che al dir di Torquemada, contavano 1561 . anni dalla fondazione d'effa fino al cominciamento del fecolo paffato: ficchè la ftimavano fondata immediatamente dopo la morte del noftro divin Redentore; ma l'oppofto confta dalle Storie delle altre Nazioni, le quali fanno i Tepanechi poco più antichi de' Meflicani in Anahuac, ed anche dalla medefima ferie de'Signori d'Azcapozalco, i cui ritratti fi confervavano fino a' noftri dì in un edifizio antico di quella Citrà. Eglino non contavano più di dieci Signori dalla fondazione della loro Citta fino alla memorabile rovina del loro Stato, cagionata dalie armi combinate de' Mefficani, e degli Acolhui, il che avvenne, come vedremo nell' anno $1425 .:$ onde bifognerebbe dare a ciafcun Signore cento quaranta anni di governo per compiere quella fomma.

I Totonachi dalla lor parte fi dicevano più antichi des Cicimechi; poichè it vantare antichità è una debolezza comune a rutte le Nazioni. Raccontavano dunque, che effendofi $e^{*}$ glino da principio per qualche tempo ftabiliti fu le rive del 
lago rezcucano, quindi fi portarono a popolare quelle monta: gne, che da loro prefero il nome di Totonacapan: che quivi furono retri da dieci Signori, cialcuno de'quali reffe ottanta anni in punto la Nazione, finchè arrivati $\mathrm{i}$ Cicimechi in A. nahuac al tempo di Xatoncan fecondo Signore della Nazion Totonaca, gli fottomifero al loro dominio, e che poi furono finalmente fortopofti a' Re Mefficani. Torquemada, il quale rapporta quefto ragguaglio de Toronachi nel lib. 3. della fua Monarcbia Indiana, foggiunge, ciò effere certo, $e$ comprovato con ifrorie autentiche, e degne di fede; ma checchè egli dica, è certo, che non fi $f a$, nè fi può fapere il tempo dell' arrivo di quella Nazione in Arahuac, e che il racconto de'dieci Signori, che governarono la Nazione ottanta anni in punto è Coltanto buono per trattenere 1 fanciulli

Men fi fa il tempo, in cui arrivarono gli Olmechi, ed a Xicallanchi. Il Cav. Buturini dice, che non porè trovar ve. suna pittura, nè memoria concernente quefte Nazioni; contutrociò egli le crede più antiche de'Toltechi; ma checcheffia egli è indubitabile, che fono tate antichiffime.

Non facciamo quì menzione delle altre Nazioni, perchè affolutamente s'ignora la loro antichità; ma non dubitiamo, atrefo ciò che abbiamo altrove efpolto, che i Chiapanefi furo: no de' più antichi, e forle $i$ primi di tutte quante le Nazioni che popolarono il paefe $d^{9}$ Anahuac.

\section{§. I I.}

Sulla corrifpondenza degli anni mefficani a nofirali, e full' epoca della fondazione di Meffico.

Tutti gli Scrittori, tanto Meffrcani, quanto Spagnuoli, che hanno fatto menzione della cronologia mefficana, fon d'ac. cordo intorno al metodo, che aveano quelle $\mathrm{N}_{\mathrm{azi}}$ zion nel contare $\mathrm{i}$ lor fecoli, ed i lor anni, da noi efpreffo nel libro VP. della Storia, e nelle tavole meffe nel fine del tomo H. Qua. lora dunque fi trovi la corrifpondenza d' un anno mefficano a 
qualcuno degli anni criftiani, tofto fi faprà la corrifpondenzà di cutti gli altri. Se io per efempio fo, che queft anno 1780. é ficcome è infarti, II. Terpatl, fon ficuro, che il $178 \mathrm{r}$. è III. Calli, il 1782. è iV. Tocbtli \&s. Tutta la difficoltà confifte nel trovare un anno mefficano, la cui corrifpondenza a qual: che anno criftiano fia affatto certa, ed indubitabile; $m a$ troviam già vinta quefta difficoltà, mentre fiam ficuri non meno per le pitrure degl' Indiani, che per la teftimonianza d'Acofta, di Torquemada, di Siguenza, di Betancurt, e di Boturini, che $l_{\text {'anno }} 1519 .$, nel quale entrarono in Meffico gli Spagnuoli, fu I. Acatl, e per confeguenza che l'anno 1518. fu XIII. Tocbtli, l' anno 15I7. XII. Calli \&ce. Sicchè non fi può dubitare della efartezza della noftra tavola cronologica meffa ful fine del tomo II. in ciò che riguarda la corrifpondenza degli anni mefficani a' criftiani. Quegli Autori, che da effa difcordano, sbagliarono nel calcolo, e fi contraddiffero. Betancurt per farci comprendere la maniera di computar gli anni, che aveano i Mefficani, ci prefenta una tavola degli anni mefficani rifcontrati cogli anni criftiani dall' anno 1663 . fino al 1688.; ma quefta tavola falla da capo a piè, perchè l' Autore Cuppone l'anno 1663 . effere ftato I. Tocbtli, il che fi dimottra falfo continuando la noftra tavola fino a quell' anno. Egli afferma, che il 1507 . fu anno fecolare: ammeffo quefto errore non può a meno di non fallare in cutra la fua cronologìa. Se l'anno I5I9. fu I. Acatl, come egli fuppone cogli altri Scrittori, troveremo retrocedendo nella noftra tavola, che non fu anno fecolare il $1507 ., \mathrm{ma}$ il 1506. Egli per confermar la fua cronologia, allega il teftimo. nio del fuo amico e compatriota il Dotr. Sigueaza, il quale dice, avea trovato, che l'anno I684. era ftato IX. Acatl. Se quefto foffe così, il fuo calcolo fenz' altro andrebbe bene; ma ancorchè non dubitiamo delia fua veracità nella citazinne del Siguenza, abbiamo pure ragion di credere, che quefto dorto Mefficano correggeffe la fua cronologìa : nè poted fare altrimenti, fapendo, ficcome in fatti fapeva, che l'anno Ijig. era fta. to I. Acatl principio certo, ful quale dee appoggiarfi tutta la cronologì mefficana, e dal quale fi deduce chiaramente l'an. 
80 1684. non effere ftato IX. Acatl, ma X. Tecpatl. Torque: mada nel ragionare, che fa nel lib. 3. de' Totonachi, dice d'un Nobile di quella Nazione, cb'era nata l'anno $I I$. Acatl, e cbe l'anno i'manzi IsIg., nel quale erano giunti in quel paefe gli Spagnuoli, era prefJo: M:J]icani 1. Acatl . Quando Torquemada fcrilfe quelto o era aggravato dal fonno, 0 diftratto in un altro penfiere; poichè egli fapeva bene, ficcome tutti il fappiamo, che l'anno, il quale preffo i Mefficani vien dietro a quello di I. Acatl non è il II. Acatl, ma il II. Tecpatl, e tal fu l'anno 1520 . di cui parla.

Suppolto dunque, che l'anno 1519. fu I. Acatl, e faputo il rapporto degli anni mefficani ai crittiani, non è difficile di rintracciare l'epoca della fondazione di Meffico. Tutti gli Storici, che hanno confultate le pitture dei Mefficani, o fi fono informati a bocca da loro, fon d'accordo nel dire, che quella celebre Citta fu fondata dagli Aztechi nel fecolo XIV. del Criftianefimo; ma difcordano quanto all' anno. L'Interprete della Raccolta di Mendoza fiffa la fondazione nell' anno 1324., Gemelli tenendo dietro al Siguenza nel 1325. , Siguenza citato da Betancurt, ed un Mefficano anonimo, citato da Boturini, nel 1327., (a) Torquemada, giufta il calcolo fatro da Betancurt, ful ragguaglio di lui, nel 1341., ed Arrigo Martinez nel 1357. I Mefficani mettono tal fondazione nell' anno II. Calli, come fi vede nella prima pittura della Raccolta di Mendoza, ed in altre citate dal Siguenza. Eflendo dunque cerro, che quella Citta fu fondata nel fecolo XIV., e nell ${ }^{2}$ anno I1. Calli, ciò non potè effere nel 1324. , e neppure nel I327., o nel 1341., o nel 1357., perchè niuno di quefti anni fu II. Calli. Se vogliamo retrocedere dall' anno 1519. fino al fecolo XIV. troveremo in quefto due anni II. Calli, cioè il 1325. , ed il 1377 . Ora in quelt anno certamente non accadde $t d l$ fondazione; perchè allora farebbe d' uopo di raccorciare troppo i regni dei Monarchi Meflicani, contraddicendo alla

(a) Il teftimonio del Mefficano anonimo fi trova, in una copia d'una pittusa antica cayaca l'anno 163 I. 
Ia cronologia delle pitture antiche. Non refta dunque altro ri: piego, fe non quello di dire, che quella celebre capitale fi fon. dò nel I 325. dell' era volgare: e quefto fu fenz' altro il fentimento del Dott. Siguenza; perciocchè Gemslli, il quale non ebbe in quelto loggerto altra iftruzione, fe non quella, che gli fu data da quel Letterato mefficano, mette tal fondazione nell' anno I 325 ., il quale dice, fu II. Calli. (b) Se prima fu d'un altro parere, lo cambiò poi accorgendofi, che non s' accorda. va bene con quel principio certo d'effere ttato I. Acatl l'an: no 1519 .

\section{I I I.}

Su la Cronologia de' Re Mefjicami。

E' difficile il mettere in chiaro la cronologia de' Re Mefo ficani a cagione della difcordanza degli Autori. Noi ci ferviremo d'alcuni punti certi per rintracciare gl' incerti. Per dare ai Lettori qualche idea della varietà delle opinioni bafta metter gli occhj fu la feguente tavola, nella quale accenniamo l'anno, in cui fecondo l' Acolta, l'Interprete della Raccolta di Mendoza, ed il Siguenza cominciò a regnare ciafcuno dei Re. (c)

\begin{tabular}{|c|c|c|c|c|}
\hline & Acolta & $L^{\prime}$ interpr. & Siguenz & \\
\hline & 1384 . & 1375. & 3. Mdggio & $136 \mathrm{I}^{\circ}$ \\
\hline Huitzlihuitl. & 1424. & 3396. & 19. Aprile & 1403. \\
\hline Chimalpopoca. & 1427. & 1417 & 24. Febbr. & 1414. \\
\hline Itzcoatl. & 1437. & 1427. & $\cdot \cdot \cdot \dot{ }$ & 1427 \\
\hline Motezuma I. & 1449. & 1440. & I3. Agofto & 1440. \\
\hline Axajacatl. & $148 \mathrm{I}$ & 1469. & 2 I. Nov. & 1468. \\
\hline Tizoc. & 1477. & 1482. & 30. Ottubre & $148 \mathrm{I}$ \\
\hline Ahuitzotl . & 1492. & 1486. & 13. Aprile & 1486 \\
\hline Motezuma & 1503. & 1502. & I 5. Sett. & $\begin{array}{l}150^{2} \\
\text { Aco. }\end{array}$ \\
\hline
\end{tabular}

(b) Abbiamo altrove accennata l'equivocazione di Gemelli hell' avere fcritto: Sanno 1325 . della creazion del Mondo, in vece di fcrivere: dell' era volgare.

(c) Gli anni meffi nella tavola fecondo l'interprete della Raccolta di Mendoza fon quelli che fi leggono nell' edizione di Tedi Purchàs, la quale non abbiamo potuto trovare. 
Acofta, e dietro a lui Arrigo Martinez, ed Herrera, non folamente difcordano dagli altri Autori nella cronologì, ma anche nell' ordine dei Re, metrendo Tizoc ful trono prima d'Axajacatl, laddove confta il contrario non meno per la teftimonianza dei Mefficani, che per quella degli altri Autori Spagnuoli. Gomara imbroglia i regni dei Signori di Tula con quelli dei $\operatorname{Re}$ di Colhuacan, e de' $\operatorname{Re} m \in$ flicáni. Torquemada indica gli anni degli uni, e degli altri, e la fua cronologìa difcorda da quella degli altri Autori. Solis fa Motezuma II. l'undecimo de' Re Mefficani; ma non fo donde traeffe un si pellegrino aneddoto. Il Sig. de $\mathrm{PaW}$ per dimoltrare anche in quefto la fua ftravaganza, non numera più di otto Re di Mefo fico; ma egli è affatto certo ed indubitabile, che i Mefficani ebbero undici $\operatorname{Re}$, cioè, que' nove fopra accennati, e dopo effi Cuitlahuatzin, e Quauhtemotzin. Alcuni Autori non vollero contar tra' Re quefti due ultimi, perche regnatono poco tempo; ma effendo eglino ftati legittimamente elerti, e pacificamente accettati dalla Nazione, hanno tanto dritto ad effer contati tra' Re mefficani, quanto tutti i loro anteceffori. Acofta dice, che non ne fa menzione; perciè eglino non ebbero di $\operatorname{Re}$ altro che il nome, mentre a' tempi loro era già quafi tutto il regno fottopofto agli Spagnuoli; ma quefto è affolutamente falfo, perchè quando fu eletto Cuitlahuatzin, gli Spagnuoli non aveano fotto loro, fe non la Provincia de'Totonachi, e que. fti erano piuttolto alleati, che fudditi. Quando fu eletto Quauhtemotzin, aveano aggiunti a quella provincia gli Stati di Quauhquechollan, d'Itzocan, di Tepejacac, di Tecamachalco, ed alcuni pochi luoghi di que' contorni; ma tutti quefti Stati, paragonati col refto dell' Imperio Mefficano, erano meno dì quello, che è Bologna in paragone di tutto lo ttato Pontificio.

Per rintracciare la cronologia di quefti undici $\mathrm{Re} f a$ me. Atieri d'adoperare un altro metodo, cominciando dagli ultirni, e continuando in ordine retrogrado fino a principj della Mo. narchìa.

QUAUHTEMOTZIN. Quelto Re fini il fuo regno $\mathrm{a}^{2} \mathbf{I}_{3}$ Agofto I521., effendo ftato fatto prigioniare dagli Spagnuoli, 
e conquiftata la corte di Meffico. Il giorno della fua elezione

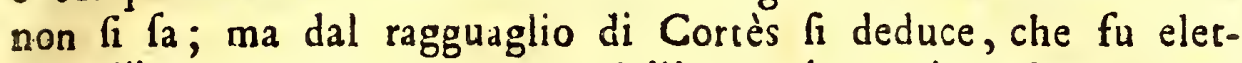
to nell' Ottobre, o Novembre dell' anno innanzi : onde non porè regnare più di nove, o dieci mefi.

CUITLAHUATZIN. Quefto Re, fucceffore del fuo fratello Motezuma, fali ful trono ne' primi gionni di Luglio 1520 , ficcome fi deduce dal ragguaglio di Cortès. Alcuni Autori Spagnuoli dicono, che non regnò più di quaranta giorni; altri affermano, che ne regnò feffanta; ma da ciò, che dice Cortés aver fentito da un Uffiziale Mefficano nella guerra di Quauhquechollan; fi fcorge, che quel $\operatorname{Re}$ era ancor vivo nell'Ottobre. Noi pertanto non dubitiamo, che il fuo regno fu almeno di tre mefi.

MOTEZUMA II. Si fa, che egli regnò I7. anni, e poco più di nove mefi, e che comincio a regnare nel Settembre 1502., e mor̀ negli ultimi giorni di Giugno 1520. La ragione d'aver meffo alcuni Autori il cominciamento del fuo regno nel 1503. fu perchè fapeano, che avea regnato diciaffette anni, e non fecero conto de' nove mefí di più.

AHUITZOTL. Acofta dà a quefto $R e$ undici anni di regno, Martinez dodici, Siguenza fedici, e Torquemada diciotto. Io credo, che potremo rintracciare gli anni del fuo regno, ed il tempo della fua efaltazione dall' epoca della dedicazione del tempio maggiore. Quefta fi fece fenza dubbio nel 1486.: nel che fon d'accordo parecchj Autori. Da un'altra parte confta, che avendo il $\operatorname{Re}$ Tizoc appena cominciata quefta fabbrica, la continuò, e conduffe a fine Ahuitzotl, e ciò non potè fare nel medefimo anno, nel quale cominciò a regnare, e neppur in due, o tre anni, effendo tal' edifizio tanto vafto, quanto fappiamo. Nemmeno potè in cos̀ breve tempo far la guerra, che fece, in tanti paefi si diftanti fra loro, e procacciarfi quel numero forprendente di prigionieri, che furono facrificati in quella gran fefta. Noi perciò crediamo, che non fi può fiffare il cominciamento del fuo regno dopo l'anno 1482 ., e nemmeno può anticiparfí fenza f́convolger l'epoche degli anteceffori di lui, come appreffo vedremo. Avendo dunque comin.

Storia Antica del Meffico Tom. IV.

H ciato 
ciato a regnare nel $\mathbf{4} 482$., ed avendo finito nel $\mathrm{I}_{502}$., dob: biamo dargli diciannove anni, ed alcuni mefi, o quafi venti anni di regno.

TIZOC. Neffuno dubita, che il regno di quefto Monap. ca non foffe affai breve, e non v'è tra gli Autori, chi gli dia più di quattro anni e mezzo di vita ful trono. Noi potremo dedurre il tempo del fuo regno, e quello ancora del fuo anteceffore da quello di Nezahualpilli $R e$ d'Acolhuacan, perchè effendo ftato quefto $R e$ tanto celebre, ed avendo avuti tanti Storici nella fua Corte, abbiamo notizie certe del fuo regno. Nezahualpilli morì nel I5I6. dopo aver regnato in Acolhuacan quaranracinque anni, ed alcuni mefi: onde dee fiffarfi il cominciamento del fuo regno nel 1470 . Si fa peraltro, che P'ottavo anno di Nazahualpilli fu il primo di Tizoc: ficchè quelti dovette cominciare il fuo regne nel 1477 ., e do. vette regnare anni quattro e mezzo, come dicono parecchj Storici. Torquemada dice, avere effo regnato men di tre; ma quelt' Autore fi contraddice apertamente non meno in quefto, che in altri articoli della fua cronologì; imperciocchè adottando egii, ficcome adorta l'accennato calcolo ful regno di Nezahualpilli, e dando meno di tre anni al regno di Tizoc, dovea fiffar la morte di luî nel $\mathbf{4} 480$., e dar per confeguenza ad Ahuizzotl non anni diciotto, $\mathrm{ma}$ ventidue di régno.

AXAJACATL. Si fa, che quefto Re cominciò a regnare rei anni prima di Nezahualpilli, cioè, l' anno I464. , e che finì; fecondo ciò che abbiamo detto, nel 1477., nel qual fali ful trono il fuo fucceffore Tizoc. $D_{a}$ ciò fi deduce, che regnò anni tredici, come affermano Siguenza, ed altri Storici. Acufta non gli dà più di undici anni, nè l'Interprete della Raccolta di Mendoza più di dodici. Il più probabile è, che gli anni tredici non foffero compiuti.

MOTEZUMA I. Tutti affermano, che quefto famolo Re compl venturto anni nel trono; ma alcuni gli danno un anno di più; perchè quefti computano un anno compiuto que' mefi, che egli regnd oltre a' ventotto anni, i quali furono dagli altri trafcurati. Cominciò dunque a regnare nel 1436. , e fiǹ nel 1464 . 
1464. Nel fuo tempo fi celebrò il toxiubmolpia, o fia anno fecolare, non già nel decimolefto anno del fuo regno, come vuol Torquemada, ma nel diciottelimo, cioè, nel r 454 .

ITZCOATL. Quafi turti gli Storici danno anni tredici di regno a quefto gran Re; foltanto Acofta, e Martinez glie ne danno dodici. La cagione d'un tal divario farà ftata quella fteffa fopra accennata, cioè che non avendo Jtzcoatl compiuti gli anni tredici nel trono, Acofta, e Martinez non curarono que' mefi di più fopra gli anni dodici, e gli altri gli contarono come fe foffe ttato un anno compiuro. Egli cominciò a regnare nel I 423.: non potè cominciare nè più prefto, nè più tardi; perchè egli falì ful trono un anno, dappoichè Maxtlaton ufurpo la corona d' Acolhuacan. Maxtlaton regnò tre anni, e fiǹ infie. me col regno de' Tepanechi. L' anno feguente, cioè tre anni poichè Irzcoatl avea cominciato a regnare fu riftabilito Neza. hualcojotl nel trono d'Acolhuacan, che gli aveano ufurpato i Tepanechi. Si fa peraltro, che Nezahualcojorl regnò quarantatre anni, ed alcuni mefi, e però avendo egli finito nel i 470 , pa$r e$, che debba fiffarfi il cominciamento del fua regno nel 1426 , la rovina de' Tepanechi nel 1425 ., il principio del reguo d'Itzcoatl nel 1423. , e quello della tirannia di Maxtlaton nel I 422 . CHIMALPOPOCA. Quefto infelice Re fu confuío da Acofta, da Martinez, e da Herrera col fuo nipote Acolnahua. catl, figliuolo d'Huitzilihuitl: onde quelti Autori fanno, che Chimalpopoca faliffe ful trono di foli anni dieci, e lo fanno tofto morire per le mani de'Tepanechi; ma l'oppofto confea dalle pitture, e relazioni degl' Indiani, citate da Torquemada, e da noi in parte vedute. Siguenza incorfe per inavvedutezza in una contraddizione; poichè dice, che Chimalpopoca fu fratello minore, come in fatti era, d'Huiczilihuitl: di quefto $R e$ afferma, che cominciò a regnare d'anni diciotto, e che regnò poco meno d'undici : ficchè dovette morire non ancar giunto agli: anni ventinove d'erà, e Chimalpopoca, il quale immedia. tamente gli fuccedette, dovrebbe aver avuto al più anni ven. totto quando cominciò a regnare; contuttociò Siguenza lo $\mathrm{fa}$ falir ful trono d'anni quaranta, e più. Nella Raccolta di Men- 
doza non fi dà a quefto $\mathrm{Re}$ più di dieci anni di regno. Tor: quemada, e Siguenza glie ne danno tredici, e quefto è fenz" altro il più probabile attefo la ferie delle fue azioni, e de' fuoi avvenimenti; ma Betancurt, tenendo dietro a Torquemada, ha in quelto punto alcuni anacronifmi notabili. Egli mette l'elezione di Chimalpopoca nel rempo di Techotlalla Re d'Acolhuacan: fupponghiamo, che ciò foffe nell' ultimo anno di quefto Re : a Techotlalla fuccedette Ixtlilxochitl, il qual regno anni fette: ad Ixtlilxochitl luccedette Tezozomoc, it qual tiranneggiò quell' Imperio anni nove, ed a quefto. fuccedette Maxtlaton, nel cui tempo mori Chimalpopoca. Secondo queAi principj, adottati da Torquemada, e da Betancurt, bifogna dare a Chimalpopoca anni fedici almeno di regno, rifultanti dai fette d'Ixtlilxochit, e dai nove di Tezozomoc; il che s'oppone alla ftefla loro cronologia, ed a quella degli altri Storici. Se poi vogliamo combinare la cronologia dei $\operatorname{Re} d i$ Meffico con quella dei Re di Flazelolco giufta il calcolo dei fuddetti Autori, appena ci refteranno anni diciannove da poter compartire tra due Re Chimalpopoca, ed Itzcoatl, ficcone appreffo vedremo. Dovendo dunque contarh anni tredici nel regno di Chimalpopoca, fecondo il parere della maggior parte degli Storici, dovremo fiffare il principio del fuo regno nel 14 1 O. Maxtlaton fuccedecte a Tezozomoc fuo Padre un anno prima della morte di Chimalpopoca, cioè nel 1422. Tezozomoc ottenne anni nove la corona d'Acolhuacan : effendo dunque morto nel 1422., cominciò la fua tirannia nel 14 3 . Per ciò che riguarda Ixtlilxochitl, legitrimo Re d'Acolhuacan, fappiamo, ch' egli regnò anni fecte finchè nel 14.13 . gli fu tol: ta dal Tiranno. Tezozomoc infieme colla corona la vita: cominciò dunque a regnare nel 1406 .

HUITZILIHUITL. Sono troppo diverí i fentimenti de gli Scritcori incorno al numerod' anni, che regno quefto Monarca. Siguenza dice, che furono dieci anni, e dieci mefi. Acolta, e Marrinez glie ne danno tredici, l'Interprete della Raccolta di Men. doza ventuno. Torquemada reftifica, che tra gli Storici Mef: 稀cani, che egli vide, alcuni gli danoo anni ventidue, ed altri 
ventifei; $m_{a}$ io non dubito, che il vero numero d'anni è quello accennato dall' tnterprete della Raccolta di Mendoza; perchè fappiamo dalle pitture ftoriche dei Mefficani, che l'anno decimoterzo di quefto $R$ e fu anno fecolare, il quale, attefo ciò, che fi vede nella noftra tavola cronologica, meffa ful fine del fecondo tomo, non potè effere altro, che il 1402.: cominciò dunque a regnare nel 1389 . Effendo poi morto nel 1410., come appare da ciò che abbiamo detto intorno al regno di Chimalpopoca, dobbiamo contar nel regno d'Huitzilihuitl anni ventuno.

ACAMAPITZIN. Suppofta la cronologia dei Re prece: denti, e ftabilita l'epoca della fondazione di Meffico, poco abbiamo a fare in ciò, che riguarda quetto Re. Torquemada afferma, che le pitture e le ltorie manofcritte dei Mefficani fiffano l'elezione d'Acamap/tzin nel ventottefimo anno della fon. dazione di Meffico. Fu dunque eletro nel I352., o nel cominciare dell' anno 1353.4 ed il fuo regno farà Itato d'anni trentafette, o poco meno. L'interregno, che vi fu dopo la morte di quefto $\operatorname{Re}$ fu, al dir di Siguenza, di quattro mefi, lad́dove tutti gli altri furono appena di pochi giorni.

\section{I V.}

Sin le epoche degl' avvenimenti della conquifta.

Non è molso difficile di rintraçciar l'epoche degli avve nimenti della conquilta, perchè le troviamo per lo più accennate dal Conquiltatore Cortès nelle fue lettere a Carlo V.; ma effendovi parechi anacronifmi preffo gli Storici Spagnuoli, - perchè non confultarono quelle lettere, 0 perchè non fi curarono di fapere in quali giorni caddero le fefte mobili di que. gli anni, delle quali fr ferve talvolia Cortès, fa meftieri di fifare alcuni punci di cronologia, tralafciandone altri di minos rilievo per rifparmiar la noja ai Lettori.

L'arrivo dell' armata di Cortès alla cofta di Chalchicuecan accadde, come tutti fanao nel Giovedi Santo del I5Ig. 
Quefto fu ai 2I. Aprile, perchè la pafqua vennequell anno ai 24.

L'entrata degli Spagnuoli nella Città di Tlafcalla avvenne non già ai 23. Sertembre, come dicono Herrera, e Gomara, ma ai 18 , come affermano Bernal Diaz, Betancurt, e Solis: ciò che può dimoltrarfi facendo il calcolo giutta il ragguaglio di Cortès dei giorni, che gli Spagnuoli ftettero in Tlafcalla, ed in Cholulla, e di quelli che impiegarono nel lor viaggio fino a Meflico. Bernal Diaz dice, che prima d'entrare in Tlafcalla ftettero giorni ventiquattro nelle terre di quella. Repubblica, e poi venti in quella Cittz, ficcome confta: anche dalla lettera di Cortès. In Cholulla entrarono ai I4. Ottobre, ed in Meffico agli 8. Novembre. Sei giorni dopo fu fatto prigione il Re Motezuma, come afferma lo fteffo Cortès. Quefo Generale fi trattenne in quella Capitale fino al principio di Maggio dell'anno feguente, nel qual tempo ando a Cem-. poalla: per opporí a Narvaez. Vi diede l'affalto, ed ottenne. Ia vittoria contra quel fuo nemico nella domenica di Penteco. fte, la quile quell' anno ( 1520. ) cadde ai 27. Maggio. La. Collevazione dei Mefficani, cagionata dalla violenza. d'Alvara a do, avvenne nella gran fefta del mele Toxat, il quale co. minciò quell' anno ai I3. Maggio. Cortès ritornò alla Capitale dopo la lua vittoria ai 34. Giugno, come fanno tutti. Nel sagguaglio degli avvenimenti accaduri negli ultimi giorni di Giugno, e ne'primi di Luglio trovo della confufione, e degli anacronifmi preffo gli Storici . Io ho tenuto dietro alle- lettere di Cortès, le quali contengono il ragguaglio più autentico della conquifta.

La morte di Motezuma pare effere accaduta ${ }^{\circ}$ 30. Giugno; perchè mori, fecondo che teftifica Cortès, tre giorni dopo aver avuta la faffara: quefta l'ebbe, mentre fi coltruivano: qualle due macchine da guerra, di cui facciam menzione nella Sto. ria: le quali furono coftrutte nella notte dei 26. Giugno, e nel giorno feguente, fecondo che fi deduce dal ragguaglio di Cortès. Non può, metrerfi quelia morte nè dopo, nè prima: dei 30. Cenza fonvolgere la ferie degli avvenimenti.

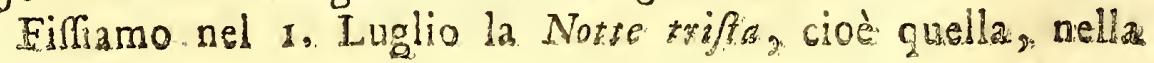


quale ufcirono fconfitti gli Spagnuoli, perchè Cortès mette fette giorni nel lor viaggio da Meffico alle terre di Tlafcalla, ed afferma, che v'entrarono agli 8. Luglio. Bernal Diaz, e Betancurt dicono, che gli Spagnuoli ufcirono di Meffico ai Io., ed entrarono ai 16 . nei dominj di quella Repubblica; $\mathrm{ma}$ in quelto fi dee maggior fede a Corrès. Gli avvenimenti acca. duti dai 24. Giugno fino al I. Luglio parranno molri per cosi poco rempo; ma non è da maravigliare, che in circoftanze di tanta Atrettezza, e di sì gran pericolo $f i$ moltiplicaffero le azioni, adoperando l'ultimo sforzo per ifcampar la vita.

La guerra fatta dagli Spagnuoli in Quauhquechollan ac'cadde nel mefe d'Ottobre, per quanto appare dal ragguaglio di Cortès. Quefta epoca c'importa per fapere il tempo, che regnò Cuitlahuatzin; poichè un Capitano Mefficano, da cui s' informò Cortès dello ftato della Corte, gli diede contezza delle diligenze adoperate allora da quel R.e contra gli Spagnuoli . Coloro, i quali vogliono, che quel $\operatorname{Re}$ non regnaffe più di qua. ranta giorni, ributtano, come falfa, quella informazione; ma ficcome non allegano veruna ragione per convincerne la falfità, dobbiamo crederla.

Intorno al giorno, nel quale fi cominciò l'áffedio di Meffico; e al rempo della fua durata, sbagliano comunemente gli Autori. Coltoro per lo più dicono, che l'affedio durò giorni novanratre; ma non fecero efartamente il loro calcolo; imperciocchè Cortès fece la rivitta delle fue truppe nella gran piaz. $z_{d}$ di Tezcuco, ed affegnò il luogo, che doveano occupare in quell'affedio le tre divifioni dell' Efercito, nel lunedi di Pentecolte dell' anno I $52 \mathrm{r}$. Ora ancorchè fupzoneffimo contra la ve. rità della Storia, che quel medefimo giorno della rivifta, fi deffe principio all' affedio, non farebbono novantatre giorni, $m a$ foltanto ottanta cinque; perchè quel luned cadde a' 20 . Maggio, e tutti fanno, che l'affedio terminò colla prefa della Ca. pitale a' 13 . Agofto. Se reputano affedio le oftilità fatre dagli Spagnuoli nelle Città del lago, doveano fiffare il cominciamen. to di tal affedio nei primi giorni di Gennajo, e numerar aon già novantatre giorni, ma ferte mefi. Cortès, il quale 
in quefto punto merita mággior fede d'ogni altro Storico, di: ce elpreffamente, che l'affedio cominciò a' 30 . Maggio, e durò fettanta cinque giorni. E' vero, che la fteffía lettera di Cortès potè cagionar queil' errore; perchè in effa $f \mathfrak{i}$ dà ad intendere, che addi I 4. Maggio erano già in Tacuba le divifroni d' Alvarado, e d'Olid, là onde cominciò l'affedio; ma c'è un manifefto errore nelle cifre; perchè egli è cerro, che que' due $\mathrm{Ca}$ pi non andarono a Tacuba prima di far la rivilta delle truppe, e fappiamo da Cortès, e dagli altri Storici, che quefta fi fece nel lunedi di Pentecolte 20. Maggio.

Torquemada dice nel lib. 4. cap. 46. che gli Spagnuoli entrarono la prima volta in Meffico agli 8. Noveonbre; ma nel cap. 14. del medefimo libro afferma, che tal entrata ac. cadde a' 22. Luglio: che vi fi trattennero 150 . giorni li 95. in amicizia co' Mefficani, e li quaranta in guerra, la quale fi cagionò dalla Atragge fattavi da Alvarado nella fefta del mefe Toxcatl, corrifpondente, fecondo che egli crede, al noftro Aprile \&c. L'ammaffo d'anacronifmi, d'errori, e di contraddi* zioni, che ha il fuddetto Autore nel citato capitolo, bafta a dare idea della fua fpropofitata cronologia. Ci perfuadiamo, che la diligenza da noi adoperata nel rifchiarare sì fatti punti, ci abbia fatti fchivare, fe non tutti, almeno molti errori.

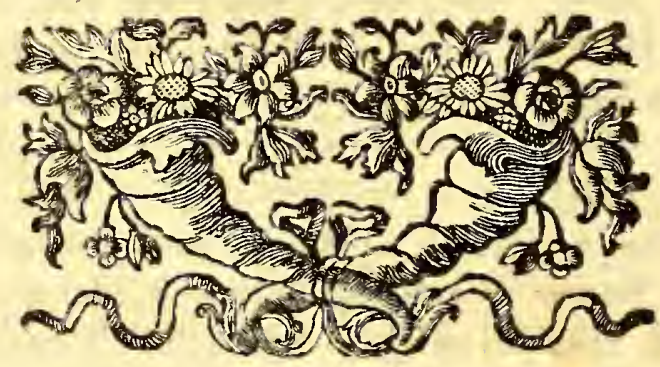




\section{DISSERTAZIONE III.}

\section{SU LA TERRA DEL MESSICO.}

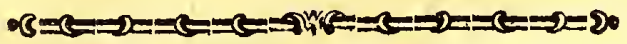

CHiunque legga l'orribile defcrizione, che fanno alcuni Europei dell' America, o fenta l'ingiurio fo difpregio, con cui fparlano della fua terra, del fuo clima, delle fue piante, de'fuoi aniınali, e de' fuoi abitatori, tofto fi perfuaderà che il furore, e la rabbia hanno armato e le loro penne, e le loro lingue, o pure che il nuovo Mondo è veramente una terra maladetta, e dal Cielo deftinata ad effere il fupplizio de'malfattori. Se diamo fede al Sig. de Buffon, l'America è un paefe affatto nuovo, appena ufcito di fotto le acque, che l'aveano allagato: $\left(^{*}\right)$ un continuo pantano nelle fue pianure: una terra incolta, e coperta di bofchi, anche dappoi che è ftata popolata dagli Europei, più induftriofi degli Americani, o ingom. brata da montagne inacceffibili, che non lafciano altro, che un piccolo fpazio di terreno per la coltivazione, e per l'abitazione degli uomini : terra infelice fotto un cielo avaro, nella quale turti gli animali trafportati dal vecchio Continente fi fon digradati, e quelli, ch'erano propri del fuo clima, fono piccoli, deformi, invalidi, e privi d'armi per la loro diféa. Se vo. gliamo dar fede al Sig. de P. ( il quale in gran parte copia i fentimenti del Sig. de Buffon, e dove non gli copia, moltiplica ed ingroffa gli errori) l'America ì fata generalmente, ed $\dot{e}$ anche oggidi un parfe troppo fterile, nel quale tutte le piante d'Europa hanno degenerato, fuorchè le aquatiche, e fugofe: il fuo terreno puzzolente porta maggior numero di piante velenofe, che tutte le altre parti del Mondo... La fua terra o in. gombrata di montagne, o coperta di bofcbi $e$ di pantani non altro da a divedere, che un immenfo e feril deferto: il fuo

Storia Antica del Meffico Tom. $1 V$. I cli-

$\left(^{*}\right)$ Hift. Natur, tom. 6. 
clima troppo contrario alla maggior parte de' quadrupedi, e fo: pratutto perniciofo agli uomini imbeftiati, indeboliti, e viziati d' una maniera forprendente in tutte le parti della loro organiz. zazione. $\left(^{*}\right)$

Il Cronichilta Herrera, quantunque peraltro tanto giudiciolo, e moderato, nondimeno quando fi mette a fare il paragone del cielo, e della terra dell' Europa con quelli dell'Ame. rica, fi moltra tanto ignorante anche de' primi elenenti della Geografia, e prorompe in tali.fpropofiti, che neppure in un fan. ciullo fi potrebbono tollerare: Il noftro Emisferio, dice, $\grave{e}$ miglior del. nuovo per rapporto al Cielo. Il noftro polo è piu abbellito di ftelle, percbè ba il fettentrione a gradi $3 \frac{1}{2}$ con molte fellé rifplendenti. Nel che fuppone $\mathrm{r}$. che l'Emisferio Auftrale è nuovo; laddove fono già tanti fecoli, ch'effo è conofciuto nell' Afia, e nell'Africa . 2. che tutta l'America appariene all' Emisferio. Aultrale, e che l'America fettentrionale non riguarda il medefimo polo, e le medelime Atelle degli Europei. Abbiamo, foggiunge, un altra preminenza, cioe che il Sole fi trattiene fette giorni piu verfo il Tropico di Cancro, che verfo quello di Capricornio: come fe l'ecceffo della dimora del Sole nell'Emisferio Boreale non foffe lo Ateffo nel nuovo, che nell' antico Continente. Pare, che il noftro buon Cronichilta fi faffe perfuafo, che il maggior amore, che porta quel Pianeta alla bella Europa, fia la cagione di trattenerfi più nell' Emisferio boreale. Penfiero galante, e degno d' un poema francefe! $E$ di qua viene, fegue il noltro Chronichilta, che la parte Artica è piis fred. da dell' Antartica; percbè $f i$ gode meno del Sole; ma come può goderfi meno del Sole nella parte Artica, trattenendofi quefto Pianeta fette giorni più nell' Emisferio boreale? La nofra terva. $\int$ fende piu da Ponente a Levante, e pertanto d piu comoda per la vita umana, che non l'altra, la quale fringerdofi da Po. nente a Levante, $f i$ slarga troppo dall' uno all'altro polo; poibe la terra, cbe fi slunga da Ponente a Levante $d$ in una piir uguale diftanza dal freddo del Settentrione, e dal caldo dell' Oftro. Ma

(*) Rech. Philo[oph. part. x. 
fe il Settentrione è la regione del freddo, e l'OAtro quella del caldo, come vuole il noftro Chronichilta, i paefi equinoziali faranno fenz' altro, giufta i fuoi principj i più comodi per la vita umana, come quelli che fono ugualmente diftanti dal Settentrione, e dall' Oltro. Nell' altro Emisferio, conchiude finalmente il noftro Autore, non v' erano Cani, neे Afini, neे Peco$r e, n \grave{e}$ Capre $\mho^{\prime} c$. Non v' erano Limoni, nè Melaranci, nd ficbi, nè Melocotogni $\sigma_{c} .\left(^{*}\right)$

Quefti, ed altri sì fatti Spropofiti di Parecchj Autori, fono effetti d'un cieco, ed ecceffivo patriotifmo, il quale ad effi fa concepire certe immaginarie preminenze del proprio lor paefe fopra tutti gli altri del Mondo. Non ci farebbe difficile l'opporre alle loro invettive contra l'America le grandi lodi, che hanno fcritto di quei paefi molti chiariffmi Europei meglio inftruiti di loro; ma oltrecchè ciò farebbe alieno dal noftro propofito, farebbe ancor nojofo a' Leggitori : onde ci contenteremo d'efaminare in quefta differtazione ciò, che coftoro fcriffero contra la terra dell' America in generale, o contra quella del Meffico in particolare.

\section{§. I.}

Su la pretefa inondazione dell' America:

Quafi tutto ciò che i Signori de Buffon, e de P. fcrivo. no contra la terra dell' America intorno alle fue piante, a' fuoi animali, ed a' fuoi abitatori s'appoggia fa la fuppofizione di una inondazion generale diverfa da quella accaduta a' tempi di Noè, e molto più recente a cagion della quale reftò gran tem. po turto quel vaftiffimo paefe fotto l'acqua. Da quelta recente inondazione nafce, per quel che dice il Sig. de Buffon, la malignita del clima dell' America, la fterilità del fuo terreno, l'im. perfezione de' fuoi animali, e la fieddezza degli Americani. La Natura non avea avuto tempo di mettere in efecuzione i fuoi I 2 dife-

(*) Herrera Dec. I. Lib. I. cap. S. 
difegni, nè di prendere tutta la fua eftenfione. Da' laghi, e da' pantani reltati da quell' inondazione ha origine, fecondoche afferma il Sig. de P. l'ecceffiva uınidità di quell' aria, e l'umidità è la cagione dell' infezion dell'ambiente, della ftraordinaria moltiplicazion degl' infetti, dell' irregolarità, e della picco. lezza de'quadrupedi, della fterilità, e del fetore del terreno, dell' infecondità delle donne, dell' abbondanza di latte nelle mamnelle degli uomini, della fupidezza degli Americani, e di mille altri fenomeni fraordinarj, che egli dal fuo gabinetto in Berlino ha offervato meglio di noi, che tanti anni fiamo fati nell' America. Quefti due Autori, febbene fieno d'accordo intorno alla fuddetta inondazione, difcordano nondimeno intorno al tempo; poichè il Sig. de P. Ia crede affai più antica, che il Sig. de Buffon.

Or quefta lor fuppofizione è mal fondata, e la pretefa inondazione del nuovo Mondo è una chimera. Il Sig. de P. fi sforza d'appoggiarla fu la teftimonianza del P. Acofta, ful numero quafi infinito di laghi, e di pantani, fu le vene di metalli pecanti, trovare quafi nella fuperficie della terra, fu' corpi marini, che fi trovano ammucchiati ne' luoghi mediterranei più baffi, fu la diftruzione de'grandi quadrupedi, e finalmente full' unanime tradizione de' Meflicani, de' Peruani, e di tutti i Selvaggi, che fono dalla terra Magallanica infino al fiume di S. Lorenzo, i quali tutti d'accordo teftificano la dimora de'loro antenati fu le montagne in tutto quel tempo, ch'erano allagate le valli.

E' pur vero, che il P. Acolta nel lib. I. cap. 25 . della fua Storia dubita, fe ciò che gli Americani dicevano del diluvio, debba intenderfi di quello di Noe, o piuttofto d'alcun altro particolare nella lor terra accaduto, ficcome quelli di Deucalione, e d'Ogige nella Grecia: e pare anche, che voglia aderire a quefra opinione, la quale dice effere ftata d'alcuni uomini pratici; ma ragionando nel lib. 5. cap. 19. delle conquifte de' primi Inchi, o fia Ingas dà a divedere, che credeva fermamente doverfa ciò intendere del Diluvio di Noè : "Il pretefto, dice, col qua. "le conquiftarono ( gl' Inchi) e fi rendettero padroni della "terra fu quello di fingere, che dopo il diluvio univerfale (de? 
7) quale aveano notizia tutti quegli Indiani) eglino aveano di nuo: ,; vo popolato il Mondo, ufcendo fette di loro dalla fpelonca "di Pacaritambo, e che per tanto tutti gli altri uomini dovea" no rendere omaggio ad eff , come a lor progenitori. "Conobbe dunque il P. Acofta, che quella tradizione degli Americani era fenz' altro del diluvio univerfale, e che le favole, colle quali s' era sfigurata, erano ftate inventate dagl' Inchi per iftabilire il loro Imperio. Che direbbe quell' Autore, fe aveffe avuto in favor di quella general tradizione que'documenti, che noi abbiamo? I Meflicani, fecondochè affermano i propri loro Storici, e noi diciamo altrove, non facearo menzione del dilu. vio fenza rammemorar parimente e la confufion delle lingue, e la difperfion delle genti, e quefte tre cole rapprefentavano in una fola pittura, come fi vede in quella, ch'ebbe il chiariffimo Siguenza dal Sig. D. Ferdinando d'Alba Ixtlilxochiti, e quefti da' fuoi nobilhfimi antenati, la cui copia abbiamo data nella noftra Storia. La medefima tradizione fi trovò preffo i Chiapanefi, i Tlafcallefi, i Michuacanefi, (a) i Cubani, e gl' Indiani di Terraferma (b) coll' efpreffione d'efferfi falvati dal diluvio alcuni uomini con alcuni animali in una barca, e d'a. ver mefto in liberta prima un uccello, il quale non tornò più alla barca, perchè fi diede a mangiar delle carogne, e poi un altro, il quale ritornò con un ramo verde nella bocca; ciò che rende manifefto, ch' eglino non parlavano d'altro diluvio, fe non di quello, che inondò tutta la terra al tempo del $\mathrm{Pa}$ triarca Noè. Tutte le circoftanze, con cui trovoffi alterata preffo alcune Nazioni Americane quefta univerfale, ed antichiffima tradizione, o fono ftate allegorie, come quelle delle fette fpelonche de'Mefficani per fignificar le fette principali $\mathrm{Na.}$ zioni, che popolarono il paefe d'Anahuac, o finzioni dell' i:

gno.

(a) Vedafi ciò che abbiamo detto nel \$.2. della I. Differtazione, come pure Herrera nella Dec. 3. lib. 3. Cap. so., nella Dec. 4. lib. 1. cap. Ir., ed in altri luoghi, Torquemada, Garcła, Boturini \&c.

(b) Della tradizione, che v'era preffo gl'Indiani di Terraferma fa menziose Herrera nella Decada 4. lib. I. cap. II. Di quella che v' era preffo. I Ile fcallefi, i Chiapanefi, ed j Cubani abbiamo noj sagionato altrove. 
gnoranza, o dell' ambizione. Niuna di quelle Nazioni credeva, che foffero fcampati gli uomini nelle montagne, ma in una barca, e fe per avventura ve ne fu qualcuna, che altrimenti credeffe, ciò fu fenz' altró, perchè la tradizione del diluvio dopo tanti fecoli era ftata alterata. E' dunque affolutamente falfo, che vi foffe una tradizione unanime d'una inondazion peculiare dell' America preffo tutti que'popoli, che abitarono dalla terra Magallanica infino al fiume di S. Lorenzo.

I Laghi ed i pantani, che pajono a' Signori de Buffon, e di $P$. tracce indubitabili della pretefa inondazione, fono indubitatamente effetti de'gran fumi, delle innumerabili fonti, e delle abbondantifrme pioggie dell' America. Se que'laghi, e pantani folfero Itati fatti da quell' antica inondazione, non già dalle cagioni da noi aflegnate, fi farebbono già dopo tanti fecoli confumati, e feccati per la continua evaporazione, che cagiona il calor del Sole, maffimamente fotto la zona torrida, 0 almeno fi farebbono confiderabilmente fcemati; ma tal diminuzione non s'offerva, fe non in que'laghi, dai quali l'indultria umana ha divertito $i$ fiumi, ed i torrenti, che vi fi fcaricavano, ficcome in quelli della valle mefficana. Io ho veduti, ed offervati $\mathrm{i}$ cinque principali laghi della Nuova Spagna, che fono quelli di Tezcuco, di Chalco, di Cuisèo, di Pàzcuaro, e di Chapalla, e lon ficuro, ch' effi non fi fon formati, nè fi confervano, fe non per le copiofe acque delle pioggie, de" fu$\mathrm{mi}$, e delle fonti. Tutto il Mondo fa, che non vi fono piog: gie più copiofe, e più dirotte, nè fiumi più grandi di quelli dell" America. Perchè dunque inventar delle inondazioni, mentre abbiama alla mano delle cagioni più naturali, e più certe? Se i laghi foffero argomento d'inondazione, dovremmo crederla piutrofto accaduta nell' antico, che non nel nuovo Continente; imperocchè tutti i laghi dell" America, anche comprefi quelli del Canadà, che fono i maggiori, non fono paragonabili co'Mari Nero, Bianco, Baltico, e Cafpio, i quali benchè volgarmente chiamati Mari, fono pure, per quel che dice il Sig. de Buffon, veri laghi, formati de' fumi, che in effi sbaccano. Se a quelti s'aggiungano i laghi Lemano, Onega, Pleskıw 


\section{I S E R T A Z I O N E III.}

e molti altri, ed affai grandi della Ruffia, della Tartaria, e d'altri paefi, $\left(^{*}\right)$ tofto ci accorgeremo, quanto s' erano dimenticati del proprio lor Continente coloro, che hanno tanto efagerato i laghi Americani. Il lago di Chapalla, che nelle carte geografiche fi vede onorato col magnifico nome di Mar Cbapallico, il quale ho veduto, e colteggiato tre volte, appena avrà cento miglia di circonferenza. Ora fe i fiumi Don, Wolga, Boriftene, Danubio, Oder, ed altri dell' antico Continente, benchè affai men groffi del Maragnone, del fiume della Plata, di quello della Maddalena, di quello di S. Lorenzo, dell' Orinoco, del Miffiffipi, e d'altri del nuovo Mondo, fon nondimeno baftevoliffimi, fecondochè dice il Sig. de Buffon, per formare que' laghi cosl grandi, che fono fati lempre mai creduti mari, qual maraviglia che i groffffmi fiumi dell' America facciano laghi minori e pantani ? Il Sig. de P. dice, che quefit lagbi Sembrano ricettacoli d'acque, che non banno ancora potuto ufcire da qué luogbi già allagati per una violenta agitazione impreffa in tutto il globo terraqueo. I moltiffimi Vulcani delle Cordigliere, o fia Alpi Americane, e delle rupi del Meffico, ed $i$ tremuoti, che inceffantemente $f i$ fentono ora in una, ora in un altra parte di quelle Alpi, danno a divedere, che quella terra non è ancora in ripofo a'noftri di. Ma fe quella violenta agitazione fu generale in tutto il globo terraqueo, perchè s' inondarono le terre del Perù, e del Meffico, effendo, come in fatti fono, e come confeffano i Signori de Buffon, e de P. fommamente elevate fopra la fuperficie del mare, e non $s$ ' inondarono quelle dell' Europa, effendo molto più baffe? Chiunque abbia offervato la ftupenda elevazione de' paefi mediterranei dell' America, non potrà mai perfuaderfi, che l'acqua poteffe elevarfi fino a coprirli fenza inondar parimente tutta l' Europa. Del refto fimilmente potremmo dire, che il Vefuvio, l'Etna, l' Hecla, ed $\mathrm{i}$ moltiffimi vulcani delle ifole Moluche, delle Filippine, $e$ del

(*) Il Sig: de Bomare numera trentotto laghi nei Cantoni degli Svizzeri, e dice che in quello d'Harlem entrano valcelli d'alto bordo. Il lagho d'Aral nella Tartaria, ha, dice il medefimo Autore, cento leghe di lunghezza, cinquanta di larghezza. 


\section{DISSERTAZIONE III.}

del Giappone, ed i frequenti tremuoti di quelle ifole, dellà China, della Perfia, della Siria, della Turchia \&c. danno a divedere, che il Mondo antico non è ancora in ripofo a' noftri dì. (c)

Le vene de' metalli, foggiunge il Sig. de P., che in alcu= ni luogbi trovanfi nella fuperfizie della terra pajono indicare, che quel fuolo $f u$ allagnto, e cbe $i$ torrenti portarono via la $f u$. perficie. Ma non farebbe meglio il dire, che alcune violente eruzioni de'fuochi fotterranei, affai manifelti ne' molti/fimi vulcani delle Cordiglieve, rovinando la fuperficie d'alcuni terreni lafciarono quafi fcoperte le vene de'metalli?

Il ritrovamento de' corpi marini, ammucchiati in alcuni Iuoghi mediterranei dell'America, fe mai provaffe quella pretefa inondazione, proverebbe piuttofto una maggiore inondazione del Mondo antico; poichè laddove in America fon pochi $i$ luoghi, ne'quali fi trovano degli ammaffi di conchighie, e d'altri corpi marini petrificati, l'Europa per l'oppofto è quafr tutta piena di petrificazioni di si fatti corpi, le quali dimo. ftrano colla maggior evidenza, che effa fu già allagara dal mare. (d) Tutti lanno le maraviglie, ed i calcoli, che hanno facto parecchj Fifici francefi di quella immenfa quantita di conchiglie, che $f i$ vede in Turenna, e neffuno ignora, che sì fatti corpi marini petrificati fi trovano anche nelle Alpi. Perchè dunque da' corpi marini ritrovati in alcuni luoghi dell' America fi dee conchiudere l'inondazione di que' paefi, e non dovrà piuttofto conchiuderf l'inondazione dell' Europa da si fatti corpi ri-

tro.

(c) Lo Aefro Sig. de P. dopo aver fatto menzione del Vefuvio, dell'Etna, dell'Hecla, e del Vulcano di Lipari, dice così:, Fra i grandi Vulcani fi , contano ii Paranucan nell' Ifola di Java, il Canapis nell' Irola di Banda, il , Balaluan nell'ifola di Sumatra. L'ifola di Ternate ha un monte fiammeg, giante, le cui eruzioni non cedono a quelle dell'Etna.... Di tutte le ifo, le, ed ifolette, che compongono l'Imperio del Giappone, neppure una ve " n'è, che non abbia il fuo vulcano o più, o meno confiderabile, ficcome ", arcora nelle ifole Manile (vuol dir Filippine) nelle Azori relle ifole di Ca", po verde ec., Recherch. Philofoph. fur les Americains. Letre 11I. fur les vicifitudes du nótre globe.

(d) Il Sig. de Bourguet nel fuo Trattato delle Fetrificazioni, e il P. Torrue bia nel fuo Apparato alla Storia Natural di Spagna ci danno un lunghiflimo Catalogo dei luoghi d'Europa, e d'Afia dove fi trovano dei corpi marini petrificati. 
trovatifi in affai maggiore abbondanza in moltiffmi luoghi d'efo fa? Se 'I trafporto di quelti corpi a' luoghi mediterranei dell' Europa fi afcrive all' acque del diluvio univerfale, perchè non dovranfi afcrivere alla medefima cagione nell' America? $\left(^{*}\right)$ Per l'oppofto fe non furono già le acque del diluvio quelle, che portarono i fuddetti corpi marini ne' luoghi mediterranei dell' Europa, ma qualle di un' altra inondazione pofteriore: fe l' $E$ u. ropa in generale $\dot{e}$, per quel che dice il Sig. de Buffon, (e) un pnefe nuovo: fe non d ancora gran tempo cb'effa era coperta di bofcbi, e di pantani, perchè nell' Europa non fi vedono, nè fi vedevano due mila anni fa quegli ftupendi effetti dell' inondazione, che vedono quefti Autori nell'America? Perchè gli animali dell' Europa non fi fon digradati, come quelli dell' America? Perchè gli Europei non fono freddi come gli Americani? Perchè le donne dell' una, e dell' altra parte del Mondo non fono prefentemente, o almeno non fono già ftate ugualmente infeconde? 'Perchè effendo ttata l' Europa allagata, come l'America, e più quella, e per più lungo tempo (ficcome evidentemente fi deduce dalle ragioni del Sig. de Buffon) il terreno dell' Europa reftò fecondo, e quelio dell' America fterile: il Cielo dell' Europa è sì benigno, e quello dell' America sì avaro: all' Europa fi concedettero tutti i beni, ed all' America fi mandarono tutti $i$ mali? Chi voleffe meglio informarfi di Storia Antica del Mefjico Tomo IV.

$\mathrm{K}$

que.

$\left(^{*}\right)$ Uno dei più alti monti dell' America s'è il Defcabezado, cioè Decapitato, fruato nelle Alpi Chilefi lontano dal mare più di cencinquanta miglia. La fua altezza perpendicolare fu la fuperficie del Mare è, al dir del Sig. Ab. Molina, erudito, e diligente Storiografo di quel regno, di più di tre miglia: Or nella cima di quefto monte cosi alto s'è trovata una gran quantita di corpi marini petrificati, i quali certamente non poteano portarfi fino a quella fupenda altezza dall' acqua di una inondazione particolare, e diverfa da quella generale accaduta ai tempi di Noè. Nemmeno può dirft, che quella cim effendo ftata già letto'del mare $s$ andò poi a poco a poco innálzando dai fuochi fotterranei, feco parimente elevando quei corpi marini ; perchè quantunque cio non fia inverifimile in alcuni luoghi, che ora veggiamo non troppo elevati fu la fuperficie del mare, anzi noi lo crediamo fovente accaduto fecondo che n' abbiamo detto altrove; con tutto ciò in una si ftraordinaria altezza ̀̀ affatto incredibile : ficchè quei corpi marini in quella cima debbono confiderarfi, come certe e indubitabili tracee del diluvio univerfale.

(e) Tom. 1. Theorie de la Terre. 
quefte difficoltà, legga ciò che fcriffe il Sig. de Buffon intorno all' inondazion dell' Europa .

L'ultimo argomento del Sig. de P. è prefo dall' eftinzione, - finimento de' gran quadrupedi nell' America, i quali dice, fono i primi a perire nelle acque. Quefto Aurore crede, che anticamente eranvi nell' America Elefanti, Cammelli, Ippopotami, ed altri grandi quadrupedi, e che tutti perirono nella fuppolta inondazione. Ma chi non fi maraviglierà, che vi periffero gli Elefanti, ed i Cammelli effendo tanto veloci, e cam. paffe la Pigrizia, o fia Pigro, effendo tanto lento, e tanto inabile al moto? Che non poteffero ricoverarfi ne' monti gli Elefanti, come fi ricoverarono gli uomini, ufcendo a nuoto, nel che fono deftriffimi, o prevalendofi della velocità de' lor piedi, la quale è sì grande, che in un giorno fanno, fecondochè afferma il Sig. de Buffon, fino a cencinquanta miglia, ed aveffero agio di falire fu le cime de' monti i Pigri, i quali appena poffono, per quel che dice il fuddetto Autore, fare una pertica in un ora? Ancorchè concedeffimo, che sì fatti quadrupedi fieno ftati già nell' America, non però framo obbligati a credere, che la loro diftruzione fia Itata dalla fuppofta inondazione cagionata: poichè poteva effa afcriverfi ad altre cagioni affai diverfe. Lo fteffo Sig. de P. $(f)$ afferma, che fe fi trafportaffero in America gli Elefanti, ficcome l' hanno tentato i Portoghefi, correrebbono la medefima fortuna de' Cammelli nel Perì, che non $f_{i}$ propagberebbono, quantunque fi lafciaffero ne' bo. fcbi al proprio loro inftinto; percbè la mutazione d'alimento, e di clima d infinitamente piu fenfibile agli Elefanti, che a tuto si gli altri quadrupedi della prima grandezza. Egli ancora protefta altrove, che le cagioni difruttive di quefti animali, cioè de' gran quadrupedi nel nuovo Mondo, fono delle difficolia asfai grandi, ed infieme degli articoli piì intereffanti della fio fira del globo terraqueo. Perchè dunque decide sì arditamente che quella immaginaria inondazione fu la cagione della lor rovina?

(f) Recherch. Philofoph. part. I. 
Il Sig. de Buffua fi sforza di perfuaderci la recente inon. dazione dell' America con parecchj argomenti, a' qua!i rifponderemo in poch a parole. Se que/to Continente, dice parlando deli' America, è cosi antico, come l'altro, perckè vi fa trovarono si pocbi nomini? Gli uomini, che vi fi trovarono non poffono dirfi pochi, re non rifpetto al vaftiftimo psefe, che abita. vano. Quelli, che vivevano in focieta, ficcome $i$ Meffizani, $i$ Michuacanefi, gli Acolhui, ed altrn, che oicupavano tutto quel grandiffimo tratto di terra, che fi itende da'gr. 9. fin'a 23. di latitudine, e da' 271. fin'a 294. di longitudine, formavano de' popoli tanto numerofi, quanto quelli dell' Europa, come faremn vedere in un' altra $D$ fferrazione. $(g)$ Quelli, che vivevano difperfi formavano piccole Nazioni, o Tribù; perchè la poca moltiplicazione è un effetto neceffario della vita felvaggia in tutti i paefi del Mondo. "Se i Selvaggi fono Pa"Itori, dice il Montefquieu, hanno d'uopo d'un gran paefe " per poter fuffiftere in un certo nuinero. Se fono cacciatori "( come erano i felvaggi dell' Amsrica) fono ancora in un mi"nor numero, e compongono per mantenerfi una Nazione plù "piccola. "

Percbe", torna a domandare il Sig. de Buffon, percbè ercano quafi tutti felvaggi, e difperfi? Non è così. Come può dirfi, che foffero quafi turti felvaggi, e difperfi; mentre fapo piamo, che i Mefficani, i Peruani, e tutti i popoli a loro fortopolti viveano in focietà? I quali, ficcome confeffa il medefimo Sig. de Buffon, erano a $\iint_{a i}$ numerof $f_{i}$ e non poffono dir. fi nuovi. L'altre Nazioni is mantennero felvagge per troppo amore deila lor libertà, o per altra cagione, che ignoriamo . Nell'Afia, contuttochè fia un paefe antichifimo, vi fono an$\mathrm{x}_{2}$ che

(g) Quefti argomenti del Sig. de Buffon contra l'antichità dell' America fi trovano vel tomo 6. della fua Storia Naturale; ma poco innanzi nello fteffotomodice cosi: Trovaronfi nel Meffico, e nel Perì uomini dirozzati, e popoli colti fottomel/s a leggi, e governati da $R e$ : aveano dell' induftria, delle arti, ed una fpezie di Religione, atitavano in Città, 'nelle quali mantenevaji l'ordine, e il gowerno meriè l' autorità del Sovrano. Quefi popoli per altro afjai numeros, non poffono dirsz nuovi . ec. Se v'è qualcuno che Mubiti di tal contraddizione, leg。 ga il fuddetto tomo del Sig. de Buffon. 
che oggi de' popoli felvaggi, e difperfi. Percbe, dice, coloro; che erano uniti in focieta contavano appena dugento, o trecento anni, dappoicbè fi congregarono? Ecco un altro errore. 1 Mefficani contavano appena dugento anni dalla fondazione della lor Capitale, i Tlafcallefi qualche cốa di più dallo Atabilimento della lor Repubblica; ma tanto quelte Nazioni, e le altre a loro fotiopolte, quanto i Toltechi, gli Acolhui, ed i Michuacanefi viveano in focietà da tempo immemorabile. Nè il Sig. de Buffon, nè il Sig. de P., nè il Dott. Robertfon, nè parecchj altri Autori Europei fanno diftinguere lo fabilimento di quelle Nazioni in Anahuac, da quello, che molti fecoli prima aveano avuto ne' paefi fettentrionali del nuovo Mondo.

Perchè, torna a dire, anche quelle Nazioni, che viveano in focieta, ignoravano l'arte di tramandare alla pofterita la memoriat de'fatii per mezzo di legni durevoli, pofocbe aveano trovato da maniera di commicarfi da lontano, $e$ di friverfi annodando de cordoni? E che erano le pitture, ed i caratteri de' Mefficani, delle altre Nazioni dirozzate d'Anahuac, fe non fegni durevoli deftinati, come i nottri caratteri a perpetuare la memcria de fatri? Vedafi cid, che dice Acotta nel lib. 6. cap. 7. della fux Storia, e cid che noi produciamo nella Differtazione fulla col. tura de' Mefficani.

Perrbt, foggiunge, non aveano addimefticati gli animali, nè d' alcun altro fervivanfi, fuorrbè del Lamt, (b) e del Paco, i quali non erano come $i$ nofri animalt dimeftici, fabili, fedels, $e$ docili? Perchè non v'erano altri animali da poterfi addimenti. care. Vuole il Sig. de Buffun, che addimefticaffero le Tigit, le Pume, i Lupi, ed altre si fatte fiere? Il Sig. de P. rinfaccia agli Americani la poca loro indufria nel non efferfi ferviti dé Renni, come fanno i Lapponefi; ma quefti quadrupedi non fi rrovavano, fe non ne' paefi troppo lontani dal Meffico, e quea"

fel.

(h). Llama (non Lama) era, per quello che dice if P. Acofta it nome generico delle quattro fpezie di quadrupedi di quel genere; ma oggidi s'adopera per fignificar foltanto quello, che dagli Spagnuoli appellaf Caxnera, cioe Montone, del Perì. I e altse tre fpezie fono il Paco, il Guanaco, o Huannaco, e la Vicugnos. Il nome Llama fi pronunzia come in Italiano Gliama. 
felvaggi, nelle cui terre fi trovavano quelti animali non volle: ro fervirfene; perchè non ne aveano bifogno, o non venne lo. to in mente di addimefticarli. Oltrechè la propofizione del Sig. de Buffor prefa in quella generalità è fenz' altro falfa ; poichè egli medefimo dice, che l'Alco, o fia Tecbicbi, quadrupede ad un cagnuolo fomigliante, e comune ad ambedue le Americhe, era dagl' Indiani addimefticato. Similmente aveano i Mefficani fatti dimeftici i Conigli, l' Anitre, i Gallinaccj, ed altri animali.

Finalmente le loro arti, conchiude il Sig. de Buffon, eras no tanto nuove, quanto la lor focieta, il lor talento imperfettos le loro idee non ancora fuiluppate, $i$ lor organi rozzi, $e$ barbara la loro lingua; ma gli errori contenuti in quefte parole del Sig. de Buffon faranno efficacemente ributtati nelle Differtazioni feguenci .

Dobbiamo dunque ributtare quella pretefa inondazion dell. America, come una delle chimere filofofiche inventate dagl' in. quieti ingegni del noftro fecolo; poichè preffo gli Americani non v'era memoria d'altra inondazione, fe non di qualla univerfale, di cui fanno menzione i libri fanti. Anzi dico, che fe mai foffe vero, che il diluvio di Noè non allagò tutta la terra, niun altro paefe avrebbe rnaggior ragione di quello del Meffico a crederfi fottratro a quella gran calamità; perchè oltre alla fomma elevazione d'effo fu la fuperficie del Mare, non v'è paefe mediterraneo, dove fieno più rari i corpi mario: ni perrificati.

\section{I I. \\ Sul clima del Meffico.}

Se volefimo prenderci l'impegno di ribattere tutti gli fpro. pofiti, che il Sig. de P. fcriffe contro il clima dell' America, farebbe d'uopo di forivere in cambio d'una difertazione un gran volume. Batta dire, ch' egli ha raccolto cutto ciò, che da parecchj Autori è ftato detto a dritto, o a torto contra diver- 


\section{D I S S E R T A Z I O N E IIT.}

fi paefi particolari dell' America, per rapprefentare a' fuoi Leg: gitori un compleffo moftruofo ed orribile fenzd accorgerfi, che fe noi, camminando fu le tracce di lui, intraprendeffim s a $f_{d}$ lo fteffo de' diverfi paefi, di cui fi compone $l^{\prime}$ antico Continente ( cid che non farebbe difficile) faremmo un ritratto molto più abbominevole del fuo; ma ciò tralafcianto, come alieno dal noftro propofito, ci contenteremo di ragiosare intorno al clima del Meffico.

Quefto paefe, effendo tanto vafto, e divifo in tante Provincie affai diverfe per la loro fituazione, debbe neceffariamen. re foggiacere a differenti climi. Alcune terre, coms le marittirne, fono calde, e per lo più umide, e malfane; altre fono, come quafi tutte le mediterranee, temperate, fecche, e fane. Quefte fono troppo alte, e quelle troppo baffe. In alcune regaa il vento Mezzogiorno, in altre il Levante, ed in altre la Tramontana. Il maggior freddo di tutti i luoghi abitati non arriva a quello di Francia, nè anche a quello di Caftiglia, nè il maggior caldo può paragonarfí con quello dell' Africa, nè con quello de' canicolari in alcuni paefi dell' Europa. Il divario fra il Verno, e la State è si poco dapertutto, che anche le perfone più dilicate portano lo fteffo abito in Agofto, e in Gen: Dajo. Tutto quefto, e quel di più, che abbiamo gia detto nella Storia intorno alla benignità, ed alla dolcezza di quel clima c̀ tanto notorio, che non abbiamo d'uopo di teftimonianze, nè d'altri argomenti per convincerlo.

Il Sig. de P. per dimoftrare la malignità del clima Ame. ricano allega 1. La piccolezza, e l' irregolarità degli animali dell' America. 2. La grandezza, e l'enorme moltiplicazione degl' Inferti, e d'altri si fatti animaluzzi. 3. Le malattie degli Americani, e particolarmente il mal venereo. 4. I difetti della loro coltituzione fifica. 5. L' ecceffo di freddo ne' paefi dell' America rapporto a quelli dell' antico Continente fituati in $u$ gual diftanza dall' equinoziale.

Or la fuppofta piccolezza, e la minor ferocia degli animali Americani, di cui altrove ragioneremo, anzichè la malignità del clima, dimoltrano la dolcezza, e la bontà d'effo, fe 
diamo fede al Sig. de Buffon, alla cui fonte bevvè il Sig. de P., e della cui teltimonianza fi è prevaluto contro Don Pernetty. Il Sig. de Buffon, il quale in molti luoghi della fua Storia Naturale produce la piccolezza degli animali Americani, come un argomento certo della malignità del clima dell' America, ragionando poi delle beftie falvatiche nel tomo XI., dice cosi: "Siccome tutte le cole, anche le creature più libe"re, fono foggette alle leggi fifiche, e gli animali al paride" gli uomini foggiacciono all' influenza del Cielo, e della Ter"ra : pare, che quelle medefime cagioni le quali hanno civi" lizzata, e raddolcita la fpezie umana ne' noltri climi, abbia" no parimente prodotto fimili effetti nalle altre fpezie. Il i) Lupo, il qual è forfe il più feroce di tutti i quadrupedi " della zona temperata, è per altro incomparabilmente meno ") terribile della Tigre, del Leone, e della Pantera della zona "torrida, e dell' Orlo bianco, del Lupo cerviere, e della Je"na della zona fredda ... Nell' America, dove l'aria e la 2 terra fono più miti di quelle dell' Africa, la Tigre, il "Lione, e la Pantera non fon rerribili, fe non nel nome... ") Effi hanno degenerato, fe pur la ferocia aggiunta alla cru3) delta faceva la lor natura, o per dir meglio non altro hanno fatto, che foffrir l'influenza del clima: fotto un cielo più dolce, il lor naturale s' è raddolcito ... Da' climi ecceffivi fi prendono le droghe, i profumi, i veleni, e tutte quelle pian. te, le cui qualità fono ecceffive. La terra temperata per l'oppolto non produce, fe non cofe temperate: l' erbe più dolci, i legumi più fani, i frutti più foavi, gli animali più tranquilli, e gli uomini più umani fono proprj di quefto clima felice. Cosi la rerra $f_{a}$ le piante; la terra, e le piante fanno gli animali; la terra, le piante, e gli animali fanno l'uomo... Le qualita fifiche dell' uomo, e quelle degli animali, che d'altri animali fi cibano, dipendono benchè più rimotamente, da quelle medefime cagioni, le quali hanno in. fluenza anche ful loro naturale, e fu' loro coltumi. La mag" gior prova per dimoftrare, che ne' climi temperati tut to fi "tempera, e né climi ecceffivi tutto è ecceffivo, e che la "gran: 


\section{DISSERTAZTONE IIr.}

is grandezza, e la forma, le quali fembrano qualità fiffe e de: ") terminate, dipendono ciò non oftante, ficcorne le qualità re") lative, dall' influenza del clima: la grandezza de' noltri qua"drupedi non può compararfi con quella dell' Elefante, del Ri9 noceronte, e dell' Ippopotamn: i più grandi de' noftri uccelli , fono affai piccoli, fe fi paragonano collo Struzzo, col Còn"dore, e col Cafoare. "Fin quì il Sig. de Buffon, il cui tefto ho copiato; perchè è affai importante al mio propofito, ed affatto contrario a ciò che fcrive il Sig. de P. contro il clima dell' America, e lo fteffo Sig. de Buffon in molti altri luoghi .

Ora poi fe gli animali grandi, e feroci fon proprj de' climi ecceffrvi, e gli animali più piccoli, e più tranquilli de' climi temperati, ficcome in quefto luogo ftabilifce il Sig. de Buffon: fe la dolcezza del clima influifce nel naturale, e ne' coftumi degli animali, mal deduce il Sig. de P. la malignità del clima dell' America dalla minor grandezza, e dalla minor ferocità de'fuoi animali; anzi dedur dovrebbe da quefto antecedente la piacevolezza di quel clima. Se all' oppofto la minor grandezza, e la minor ferocità degli animali Americani, rapporto a quelli dell' antico Continente, fono prova della lor degenerazione, per la malignità del clima, ficcome vuole il Sig. de $P$. dovremo fimilmente arguire la malignità del clima dell' Europa dalla minor grandezza, e dalla minor ferocità de' luoi animali, paragonati con quelli dell' Africa. Se qualche Filofofo della Guinea intraprendeffe un' opera ful modello di quella del Sig. de P. con quefto titolo, Recberches philofopbiques fur les Européens, potrebbe prevalerfi del medefimo argomento del Sig. de P. per dimoftrare la malignità del clima dell' Europa, ed i vantaggi di quello dell' Africa. "Il clima dell' Europa, di" rebbe colle fteffe parole del Sig. de P. \& troppo contrario alle "generazione de quadrupedi, che vi $f i$ trovano incomparabil"menie minori, e piir codardi de' noftri. Che fono il Cavallo, " ed il Bue, i più grandi de' fuoi animali, comparati co' no, "Atri Elefanti, co' noftri Rinoceronti, co' noftri Ippopotami, " co' noftri Cammelli, e colle noftre Giraffe? Che fono i fuoi ") lu: 
¡) lucertoni o fia nella grandezza, o nell' intrepidezza paragonati " co' noftri Coccodrilli? I Lupi, e gli Orî, le più temute del") le fue fiere a lato de' nottri Leoni, e delle noftre Tigri fem"brano cagnuoli, Le fue Aquile, i fuoi Avoltoi, e le fue " Gru, fe fi paragonano co' noftri Struzzi, parranno altrettan"te galline." Tralafcio altre belle cofe, che dir potrebbe contra l'Europa, fervendofi degli fteffi materiali, ed anche delle freffe parole del Sig. de P. per non render nojofa quefta differtazione. Ciò poi, che i Signori de Buffon e de P. rifponderebbono a quel Filofofo Africano, rifpondiano noi a quefti Filofofi Europei; poichè i loro argomenti o non provano, che è cattivo il clima dell' America, o pur convincono, ch'è cattivo quello dell' Europa, o almeno che è migliore il clima Africano dell' Europeo.

Dalla fcarfezza, e piccolezza de' quadrupedi paffa il Sig. de P. all' enorme grandezza, e prodigiofa moltiplicazione degl' infetti, e d'altri animaluzzi nocevoli. "La fuperficie della ter" ra, dice, infetta dalla putrefazione era inondata di lucertole, " di ferpi, di rettili, e d'infetti moftruofi per la loro grandez. " $z a$, e per l'attività del loro veleno, che tiravano da' fughi " abbondanti di quefto fuolo incolto, viziato, ed a fe fteffo " abbandonato, nel quale il fugo nutritivo diveniva agro, co" me il latte nel feno degli animali, che non efercitano la "virtù propagativa. L' eruche, le piattole, le farfalle, gli fca. " rafaggi, i ragni, le rane, ed i rofpi erano per lo più d'una " corpordtura gigantefca nelle lor fpezie, e s'erano moltiplicd" ti più dı quello, che può immaginarfi... Panamà è infefta"ta da ferpi, Cartagena da nubi di enormi pipittrelli, Portobello da rofpi, Surinan da kakerlachi, o fia cucaraccie, "la Guadalupe, ed altre colonie delle ifole da fcarafaggi, Qui"to da picbi, o fia nigue, e Lima da pidocchj, e da cimicj. 2) Gli antichi $\operatorname{Re}$ di Meffico, e gl'Imperatori del Perù non " trovarono altra maniera di liberare i loro fudditi da quefti infetti, che gli mangiavano, fe non quella d'impor loro il "tributo d'una certa quantità di pidocchj, che doveano pa"gare ogni anno. Ferdinando Cortès trovò de' facchi pieni Storia Antica del Meffico Tom. IV: I $\quad$ "d'effs 


\section{DISSERTAZIONE IIT.}

3) d'effi nel palagio del Re Motezuma., Ma quefto argomen: to, pieno peraltro di falfità, e d' efagerazioni, nulla prova contro il clima dell' America in generale, e molto meno contra quello del Meffico. L'effervi alcune terre nell' America, nelle quali, perchè fono calde, ed umide, o difabitate, trovanfi degl'infetti grandi, e che fi mottiplicano ecceffivamente al più proverà, che in alcuni luoghi d'effa la fuperficie della terra è infetta, com' egli dice di putrefazione; ma non che il terreno del Meffico, o quello di tutta l'America fia puzzolente, in. colto, viziato, od abbandonato a $\int e$ fe $\iint_{0}$, ficcome fioccamente pretende il Sig. de P. Se tal confeguenza foffe buona, diremmo ancora, che il fuolo dell' antico Continente è parimente guafto e puzzolente; poichè in molti paefi d'effo $v$ 'è una prodigiofa moltitudine d'infetti moftruofi, di rettili nocevoli, $e$ d'animaluzzi vili, come nelle ifole Filippine, in molte di quelle dell' Arcipelago Indiano, in parecchj paefi dell' Afia meridionale, in molti dell' Africa, ed anche in alcuni dell' Europa. Le ifole Filippine fi veggono infeftare da certe enormi formiche, e da moftruofi pipiftrelli, il Giappone da fcorpioni, l' Afia meridionale, e l'Africa da ferpi, l'Egitto da afpidi, la Guinea, e l'Etiopia da eferciti di formiche, l'Ollanda da forci campagnuoli, l' Ukrania da rofpi, ficcome afferma lo fteffo Sig. de P. (i) Nell' Iralia la Campagna di Roma ( dopo tanti ́́ecoli, dacchè è popolata ) da vipere, la Calabria da tarantole, le cofte del Mare Adriatico da nubi di zanzare, ed anche nella medefima Francia, la cui popolazione è tanto grande, e tanto antica, le cui terre fono sì ben coltivate, ed il cui clima è tanto celebrato da' Francefi, comparve pochi anni fa, fecondochè teftifica il Sig. de Buffon, una nuova fpezie di topi campagnuoli più grandi de' comuni da lui appellati Surmulots, i quali fi fono ecceifivamente moltiplicati con gran danno de' campi. Il Sig. de Bazin, nel compendio della Storia degl' Infetti, numera fettanta fette fpezie di cimici, le quali tutte fi trovano in Parigi, e ne' contorni. Quella gran Corte, fecondo.

(i) Defenfe des Recherches Philofoph. fur les Americains, chap. 13. 
dochè dice il Signor de Bomare, formica di sì fchifofi infet: ¿i. E' vero, che vi fono de' luoghi nell' America, ne' quali la moltitudine d'infetti, e di beftiole lorde fanoo nojofa la vita; ma non fappiamo, che fia arrivata ad un tal ecceffo la loro moltiplicazione, che abbiano fpopolato qualche luogo: almeno non potranno produrfi tanti efempi, di sì fatta fpopolazione nel nuovo, quanti nell'antico Continente, di cui fanno fede Teofralto, Varrone, Plinio, $(k)$ ed altri Autori. Le rane fpopolarono un lungo nelle Gallie, ed un altro nell' Africa le lo. cuite: l'ifola Graro, una delle Cicladi, reftò fpopolata da' topi : Amiclas preffo al luogo di Terracina dalle ferpi : un altro luogo vicino all' Etiopia dagli fcorpioni, e dalle formiche velenofe, ed un altro dalle fcolopendre, e più vicino a' noftri tempi, l'ifola Maurizio fu per effere abbandonata da' fuoi abitatori a cagione della Atraordinaria moltiplicazione de' forci, per quanto $\mathrm{mi}$ ricordo d' aver letto in un Autore francefe.

Per cid che riguarda la grandezza degl' infetti, de' rettili, e di sì fatti animali, il Sig. de P. fi prevale della teltimonianza del Sig. Dumont, il quale nelle fue Memorie fu la Luigiana dice, che vi fono delle rane si grandi, che pefano trentafette libbre francefi, il cui orrendo clamore contraffa il muggito deile Vacche. Ma chi potrà fijarfi di quell' Autore, maffimamente fapendo ciò che dice lo fteffo Sig. de P. ( nella fua rifpolta a Don Pernety cap. 17. ) che tutti quelli, che banno fcritto intorno alla Luigiana da Kenepin, le Clerc, ed il Cavaliere Tonti infino a Dumont, fi fon contraddetti gli uni agli altri or Sopra quefto, or Sopra quell articolo? Io peraltro mi ma. raviglin, che il Sig. de P. abbia avuto l' ardire di fcrivere, che non efiftono si fatti molti nel, refto del Mondo. So benifimo, che non efiftono nell' antico Continente, come nè anche nel nuovo, rane di trentafette libbre; $\mathrm{m}_{3}$ efitono pure nell' Afia, e nell' Africa delle ferpi, de' pipiltrelli, delle formiche, ed altri fimili animali di sì Pupenda grandezza, che fuperano d'affai tutti quelli, che fi fono fcoperti nel nuovo Mondo. In L 2 qual

(飞) Plin. Hift. Natur. Lb. 8. cap. 29. 


\section{4 "DISSERTAZION E III.}

qual luogo dell' America s'è veduta mai una ferpe di cinquan: ta cubiti romani, come quella che moftrò al Popolo romano Augufto negli f́pettacoli, fecondochè afferma Svetonio, (l) a cosi groffa, come quella che fu ammazzata nel Vaticano al tempo dell' Imperator Claudio della quale teftifica Plinio, Autore quafi coetaneo, che nel ventre le fu trovato un fanciullo intero? Ma fopratutto dove mai s'è veduta anche ne' bofchi più folitarj dell'America una ferpe, che in verun modo paragonarfi poffa con quella enormiffima e prodigiofa di centoventi piedi, veduta nell' Africa al tempo della prima guerra $\mathrm{Pu}$ nica, ed ammazzata con macchine da guerra dall' efercito d'At. tilio Regolo, la cui pelle, e le cui mafcelle fi confervarono in un tempio di Roma fino alla guerra di Numanzia, ficcome teftificano Livio, Plinio, ed altri Storici romani? So bene, che qualche Storico dell' America dice, che in alcuni bofchi fi trova certa fpezie gigantefca di ferpi, le quali col loro fiato attraggono gli uomini, e gl'inghiottifcono; ma fo ancora, che lo feffo raccontano parecchj Storici tanto antichi, quanto moderni, delle ferpi dell' Afia, e qualche cofa davvantaggio. Megaftene, da Plinio citato, diffe, che nell'Afia fi trovavano del le ferpi cosi grandi, che inghiottivano cervi, e tori interi. $(m)$ Metrodoro, cirato dallo fteffo Autore afferma, che nel. Ponto v' erano delle ferpi, che col fiato attraevano gli uccelli, quan. tunque alto foffe, e veloce il lor volo. Tra i moderni il Ge. melli nel tom. 5. del fuo Giro del Mondo, dove ragiona degli animali delle ifole Filippine dice così "Vi fono ferpenti $"$ in quefte ifole di fmifurata grandezza. Ve $\mathbf{n}$ ' ha uno detro "Ibitin molto lungo, che appefofi per la coda ad un tronco d'ál.

(l) In Octaviano Crare.

(m) Megafbenes Jcribit, in India Jerpentes in tantam magnitudinem adolefcere, ut folidos bauriant cervos taurofque. Metrodorus, circa Rbvndacum amners in Ponio, ut fupervolantes quamvis alte, perniciterque alites baufu, raptas abforbeant. Nota eft in Punicis bellis ad Aumen Bagradam a Regulo Imper. balifis tormentifque, ut oppidum aliquod, expugnata ferpens CXX. pedrum longitudinis. Pellis ejus maxillaque ufque ad bellum Numantinum duravere Roma in templo. Faciunt bis fidem in Italia appellata boe in tantam amplitudinem exeuntes, ut Divo Claudio Principe occifa in Vaticano-Solidus in alvo fpectatus fot infans. Plin. Hirt. Nat. lib. 8. cap. 14. 
"d'albero, attende, che paffino cervi, cinghiali, ed eziandio " uomini, per trargli a fe col fiato violentamente, e divorar"gli belli, ed interi, \&c. "Onde fi vede che quefta antichiffima favola è ftata comune all'uno, ed all'altro Continen: te. $(n)$

Il Sig. de P. vorrà forfe sbrigarfi col dire, che quei mo: ftruofi animali fi vedevano già nell' antico. Continente, allorchè il fuo clima non s' era ancor perfezionato. Ma chi farà, che paragonando ciò che fcriffero gli antichi con ciò che prefentemente fappiamo dell' Afia, e dell' Africa, non vegga, che il clima di que' paefi è per lo più al prefente tale, qual' era due mila anni fa: lo fteffo caldo, la fteffa ficcità, o umidità, la fteffa fatta di piante, d'animali, e d'uomini \&c. Oltrechè anche a'noftri tempi fi vedono in quelle regioni varie forti di motruofi animali, che fuperano affai i loro analoghi del nuovo Continente. In qual paefe dell' America potrà trovare il Sig. de P. delle formiche che poffano paragonarí con quelle, che nelle ifole Filippine fono appellate Sulum, delle quali afferma il Dott. Hernandez, (o) che hanno foi dita di lunghezza, ed uno di larghezza?' Chi ha veduto mai nell' America de' pipiltrelli così groffi, come quelli delle ifole di Borbon, di Ternate, delle Filippine, e di tutto l'Arcipelago Indiano? Il più grande pipiftrello dell' America ( proprio di certe terre calde, ed ombrofe) ch'è quello il quale da! Sig. de Buf. fon è appellato Vampiro, è̀ fecondochè dice lo fteffo Autore, della grandezza d' un piccione: la Rougette ( una delle fpezie dell'Afia ) è grande quanto un corvo, e la Rouffette (un'altra (pezie dell' Afia ) è groffa quanto una gallina grande. $(p)$ Le fue ali diftele hanno dall' una punta all'altra tre piedi di Parigi, e fecondo il Gemelli, che la mifurò $(q)$ nelle ifole Filippine, fei palmi. Il Sig. de Buffon confeffa l'ecceffo nella

gran-

(n) Vedafi ciò che rapporta il Sig. di Bomare della Minia dell' Africa, della Rimberab di Ceilan.

(o) Hern. Hift. Infector. N. Hifp: cap. 30.

(p) Buffon Stor. Nat. tom: 29.

(q) Gemelii tom. s. 
grandezza de' pipiftrelli afiatici rapporto agli Americani; ma lo nega nel numero. Gemelli teftimonio oculato dice, che quelli dell' ifola di Luzon erano tanti, che coprivano l'aria, e che il rumore, il qual faceano co'lor denti nel mangiar le frutta de' bolchi, fi Centiva in diftanza di due miglia. (r) Lo fteffo Sig. de $P$. dice ragionando delle ferpi, $(f)$ non potérs affermare, che nel nuovo Mondo fienfi trovate Serpi piu grandi di quelle, che vide il Sig. Adanfon ne' deferti dell'Africa. La maggior ferpe trovata nel Meflico dopo le più diligenti ricerche fatte dal Dotr. Hernandez, era diciotto piedi lunga; ma quefta noń è da paragonarfi nè con quella delle Moluche, della quale dice il Sig. de Bomare, che ha trentadue piedi di lunghezza, $(t)$ nè coli' Anacandaia di Ceilan, che ha per quel che dice lo" Steffo Autore più di trentatre piedi, $(u)$ nè con altre dell' A. fia, e dell'Africa dal medefimo Autore mentovate. Finalmente l'argomento prefo dalla moltitudine, e dalla grandezza degli infetti americani è quafi tanto inefficace, quanto l'altro prefo dalla piccolezza, e dalla fcarfezza de' quadrupedi, e nell' uno, e nell'altro fi moltra la medefima ignoranza, o pur la medefima volontaria dimenticanza delle cofe dell' antico Continente.

Quanto a quel, che dice il Sig. de P. intorno al tribito di pidocchj in Meffico, $\mathrm{fa}_{\mathrm{a}}$ in ciò palefe, ficcome in molte altre cole, la fua mala fede: E' vero, che Cortès trovò dei facchi di pidocchi nei magazzini del palagio del Re Axajacatl. E' parimente vero, che Motezuma impofe un tal tributo; non già a tutti, ma foltanto ai mendici, non gia perchè la ftraordinaria moltitudine di si fatti infetri gli divorava, come afferma il Sig. de P.; ma perchè Motezuma, il quale non poteva Coffrir l'ozio nei fuoi fudditi, volle che anche quella gente miferabile, la qual non poteva lavorare, s'occupaffe almeno nello

(r) Ciò ch: dice Gemelli dello Aupendo rumore dei piniftrelli dell' Ifola di Luzon, 'mi vien confermato da parecchie perfone degne di fede, che fono Atati alcuni anni in quell' ifola.

(s) Defunfe des Recherch. philofoph. chap. 22.

(t) Bomare Diczionn. univ. d'Hijoire natur. $V$. Coulewors.

(u) Id. V. Anacandaja. 
sello fpidocchiarf . ( $x$ ) Quefta è ftata la vera cagione d'un sì Atravagante tributo, cóme affermano Torquemada, Betancurt, ed altri Storici, e neffuno vi è ftato finora, a cui veniffe in mente ciò, che afferma il Sig. de P. foltanto perchè gli tornava a conto pel fuo fpropófitato fiftema. Del refto abbondano tanto quegli Cchifofi infetti nei capelli, e negli abiti dei mendici Americani, quanto nella gente miferabile ed inmonda di qualfivoglia paefe del Mondo, e non v' è dubbio, che fe qualche Sovrano dell'Europa efigeffe un tal tributo dai poveri del fuo Stato, non che facchi, ma ne potrebbe anche em. pier dei Vafcelli.

Finalmente riferbando per un' altra differtazione l'efamina delle prove del cattivo clima dell' America, fondate fu le malattie, e fopra i diffetti della coftituzione fifica degli Americani, nella quale dimoftreremo gli errori, ele prevenzioni puerili del Sig. de P., veggiamo ora ciò che dice fu l' ecceffo del freddo nei paefi del nuovo Mondo rifpetto a quelli dell'antico, fituati in ugual diftanza dall' equinoziale. "Paragonando, dice, "L'efperienze fatte coi termometri nel Perù dai Signori de la "Condamine, e Giovanni d' Ulloa (non fi chiama Giovanni, " ma Antonio ) con quelle dell' infaticabile Sig. d'Adanfon nel "Senegal, fi può facilmente intendere, che l'aria è men cal. "da nel nuovo Mondo, che nell' antico. Calcolando colla mag") gior efattezza poffibile il divario di temperamento, io cre" do, che vi farà di dodici gradi di latitudine, cioè, che fa " tanto caldo nell' Africa ai trenta gradi dall' Equatore, quan. "to ai diciotto gradi dalla medefima linea nel!' America. Il " liquore non è montato a tanta altezza, nel termometro nel "Perù nel centro della zona torrida, a quanta è montato " nella Francia nel maggior caldo della State. Quebec con. "tuttochè fia quafi nella medefima altezza polare di Parigi, " ha pure un clima incomparabilmente più afpro, e più fred. " do

(x) E' certo, che Motezuma era tanto portato per la pulitezza, quanto er nemico dell'ozio: ond'è da crederf, che per l'uno, e per l'altro motivo s' in. ducelle ad imporre quello Atraordinario tributo. 
„) do di Parigi. La differenza è parimente fenfibile nella Babia "dHudfon, e nel Tamigi, che hanno la medefima laticu-i " dine."

Quantunque tutto ciò concedeffimo al Sig. de P., nulla gli gioverebbe per dimoltrare, la malignità del clima america no. Perchè dall' ecceffo del freddo nelle terre americane fi vuol dedurre il fuo cattivo clima, e non fi dovrà piuttolto dedurre il cattivo clima dell'antico Continente dall' ecceffo del caldo ne' paefi fituati in ugual diftanza dall' equinoziale? Non potrà il Sig. de $P$. formare in quelta materia verun argomento contra l'America, che non lo rivolgano efficacenente gli Americani contro l'Europa, o contra l'Africa. Ma a dire il vero, tutte le offervazioni fattefi non fono fufficienti per iftabilire, come un principio generale, che $i$ paefi del nuovo Mondo fon più freddi di quelli del Mondo antico, fituati nella medefima latitudine, e molto meno per credere, come crede il Sig. de P. che vi fia tanto caldo nell' ántico Continente a trenta gradi d'altezza polare, quanto nel nuovo Continente a diciotto gradi. Se ciò foffe vero, farebbe in America così intenfo il freddo a'gradi 67. di latitudine, come nel Continente antico a' gradi 80. Ora il Sig. de $\mathrm{Paw}$ dice, $\left(^{*}\right)$ che il freddo nell'. antico Continente dee divenire in Novembre di là dal gr. ottantefimo così efiziale agli uomini, che neffuno vi potrebbe vivere: dunque nemmeno faprebbe vivere in America di là dal grado feffantefimo fettimo. Come dunque egli medefimo ivi afferma, che ne' paefi degli Efquimaux fi trovano degli abitatori di là dal gr. fettantefimo quinto? $\mathrm{E}$ fe $\mathrm{i}$ deboli Americani poffono in quella latitudine fuffiltere; dobbiamo credere, che $i$ fortiffimi Europei farebbono capaci di reggere al freddo del gr. ottantefimo. Inoltre fe quel principio foffe vero, farebbe tanto caldo in Gerufalemme fituata in poco meno di 32 . gradi, quanto nella Veracroce fituata in poco meno di 20. gradi: il che niun altro, che il Sig. de $P$. è capace di penlare. Similmente portrebbonfi dedurre altre così fpropofitate con-

(*) Recherch. philor. part. 3. fect. 1. pag. mihi 304. 
feguenze, maflimamente fe fi adottaffe il calcolo del Dott. Miehell, il quale, per quel che dice il Dott. Robertfon conchiufe dopo trenta anni d'offervazioni, che la differenza fra il clima del nuovo Mondo, e quello dell' antico è da 14. in 15 . gradi, cioè che fa tanto caldo ne' paefi dell' antico Continente, che fono a 29. ovvero 30. gradi, quanto ne' paefi del Continente nuovo, che fono a 15: gradi. Egli è certo, che ficcome fono molti paefi nell' America più freddi d'altri dell' antico Continente ugualmente diftanti dall' equinoziale, così ancora ve ne fono molti altri più caldi. Agra Capital del Mogol, ed il porto del Loreto nella California fi trovano quafi nella medefima latitudine, e pure non è paragonabile il caldo di quella Città afiatica con quello di quefto porto americano. Hue, Capital della Cochinchina, ed Acapulco fono quafi ugualmente diftanti dall' equinoziale, e pur l'aria d'Hue è frefca in paragone di quella d'Acapulco. Affai più falfa ed improbabile è quell'altra propolizione del Sig. de P., cioè, che nel centro della zona corrida non monta a tanta altezza il liquor del termometro, a quanta monta in Parigi nel maggior caldo della ftate. Se ciò foffe vero, il divario fra il clima Americano, e l'Europeo non farebbe già di dodici foli gradi, come vuol il Sig. de P. $\mathrm{ma}$ bensì di 49. , cioè quanta è la differenza di latitudine fra il centro della zona torrida, e di Parigi. E' vero, che attefo le offervazioni fatte in Quito, e paragonate con quelle, che fi fecero in Parigi, non arriva mai il caldo di quella Citra equinoziale a quello di Parigi nella ftate; ma è del pari certo, attefo le offervazioni fatte da' medefimi Accademici co'medefimi termometri nella Città di Cartagena, la quale non è centro della zona torrida; ma ben 10 . gradi dall' equinoziale difcofta, che il caldo ordinario di quefta Città è uguale al piir gran caldo di Parigi, ficcome ne $f_{a}$ fede $D$. Antonio Ulloa uno di quegli Offervatori. $(y)$

Storia Antica del Meffico Tom. IV. M So-

(y) Nell' anno 1735. fi tenne regolarmente il liquor del termometro del Sig. de Reaumur in Cartagena in $1025 \frac{1}{2}$ fenz'altro divario qualche volta, che quello di fcendere a 1024 , 0 di montare a 1026. In Parigi quello ftefto anno non 


\section{DISSERTAZIONE III.}

Sono molte le cagioni, che oltre a quella della vicinan: za, o della diftanza dall' equinoziale, fanno il caldo, o il freddo d'un paefe. L'elevazione del terreno, la vicinanza di qualche alta montagna coperta di neve, l'abbondanza delle pioggie \&c. contribuifcono affai alla freddezza dell' ambiente, e per l'oppofto la depreffione del terreno la fcarfezza d'acqua, i renai \&c. aumentano il caldo. Città Reale, capital della Diocefi di Chiapa, perchè è fituata in un luogo alto, è fredda, e la Città di Chiapa degli Indiani poco da quella diftante, è caldifima, perchè fituata in luogo baffo. Chachicomula borgo grande fituato appiè dell' altiffima montagna d'Orizaba, è freddo, e la Veracroce pofta nella medefima latitudine è calidiffma: e quel che è ancor più, effendo fredda l' aria di Citta Reale nella latitudine di $16 \frac{\mathrm{t}}{2}$ gradi, è calidiffima quella di Lo. reto nella California nella latitudine di $25 \frac{\mathrm{I}}{2}$ gradi .

I.e medefime offervazioni allegate dal Sig. de P. convin: cono, che il clima dell' America non è così vario come quello dell' Europa, che gli abitatori del nuovo Mondo non fono, eome quelli della maggior parte dell' Europa, coftretti a paffare dall' eftremo d'un freddo ecceffivo a quello d' un caldo intollerabile. Quanto più è uniforme il clima, tanto più facil. mente s'avvezzano ad effo gli uomini, e fchivano que' perniciofi effetti, che cagiona la vicenda delle ftagioni. In Quito non monta il liquore nel termometro tanto, quanto in Parigi nella ftate, ma nè ineno cala tanto, quanto ne' paefi più temperati della Europa nell'Inverno. Che cofa può effere più defiderabile in un clima, che un tal temperamento nell' aria, il qual fia ugualmente diftante dall' uno, e dall' altro eftremo, come è quel. lo di Quito, e della maggior parte del Meffico? Che clima più dolce, e più confacevole alla vita, che quello, nel quale fi gode tutto l'anno delle delizie della campagna, e la terra fi vede fempre adorna d'erbe, e di fiori, i campi coperti fono di biade, e gli alberi carichi di frutto: gli armenti, e le gregge

montò a più di $8025 \frac{\mathrm{I}}{2}$ nel maggior caldo di Luglio, e d'Agofto. Ulloa Rela. cion del Viage a la America Meridional part. I. tom. I. 
rifparmiando fatiche all"uomo nè hanno d' uopo della fua prov: videnza per mantenerfi, nè del fuo tetto per refiftere all'inclemenza della Atagione: nè la neve, o la brina forza l'uomo a Atarfi al fuoco, nè il bruciante caldo della ftate lo fcaccia dalla popolazione; ma fperimentando ognora benigna verfo di fe la natura, gode indifferentemente in tutte le tagioni, o della compagnìa degli uomini nella Città, o degl' innocenti piaceri della campagna? Quefta è l'idea, che hanno gli uomini d'un clima dolce, e però i Poeti volendo ne'lor verfi innalzar con lodi alcuni paefi, dicevano, che vi regnava una perpetua primavera, ficcome diffe Virgilio della fua Italia, $(\boldsymbol{z})$ ed Orazio delle ifole fortunate, $(A)$ là dove invitava i fuoi Compatrioti Così rapprefentavano gli Antichi i Campi Elisj, ed anche ne' libri fanti per darci qualche idea della felicità della Gerufalemme celefte $f_{i}$ dice, che non $v^{2}$ è nè freddo, nè caldo.

II P. Acofta, la cui Storia è appellata dal Sig. de P. $O$ pera eccellente, il qual era pratico de'climi d'ambidue i continenti, e per altro nun era parziale dell' America, nè avea verun intereffe d'ingrandirla, ragionando del clima dell' America parla cosi: "Vedendo io la dolcezza dell aria, e la piacevo" lezza del clima di molri paefi dell' America, dove non fi " fa che cofa fia inverno che ftringa, nè ftate che angofci col " caldo; dove con una Atuoja fi difendono da qualunque incle. " menza del tempo: dove appena è d'uopo mutar l' abito in " tutto l'anno: confiderando, dico, tutto ciò m'è paruto fpef"fe volte, e mi pare anche oggidi, che fe gli uomini volef" fero sbrigarfi de' laccj, che lor tende l'avarizia, e lafciare " certe pretenfioni ioutili e nojofe, potrebbono menar nell' A. " merica una vita affai tranquilla e piacevole; perchè ciò che "que' Poeti cantarono de' Campi Elisj, e della famofa Tem. " pe, e cid che Platone raccontava, o fingeva di quella fua i) ifola Atlantida, tutto il troverebbono gli uomini nelle fudM 2 " det-

(z) Hic ver affiduum, atgu: alienis monfibus aftas:

Bis gravida pecudes, bi pemis $u$ ilis a.bos. Virg. Georg. 2.

(A) Ver ubi longum, tepida sque prebet

Jupiter brumas. Horat. lit. 2. ude 4. 


\section{D I S S E R T A Z I O N E III.}

" dette terre \&c., (B) Lo fteffo che Acolta dicono dell' Ame: rica altri Storici, e particolarmente del Meffico, e delle Provincie circonvicine, i cui paefi mediterranei quafi dall'iltmo di Panamà infino al grado quarantefimo di latitudine (poichè quelli, che fono di la da tal grado non fi fono ancora fcoperti ) godono di un' aria dolce, e d'un clima favorevole alla vita, fuorchè pochi luoghi, i quali o per la lo:o depreffione fono caldi ed umidi, o per la fomma loro altezza fono d' un clima afpro. Ma quanti non fono nel Mondo antico afpri, o nocevoli?

\section{I I I.}

\section{Su la qualita della Terra del Mefjico.}

Egli è certo, dice il Sig. de Pavv, che l'America in gearevale è fata, ed è anche oggidi un paefe troppo fterile; ma egli è piuttofto certo, che quefta in generale è una gran falfita, e fe il Sig. de P. vuol renderfene ficuro, può informarfi da molti Tedefchi venuti di frefco dall' America, dove alcuni an. ni fi trattennero, i quali prefentemente fi trovano nell' Auftria, nella Boemia, nel Palatinato del Rero, ed anche nella fteffa Pruffia, o pur rilegga $l$ 'eceellente opera del P. Acolta, e vi troverà nel lib. 2. cap. I4., che fe v'è qualehe terra al Mondo, cui convenga il nome di Paradifo, deffa è quella dell' America. Quefto dice un Europeo dotto, giudiziofo, ed imparziale, e nato in Ifpagna, uno de' migliori paefi d' Europa, e parlanda nel lib. 3. de' paefi dell' Imperio Mefficano, dice, che la Nuova Spagna è il miglior paefe di tutti quanti ne circonda it Sole. Certamente non parlerebbe così il P. Acolta dell' America ia generale, e della N. Spagna in particolare, fotto il cui nome comprende tutto il Continente dell' America fettentrionale Spa. gnuola, fe l'. America foffe in generale un paefe fterile. Non parlano altrimenti dell'America, e maffmamente del Meflico moltiffmi altri Europei, le cui teftimonianze tralafcio per non recar

(B) Stor. Nat, e Mor. lib, 2, Gapo do. 
recar noja a' Leggitori. ( $\left.{ }^{*}\right)$ Pel medefimo motivo tralafcieremo ancora cio che fcrive il Sig. de P. contro altri paefi del Nuovo Mondo; poichè farebbe impoffibile d'efaminar le ragioni $\mathrm{da}$ lui allegate contro ciafcun d'effi fenza fare un gran volume, e ci contenteremo di ciò che appartiene alla terra del Meffico.

I Signori de Buffon, e de P. fi fon perfuafi, che tutto il terreno dell' America fi riduca a monti inacceffibili, a bofchi impenetrabili, ed a pianure allagate, e pantanofe. Leffero quefti Filofofi nelle defcrizioni dell' America, che i famofi Andes, - alpi americane formavano due lunghiffime catene di montagne altiffime, e coperte in gran parte di neve, che il vafto deferto delle Amazoni fi compone di folti bofchi: che Guayaquil, e qualche altro luogo è umido e pantanofo, e ciò baftò loro per dire, che nell' America non c'è altro, che monti, bofchi, e pantani. Leffe il Sig. de P. nella Storia di Gumilla ciò, che dice queft' Autore intorno al modo, che aveano gl'Indiani dell'Orinoco di preparar quel terribile veleno delle loro frecce, e nella-Stcria d'Herrera, o d'altri Autori, che i Cannibali ed altre Nazioni barbare ufavano delle frecce avvelena. te, e quefto gli baftò per affermare, che il nuovo Continente produce un maggior numero d'erbe velenofe, che il refto del Mondo. Leffe, che nelle terre troppo calde non nafce il frumento, e nè meno le frutta dell' Europa, e ciò gli baftò per dire, che le perficbe, e gli albercoccbi foltanto banno fruttifianto nell ifola di Giovanni Fernandez (C), e che il frumento, e l'or-

(*) Tommafo Gages, oracolo degl' Inglefi, $\in$ dei Francefi in ciò che riguarda l'America, parlando del Meffico dice cosi:, Il ne manque rien a Mexi"que de tout ce qui peut rendre une ville heureufe: \& fi ces Ecrivains, qui " ont employè leurs plumes a louer les Provinces de Grenade en Espagne, \& ", de Lombardie, \& de Tofcane en Italie, dont ils font des paradis terre"ftres, auroient vâ ce nouveau Monde, \& la Ville de Mexique, ils fe de", dirojent bientot de tout ce qu'ils ont dit en faveur de ces lieux la., ,Part. 1. cap. 22. Cosi parla del Meffico quefto Autore, che non feppe parlar bene di nefruno.

(C) Acciocchè fi veda quanto fiafi dal vero difcoftato il Sig. de P. è d'uopo fapere, che nella miferabile Ifola di Gio: Fernandez, dov'egli dice che vengono cosi bene le perfiche, vengono affai poche e cattive, ficcome l'ho intelo dal Sig. $\Lambda$ b. D. Giureppe Garcia, Valenzano, il quale yi Aterte fei me- 


\section{DISSER T. A Z I ONE III.}

- l'orzo non banno allignato, fe non in alcune contrade del fertentrione. Tal è la Logica adoperata dal Sig. de P. in tutta la fua opera, ficcome talora lo faremo vedere in quelte differtazioni:

Ma niente è vero rapporto al Meffico di tutto cio, che egli dice contra la terra dell'America. Sono certamente nel Meffico delle montagne altifime, ed eternamente coperte di neve: vi fono de'grandi bolchi, e vi fono ancora alcuni luoghi pantanofi; ma è fenza paragone più grande il terreno fertile e coltivato, ficcom' è noto a tutti coloro, che fono fati in que' paefi. In tutto quell' immenfo Tpazio di terra dove prefentemente fi femina il frumento, $l^{2}$ orzo, il frumentone, ed altre fpezie di biade, e di legumi, di cui abbonda infinitamente quel regno, fi femirava gia del frumentone, del peverone, de'fagiuoli, del caccao, della chia, del cotone, e si fatte piante, che fervivano al foltencamento, al veftimento, ed alle delizie di que'Popoli, i quili effendo tanto numerofi, quanto abbiamo accennato nella Storia, e dimoftreremo altrove, non avrebbe mai la terra poturo provvedere a lor bifogni, fe non vi foffe altro, che monti, bofchi, e pantani. Il Sig. de Buffon, il quale nel fuo tomo 1 . dice, che $l$ 'America non è altro che un continuato pantano, e nel tomo 5. afferma, che le montagne inacceffibili dell' America appena lafciamo de' piccoli Spazj all' agricoltura, ed all' abitazione degli uomini, nello fteffo tomo 5. confeffa, che $i$ Papoli del. Meffico, $e$ del Perì erano affai numerofi. Ma fe quelti Popoli, i quali occupavano una grandiffima parte dell' America erano affai numerof, e viveano, come egli dice in focieta, e fotto la direzion delle leggi, non d certamente l'America un continuato pantano: fe quefti Popoli tanto numerofi fi foltentavano, ficcome è certo, delle biade, e de'frutti, che coltivavano, non fono piccoli gli fpazj, che Le

fi, e vi fi trovo nella fagione delle frutta. Per linppofto in quafi tutti i paefi temperati, e freddi dell' America Spagnuola, dov'egli crede che non fruttificano le perfiche, vengono beniffimo, ed in molti paefi, ficcome in quelli del regno del Chile, ed in alcuni della $N$. Spagna aflai meglio che in Earopa. 
le montagne inacceffibili lafciano all' agricoltura, ed all' abita. zione degli uomini.

La moltitudine, la varietà, e la bontà delle piante del Meffico non lafciano verun dubbio della prodigiofa fertilita di quelle terre. Nè pafcoli, dice il P. Acolta, è eccellente la Nuova Spagna, e però vi fi alleva una moltitudine innumerabile di Cavalli, di Vaccbe, di Pecore, e d'altre beftie. E' ancora afjai abbondante tanto di frutta, quanto d' ogni forta di biade. In tatti non' v' è biada, legume, ortaggio, o frutto, che non venga bene in quella felice terra. Il frumento, il quale appena concede il Sig. de P. ad alcune contrade del fettentrione dell' America, non viene per lo più nelle terre troppo calde della N. Spagna, come neppure nella maggior parte dell' Africa, ed in molti altri paefi dell' antico Continente; ma nelle terre fred. de e temperate di quel regno viene eccellente, e più abbondante che in Europa. Bafta dire, che quello che fi raccoglie nella Diocefi d'Angelopoli è tanto, che di quello che avanzava dappoichè erano ftati provveduti tutti i fuoi innumerabili abitatori, fi provvedeva alle ifole Antille, ed alla flotta di Vafcelli, che era già nell' Havana col nome d'Armata di Barlovento. In Europa non v'è più d'una fementa, e d'una raccolta: nel. la N.Spagna, ve ne fono parecchie. In quelle zerre, dice Torquemada Autor Europeo, che Atette molti anni in quel regno, e viaggiò per tutto, in quelle terre, nelle quali fi coltiva it frumento, $\sqrt{i}$ vede in ogni ftagione dell' anno un frumento, che $f_{i}$ fa tagliando, un altro che va maturando, un altro $c b^{\prime} \dot{d}$ ancor piis verde, ed un altro cbe $f i$ va feminando, ed ora, $c b$ ' il mefe di Novembre, veggiamo cio avverato nel frumento tem. poral, che fi fa tagliando, ed in quello di riego, $(D)$ ibe va crefcendo in Atrifco, ed in altri luogi, ed in quello che fi va feminando: ciò cbe dà a divedere la maravigliofa fertilutà della

(D) Il frumento appellato di Riego fi femina in Ortobte, in Novembre, o in Decembre, e fi fa la raccolra in Maggio, o in Giugno. Il Temporal fi remina in Giugno, e fi taglia in Ottobre, ed il frumento Aventurero fi femine in Novembre, e fi fa la raccolta or più prefto, or più tardi. 
la terra. (E) Lo fteffo Autore fa menzione di parecchie terre; che rendevano or feffanta, or ottanta, or cento per uno, ed a' noftri dì s' è veduta una sì gran moltiplicazione del frumento in alcuni campi di que' paefi, $(F)$ la quale, parlando in gererale, è certamente più grande di quella dell' Europa con a $\int_{\text {. }}$ fai minor coltivamento, ficcome è notorio agli Europei intendenti d'Agricoltura, che fono ftati in quella parte dell' America. Ciò che diciamo del frumento pofiamo ancor dirlo dell' orzo, benchè quefto non fi femini, fe non a proporzione del confumo che fe ne fa nel foftentamento de cavalli, e delle mule di ftalla, e de' porci. Affai più potrebbe dirfi del frumenrone, come quello, ch'è la biada più propria dell' America.

Il Sig. de P. pretende, che tutte le piante d'Europa hanno tralignato nell' America, fuorchè le aquatiche e fugofe, $e$ per provare si fatto fpropofito dice, che le perficbe, e gli albercoccbi foltanto banno fruttificato nell' ifola di Giovanni Fernandez. Ancorchè gli concedeffimo, che in niun paefe dell'America vengono quelle frutta, nulla gli gioverebbe per convincere ciò che vuole; ma tanto falfa è quefta propofizione particolare, quanto quella univerfale. Il P. Acofta ragionando di tali frutte in particolare, dice così: Vengono ancora bene ( nell' America) le perficbe, $i$ melocotogni, e gli albercoccbi, bencb̉è piì nella Nuova Spagna. (G) In tutta la Nuova Spa-

gna,

(E) Torquemada lib. r. della Monarcbia Indiana cap. 4. Vedafi ancora ciò che dice queft' Autore della varietà, ie dell'abbondanza di frutta, che vi fi hanno in tutte le flagioni, ed Herrera in molti luoghi della fua Storia.

(F) Io fono tato in un paefe, nel quale foleva la terra render cinquanta per uno, ed ho faputo d'un altro che rendeva talvolta infino a cento. Nella Cinaloa contuttochè fia paefe ben caldo, fuol far la terra dugento per uno, fecondochè mi ha informato una perfona autorevole, e degniffima di fede, che vi ftette alcuni anni. Il mio erudito amico il Sig. Ab. D. Giovanni Ignazio Molina dice nella Storia compendiofa del regno del Chile, che pochi anni fa Aampò in Bologna, che in quelli paefi fuol fare il frumento 150 . per uno. E' tanta i'abbondanza di quefta biada, che vi fi vende la fanega a cinque paoli, ed ogn'anno vanno al Perù 30. valcelli incirca carichi d'effa, ed ancor avanza.

(G) Acofta lib. 4. cap. $9 r$. E' tanta l'abbondanza di perfiche nella N. Spagna, che ordinariamente fi rendono a ventine, ef danno due, tre, ed an- 
gna, fuorchè nelle terre troppo calde, hanno allignato affai bene quelle frutte, e tutte le altre, trafpiantate dall' Europa, e vengono in grand'abbondanza, ficcome il fanno tutti gli Europei, che fono ftati in que paefi. $(H)$ Finalmente, dice A. colta parlando dell' America in generale, quafi tutto il buono, che fi produce in Ifpagna, $v$ 'd là, in parte migliore, ed in parte no: frumento, orzo, infalate, ortaggio, legumi orc. (I) Se egli aveffe parlato foltanto della N. Spagna, avrebbe ommeffo quel quafi.

$V^{\prime} \dot{e}$ ancora un altro vantaggio, dice Acofta, cioè, che nell' America vengono meglio le coje d' Europa, che in Europa quelle dell' America. E parrà piccolo sì fatto vantaggio al Sig. de P.? Quefto folo bafterebbe per dimoftrare, che fe v' è qualche eccello, deffo è dal canto dell' America. Nella N. Spagna vengono affai bene, come fanno fede molti Autori Europei, e fanno tutti coloro, che fono ftati là, il frumento, l'orzo, il rifo, e tutte l'altre biade dell' Europa: i ceci, i pifelli, le fave, e tutti gli altri legumi; le lattughe, i cavoli, i navoni, gli fparagi, ed altre infalate, e radici, ed ogni forta d'ortaggio; le perfiche, le mele, le pere, i melocotogni, e le altre frutte; i garofani, le rofe, le viole, $i$ gelfomini, il baffilico, la menta, la majorana, la meliffa, ed altri fiori, e piante odorofe trafpiantate dall' Europa; ma in Europa non allignano per lo più, nè poffono allignare le piante americane. Il frumentone viene nelle terre dell' Europa; ma più piccolo, e molto men buono di quello dell' America. Delle molte deliziofe frutte del Nuovo Mondo alcune, come la Mufa, e Storia Antica del Meffico Tom. IV. N I Ana-

che quattro ventine per la più piccola moneta, che v'è là. Nel regno del Chile contano fin'a dodici fpezie di perfiche, $e$ ve ne fono st grandi, che alcune pefano una libbra Spagnuola: o fia I6. oncie. Molina Stor. del Chile. Vedafi ancora ciò che dice il P. la Feuillèe del lor gufo delicatiffimo.

(H) Le pere fi vendono ancora a ventine in Meffico; e ve ne fono più di cinquanta fpezie. L'Italiano Gemelli teftifica, che i Carmelitani di S. Angelo, borgo diftante fette miglia da Meffico tiravano ogni anno 6500. zecchini dalle frutta Europee del loro giardino, ed i Domenicani cavavano 3. mila zecchini dall'ortaggio del loro piccol orto di S. Giacinto, in un fobborgo di Meffico. Gemelli Giro del Mondo tom. 6, lib. r. cap.8.

(I) Acotta lib. 4. cap. $3 \mathrm{I}$. 


\section{DISSERTAZION E III.}

l'Ananàs fon riufcite ne' giardini de' Principi dell' Europa mercè delle ftufe, e d'una gran cura e diligenza; ma nè cosi bene ftagionate, nè in quell' abbondanza del proprio lor paefe; altre poi più d'effe pregiate, come la Chirimoya, il Mamey, ed il Chicozapote non fo, che abbiano potuto finora allignare a difperto dell' induftria Europea. La cagione di quefto gran divario tra l'America, e l' Europa è quella, che accenna il fuddetto Acofta, cioè, percbè in America v'd maggior varietà di semperamenti, che in Europa: ond' $d$ piv facile di dare a ciafouna pianta quel temperamento, che le conviene. Or come non è argomento della 'fterilita dell' Europa, che in effa non allignino le piante proprie dell' America, nè meno è argomento della fterilità d'alcuni paefi dell'America, che in effi non allignino alcune piante dell' Europa; perchè non omnis fert omnia tellus. Hic fegetes, ibi proveniunt felicius uve; anzi que' paefi caldi, ne' quali non viene il frumento, nè vi riefcono le frurte europee, fon peraltro $i$ più ameni, ed $i$ più fecondi, come fanno bene gli uomini pratici di que' paefi.

Io per altro non dubito, che fe vuol farfi il paragone dell' America con tutto l'antico Continente, fi troveranno quafi uguali nelle loro produzioni; perciocchè nell' Afia, e nell'Africa vi fono delle terre, e de'climi proporzionati a tutte le piante dell' America, le quali a cagione della diverfità del clima non poffono riufcire in Europa. Ma che utile potrà mai recare a' Signori Europei l'abbondanza dell' Afia in sì gran lontananza? per l'oppofto i Mefficani circondati da' paefi d' ogni forta di clima, godono di tutti i loro differenti frutti. La piazza di Meffico ( ficcome quelle di moltiffime altre Città dell' America) è il centro di tutti i doni della Natura. Quivi fi trovano le mele, ie perfiche, gli albercocchi, le pere, le uve, le vifciole, le ciriegie, $\mathrm{i}$ camoti, le xicame, le noci, ed altre innumerabili frutte, radici, ed erbe faporite, che rendono le terre fredde e temperate: $l$ ' ananàs, le mufe, $i$ cocchi, le anone, le chirimoye, i mamei, i chicozapoti, i zapori neri, ed altre moltiffime, che portano le terre calde: i mello: ni, i cucumeri, i melarancj, le melagrane, gli ahuacati, i 
zapoti bianchi, ed altre, che vengono indifferentemente ne' pae: fi caldi, e ne'freddi. In tutte le ftagioni dell' anno fi vede quel mercato abbondancemente fornito di varie eccellenti frutta, anche in quel tempo, nel quale gli Europei fe la paffano colle loro caftagne, o al più colle mele, e colle uve, che la loro induftria conferva.. Tutto l'anno, anche nel rigor dell' inverno, entrano in quella piazza per uno de' canali innumerabili barche cariche di tanta varietà di frutte, di fiori, e d'ortaggio, che pare che fieno ad un tempo venute tutte le ftagioni dell' anno, concorrendo in quel lwogo le piante più pregevoli dell' Europa co' propri vegerabili di çuel paefe : ciò che poffono teftificare tutti quegli Europei, che hanno avuto il piacer di vederlo.

Non è minore l'abbondanza di quella terra in piante medicinali. Baft d per ciò vedere l'opera del celebre Naturalifta Hernandez, nella quale li defcrivono, e fi difegnano più di novecento piante ( prodotte per lo più nelle vicinanze di Meffico ) la cui virtù è ftata conofciuta per la fperienza, oltre ad altre trecento l' ufo delle quali non fi accenna: e non v'è dub. bio, che ve ne mancano moltiffime, e innumerabili. Il Sig. de P. per lo contrario dice, che l'America produce un maggior numero di piante velenofe, che tutto il relto del Mondo. Ma che fa egli delle piante, che nafcono ne paefi mediterranei dell' Africa, e dell' Afia per poter farne si fatto paragone? effendo tanto grande la fertilità del fuolo. Americano, non farebbe da maravigliare, che di tutto vi foffe abbondanza. $M_{2}$ a dire il vero io non fo, che finora fiafi fcoperta nella N. Spagna nè anche la ventefima parte di quelle pianre velenofe nate aell' antico Continente, delle quali fanno fpeffo menzione ne' loro libri i Noturalifti, ed i Medici Europei.

Quanto alle gomme, alle ragie, agli olj, ed altri fughi, che mandano fusuri gli alberi o fpontaneamente, o a jutati dall' indultrid: umana, è eccellente, al dir d'Acoftd, la N. Spagna. In fatu vi fono bofchi interi d'Acacia, che rende la vera gomma arabica, la quile per la lua abbondanza non è quivi pregiara. V'è inoltre del balfamo, dell' incenfo, del copal di molte fpe- 
zie, del liquidambra, della tecamaca, dell' olio d'abete, ed altri molti fughi ftimabili pel loro foaviffimo odore, o per la loro virrù medicinale.

Anche que' medefimi bofchi, de' quali è coperta la terra dell' America fecondochè affermano i Signori de Buffon, e di P. dimoltrano la fua fecondià. Sono pure mai fempre ftati, e fono ancora in quelle valtiffime regioni de' gran bofchi; ma non fono tanti, che non fi poffa fare un viaggio di cinquecento, o di feicento miglia, fenza neppur trovarne uno. E che bofchi? Per lo più o d'alberi fruttevoli, ficcome di mufe, di mamei, di chicozapori, di mele, di melarancj, di limorri, quali fon quelli di Coatzacoalco, della Mifteca, e di Michuacan, o d'alberi pregevolí pel loro legno, o per le loro ragie, ficcome fon quelli, che feparano la Valle Mefficana calla Diocefi d'Angelopoli, e quelli di Chiapa, de'Zapotechï 3xc. Oltre a' pini, a' roveri, a' fraflini, alle noci, agli abeti, e ad altri moltiffimi comuni a tutti $i$ due Continenti, vi fono in molto maggior numero gli alberi proprj di quella terra e più pregevoli. Di cedro vi fono, ficcome abbiamo detto altrove, de' bof chi interi. Il Conquiftatore Cortès fu accufato da' fuoì emoli davanti all' Imperatore Carlo V. d'aver meffo nel palagio, che fi fece fare in Meffico, ferte mila travi di cedro, ed egli fi fcusò dicendo, ch' effo era un legno comune in quel paefe. In fatti è tanto comune, che di quetto legno preziofo fan. no gli fteccati per le fondamenta delle cale nel fuolo paludofo: delia Capitale. Del famoro, ed a ragione celebrato ebano vi fono parimente de'bofchi in Chiapa, in Jucatan, ed in Cozumel, del brafile nelle terre calde, e dell' odorofo legno aloè nella Mifeca. Il Tapinceran, il granadillo o ebano roflo, il camote, e gli altri da noi mentovari nella froria fornifcono de' legni affai migliori di quelli, che fi adoperano in Europa. Finalmen. te per non trattenermi in una lunga, e nojofa enumerazione? mi rimetto al P. Acolta, al Dott. Hernandez, a Ximenez, ed altri Autori Europei, che fono ftari nella N. Spagna, benchè wutto ciò, che eglino dicono non bafti a dare una compita idea de्lla fertilità di quella terra. Il P. Acofta afferma, che cosk 
qudnto al numero, come quanto alla varieta d'alberi prodotti dalla Aeffa natura, v'è maggior copia in America, che in $A$ fia, in Africa, e in Europa. (K)

Ecco quì un nuovo argomento atto a convincere $i$ vantaggj della terra dell' Anerica fopra quella dell' Europa. La natura, e qualità d' un terreno fi dà meglio a divedere dalle piante, ch'elfo produce per fe lteffo, Cenza l'ajuto dell' arte. Paragoniamo dunque le produzioni proprie dell' Europa con quelle non già di tucta l'A America, ma foltanto della N. Spagna ., La "cagione, dice Montefquieu, $(\boldsymbol{L})$ d'effere tanti relvaggi nell'A" merica è che la terra vi produce per fe fteffa molci frutti, " di cui poffono cibarfi... Io credo che quelti vantaggj non fi " potrebbono aver nell' Europa, fe la terra foffe lafciata fenza " coltura: non altro produrrebbe, che felve di quercie, e d'al"tri alberi inutili. "Efaminando, dice il Sig.de P., la to"ria, e l'origine de' noltri legumi, de' nottri ortaggi, de' noltri ") alberi fruttevoli, ed anche delle noltre biade, fi conofce, che ") tutre fono Atraniere, e che fono ftate da un altro clima tra" fpiantate nel noftro. Può facilmente intenderfi quanto grande " farà Itata la miferia degli antichi Galli, ed anche quella de" "Germani, wella cui terra non fi produceva nè anche al tempo "di Tacito verun albero fruttevole... Se la Germania dovelfe , reltituire i vegetabili ftranieri, che non appartengono originalmente al fuo terreno, ovvero al fuo clima, quafi nulla le rimarrebbe, nè altro conferverebbe tra i femi, che fervono pel foltentamento, fe non il papavero falvatico, e la vena "ancor falvatica. "( $(M)$ Cid che il Sig. de P. confeffa fchiettamente delle Gallie, e della Germania, potrebbe ancor dirfi degli altri paefi dell'Europa, ed anche della Grecia, e deil' Italia, le quali provvidero gli altri paefi. Se l'Italia forfe obbligata a reftituire tutri que'frutti, che non appartengono originalmente al fuo terreno, che le relterebbe dalle ghiande in

fuo.

(K) Acolta lib. 4. cap. 30.

(L) Monterquieu L'esprit des Loix lib. I8. cap.9،

(M) Recherch. Philoloph. part. I. 
fuora? Que' nomi Malum perficum, Malum medicun, Malum. assyrium, Malum punicum, Malum cidonium, Malum armenia: cum, Nux pontica Ooc. fervono a ricordarle, che tali frutta le fon venute dall' Alia, e dall" Africa. "Si fa, dice il Sig. de "Bufching, $(N)$ che le più belle, e migliori frutte d' alberi paffa9. rono dall" Italia in que paefi, dove prefentemente fi produ: "cono. L'Iralia le ricevè dalla Grecia, dall' Afia, e dall'A9. frica. La mela le venne dalla Soria, dall'Egitto, dalla Gre. , cia \&c., l' albercocco dall' Epiro, la pera da: Aleffandria, " dalla. Sorì, dalla Numidia, e dalla Grecia, il limone, ed 22 il melarancio dalla Media, dall' Affria, dalla. Perfia, il fi. " co dall'Afia, la melagrana da Cartagine, la caftagna da Ca"Atania nella Magnefia, Provincia della Macedonia, la cirie" gia da Cerefunto del Ponto o. la: mandorla dall' Afia alla Gre. - cia, ed indi all' Italia, la noce dalla Perfia, la nocciuola dal 3. Ponto, l'oliva da Cipro, la fufina dall'Armenia, e dalla. 9. Soría, la perfica dalla Perfia, il melocotogno da Cidonia in 2. Cardia alla Grecia, ed indi all" Italia o, ,

Plinio dice, che gli uomini da principio non fi foltenta. vano d'altro, che di ghiande. (O) Ciò, benchè fia fallo rap. porto al comun degli uomini, pare effer certo rifpetto a primi popolatori dell' Italia, almeno tal era l''opinione degli antichi, come appare da' loro fcritti. Plinio foggiunge', che anche al fuo tempo: molti popoliper mancanza di biade: fi: timavano ricchi a proporzion della quantità di ghiande che aveano, del. la cui farina faceano pane, ficcome il fanno oggidi nella Nor. vegia di fcorza di pino, ed in altri paefi fettentrionali d'Eu. ropa d'offà di pefci: il che è fenza dubbio la più gran miferia. Il Sig. de Bomare protelta, che tutte le bellezze de' giardini Eutopei fono ttraniere, $(P)$ e che i più bei fiori, che hanno, fon venuti da Levante. (Q) Lo Iteffo Sig. de P. fa una prù generale confeffione dell' antica: mileria degli Europei, dove af-

fer-

(N) Bufrhing. Geograf. tom. I.

(O) Plin. Hift. Nat. lib. 2. cap. 56.

(P) Bomare DiZzion. Univ. d' Hifoir. Natur. v. Plante.

(Q) Id. r. Flewr. 


\section{IS SER T A Z I NE III. 103}

ferma, che le piante utili, che hanoo prefentemente paffarono dall' Afia meridionale nell' Egitto, dall' Egitto alla Grecia, dal. Ja Grecia nell' Italia, dall' Italia nelle Gállie, e dalle Galiie nella Germania, $(R)$ ficchè il terreno dell' Europa quanto alle fue proprie ed originali produzioni è de' più poverì, e fterili del Mondo. Per l'oppofto quanto ubertofo ed abbondante non è il fuolo Americano, e maffimamente quello del Meifico di piante proprie, ed utili al foftentamento, al veltiario,e ad altri ufi della vita? Vedanfi l'opere degli Autori Europei, che hanno fcritto della Storia naturale di quel Nuovo Mundo.

Ecco dunque la rifpolta, che potrebbono dar gli Americani a quel ridicolofo paragone, che $\mathrm{fa}$ il Chronichilta Herrera nella fua prima Decada, del quale abbiam fatta menzione nel principio di quelta differtazione. "In America, dice, non "erano, come in Europa, limoni, nè melarancj, nè melagra" ne, nè fichi, nè melocotogni, nè melloni, nè uve, nè oli"ve, nè zucchero, nè rifo, nè frumento. "Diranno dunque gli Americani. I. che neppur in Europa era veruno di tali frutti, finattantochè non vi furono trafpiantati dall' Afia, e dall' Africa . 2. che oggidi fono in America, come in Europa, e per lo più fono là migliori, ed in maggiore abbondanza, maffimamenre i melaranci, i limoni; $i$ melloni, e le cannameli 3. che fe l'America non avea frumento, nè meno l' Europa avea frumentone, il quale non è men utile, nè men fano; fe l'America non avea melagrane, limoni, \&c. almeno oggidì gli ha; ma l'Europa nè ha avuto, nè ha, nè può avere Chirimoye, Ahuacati, Mufe, Chicozapori \&c.

Finalmente i Signori de Buffon, e de P. ed altri Filofofi, e Storici Europei, che tanto Cchiamazzano per la tterilità, per li bofchi, per li panrani, e per li deferri dell' America, ricordinfi per grazia, che i miferabili paefi della Lapposia, della Norvegia, dell' Islandia, della Nuova Zembla, dello Spitzbergo, ed i valti ed orrendi deferti della Siberia, deila Tartaria, dell' Arabia, dell' Africa, ed altri fon puse paefi dell'an. tico

(R) Recherch. Philofoph. part. I. 


\section{IO4 DISSER TAZIO NE III.}

rico Continente, e fanno almeno una quarta parte della fua eltenfrone. Ma che paeli? Vedafi la delcrizione, che d'effi fanno gli iteffi Europei. Vedafi aimeno l'eloquente defcrizione; che fa il Sig. de Buffon de' deleru dell' Arabia., Un paefe, " dice, fenza verdura, e fenźdacqua: un Sole tuttora brucian"te, un Cielo tuttora fecco, planure arenofe, montagne an"che prù aride, fu le quali fi ftende quanco può la vilta fen: "za incontrar verun oggetto vivente: und terra, per cosi:di"re, morta è fcorticata da'venti, la quale altro non pré́en. " ta, che offami, falfi fparfi, e rupi innalzate, o rovefciate: " un deferto affitio fcoperto, nel quale il viandante non refpi"ra mai fotco lombra, dove niente gli fa compagnia, e nul"la v'è, che gli ricordi la natura viva : folitudine affoluta " affai più fpaventevole di quella de' bofchı; poichè almero gli " alberi fono creature viventi, che recan qualche follievo all' uo"mo, il quale fi trova folo, ifolato, più ignudo, e più fmar. , rito in quelti luoghi vuoti e fenza termine. Tutto il terre: "no, che gli fi prefenta, lo vede come il fuo fepolcro: la "luce del giorno più malinconica, che le ombre: della notte, "non rinafce, fe non per fargli vedere la fua nudita, e la fua " impotenza, e per mettergli d'avanti agli occhi la fua orren" da fituazione, allontanando dalla fua vifta i limiti del va" cuo, ed ampliando attorno a lui l'abiffo dell' immenfità, ") che lo fepara dalla terra abitata: immenfita si grande, che " indarno cercherebbe di paffare; perchè la fame, la fete, ed " il caldo bruciante gli raccorciano que momenti, che gli re: "Atano fra la difperazione, e la morte." (S)

(S) Buffon Hiftoire Natur. tom. 22. 


\section{DISSERTAZIONE IV.}

SU GLI ANIMALI DEL MESSICO.

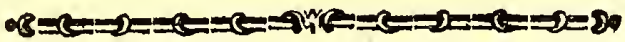

T $\mathrm{Na}$ delle cofe più inculcate da'Signori de Buffnn, e de Paw per dare a divedere l'infelicità del fuolo americano, e la malignità del fuo clima è quella della pretela digra. dazione degli animali, tanto di quelli, che fon proprj di quella terra, quanto di quelli, che vi fono ftati trafportati dall' antico Continente. In quefta differtazione difamineremo le loro ragioni, e dimoftreremo alcuni de' molti loro sbaglj, e con. traddizioni .

\section{\$. I.}

Su gli animali propri del Mefjico:

Tutti gli animali, che fi trovano nel Nuovo Mondo, vi paffarono dal Mondo antico, ficcome abbiamo ftabilito nella prima differtazione, e ciò lo confeffa lo fteffo Sig. de Buffon, nel tomo 29. della fua Storia Naturale, e debbono confeffarlo tutti coloro, che portano rifpetto a'facri libri. Noi dunque diciamo proprj del Meffico quegli animali, che vi trovarono gli Spagnuoli, non perchè traggano in fatti la prima lor origine da quella terra, ficcome danno ad intendere il Sig. de P. in tutta la fua opera, ed il Sig. de Buffon ne' primi ventotto tomi della fua Storia; ma foltanto per diftinguere quelle beftie che fino da tempo immemorabile s'allevavano in que'paefi da quelle altre, che furono poi trafportate colà da Europa: appelleremo dunque quefte europee, e quelle americane.

Il primo capo di rimprovero contra l'America è, preffo il Conte de Buffon, il piccol numero de'fusi quadrupedi paragonati con quelli dell' antico Continente. Egli numera dugen:

Storia Antica del MejJico Tomo IV.

Q

to 


\section{TOG DISSER TAZION E IV.}

to fpezie di quadrupedi in tutta la terra finora fcoperta, delle quali cento trenta fi trovano nell antico Continente, e folamente fettanta nel nuovo Mondo: e fe da tal numero if le. vano quelle, che fon comuni ad ambidue i Continenti, appe. na avremo, dice, quaranta fpezie di quadrupedi propriamente americani. Da quelto antecedente egli deduce, che nell' America ba fareggiato prodigiofamente la materia. (a)

Ma perchè vuol togliere all America delle fettanta fpe. zie, che ha di quadrupedi, quelle trenta, che fon comuni ad anendue i Continenti, mentre quefte per l'antichifitma loro abitazione in que' paefi fono così propriamente americane, come le altre? Oltracciò fe quelle beftie, ch'egli appella propriamente americane, foffero tate create da principio nell' $A$ merica, potrebbe forfe con qualche minore inverifimilitudine da lui affermarfi la pretefa fcarfità della materia in quella parte del Mondo; ma effendo ftate afiatiche nella prima lor origine tutte le beftie, com' egli. Ateffo confeffa, non fo, come eb. be ardire di dedurre una tal confeguenza. "Ogni animale, " dice il medefimo Conte de Buffon, abbandonato al fuo iftin"to, fi cerca una zona, ed una regione proporzionata alla fua "natura. "(b) Ecco dunque la cagione del minor numero delle fpezie di quadrupedi nell' America; perchè abbandonati al loro iftinto, dappoichè ufcirono dall' arca di Noè, fi cercarono, e trovarono ne' paefi dell' antico Continente una zona ed una regione convenevole alla lor natura: ficchè nan ebbero bifogno di fare quel lungo viaggio all' America. Se l'Arca di Noè in vece di fermarfi nelle montagne dell' Armenia, fi foffe fermata nelle alpi americane, farebbe allora ftato per la medefima ragione minore il numero delle fpezie de' quadrupedi nel Continente antico, e farebbe degno di biafimo il Filofofo ame. ricano, che da un tal antecedente pretendeffe arguire la prodigiofa fcarfita della materia, e il cielo avaro di quello, che ora chiamiamo Continente antico.

(a) Hift. nat. tom. 23.

(b) Hift. Nat. tom. 29 。 
Ma ancorchè tutti que' quadrupedi foffero veramente ori. ginarj dell'America, non dovrebbe quindi dedurfi la pretefa fcarfità della materia; perchè non può dirfi fcarfeggiante la materia in un paefe, il quale ha un numero di fpezie di quadrupedi proporzionato alla fua eftenfione.' L'eftenfione dell' America è la terza parte di quella di tutta-la terra: dunque non può dirfi, che vi fcarfeggia la materia, qualora vi fia la terza parte delle fpezie de'quadrupedi. Or le fpezie de' quadrupedi fono al dir del Conte di Buffon dugento, e l'America ne ha fettanta, cioè un poco più della terza parte: non può dunque dirfi, che vi fcarfeggia la materia.

Finora abbiamo ragionato nella fuppofizione che fia vero quanto dice il Conte de Buffon intorno al numero delle fpezie de'quadrupedi; ma chi lo fa, mentre non s'è ancora trovato il vero carattere diftintivo delle fpezie? Cosi il C. de Buffon, come parecchj altri Naturalifti, che hanno fcritto dopo di lui, credono, che l'unico indubitabile argomento della diverfira fpecifica di due animali fimili peraltro in molti accidenti e proprietà, è quello di non potere il máchio coprir la femmina,e produrre per mezzo della generazione un individuo fecondn, ed a loro fomigliante. Ma quelto argomento della diverfita, oltrechè falla in alcuni animali, è rapporto ad altri affai difficile da verificare. Acciocchè fi veda l'incertezza d'effo, mettiamo da una parte un afino, ed una cavalla, e da un' altra un ma. Atino, ed una levriera, due razze affai differenti di cani. Da quefta coppia nafce un cane, che ha del maftino, e della levriera; da quella nafce una mula, che ha fimilmente dell'afino, e della cavalla. Ora io voglio fapere, perchè l'afino, e la cavalla fono due fpezie diverfe di quadrupedi, e il maftino, e la levriera fono folamente due differenti razze d'una medefima fpezie. Perchè ouefta coppia, dice il C. de Buffon, genera un individuo fecondo, e quella no. Ma come? Lo fteffo $\mathrm{C}$. de Buffon nel tomo 29. della fua Storia, afferma fihiettamente, che il non concepire per l'ordinario le mule non è già per cagione di qualche affoluta impotenza, ma foltanto per l'ecceffivo caldo, e per le ftraordinarie convulfioni, che paticono 
nel coito. Il Sig. de Bomare (c) dopo aver citato il teftimo: nio d'Ariftotele, il quale nella Storia degli animali racconta, che a' tempi fuoi i muli della Siria provenienti da cavalli, ed afine generavano de'muletti loro fomiglianti , foggiunge: "Que"fto fatto, rapportato da un Filofofo affai degno di fede, pro"va, che le mule fono animali fpecificamente fecondi in fe "Atefi, e nella lor pofterita. "Simili fatti dimoftranti la fecondita delle mule trovanfi teftificati da molti Autori altrettanto degni di fede così antichi, come moderni, ed alcuni fono ancora accaduti a' noftri dì nel Meffico. (d) Non v'è dunque altra difparita tra quelle due coppie di quadrupedi, fe non che $\mathrm{i}$ parti delle cagne generate da quella coppia di cani fon più frequenti di quei delle mule.

Oltraccid da chi ha faputo il C. de Buffon, che il Gib. bone, ed il Magoto, il Mammone, ed il Pappione ( quattro for. ti di (cimie) non s'accoppiano fra loro, e generano un individuo fecondo? Nè quefto Autore ha fatto intorno a ciò veruna Sperienza, nè cita alcun altro Naturalita, che l'abbia fatta, e ciò non oftante decide, che turti $\mathrm{i}$ fuddetri quadrupedi fono altrertante fpezie diverfe. $E$ ' dunque in gran parte dubbiofa, ed infuffiftente la divifion delle fpezie de' quadrupedi da lui fatta, e non fappiamo, fe certi quadrupedi, che egli mette come fpezie differenti, fieno in fatti una fola fpezie, e per l'oppolto fe altri, che egli crede d'una fpezie, fieno invero fpecificamente diverf:

$\mathrm{Ma}$ lafciando quefto bafterebbe pure a cagionare una gran diffidenza della divifione, che il C. de Buffon fa de' quadrupedi, l'accorgerfi delle contraddizioni, che trovanfi tanto in que. fto, quanto in altri punti della fua Storia, benchè per altro affai pregevole. Nel difcorlo, che fa nel tomo 29. fulla degenerazione degli animali, afferma, che $\int e f i$ vuol fare l'enume. razio-

(c) Dictionn. d'Hiftoir. Natur. V. Mulet.

(d) Tra gli altri è degno di particolar menzione il parto replicato d'una Mula generata da afino, e da cavalla, accaduto nel groffo podere appellato il Salto di Zurita preffo alla Città di Lagos, appartenente al Cav. D. Fulgenzio Gonzalez Rubalcaba. Quefta Mula concepì da un afino,e partori un muletto nel 1762. e un altro nel 1763. 
razione de quadrupedi propri del nuovo Continente, troveremo cinquanta fpezie differenti, e nell' enumerazione, che fa de' quadrupedi d'ambidue i Continenti, dice, che quelli dell' America appena fanno quaranta fpezie. Nella fuddetta enumerazione mette, come fpezie differenti, quelle della capra domeftica, della camozza, e del buquetrino, o fia caprone falvatico, e nel tomo 24. trattando de'fuddetti animali, dice, che quefti tre quadrupedi, e le altre fei, o fette fpezie di capre, che diftinguono i Nomenclatori, fon turti d'una medefima fpezie: ficchè dovranno levarfi quefte otto; o nove fpezie da quelle cento trenta, che egli numera nell' antico Continente. Nella fuddetta numerazione conta il cane, il topo, e la marmotra, e foggiunge, che niuno di quefti quadrupedi era nell' America; ma ragionando poi degli animali comuni ad amendue $i$ Continenti, dice, che le marmotte, ed $\mathrm{i}$ topi fono fpezie comuni all'uno, ed all' altro Continente, benchè fia difficile da decidere, fe tali quadrupedi americani fono della medefima fpezie di quelli del Continente antico; e nel tomo I6. afferma, che i topi furono portati all' America fu'vafcelli dell' Europa . Quanto poi a'cani, che nella fuddetta enumerazione nega all' A. merica, gli accorda ad effa nel tomo 30.; poichè afferma, che il Xoloitzcuintli, l'Iizcuintepotzotli, ed il Tecbicbi erano tre differenti razze della medefima fpezie de' cani dell' antico Continente. Bafta quefto faggio per dare a divedere, che il C. de Buffon, malgrado il fuo grand' ingegoo, e la fomma fua diligenza, fi dimenticò talvolta di ciò, che avea fcritro.

Fra le I30. Ipezie di quadrupedi dell' antico Continente numera fette fpezie di pipiftrelli comuni nella Francia, ed in altri paefi d'Europa, delle quali le cinque, che erano innanzi fconofciute, o confufe, furono noviffmamente fcoperte, e diftinte dal Sig. Daubenton, ficcome egli afterma nel tomo 16. della fua Storia. Ora fe nella dotta Francia, dove tanti feco. li fa fi Atudia la Storia della Natura, furono finora ignorate cinque fpezie di pipiftrelli, qual maraviglia, che nelle vafte regioni dell' America, dove non fono ttati sì bravi Naturalifti, e neppur è gran tempo, che è in pregio un tale ftudio, vi fieno. 


\section{IO \\ DISSER TAZION E IV.}

fieno parimente fconofciute molte fpezie di quadrupedi. Io now dubito, che fe foffero alcuni Buffon, e Daubenton al nuovo Mondo, fi potrebbono contare affai più fpezie di quadrupedi di quelle, ch"egli numera da Parigi, dove non può avere intorno agli animali americani tutti que' lumi, the ha intorno agli europei. Mi fa invero pietà, che un Filofofo così celebre, così ingegnolo, così erudito, e così eloquente, il quale s' è meffo a frivere di tutti i quadrupedi del Mondo, diftingue le loro fpezie, famiglie, e razze, defcrive il lor carattere, la loro indole, ed $i$ loro coftumi, numera $i$ lor denti, ed anche mifura le lor code, fi moltri peraltro ignorante degli animali più comuni del Muffico. Qual beltia più comune, e più conofciura nel Meflico, che il Cojote?' D' effa fanno menzione tutti gli Storici di quel regno, e ne $f_{d}$ un" efatta e minuta defcrizione il Dott. Hernandez, la cui Storia troviano fpeffiflimo citata dal C. de Buffon; eppur quefto Autore non ne fa motto nè fotto que!lo, nè fotto verun altro nome. (e) Chi non $\mathrm{fa}$, che il coniglio era un quadrupede comuniffimo ne" paefi dell'Imperio Mefficano fotto il nome di Tocbtli, che la figura d'effo era uno de' quattro caratteri degli anni mefficani, e che del pelo della fua pancia fi teffevano giubboni per ufo de' signori nell' inverno? Conturtociò. il C. de Buffon vuole, che il Coniglio fia uno di que quadrupedi, che da Europa furono trafportati in America; ma fra tanti Storici europei del Meffico non ne ho trovato neppur uno, che il dica; anzi tatti fuppongono, che effo abiti da tempo immemorabile in que' paefi, ed io non dubito, che i Melficani in leggendo quelto fingolare aneddoro del Co. de Buffon, fi rideranno di lui.

Il Dott. Hernandez numera nella Storia dei quadrupedi quattro animali meflicani della claffe dei cani, da noi accennati.

(e) Cli animali dell'antico Continente, cui più ralfomiglia il Coiote, fono il Cbacal, ''Adive, e I'I Ifatis; ma è da effi diverfo. Il Chacal de della grandezza d'una volpe, il Cojote è al doppio maggiore. I Chacali vanno quafi fe npre accompagnati o trenta o quaranta; il Coiote va per lo niu folo. L'Adive è anche più piccolo, e più debole del Chacal. L'Iratis è proprio della Zona fredda, e fugge dai borchi; il Cojote ama i bolchi, ed abita nei paef caldi e temperati. 


\section{I S E R T A Z I O N E IV. II}

sati nel lib. I. della Storia: il primo il Xoloitzcuintli, o cane pelato, il fecondo l'Itzcuintepotzotli, o cane gobbo, il terzo il Tecbicbi, o cagnuolo commettibile, ed il quarto il $T e$ peitzcuintli, o cagnuolo montano. Quefte quattro diverffftme fpezie di quadrupedi fono ftate ridotte dal Co: de Buffon ad una fola. Egli dice, che il Dott. Hernandez s' ingannò in ciò, che Ccriffe del Xoloitzcuintli; perchè niun altro Autore ne fa menzione, e pertanto dee crederfi, che quel quadrupede vi fu trafportato da Europa; poichè il medefimo Hernandez afferma, averlo prima veduto in Ípagna, ed effo non avea nome nel Meffico; mentre Xoloitzcuintli è il nome proprio del Lupo, impofto dall' Hernandez a quell' altro quadrupede : che tuti quei cani erano conofciuti nel Meffico col nume generico d'Alco. Ecco in poche parole un ammaffo d' errori. Il nome Alco, o fia Allco non è mefficano, nè è ftato giammai ufato nel Meffico, ma nell' America Meridionale. Quello di Xoloitzcuintli non è pure il nome del Lupo, ne fo, che fia ftato mai veruno al Meffico, che l'abbia cosi appellato. I Mefficani, chiamano il Lupo Cuetlacbtli, ed in alcuni luoghi, dove non fi parla colla maggior proprietà il meficano, l'appellano Tecuani, il qual è il nome generico delle fiere. Confta inoltre dal medefimo tefto dell' Hernandez, che mettiamo quì fotto, $(f)$ che nè il Xoloitzcuintli fu trafportato dall' Europa al Meffico, nè tal nome fu ad effo impotto dall' Hernandez, ma era quello, con che l'appellavano i Mefficani. L'Hernandez avea veduto quel quadrupede in Ispagna; perchè v' era ftato trafportato dal Meffico, come egli medefimo accenna, ficcome avea veduto ne' giardini di Filippo II. parecchie piante mefficane. Ma perchè niun altro Autore ha fatto menzione del Xoloitzcuintli? perchè niun altro nè prima, nè dopo di lui ha intraprefo la Storia dei quadrupedi mefficani, e gli Storici di quel regno fi fon

con-

(f) Prater canes notos nofro orli, qui cmnes pene ab Hifpanis translati ab Indis in bis plagis bodie exucantur, tria alia offendas genera, quorum pimum, antequam buc me conferrem, vidi in patria; cateros vero neque confpextram, neque adbuc è delatos puto. Primus Xoloitzcuintli vocatus alios corporis vincit magnitudine or. Hernandez Hift. Quadrup. Novx Hifp. cap. 20. 
contentati di fare un motto degli animali più comuni. Del re: fto ogni uomo favio ed imparziale dovrà dar maggior creden: za al Dott. Hernandez nella Storia Naturale del Meffico, come a quello, che tanti anni in effa s'impiegò per ordine del Re Filippo II., e che coi fuoi proprj occhj offervd gli animali, di cui fcriffe, e s'informò a bocca dagli fteffi Mefficani, la cui lingua imparo, che non al Co: de Buffon; il quale quantunque più ingegnofo, e più eloquente, non ebbe altre notizie degli animali mefficani, fe non quelle, che fi procacciò nell'opera del medefimo Hernandez, o nelle relazioni di qualche altro Autore non tanto degno della noltra fede, quan: to quel dotto e pratico Naturalita.

Vuole il Co: de Buffon, che il Tepeitzcuintli dell' Her: nandez non fia altro, che il Ghiottone, quadrupede comune nei paefr più fettentrionali d'ambidue $i$ Continenti; ma chiunque voglia confrontar la defcrizione, che il Co: de Buffon fa del Ghiottone con quella, che il Dott. Hernandez fa del $\boldsymbol{T}_{e-}$ peitzcuintli, fcorgerà tolto un' enorme diversità tra quei due quadrupedi. (g) Il Ghiottone è , fecondo il Co: de Bulfon, proprio dei paef freddi del Settentrione; il Tepeitzcuintli è della Zona torrida: il Ghiottone è al dir del Co: de Buffon più grande al doppio del Taffo; il Tepeitzcuintli è, come dice l'Hernandez, parvi canis magnitudine. Il Ghiottone s' appella così a cagione della fua ftupenda, ed inaudita voracità; la quale lo porta all' ecceflo di diffotterrare i cadaveri per mangiarfeli; nulla di fomigliante dice l'Hernandez del Tepeitzcuintli, e non l'avrebbe tralafciato effendo quefto il principal carattere del Ghiottone; anzi afferma, che il Tepeitzcuintli s'addimeftica, e fi ciba di tuorli d'uova, e di pane disfatto in acqua calda, e non potrebbe certamente foftentarfene una beftia tanto avida di carne, quanto n'è il Ghiottone. Finalmente tralafciando altri argomenti della lor diverfita la pelle del Ghiottone è, fecondo che dice il Co: de Buffon, quafi tanto

(g) Buffon Hift. Nat. tom. 27. Hernandez Hif. Quadrup. N. Hifpania sap. 2I. 
tanto pregevole, quanto il zibellino; $(b)$ e non fappiamo, che quella del Tepeitzcuintli fia mai ltata pregiata, o ufata.

Effendo dunque il Xoloitzcuintli diftinto dal Lupo, ed il Tepeitzcuintli dal Ghiottone, ed effendo que' quattro quadrupedi americani della claffe de' cani affai fra loro diverfi nella grandezza, nell' indole, ed in molti altri accidenti notabili, nè conftando peraltro, che poffano fra loro accoppiarfi, e produrre un terzo individuo fecondo, dobbiamo conchudere, che fono quattro Spezie diverfe; e però debbono reftituirí all' America quelle tre fpezie levatele a torto dal C. de Buffon.

Non finiremmo mai. fe voleffimo accennar tutti gli abbagli di quefto Autore intorno a' quadrupedi Americani; ma per dimoltrare vieppiù, che il numero di fettanta lpezie da lui prefcritto all' America, non è giufto, ma affai mancante, e contrario altresì a ciò, che egli medefimo fcriffe nel decorfo della fua Storia, daremo alla fine di quefta differtazione una lifta de' quadrupedi americani cavata dalla fuddetta Storia, alla quale aggiungeremo i quadrupedi da lui confufi con altri alfa1 diverfi, e quegli ancora da lui affatto ommelfi, nel che faremo vedere, quanto fiafi allontanato dal vero nel dire, che nell' America ba faraseggiato prodigiofamente la materia. Oltrechè per conchiudere una tale fcarfita non balta farci conofcere, che fon poche le fpezie, ma bifognerebbe inoltre dimoftrare, che fon pochi gl' individui di tali fpezie; poichè fe gl'individui delle fettanta fpezie di quadrupedi americani fono più di quelli delle cento trenta 'pezie dell' antico Continente, farà bensì nell' America men varia la natura, ma non più fcarfa la materia. Bifognerebbe altresì dimoftrare, che fon parimente poche, e poco numerofe le fpezie de' retrili, e degti uccelli, poichè non meno quefte, che quelie, fervono a inanifeAtare la fcarfezza, o l'abbundanza della materia; ma chi è sì ignorante delle cole dell' America, che non abbia contezz dell,

Sioria Antica del Mejfico Tom. $I V$. P in-

(h) Il Sig. de Bomare dice, che la pelle del Ghiottone è più pregiata dai Kamtichatkefi, che il Zibellino, e che nella Srezia è afai ricercata, e molto cara. 


\section{II4 DISSER TAZION E IV.}

incredibile varietà, e della forprendente moltitudine degli uc: celli americani ? Or mi fi dica, perchè effendo ftata la Natura sì avara ne' quadrupedi verfo l'America, come vogliono il C. de Buffon, e il Sig. de Paw, è ftata si prodigiofa negli uccelli?

Non contenti quefti Autori di fminuir le fpezie de'quadrupedi americani, fi sforzano ancora d'appiccolire la loro ftatura. "Tutti gli animali dell' America, dice il C. de Buf"fon, (i) tanto quelli, che furono trafportati dagli nomini, "ficcome i Cavalli, gli Afni, i Tori, le Pecore, le Capre, "i Porci, i Cani \&c. quanto quelli, che vi paffarono per f: "Iteff, come i Lupi, le Volpi, i Cervi, e gli Alci, vi fono " confiderabilmente più piccoli, che in Europa: e quefto, fog" giugne, Senza veruna eccezione: "Il quale Itupendo effetto egli afcrive al cielo avaro dell' America, e alla combinazione degli elementi, e d'altre caufe fifiche. "Non v' era, dice il "Sig. de $P_{0}$, fotto la zona torrida del nuovo Continente verun 2) quadrupede grande. Il più gran quadrupede ( tra' proprj di ") que' paefi ) che efifte prefentemente nel nuovo Mondo fra' "tropici, è il Tapir il qual è della grandezza d' un Vitello. (k) "La beftia più corpacciuta del Nuovo Continente, dice il "C. de Buffon, è il Tapir, il qual è grande come una pic" cola mula, e dopo effo il Cabiai, il qual è della grandezza 9) d'un porco mediocre. "

Abbiamo gia dimoftrato nell' antecedente differtazione, che ancorchè concedeflimo a quelti Filofofi la pretefa piccolezza de' quadrupedi americani, nulla fi conchiuderebbe contra la terra, o contra il clima dell' America; poichè giufta i principj ftabiditi dal C. de Buffon, e da noi altrove citati, gli animali più grandi fon propri de'climi ecceflivi, ed i men grandi de' climi temperati e dolci: e fe dalla grandezza de'quadrupedi doveffero arguirfi i vantaggi del clima, diremmo fenz'altro, che il clima dell'Africa e dell' Afia Meridionale è molto migliore di quello dell' Europa. Ma fe nell' America, allorchè fu fco:

(i) Hiftoir. Natur. tom. 18.

(k) Recherch. Philofoph. part. 3. fec. 2. 


\section{DISS ER T A ZIONE IV. 115}

perta dagli Europei, non erano Elefanti, Rinoceronti, Ippopo. tami, Cammelli, nè Giraffe, almeno vi furono un tempo, fe diarno fede a' Sige de $P_{i}$, Sloane, du Pratz, Lignery, e parecchj altri Autori, i quali affermano l'antica efiftenza di quei gran quadrupedi nell America, fondati nel ritrovamento delle offa foflili, e de'carcami interi di fmifurata grandezza in diverfi luoghi di quel nuovo Mondo; anzi fe crediamo ciò, che il C. de Buffon furiffe nel tomo I8. della fua Storia, fu già nell' America un quadrupede fei volte più groffo dell'Elefante, appellato Mammout dal Sig. Muller ; $(l)$ ma nell' Europa nè fu mai, nè può effervi alcun quadrupede di prima grandezza. Nell' America non erano Cavalli, nè Afini, nè Tori, $(m)$ prim ma che vi foffero trafportati dall' Europa; ma nemmeno erano in Europa, prima che vi foffero trafportati, o menati dall' Afia. Turti gli animali traggono la lor origine dall' Afra, e quindi fi Cparfero per altri paefi : la vicinanza dell' Europa, ed il commercio dépopoli afiatici cogli europei agevolarono il paffag. gio di que' quadrupedi in Europa, e con effi vi furono anche portate alcune ufanze ed invenzioni utili alla vita, delle quali furono privi gli Americani per cagione della lontananza de'paefi, e della mancanza di commercio.

Allorchè il C. de Buffon affermò, che il più groffo qua. drupede del nuovo Mondo era il Tapir, e dopo effo il Cabiai, s'era affatto dimenticato delle Morfe, delle Foche, de'Bifonti, de'Renni, degli Alci, degli Orfi, e de' Huanachi. Egli Reffo

$$
\text { P } 2 \text { con- }
$$

(1) Artefo cid, che dice il Sig. Muller del fuo Mammout, quefto quadrupede farebbe ftato lungo piedi 133 , ed alto piedi 105 . II C. de Buffon ne parla cosi nel tomo r 5:," Il prodigiofo Mammout, le cui offa enormi noi abbiamo. "Fovente confiderate, e che abbiam giudicato fei volte almeno più grande " del più groffo Elefante, non efifte più.," Nel tomo 22. dice d'efrérfi afircurato, che quelle ofla fmifurate fono già ftate d'Elefanti fette orvero otto volte più grandi di quello, il cui carcame egli avea offervato nel Real gabinetto di Parigi; ma nella nuova fua opera intitolata. L'Epocbe della Natura torna ad affermare l'antica efiftenza di quell' enorme quadrupede in America.

(m) Quando diciamo, che non erano dei Tori nell' America, difcorriamo foltanto della razza comune, che s'impiega nell'agricoltura; poichè v'erano dei Bifonti, dei quali talora crede il C. de Buffon, che fono della medefima. fpezie dei Tori comuni, e talora ne dubita. 


\section{I6 DISSERTAZIONE IV.}

confoffs, (n) che la foca veduta da Lord Aadron, e da Rog: gers nell' America, e da Joro chiamato Leone marino, era incomparabilmente più grande di tutte le fuche del Mondo antico. Chi oferà paragonare il Cabiai, il qual non è più gran. de d'un mediocre porco, co'Bifonti, e cogli Alci? I Bifonti fon per lo più uguali a'Tori comuni d'Europa, e fovente gli eccedono in grandezza. Vedafi la defcrizione, che fa il Sig. de Bomare (o) d'un di quefti quadrupedi, trafportato dalla Luigiana in Francia, ed efattamente mifurato dal medelimo $\mathrm{Na}$ turalifta in Parigi nel I 769 . Havvi un' innumerabile moltitudine di quefti groffi animali nella zona temperata dell' America fettentrionale. Gli Alci del nuovo Meffico fon della grandezza d'un buon Cavallo. Vi fu un Cavaliere nella Città di Zacatecas, che fi fervi d'effi per la fua carrozza in vece di cavalli, ficcome ne fa fede il Betancurt, $(p)$ e talvolta fono ftati mandati alla Corte di Spagna da prefentarfi al Re Cat. tolico.

La propofizione univerfale, nella quale afferma il C. de Buffon, che turti i quadrupedi comuni ad ambidue i Continenti, fon più piccoli in America fenza veruna eccezione, è Itata Imentita da parecch; Autori Europei, che videro quegli animali co' propri lor occh;, ed anche dal medefimo C. de Buf. fon in altri luoghi della fua Storia. Dol Miztli, o Leone americano dice il Dott. Hernandez, che è più grande del Leo. ne della medefima fpezie dell' antico Continente. (q) Della Ti-

gre

(n) Hift. Nat. tom. 27.

(o) Diction. d'Hiftoir. Nat. V. Bifon. Il Sig. de Bomare appella quella beftia americana per la fua grandezza quadrupede colofjal: dice che la fua lunghezza dal mufo infino all'origine della coda mifurata per li fianchi era di piedi nove, ed oncie due: la fua altezza dalla fommita della gobba infino all' unghia di piedi cinque, ed oncie quattro: la fua groffezza mifurata nella gob12 , e nella giogata di piedi dieci di circonferenza. Soggiunge poi, aver egli intefo dal padrone di quella beftia, che le femmine erano ancor più grandi.

(p) Affai grandi dovrebbono effere quegli Alci per poier tirare le carrozze. che in quet regno s'ufavano nel fecolo paffato.

(q) Leoni nofrati minime jubato aut idem ef Miztli, aut congener, in infantia fuscus, \& fulvus in juvenia, interdumque rubeus, aut fubalbidus, in majarem tamen afsurgens molem, quod ob regionis diverfatatem poteft evenire. Hiftor. Quadrup. N. Hifp. cap. XI. 


\section{DIS S ER TAZIONE IV. IY}

gre mefficana afferma lo Ateffo. ( $r$ ) Nè il C. de Buffon, nè il Sig. de P. hanno una giufta idea di quella fiera. Io ne vidi una tra l'altre, poche ore innanzi ammazzata con nove archibufate, affai più grande di quello, che vuol farci credere il C. de Buffon. Quetti Autori poichè non fi fidano del ragguaglio degli Spagnuoli, dovrebbono almeno dar credenza al Sig. de la Condamine, Francefe dotto, e fincero, il qual dice, che le tigri da lui vedute ne'paefi caldi del nuovo Mondo non gli parvero diverfe dalle tigri africane, nè quanto a' bei colori della pelle, nè quanto alla grandezza \&c. Del Lupo meílicano dice il fuddetto Hernandez, che tanto nella figura, nel colore e nelle inclinazioni, quanto neila grandezza è fomigliante al Lupo europeo, fuorchè nell' avere quello la telta più groffa. $\left(\int\right)$ Lo fteflo afferma de' Cervi comuni, ed Oviedo de' cervi, e de" Daini. Il medefimo C. de Buffon malgrado la generalità del principio da lui ftabilito fenza veruna eccezione fu la minor grandezza de' quadrupedi americani, ragionando poi nel tomo 29. fu la degenerazione degli animali dice, che il Daino, ed il Capriuolo Cono tra'quadrupedi comuni ad ambidue $i$ Continenti que' foli, che fono più grandi, e più forti nel nuovo Mondo, che nell' antico; e difcorrendo nel tomo 27. della Lo. dra del Canadà confeffa, che efla è affai più grande di quelle dell' Europa, e lo fteffo dice del Caftore americano: Gicchè quegli, che non ammetteva veruna eccezione nel fuo principio; l'arnmette pure ne' Daini, ne' Capriuoli, nelle Lodre, ne' $\mathrm{Ca}_{2}$ ftori, e nelle Foche. Se a quefti poi s'aggiungono le Tigri, i Leoni fenza chioma, ed i Cervi giufta la teltimonianza d'Hernandez, e d'Oviedo, avremo almeno ot:o fpezie di quadrupedi comuni ad ambidue $i$ Continenti, che fon più grandi ne! Mondo nuovo, che nell' antico. A'fopradetti debbono fimilmen. te aggiungerfi que' quadrupedi, che fono ugualmente grandi nell' udo, e nell'altro Continente; poichè tanto quefti, quan.

to

(r) Vulgaris ef buic orbi Tygris, fed nofrate major. Hift. Quadr. N. Hifp. cap. X.

(s) Forma, colore, moribus, ac mole corporis Iupo nofarati fimilis eft Cuetlact:= Ii, atque adeo ejus, ut mibi videtwe, Jpeciei, fed ampliore capite.lbid. cap. XXISL. 


\section{8}

DISSERTAZIONE IV.

to quelli dimoltrano falfo il principio del $C$. de Buffon. Il D. Hersandez afferma, che il Lupa mefficano è della medefima grandezza dell Eurupzo. Il C. de Buffon dice, che fra l'uno e l'altro con v'è divario, fe non che il Lupo mefficano ha più bella la pelle, e cinque dita ne' piedi anteriori, e quattro ne' polteriori. Per ciò poi, che riguarda gli Orfi, fono prefentemente in Italia moltiffimi Europei, che hanao veduti gli Oifi del Meffico, e quelli delle Alpi. Non credo, che fra tanti teftimoni vi fia neppur uno, che abbia riconofciuto verun ecceffo negli Orfi europei. Io almeno finceramente protefto, che tutti quelli, $\mathrm{i}$ quali ho veduti nel Meffico mi fon paruci più grandi di quelli, che ho veduti in Italia a $(t)$

E' dunque fallo, che tutti gli animali del nuovo Mondo fono più piccoli di quelli del Mondo antico fenza veruna eccezione. $E^{\prime}$ pur falfiflimo, che fono tutti a f $a i$ più piccoli, e che la Natura s'è Servita nel nuovo Mondo d' una differente fcala di grandezza, ficcome afferma in un altro luogo il C. de Buffon. (u) Similmente fi può dimoltrar l'errore del Sig. de Pavv nel dire, che turti i quadrupedi dell" America-fono una fefta parte più piccoli dei lor analogi dell'antica Continente. La Tu$z a$ del Muffico è analoga della Talpa europea, ed è più gran. de di quefta, fecondo che dice il C. de Buffon. Quel quadrupede mefficano, che il C. de Buffon appella Coquallino, e noi Tlalmorotli, è analogo dello Scojattolo d' Europa, ed è al dir del medefimo Autore al doppio più grande: Il Toporagno del Brafile è analogo del Toporagno Europeo, e pur è più grande di quefto, come confeffa il fuldetto. Autore. Il Cojote analogo del Chacal è al doppio più grande. La Llama, o Montore del Perù analogo del Montone d' Europa è fenza paragone più grande \&c. Ma quefti Filofofi troppo impegnati nell' avvilixe l'America, e nel difcreditare i fuoi animali, trovano anche. da.

(t) Il C. de Buffon diftingue la rpezie degii orf neri da quella dei bruni, - afferma, che gli orf neri non fono panto feroci; ma gli Orfi meflicani, i quali fon tutti neri, fono pure ferociflimi ${ }_{2}$ com'è notorio nel Mellico, ed ia me pofo effer teftimonio.

(u) Hit. Nato tom. I8. 
da cenfurare nelle lor code, nei lor piedi e nei loro denti.

Non folamente, dice il C. de Buffon, fcarfeggiò prodigiolamente la materia neil nuovo Continente, ma eziandio fono imperfette le forme dei fuoi animali, e pajono effere ftate neglette. (x) Gli animali dell' America Meridionale, che fon quelli i quali propriamente appartengono a quefto nuovo Continente, fono quafi tutti privi di zanne, di corna, e di code: la lor figura è ftravagante, e le lor membra fproporzionate, e mal accozzate, ed alcuni, come i Formicari, ed i Pigri, d'una natura tanto miferabile, che hanno appena la facoltà di muoverfi, e di mangiare., (y)" Gli animali propri del nuovo Mondo, dice il Sig. de Pavv, fon per lo più d'una forma sgraziata, ed in alcuni così mal difpolta, che $\mathrm{i}$ primi Difegnatori non poterono, fe non a ftento, far fenfibili i lor caratteri. E' ftato offervato, che alla maggio: parte delle Tpezie manca la coda, e che hanno una certa irregolarita nelle zampe: il che è notabile nel Tapir, nel Formicaro, nel Glama di Margraf, nel Pigro, e nel Cabiai . Gli Struzzi, i quali nel nottro Continente non hanno più di due dita unite con una membrana, hanno turti quattro dita Ceparate nell' America.," (z)

Quefti difcorfi, a dire il vero, fono piuttolto una cenfura della condotta del Creatore, che del clima dell' America, non diffimile a quella beftemmia, che da alcuni fi attribuifce al $\operatorname{Re}$ Don Alfonfo il favio fulla difpofizione de' corpi celefti. Se i primi individui di quelle fpezie d'animali non vennero così dalla mano del Creatore, ma il clima del nuovo Mondo è ftato, la cagione delle pretefe loro irregolarità, qualora tali animali foffero trafportati in Europa, fi perfezionerebbe la loro forma, la loro indole, e il loro iftinto : almeno dopo dieci, o dodici generazioni quei miferabili animali, ai quali il maligno clima dell'America ha tolto la coda,

(x) Hift. Nat. tom. 18.

(y) Hiftoir. Nat. tom. 23.

(2) Recherch. fur les Americains part. I. 
da, le corna, e le zanne, le ricupererebbono fotto un clima più benefico. Nò, diranno quei Filofofi, perchè non è tanto facile il ricuperar nella natura ciò che fi perde, quanto il perdere ciò che fi ha: onde ancorchè quegli animali non poreffero riavere nell' antico Concinence la coda, le corna, o le zanne, turtavia potrebbe dirfi, che il clima dell' America è ftato la cagione di tal perdita. Sia pur cosi, e per tanto non difcorriamo ora delle irregolarita confiftenti in qualche difetto, ma di quelle, che fon tali per eccefio della materia. Dif́corriamo degli ftruzzi, i quali hanno per vizio della $\mathrm{Na}$ tura al dir del Sigo de Pavv, $\left({ }^{*}\right)$ due dita più in ciafcun piede, o per non lafciare i quadrupedi difcorriamo piuttofto dell' Unau, Spezie di Pigro americano, il qual tra le altre irregolarità, ha quella d'aver quarantafei colte. "Il nume. "ro di quarantalei cofte in un animale di si piccol corpo " è, dice il C. de Buffon, una fpezie d'errore, o d'ecceffo "della Natura; poichè niun animale nè anche dei più gran"di, o di quelli che hanno il corpo più lungo a proporzion " della lor groffezza, ne ha tante. L'Eiefante non ha più di " quaranta colte, il Cavallo trentafei, il Tafo trenta, il ca" ne ventifei, e l'Uomo ventiquattro. ,Ora fe il primo Unau, che fu al Mondo ebbe dalla mano di Dio quello fteffo numero di colte, che hanno prefentemente i fuoi difcendenti, il difcorfo del C. de Buffon è una cenfura del Creatore, e il dire, che quell' eccelfivo numero di cofte è ftato un errore delLa Natura, vorrà dire, che è tato un error di Dio, il qual è la vera Natura effetrice. Son ben ficuro, che una tal beftemmia è troppo aliena dalla fublime mente, e dal cuor crifitano del $C$. de Buffon; ma lo fpirito filofofico, che regna da pertutto nelle fue opere, l'induffe talvolta a far ufo di tali efpreffioni, che ben difaminate non piaceranno ai buoni Criftiani.

(*) Il Sig. de Pavr s'inganno nel numero delle dita del Tourou o fia Struzzo americano; poichè non ne ha più di tre; ma nella parte di dietro dei piedi ha un tubercolo tondo, e callofo, che gli ferve in vece di tallone, dal Volgo è Aato creduto un dito. 


\section{IS S E R T A I O N E IV.}

ftiani. (A) Se per l'oppofto credono quefti Filofofi, che l'Unau nella fua prima origine ebbe un numero di colte proporzionato alla grandezza del fuo corpo, e che il maligno clima dell America, glielo andò poi accrefcendo, douremo perfudderci, che qualora foffe trafportata quella fpezie di quadrupede nell antico Continente, e s'allevaffe fotto un clima più favorevole, fi ridurrebbe finalmente alla fua priftina perfezione. Facciafi dunque la fperienza: trafportinfi nel Mondo antico due o tre mafchi di quella fgraziata fpezie, ed altrettante femmine, e fe dopo venti, o più generazioni fi riconofce, che comincia in effi a diminuirfi il numero delle cofte, tofto confefferemo, che la terra dell' America è la più infelice, e il clima il più cattivo del Mondo. Se avviene altrimenti, diremo allora, ficcome infin da ora diciamo, che la logica di quefti Signori è più miferabile di quel quadrupede, e che i loro raziocinj fono meri paralogifmi. Del relto è veramente da maravigliare, che in un paefe dove tanto ha fcarfeggiato la materia, abbia la Natura peccato per ecceffo d'effa nelle cofte de' l'igri, e nelle dita degli Struzzi.

Ma per dare a divedere, che quefti Filofofi impegnati nel far comparir maligno il clima del nuovo Mondo, fi erano affatto dimenticati delle miferie del proprio lor Continente, addimandiamo loro, qual è l'animale più miferabile dell' America? Il Pigro, rifponderanno incontanente; perchè quefto quadrupede è il più imperfetto nella fus organizzazione, il più inabile pel moto, il più fprovveduto d'armi per la fua difefa, e foprattutto quello, che pare aver minor fenfazione di tutti gli altri animali: animale veramente infelice, condannato dalla Natura alla inerzia, alla fame, ed al pianto, col quale defta ognora negli altri animali la compaffione, e l'orrore. Ma quefta claffe di quadrupedi tanto faınofi per la lor mileria è Storia Antica del Meffico TomoIV.

(A) Volendo it C. de Buffon render la ragione, perchè l'uomo refifte più che gli aninali alle influenze de'cluni, dice così nel tomo 18 . L'uomo è in twito cpera del Cielo; gli animali non fon per molti riguardi fe nex produzioni della terra. Qlefta propofizione pare un pò troppo dura; ma aflai più dure fi liggono nella fua opera delle Epocbe della Natura. 
comune ad ambidue i Continenti. Il C. de Buffon non vuol crederlo, perchè non gli torna a conto, e dice, che fe qualche Pigro fi trova nell' Afia, quello v' è Atato tralportato dall' America; ma checche dica, egli è cerro, che l'Unau, una delle fpezie di Pigri, è animale afratico, come ne fanno fede Klein, Linneo, Briffon, il pubblicatore del Gabinetro di Seba, e Coprattutto Vofmaër, dotto e diligente Naturalifta Olan. defe. (B) L'Unau di Bengala veduto, allevato, ed efátamente defcritto da quefto Autore non può effere ftato trafportato dall' America; perchè non v' è ftato mai verun commercio tra l'America Meridionale, e l'Afia per poterlo trafportare. Oltrechè l'Unau di Bengala è diverfo dall' a mericano: quelto ha due fole dita nelle zampe, e quallo cinque. Se il C. de Buffon fi perfuade, che il clima dell' Afia potè accrefcere il numero delle dita nel quadrupede americano, diremo allora, che il clima dell'antico Continente farebbe anche capace di reftituire la coda, le corna, e le zanne a que' quadrupedi, a' qua. li le avea tolte il clima efiziale dell'America. Del refto chiunque voglia leggere, e confrontare l'eloquente defcrizione, che il C. de Buffon fa de' Pigri americani, e quella che il Signor de Vofmaër fa del Pigro pentadattilo di Bengala, tofto cono. fcerà, che quelto quadrupede afiatico è tanto miferabile, quanto quegli americani.

Ma efaminiamo flofoficamente cio, che dicono quelti Autori intorno alla pretela irregolarità di que' quadrupedi. La ve. ra irregolarità negli animali è la fproporzione nelle membra, - la fconvenenza nella forma, o neli'indole d'alcuni individui rapporto al comun della fpezie, non già quella, che s'offerva in una nuova fpezie paragonata con un altra conolciuta. Sarebbe fenz altro fciocco chiunque riputaffe irregolare il Tecbicbi, perchè non abbajz. Quefto è un quadrupede americano, il quale, perchè fomigliante a' cagnuoli europei, fu appellato cane dagli Spagnuoli, non perchè foffe della medefima fpezie: e quindi ebbe origine quella favola fpacciata da non pochi Autori

(B) Defoription de plufaeurs Animaux. Opera ftampata in Amferdam. 
tori europei, che nell' America erano i cani muti. I Lupi fo: no fomigliantiffimi a' cani, eppur non abbajano. Se i primi Spagouoli, che andarono in Meffico, non aveffero mai veduti de' Lupi nell' Europa, in vedendo quelli del Meffico avrebbono pubblicato, che v'eraro de" cani grandi, che non fapevano addimefticarfi, e che in vece d" abbajare urlavano: ed eceo quit un nuovo argomento, di cui fi farebbono prevaluti il C. de Buffon, e il Sig. de PaW per provare la degradazione, e l'ir regolarità degli animali americanio.

In fatti non è d'altro calibro l'argomento del Sig. de: $\mathrm{Paw}$ fu gli Struzzi americani. Il Touyou $\left({ }^{*}\right)$ è un uccello ame. ricano fpecificamente diverfo dallo Struzzo: ma perchè è affai grande, e molto fimile a quel grande uccello africano, $\grave{e}$ ta. to volgarmente appellato fruzzo. Quefto bafta al Sigo de $P$. per affermare, che v'è dell'irregolarità in quegli uccelli americani; ma ancorchè gli concedeffimo: per grazia, che il Touyou è un vero ftruzzo, non potrebbe mai convincere ciò che vuole. Egli vuol farci credere irregolare lo ftruzzo americano, perchè in cambio d'aver due fole dita unite con ura membra. na, come l'africano, ne ha quattio feparate. Ma un americano potrebbe dire, che lo ftruzzo africano è piuttofto irregola. re; perchè in voce d'aver quattro dita feparate, ne ha foltanto due, e quelle unite per mezzo d'una membrana. "No, "replicherebbe tutto in collera il Sig. de P., non è così: l'ir"regolarità è certamente ne"voftri ftruzzi; perchè non fì con "formano cogli ftruzzi del Mondo antico, che fon gli efem" plari della f́pezie, né col ritratto, che di tali uccelli ci la" Cciarono i più famofi Naturalifti dell' Europa ." "Il noftro. "Mondo, rifponderebbe l'Americano, che voi appellate nuo "vo; perchè: tre fecoli fa non era ancor da voi conofciuto, e ". tant'antico, quanto il Mondo voftro, ed i noftri animali fon "parimente coetanei de"voftri. Nè quelli hanno verun obbligo 3s) di confurmarfi co'voltri animali, nè noi abbiamo la colpa, Q 2: "che

${ }^{*}$ ) Nel Perù è conofeiuto lo Struzzo col nome di Suri; naa io adopro quit quello di Touyou per condifcendere ai noftri Naturalifti. 
3) che le fpezie de'noftri animali fieno ftate ignorate $\mathrm{da}^{2}$ voftri "Naturalifti, o confufe per la fcarfezza de' lor lumi. Sicchè o " fono irregolari $i$ voltri ftruzzi, perchè non $\mathfrak{f}$ conformano "co' noltri, o almeno i noftri non debbono dirfi irregolari, per") chè non fi conformano co'voitri. Finattantochè non mi di"'moltriate con documenti innegabili, che $\mathrm{i}$ primi ftruzzi ven3) nero dalla mano del Creatore con due fole dita unite con una " membrana, non mi perfuaderete mai l'irregolarirà de' noltri "Touyou. , Quefta ragione fenza dubbio efficaciffima ferve ancora per diffipare altri sì fatti difcorf de' noltri Filofofi, cagionati dall'imperfezione delle idee, 0 dalla lor prevenzione in favor dell' antico Continente.

Non fono in vero più felici i noftri Filofon ne'lor difcor. fi fu le code de'quadrupedi, che in ciò che fcriffero intorno a'piedi degli ftruzzi. Eglino dicono francamente, e fenza ve. run riguardo alla verita, che la maggior parte de' quadrupedi del nuovo Continente è affatto priva di coda : to che ficcoma tutti gli altri effetti da loro offervati in quegli fventurati paefi, eglino afcrivono all' avarizia del Cielo americano, all' in. fanzia della Natura in quella parte del Mondo, al cattivo clima, ed a non fo quale combinazione degli elementi. Così ragionano queti celebri Filofof del fecolo illuminato. Ma effendo al dir del C. de Buffon fettanta le fpezie de' quadrupedi americani, bifognerebbe, che almeno quaranta foflero fenza coda, acciocchè folfe vero, che la maggior parte è priva di tal membro, come dice il Sig. de $P_{0}, e$ molto più per verificare, che quaje tutti i quadrupedi fono sforniti di coda, come afferma il C. de Buffon. Or fiffati quadrupedi nell' America fono al più fei, ficcome appreffo vedremo: dunque la loro propofizione è una fmifurata iferbole, per non dire una groffa bugia.

Pare, che a' rempi di Pinio non foffero conofciuti da'. Naturaliti europei altri animali fenza coda, che l' Uomo, e la Sci. mia. (C) Se da allora in qua non folfero ftati fcoperti nell'ansico

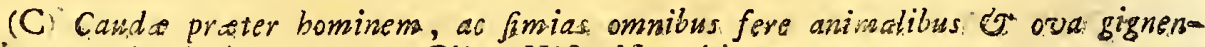
tikus pro defuderio corporum. Plin. Hiko. Nata lib. 8I. cap. 5o. 


\section{I S S ER T AZ IO NE IV. I2S}

tico Continente parecchj altri quadrupedi parimente sforniti di

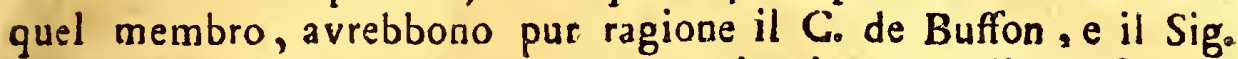
de P. di tacciarne i quadrupedi americani; ma dalla fteffa Storia del C. de Buffon contta, che fono più le fpezie de'quadrupedi fenza coda nell' antico Continente, che nell' America. Ecco la lifta degli uni, e degli altri cavata dalla fuddetta Storia.

\section{QUADRUPEDI SENZA CODA NEL CONTINENTE ANTICO.}

I. Il Pongo, offia l' Orang.outang, o Satiro, ovvero nomo falvaggio.

2. Il Pireco, o Scimia propria .

3. Il Gibbone, un'altra Ipezie diverfa di fcimia

4. Il Cinocefalo, o Magoto.

5. Il Can turco.

6. II Tanrec di Madagafcar.

7. 11 Loris di Ceilan.

8. Il Porcellino dell' India.

$\left.\begin{array}{l}\text { 9. La Ruffetta } \\ \text { 10. La Rugetta }\end{array}\right\}$ due fpezie di groffi pipiftrelli dell' Afia:

II. La Talpa dorata della Siberia.

A' quali debbono aggiungerfi $i$ tre feguenti.

I2. Il Pigro pentadattilo di Bengala, defcritto dal Sig. di Vofmä̈ .

13. La Klipda, o Marmotta baftarda del Capo di Buona fperanza, defcritta dal medefimo Siz. de Vofmaër.

14. Il Capiverd, o Capivard del C. di Buona Speranza, defcritto dal Sig. de Bomare.

\section{NELL' AMERICA.}

ฐ. L'Unau, Spezie di Pigro.

2. Il Cabiai, o Porco anfibio.

3. L'Aperea del Brafile.

4. Il Porcellina dell'India. 
5. II Saino, Pecar, o Cojametl.

6. Il Tapeto.

Sicchè nell' antico Continente fono almeno quattordici fpe. zie $(D)$ di quadrupedi sforniti di coda, e nell'America fono foltanto fei, dalle quali potremmo levar le due ultime, perchè fono incerte. $(E)$ In tutti i trenta tometti della "Storia de'quadrupedi del C. de Buffon non ho trovato altro animale americano fenza coda, fuorchè $\mathrm{i}$ fuddetti . E ciò non oftante osò affermare, che nel nuovo Mondo quafi tutti gli animali erano privi di coda. Nel che fi vede, che sì fatte propofizioni uni. verfali fono tanto facili da profferire, quanto difficili da provare.

Se il clima dell' America è tanto perniciofo alle code degli animali, perchè effendo affatto prive di tal membro quattro f́ezie di fcimie dell'antico Continente, cioè il Pongo, il Piteco, il Gibbone, il Cinocefalo, l' hanno pure tutte le fpezie di fcirnie del nuovo Mondo, ed alcune, ficcome i Saki, hanno coda tanto lunga che è fefquidupla del loro corpo ? Perchè abbon. dano tanto nell' America gli Scojattoli, i Coquallini, i Formicari, ed altri fiffatti quadrupedi forniti d' un enorme coda a proporzione de'loro corpi? Perchè la Marmottadel Canadà, conturtochè fia della medefima fpezie di quella delle Alpi, ha pur Ia coda più grande, come confeffa il medefimo C. de Buffon? Perchè il Cervo, e il Capriuolo dell' America, conturtochè fieno pì̀ piccoli di quelli dell' antico Continente, hanno: pure la coda.

(D) Alle quattordici ruddette fpezie potrei aggiugnere. 'IJnau didattilo di Ceilano, di cui fanno menzione parecchi Autori, e il Porte-mufe o fia Portamofco defcritto dai. Sig. d'Aubenton, e Bomare; ma tralafciai il primo; perchè non. fon ficuro, che fia diverfo dal Loris, del C. de Buffon : tralafciai ancora il fecondo; perchè può effere che abbia qualche piccola coda, febbene non potè trovarla il diligente Sig. d' Aubenton: onde parimente dorrebbono levarfi come incerte quelle due ultime fpezie di quadrupedi americani.

(E) Il Pecar fi trova defcritto da. Oviedo, da: Hernandez, e da Acofta fotto i nomi di Saino, e di Cojatmetl, e nulla dicono quefti Autori della mancanza di coda. Io però m' informai da perfone critiche e fincere, che hanno ve duti molti Saini, e mi differo, chieffi aveano: la lor coda, benchè piccola . Intorno al 'Tapeto crede il C. de. Buffon, che effo fia il Citli d' Hernandez. Or tutti i Meficani fanno, che il Citli'd'Hernandez è la Lepre del" MeflicQ a fam ficuri, ch'effa ha la. Lua coda come la Lepre comune d' Euraga 
coda più lunga, fecondo che afferma lo fteffo Autore? $(F)$ Se mai foffe nell' America qualche principio diftruttivo delle code degli animali, quelli che vi tráportò Colombo dall' Europa, e dalle ifole Canarie nel I493. farebbern già rimafti affatto fcodati, maffimamente i Porci, che portarono delle code si piccole, o almeno lor fi farebbono notabilmente raccorciate dopo anni 288.; ma fra tanti Europei, che hanno veduto le Pecore, i Cavalli, i 'Buoi \&c. nati nell' America, e quelli, che prefentemente s'allevano in Europa, non vi fatà ne anche uno, che poffa trovar qualche divario tra le code degli uni, e degli altri.

Quefto medefimo argomento vale parimente contra ciò, che dice il C. de Buffon fu la mancanza di corna, e di zanne nella maggicr parte de'quadrupedi americani, poichè $i$ Buoi, le Pecore, e le Capre confervano invariabili le loro corna, i Cani, ed $\mathrm{i}$ Porci $\mathrm{i}$ lor denti, ed i Gatti le lor unghie, come Tanno tutti quelli, che gli hanno veduti, e paragonati con quelli d'Europa. Se il clima americano foffe tanto efiziale a'denti, ed alle corna degli animali, avrebbono già perduto almeno una buona parte d'effe i difcendenti di que' quadrupedi europei, che vi furono trafportati quafi tre fecoli $f_{2}$, e molto più la pofterità de' Lupi, degli Orfi, e d'altri fimili quadrupedi, che vi paffarono dall' Afia forfe fin dal primo fecolo dopo il diluvio univerfale. Se per lo contrario la zona remperata dell' Europa è più propizia a' denti degli animali, che la zona torrida del nuovo Mondo, perchè la Natura diede a quefta, e non a quella i Tapir, ed i Coccodrilli, i quali nel numero, nella grandezza, e nell' atrocita de'denti eccedono rutti i qua. drupedi, ed i rettili europei ?

Finalmente fe fono alcuni animali nell' America fenza corna, fenza denti, $(G)$ e fenza coda, non è già per cagione del

(F) Hif. Nat. tom. I 8 .

(G) Fra tutri i quadrupedi del nuovo Mondo non ve ne fono altri privi di denti, fuorchè i Formicari, ficcome vi fono nel Continente antico il Pangolino, e il Fatagino, quadrupedi dell'India Orientale coperti di fquame in vece di pelo. Tutti quefti quadrupedi, come quelli che non fi cibanod altro, che di formiche, non hanno bifogno di denti per foftentarfi; ma peraltro fo- 
del clima, o del Cielo avaro dell' America, o di quella imma: ginaria combinazione degli elementi; ma perchè il Creatore, le cui opere fon perfette, ed i cui configli dobbiamo umilmente riverire, gli volle far così, perchè tal varietà ferviffe all'abbellimento generale dell'Univerfo, ed a fare fpiccar vieppiù la fua fapienza, ed il fuo potere. Quello fteflo, che rende belli alcuni animali, altri farebbe deformi. Nel Cavallo è perfezio. ne l'avere la coda grande, nel Cervo l'averla piccola; nel Pongo l' efferne affatto privo.

Quanto a ciò, che dicono i noftri Filofofi intorno alla bruttezza degli animali americani è vero, che fra tanti ve ne fono alcuni, la cui forma non corrifponde all'idea, che abbiamo della bellezza delle beltie. Ma chi ci ha afficurati, che tal idea fra giulta, anzichè imperfetta, e cagionata dalla limitazione della noltra mente? E quanti altri animali non potremo trovar nell'antico Continente ancor peggio formati di tutte le beftie americane? (Difcorro qui giufta le idee di que' $F_{i}$ lofofi ; poichè del refto rifpetto la mano del Creatore in turte le fue opere.) Che quadrupede è nell' America, che poffa paragonatfi nella deformità, e nella fproporzione delle membra coll'Elefante, chiamato Mofro di materia dal medefimo C. de Buffon? (H) Quella valta mole di carne più alta che lunga : quella pelle fchifola priva di pelo, e folcata di grinze : quell' enorme tromba in vece di nafo: que'lunghi denti meffi fuori da quella bruttiffima bocca, e rivolti all' insù, al contrario di quello, che $f$ offerva in altri animali, per aumentar vieppiù la deformità della fua faccia: quegli orecchi valti, e poligoni: quelle gambe groffe, Atorte, e fproporzionatamente piccole: que'. pie-

no Aari dal Creatore provveduti d'una lingua affai lunga, colla quale pigliano deframente le formiche, e le inghiottifcono.

(H) "En confidérant cet animal ( dice dell' Elefante il Sig. de Bomare) re, lativement a l'idée, que nous avons de la iufteffe des proportions, il fem") ble mal-proportionné, à caufe de fon corps gros a court, de res iambes ", roides \& mal-formées, de fes pieds ronds \& tortus, de fa grofte tête, da " res perits yeux. \& de fes grandes oreilles: on pourroit dire auf que l'ha", bit dont il eft couvert eft encore plus mal taillè \& flus mal fait. Sa trom" pe, res défenfes, fes pieds le rendent auffi extraordinaire, que la grandeur „, de ra taille, 
piedi informi colle dita appena abbozzate, e finalmente que' minuti occhietti, e quella ridicola codetta in un corpo sì fmifurato, non rendono I' Elefante il quadrupede più irregolare? Sfido i noftri Filofofi a trovarmi nel nuovo Mondo un quadrupede più fproporzionato, e la cui forma fia più fgraziata. Simili rifleffioni fi potrebbono ancora fare ful Cammello, fu la Giraffa, ful Macaco, del quale dice il C. de Buffon, che è d' una deformita Spaventevole, e fopra altri animali dell' antico Continente, e non però ofiamo biafimare il clima, che gli alleva, e nemmen cenfurare il fupremo Artefice, che gli formò.

Ciò poi, che dicono i noftri Filofofi intorno alla minor ferocia delle fiere americane, in vece di giovar loro per pro. vare la malignità di quel clima, non ferve ad altro, che a dimoltrare la fua dolcezza, e bontà. "Nell' America, dice il „C. de Butfon, (I) dove l'aria e la terra fono più miti di "quelle dell' Africa, la Tigre, il Leone, e la Pantera non "fon terribili fe non nel nome... Effi hanno degenerato, fe " pur la ferocia aggiunta alla crudeltà faceva la lor natura, o " per dir meglio, non altro hanno fatto, che foffrir l'influen. "za del clima: fotto un cielo più dolce il lor naturale s' è "raddolcito. "Che puod davvantaggio defiderarfi in favor del clima dell' America? Come dunque s'allega la minor ferocia delle beftie americane, come un argomento della lor degenerazione, cagionata dalla malignità di quel clima? Se il clima dell' antico Continente dee reputarí miglior di quello del nuo. vo Mondo, perchè fotto quello s'allevano le fiere più terribili; dovrà crederfi per la medefima ragione il clima dell' A frica incomparabilmente più eccellente di quello dell' Europa. Que. fto argomento da noi altrove adoperato debbe inculcarfi per maggior confufione de' noftri Filofofi.

Ma quefti Autori non hanno una giufta idea delle fere americane. E' vero, che il Miztli, o Leone mefficano non paragonabile co' celebri Leoni dell' Africa: quefta ́pezie o non pafsò mai nel nuovo Mondo, o fu eftinta dagli uomini; ma non

Storia Antica del Me/fico Tom. IV.

$\mathbf{R}$ la

(I) Hiftoir. Nat. tom. I2. 
la cede quella beftia americana a quelle della fua fpezie, o fia a'Leoni fenza chioma dell'antico Continente, ficcome depone l'Hernandez, il quale conofceva bene e le une, e le altre. La Tigre mefficana o fia, o non fia della medefima fpezie delle Tigri Reali dell' Africa, poichè ciò non c'importa nulla, è d'una forza, e d'una ferocia forprendente. Non v'e quadrupede nè tra gli europei, nè tra gli americani, che poffa contraftar con effa. Affaiifce intrepidamente, e fquarcia gli Uomini, i Cervi, i Cavalli, i Tori, ed anche i più orrendi Coccodrilli, come ne fa fede l'Acolta. (K) Quefto dotto Autore vanta l'intrepidezza, e la velocità di quella fiera. Gonzalo d'O. viedo, il quale avea viaggiato per molri paefi dell' Europa, e non era ignorante della Storia Naturale, ragionando di quelle Tigri americane, dice $(L)$ così : "Sono animali affai forti di " gambe, ben armati d'artigli, e così terribili, che a mio " giudizio, non v'è Leone Reale de' più grandi, che poffa con effi "gareggiare nella forza, e nella ferocità . "La Tigre è il terrore de" bolchi dell'America: non è capace d'appiacevolirfi, nè di lalciarfi pigliare, quando è adulsa: quelle, che fi pigliano ancor piccole, non poffono cuitodirfi fenza pericolo, fe non racchiufe in gabbie fortiffime di legno, o di ferro. Tal è il carattere di quelle beftie, che fono appellate poltroni dal Sig. de P., e da altri Autori, che non feppero difcernere le fpezie de'quadrupedi di pelle macchiata.

Egli e peraltro certo, che quegli Autori fi moftrano tanto facili nel credere tutto ció, che trovano fcritto intorno al. Ia grandezza, alla forza, e all'intrepidezza delle Tigri Reali dell' antico Continente, quanto oftinati nel negar fede a ciò, che dicono delle Tigri americane parecchj teftimonj oculati . Il C. de Buffon crede fu la fede di non fo quali Autori, che la Tigre Reale ha infino a tredici o quattordici piedi di lunghezza; e cinque d'altezza: che combatte infieme con tre Ele-

(K) Stor. Nat., e Morale lib. 3 cap. I 7.

(L) Sommario della Stot. Natur. cap. Ir. vedafi ancora cio che ne dice Sig. Ab. Gilij nel tomo I. della Storia dell'Orinoco lib. 5. cap. 6. 
Elefanti: che ammizza un Bufalo, e lo Arafcina facilmente fin dove vuole, ed altre fiffatte maraviglie, le quali non poffono crederfi da coloro, che non. fono tanto prevenuti in favor dell'antico Continente. Se alcuni Autori degni di fede raccontaffero delle Tigri americane: una piccola parte di quello, che eglino dicono delle Tigri afiatiche, farebbono tofto fenz' alcun efame rigetrati, come millantatori. ( $M$ ) Il ragguaglio, che $\mathrm{fa}$ Plinio $(N)$ della indutria de' Cacciatori nell' involare alla Tigre i fuoi figli, e della flemma, colla quale la Tigre: gli va ad uno ad uno ricuperando, e quello, che $f_{a}$ il Sig. de Bo. mare $(O)$ del combattimento accaduto l'anno 1764 . nella fel. va. di Windfor in Inghilterra tra un Cervo, ed una Tigre portata dall' Indiza al Duca di Cumberland, nel quale reftò vincitore il Cervo, dà a divedere, che la ferocita di quelle beftie afiatiche non è tanto grande, quanto la rapprefentano il Co de Buffon, e il Sig. de $P_{\text {o. }}$

I Lupi americani non fono nè men forti, nè men arditi di quelli dell'antico Continente, come fanno bene tutti quelli, che hanno fperienza degli uni, e degli altri Anche i Cervi, i quali fono al dir di Plinio $(P)$ gli. animali più tranquilli, fon nel Meffico così audaci, che fovente affalifiono i. Caccia. tori, come ne fa fede l'Hernandez (Q), ed è notorio in quel Regno. Io ho veduto co' miei occhi la ftrage fatta in cala mia. da un Cervo, divenuto quafi. domeftico, in una povera Ame. ricana.

Ma fieno pur più piccoli, più fgraziati, e più pufillanimi i quadruped a mericani. Concediamo ancora a que" Filofof, che da un tal antecedente poffa dedurfi la bontà del clima dell' antico Continente; non però potranno mai perfuaderci, che effa fia. una pruova compiuta, ed un argomento certo della mali-

$$
\text { R. } 2 \text {. }
$$

gnità

(M) Bafta fapere il conto, che fanno quegli Autori della reftimonianza del Sig. de la Condamine intorno alle Tigri americane, malgrado della Atima, neila quale è preffo loro, e prelfo tutti, quel dotto Matematico.

(N) Hiftor. Natur. lib. 8. cap. I 8:

(O) Bomare Dictionn. d' Hiftoire Nat. v. Tigre.

(P) Hift. Nat. lib. 8. cap. 32.

(Q) Hift, Nat. lib. 9. cap. I4. 
gnità del clima americano, mentre non ci facciano vedere ne" retrili, e negli uccelli dell'America $(R)$ quella fteffa degradazione, ch'eglino fuppongono ne' quadrupedi. Il Sig. de P. dice de' Coccodrilli americani, la cui ferocità è tanto notoria, che pare per le offervazioni del Sig. du Pratz, e d'aliuni altri, cb'effe non banno il furore, e l' impetuofita di quelli dell Africa; ma il Dort. Hernandez, il quale conofieva bene e gli uni, e gli altri, non trovd alcun divario fra loro. (S) Acolta dice, che gli americani fono ferociffimi, na lenti; ma quelta lentezza non è già nel moto progreflivo per linea dritta, nel che fono affai veloci, ed agili, ma foltanto nel voltarf, o pie:garfi all' una, o all' altra parte, ficcome arviene ancora ne'coc:codrilli africani fenz' altro per cagione dell' infleffibilità delle lor vertebre. Il Dote. Hernandez afferma, che l'Acuetspalin, - Coccodrillo mefficano fugge da coloro, che l'affalifcono, perfeguita coloro, che fuggono (benchè quefto accada più comunemente di quello.) Plinio dice lo fetfo de Coccodrilli a fricani. ( $T$ ) Finalmente fe fi paragona cid, che racconta Plinio di quelti, con quello, che dice Hernandez di quelli, fi troverà, che neppur nella grandezza 'v' è differenza fra loro. ( $V)$

Quanto poi agli uccelli, il Sig. de $P$. non ne fa menzione, fuorchè degli Struzzi, e ciò tanto fconciamente, quanto ab-

(R) Il Sig. de Buffon potrebbe dire ficcome accenna nel tomo 18 . che non 6 dee far conto degli uccelli per quello, che riguarda il clima; perchè poten-. do eff facilmente pajJare dall' un Continente all altro farebbe quafi impo/fibile di difinguere, quali appartengano propriamente all'uno, o all'altro. Md ficcome ia. cagione de'viaggi, che fanno gli uccelli, e il freddo, o il caldo delle ftagiani, che cercano di fchivare, così non hanno bifogno gli uccelli americani di ufcire dal lor Continente; perchè hanno quivi paefi d"ogni forta di climi per difenderfi dalla ftagione lor nocevole, e cercarfi. il lor vitto. Siamo affatto ficuri, che gli uccelli mefficani non fanno viaggi all'antico Continente.

(S) Hern. Hirt Nat. lib. 9. cap. 3.

(T) Terribilis bee contra fugaces bellue ef, fugax contra infequentes. Plin. Hift. Nat. lib. 8. cap. 25 .

(V) Plinio dice, che il Coccodrillo afticano ba fovente più di dicioto cubiti di lunghezza o fia piedi romani ventifette. Il Dott. Hernandez afferma, che il Coccodrillo mefficano fuol effer lungo più di fette paffi. Se egli parla di paffi caftigliani, fanno piedi romani quafi ventotta; fe parla di paffi romani faranno piedi trentacinque: ficchè it divario è piccolo, 0 fe vi è qualthe ecceffo, efro è dalla parte del coccodrillo americano. 
abbiam veduto. Prefe fenz' altro il partito di tacere, perchè riconobbe perduta da quefta parte la fua caufa; imperocchè or fia nel numero, o nella varieta delle fpezie, or nell' intrepidez. $z a$, or nella vaghezza delle penne, or nell' eccellenza del canto non poffono certamente gareggiare cogli uccelli americani quelli dell' antico Continente. Della forprendente lor moltitudine abbiamo ragionato altrove. I campi, i bofchi, i fiumi, i laghi, ed anche i luoghi abitati fono pieni $d^{\prime}$ innumerabili fpezie. Il Gemelli, il quale avea fatto il giro del Mondo, ed era ftato ne' migliori paefi dell' Afia, dell' Africa, e dell' Euro$\mathrm{pa}$, protefta, che non v'è paefe al Mondo, il qual poffa paragonarfi collia Nuova Spagna nella vaghezza, e nella varietá degli uccelli. $(U)$ Vedafi ancora ciò che ne dicono gli Storici della Nuova Francia, della Iuigiana, del Brafile, e d'altrỉ paefi del nuovo Mondo.

Della forza, e dell' animofità degli accelli americani fan. no teftimonianza molti Autori Europei affai degni di fede. Il Dott. Hernandez, il quale avea avuta tanta fperienza degli uccelli di rapina nella Corte di Filippo II. Re di Spagna, allorchè più che mai era quivi in pregio la frafconaja, ed avea ancora offervate quelli del Meffico, confeffa, quando ragiona del Quaubtotli, o Sagro mefficano, che tutti gli uccelli di quefta claffe fono migliori, e più coraggiofi nella Nuova Spagaa, che nell' antico- Continente. $(X)$. Per cagione d'effere fta. ta conofciuta fin dal principio l'eccellenza de" Falconi america. ni, fu ordinato da Carlo V., che turti gli anni gli foffero mantdati alla Corte cinquanta Falconi dalla Nuova Spagna, ed altrettanti dall' ifola Spagnuola, ficcome ne fa fede lo Storico Herrera, $(\mathcal{X})$ e il P. Acolta racconta, (Z) che i Falconi del Meffi-

(U) Ella è tanta la vagbezza, e la varietd degli uccelli della Nuova Spagna, che non $v$ ' $\dot{e}$ paefe al Mondo, Gbe ne abbia: pari. Giro del Mondo tom. 6. lib.2. cap. 9.

(X) Fateor Accipitrum omne genus apud hanc Novam Hifpaniam, Jucatanicamve provinciam repertam praftantius effe atque animofisus vetere in orbe natis. Hernandez De Avibus N. Hifp: cap. 92.

(Y) Herrera Dec. 3. lib. 6. cap. L.

(Z) Hift. Nat. y Mor. de las Indias lib. 4. cap. 3.5. 
Mefficn, e del Perù, percbè erano affai pregiati, fi mandavano a prefentare à Magnati di Spagna. Lo fteffo Acofta dice, (aa). che i Condorj, o. Avoltoj Americani fono d' una immenfa grandezza, ed banno tanta forza, cbe non folo jquarciano un montone, ma eziandio un vitello; e D. Antonio Ulloa teftifica, (bb) che d'uo colpo d'ala martono. a giacere un uomo. (cc) Il Dort. Hernandez dice, ch: l'Itzquaubtli, o Aquils Reale del Maffico affalifce gli uomini, ed anche i piu feroci quadrupedi.(dd) Se il clima dell' America aveffe tolto a' quadrupedi la forza e. il coraggio, aurebbe fenza dubbio cagionato il medefimo effetto negli uccelli; ma per la teftimonianza de'fudderti Autori, e d'altri tutti Europei, e degni di fede conta, che effi non fono deboli, e pufillanimi, ma che fuperano nella forza, e. nell' intrepidezza quslli dell" antico. Continente.

In ciò che riguarda la vaghezza degli uccelli non contraftano i vantaggi all' America quagli Autori, che per altro $f$ fon impegnati nell' avvilire quel nuovo Mondo. Chi ne volerfe formare una qualche idea, veda le opere d'Oriedo, d'Hermandez, d'Acolta, d'Ulloa, e d'altri Autori europei, che hanno veduti co' lor occhj gli uccelli americani. Nella N. Spagna, dice Acofta, v'è una gran copia d'uccelli adorni di si eccel. lenti penne, e si fine, che non fe ne trovano pari in Euro. pa. (ee)

E' vero, dicono alcuni Autori europei, che gli uccelli americani fono fuperiori a' noftri nella vaghezza delle penne, ma non già nell' eccellenza del canto, nel quale fon da' nottri

fu.

(aa), Hift. Nat. y Mor. lib. 4. cap. 3\%.

(bb) Relazione del viaggio fatto all' Amer. Merid: part. I. lib. 6. cap. 8.

(cc) Il Condor è tanto grande, che ha da quattordici fino a fedici piedi dall' una all' altra eftremita delle ali, diftefe. Il Sig. de Bomare dice, che effo è comune ad ambidue i Continenti, e che gli Svizzeri, l'appellano Laemmer-geyer; ma checcheffia di quefto, egli. è certo, che non s'è trovata finora mell' antico Continente un uccello, di rapina, che: poßra agguagliarfi nella Erandezza, e. nella forza col Condor dell' America.

(dd) Hernandez. De Avibus. N. Hisp. cap. 100.

(ee) Hift. Nat. y Mor. lib. 4. c. 320. 
fuperati. Così la penfano due Moderni Italiani (ff) tanto dotti in certe materie fpeculative, quanto ignoranti delle cole dell' America. Bafterebbe pure a confondere quelti Aurori il teftimonio del Dott. Hernandez, che copiamo qui fotto, $(g g)$ il quale dopo aver fentiti $i$ migliori Roflignoli nella Corte di Filippo II. fenti molti anni i Centzontli, o Poliglotti, i Cardinali, i Tigretti, i Cuitlaccocbi, ed altre innumerabili fpezie d'uccelli canori volgari nel Meffico, e non conolciuti in Europa, oltre a'Roffignoli, a' Calderini, alle Calandre, e ad altri comuni ad ambidue i Continenti. Fra tutti gli uccelli cantori il più pregiato in Europa è il tanto rinomato Roffigno. lo, eppur quefto è affai migliore in America, fecondo che afferma il Sig. de Bomare. "Il Roffignolo della Luigiana, dice e" gli, è il medefimo dell' Europa; ma quello è più famiglia" re, canta tutto l'anno, e il fuo canto è più variato.", Ecco tre gran vantaggi dell' americano full' europeo. Ma ancorchè non foffero nell' America de' Rolfignoli, nè de' Calderini, nè alcun altro uccello di quelli, che fono ftimati in Europa pel loro canto, le bafterebbe il folo Centzontlo, o Poliglotto $\left({ }^{*}\right)$ per non aver da invidiare a verun paefe del Mondo. Protefto a' no. ftri Filofofi antiamericani, che quanto dice il Dott. Hernandez intorno al grand' ecceffo di merito del Poliglotto fopra il Rolfignolo è affai vero, e conforme al giudizio degli Europei, che fono ltati nel Meffico, ed a quello de' Mefficani, che fono ftati in Europa. Oltre alla fingolar dolcezza del luo canto, alla

pro-

(ff) L'Autore di certa Differtazione metafifico-politica fulla proporzione de' talenti, $e$ del loro ufo, nella quale fcriffe tali fpropofiti intorno all' America, e fi moftò tanto ignorante della terra, del clima, degli animali, e degli uomini di quel nuovo Mondo, come un fanciullo. L'altro è l' Autore di certe belle favolette italiane, in una delle quali mette un uccello americanodifcorrendo con un Rosfignolo.

(gg) In caveis, quibus detinetur, fuavifime cantat : nec eft avis ulla, animalve, cujus vocem non reddat luculentifime, or exquijitifjme cmuletur. Quid? Pbilomelam nofram longo (uperat intervallo, cujus fuaviffimum concentum tantopere laudant celebrantque vetufti Auctores, \& quidquid avicularum apud nofrum orbem cantu auditur fuavifimum. Hernandez De Avibus N. Hifp. cap. 30. de Centzontlatole, five Centzontli.

$\left.{ }^{*}\right)$ Linneo appella il Centzontli Orfeo. Altri Autori l'appellano Mocqueur, - fra Beffardo. 


\section{I36 D I S S E R T A Z IO N E IV.}

prodigiofa varietà de' fuoi tuoni, e alla graziola proprietá nel contraffare le differenti voci degli uccelli, e de' quadrupedi, che fente, $(b b)$ ha ful Rolfignolo il vantaggio d'effer men rultico, e più comune; poichè la fua fpezie è una delle più numerofe. $\mathrm{Se}$ io voleffi ragionare alla maniera del Sig. de Pavv, potrei aggiugnere per dimoftrare la bontà del clima dell'. America, che alcuni uccelli, $i$ quali non fon pregiati in Europa pel lo. ro canto, cantano affai bene nell' America. I pafferi, dice Valdecebro Autore europeo, $i$ quali in I/pagna non cantano, fon nella Nuova Spagna migliori de' Calderini. (ii)

Ciò che diciamo degli uccelli cantori, poffiamo altresì dire di quelli, che contraffanno la loquela umana; poichè non vi fono nell' Afia, e nell' Africa tante fpezie di Pappagalli, nè così numerole, come nell' America. (kk)

Ma poichè framo ful difcorfo degli uccelli, voglio prima di metter fine a quelto a rticolo, fare un' opportuna rifleffione. Non v'è animale americano, fül quale facciano più grande fchiamazzo i nottri Filofof, che ful Pigro a cagione di quella ftupenda lentezza ed inabilità pel moto. Or che direbbono, fe $v i$ foffe un uccello di quella fatta? Quefto farebbe fenz'altro l'animale più irregolare del Mondo; poichè una tal tardità, o inerzia è più difdicevole ad un uccello, che ad un quadrupe. de. Ma dove mai è quelt'uccello? Nell' antico Continente, ed è ftato defcritro dal medefimo C. de Buffor, il quale dice, che i) Dronte, uccello dell' India Orientale più grande del Cigno, è tra gli uccelli quello, che è il Pigro tra' quadrupedi, "Pa. "re,

(bh) Il Sig. Barrington, Vice-Prefidente della Società Regia di Londra, dice in una fua curiofa opera ful canto degli uccelli, prefentata a quella dotta Accademia d'avere egli offervato un Poliglotto, il qual nello fpazio d'un fol minuto contrafiece il canto dell' Allodola, del Fringueilo, del Merlo, del $\mathrm{Pa}$ fere, e del Tordo.

(ii) Valdecebro nell'opera fpagnuola intitolata, Gobierno de las Aves lib. 5. cap. 29. Ma abbiamo già detto nel lib. I. della Storia che i Pafferi mefficani, benchè fomiglianti a'veri Pafferi, fono di diverfa fpezie.

(kk) $V$ 'è nell' America una grande abbondanza di Pappagalli, masimamente nelle Andi del Perù, e nelle ifole di Portorrico, e di San Domenico. Acofta lib. 4. cap. 35. Nelle cofte mefficane del Mar Pacifico è ancor più grande, che in quelle irole, il numero de' Pappagalli. 
5) re, dice, una teftuggine veftita delle fpog'ie d'un uccello, $e$ " la Natura concedendogli quelti inutili ornamenti (delf' ali, "e della coda) pare aver voluto aggiugnere l'impaccio alla " pefantezza, e l'irregolarità de'fuoi movimenti all' inerzia del " corpo, e far la lua pefante groffezza più ributtante ricordan. " dogli, che è uccello." "

Da ciò, che abbiam fin quì detto, fr conchiude evidentemente, che nè il Cielo dell'America è avaro, nè il fuo cilma è contrario alla generazione degli animali : che nè la materia vi ha fcarfeggiato, nè la Natura s'è fervita d'una differente fcala di grandezza: che è un errore, o per dir meglio un ammaflo d'errori, quanto il C. de Buffon, e il Sig. de Paw dicono fu la piccolezza, fu la irregolarità, e fu i difetti dei quadrupedi americani, e avvegnachè foffe vero, niente lor gioverebbe per dimoftrare la malignità del clima dell' America; ma ora vediamo, fe fanno un minor torto al nuovo Mondo in quello, che dicono fu la pretefa degradazione dei .qua: drupedi trafportativi dall' Europa.

\section{IT.}

Su gli animali Europei trafportati nell' America:

"Turti gli animali trafportati nell' America, come i Cä y) valli, gli Afrni, i Tori, le Pecore, le Capre, i Porci, ed " i Cani, fono, dice il Co de Buffon, (li) confiderabilmente "più piccoli quivi, che in Europa, e ciò fenz' alcuna eccezio. "ne. "Se cerchiamo la pruova d' un'afferzione tanto univerfale, non ne troveremo altra in tutta fa Storia Naturale di quel Filofofo, che quella d'effer più piccole in Canadà, che in Francia, le Vacche, le Pecore, le Capre, i Porrci, ed i Cani., Gli animali Europei o afratici, dice il Sig. de PaW "( $\mathrm{mm})$, trafportativi nell' America, immediatamente dopo la Storia Antica del Meffico Tom. IV. S "fua

(ii) Hir. $\mathrm{N}$ t. tom. 18.

( $\mathrm{mm}$ ) Recherch. philoroph. part. 1. 


\section{${ }_{3} 8$ DISSERTAZIONE IV.}

9) fua fcoperta, v'hanno tralignato, la lor corporatura fi è fce: " mata, ed effi hanno perduto una parre del loro iftinto, e " Uella lor indole: le cartilagini, e le fibre della lor carne fi "fon rendute più rigide, e più groffe. "Tal è la conclufion generale del Sig. de $\mathrm{PaW}$; vediamo ora le pruove. I. La carme di bue è tanto, fibrofa., che appena fo può mangiare nell' ifola Spagnuola. 2. I Porci nell' ifola di Cubagua cangiarono in breve a tal fegro la lor forma, che non $f_{1}$ potevano ravvifare: lo lor unghie crebbero tanto, che aveano un mezzo palmo di lungbezz. 3. Le Pecore fofferfero una grande alterazione nella Barúada. 4. I Cani trafportati dai noftri paeji perdono la voce, $C$ cefJano d' abbajare nella maggior parte delle regioni del nuovo Continente. 5. Il freddo del Perì fconcertò nei Cammelli traSportativi dall' Africa gli organi della generazione. 'Tali fono gli argomenti, di cui fi prevalgono quelti Filofofi per promuovere la degradazione degli animali dell'antico Continente nell' America: argomenti tali, che ancorchè foffero veri, non bafterebbero a provare una conclufione tanto univerfale; imperciocchê, che importa, che la carne di bue fia tanto fibrofa nell' ifola Spagnuola, fe in quafi tutri gli altri paefi dell' America è buona, ed in molti, ficcome in tutti quei del Meffico, fituati nelle cofte del Mar Pacifico, è tanto eccellente, quanto la miglior d'Europa, e forfe ancor più? Che importa, che le Pecore abbiano fofferto qualche alterazione nella Barbada, ed in alcuni paefi troppo caldi, fe nei paefi temperati del Meffico, e dell' America meridionale fi confervano tali, quali vi paflarono da Spagna? Che importa, che i Porci fianfi sfigurati in Cubagua, Ifoletta miferabile, "priva d'acqua,e di tutto il bifognevole alla vita, fe nel refto dell' America banno acquiftato, come dice lo fteffo Sig. de Pavv, una corporatura Atraordinaria, a la lor carne s'é perfezionata affai a tal fegno, che $i$ Medici la prefcrivono, ai loro ammalats con preferenza ad ogni altra carne. Ora, fe l'afferfi sfigurati $\mathrm{i}$ Porci in Cubagua non prova, che il clima dell'America non fia loro il più convenevole, perchè l'aver patito qualche detrimento le Pecore nella Barbada, l'effer divenuta più fibrofa la carne dei buoi nella 
Spagnuola, e l'efferfi alquanto appiccoliti alcuni quadrupedi nel Canadà dovrà provare, che il clima dell' America in generale è contrario alla generazion degli animali, alla lor corporatu$r a$, e al loro iftinto?

Se tal Logica foffe: tollerabile, potremmo noi adoperar degli argomenti affai migliori contro il clima dell'antico Continente fenza fervirci d'altri materiali, che di quelli, ci fomminiftra il medefimo. C. de Buffon nella fua Storia Naturale. I Cammelli non hanno potuto moltiplicarfi in Ifpagna, ficcome dice lo fteffo. Autore, con tutto che quel clima fra tutti quelli dell Europa fia il meno contrario al loro naturale. I Buoi hanno tralignato in Barberìa, e in Islanda hanno perdute le loro corna. Le Pecore, dice il C. de Buffon, banno de. generato dal primo lor effere nei noftri paefi, ed in tutti i paefi caldi dell' antico Continente cangiano la lana in pelo. Le Ca. pre fi fono appiccolite nella Guinea, ed in altri paefi. I Cani nella Lapponia: fon divenuti piccoliffimi e deformiffimi, e quelli dei climi temperati, allorchè trafportanfi ne'climi freddi, ceffano d'abbajare, e dopo la prima generazione: nafcono cogli orecchi ritti . "Dalle relazioni dei Viaggiatori ci confta, che i "Maftini, i Levrieri, ed altre razze di Cani europei: trafpor23 tati in Madagafcar, in Calicut, in Madurè', e nel Malabar "tralignano dopo la feconda o terza generazione, e che nei 2) paefi ecceffivamente caldi, come fono la Guined, e il Se" negal, quefta degenerazione è più pronta; poichè appena paf"Cati tre o quattro annil, perdono il pelo, e la voce., I Cervi nei paefi montuofi caldi e fecchi, come quelli di Corfica, - di Sardegna, hanno perduta la metá della lor corporatura. Se a quefte, e ad altre notizie, che ci dà il C. de Buffon, volefimo aggiugnere quelle, che ci fomminiftrano moltifimi altri Autori, che copia d'efempi della degenerazione degli animali neil ${ }^{2}$ antico Continente: non avremmo, affai più grande, e più vera di quella de' noftri Filofof? $\mathrm{Ma}$ acciocchè fi veda l' efagerazione e la falfita, che v'è ne'loro efempi, efaminiamo ad una ad una tutte le fpezie d'animali afiatici, ed europei trafportati nel nuovo Mondo, che da loro diconfi degenerate. 


\section{DE' CAMMELLI 。}

99. Tra tutti i quadrupedi trafoortati nell'America, dice is " Sig. de P., quelli, che meno fono riufciti, fono tati fenz' al: 39. tro i- Cammelli. Sul principio del fecolo XVI. ne furono at. i) cuni tralportuti dall' Africa nel Perù, dove il freddo fconceris to $\mathrm{i}$ lor organi deltinati alla riproduzione, e non lafciarono "alcuna pofterità." Ma diffimulando ora l'errore cronologico, nel qual incorre, come quello, che importa poco al noftro propolito, $(n n)$ fe il freddo fu, che diftruffe la lpezio dei Cam. melli nell' America, lo fteffo accaderebbe in Europa fpecial. mente nei paefi fettentrionali, nei quali il freddo è fenza pa. ragone più grande, che in qualfivoglia paefe del Perù. Se il Freddo fu la cagione della loro eftinzione, incolpi il Sig. de Jaw coloro, che Atabilirono quei quadrupedi nei luoghi non confacevoli alla lor natura, non già l'America, nella quale vi fono delle terre calde e fecche, quali fi vogliono per la fuffi. Atenza dei Cammelli. La fteffa fperienza, che fi fece nel Perù coi Cammelli, fi fece ancora in Ifpagna, e riufci fimilmente infruttuofa; e non però vi farà chi dubiti, che il clima di quefra penifola è dei più temperati, e dei più dolci dell' Euro. pa. Il C. de Buffon dice, che non meno in America, che in Ifpagna fi potrebbono propagar quei quadrupedi, fe fi prendeffeso tutce le precauzioni neceffarie; ed io non dubito che nelle contrade della. Nuova Gallizia riulcirebbono affai bene. Del refto è falfo, che-i Cammelli trafportati nel. Perù, non lafciarono alcuna polterità; poichè il P. Acolta, il quale vi andò alcuni anni do. po , teftifica, d'averli veduti moltiplicati, benchè poco.. (ooz)

\section{DE' BUOY:}

Quefta è una di quelle fpezie d'animali, che dai noftì Filo.

(nn) Il trafporto de' Cammelli nel Perù non fu fatto, nè potè farfífulprincipio del: fecolo XVI.; perchè allora ton s'era ancora fcoperto quel paefe; ma verfo la metà di quel fecolo, come ne fa fede Herrera nelle fue Decadj.

(ov) Hiftor. Nat. y: Mor. lib. 4. cap. 33. 
Filofofi credonfi degradate nell'. America, ed alle quali fi fup: pone contrario il clima. Ma fe forfe nel Canada hanno perdura i Buoi una parte della lor corporatura, come afferma il $\mathbf{C}_{\text {. }}$ de Buffon, e fe nella Spagnuola è divenuta più fibrofa la loro carne, come vuol il Sig. de Paw, almeno non è così nella maggior parte dei paefi del nuovo Mondo, nei quali la moltitudine, e la grandezza di quegli animali, e la bontà della lor carne danno a divedere quanto favorevoli fieno quei climi alla lor generazione. La prodigiofa loro molriplicazione in quei. paefi fi trova teftificata da moltiffimi Autori Europei tanto antichi, quanto moderni. Il P. Acofta racconta $(p p)$, che nel. la flotta venuta dalla Nuova Spagua all' antica, nella quale egli ritornò in Europa, nel 1587. , cioè feffanta anni incirca, dappoichè erano ftati trafportati nel Meffico i primi Tori e Vacche, fi portarono da quel paefe 64. 360. cuoi bovini, e dalla fola Spagnuola, la quale crede il Sig. de Paw tanto contraria alla gererazione di quefti quadrupeti, 35.444 . Io non dubito, che fe fi paragonaffe il numero dei Toti, e delle Vacche portato dall' antico Concinente nel nuovo col numero di cuoi, che l'America ha mandato in ricompenfa all' Europa, fi troverebbono più di cinque miglioni di cuoj per ciafcun di ques gli animali. Valdecebro, Domenicano Spagnuolo, che viffe alcuni anni in Melico verfo la metà del fecolo paffato, racconta, come una cofa notoria, che a D. Giovanni Ordugna, Ca. valier Mefficano, diedero le fue Vacche in un anno trentafei mila vitelli: $(q q)$ il che non potè avvenire, $\mathrm{fe}$ non in un armento di dugento mila fra Tori, e Vacche. Oggidi vi fono dei Particolari, che fon padroni di 50.000. Ma niun'altra cofa dà più a divedere la ftupenda moltiplicazione di tali quadrupedi, quanto il venderfi a si buon mercato in quei paefi, nei quali: fon neceffari pel foftentamento degli uomini, e per li Lavori della campagna, e dove a cagione dell'abbondanza dell'

ar-

(p.p) Lib. 4. cap. 33.

(qq) Valdecebro nell' opera Spagnuola intirolata, Gobierno de animales lib. Ax a.p. 34: 
argento tutto fi vende caro: $(r r)$ e per dirlo in poche parole i Tori fi fono molriplicati nel Meffico, nel Paraguai, ed in al. tri paefi del nuovo Mondo più, che nell' armentofa Italia. (J)

Per cio che riguarda la grandezza de'buoi americani, è affai facile l'averne informazione, poichè approdanofpeffo a $\mathrm{C}_{2}$ dice, ed a Lisbona de' vafcelli carichi di corami bovini. $\left(^{*}\right)$ Facciafi dunque mifurar cinquanta, o cento cuoj il Sig. de P., o qualcun altro di coloro, che foltengono la degradazione degli animali europei nel nuovo Mondo, e fe frovano più piccoli de' buoi comuni dell' Europa, tolto confefferemo, che il clima: dell'America ha lor raccorciato il corpo 2 che vi ha fcarfeggiato la materia; altrimente dovranno eglino confeffare, che fon falle le loro notizie, mal fondate le loro offervazioni, e fantaftico: il lor fiftema: ed acciocchè $f$ veda, quanta ragione abbiamo di non fidarci delle loro notizie, Gonzalo d'Oviedo, il qual fu uno degli antichi popolatori dell'ifola. Spagnuola, o fia di So. Domenico, e vi dimorò alcuni anni, ragionando de" buoi di quell'ifola, la carne de quali non può mangiarfi al dir del Sigo de P., perchè troppo fibrofa, dice. (tt) cbe glíar. men-

(rr) Ne'contorni di Meffico, capitale della N. Spagna, contuttochè fieno aftai popolati fi vende un buon paio di buoi per l'aratro zecchini dieci, ed i tori all ingroffo paoli 45. l' uno: Nei contorni di Gizadalaxara, capital della N. Gallizia vale un buon paio di buoi da 6. in 7 . zecchini, una vacca paoli 25.9 ed. un vitello dl più di due anai ro.. o 12. paoli. In molti altri paefí di quel Regno fi vendono affai meno tutti queft animali. In moltiffimi luoghi delle Provincie del Fiume della Plata fi ha una vacca per paoli cinque. Secondo il dettaglio fattomi da una: perfona affa $\mathrm{i}$ pratica, e fincera, nelle Provincie del fuddetto fiume faranno quafi cinque milioni $\mathbf{i}$ buo i ridottiad armenti, e fi crede che de' falvatici vi faranno due milioni incirca.

(f) Timeo Autor greco e Varrone citati da Aulo Gellio (Noct. Attic.

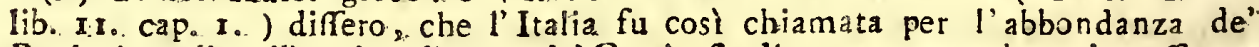
Buoi, i quali nell' antica. lingua de' Greci. fi. diceva no, $17 \times x_{0} i$ : unde afferma Gellio, che Italia vuol dire Armentofif/ima'。.

$\left({ }^{*}\right)$. Tutti fanno, che non $v^{\prime}$ è paefe, che faccia un più gran commerciecon: Ifpagna in cuoi bovini, che il paraguai. là onde vengono de' vafcetli carichi di effi. Io pur fo per informazione avuta da perfona pratica di quel paefe, e affai degna di fede, che i cuoi, che di là fi portano in Ifpagna fono lunghi almeno tre varas ( mifura di Spagna) e molti fin' a quattro, o fia più di dieci piedi di Parigi. Non credo, che fieno, tre paefi in Europa, ne'quali giungano talvolta i Buoi a si fmifurata grandezza.

(tr) Sommario della Storia naturo dell' Indie cape. 2. 
menti fono quivi piu grandi, ed affai piu belli di tutti quelli di Spagna, e ficcome $i$ aria in quelle parti $\&$ dolce, $e$ non mai fredda, non diventano $i$ buoi giammai magri, ne la lor carne $\dot{e}$ giammai di castivo gufto. Il C. de Buffon afferma, che i paefi freddi fono più confacevoli a' Buoi, che i caldi; ma non è così nella Nuova Spagna; poichè quantunque buoni vi fieno $\mathbf{i}$ Buoi de' paefi freddi e temperati, fono nondimeno mighiori quelli de' paefi caldi. La carne di queft' animali nelle terre marittime, le quaii fono affai calde, è tanto eccellente, che fi man. da come regalo alla Capitale anche da' luoghi da effa difcofti 250., e 300. miglia.

\section{DELLE PECORE.}

Il C. de Buffon confeffa (un) che le Pecore fon ben riu: Fcite tanto ne'paefi caldi, quanto ne'freddi del nuovo Continente; ma foggiunge, che quantunque fienfi affai moltiplicate, fono ciò non oftante più magre, e la lor carne ce men fugofa, e men tenera, che in Europa: nel che $f_{a}$ palefe, che n'è ftato mal informato. Ne' paefi caldi del nuovo Mondo non riefcono bene per lo più le Pecore, e la carne de'caftrati è cattiva : della qual cofa non è da maravigliare, mentre il clima caldo è tanto loro contrario anche nell' antico Continente, ficcome dice lo fteffo C. de Buffon, che fotto effo fi coprono di pelo in cambio di lana. Ne'climi freddi, e temperati della nuova Spagna fi fon moltiplicati a proporzione più, che i Tori la loro lana è in molti luoghi tanto fina, quanto quella delle pecore di Spagna, e la loro carne tanto buona, quanto la miglior d'Europa, come poffono teflificare quegli Europei, che fono ftati in que' paefi. La moltiplicazion delle Pecore nell' A. merica è ftata forprendente. Il P. Acofta teftifica, $(v v)$ che prima ch' egli vi andaffe, erano nell' America de' beneftanti, che poffedevano fettanta, ed anche cento mila Pecore, ed oggidi vi è

(uu) Hift. Nat. tom. I 8.

(vv) Stor. Nat. e Mor. lib. 4. cap. 33. 


\section{I44 DISSERTAZIONE IV.}

vi è nella nuova Spagna chi ne ha quattrocento, cinquecento; ed anche fettecento mila. ( $x x)$ Valdecebro dice, $\left(y^{\prime} y\right)$ che $\mathbf{D}$. Didaco Muñòz Camargo, Nobil Tlafcallefe, di cui abbiamo farta menzione nella Notizia degli Scrittori della Storia antica del Meffico, da dieci fole Pecore n'ebbe in dieci anni quaranta mila. Or come potrebbono si ecceflivamente moltiplicarfi quegli animali, fe il clima foffe contrario alla lor generazione? Intorno poi alla lor gradezza, protefto fnceramente di non aver veduti finora in Europa montoni più grand di quelli del Meffico.

\section{DELLE CAPRE.}

Il medefímo C. de Buffon costuttochè tanto s'impegni nel tacciar gli animali dell' America, tuttavia confeffa, che le capre fon riufcite bene ne' climi americani, e che la loro moltiplicazione è quivi affai più grande, che in Europa; $(z z)$ poìchè laddove in Europa fanno in ogni parto un fol capretto, o al più due, nell' America ne fanno tre, quattro, e talvolta cinque. Il Sig. de P., il qual àà degnamente al C. de Buffon il titolo di Plinio della Francia, e vuole, che in foggetto d'animali fi deferifca all'autorità di lui, come a quello, che ha fatto la raffegna di tutti gli animali della terra, dovrebbe aver ponderate quefte, ed altre confeffioni di quel dotto Filofofo prima di metterfs a fcrivere fu gli animali americani.

\section{DE' PORCI.}

Non fon d'accordo in quefto articolo i noftri Filofofi; poichè laddove il C. de Buffon mette i Porci. tra gli animali de-

(xx) Quegli Europei, che non fono ftati maj in America, non vogliono credere ciò che lor diciamo intorno al numero dei Buvi, di Cavalli, di Pecore, e di Capre che hanno molti Signori Americani ne loro poderi; ma fe non foffe vero, non oferemmo pubblicarlo innanzi a tanti, che ci potrebbero fmentire.

(yy) Nell'opera intitolata: Gobierno de animales lib. 4. cap. 34. 11 ragguaglio di Valdecebro fu la moltiplicazione delle pecore di Carnargo è Alato confermato da parecchi altri Storici del Meflico.

(zz) Hilt. Nat. tom. 18. 
degradati nell' America, il Sig. de P. per contrario afferma; che quefti fono $i$ foli animali, che hanno acquittato nel nuo. vo Mondo una corporatura Itraordinaria, e la cui carne s'è perfezionata. Quefta contraddizione nacque fenz'altro dal non diftinguere, come dovrebbono, i diverfi paefi dell' America . Pud effere, che ve ne fieno alcuni, benchè io nol fappia, ne' quali i Porci abbiano perduta una parte della loro grandezza; ma egli é certo, che nella N. Spagna, nelle ifole Antille, in Terraferma, ed in altri paefi dell' America fono tanto grandi, quanto quelli d' Europa, e nell'ifola di Cuba v' è uua razza di Porci più grandi al doppio degli Europei : lo che è coltante e notorio a tutti quelli, che fono ftati in que' paefi. 1 noAtri Filofofi poffono, fe lor piace, prendere informazione da parecchj Autori Europei, che hanno veduti i Porci di Toluca, e d'Angelopoli nella N. Spagna, di Carragena, di Cuba \&c. intorno alla loro eccefliva moltiplicazione, e all' eccellenza del. la loro carne. $(A a)$

\section{DE' CAVALLI, E DELLE MULE。}

In niun' altra cofa di tutto cio, che dicono contro gli animali americani il C. de Buffon, e il Sig. de Paw, fanno un più gran torto all' America, e alla verita, che nel fupporvi ancora degradati i Cavalli. Di quefti ne dice $(B b)$ il P.A. coita, " che in molti paefi dell' America, oppur nella maggior "parte, fon riufciti, e riefcono aff $¥ i$ bene, e ve ne fono alcune "razze tanto buone, quanto le migliori di Spagna, non mea "no pel corfo e per la comparfa, che per li viaggi, e le fati. Storia Antica del Meffico Tom. IV. T " che

(Aa) Bafta leggere cid, che fcritle il P. Acofta nel lib. 4. cap. 38. della fua Storia: ,Egli è certo, dice, che i Porci ti fono in grand' abbondanza "moltiplicati da per tutto nell' America. In molti paefi fi mangia la loro "carne frefca, e fi ftima tanto fana, quanto quella di Caftrato, ficcome in "Cartagena.... In alcuni luoghi s' ingrafiano con frumentone, e divengono ec", cefrivainente grafi. In altri fe ne fanno eccellenti lardi, e prefciutti, come " in Toluca della N. Spagna, e in Paria. "Il Co. de Buffon nel medefimo tomo 18. nel quale mette $i$ Porci tra gli animali degenerari in Anerica, dis. ce efpreffamente, che $i$ Porci trafportati in America vi fono ben riufciti.

(Bb) Hift. Nat. y Mor. lib. 4. cap. 33. 


\section{I46 DISS ER T A Z IO N E IV.}

3) che." Siffatta teftimonianza d'un Europeo tanto critico, tanto imparziale, e tanto pratico delle cofe d'America, e d' Eu. ropa, vale affai più di tutte le declamazioni di que' Filofofi contro il nuovo Mondo. Il Tenente Generale D. Antonio Ulloa, dotto Matternatico Spagnuolo ancor vivente, (Cc) parla con iftupore de Cavalli americani, che egli vide nel Chile, nel Perù, e celebra con gran lodi i Chilefi pel loro paffo, quelli che fi appellano Aguilillas per la Atraordinaria lor velocita, quelli detti Parameros per la ftupenda loro agilità nel correre che fanno nella caccia de' Cervi co' Cavalieri Sopra, per le pendici, per li luoghi più fcofcefi, e dirupati delle montagne. E. gli teftifica d'aver fatto fpeffe volte fopra uno de' Cavalli detti Aguilillas, il quale foggiunge, non era de' più veloci della ruz razza, più di quindici miglia in 57.058 . minuti. Nella Nuova Spagna v'è un' indicibile quantità di Cavalli, e di Mule. La lor moltitudine può conghietturarfi dal loro prezzo, poichè laddove à tempi della conquilta valeva un cavallo ordinario fin mille fcudi, oggidi fe ne acquifta un buono per ifcudi dieci, o dodici. $(D d)$ La lor grandezza è quella de' cavalli comuni d' Europa; di rado fi vede nel Meffico un Cavallo sì piccolo, come gli fchiavoni, the veggiamo in Italia, e molto meno come quelli d'Islanda, e d'altri paefi fettentrionali al dir d'Anderfon, o quelli dell' India al dir di Tavernier, e d'altri Autori. La lor fortezza è tale, che è affai comune negli abitatori di que' paefi il fare a cavallo fettanta, ovvero ottanta miglia, e talvolta ancor più, camminando tutrora di buon paffo, fenza mai fer-. marfi, nè mutar cavallo per iftrade fovente malagevoli. I $\mathrm{Ca}$ valli. da fella, contuttochè fieno per lo più caltrati, hanno un fuoco Atupendo. Le Mule, le quali in tutto quel Regno fervono per li cocchi, e per la foma, fono ancora quanto alla

gran-

(C) Viaggio all' America Meridionale part. 1. lib. 6. cap. 9.

(Dd) Nella Nuova Gallizia s'ba un cavallo mediocre per zecchini due, una mula per tre, o due e mezzo, ed una mandra di ventiquattro cavalle col fuo fallone per zecchini venticinque. Nel Chile fi può avere anche per mezzo zecchino, o fia per uno fcudo un cavallo di quelli che vanno a trotto, $i$ quali fono i più pregiati 'ai Contadini per la lor fortezza, e la fomma loro agi-, lità nel corfo, e una cavalla fuol comprarfi per bajocchı venticinque. 
grandezza tali, quali le comuni d'Europa. Quelle da foma, che camminano in condotta, portano addoffo cinquecento libbre incirca: non fanno ogni giorno più di dodici, o quattordici miglia fecondo l'ufanza di qué Vetturali; $m a$ in tal maniera fanno de'viaggi d'ottocento, di mille, ed anche di mille cinquecento miglia. Quelle da cocchio vanno del paffo delle pofte d'Europa, benchè $i$ cocchi portino un pefo affai più grande per cagione dell' equipaggio dei paffaggieri, e così fanno le medefime Mule dei viaggi affai lunghi camminando ozni giorno trenta miglia almeno. Quelle da fella fervono per li più lun. ghi viaggi. E'comune il fare in una Mula il viaggio da Meffico a Guatemala di mille miglia in circa, e per iftrada in gran parte montuofa e cattiva, camminando trenta o più miglia ogni giorno. Tutto quefto da me addotto per palefar l'inganno dei noftri Filofofi intorno alla pretefa degradazione di quei quadrupedi è pubblico e notorio in quel Regno, e conforme al ragguaglio, che ne fanno parecchi Autori europei . Ma: nulla a mio avvifo dà più chiaramente a divedere la moltitudine, e l'eccellenza dei cavalli americani, quanto un' offervazione da me fatta. Fra tante cofe, che fi fanno venir da Spagna a quaIunque fpefa gli Spagnuolu ftabiliti in. America per l'amor, che confervano alla lor patria, non fo (almeno rapporto al Meffico) che da dugento anni in quà fi. fiano mai fatto condurre da Spagna dei Cavalli; e per contrario fon ficuro, che fpeffe volte hanno mandato in I pagna dei cavalli americani da prefentarfi ai Magnati della Corte, ed anche allo fteffo Re Catrolico a

\section{DE CANY 。}

Tra" grandi fpropofiti pubblicati dal Sig de Paw, i quali nov fon pochi, è affai grande quello, che fcrive intorno ai $\mathrm{Ca}_{2}$. ni. I Cani dice (Ee) trafportati dai noftri paefi perdono fubito la voce, e ceflano d'abbajare nella maggior parte delle regioni $\mathbf{T} 2$

del

(Ee) Recherch. Philofoph. part. i. 


\section{DISSERTAZIONE IV.}

del nuovo Continente. Gli Americani hanno per altri "capi trop. po da ridere nell'opera del Sig. de PaW; ma in leggendo queito paffo dei Cani rideranno fgangheratamente. Ancorchè concedeffimo al Sig.de P., che nella maggior parte abbiano degenesato i Cani, niente però fi conchiuderebbe contro quel nuovo Continente, che non poteffe parimente conchiuderfi contro il Mondo antico; poichè, fecondó che afferma il C. de Buffon, i Cani trafportati dai climi temperati nei freddi dell' antico Continente perdono la voce, e trafportati nei paef ecceffiva:mente caldi perdono oltre alla voce anche il pelo. Quelt' afferzione del C. de Buffon s'appoggia fu la fperienza fatta nei $\mathrm{Ca}_{\text {- }}$ ni europei tralportati in Afia, ed in Africa, la cui degenerazione, dice il fuddetto Filofofo, è cosi pronta nella Guinea, $e$ in altri paefi troppo caldi, che dopo tre, o quat. tro anni reftano affatto muti e pelati. Nor ardifce il Sig. do P. di dire altrettanto dei Cani trafportati in America; ma ancor quello che afferma è falfifimo; perchè quali fono quei paefi dell' America, dove i Cani hanno perduta la voce? Su la fede di che. Autori ha olato pubblicar fiffatta favola? La maggior parte dei paefi americani, nei quali fono ftati. trafportati - Cani europei, fono, fottopofti al Re Cattolico, o- in niuno d' effi è accaduta ai Cani una tal difgrazia. Nè tra gli Autoxi. Europei, che hanno notate le particolarita dell' America: zè tra moltiffimi Americani, che fon quì prefentemente venuti. da tutti i paefi dell' America fpagnuola ho trovato neppur uno, che confermi l'aneddoto del Sig. de P. Quello bens'i, che fappiamo e da parecchj Scrittori dell' America, e da. pa; recchie perfone pratiche di quei paefi, da cui ci fiamo infor mati, fi ̀̀, che i Cani non hanno mai la rabbia nel Perù,, nel Quito, nel Chile, e in altre contrade di quel nuovo Mondo. Se forfe nei dominj dell' Inghilterra, o della Francia v'è qualche paefe ( $l o$ che io non credo), nel quale fien divenuti (muti i Cani, dovrà parò dirfi, che banno perduta la voce nelís maggior parte delle regioni del nuovo Continente? Leffe perav, vencura il Sig. de 'Po, che in alcupi paefi. dell' America v' era. no dei Cani, che non abbajavano, e quefto gli bafto per pub. 
blicare, che $\mathrm{i}$ Cani europei trafportati in America tofto perdevan la voce. Similmente potrebbe dire, che i fichi rrafportati dall' Europa in America divengono fubito fpinofi, perchè ha delle fpine la Nocbili, o fra Tuna, la quale per non fó che fomiglianza col fico, fu dagli Spagnuoli chiamato fico d' Indie, ficcome fu da loro appellato cagnuolo del Meffico it Techichi perchè fomiglidote ai cagouoli; ma nè quefto quadrupede è vero Cane, nè quel fratto èं vero fico. E' troppo facile il precipitarli in tali errori, qualora non fi regolano le idee, nè fi moderano le paffroni. Il C. de Buffon per l'oppofto afferma, $(F f)$ che i Cani europei fono ben riufciti tanto sei paefi caldi, quanto nei freddi del nuovo Mondo: nel che accorda fenz' altro un gran vantaggio al clima dell' America. fopra quello dell' antico. Continente.

\section{DE' GATTI。}

Niente dicono in particolare $i$ noftri Filofof fa la degeo nerazione dei Garti nell' America; ma debbono intenderfi comprefi nelle loro afferzioni univerfali. Contuttocid il C. de Buffon, i! quale nel paffo fopra citato non ammette alcuna eccezione in ciò, che dice intorno alla degenerazione degli animali in America, ragionando poi in particolar dei Gatti, dopo aver vantati quelli di Spagna, come $i$ miglicri di tutti, afferma, che quefti Gatti Spagnuoli trafportati in America banno confervati i loro bei colori, e non banno punto degenerato $(G g)$.

Quefti fono i quadrupedi trafportati $(H b)$ dall' antico al nunvo Continente, i quali tutti, fuorchè i Cammelli, fi fono ecceffivamente moltiplicati, ed hanno confervato fenza alterazione la lor corporatura la lor figura, e tutte le

per-

(Ff) Hiftoire Nat. tomo ro.

(Gg) Hit. Nat. tom. II.

$(\mathrm{Hh}) 11 \mathrm{C}$. de Buffon aggiunge a' fuddetti quadrupedi trafportati nell America il corco di Guinea, e il Coniglio; ma afferma, che quefte due fpezie fon ben riulcite. Quanto a' topi farebbe certamente un gran bene per I'America, che non poteffero vivere forto quel clima. 


\section{I50 DISSERTAZIONE IV.}

perfezioni dei loro afaendeni, ficcome confta parte dalla confeflione dei medefrmi Filcfof, parte dalla depofizione d'Autori europei imparziali, giudiciofi, e pratici di quei paefi, e parte ancora dalla notorietà, che alleghiamo fenza paura d'effere fmentiti . Non dubitiamo, che i lettori imparziali conofceranno da ciò, she fin quì abbiamo finceramente efpofto, gli errori e le contradáizioni dei noftri Filofofi cagionate dal ridicolofo impegno d'infamare il nuovo Mondo, la falfità delle loro offervazioni, l'infuftiftenza dei loro raziocini, e la temerità dela la loro cenfura.

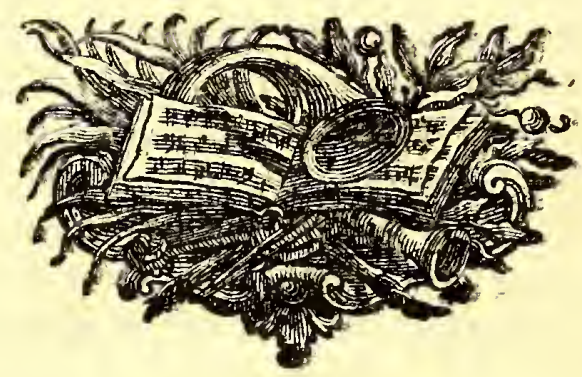




\section{CATALOGO}

DEI QUADRUPEDI AMERICANI。

๑. I.

Spezie riconofciute, e ammeffe dal C. de Buffon。

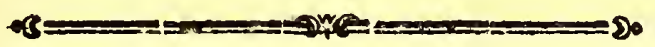

Il numero aggiunto a ciafcuna Spezie denota il tomo, nel quale il Suddetto Aitore ne parla.

A COUTI, piccol quadrupede del Paraguai, e del Brafile fimile al Coniglio. Il vero nome in lingua guarani, o paraguajefe, e quello di Ácutio. 17.

AI, Spezie di Pigro fornito di coda. 26.

AKOUCHI, piccol quadr. della Guayana, o Gujana. 30 .

ALCE, appellato volgarmente Granbeftia, (a) da' Francefi Elan, da' Canàả defi Orignac. 24.

ALCO, preffo i Peruani Alleo, preffo i Meflicani Tecbicbi, quadr. mua to e commettibile fimile ad un cagnuolo. 30 .

APAR, fpezie di Tatì, o Armadillo, fornito di tre fafcie o bande mobiti. : 21 :

APEREA ( in guaraǹ Apered) quadr. fomigliante al coniglio, ma fen za coda. $33^{\circ}$.

BISONTE, o Toro gobbo appellato nel Melfico Cibolo, quadr. grande dell' America Settentr. 23.

CABASSOU, fpezie di Tatù coperto di due lamine o conche, e di doa dici bande mobili. 2 I.

CAB'AI, o Capibara, (b) quadr. anfibio fimile al porco. 25.

CACHICAMO (in Ital. diremo Caccicamo, e cosi negli altri nomi fcritti con $c b$ ) Spezie di Tatù coperto di due lamine, e di nove bande mobili. 21.

CAMOZZA, in francefe Chamois. 24.

CAPRIUOLO, in francefe Chevreuil. 29.

CA.

(a) In Ámerica danno il nome di Granbefia al Tapir, o Danta.

(b) Il Cabiai del C. de Buffon s' appella Capibara o Capiguara da' Tucumanefi, Capiiba o Capibarà da' Guaranies, o Paraguaiefi, Cappinà da' Tamana chi, da' Chiquiti Oquis, e da alse Nazioni Cbiaco; Ciguiri, Irabusi. 
CASTORE. IT.

CERVO. II.

CHINCHE, fpezie di Puzzola americana. (c) 27.

COAITA, fpezie di Cercopiteco, o Scimia fornita di coda . 30.

COASO, fpezie di Puzzola. (d) 27.

COATI, o piuttolto Cuati, piccolo, e suriofo quadr. de' paefi meridió nali d'America. 17.

COENDU', o piuttofto Cuandì, Iftrice della Gujana, e del Paraguai, apa' pellato nell' Orinoco Arura. 25.

COJOPOLLIN ( non Cayopollin, come fcrive il C. de Buffon) piccol quadr. del Meffico. 2 I.

CONEPATA, in mefficano Conepatl, la più piccola fpezie o razza di Puzzola. 27.

COQUAllino. Cosi appella il C. de Buffon il Cozocotecuillin de'Mef. ficani, quadr. fimile allo Scojattólo, ma diverfo. 26.

COUGUAR, o fia Cuguar, fiera macchiata del genere delle Tigri. Ig. DAINO. 12.29.

ENCOBERTADO, Tatì coperto di due lamine o conche, e di fei bande. 21 .

EXQUIMA, fpezie di Cercopiteco. 30.

FALANGER, nome dato ad un piccol quadr. fimile al Topo. 26.

FER DE LANCE ( in Ital. diremo Ferdilancia) fpezie di pipiftello così appellato dal $C$. de Buffon per cagione d'una membrana che effo ha fimile nella forma al ferro d'una lancia. 27.

FILANDRO di Surinàn, quadr. fimile alla Marmofa, e al Tlacuatzin, ma diverfo. 30 .

FORMICARO (e) quadr. de' paefi caldi d'Amer. 20.

GHIOTTONE, detto de' Canadefi Carcajì, fiera de' paefi Settentr. 27.

(c) Cbincbe in Iroagnuolo fi appella la Cimice: onde potrebbe effere, che il nome di quefto inferto foffe ancor dato alla Puzzola per cagione di quell' intollerabile fetore, che rende per la parte dereiana; ma io non dubito, che il Conte de Buffon alterò piuttofto il nome Cbinghe, col quale è conofciuta la Puzzola nel Chile; poichè non troviams ufato in verun paefe d'America il nome Chincbe per fignificar quel quadrupede.

(d) Vedafi cid che abbiam detto nel lib. $I$. della Storia intorno al nome $\cos \int a$.

(e) II Formicaro è appellato dagli Spagnuoli ofo ormiguero, o fia Orfo formicaro, benchè fia più diverfo forfe dall'Orfo, che non è il Cane dal Gatto. Il C. de Buffon ne diftingue tre fpezie in America: il primo è da lui chiamaro femplicemente Fourmillier, o Formicaro: il 2. Trammannoir, e il 3 . Tamandua. I Peruani. gli appellano Hucumari, i Quitef Huauniri, e'Cuchicbi. i Tamanachi nell' Orinoco traraca. Nel Brafile chiamano i Formicari grandi Tamanduà-guazu, e i piccoli Irara, e Guatimondè. 
JAGUAR (A) o Tigre Americana. I 9.

JAGUARETE $(g)$ o piuttofto Jaguaretè, fiera del genere delle Tigri.r8. ISATIS, fiera de' paefi freddi. 27.

LAMENTIN : così appellano i. Francefi il Manatì, beftia grande del Ma. re, de' laghi, e de' fiumi meffa dal C. de Bufton tra i quadrupedi, benchè appena poffa dirfi bipede, o piuttofto bimano. 27.

IEONE MARINO: così appellò Lord Andfon la Foca maggiore, la quale ha preffo i Chilefi il nome di Lame. 27.

IEPRE comune. I3.

LINCE, o Gatto cerviere. 19.

LLAMA, no Lama, come dice il C. de Buffon, nè Glama, come forive il Sig. de Pavv. Quefto nome, il qual in Ital, diraffi Gliama, è quello del Montone peruano. 26.

LONTRA, detta da' Peruani Miquilo. I4.

LUPO comune, det to da'Mefficani Cuetlacbtli. I4. Ig.

LUPO MARINO, O Foca minore. 27.

LUPO NERO affai diverfo dal comune. Ig.

MAPACH, quadr. curiofo del Meffico. I7.

MARGAI, o Gatto-tigre, fiera. Può effer che quefto nome fia itato prea fo dal Mbaracajà de' Paraguajefi . 27.

MARIKINA, o Scimia leonina, fpezie di Cercopiteco. 30.

MARMOSA, piccolo e curiofo quadrup. de'paefi caldi e temperati d'Ad merica. 2 I.

MARMOTTA, appellata Muax, da' Canadefi . 26.

MICO, Ia fpezie più piccola de' Cercopitechi. (b) $30 .{ }^{\circ}$

MORSO, in franc. Morfe, beftia gr. antibia del mare. $27 \%$

OCELOTL, (i) o Gatto-pardo del Meffico. 27.

ONDATRA ( Rat mufque du Carad'a ) quadrupede alquanto fimile al

Topo. 20.

ORSO BRUNO. I7.

ORSO NERD, ipecificamente diverfo dal bruno. I\%.

PACA, quadr. fomigliante al Porcellino nel pelo e nel grugnito, e nelo la forma della tefta al Coniglio. Nel Brafile s'appalla Paca, nel Pa. raguai $P$ ag, nel Quito Picuru, e nell' Orinoco Accuri. 2 I .

PACO, quadr. dell' America Merid. dello fteffo genere, non però della Storia Anvica del Miefico Tom. IV. ftef-

(f) Jagua in lingua guaranı̀ è nome comune alle Tigri, alle Pume, ed a? Cani. I Peruani appellano la Tigre Uturuncu, e i Mefficani Ocelotl.

(g) Jaguar-etè è in guaraǹ̀ it nome generico delle Tigri .

(h) Mico in Ifpagnuolo è nome generico de'Cercopitechi; ma il Conte de Buffon lo dà alla più piccola rpezie.

(i) Ocelotl in Mefficano è il nome della Tigre, ma il C. de Buffun lo dà al Gatto-pardo. 
Iteffa fpezie della Llama. Il nome Indiano è Allpaca. 26.

PECARI, quadr. il quale ha fulla fchiena una glandola d'umor puzzo: lente creduta da molti ombellico. I veri nomi d'effo in diverfi pae. fi d'A merica fono quelli di Saino, Cojametl., Tàtabro, e Pacbira $(l) .20$. PEKAN, o Martora americana. 27.

PETIT-GRIS. Cosi appella il C. de Buffon un piccol quadr, de' paefi freddi fimile allo Scojattolo. 20.

PILORI ( Rat mufque des Antillies) piccol quadrup. fimile al Topo, e diverfo dall' Ondatra . 20.

PINCHIS (prefo il C. de Buffon $\boldsymbol{P}$ inche ) fpezie di piccol Cercopiteco, 30. POLATUCA, quadr. fimile in parte allo Scojattolo, da'Meficani appellato Quimichpattan, o Sorcio volante. 20.

PORCELLINO D' INDIA ( in franc. Porc $d$ 'inde ) piccol quadr. dell' Amer. Merid. fomigliante in parte al porcellino, $e$ in parte al co. niglio, ma fenza coda. I 6 .

PUMA, o Leone americano, appellato da'Meíficani Miztli, e da' Chile. fi Pagi. 18.

QUIRQUINCHO ( in Ital. diremo Chircbincio ) fpezie di Tatù eoperto d'una conca, e di bande diciotto. $(m) 2 \mathrm{I}$.

RENNO, da' Canadefi detto Caribit. 24.

SAI, (n) fpezie di Cercopiteco. 30.

SAIMIRI, o piuttofto Caimiri, fpezie curiofa di Cercopiteco. 30.

SAKI, fpezie di Cercopiteco fornito di coda affai lunga 30.

SARICOVIENNA, Lontra particolare del Paraguai, del Brafile, della Gujana, e dell' Orinoco. Nel Paraguai l'appellano $\boldsymbol{K} i j a ̀$, e nell'Orinoco Cairo, e Nevi. 27.

SAYU'.( forfe Cajic) fpezie di Cercopiteco. 30.

SORCIO d'acqua. 30 .

SURICATE, quadr. dell'America Merid. il qual ha, come la Jena; quattro dita in tutti i quattro piedi. 26.

SVIZ.

(1) E' da credere che Pacari fia cosi detto dal C. de Buffon da' Pachira, il gual nome è in ufo nell'Orinoco per fignificar quello fteffo quadrupede. Il C. de Buffon l'appella ancora Tayaffon; ma Tajazù ( così dee fcriverfí) netLa lingua guarani è nome comune a tutte le fpezie di porci.

(m) Quirquincbo preffo i Peruani, Ajotocbtli preflo i Mefficani, Tatù preffo i Paraguajefi, e Armadillo ( cioè Bardato) preffo gli Spagnucli fono tutti nomi generici di quelle (pezie di quadrupedi. Il C. de Buffon riftigne il nome Quirquincbo. (no Cirquincon, come egli fcrive) ad una fola fpezie, ficcome pure quello d'Ajotechtli .

(n) Cai, non Sai, come fcrive il C. de Buffon, è nella lingua guaraul il nome generica di cutti i Cercopitechi; ma egli fimilmente lo dà ad una fola spezie. 
SVIZZERO, detto da'Mefficani Tlalmototli, quadr. fomigliante nella forma allo Scojattolo, ma diverfo nella maniera di vivere, e quafi il doppio più grande. 20.

TAIRA, o Donnola della Gujana .

TAMANDUA, o piuttofto. Tamanduà, la fpezie mezzana tra quelle de Formicari . 26.

TAMANNOIR, la fpezie più grande de' Formicari . 26.

TAMARINO, fpezie di piccol Cercopireco. 30.

TAPET, o Tapeto, quadr. dell' America Meridionale fimile in parte al la Lepre, e in parte al Coniglio. Il vero nome in lingua guaranì $\grave{c}$ Tapiiti. 30 .

TAPIR $(o)$ quadr. zrande dell' Amer. appellato dagli Spagnuoli, Anta, Danta, e Granbeftia, e in diverfe lingue americane Tapii. Tapiira, Beori, Tlacaxolotl, Huariari, Sacha-Vacea Orc. 23.

TARSIERE, quadr. alquanto fimile alla Marmofa, e al Tlacuatzin. 29. TATUETO, nome dato dal C. de Buffon a quella fpezie di Tatù, che è coperto di due conche, e d'ot to bande. 2 I.

TLACUATZIN, quadr. curiofo, la cui femmina porta i figliuoli, dopo averli partoriti, dentro una borfa, o membrana, che ha fotto il ventre. Ha in diverfi paefi d'America tutti quefti nomi: Cburcha, Chucha, Mucamuca, Jariquè, Fara, ed Auare. Gli Spagnuoli del Meffico 1'appellano Tlacuacbe. Alcuni Naturalifti gli danno il nome improprio di Filandro, ed altri quello propriffimo di Didelfa. Il C. de Buffon l'appella Sarigue, e Cariguei alterando il nome. Jariguè., col quale è conofciuto quel quadr. nel Brafile. 21 .

TOPORAGNO ( in Ifpagnuolo Mufaraña) 30.

TUZA, ( no. Tucan come fcrive il C. de Buffon) in Mefficano Tozan, $(p)$ quadr. del Meffico del genere delle Talpe, ma più groffo, e piì bello. 30 .

VAMPIRO, gran Pipiftrello d'America . 20.

UARINA, preffo il C. de Buffon Ouarine, $(q)$ gran Cercopiteco barbuto; appellato nel Quito Umeco. 30.

$$
\text { V. VI. }
$$

(o) Io adopro volentieri il nome Tapir; perchè è già in ufo prefro i Zoologifti moderni, e peraltro non è equivoco. Quello di Granbeftia è proprio dell' Alce: quello d'Auta, o. Danta fi dà ancora al Zebù, quadr. dell' Africa affa diverfo dal Tapir.

(p) Non ro fe la Tuza fia della medefima fpezie di quel quadrupede, che i Peruani appellano Tupu-tupu.

(q) Il C. de Buffon dubita fe l'Aluata, Cercopiteco, anche effo grande, fia della medefsma fpezie dell'Uarina; ma io gli concedo che fia in fatti della medefina Spezie, e però non metto l'Aluata ( preffo il C. de Buffon Alona. to ) in quefto ca talogo. 
VISON, o Faina americana. $2 \%$.

UISTITI, fpezie di piccol Cercopiteco. 30.

UNAU, fpezie di Pigro fenza coda $(r) .26$.

VULPE comune. 14.

URSON, quadr. de' paefi freddi fimile al Caftore, ma diverfo. 25.

ZORR!LLO, o fia Zorriglio, fpezie di Puzzola. (S) 27.

Sicchè il C. de Buffon, il quale non trovava in tutta l' America più di fettanta fpezie di quadrupedi, nel decorfo della fua Storia Natirale ne riconofce e diftingue almeno novantaquattro. Diffi almeno; poichè alle fopradette doveano aggiungerfi ciuelle del Porco comune dell'Ermellino, ed altre, le quali benchè negate dal C. de Buffon. all' America in un luogo della fua Storia, de fono pure ftate accorto date in altri luoghi.

\section{II.}

\section{Spezie confufe dal $C$. de Buffon con altre diverfea}

IL Guanaco colla Llama, o Gliama (i)

Ia Vicugna col Paco..

Il Citli col Tapet, o fia Tapiitì . $(u)$

L'Huitztlacuatzin o Iftrice mefficano col Cuandù , o Iftrice della Gujana. $\{x\}$ If Tlacocelotl coll' Ocelotl. $(y)$

- (r) Il Co de Buffon diftingue a ragione due fpezie di Pigri, l'una fornita di coda, e l'altra priva d'effa; poichè oltre a quefto hanno altri caratteri dìverfi. Nel' Quito appellano i Pigri Quillac, o. fia Quiglac, e nell' Orinoco Pro10. Gli Spagnuoli gli chiamano Pereza, cioè Pigrizia, e Perico ligero, cioè Can veloce, per antifrafi.

(f) Z.orrilla, cioè Volperta, è il nome generico che danno gli Spagnuoli, ed altri paefi alle Puzzole. I Niefricani le appellano Epatl. Nel Chile Cbingbe e in altri paefi dell' Amer. Merid. Mapurito, Agnatuja \&xc.

(t) Oltre ad altri capi di diverfità fra la Gliama, il Guanaco, la Vicugna, e. il Paco, non fi fon veduti mai accoppiarfi fra lora, ancorchè meffi infieme in un luogo. Or fe quefto ba凡a per conchindere la diverfità fpecifica fra il Cane, e il Lupo, quadrupedi peraltro affai fimili tanto negli accidenti efterni, quanto nella interna organizzazione, che dovremo dire di quelli quattro quadrupedî più fra loro differenti, che non è il Cane dal Lupo?

(u) Per renderfi certo della diverfità tra il Citli, ed il Tapete bafta paragunare le defcrizioni, che fanno dell' uno, e dell' altro il Dott. Hernandez, e il C. de Buffon.

(x) Vedafi ciò che abbiamo detro nel lib. I. della Storia intorno alla diverfità tra l'Iftrice mefficano, e quello della Gujana.

(y) Il Co de Buffon vaol perfuaderci, che il Tlasoceloth e l'Ocelotl fono un faw 
Il Tepeitzcuintli, o Can montano del Meffico col Gghiottone. (z)

Il Xoloitzcuintli, o Can pelato col Lupo.

L'Itrcuintepozotli, o Can gobbo coll'Alco, o Techichi.

Debbon dunque aggiugnerfi queft' otto fpezie da lui confufe a quelle na: vantaquattro di fopra, e faranno centodue.

\section{II I.}

Spezie ignorate, o negate a torto dal C. de Buffors.

ACHUNI, cercopiteco del Quito fornito di mufo lungo, e di fortiflimi denti, e veftito di pelo groffo come fetole. M.S. appreffo di me.

AHUITZOTL, piccol quadr. anfibio del Meffico da noi defcritto nel lib. I. della Storia.

AMIZTLI, quadr. anfibio del Meffico da noi defcritto. (A)

CACOMIZTLE, quadi. del Meffico fimile alla Faina nella maniera di vivere, ma diverfo nella forma, defcrit to nel lib. I. della noftra Storia .

CANE di Cibola, o Can fomajo, quadr. del paefe di Cibola, fimile nella forma a un maftino, del quale fi fervono quegl' Indiani per por. $\operatorname{tar}$ fome. Fanno menzione di quefto robufto quadr. parecchj Storici del Meflico.

CHICHICO, cercopireco del Quito tanto piccolo, che fi pud tenere nel pugno. Si trova di diverfi colori. M. S.

CHILLIHUEQUE, quadr. grande del Chile fimile.al Guanaco, ma di* verfo. Stor. del Chile del Sig. Ab. Molina.

CHINCHILLA $($ in Ital. Cinciglia) fpezie di forcio campagnuclo la. nuto. Ne parlano moltiffimi Storici dell' America Merid.

CHINCHIMEN, o Gatto marino, quadr. anfibio del Mar del Chile. Stor. Nar. del Chile.

CINOCEFALO CERCOPITECO, quadr. del Mefico del qual fanno men* zione Hernandez, Briffon, ed altri.

$\mathrm{CO}$.

lo animale: che quefto è il mafchio, e quello la femmina: che Ocelotl è lo fterfo nome del Tlacocelotl fincopato. Così potremo dire, che il Canis latino nor ̀ diverfo dal Semicazis, e che Tygris, e lo ftefoche Semitygris; poichè il mefficano Ocelotl vale lo feffo, che Tygris, e Tlacocelotl non vud dire altro che Semitygris. 11 C. de Buffon non può incolparfi di non fapere il mefficano; ma nemmeno può fcufarfi d'aver ofato decidere di quello che non fa. Il Dott. Hernandez, il quaie ebbe fotto gli occhj, ed offeryd da Naturalifta quelle due fiere merita fenza dubbio maggior fede.

(z) Vedafi intorno alla diverfità di quefti tre ultimi quadrapedi ciò che abbiam derto nella IV. Differtazione.

(A) Diff in una nota da me meffa nel lib. I. della Storia, che l'Amiztli mi pareva quello ftefro quadrupede che il C. de Buffon appella Saricovienne; ma avendona fatte nuove sifleflioni, ho trovari fpecificamente diverfi que' due gira. drupedi. 


\section{$15^{8}$}

COJOTE ( in Mefficano Cojotl) fiera da noi deferitta .

CUNIGLIO comune, appellato da' Mefficani Tocbtli .

CUL, o fra Coniglio peruano, piccol quadr.affai fimile al Porcellino d'Ina dia, di cui fan menzione parecchj Storici del Perù.

CULPEU, fpezie particolar di Volpe grande del Chile. Stor. del Ch.

DEGU, o Ghiro del Chile. Stor. del Chile.

FOCA PORCINA, o Porco marino aníbio, fpezie particolar di Foca del Chile. Stor. del Chile.

FURETTO del Chile, e del Paraguai appellato in guaranì Jaguarobape. Stor. Nat. del Chile, e M. S. appreffo di me.

GATTO MELERO. Così appellano gli Spagnuoli un quadr. della Provincia del Chaco nell' Amer. Merid. fimile nella forma al Gatto, il quale da la caccia agli uccelli negli abberi, ed è affai ghiotto del mele delle api. M. S. appreffo di me.

GUANQUE, fpezie di Sorcio campagnuolo turchino del Chile. Stor. Nat. del. Chile.

HORRO, Cercopiteco grande del Quito, e del Meffico tutto nero fuorche il collo, il qual è bianco. Grida fortemente nex bofchi, e meffo ritto fu due piedi ha l'altezza d'un uomo. M. S. appreffo di me.

HUEMUL, o fia Cavallo bifulco del Chile. Stor. del Chile.

JAGUARON, in guarani Jagua-rì, fiera anfibia del Paraguai, chiamata da alcuni Tigre acquatica. M. S. appreffo di me.

KIKI, quadr. del Chile del genere delle Donnole. Stor. del Chile.

MAJAN, quadr. fimile a un porcello, il qual ha il corpo tondo e le fetole arricciate. Abita nel Paraguai. M. S. appreffo di me.

PISCO.CUSHILlo, cioè Cercopiteco uccello, Cercopiteco del Quito, il qual è coperto dal collo fino alla coda di certa fpezie di penne. M. S. appreffo di me.

RICCIO conune nel Paraguai. M. S. appreffo di me .

SORCIO comuniffimo nell'A America prima che vi approdaffero gli Spa. gnuoli, e appellato da'Mefficani Quimichin. Stor. del Meffico.

SORCIO CAMPAGNUOLO volgare nel Meffico, e in altri paefi d'A merica.

TAJE', quadrup. della California, del qual fi fa menzione tanto nella Storia fampata, quanto nelle relazioni manufcritte di quella penifola. Il Tajè è fenza dubbio, l' Ibex di Plinio, defcritto dal C. de Buffon fotto il nome di Bouquetin .

TAITETU', quadr. del Paraģuai del genere de' porci, la cuí femmina fa fempre due figliuoli, i quali nafcono uniti fra loro per mezzo della corda umbillicare. M. S. preffo di me.

TASSO BIANCO della Nuova Yorch defcritto dal Sig. de Briffon.

THOPEL.LAME, quadr. anfibio del mar di Chile fpezie di Foca affai più fomigliante al Leone, che non è quella veduta da. Lord Andfon. Stor. Nat. del Chile.

TLAL. 
TLALCOJOTE, in Meflicano Tlalcojotl. quadr. comune del Meffico da noi defcritto nel lib. I. della Stor.

TOPO BIANCO campagnuolo comune nel Meffico.

TOPO comune campagnuolo comune nel Meflico, e in altri paefi d'A: merica.

TOPO DI MAULE, quadr. di quella Provincia nel Regno del Chile affai fomigliante alla Marmotta, ma più grande d'effa al doppio. Stor. Nat. del Chile.

TREFLE, ofia Trifoglio, quadr. grande dell' America Settentr. defcrit. to dal Sig. de Bomare.

VISCACHA campagnuola, quadr. fomigliante al Coniglin, ma fornita d'una gran coda ripiegata all' insù. Acofta, ed altri Storici dell' A. mer. Merid.

VISCACHA montana, quadr. affai bello del medefimo genere della Vifcacha campagnuola, ma di diverfa fpezie. M. S. appreffo di me. USNAGUA, o Cercopiteco notturno del Quito. M. S. \&c.

Aggiunte queite quaranta fpezie a quelle cento due fopra accennate fanno cento quarantadue fpezie di quadrupedi americani. Se poi a quefte s'aggiungono quelle de' Cavalli, degli Afini, de'Tori, delle Pecore, delle Capre, de' Porci comuni, de' Porci di Guinea, de' Cani, de' Gatti, e de'Topi domeftici, trafportatevi dopo la conquifta, ne avremo prefente. mente in America fino a cencinquantadue fpezie. Il C. de Buffon, il quale in tutta la fua Storia Naturale non conta mai più di dugento fpezie di quadrupedi ne' paefi del Mondo finora fcoperti, ora nella nuova fua 0 o pera delle Epoche della Natura, ne numera trecento. Tanto fi accrebbe quel numero in pochi anni! Ma fieno pur trecento ecco l'America contuttochè non faccia più della terza parte del noftro globo, ha nondimeno la metà almeno delle fpezie di quadrupedi. Torno a dire almeno; perchè ne ho tralafciate tutte quelle, delle quali dubito, fe fieno, o no, diverfe da quelle defcritte dal C. de Buffon. Il fine principale prefiffomi nella formazione di quefto Catalogo non è ftato quello di dimoftrar to sbaglio del C. de Buffon nell' enumerazione de' quadrupedi americani, e la falfità di ciò che fcriffe intorno alla immaginaria fcarfità della materia nel nuovo Mondo; ma bensi quello di fervire a' Naturalifi Europei, indicando loro alcuni quadrupedi finora incogniti, e fpianando alquanto quelle difficoltà, che ha cagionato una mal intefa nomenclatura. Coftoro vorrebbono che $\mathrm{i}$ nomi di que' quadrupedi foffero accompagnati da una efatta defcrizione, ed io lor compiacerei volentieri in quanto mi foffe poffibile, fe ciò non forfe alieno dal mio propofito. Per far quefto catalogo oltre al grande fudio da me impiegato, ho prefo informazioni in ifcritto da perfone dotte, efatte, e pratiche di diverfi paefí d'America, alla cui fino solar bonià mi confeffo fommamente obbligato.

DIS. 


\section{DISSERTAZIONE V.}

SU LA COSTITUZIONE FISICA E MORALE DEI MESSICAN1。

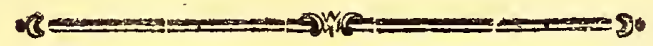

Uattro claîfi d' Uomini poffono diftinguerfi nel Melfico, e in altri paefi dell' America. 1. gli Americani propri, volgarmente appellati Indiani, cioè coloro, che difcendono dagli antichi popolatori di quel nuovo Mondo, e non hanno mifchiato il lor fangue con quello dei popoli deli' antico Continente. 2. Gli Europei, gli Afratici, e gli Africani Atabilitifi in quei paefi. 3. 1 figli, o difcendenti di coftoro, i guali fono appellati dagli Spagnuoli Criollos, e noi diremo Creogli, benchè tal nome fi dia principalmente a quei figli, o difcendenti d'Europei, il cui fangue non fiafi mifchiato con quello degli Americani, degli Afiatici, o degli Africani. 4. Le râzze mifchiate, appellate caftas dagli Spagnuoli, cioè quelli, che fon nati, o difcendono da Europeo, ed Americana, o da Europeo ed Africana, o da Africano ed Americana ec. Tutte quefte claffi d'uomini vengono infamate e ftrapazzate dal Sig. de P. Egli fuppone, o finge tanto maligno il clima del nuovo Mondo, che fa degenerare non folo i Creogli, e gli Americani propri quivi nati, ma eziandio gli Europei abitatori di quei paefi, contuttochè fieno nati fotto un cielo più mite, e fotto un clima più favorevole, come egli crede, a tutti gli animali. Se il Sig. de R. aveffe fcritto le fue Ricercbe Filofoficbe in America, potremmo a ragion fofpertare la degenerazione della Pezie umana fotto il clima americano; ma ficcome vediamo, che tal opera, e moltiffime altre dello fteffo calibro fi fanno in Europa, ci confermiamo vieppiù nella verità di quel proverbio italiano prefo dai Greci, Tutto il mondo è paefe. Ma lafciando ora gli fpropofiti di quel Filofofo, e dei fuoi partigiani contra le altre claffi d'uomini, ragioneremo foltanto di quelli, che egli fcriffe contro gli Americani prepri, mentre co: ftoro 
ftoro fono e $\mathrm{i}$ più ingiuriati, e $\mathrm{i}$ più indifefi. Se nello fcrivere quefta differtazione fulfimo indotti da qualche pafiune $o$ interefle, avremmo piutcofto intraprefa la difefa dei Creogli, come quella, che oltre all' effere affai più facile, dovrebbe intereffarci più. Noi framo nati da genitori Spagnuoli, e non abbiamo veruna affrità o confanguınità cogl'Indiani nè pofiamo fperar veruna ricompenfa dalla loro miferia. Sicchè niua altro motivo, che l'amor della verità, e lo zelo per l' umauità, ci fa ab. bandonar la propria caufa per difeader l'altrui con manco peri. colo d'errare.

\section{§. I.}

Su ic qualità corporali dei Mefjecani.

Il Sig. de P., il quale biafima la ftatura, la forma, e le pretefe irregolarità degli animali americani, non è tato più in dulgente verfo gli Uomini. Se gli animali gli parvero una fefta parte più piccoli di quelli d'Europa, gli uomini fono ancora, al dir di lui, più piccoli dei Caftigliani. Se negli animali notò la mancanza di coda, negli uomini pur cenlura la mancanza di pelo. Se negli animali trovò delle deformità notabili, negli uomini vitupera il colore e le fattezze. Se credette, che gli animali v'erano men forti di quelli dell' antico Continente, afferma parimente, she gli uomini vi fono deboliffimi, e che foggiacciono a mille malattie cagionate dalla corruzione di quell' aria, e di quel terreno puzzolente.

Intorno alla ftatura degli Americani dice in generale, she benchè effa non fra uguale a quella dei Caftigliani, v'è poco divario. Ma noi framo affatto ficuri, ed è notorio in turta la nuova Spagna, che gl'Indiani abitatori di quei paefi, cinè di quelli, che fono dai gradi nove fino ai quaranca di latitudi. ne fettentrionale, fir dove fon giunte le fonperte degli Spagnuoli, hanno d'altezza più di cinque piedi parigini, e che que!li, che non oltrepaffano quella mifura, fono affai più rari fra $\mathrm{gl}^{3}$ Indiani, che fra gli Spagnuoli. Siamo inoltre certi, che molte

Storia fintica del Meffico TomoIV. $\mathrm{X}$ 
di quelle Nazioni, ficcone gli Apaches, $\left({ }^{*}\right)$ gli Hiaquef, i Pi. mefi, ed i Cochimi, fono almeno tanto alti, quanto i prù alti Europei, e non fappiamo, che in turta la valta eftenfione del nuovo Mondo fi trovi alcun popolo, fuorchè quello degli Eskimaux, che fra d'una itatura tanto piccola, quanto quella dei Lapponefi, dei Samojedi, e dei Tartari fettentrionali nell' antico Continente. Onde da quefta parte fono uguali gli abitatori d'ambidue i Continenti.

Quanto alla regolarită, e proporzione delle membra dei Mefficani non bilogna dir più di quello, che abbiam detro nel lib. I. della noftra Storia. Ci perfuadiamo, che fra coloro, i quali in America leggeranno quella noftraopera, non vi farà chi contraddica alla defcrizione della forma, e del carattere degl' Indiani, fe già non abbia gli occhi addietro, o ftravolto il cervello. E'vero, che D. Antonio Ulloa dice (a) ragionando degl' Indiani del Quito, efferfi offervato, che „, fra loro abbondano gl' im"perferti, o perchè hanno $i$ corpi irregolari e moftruofi per " Cagione della loro piccolezza, o perchè divengono infenfati, "muti, o ciechi, o perchè lor manca qualche membro;" ma avendo io fatro delle ricerche intorno a quelta fingolarita dei Quitefi, ho faputo per informazione avuta da perfone degne di fede, e pratiche di quai paefi, che tali difetti non fono cagionati nè da cattivi umori, nè dal clima, ma dalla mal intefa e crudele umanità dei lor genitori, i quali per fottrarre i lor figliuoli dalle gravezze, e dalle fatiche, a cui fono dagli Spagnuoli fottopofti gl'Indiani fani, gli fanno a bella poIta diventare inutili, o imperfetti: lo che non avviene in aleri paeli d'America, e nemmeno in quei luoghi del medefimo regno di Quiro, dove gl'Indiani fon liberi da quelle fariche. Il Sig. de P., e dietro a lui il Dott. Roberton dicono, che fra i Selvaggi dell'America non fi trovano dei deformi ; perchè fanno morire, come faceano già i Lacedemonj, quei

bam-

$\left.{ }^{*}\right)$ Ciò, che diciamo di quelle Nazioni dell' America íttentrionale fi potrebbe anche dire de'Chilef, de'Patagoni, e d'altri popoli dell' America meridionale.

(a) Relacion del Viage d̀ la Americ Meridional oco 
bambini, che nalcono gobbi, ciechi, o mancanti di qualche menbro; ma che in quei paefi, nei quali fono ridotti a focieta, e'dove la vigilanza di coloro, che gli reggono, non permette loro fiffatti infanticidj, il numero degl' individui irregolari è più grande di quello di qualfifia paefe d'Europa . Quefto farebbe un bello fcampo alla difficoltà , fe foffe vero; ma fe per avventura v'è ftata in America qualche tribù di felvaggi, che abbia imitato il barbaro efempio $\left({ }^{\star \star}\right)$ dei tanto rinomati Lacedemonj, egli è certo, che quegli Autori non hanno ragion d'imputare una tale inumanicà al refto degli $\mathbb{A}$. mericani; poichè effa non è ftata in ufo almeno preffo la maggior parte di quelle Nazioni, ficcome può dimottrarfi per la depofizione degli Scrittori più bene inftruiti dei loro coftumi. Oltracciò in tutti i paefi del Meffico, o fia della N. Spagna, i quali fanno almeno una quarta parte del nuovo Continente, vivono gl' Indiani uniti in focierà, e congregati in Città, borghi, e Villaggj fotto la cura dei Magiftrati, e dei Parrochi Spagnuoli, o Creogli: non vi fi vedono mai, ne fi fentono quagli efempi di crudelta verfo i teneri fanciulli, e ciò non oftante fonovi cos̀ rari $\mathrm{i}$ deformi, che tutti quegli Spagnuoli, e Creogli, che nel 1768. vennero dal Meffico in Italia, reftarono allora, e lono anche oggid' maravigliati dall' offervare nelle Città di quefta coltiflima penifola un si gran numero di ciechi, di gobbi, di zoppi, d'attratti ec. E' dunque affai diverfa da quella, che s'immaginano i fuddetii Autori, la cagione di quel fenomeno offervato da tanti Scrittori negli Americani.

Dal color poi dei Popoli Americani non fi può formaré alcun argomento contro il nuovo Mondo; perchè quel colore è meno diftante dal bianco degli Europei, che dal negro do gli Africani, e d'una gran parte degli Afiatici. I capelli dei Mefficani, e della maggior parte degl' Indiani fono, come ab.

$$
\mathrm{X} 2 \text { biam }
$$

$\left({ }^{* *} L\right.$ Quell' inumanità d'ammazzare i figliuoli che nafcevano deformi non che permelfa in Roma, ma fu anche prefcritta dalla legge delle dodici $\mathbb{T}_{3}$ yole: Pater injignem ad deformitatem purrum cito necato. 


\section{I $\sigma_{4}$ DISSERTAZIONE V.}

biam detto alirove, groffi, e folti, il loro pelo fcarfo nella faccia, e niuno per lo piir (b) nelle braccia, e nelle gambe; ma è un errore il dire, come dice il Sig. de P., che effi fano affatto privi di pelo in tutte le altri parti del corpo. QueSto è uno di quei molti palli delle Ricercbe flofoficbe, nei quali non potranno trattener le lor rifate i Mefficani, ed altre Nazioni americane in vedendo un Filofofo Europeo tanto impegnato nello fpogliarli di quello, che hanno avuto dalla natura. Egli leffe fenc'altro quella ignominiofa defcrizione, che fa il Sig. Ulloa d'alcuni popoli dell' America Meridionale, (c) e da quefta premeffa particolare deduffe giulta la fua Logica quella conclufione univerfale.

L'afpetto folo di qualche Angolano, Mandinga, o Congo dovrebbe avere impaurito il Sig. de P., e diftoltolo dalla cenFura, che egli fa del colore, delle fattezze, e del pelo degli Americani. Qual cola può immaginarfi più oppofta all' idea, che abbiamo della bellezza, e della perfezione del corpo uma: no, che un uomo puzzolente, la cui pelle è negra, come l' inchioftra, il capo e la faccia coperti di lana negra in vece di pelo, gli occhi giallicii, o fanguizni, le labbra groffe e nericcie, e il nafo fchiacciato? Tali fono gli abitatori d'una grandiffima parte dell' Africa, e di parecchie ifole dell' Afia. Che uomini più imperfetti di quelli, $i$ quali non hanno più di quattro piedi di ftatura, la fáccia lunga e piatta, il nafo lchiacciato, l' iride degli occhi gialla nericcia, le palpebre ritratte verfo le tempie, le gote oltremodo elevare, la bocca troppo grande, le labbra groffe e prominenti, e la parte inferior del vifo troppa Atretta? Tali fono, al dir del $C_{a}$ de Buffon, (d) i Lap.

pone-

(b) Difli per lo più; perchè vi fono nel Meffico de' popoli americani barbati, e forniti di pelo nelle braccia, e nelle gambe.

(c) Il Sig. Ulloa nella defcrizione che fa degl'Indiani del Quito nel tom. . $_{\text {. }}$ della Relazione del fuo Viaggio in America, dice, che nè agli uomini, nè alle donne viene il pelo, come al refto degli uomini, quando arrivano alla: pubertà; ma checcheffia di quefta fingularita de' Quitefi, e della cagione di efra, non v'è dubbio che preffo il comun degli Americani la pubertà viene accompagnata da'medefimi fintomi, che preffo le altre Nazioni del Mondo.

(d) Hift. Natur. tom. 6. 
ponefi, i Zemblef, i Borandiani, i Samojedi, ed i Tartari Orientali. Quali obbietti più deformi, che uomini, la cui fac. cia fia troppo larga e grinza anche nella lor gioventù, il nafo fchiacciato e groflo, gli occhi piccoli ed affondati, le gote troppo alzate, la parte fuperior delle mafcelle abbaffata, $i$ denti lunghi e difuniti, $\mathrm{i}$ cigii tanto carichi, che ingombrano gli occhi, le palpebre groffe, qualche fecola nella faccia in vece di barba, le cofcie grandi, e le gambe piccole? Tal è il ritratto, che $f_{d}$ il C. de Buffon dei Tartari, cioè di quei popoli, che ficcome egli fteffo dice, abitano un tratto di paefe dell' Afra lungo più di mille dugento leghe, e largo più di fettecento cinquanta. Tra quefti $\mathrm{i}$ Calmuchi fono i più notabili per la lor deformità, la quale è fi grande, che effi fono, a detta del Tavernier, gli uomini più brutti di tutto l' Univerfo. La lor faccia è tanto larga, che v'è tra due occhi uno fpazio di cinque, o fei dita, fecondochè afferma il medefrmo C. de Buffon: In Calicut, in Ceilan, ed in altri paefi dell' India, evvi, dicono Pyrard, ed altri Scrittori di quelle regioni, una razza d'uomini, che hanno una delle gambe, o amen. due cos̀̀ grofle, come il corpo d'un uomn, la cui deformità è preffo loro ereditaria. Le Ottentote hanno, oltre ad altre grandi imperfezioni, quella moftruofa irregolarita di un' appendice callofa, che fi ftende dall' offo pubes in giù, ficcome ne fanno fede gli Storici del C. di Buona Speranza. Marco Polo, Struys, Gemelli, ed altri Viaggiatori atfermano, che nel regno di Lambry, nell' Ifola Formofa, e in quella di Mindoro fi trovano degli uomini forniti di coda. Il Sigg. de Bomare (e) dice, che una tal coda in quegli uomini non è altro, che un allungamento dell' offu coccyx; ma che è la coda nei quadrupedi fe non un al. lungamento di quell' offo, benchè divilo in parecchie arricolazioni ? $(f)$ Checcheffia, egli è certo, che quell'allungamento tanto vale, quanto la vera coda a render quegli Afiatici troppo irregolari.

Se

(e) Dictionn. d'Hiftoire Natur. V. Homme

(f) Vedafi L'Hzilter nel fuo Comp. Anat. de Ofdbus trunci. 


\section{DISSERTAZION E V.}

Se voleffimo nello fteffo modo percorrere le altre Nazioni dell' Afia, e dell' Africa, appena ne troveremmo una parte non troppo grande, che non fia di color più ofcuro, e nella quale mon fir fcorgano delle irregolarità più enormi, e dei difetti più grandi di quanti ne cenfura il Sig. de P. negli Americani. Il color di coftoro è affai più chiaro di quafi tutti gli Africani, e degli abitatori dell' Afia 'meridionale. La fcarfezza di barba è comune agli abitanti delle Ifole Filippine, e di tutto l'Arcipelago Indiario, ai famofi Chinefi, ai Giapponefi, ai Tartari, ed a molte altre Nazioni dell' antico Continente, ficcome è nota a tutti quelli, che hanno qualche notizia della varietà della fpezie umana nei diverfi paefi della Terra. Le imperfezioni degli Americani, quantunque grandi fi vogliano rapprefentare, non fono certamente da paragonare coi difetti di quegl' immenfi Popoli, il cui carattere abbiamo abbozzato, e d'altri, che tralafciamo. $(g)$ Tutto quefto dovrebbe aver trattenuta la penna del Sig. de P., ma egli fe n'era dimenticato, o pur maliziofamente il diffrmulò.

Il Sig. de P. rapprefenta gli Americani deboli e malaticci; Il Sig. Ulloa per l'Oppofto afferma, (b) che effi fon Sani, robufti, e forti. Chi dei due meriterà da noi maggior fede: il Sig. de P., che da Berlin fi mife a filofofar fu gli Americani fenza conofcerli, o il Sig. Ulloa, che per alcuni anni li vide, e gli praticò in diverfi paefi dell' America meridionale? Il Sig. de P., che fi prefe l'impegno di vilipenderli, e d'avvilirli per iftabilire il fuo fpropofitato fiftema della degenerazione, o il Sig. Ulloa, il quale benchè peraltro poco favorevole ag!' Indiani, non fi curò di formar verun fiftema, ma foltanto di fcrivere quello, che giudicava vero? Il Lettore imparziale deciderà quefto dubbio.

Per dimoftrare il Sig. de P. la debolezza, e lo fconcerto della cofituzione fifica degli Americani allega parecchie ragio: ni,

(g) Vedafi il tom. 6. della Stor. Nat. del C. de Buffon, come pure gli Storici dell' Afia, e dell' Africa .

(b) Relacion del Viage ì la America Meridional tom. I. lib. 5. sap. 5. 


\section{DISSER TAZIONE V. 167}

ni, che noi non dobbiamo diffimulare, e fono quefte. I. Che i primi Americani condotti in Europa arrabbiarono nel viag. gio, e la rabbia lor durò fino alla morte. 2. che gli uomini adulti in molti paefi dell' America hanno del-latte nelle lor mammelle. 3. Che le Americane fi Gravidano con troppa facilità, hanno una ftraordinaria abbondanza di latte, ed è in loro fcarfa, e irregolare la periodica evacuazion del fangue. 4. Che il men vigorofo Europeo vinceva nella lotta qual fi foffe Americano. 5. Che gli Americani non poteano reggere al pefo d'una foma leggiere. 6. Che foggiacevano al mal venereo, e ad altre malartie endemiche.

Quanto alla prima prova, noi la neghiamo come affolutamente falfa e infuffiftente. Il Sig. de P. fu la fede del Fiammingo Dappers dice, che $\mathrm{i}$ primi Americani, che conduffe feco Colombo nel 1493. fi vollero ammazzare nella navigazione; ma che avendolı legati per confervarli, divennero rabbiofi, e la lor rabbıa durò finchè viffero: che allorchè entrarono in Barcellona fpaventarono a tal fegno quei Cittadini col loro urli, colle loro contorfioni, e coi loro movimenti convulfivi, che furono fimati farnetici. Io non ho veduta l'opera di Dappers; ma non dubito, che tutto il fuo ragguaglio è un ammaffo di favole; poichè non ho trovato chi faccia menzione di quel fucceffo nè fra gli Autori contemporanei, nè fra quelli, che fcriffero negli anni fuffeguenti; anzi da ciò, che teftificano coftoro fi può dimoftrar la falfità di quella relazione. Gonzalo Fernandez d'Oviedo, il quale fi trovava allora in Barcellona, quando vi giunfe Colombo, vide e concbbe que. gli Americani, e fu teltımonio oculare di quanto avvenne, nulla dice della lor rabbia, nè dei lor urli, e contorfioni, e non l'avrebbe tralafciato, fe foffe vero, effendo egli piuttofto contrario agl' Indiani, come altrove direme, e parlando si individualmente della loro entrata in quella Città, del lor battefimo, dei lor nomi, e in parte del lor fine. Dice cunque (i), che Colombo conduffe feco dall' ifola Spagnuola dieci Americani,

(i) Stor. gener. delle Ind. Occid. lib. 2, cap. 7. 


\section{I68 DISS E R T A Z IO N E V.}

cani, dei quali uno mori nel viaggio, tre reltarono ammalati in Palos, porto d'Andaluzia, dove per quanto egli congettura, indi a poco morirono, e gli altri Cai andarono a Barcellona, dove allora fi trovava la Corce: ch'effi furono bene inftruiti nella dotrina criftiana, e folennemente battezzati, effendo lor Patrini i Re Cattolici, e il Principe D. Giovanni: che il principal d'effo loro, il qual era parente del Re Guacanagari, prefe nel battefimo il nome del Re Cattolico, e fi chiamò Don Ferdinando d'Aragon, e al fecondo fu impoto il nome del Principe, appellandofi d'allora innanzi Don Giovanni di Caftiglia, il quale fu alloggiato dal Principe nel fuo palazzo, e coftui to fece inftruire: che egli imparò aftai bene la lingua Spagnuola, ed indi a due anni morì. Pietro Martire d'Anghiera, il qual era anch' egli in Ípagna, allorchè vi arrivò Colombo, fa pur menzione (k) degl'Indiani, che menò feco lui quel famofo Almirante, e non $f a$ un motto della lor rabbia; anzi racconta, che allorchè Colombo ritornò nella Spagnuola riconduffe feco tre di quegl' Indiani; poichè twti gli altri eran già morti per la mutazione dell' aria, $e$ dei cibi, $(l)$ e che fi prevalfe d'uno d'effi per informarfi dello ftato, in cui trovavanfi gli Spagnuoli, che avea lafciati in quell'ifola. Ferdinando Colombo dotto e diligente Scrittore della vita di Criftofo. ro Colombo fuo Padre, il quale trovavaf parimente allora in Í́pa-

(k) Sommar. della Stor. delle Ind. Occid. cap. 4.

(1) Al!e cagioni della morte di quegli Americani addotte da Pietro Martire fi doveano aggiungere gli ftraordinari difagi, che patirono in quell' orrenda navigazione, le cui circoftanze poffono leggerfi nelle medefime lettere dell'Almirante Colombo rapportate dal fuo dotto figlio D. Ferdinando. Da quel numero de' morti accennato da Pietro Martire fi dee eccettuare quell' Americano, che ritenne feco il Principe $D$. Giovanni; poichè egli non morì fe non due anni dopo, come ne fa fede Oviedo. Ma quantunque foffero tutti rnorti nel viaggio, o divenuti frenetici o maniaci, non farebbe da maravigliare attefo ciò che racconta lo fteffo Sig. de Pavy nella part. 3. rez. 2. delle rue Ricerche: Les Academiciens Francois, dice, enlezerent au de-là de Torneo devx Lappons, qui, objedés or martyrifés par ces pbilolopbes, moururent de défefpoir en rouste. Ora nè il paere, che lafciavano qué Lapponefi, nè il viaggio, che ebbero a fare può paragonarfi col paefe, e col viaggio di quegli Americani, nè io poffo credere tanto umani i Marinai'Spaguuoli del fecolo XV., quanto gli Accademici Francefi dol fecolo XVIII. 


\section{I S E R T A Z I O NE V. 169}

Ifpagna, fa un minuto ragguaglio dei viaggi, e delle azioni del fuo gloriofo Genitore, parla di quegl' Indiani da lui veduti, e non dice altro da quello, che racconta Pietro Martire. $E^{\prime}$ dunque fallo il ragguaglio di Dappers, o pur diremo, che i Re Cattolici vollero tenere al battefimo degli uomini rabbiofi, che il Principe volle aver feco un rabbiofo per ricrearfi coi fuoi urli fpaventevoli, che un rabbiofo imparò affai bene la lingua Spagnuola, e finalmente che il prudente Colombo fi fervì d' un uomo rabbiofo per informarfi di tutto cio, ch' era accaduto alla Spagnuola, mentre egli era affente.

L' aneddoto del latte nelle mammelle degli Americani è uno de' più curiofi, che fi leggano nelle Ricercbe Filofoficbe, e de' più degni di celebrarfi colle noftre rifate, e con queile di tutti gli Americani; ma bifogna confeffare, che il Sig. de P. fr mổrò in ciò più moderato d'altri Autori da lui citati. Il celebre Naturalifta Jonfton afferma nella lua Tbaumatograpbia fu la fede di non fo che Viaggiatori, che nel nuovo Mondo quafi tutti gli uomini abbondano di latte ne!le mammelle. In tutto il Brafile, dice l' Autor delle Ricerche Storicbe, gli uomini foli allattano i bambini; perchè le donne non hanno qua. fi niente di latte. O che bei materiali per una Thaumatografid! Io in vero non fo che debba ammirar più, fe la temerità, $e$ la sfacciataggine di que' Viaggiatori, che fpacciano fiffatte favole, o la troppa femplicita di coloro, che le adottano. Se foffe mai nel nuovo Mondo qualche Popolo, nel quale foffe ftato veramente offervato un tal fenomeno (ciò che il Sig. de P. non potrà giammai verificare) quello certamente non farebbe fufficiente per dire, che in molti luoghi dell' America abbonda il latte nelle mammelle degh uomini, e molto meno per affermarlo, come l'afferma Jonfton, di quafi tutti gli womini del nuovo Mondo.

Quelle fingolarita, che nota il Sig. de P. nelle America: ne, farebbon loro gradevoliffime, fe folfero vere; perchè che faprebóono più defiderare, che di vederfi libere da que'gran dolori e ftenti, che hanoo nello fgravidare, d'abbondare di quel liquore, con cui debbono nudrire i lor figliuoli, e di rifpar: Storia Antica del MeJjico Tom. IV. Y 
I70 DISSERT TIONE V.

miarfi in gran parte gl' incomodi, che lor cagiona quella periodica e fchifofa evacuazione? Ma ciò, che da loro crederebbefi una gran felicità, è reputato dal Sig. de P. argomento di degenerazıone; perchè quella facilità nello fgravidarfi dimoftra, dice egli, l'efpanfione del condotto vaginale, e il rilaffamento de'mufcoli della matrice per cagione de'fluidi troppo copiofi : l'abbondanza di latte non puó derivare, fe non dall' umidità della lor compleffione, el del refto elleno non fi conformano colle donne dell' antico Continente; poichè quefto debbe effere fecondo la legislazione del Sig. de P., il modello di tutto il Mondo. Ma a chi non farà meraviglia, che Jaddove l'Autor delle Ricercbe Storicbe nota nelle Americane una tale fcarfezza di latte, che per cagione d'effa fono gli uomini coltretti ad allattare eglino Ateffi $i$ lor figliuoli, l'Autor delle Ricercbe Filofoficbe per lo contrario rilevi in effe una si Atraordinaria abbondanza di quel liquore? $E$ chi farà, che in leggendo quefte, ed altre fimili contraddizioni, e frottole pubblicate in Europa, fpezialmente da pochi anni in qua, non s'accorga, che i Viaggiatori, gli Storici, i Naturalifti, ed i Filofofi Europei (*) hanno fatto nell' America il magazzino delle lor favole, e delle lor ciancie, e per render più amene le loro opere colla novità maravigliofa delle loro fuppofte offervazioni, attribuificono a tutti gli Americani ciò ch'è ftato offervato in alcuni individui, ovvero in neffuno?

Le Americane, come quelle che foggiacciono alla comun condanna, non fi fgravidano fenza dolore; ma neppur con quell' apparato delle donne europee; perchè fon meno delicate, e più avvezze a'difagi. Tevenot dice, che le donne Mogolefi fi difgravidano con fomma facilita, e che il giorno appreffo fi vedono girar per le ftrade delle Città, e non però fi dee contraddire alla lor fecondirà, o biafimar la loro compleffione.

La quantità, e la qualità del larte delle Americane fono nel

$\left(^{*}\right)$ Cid, che dico degli Europei fcrittori delle cofe d'America, non voglio, che s'intenda di tutti; poichè tra loro vi fono degli uomini veramente raggi, e amanti della verità. 
nel Meffico, e in altri paefi d'America ben note alle Dame europee e creoglie, come quelle, che comunernente le prendon no per balie de'lor figliuoli; perchè fanno bene, che effe fon fane, e affai fedeli, e diligenti in tal minifterio. Nè vale dire, che fi parla delle Americane antiche, e non delle moderne, come talvolta rifpofe il Sig. de 'P. al fuo avverfario Don Pernery; poichè, oltrecchè le fue propofizioni contro l'America fono quafi tutte di prefente, ficcome è noto a coloro, che hanno letta la fua opera, quella diftinzione non ha luogo in molti paefi dell'America, e particolarmente nel Meffico. I Mefficani ufano per la maggior parte degli fteffi cibi, che us favano prima della conquifta. Il clima, fe forfe s'è mutato ia altre regioni per cagione dell'abbattimento de' bofchi, e dello fcolo delle acque ftagnanti, nel Meffico è fenz' altro il medefimo. Coloro che hanno paragonato, come ho fatto io, le relazioni de' primi Spagnuoli collo ftato prefente di quel Regno, fanno colla maggior evidenza, che fuffiftono gli fteffi laghi, gli fteffi furni, e per lo più gli fteffi bofchi.

Intorno a' meftrui delle Americane nè io poffo darne con. tezza, nè fo, che vi fia alcuno, che poffa darla? Il Sig. de P., il quale da Berlino ha vedute tante cofe in America, che non vedono gli ftelfi abitatori di que' paefi, avrà forfe trovata preffo qualche Autore francefe la maniera di faper ciò, che noi nè poffiamo, nè vogliamo ricercare. $\mathrm{Ma}$ dato che la meftruale evacuazione delle Americane fia ftata fcarfa e irregolare, quanto vuole il Sig. de $P_{0}$, nulla potrebbe conchiuderfi contro la loro compleffione; perchè la quantita di tal evacuazione dipende, ficcome dice bene il C. de Buffọn, $(m)$ dalla quantita dell' alimento, e della trafpirazione infenfibile. Le donne, che manginno troppo, e fanno poco efercizio, hanno i me. frui abbondantiffimi. Ne paefi caldi, néquali la trafpirazione ¿ piu copiofa, che ne' freddi, è piì fartfa quell' evacuazione. Ora te la fcarfirà di fiffatta evacuazione può provenire dalla fobrietà nel vitto, dal calor del clima, e dall' efercizio, perchè $\mathrm{Y} 2$

(m) Stor. Natur, tom. 6. 


\section{D I S S E R T A Z I O N E V.}

fi vuol produrre come argomento di cattiva complefione? Olirechè io non fo, come aggiuftare quella fcarfità del meftruo con quella foprabbondanza di fluidi, che il Sig. de P. fuppone nelle Americane, come una forgente dello fconcerto della fifica lor coltituzione.

Non fono più efficaci le fapraccennate prove della debolezza degli Americani. Dice il Sig. de P., che effi erano vinti da qualunque europeo nella lotta, e che venivano meno fotto un pefo mediocre: che fattone il computo, fi trovò effer periti in un fol anno nel trafporto de' bagagli dugento mila Americani. Quanto alla prima, bifognerebbe, che la fperienza della lotta fi foffe fatta tra molti individui dell' uno, e dell' altro Cuntinente, e che la vittoria veniffe teftificata non meno dagli Americani, che dagli Europei. Ma comunque fia, io non pretendo di far comparir gli Americani più forti degli Europei. Poffono efser men forti fenza però effere pofitivamente deboli, e fenza aver degenerata in loro la fpezie umana. Gli Svizzeri fon più forti degl'Italiani, e non peræ̀ crederemo gl' Italiani degenerati, e nemmeno taccieremo il clima dell' Italia. L'efempio de' dugento mila uomini morti in un fol anno fotto il pefo de' bagagli, fe mai fof́se vero, non convincerebbe tanto la debolezza degli Americani, quanto l'inumanità degli Euro. pei. Come perirono que' dugento mila Americani, cosi farebbono periti dugento mila Pruffiani, fe fofsero ftati coltretti a fare un viaggio di trecento, di quattrocento e più miglia con cento libbre di pefo addofso: fe meffi loro de' collari di ferro uniti con grofse catene folsero ftati obbligati a portar quella foma per monti e balze, ed a coloro che fi ftancavano, o fi rompevano $\mathrm{i}$ piedi, folsero ftate tagliate le tefte, perchè non ritardafsero il pafso agli altri: fe non fofse ftato loro dato, che un cibo afsai tenue da poter reggere a sì intollerabile fatica. Lo ftefso Autore $\left(^{*}\right)$ da cui prefe il Sig. de P. quel computo de" dugento mila Americani morti fotto il pefo de' bagagli, rapporta anche tutte le predette circoltanze: onde fe egli gli da fe-

(*) Monfig. de las Calas. 
de in quello, dovrà dargliene anche in quefto. $\mathrm{Ma}$ un Filofo: fo, che vanta tanto le qualita fifiche e morali degli Europei fopra quelle degli Americani dovrebbe piuttofto aftenerfi dal far menzione di que' fatti tanto ad efsi Europei obbrobriofi .E' vero, che nè l'Europa, nè veruna Nazione d'effa può incolparfi di quegli ecceffi, ne' quali incorrono alcuni de' fuoi individui, maffimamente in paefi tanto lontani dalla Metropoli, e contro l'efpreffa volontà e gli ordini replicati de' loro Sovrani; ma fe gli Americani voleffero fervirfi della Logica del Sig. de PaW, potrebbero da tali antecedenti particolari dedur delle confeguenze univerfali contra tutto l'antico Continente, poichè egli forma ad ogni tre parole degli argomenti contra tatto il nuovo Mondo da ciò, che è ftato ofservato in qualche popolo, o in qualche individuo, come fi può vedere in leggendo la fua opera .

Egli concede agli Americani una grande agilità e velocita nel corfo; perchè da fanciulli s'avvezzavano a tal efercizio: dunque nemmeno dovrà negar loro la forza; poichè, ficcome confta dalla loro Storia, e dalle fteffe loro pitture, appena che comminciavano a camminare i bambini, gli fottomettevano all'efercizio della carica, nel quale doveano impiegarfi tutto il tempo della lor vita: anzi giufta i fuoi principj niun'altra Nazione dovea effere più vigorofa per la carica; perchè niun'altra s'efercitava tanto, qquanto gli Americani nel portar cariche addoffo per cagione di mancar loro le beftie da foma, (n) di cui fi fervono altre Nazioni. Se il Sig. de $\mathrm{PaW}$ aveffe veduto, ficcome ho veduto io, gli enormi pefi, che portano fu le loro Ppalle gli Americani, non avrebbe avato coraggio di rinfacciar loro la debolezza.

Ma nulla dimoftra così chiaramente la robultezza degli Ame-

(n) Benchè i Peruani aveffero beflie da foma, non crano tali da poterfene rervire pel trafporto di quelle gran pietre, che fi trovarono in alcuni de'loro edifizj, come pure in quellt del Mefíco: onde neppure avendo delle macchine per agevolare an tal trafporto, quetto dovea farfí colle fole forze degli uomini. 


\section{DISSER T A Z ION E V.}

Americani come qualle molte e grandi fatiche nelle quali fono continuamente impiegati. Il Sig, de Paw dice (o), che al. lorchè fu fcoperto il nuovo Mondo, non vi fi vedeva altro che folti bofchi : che oggidi vi fono alcuni terreni coltivati, ma dagli Africani, e dagli Europei, non dagli Americani; e che il terreno coltivato rapporto all' incolto è nella proporzione di due mila a due milioni. ( $p$ ) Quefte tre afferzioni fono altret. tanti errori; ma riferbando ora per un' altra differtazione ciò che appartiene alle fatiche degli antichi Mufisani, e parlando foltanto dei tempi pofteriori, egli è certo, che dalla conquifta in quà gli Americani foli fono Itati quelli, che hanno fopportate tutte le fatiche dell' Agricoltura in tutti $i$ vafti paefi del Continente dell' America Settentrionale, e nella maggior parte di quelli dell'America Meridionale fottopoiti alla Corona di Spagna. Non vi fi vede mai un Europeo impiegato nei lavo. ri della campagna. I Mori, i quali nel vafto regno della $\mathbf{N}$. Spagna fon pochiffimi in paragone degli Americani, fono incaricati della coltura della cannamele, e del tabacco, e della

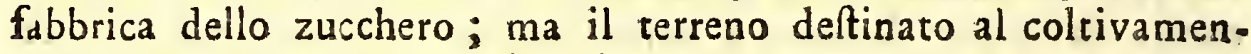
to di quelle piante non è rifpetto a tutta la terra coltivata neppur nella proporzione d'uno a due mila. Gli Americani fon quelli, che vi lavorano la terra. Eglino foli fono gli aratori, i feminatori, i farchiatori, ed i mietitori del frumento, del frumentone, del rifo, della fava, dei fagiuoli, e delle altre biade, e legumi; del caccao, della vainiglia, del cotone, dell' indaco, e di tutte le altre piante utili al foftentamento, al veftire, $e$ al commercio di quelle Provincie, e fenza loro niente $f i f_{a}$ a tal feguo, che- $l^{2}$ anno 1762 . 's'abbandonò in molti luoghi la raccolta del frumento per cagione dell' infermità che vi fu, e non permife agl' Indiani di far la miecitura. Ma quetto è poco: eglino fono quelli, che tagliano e trafpor-

tano

(o) Defense des Recberckes cap. I2.

(p) Sarebbe ftato meglio dire. nella proporziome d' uno a mille, mentre queto vale lo felfo con numeri più femplici. 


\section{I S S E R T A Z I O N E V.}

tano dai bofchi tut:o il legname neceffario: quelli che taglia: no, trafportano, e lavorano le pietre, e quelli che fanno la calcina, il geffo, ed i mattoni. Eglino fono quelli, che $f_{d} b$ bricano tutti gli edifizj di quel regno, fuorchè in pouhi luoghi, dove effi non abitano. Eglino fono quelli, che aprono, ed acconciano le ftrade, quelli che fano le gore, e le pefcaje, e quelli che nettano le Citta. Eglino lavorano in moltiffime miniere d'oro, d'argento, di rame ec. Eglino fono i Paftori, e gli Armentarj, i Teffitori, i Pentolai, i Panattieri, i Fornaj, i Corrieri, i Facchıni ec. In una parola eglino fon quelli, che portano tutro il pefo dei travagli pubblici, ficcome è notorio in tutte le Provincie di quel gran regno. Quefto $f_{d} n$. no $\mathrm{i}$ deboli, $\mathrm{i}$ poltroni, e gl' inutili Americani, frattantochè il vigorofo P., ed altri infaticabili Europei s'occupano nello fcrivere delle invettive contra loro.

Quefti travagli, nei quali s'impiegano continuamente gl'Indiani, danno a divedere la lor fanità e robuftezza; poichè non potrebbono reggere a sì gran fatiche, fe foffero malaticci, e fe per le lor vene giraffe un fangue guafto, come vuole il Sig. de P. Egli per far credere viziofa la lor compleffione allega tutto ciò o vero o falfo, che potè trovar preffo gli Scrittori d'America intorno alle malattie, che regnano in alcuni paefi particolari di quel gran Continente, e fpezialmente intorno al mal venereo, che egli crede veramente americano. Or quanto al mal venereo, ne ragioneremo diffufamente in un'al: tra differtazione; intorno poi alle altre malattie, io gli accordo, che nella vafta eftenfione dell' America vi fono alcuni pae. fr, nei quali gli uomini fono più che altrove efpofti ad alcune malattie cagionate o dall' intemperie dell' aria, o dalla cattiva qualità degli alimenti; ma egli è certo attefo la depofizione di molci gravi Autori pratici del nuovo Mondo, che i paefi americani fon per la maggior parte fani, e che fe gli Americani voleffero render la pariglia al Sig. de P., e ad aitri Europei, che fcrivono come lui, avrebbono una copia affai più grande di buoni materiali per ifcreditare il clima dell' antico Continente, e la compleffione dei fuoi abitatori, in tante ma: lattie 


\section{IF 6 DISS ER T A ZION N V.}

lattie endemiche, che vi fono, come l'Elefanzia, e la Lebbrà dell' Egitto, e della Soria, $(q)$ il Verben dell' Afia Meridionale, il Dragoncello, o fia verme di Medina, il Pircal del Malabar, l' Yaws, o mal di Guinea, la Tiriafi, o Morbo pedicolare della piccola Tartaria, lo Scorbuto, e la Diffenteria boreale dei paefi fettentrionali, la Plica di Pollonia, il Gozzo del Tirolo, e di molti paefi alpigni, la Rogna, la Rachitide, il Vajuolo, $(r)$ e foprattutto la Pefte, che tante volte ha Spopolate moltiffime Città e Provincie intere dell' antico Continente, e che ogni anno fa una grande ftrage nell' Oriente: flagello terribile, dal quale è ftato finora prefervato il nuovo Mondo.

Finalmente non fí può accordare la pretefa debolezza, e viziofa abitudine degli Americani colla lunga lor vita. Tra quegli Americani, cui le gran fatiche, e gli ecceffivi travagli, ovvero le malattie epidemiche non anticipano la morte, vi fono non pochi, che giungono agli ottanta, novanta, e cento ạnni, e ciò, ch'è più da maravigliare, fenza offervarfí in loro quella Atrage, che fa comunemente il tempo ne'capelli, ne' denti, nella pelle, e ne'mufcoli del corpo umano. Quefto fenomeno tanto ammirato dagli Spagnuoli abitatori del Mefico

non

(q) L'Elefanzia nıวbo endenico dell' Egitto, ed affatto incognito nell' America, fu si comune in Euroja nel recolo decimuterzo, che v'erano, per quel che dice Matteo Paris, Scrittore efatto di quel tempo, diciannove mila fpedali.

(r) Il Vajuolo $\mathrm{fu}$ portato in America dagli Europei, come fanno tutti, e $\checkmark i$ ha fatto una Arage arrai più grande di quella, che ha fatto in Europa il mal venereo. La Rachitide, o fra Nodatura non è conofciuta nel nuovoMondo, e quefta è a mio giudizio la principal cagione di non efrervi quel numeso d'uomini imperfetti, che fi vede in Europa. La Rogna o non v'è, o è si rata, che effendo io tato tanti anni in parecchj paefi del Meffico, nè vidi mai verun rognofo. nè feppi che vi foffe. Il vomito prieto, il qual pare ancora un mal endemico, è aftai moderno, e non fo patifce; fe non in alcuni porti della zona torrida frequentati dagli Europei. I primi a fentirlo furono i Marinaj d'alcuni vafcelli europei, $i$ quali dopo $i$ cattivi alimenti della navigazione mangiavano in que' porti avidamente delle frutta del paefe, e beevano dell'acquavita. D. Antonio Ulloa afferma, che in Cartagena, uno de' più infalubri luoghi dell' america non fu concfciuto il vomito prieto prima dell'anno r 729., e comincio dalla marineria dell' armatz europea, che vi approdd quell' anno fotto il comando di D. Domenico.Giuftiniani. 
non può afcriverfi, che alla fanita della lor compleffione, alla lor fobrietà nel vitto, e alla falubrità del clima. Lo iteffo raccontano d'altri paefi del nuovo Mondo gli Storici, ed altre perfone che vi fono alcuni anni dimorate. Ma fe per avventura vi è qualche regione, nella quale non fi prolunghi tanto la lor vita, almeno non havvene alcuna, bella quale fi raccorci tanto, quanto nella Guinea, nella Sierra-Leona, nel C. di Buo. na Speranza, $e$ in altre contrade dell' Africa, nelle quali comincia comunemente la vecchiaja agli anni quaranta: e colui, che arriva a' cinquanta, e riguardato, come larebbe preffo noi un ottogenario. $(f)$ Di quefti $f_{1}$, che potrebbe dirfi a ragione, che hanno ii fangue guafto, e fconvolta la fifica lor coftituzione.

\section{G. I I.}

\section{Su le anime Dei Mefficani.}

Fin quì abbiamo efaminato ciò, che dice il Sig. de Paw fu le qualità corporali degli Americani. Vediamo ora gli fpropofiti, che fcriffe contra le loro anime. Egli non ha potuto in effe trovare, fe non una memoria tanto debole, che oggi non fi ricordano di ciò, che fecero jeri, un ingegno così ottufo, che non fon capaci di penfare, nè di mettere in ordine le loro idee, una volontà tanto fredda, che non fentono gii ftimoli dell' amore, un animo dappoco, e un genio Atupido, ed indolente. Finalmente con tali colori dipinge gli Americani, ed avvilifce a tal fegno le loro anime, che benchè talvolta Storia Antica del Meffico Tom. IV. Z Z invei-

(f) Gli Ottentoti, dice il C. de Buffon, vivono poco; poichi appena oltrapaffano gli anni quaranta. Drack teftifica, che certi Popoli, che abitano nelle frontiere de' deferti d'Etiopia, per cagione della fcarfezza di viveri ficibano di locufte falate, e che un sì cattivo alimento lor cagiona un terribile effetto, cioè che allorchè s'accoftano agli anni quaranta, fi generano ne'lor corpi certi inietti volanti, i quali lor arrecano in breve la morte divorando loro prima il ventre, indi il petto, e poi finalmente anche l'offa. Quefti inferti, ficcome pure quelli, da' quali fon divorati gli abitatori della piccola Tartaria, fecondochè confeffa il medefimo Sig. de Parr, baftano agli Americani per contraccambiare foprabbondantemente ad effo lui que'vermi afcaridi, che egll dice aver trovati preffo non fo che Popoli dell' America. 


\section{I78 DISS ER T AZION E V.}

inveifca contra coloro, che mifero in dubbio la lor razionalità, io non dubito, che fe allora ne foffe ftato confultato, fi farebbe dichiarato contro il parere dei Razionalifit. So bene; che molti altri Europei, e cio ch'è più da maravigliare, molti ancora di quei figli, o difcendenti d' Europei, che fon nati nella feffa America, la penfano come il Sig. de Paw, alcuni per ignoranza, altri per mancanza di rifleflione, ed altri per una certa paffione o prevenzione ereditaria. Ma tutto ciò, molro ancora, che vi folfe davvantaggio, non bafterebbe a fmentire la propria noftra fperienza, e la teftimonianza d'altri Europei, la cui autorità vale affai più, e perchè erano uomini di gran giudizio, dotrrina, e pratica di quei paefi, e perchè teltificarono in favor d'uomini Atranieri contra i propri lor nazionali. Sono tante le teftimonianze, e le ragioni, che potremmo addurre in favor delle anime degli Americani, che fe ne potrebbe formare un groffo volume; ma lafciandone ora la maggior parte per non render troppo diffufa, e nojofa quefta differtazione, ci contenteremo di pochi teftimoni, che valgono in cambio di mille.

Giovanni di Zummarraga, primo Vefcovo di Meficco, Prelato di felice memoria, e fommamente ftimato dai $\mathrm{Re}$ Cattolici per la fua dottrina, per la fua vita immacolata, pel fuo zelo paftorale, e per le fue appoftoliche faciche, nella fua lettera f́crita l'anno $153 \mathrm{I}$. al Capitolo General dei PP. Francefcani congregato in Tolola parla cosi degl' Indiani : Sono cafti, ed affai ingegnofi, maffimamente per $l$ arte della Pittura. Eglino banno fortito delle anime buone. Lodato fia per tutto il Signore.

Se il Sig. de P. non pregia la teftimonianza di quefto venerabilifimo Prelato, cui egli appella Sumarica e barbaro per cagione di quell' autorita, che fi è arrogata per ingiuriar coloro, i cui fentimenti non fono conformi al fuo fpropofitato fiftema della degenerazione, legga cio che fcriffe degli Americani Monfig. Bartolommeo de las Calas, primo Vefcovo di Chiapa, il quale gli conofceva affai bene, come quegli che n'ebbe una gran pratica per tanti anai in diverfi paefi dell' America. Quefti dun- 
que in un fuo memoriale prefentato a Filippo II. ne parla co: sì: "Sono altresi (gli Americani) di chiari e vivi ingegni, " affai docili e capaci d'ogni buona dottrina, attiffmi a rice"vere la noftra fanta Fede, ed i virtuofi coftumi, e quelli fra "tutri i Popoli del Mondo, che banno per ciò manco impe"dimento." Preffochè gli ftefi termini adopra nella fua impugnazione delle rifpotte del Dott. Sepulveda : "Hanno, dice, " gl'Indiani la mente tanto buona, e l'ingegno tanto acuto, " e tanta docilità e capacità per le fcienze morali e fpecula" tive, e fono per la maggior parte tanto ragionevoli nel po"litic lor governo, ficcome fi vede in molte delle loro leg"gi giuftifime, e fonofi tanto avanzati nelle cofe della fanta " noitra Fede e Religione, nei buoni coltumi, e nella corre"zione, dovurque fono ftari ammaeftrati da Religiofi e per"fone di buona vita, e tanto s'a vanzano oggidl, quanto qua"lunque altra Nizione dai tempi appoftolici in quà." Ora poichè il Sig. de $\mathrm{PaW}_{\mathrm{W}}$ crede tutto cid, che quefto dotto, efem. plare, ed infaricabile Prelato fcriffe contro gli Spagnuoli, contutcochè non foffe ftato prefente alla maggior parte dei fatti, che racconta, dourà molto più creder ciò, che il medefimo Vefcovo, come teftimonio oculare, e tanto pratico, depofe in favor degli Americani; mentre affai meno vi vuole per perfuaderci, che gli Americani fon di buon ingegno e di buona indole, che per farci credere quegli orrendi ed inauditi attentati dei Conquiftatori Spagnuoli.

$M_{d}$ fe tuttavia non vuole ammettere la teftimonianza di quel fumofo Vefcovo, perchè il reputa, benchè a gran torto, raggiratore ed ambiziofo, legga la depolizione, che ne fa Monfignor Giuliano Garcès, primo Vefcovo di Tlafcalla, uomo dottiffimo, ed a ragione ftimato e lodato dal fuo famolo Maeftro Antonio di Nebrija, reftauratore della letteratura in ITpagna. Quefto infigne Prelato nella fua grave lettera latina a Papa Paolo III. fcritta nel 1536. dopo dieci anni di continua pratica, e d'offervazione oculare degli Americani tra le molte lodi, con cui celebra la buona lor indole, e le doti delle lor anime, loda il loro ingegno, e in qualche foggia l'inaalza fo- 


\section{DISSERTAZIONE V.}

pra quello dei fuoi. Spagnuoli, ficcome può vederfi nel paffo di quella lettera, che copiamo quì fotto. ( $t$ ) Chi farà mai, che non dia maggior fede a quetti tre venerabili Vefcovi, $i$ quali oltre ai pregi della lor probita, della lor dottrina, e del lor carattere, ebbero pur quello della lunga lor pratica degli Americani, che non a tanti altri Scrittori, $i$ quali o non videro mai gli Americani, o gli videro fenza rifleffione, o deferirono più che non fi conveniva alle informazioni d'uomini ignoranti, o prevenuti, o intereffati?

Ma fe finalmente il Sig. de P. rifiuta la depofizione di quefti tre teftimonj, quantunque autorevoli, perchè erano Religiofi, dei quali crede egli propria l'imbecillità di mente, non putrà a mno di non arrenderfi al giudizio del famofo Vefcovo d'Angelopoli Monfig. Palafox. Il Sig. de P., benchè Pruffiano e Filofofo, appella pure quel Prelato venerabil Servo di Dio. (u) Or fe egli dà tanta fede a queito Venerabil fervo di Dio in ciò, che egli fcriffe contro i Gefuiti nella fua propria caufa, perchè non dovrà credergli in quello, che fcriffe in favor degli Americani? Legga dunque l'opera da quel Prelato compolta per dimoltrare l'indole, l'ingegno, e le virtù degl' Indiani. (x)

Malgrado l'odio implacabile, che porta il Sig. de Paw agli Ecclefraftici della Chiefa Romana, e foprattutto ai Gefuiti, egli nondimeno loda la Storia Naturale, e Morale dell' Acolta,

(t) "Nunc vero de horum figillatim hominum ingenio, quos vidimus ab ", hinc decennio, quo ego in patria converfatus eorum potui perfpicere mo"res, ac ingenia perícrutari, teftificans coram te, Beatiflime Pater, qui Chri"Ati in terris Vicarium agis, quod vidi, quod audivi, \& manus noftræ con" trectaverunt de his progenitis ab Ecclefia per qualecumque minifterium meum $\Rightarrow$ in verbo vitx, quod fingula fingulis referendo, id eft, paribus paria., ratio", $n$ is optimx compotes funt, 2 integri fenfus ac capitis, fed infuper noftra" tibus pueri iftorum \& vigore fpiritus, \& fenfuum vivacitate dexteriore in " omni agibili, \& intelligibili, prxftantiores reperiuntur. "Quefta lettera fi trova in latino nel primo tomo de'Concili Mefficani pubblicati in Meffico I'anno r 769. , e in francefe nella ftefra Storia dell' America del P. Touron, che il Sig. de P. allega contro gli Americani.

(u) Recherch. Philofoph. part. 6. lettre 4.

(x) Opera di Monfig. Palafox intitolata: Las virtudes del Indio, o Naturaleza, y coltumbres de los Indios de la $N$. Efp., e fperTe volte ftampata. 


\section{DIS S E R T A ZIO N E V. 181}

colta, e l'appella a ragione opera eccellente. ( $y$ ) Or quefto giudiciofo, imparziale, e dottiffimo Spagnuolo, il quale vide ed offervò coi fuoi propri occhj gli Americani tanto nel Perù, quanto nel Meffico, impiega tutto il libro fefto di quell'opera eccellente nel dimoftrare la buona ragione dei medefimi Americani mercè l'efpofizione del lor governo antico, delle lor leggi, delle loro Storie in pitture e cordoni, dei lor Calendari ec. Balta per informarfi del fuo giudizio in quefta materia leggere il primo capitolo di quel libro. Prego tanto il Sig. de PaW, quanto i miei Lettori di leggerlo attentamente; perchè vi fono delle cofe degne di faperfi. In effo riconofcerà il Sig. de P. l'origine dell'errore, in cui fono ineorfi egli e moltiffimi Europei, e vi fcorgerà il gran divario, che v'è tra il veder le cofe con occhi ofcurati da qualche paffione, e l'efaminarle con giudizio ed imparzialità. Il Sig. de P. reputa beftiali gli Americani; Acolta per l'oppofto reputa fciocchi e prefuntuoli coloro, che ne penfano così. Il Sig. de $\mathrm{P}$. dice $(z)$, che $\mathbf{i}$ più accorti Americani erano inferiori in induftria $e$ fagacita alle più groffolane Nazioni dell'antico Continente; Acolta innalza con lodi il governo politico dei Mefficani fopra quello di molte Repubbliche d'Europa. Il Sig. de P. non trova nella condotra ragionevole e politica degli Americani, fe non barbarie ftravaganza, e beftialità, e Acolta vi trova delle leggi ammirabili e degne di confervarfi anche nel loro Criftianefimo. A qual di quefti due Autori dovremo dar fede? L'imparzialità dei noftri Lettori deciderà quefto problema.

Io frattanto non poffo difpenfarmi dal copiar qui un paffo delle Ricercbe Filofoficbe, nel quale fi moltra quelto Aurore non meno maldicente, che nemico della verità." $D_{a}$ pri"ma, dice, non furono gli Americani creduti uomini, ma "piuttofto Satiri, o fcimie grandi, che potevano effere am. "mazzati fenza rimorfo, o rimprovero. Alla fine per aggiu"gnere il ridicolo alle calamità di quelti rempi un Papa fece

(y) Recherch. Philofoph. part. I.

(z) Recherch. Philof. part. 5. fed. I. 


\section{DISSERTAZIONE V.}

" una bolla originale, nella quale dichiarò, che bramando egli " di fondar de' Vefcovadi nelle contrade più ricche d'America, " piacque a lui, ed allo Spirito Santo di riconofcere per veri " uomini gli Americani : ficchè fenza quefta decifione d' un Italiano " gli abitarori del nuovo Mondo farebbono anche oggidi agli " occhi de' Fedeli una razza d'uomini equivoci. Non v'è efem" pio di fiffatta decifione, dacchè quefto globo è abirato da "Lomini, e da fcimie "Dio voleffe, che neppur vi foffe al mondo un altro efempio di tali calunnie, ed infolenze, come quelle del Sig. de $P_{0}, \mathrm{ma}$ acciocshè fi renda più manifelta la fua malignita, daremo una copia di quella decifione papale do. po avere efpolta la cagion d'effa.

Alcuni di que'primi Europei, che fi ftabilirono in America, non meno potenti, che avari, volendo arricchirfi vieppiù con detrimento degli Americani, gli tenevano continuamente occupati, e fi fervivano d'effi, 'come di fchiavi, e per ifchivare $i$ rimproveri, che lor facevano i Vefcovi, ed i Miffionari, affinchè trattaffero con umanità que' Popoli, e lafciaffero lo. ro qualche tempo almeno per effere ammaeftrati nella religione, e per foddisfare a'lor obblighi verfo la Chiefa, e verfo le Joro famiglie, coloro promovevano, che gl' Indiani erano dalla lor natura fervi, e che erano incapaci d'intruzione, ed alzri ffffatti Spropofiti, di cui $f_{a}$ menzione il Cronichifta Herrera. Ora non potendo que'zelanti Ecclefiaftici nè colla lor autorità, nè colle loro prediche fottrarre que' miferi Neofiti dalla tirannìa di quegli avari, ricorfero a' Re Cattolici, e finalmente ottennero dalla lor equità e clemenza quelle leggi tanto favo. revoli agli Americani, e tanto onorevoli alla Corte di Spagna, che $f(i)$ leggono nel Codice Indiano $\left({ }^{*}\right)$, le quali principalmente fi dovettero allo zelo infaticatile di Monfig. de las Cafas. Da un' altra parte Monfig. Giuliano Garcès, Vefcovo di Tlafcalla, fapendo, che quegli Spagnuoli malgrado la loro malvagità portavano un gran rifpetio alle decifioni del Vicario di G. C. fece ricorfo l'anno 1536. a Papa Paolo III. con quella famofa let-

(") Nuerva Recopilacion de las Leyes de Iradias. 


\section{IS S E R T A Z I O N E V. 183}

lettera di cui abbiam fatta menzione, rapprefentandogli $i$ mali, che da quegli fcellerati Criftiani foffrivano gl'Indiani, e pregandolo d'interporvi la fua autorita. Il Papa inoffo da sì gravi rimoftranze fped l'anno feguente quella bolla originale, la cui copia fedele diamo quì fotto: $(A)$ la quale non fu fatta, com'è manifelto, per dichiarar veri uomini gli Americani; mentre quelto farebbe una fciocchezza troppo aliena da un tale, da qualunque altro Pontefice; ma foltanto per foltenere i dritti naturali degli Americani contro i tentativi de'lor perfecutori, e per condannar l'ingiuttizia, e l'inumanità di coloro, che fotto preteito d'effer quegli uomini idolatri, o incapaci d'initruzione, lor toglievano la roba, e la libertà, e fi fervivano d'effi come di beftie. Gli Spagnuoli in vero fareb. bono ftati ancor più balordi de'più ruzzi Selvaggj del nuovo

Mon-

(A) Paulus Papa III. univerfis Chifti Fidelibus prefentes Litteras infpecturis Salutem \& Apoftolicam Benedictione:n -- "Veritas ipfa, qua nec falli, " nec fallere poteft, cum Prædicatores Fidei ad officium prædicationis defti"naret, dixiffe dignofcitur: Euntes docete omnes gentes: omnes dixit abfque " omni delectu, cum omnes Fidei difciplinz capaces exiftant. Quod videns " \& invidens ipfius humani generis zmulus, qui bonis operibus, ut pereant, " femper adverfatur, modum excogitavit hactenus inauditum, quo impediret, " ne Verbum Dei Gentibus, ut falvæ fierent, prædicaretur: ac quofdam fuos ", fatellites commovit, qui fuam cupiditatem adimplere cupientes, Occiden" tales \& Meridionales Indos, \& alıas Gentes, qux temporibus iftis ad no" Aram notitiam pervenerunt, $\{u b$ pratextu quod Fidei Catholica expertes , exiftant, uti bruta animalia, ad noftra obfequia redigendos effe, paffim af"Serere prafumant, \& eos in rervitutem redigunt tantis aflictionibus illos ," urgentes, quantis vix bruta animalia illis fervientia urgeant. Nos igitur, qui eiufdem Domini noftri vices, lice indigni, gerimus in terris, \& Oves gregis fui nobis commiffas, qux extra eius Ovile funt, ad ipfum Ovile toto nixu exquirimus, attendentes Indos ipfos, utpote veros homines, non folum Chriftianx Fidei capaces exiftere fed, ut nobis innotuit, ad Fidem , ipfam promptiffime currere, ac volentes fuper his congruis remediis providere, prædictus Indos, \& omnes alias gentes ad notitiam Chriftianorum in , pofterum deventuras, licet extra fidem Chrifti exiftant, fua libertate \& do", minio huiufmodi uti, \& potiri, \& gaudere libere \& licite polfe, nec in " fervitutem redigi debere, ac quidquid fecus fieri contigerit irrirum \& inane, ", ipfofque Indos, \& alias Gentes Verbi Dei pradicatione, \& exemplo bon ", vitz ad dictam Fidem Chrifti invirandos fore, Auctoritate Apoflolica per " præfentes litteras decernimus, \& declaramus, non obftantibus præmiffis, cx", terifque contrariis quibufcunque -- Datum Rom $x$ anno I537. IV. Non. Iun. Pontificatus neftri anno IIl. Quefta, e non altra è gr:olla famora bolla, per la quale $b^{\prime}$ è fatto un sì grande fchiamazzo. 


\section{I84 DISSERTAZIONE V.}

Mondo, fe per riconofcere per veri uomini gli Americani, a: veffero dovuto afpettar la decifione di Roma. Egli è certo, che molto prima, che il Papa fpediffe quella bolla, i $\operatorname{Re} C a t-$ tolici aveano caldamente raccomandata l'inftruzione degli $\mathrm{A}$ mericani, aveano dati gli ordini più premurofi, perchè foffero ben trattati, e non fi faceffe loro verun torto ne' loro averi, o vella lor liberià, $(B)$ ed aveano mandari al nuovo Mondo parecchj Vefcovi, ed alcune centinaja di Miffionari a fpefe del regio erario, acciocchè predicaffero a que' Satiri la Fede di G. C., e gli ammaeftraffero nella vita criftiana. Nel I 53 I. fei anni prima, che veniffe fuori quella bolla, i foli Miffionari Francefcani aveano nel Meffico battezzato più d'un milione di que' Satiri, come ne fa fede Monf. Zumarraga, $(C)$ e nel 1534 . s' era già fondato in Tlatelolco il Seminario di Santa Croce per l'inftruzione d'un buon numero di Scimiotti, dove effi imparavano la lingua latina, la Rettorica, la Filofofia, e la Medicina. (D) Se da principio furono ftimati Satiri gli Americani, neffuno potrà meglio dirlo, che Criftoforo Colombo loro fcopritore. Senta dunque, come parla quel celebre Almirante nel fuo ragguaglio a' $\operatorname{Re}$ Cattolici Ferdinando ed Ifabella dei primi Satiri da lui veduri nell'ifola Haitì, o fra Spagnuola: "Giuro, dice, a "VV. AA., che non v'è al Mondo gente miglior di quefta, " nè cosi amorola, affabile, e manfueta. Amano $i$ lor proffi" mi, come fe fteffi: il loro linguaggio è il più foave, il più " dolce, il più allegro, mentre parlano fempre forridendo, e " benchè vanno nudi, mi credano VV. AA., che hanno dei 2) coftumi affai lodevoli, e che il lor $\mathrm{Re}$ è fervito con gran "maettà, il quale ha delle maniere fi avvenevoli, che reca " gran piacere il vederlo, ficcome pure il confiderar la gran ,? re-

(B) Gli ordini dati da' $\operatorname{Re}$ Cattolici intorno alla converfione degli Americani prima di quella bolla, e le leggi da lon pubblicate in favor di quelle $\mathrm{Na}$ zioni polfono vederfi nelle Decadi d'Herrera, e nel Codice Indiano.

(C) Lettera fcritta da Mong. Zumarraga al Capitolo General de' Francefcani congregato in Tolofa.

(D) Torquemada nel lib. I5. cap. 43. della Monarchia indiana racconta l'erezione rolenne del Seminario di Santa Croce fatto dal primo Vicerè delMeffico coll' intervento di due Vefcovi. 
7) retentiva di quel Popolo, e la brama di faper tutto, la qua" le gli fpinge a addimandare le caufe e gli effetti delle co"fe." (E) Quanto meglio farebbe per noi, che il Mondo forfe abitato da fiffatti Satiri, che non da uomini bugiardi e calunniatori! Del refto poichè il Sig. de P. impiegò dieci anni continui nel ricercar le cofe d'America, dovrebbe fapere, che nei paefi del nuovo Mondo fottopofti agli Spagnuoli non fi for mai fondati altri Vefcovadi, che quelli che ha voluto il Re Cattolico. Ad effo lui tocca pel Jufpatronato, che egli ha, nelle Chiefe Americane, autorizzatogli fin dal I 508. da Papa Giu. lio II. la fondazione dei Vefcovadi e la prefentazione dei Vefcovi. Dunque l'affermare, che Paolo III. volle riconofcere per veri uomini gli Americani per fondar dei Veícovadi nelle contrade più ricche del N. Mondo, è una temeraria calunnia di un nemico della Chiefa Romana: altrimenti fe egli non aveffe la mente tanto accecata dall' odio, dovrebbe piuttofto lo. dar lo zelo e l' umanità, che fa f́piccar quel Papa nella men. tovata bolla.

Il Dott. Robertfon, il qual adotta in gran parte gli ftravolti fentimenti del Sig. de Paw, parla così degli Americani nel lib. 8. della fua Storia d'America :, Alcuni Miffionari, at" toniti ugualmente alla loro lentezza di comprenfione, e alla " loro infenfibilità, gli fentenziarono per una razza d'uomini "tanto degenerante, da effere incapaci d'intendere $\mathrm{i}$ primi ru"dimenti di religione." Ma chi fieno tali $M$ fronari, e quan. to debba valutarfi la loro fentenza, da niuno porrà meglio intenderfi, che da Monfig. Garcès nella fopraccencata lettera a Papa Paolo III. Leggafi dunque il paffo d'effa, che copiamo qui fotto, $(F)$ nel qual fi vede, che le cagioni d'un tal errore fono l'ignoranza e la defidia di quei Miffiouarj; ed io Storia Antica del Meffrco Tom. IV. A a agg un-

(E) Cap. 32. della Stor di D. Crifoforo Colombo fcritta dal fuo figliuolo D. Ferdinando.

(F) Quis tam impudenti animo ac perfricata fronte incapaces fidei afferere auder, quos mechanicarum artium capaciffimes intuemur, ac quos etiam ad min fterium nofrum red actos bonæ indolis, fideles, \& folertes exnerimur? Et fi quando, Beatiflime Pater, Tua Sanctitas aliquem religiofum virum in hanc 
aggiungo, che anche le falfe idee inttillate loro fin dalla prima età. Quafi lo fteffo, che Monfig. Garcès, dicono Monfig. de las Calas, Acolta, ed altri gravi Scrittori d'America.

"Un Concilio tenuto a Lima, fiegue il Dott. Robertfon, ") decreid, che a conto di quelta loro imbecillita doveano effe"re efclufi dal Sacramento dell' Eucariftia. E quantunque Paa"lo III. colla fua bolla emanata l'anno 1537. gli dichiaraffe " creature ragionevoli, e capaci di tutti i privilegi dei Cri"Atiani, nulladimeno dopo il corfo di due fecoli fono cosi im") perfetti $i$ loro progreffi in cognizione, che pochiffirni poffeg"gono tal porzione di fpirituale difcernimento per effere giu" dicati degni d accoftarfi alla facra menfa... Anche dopo la " più affidua initruzione la loro credenza è tenuca per debole, "e per dubbiofa, e benchè alcuni d'effi fiano giunti fraordina3) riamente a imparare le dotie lingue, ed abbiano paffato con 3) applaufo il corfo d'accademica educazione, la loro debolezza ") é fempre cos) fofpetra, che neffun individuo è mai ordina"to Prete, ed è ricevuto di rado in un ordine religiofo." Ecco in poche parole quattro errori almeno: 1. che un Concilio di Lima abbia efclufi gl' Indiani dal Sacramento dell' Eucariftia a cagione della loro imbecillitia. 2. che Paolo III. di. chiarò gl' Indiani creature ragionevoli . 3. che pochiffimi Indiani polfeggono tal porzione di fpirituale difcernimento per potere

declinare fententiam audierit, etfi eximia integritate vitæ, vel dignitate fulgere videatur is, non ideo quicquam illi hac in re praftet auct ritatis, fed eumdem parum aut nihil infudafe in illorum converfone certo certius arbitretur, ac in eorum addifcenda lingua, aut inveftigandis ingenis parum ftuduiffe perpendat: nam qui in bis caritate chriltiana. laborarunt, non frufta in eos jactare retia caritatis affirmant; illi vero qui folitudini dediti, aut ia gnavia prapediti neminem ad Chrifti cultum fua indufria reduxerunt, ne inculpari poffint quod inutiles fuerint, quod propriz negligentix vitium eft, id Infidelium imbecillitati adferibunt, veramque fuam defidiam falfx incapacitatis impofitione defendunt, ac non minorem culpam in excufatione committunt, quam erat illa, a qua liberari conantur. Ladit namque fumme ifud hominum genus talia afferentium hanc Indorum miferrimam turbam : nam aliquos religiofos viros retrahunt, ne ad eofdem in fide inftuendosproficifcantur: quamobrem nonnulli Hifpanorum qui ad illos debellandos accedunt, horum freti iudicio, illos nẹgligere, perdere, ac $m$ ctare opinari folent non effe flagitium. "Ex litteris Julliani Garcès Ep. Tlaj. ad Paulum III. Pont. Max. 
tere effere giudicati degni d'accoftarfi alla facra menfa. 4 . che neffun Indiano è mai ordinato Prete.

Quanto al t. è vero, che in una Congregazione d'Ecclefiaftici tenuta in Lima l'anno 1552 . la quale fu chiamata Primo Concilio Lime e; ma nè fu Concilio, nè ebbe mai autorità conciliare, fu ordinato, che non s'amminiftrafie l' Eucariftla agl' Indiani finattantochè non foffero perfettamente inftruiti e perfuafí nelle cofe della fede; perchè quel fagramento è cibo de'perfutti, non già perchè effi foffero "ftimati imbecilli. Ciò confta dalla teftimonianza del primo Concilio Provinciale (detto volgarmente II.) tenuto in Lima l'anno I567. il quale ordinò a' Parrochi d'amminiftrare tal facramento a tutti quegl'Indiani, che trovaffero ben difpofti. (G) Ma non baftando quell' ordine per far piegare a quegli Ecclefiaftici, del che fis lagnò a ragione il $\rho$. Acofta, il fecondo Concilio Limefe tenuto net 1583 . nel quale prefiedette S. Toribio Mogrobejo, cercò di rimediare a tali difordini co'decreti, che diamo quì fotto: $(H)$ ne'quali li vede che ugualmente, e per li medefi. mi morivi negavano l' Eucariftì agl' Indiani, ed a' Mori fchiaA $a$ vi

(G) Quamquam omnes Chriftiani adulti utriufque fexus teneantur Sanctiffmum Euchariftiz Sacramentum accipere fingulis annis faltem in Pafchate, hujus tamen Provincix Antiftites cum animadverterent gentem hanc Indorum \& recentem effe $\&$ infantilem in fide, atque id illorum faluti expedire iudicarent, ftatuerunt ut ufque dum fidem perfecte tenerent, hoc divino facramento, quod eft perfectorum cibus, non communicarentur, excepto fs quis ei percipiendo fatis idoneus yideretur.... Placuit huic Sanctze Synodo monere, prout ferio monet, omnes Indurum Parochos', ut quos audita jam confeffione perspexerint, hunc cæleftem cibum a reliquo corporali difcernere, atque eumdem devote cunere $\&$ pofcere, quoniam fine caufa neminem divino alimento privare poffumus, quo tempore cxerers Chriftianis folent, Indis omnibus adminiftent ., Conc. Lim. I. vulgo 11 , cap. 58.

(H) Crelefte viaticum, quod nulli ex hac vita migranti negat Mater Ecclem fia multis abhinc annis Indis atque AEthiopibus, cæterifque perfonis miferabilibus praberi debere Concilium Limenfe conflituit. Sed tamen Sacerdotum plurium vel negligentia, yel zelo quodam prapoftero atque intempeftivo illis nibilo magis hodie præbetur. Quo fit, ut imbecilles animæ tanto bons, tamque neceffario priventur. Volens igitur Sancta Synodus ad executionem perducere, quæ Chrifo duce ad falutem Indorum ordinata funt, fevere præcipit omnibus Parochis, ut extreme laborantibus Indis atque Ethiopibus viaticum miniftrare non pratermitrant, dummodo in eis debitam diffofitionem agno-

SEant, 


\section{DISSER TAZIO NE V.}

vi condonti dall' Africa: che le vere cagioni di negarla erano: a giulizio del Concilio la negligenza o defidia, e lo zelo indifcreto e mal inteío di que'Parrochi, e che il Concilio ficredette obbligato di por rimedio a un si grave difordine con nuovi decreti, e con feveri gaftighi. So bene, che neppur queIti rifpetrabili decreti furono efattamente efeguiti, e bifognò, che foffero di bel nuovo inculcati da'Sinodi diocefani di Lima, della Plata, della Paz, d'Arequipa, e del Paraguai ; ma: ciò dimoftrá più la pervicacia di quegli Ecclefiaftici, dhe l'incapacità degli Americani.

Intorno alla bolla di Paolo IIr. abbiamo già dimoftrato, che egli non li prefe a dichiarare uomini gli Americani, ma: fuppoita la lor razionalita, di cui non poteano dubitare, fe non le beftie, fe foffero capaci di dubbj, condannò l'ingiultia zia de'loro oppreffori.

Quanto poi al terzo errore del Roberton da me fopraca cennato, tralafciando ora cio che appartiene agli altri paefi d'America, perchè non è neceffario, egli è certo e notorio, che in tutra la Nuova Spagna gl'Indiani fono al pari degli Spa gnuoli obbligati a ricever la Sacra Eucariftia nella Pafqua, fuorchè i Neofiti delle rimote contrade, i quali fono ammeffr, o no, alla facra menfa giufta il giudizio de'loro Miffionarj. Ora nelle tre udienze, nelle quali é divifa la Nuova Spagna, vi fono, dice il Roberton, almeno due milioni d'Indiani. (l) Io fon ficuro, che un tal numero è di lunga mano inferiore al vero; ma fia pur tanto, e non più. Non fono dunque pocbif:-

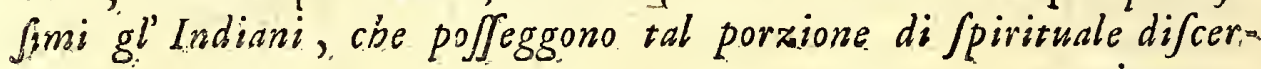
nimen.

Scant, nempe fidem in Chrifum, \& pœnitentiam in Deum fuo modo Porro Parochos qui a prima hujus decreti promulgatione negligentes fuerint, soverint fe, præter divina ultionis iudicium, etiam pœnas Arbitrio Otdina siorum, in quo conf́cientiz onerantur, daturos: atque in Viftationibus in ils los de bujus faturi obfervatione fpecialiter inquirendum." Conc. Lim. II sulgo 1.11. A.ct. 2. cap. 19.

, In $\mathrm{Pa}$ fchate faltem eucharifiam miniftare Parochus non pratermittat: iis, quos \& fatis inftuctos, \& correctione vitæ idoneos judicaverit: ne \& ipfe alioqui ecclefiaftici præcepti violati reus fit. $z$ Ibid, cap. 20.

(1) Storia dell' America lib. 8. 


\section{DISSER T A ZIO NE V. 189}

nimerito per effere giudicati degni d' accoftarfi alla facra menfa; fe già due milioni non pajono pochiffimi al Robertfon, o non reputa temerari que'tanti Velcovi e Parrochi, che non folo ammettono, $\mathrm{ma}$ obbligano ancora quegl' Indiani a comunicarfio. E che farà, fe a quel numero s'aggiungono gl' Indiani di molte Provincie dell' America Meridionale, che fono parimente obbligati a ricever la facra Eucariftia .

Non è men groffo il quarto fuo errore nell' affermare, che neffun Indiano è mai ordinato Prete. E' da maravigliare, che uno Scritrore, il quale ammafso una si gran librerìa di Scrittori d'America, e cui furono fatti da Madrid tanti ragguagli: delle cofe del nuovo Mondo, fia ftato tanto in quefto, quanto in altri punti, cosi male informato. Sappia dunque il Dott. Robertfon, che benchè il primo Concilio Provinciale celebra. to in Meffico l' anno 1555. vietaffe, che foffero ordinati gli Indiani non già per cagione della loro inczpacità, ma perchè fi credeva, che dall' avvilimento della lor condizione ridondaffe qualche infamia nello Stato Ecclefiaftico; $\left({ }^{*}\right)$ nulladimeno il terzo Concilio Provinciale tenuto nel 1585 . il quale fu il più celebre di tutti, e le cui decifioni fono in vigore, permife, che effi $f_{1}$ faceffero Preti, purchè s' aveffe gran cura nell' ammetterli a' facri ordini. $(K)$ Ma convien fapere, che i decreti dell' uno, e dell' altro Concilio comprendono ugualmente, e fotto i medefimi termini, e gl' Indiani, ed i Mulati, cioè coloro, che fon nati, o difcendono da Padre Europeo, e Madre Africana, - al contrario: eppur niuno dubita del gran talento e della capacità de' Mulati per imparar turte le frienze. Torquemada it quale fcriffe la fua Sroria ne' primi anni del fecolo paffato, dice, $(L)$ che non folevano ammetterfi gl'Indiani negli Ordini religiofi, nè ordinarfi Preti per cagione della violenta loro inclio nazione all' ubbriacchezza; ma egli medefimo teftifica, che $a^{2}$ tempi fuoi alcuni Indiani Sacerdoti erano affai fobri ed efem. plari:

(*) Conc. Mexic. Provinc. I. cap. 44.

(K) Conc. Mexic. III. lib. I. tit. 4.

(L) Monarcbia Ind. lib. I7. cap. Iz. 
plari: ficchè fono almeno cento fettanta anni, che cominciarono a farfi Preti gl' Indiani. D'allora in qua fono ftati tanti i Sacerdoti Americani nella Nuova Spagna, che potrebbonocontarfi per migliaja fra $\mathrm{i}$ quali vi fono itate alcune centinaja di Parrochi, parecchi Canonici, e Dottori, $(M)$ ed anche, per quanto fi crede, un Velcovo dottiffimo. $(N)$ Prefentemente ve. ne fono moltifini Preti, non pochi Parrochi, tra i quali fo. no tre o quattro de'miei Allievi. Ora $f e$ in un punto dique. fta fatta errò sì groffamente il Robertion, che farà in quelli, che non poffono cosi facilmente rifchiararfi da un Autore, che firive tanto lontano da' que' paefi fenza averli mai veduti.

Io per lo contrario trattai intimamente-gli Americani: viff alcuni anni in un Seminario deftinato alla loro iftruzione: vidi l'erecione, ed i progreffi del Real Collegio di Guadalupe, fondato in $M=i ́ f i c o$ da un Gefuita Mefficano per l' educazione delle fanciulle indiane: ebbi poi alcuni Indiani tra miei difcepoli: trattai molti Parrochi Americani, molti Nobili, moltiflimi Artigiani: offervai attentamente il lor carattere, il lor ganio, le loro inclinazioni, e la lor maniera di penfare: ed oltracciò ho efaminato con fomma diligenza la loro ftoria antica, la lor religione, il lor governo, le lor leggi, ed i loro coltumi. D po una si gran pratica, ed un sì proliffo itudio, per lo quale mi credo in iftato di poterne decidere con manco pericolo d'errare, protefto al Sig. de Paw, e a tutta l'Europa, che le anime degli Americani non fono punto inferiori

(M) Tra quefti Dottori Americani è degno di particolar menzione D. Sebaftiano Grijalva, nativo d'Ocozoquaubtla, luogo grande della Diocefi di Chiapa. Quefti venuto in Ifpagna fi fece Dottore in Sacra Teologia nella famora Univerfita di Salamanca, e vi s'acquiftò una gran riputazione per la fuz dottrina. Ritornato poi in America fu fatto Parroco della fua patria, e vi fece tali regolamenti per la civile e criftiana condotta de'fuoi concittadini, che la fua Parrochia potrebbe effere il modello di tutte quelle d'America, e finora fe ne vedono gli effetti. Scriffe una dotta opera teologica fu l'Immacolata Concezione della B. V. il cui originale fi confervava nella libreria del Collegio de'Geruiti di Ciudad Real, Capitale di quella Diocefr.

(N) Monfig. Gioranni de Merio, Vefcovo d'Honduras, e dotriflimo neiSa. cri Canoni, il qual fu Vicario Generale di Monfig. Palafox. Non ho potuto trovar quì verun Autore, che faccia menzione della nafcita di quel Velcoro: ma l'opinione generale' lo crede Indiano. 
riori a quelle degli Europei : che eglino fon capaci di turte le fcienze, anche delle più aftratte; e che fe feriamente fi pren. deffe cura della loro educazione, re da fanciulli s' allevaffero in Ceminarj fotto buoni. Maeftri, e fe foffero protetti e al ettati con premi, fi vedrebbono tra gli Amerncanı de' Filofofi, de' Matematici, e de' Teologi, che potrebbono gaiegglare co' più famofi d'Europa. Ma è affii difficile, pet nin oire imporfibile, far de' gran progreffi nelie fcienze in $\mathbf{m} z z 0$ ad und vita miferabile e fervile, ed a continu difas'. (hi contempla lo ftato prefente della Grecia non potrabb: perfuzderfi, che vi folsero già ftati que' grand' uomini, che fappi mo, le non nee fofse ficuro, e dalle loro opere immorrah, e dal confenfo di tutti i fecoli. Eppure gli oftacoli, che harno prefentemente da fuperare i Greci per farfi dotti, non fono paragonabili con quelli, che hanno mai fempre avuti, ed hanno tuttora gli $\mathrm{A}$ mericani. Con tutto ciò io vorrei, che il Sig. de $\mathrm{PaW}$, e quanti altri penfano come lui fi trovaffero prefenti, fenza efsere ofservati, in que' configli, o ragunanze, che fanno in certi giorni per deliberar fu gli affari occorenti quegli Americani, che hanno qualche apparenza di fuperiorità ne' lor villaggi, e fentifsero come aringano, e difcorrono que' Satiri del Nuovo Mondo.

Finalmente tutta la Storia antica de' Mefficani, e de' Peruani, da a divedere, che fanno penfare, ed ordinare le loro idee: che fono fenfibili alle paffioni dell'umanità, e che gli Europei non hanno avuto altro vantaggio fopra loro, che quello d' effere meglio inftruiti. Il governo politico degli ant chi Americani, le loro leggi, e le loro arti dimoltrano evidencemente il loro buon ingegno. Le loro guerre fanno vedere, che le Joro anime non fono infenfibili agli ftimoli dell' amore come penfano il Sig. de Buffon, e de Pavv; poichè talvolta prefero le armi per intereffi a norufi.

Perciò poi, che riguarda il loro coraggio, abbiamo efpofo finceramente, laddove raggionammo del loro caratrere, cio che abbiano offervato negli Americani prefenti, e ciò che giudichiamo degli antichi. Ma perchè ii Sig. de Pavv allega la 
conquilta del Meffico, come una prova convincente della loro codardia, conviene illuminar la fua ignoranza, o piuttofto convincere la fua mala fede.

, Cortès, dice egli, $\left({ }^{*}\right)$ conquiftò l'imperio de' Mefficani " con quattrocento cinquanta vagabondi, e quindici cavalli mal " armati: la fua miferabile artiglieria confilteva in fei falconet"ti, i quali non farebbero oggidi capaci di far paura ad un " fortino difefo da invalidi. Egli durante la fua affenza man. "tenne in rifpetto la Capitale colla metà delle fue truppe. "Che uomini! Che avvenimenti!

"Egli è coltante, loggiunge, per la depofizione di tutti "gli Storici, che gli Spagnuoli enirarono la prima volta in "Muffico fenza fare un folo fparo della lor artiglieria. Se il ") titolo d'Eroe conviene a colui, che ha la difgrazia di far 9) morire un gran numero d'animali ragionevoli, Ferdinando "Cortès potrebbe precenderlo: del refto io non veggo, qual " vera gloria abbia egli acquitata, mettendo in conquaffo una " monarchì vacillante, che parimente potrebbe effer conquaf"fata da qualungue affafino del noftro Continente." Quefti paffi delle Ricercbe Filofoficbe fanno palefe, che il Sig. de Paw ignorava la Storia della Conquilta del Meffico, o ciò che è più verifimile, maliziofamente tacque quello, che apertamente fmentiva il fuo fiftema: poichè tutti quelli, che hanno letta la fuddetta Storia, fanno bene, che la Conquifta di Meffco non fi fece con quattrocento cinquanta momini, ma bensì con più di dugento mila. Il medefimo Cortès, al quále più, che non al Sig. de P., importava fminuire il numero dei Conquiftatori per far comparir più grande !a fua prodezza, e più gloriola la fua conquilta, confeffa pure l'ecceffivo numero degli Alleati, che erano fotto i fuoi ordini nell' affedio della Capitale, e combattevano con maggior furore contro i Mefficani, che gli fteffi Spagnuoli. (O) Conlta dal ragguaglio, che fece

Cor-

(*) Recherch. Philofoph: part. I.

(O) Vedafi la lettera di Cortès a Carlo V. Ccritta da Cojoacan l' anno r saz. come pure la Stor:a di Bernal Diaz. 
Cortès all' Imperatore Carlo V., che l'affedio di Meffico fi cominciò con ortanta fette Cavalli, ottocento quarantotto pedoni Spagnuoli, armati di fchioppi, balifte, fpade, e lance, e pis di fettantacinque mila Alleati Tlafcallefi, Huexozinchi, ChoJullefi, e Chalchefi armati di varie forti d'armi: con tre gran cannoni di ferro, e quindici piccoli di bronzo, e con tredici brigantini. Nel decorío dell' affedio vi s'aggregarono te nuınerofe Nazioni degli Otomiti, dei Cohuixchi, e dei Matlazin. chi, e le truppe delle popolofe Citrà dei laghi: ficchè l'efercito degli Affediatori non folo forpafsò i dugento mila, ma arrivò a dugento quaranta mila, fecondochè appare dalla fteffa lettera di Cortès, ed oltracciò tre mila barche, o canoe, che vennero in loro ajuto. Io dunque domando al Sig. de P. , re gli pare codardia l'aver foftenuto per ben fettantacinque gior. ni l'affetio d'una Citta aperta, combattendo giornalmente con un efercito tanto grande, $e$ in parte armato d'armi tanto fuperiori, e fopra tutto combattendo colla fame e colla fete? Meritano il rimprovero di codardi coloro, che dopo aver perdure delle otto parti della Città le fette, e cencinquanta mila in. circa dei lor Cittadini, parte uccifi a fil di fpada, e parre morti di fame, e d'infermità, continuarono a difenderfí fino ad effere furiofamente affaliti e fopraffatti nell' ultimo cantone, che lor reftava? $(P)$

Egli e certo, dice il Sig. de P., per la depofizione di thi:ti gli Storici, che gli Spagnuoli entrarono la prima volta in Meffico fenza fare nè anche un folo fparo della loro arriglieria. O che bell'argomento, proprio certamente della logica del Sig. de P. Se i Meflicani furono codardi, perchè gli Spagnuoli entrarono la prima volta in Meffico fenza fare un folo fparo della loro artiglieria, potremmo ancora dire, che fon codardi i Pruffiani, perchè gli Ambafciatori di parecchie Corti d'Europa entrano in Berlino fenza fparare nè anche un fucile. Chi non fa, che gli Spagnuoli furono allora ammeffi in quel: Storia Aintica del Melsico TomoIV.

B b la

(P) Tutto cid, che diciamo qui intorno all' affedio, e alla conquifta di Me[fico, "è prefo dalla lettera del Conquiftatore Cottès a Carlo V. 


\section{DISSERT A I ON E V.}

la Capitale, come Ambarciatori del Monarca di Levante? Ve. dafi ciò, che ne raccontano gli Storici, e foprattut to il mede. fimo Cortès, che fi finfe Ambafciarore del Re Cattolico. Se i Mefficani aveffero voluto allora opporfi, come fi oppofero la feconda, volta, quando mai farebbono ftati capaci gli Spagnuo. li d'entrarvi con foli fei mila uomini, effendo itato loro tanto difficile la feconda entrata con dugento mila? (Q)

Intorno a cid, che aggiunge il Sig. de P. contra Cortès, io nè voglio far l'apologia di quefto Conquiftatore, nè poffo foffrire quel panegirico, che in cambio di Storia foriffe il Solis; ma qualunque uomo imparziale, e bene iftruito nella Storia delle militari di lui azioni, dovrà confeffare, che nel coraggio, nella coftanza, e nella prudenza militare può egli ga. reggiare coi più famofi Generali, e che ebbe quella !pezie d'eroifmo, che riconofciamo negli Aleffandri, e nei Cefari, nei quali fi loda la magnanimità malgrado $i$ vizi, di cui erano per altro infetti.

Le cagioni della rapidità, colla quale conquitarono gli Spagnuoli l'America, fono tate in parte accennate dal medefimo Sig. de P. Io confeffo, dice, che l' artiglieria era sno fromento diftruttivo, ed onnipotente, che dovea neceffariamente do. mare $i$ Mefficani. Se all'artiglieria s'aggiungono le altre armi fuperiori, i cavalli, e la miglior difciplina militare dalla parte dei Conquiftatori, e la divifione da quella dei Conquiftati, fi vedrà, che non v'è ragione di tacciar gli Americani di pufillanimità, nè di maravigliarfi del violento conquaffo del nuovo Mondo. S'immagini il Sig. de $P_{0}$, che ai tempi delle ftrepitofe e crudeli fazioni di Silla e di Mario, aveffero gli Ateniefi inventata l'artiglieria, e le altre armi da fuoco, e forniti d'el-

(Q) "Non è men certo, dice Acofta, che nella Nuova Spagna l'ajuto de' "Thafcallefi fu quello, che diede a Cortès, ed a' fuoi la vittoria, e la con" quifta di Meffico, e renza loro faretbe ftato impoffibile non che d'impa, dronitfi di quella terra, ma neppure di trattenervifi più tempo. Coloro, che ", fanno poco conto degli Indiani, e fi perfuadono, che gli Spagnuoli potea" no conquiftar qualunque paefe e nazione, mercè il folo vantэggio delle lor "perfone, de'lor cavalli, e delle loro armi offenfive, s'ingannanu troppo. "s Stor. Nat. e Mor. lib. 7 . cap. 28. 
fe non più di fei mila uomini, e aggiuntifi non già a tutto l'efercito di Mario, ma foltanto ad una parte delle fue truppe, aveffero intraprefa la conquifta dell' I talia. Crede il Sig. de P., che non farebbono riucciti a difpetto della poffanza di Silla, del coraggio, e della difciplina delle truppe romane, del numero delle legioni, e della Cavallerìa, della moltitudine delle lor armi, delle lor macchine, e delle fortificazioni delle lor Città? Quanto terrore non aurebbero meffo negli animi dei più coraggiofi Centurioni e l'orrendo ftrepito dell' artiglieria, e la violenza diftruttiva delle palle, colle quali fi vedevano levar delle file intere? Or che farà ftato preffo quelle Nazioni del nuovo. Mondo, che non aveano nè le armi, nè la Cavallerìa, nè la difciplina, nè le macchine, nè le fortificazioni dei Romani? Quello per l'oppofto, che è veramente da maravigliare, fi ̀̀, che i prodi Spagnuoli con tutta la loro difciplina, la loro artiglieria, e le armi da fuoco, non abbiano potuto in più di due fecoli foggiogare nell' America Meridionale i guerrieri A. ralicani armati foltanto di lance, e di mazze, nè nell' America Settentrionale gli Apacbes armati d'arco e di frecce, e foprattutto cid che pare incredibile, ma è pur certo, cinquecento foli Uomini della Nazione dei Seris fono ftati per molti anni il flagello degli Spagnuoli di Sonora e Cinaloa.

Finalmente tralafciando molti altri fpropofiti del Sig. de Paw contro gli Americani per non iftancar la pazienza dei Leto tori, non poffo diffimulare l'atroce ingiuria, che lor fa in materia di coftumi. Quattro fono i principáli vizi, di cui infama tutti gli Americani, la Ghioriornia, l' Ubbriachezza, l'Ingratitudine, e la Pederaftia, ovvero Sodomia.

Io in vero uon avea mai fentito rimproverar la Ghioetornia agli Indiani prima che $m$ ' imbatteffi nel paffo de! Sig. de la Condamine, citato e adottato dal Sig. de $\mathrm{PaW}$. Non ho trovato alcun Autore alquanto iftruito nelle co. fe d'America, che non lodi la fobrietz degli Americani nel mangiare. Veda chi vuole quanto ne dicono Monfig. de las Cafas, Monfig. Garcès, il Conquiftatore Anonimo, $\mathrm{O}$. viedo, Gomara, Acolta, Herrera, Torquemada, Betancurt . B b 2

ec. 
ec. ec. $(R)$ Quafi tutti gli Storici raccontano la meraviglia; che fece agli Spagnuoli la parfimonia degl' Indiani, e per l'oppofto la meraviglia degl' Indiani in vedendo gli Spagnuoli mangiar più in un giorno, che eglino in una fettimana: e per dirlo in poche parole, la fobrietà dégli Americani è cosi notoria, che farebbe foverchia la loro difefa in quefto foggetto. Il Sig. de la Condamine vide forfe mangiare avidamente alcuni Indiani affamati nel fuo viaggio pel fiume Maragnone, e quindi fi perfuafe, come accade fpeffo ai Viaggiatori, che tutri gli Americani erano ghiottoni. Egli è certo, che D. Antonio UUlloa, il quale ftette in America col Sig. de la Condamine, vi dimorò più tempo, e s'informò più dei coftumi degl' Indiani, nè parla tutto al contrario di quel, Matematico francele.

L'Ubbriachezza è il vizio domiante di quelle Nazioni. Io il confeffo ingenuamente nel primo libro della mia Storia, n'efpongo gli ecceffi, e n'addito le cagioni, ma vi foggiungo ancora, che non era così nei paefi d'Anahuac prima che v'en. traffero gli Spagnuoli per cagione del gran rigore, con cui fi caftigava quel vizio, il quale nella maggior parte dei paefi dell'antico Continente refta impunito, e ferve anche per eccezione o fcufa d'altri delitti più gravi. Confta pure dalla teftimonianza degli Scrittori, che fecero delle ricerche intorno al governo politico dei Mefficani, delle fevere leggi, che v'erano contra l'ubbriachezza tanto in Meflico, quanto in Tezcuco, in Tlafcalla, e in altri Stati, le quali ho vedute rapprefentate in pitture antiche. La pittura feffantefimaterza della Raccolta di Mendoza rapprefenta due giavani d'ambidue i fefti

(R) Monfig. de las Cafas in quel fuo Memoriale a Filippo II. , che porta il titolo Della Diffruzione delle Indie afferma, che il mangiar degli Americani, à tale, che quello degli antichi Santi Padri della Tebaida non poteva effere nè men dilettevole, nè più fcarfo, o più miferabile. Monfig. Garcès nella fua lettera a Papa Paolo III. dice, che la loro fobrietà non"può darfi abbaftanza ad intendere. Il Conquiftatore Anonimo afferifce nella fua Relazione, cheli Americani fono fra tutti i popoli del mondo quelli, che fifoftentano con meno. Cosi parlano tutti i teftimonj oculari de'lor coftumi. Sappiamo da Torquemada che i primi aftinentiffimi Religiofi, che annunziarono i! Vangela $a^{\prime}$ Meflicani, ebbero non poco d' ammirare, ed anche da imparare dalla loro robrietà. 
condannati alla morte per cagione d'efferfi imbriacati, ed infie: me un vecchio fettuagenario, cui le leggi per riguardo alla fua età permettono di bere quanto voglia. Pochi Stati troveranfi al Mondo, nei quali fia itato più grande lo zelo dei Sovrani per la correzione degli ecceffi di quefta fatta.

Nel fuddetto libro primo della Storia antica abbiamo parimence ribattuto il comun errore intorno alla gratitudine degl'Indiani; ma perchè quanto fu ivi detto non bafterà per convincere coloro, che fon contra effi prevenuti, vogliamo quì rapportare un fingolar efempio di gratitudine, il quale farà per fe folo fufficiente a diffipare qualunque oppofta idea. Nell' anno "I 556. morì in Uruapa, luogo confiderabile del Regno di Michuacan, vifitando la fua Diocefi nell' età d'anni novantaeinque, Monfig. Vafco de Quiroga, Fondatore e primo Vefcovo di quelia Chiefa, il quale ad efempio di S. Ambrogio fu trasferito dal giudicato fecolare alla dignità velcovile. Quefto infigne Prelato deguo di effere paragonato co' prirni Padri del Criftianefimo, faticò infinitamente in favor de' Michuacanefi, iftruendoli da Apoftolo, ed amandoli da Padre: fabbrico de' tempi, fondò degli Spedali, ed affegnò a ciafcun luogo degl' Indiani un ramo principale di commercio, affinchè la reciproca loro dipendenza gli teneffe uniti in carità, fi perfezionaffero le arti, e a neffuno mancaffe la maniera di vivere. La memoria di tali benefizj fi conferva sì viva preffo quegli Americani dopo più di due fecoli, come fe tuttor foffe vivo il loro Bene. fattore. La prima cura, che hanno le Indiane, tofto che ilor figliuoli cominciano ad aver qualche giudizio, è quella di dar loro contezza del loro Tata Don Vafco (cosi l'appellano an. che oggidi a cagione dell'amor figliale, che gli confervano) il danno loro a conofcere ne' fuoi ritratti, dichiarando loro ciò, che egli fece in favor della lor Nazione, e non paffano mai davvanti al fuo ritratto feaza inginocchiarfi. Oltracciò fondo quel gran Prelato nel 1540. un Seminario nella Città di Pazcuaro per l'iftruzione della Gioventù, ed incaricò gl' Indiani di Santa Fe ( luogo fondato da lui medefimo nella riva del lago di Pazcuaro) di mandare ogni fettimana un uomo a fer- 


\section{DISSERT A Z I O N V.}

vire a' Seminarifti. Fu ubbidito,e fino ad oggi dopo anni 230 . e più, non è mancato l' Indiano che vi dee fervire, fenza effervi mai bifogno di coltrignerlo, o di chiamarlo, foltanto per corrifpondere con quell' oflequio al gran bene, che lor fece quel Vefcovo impareggiabile. Poffeggono nella Cirtà di Pazcuaro le fue ofsa con tal venerazione, che una volta, che tentò trasferirle a Valladolid il Capitolo di quella Cattedrale, s' inquietarono gl' Indiani, e fi preparavano a impedirlo a forza d'armi, ficcome infatti farebbe avvenuto, fe il Capitolo per ifchivare i difordini di quella contefa non fi foffe diftotto dalla prima rifoluzione. Può immaginarí una prova più concludente della gratitudine d'una Nazione? Simili dimoftrazioni fi fon fatte dagl' Indiani in molti altri luoghi di quel regno per ritenere i Miffionarj, che gli aveano ammaeftrati nella Fede. Quelle de'due fecoli pafsati pofsono vederfi nel tomo 3. di Torquemada, e nel Teatro Mefficano di Betancurt. Di quelle, che fi fon fatte $a$ ' noftri di, vi fono ancor viventi moltiffimi teftimonj oculari, ed io ancora ne polso teftificare. Se talora non fi moftrano gli Americani grati a' benefattori, cid è perchè la continua fperienza de'mali, che lor fi fanno, rende loro fofpetti i benefizj; ma ogni volta che fon ficuri della fincera benevolenza del benefattore, fon capaci di facrificare tutti $i$ lor beni alla gratirudine, ficcom'è notorio a tutti quelli, che gli hanno praticati e ofservati fenza prevenzione.

$\mathrm{Ma}$ in niun' altra cofa di quante pubblicò il Sig. de $\mathrm{P}$. contra gli Americani è loro tanto ingiuriofo, quanto nell' affermare, che la Pederaftia eva in gran voga nelle ifole, nel Perit, e nel Mefjico, $e$ in tutto il nuovo Continente. (S) Io non fo, come dopo aver pubblicata una sì atroce calunnia, baftd l'animo al Sig. de P. per dire, come dice nella fua rifpofta a Don Pernety, che tutta la fua opera delle Ricercbe Filofofiche refpira umanità. E' forfe umanità l'infamare a torto tutte le Nazioni del nuovo Mondo d'un vizio tant' enorme, e tanto obbrobriofo alla Natura? E' umanità lo fdegnar.

(5) Rech. Philoloph. part. I. 


\section{I S E R T A Z I O N E V.}

fi, che egli fa contro l' Inca Garcilafso, perchè difende i Pe: ruani da una tal imputazione? Ancorchè vi folsero de' gravi Autori, che ne attribuiffero quel delitto 2 tutti i Popoli d' America, elsendo, come in fatti vi fono, molti Autori pur gravi, che affermano tutto il contrario, doveva il Sig. de PaW giufta le leggi dell' umanità aftenerfi da una sì grave accufa. Quanto più non dovrà altenerfene non efsendovi veruno Scrittore autorevole, fu la cui teftimonianza pofsa egli appoggiare una sì univerfale afserzione. Troverà egli bensì alcuni Autori, come il Conquiftatore Anonimo, Gomara, ed Herrera, che hanno incolpa to di tal vizio alcuni A mericani, o al più qualche Popolo d'A merica; ma dove mai trovare un o Storico riguardevole, che abbia ofato dire, che la Pederaftla era in gran voganelle Ifole, nel Peri, nel Mef. fico, $e$ in tutto il nuovo Continente? Anzi tutti gli Storici del Meffico dicono ad una voce, che tal vizio era fommamente abbominato da quelle Nazioni, e fanno menzione delle terribili pene prefcritte dalle lor leggi contra efso, ficcome può vederfi nelle opere di Gomara, d'Herrera, di Torquemada, di Betancult, e d'altri. Monf. de las Calas teltıficò in un fuo fcritto prefentato a Carlo V. nel 1542., che avendo egli fatte diligenti ricerche nelle Ifole Spagnuola, Cuba, Giamaica, e Portorrico, e nelle Lucaje, trovò, che non vi fu mai memoria di quel delitto prefso quelle Nazioni. Lo ftefso afferma del Perù, di Jucatàn, e di tutti i paefi d'America in generale, fe non fe in qualche luogo, dove fi dice, che ve ne fono alcuni colpevoli; $m a$ non peró, foggiunge, dee incolparfi tutto quel Mondo. ( $T$ ) Chi

dun-

(T) "Gli Spagnuoli ( paria Monfig. de las Cafas d'alcuni non di tutti ) " hanno infamati gl Indiani de' più gran delitti non per altro, che pel tem"porale loro intereffe... Dappoi che s'accorfero, che la lor ricchezza con" fifteva nell impadronirfi della roba, e delle Perfone degli Indiani, gli " hanno mille volte infamati, ed accufati, che effi erano infetti di fodomia; "ma tal imputazione è una gran falfità, e malvagità degli Accufatori; poi" chè in tutte le Ifole grandi Spagnuola, Cuba, S. Giovanni, e Giamaica, " $e$ in Ceffanta Itole Lucaje, nelle quali erano de' Popoli affai numerof, non "vi fu mai memoria di tal vizio, ficcome noi ne poffiamo teftificare, aven" done fatte delle ricerche infin da principio. Nemmeno rel Perù, in Juca, tàn non fi trovò tal vizio, e così generalmente da pertutto, fuorchè in al"cuni luoghi, dove fi dice, che fono alcuni ec. "Cafas nel memoriale futo la libertà pretela dal Supplice Indiano, Ragione 6. 
dunque ha autorizzato il Sig. de $P$. per infamare in materia si grave tutro il nuovo Mondo? Ancorchè gli Americani foffero veramente, come egli crede, uomini fenza onore, e fenza vergogna, le fteffe leggi dell' umanità richiederebbono da lui, che non gli calunniaffe. A tali ecceff lo porta quel ridicolo impegno d'avilir l'America, e tali fono le confeguenze della fua fcellerata logica, deducendo fpeffo, come abbiam già dettó, conclufioni univerfali da premeffe particolari. Se perchè i Panuchefi, - altri Popoli americani erano forfe infetti da quel vizio, fi puó affermare, che la Pederaftia era in gran voga in tutto il nuovo Mondo, potranno fimilmente a dritto gli Americani infamare con fiffatra imputazione tutto l' antico Continente, mentre la Pederaftia fu in gran voga preffo alcuni antichi Popoli dell'-Afia, e troppo comune preffo i Greci, ed i Romani : Oltrecchè non fi fa che in America fia prefentemente veruna Nazione da quel vizio infetta; laddove fappiamo per la depofizione di parecchj Autori, che alcuni Popoli Afratici non hanno ancor diImeffa quell' abbominazione, e che anche in Europa, fe mai è vero ciò che dicono $\mathrm{i}$ Signori de Locke, e de $\mathrm{P}$. è comune fra que' Turchi, che fanno profelfione di Santocchieria, un altro vizio più efecrabile del medefimo genere, e che in vece d'effere feveramente puniti, fono da quella Nazione tenuti in conto di Santi, e tutti a gara fanno verfo di loro le più gran dimoltrazioni di rifpetto e venerazione. (U)

Tra i delitti, che rinfaccia il Sig. de P. agli Americani, d'a egli ancor luogo al fuicidio. E' pur vero, che furono molti quelli, che a' tempi della conquifta s'appiccarono, o fi precipitarono, o coll' inedia pofero fine all' amara lor vita; ma che maraviglia, che degli uomini privi del lume della vera religione, e difperati per cagione delle intollerabili veffazioni, che foffrivano da' Conquiftatori, efeguiffero ciò, che faceafi cosi frequentemenre da'Romani, da' Greci, e dagli. Spaguuoli antichi, e dagl' Inglefi, da' Francefi, e da' Giapponefi moderni per un lie-

(U) Recherch. Philor. part. 4. fect. 4. 


\section{DISSER TAZIO N E V. 20I}

ve motivo; per un'idea ridicola d'onore, o per un capriccio? $(X)$ Chi fi perfuaderebbe mai, che un Europeo foffe per rimprove. rare agli Americani il Suicidio in un fecolo, nel quale effo $c$ divenuto moda in Inghilterra, e in Francia, $(r)$ dove fcancellardo dalla mente quelle più giulte idee, che abbiamo dalla Nacura, e dalla Religione, s'inventano ragioni, e fi pubblica. no libri per giuftifizarlo? Tanto grande è l'impegno d'infamar l'America, e gli Americani.

Un fiffato impegno fembra avere avuto quello Spagnuolo chiccheffia, che ordinò l' indice generale delle Decadi del Cro. nichilta Herrera, imputando inconfideratamente a tutti gli Americani ciò, che Herrera dice nella fua opera d'alcuni particolari con varie eccezioni. Vonglio copiar qui cid, che fi leg. ge in quell'indice, acciocchè fi vergognino gli uomini di fcrivere tali fpropofiti: "Gl'Indiani, dice, fono afjai pigri, vizio. fiffimi, grand" ubbriacbi per genio, infingardi, deboli, bugiardi, truffatori, novatori, inconftanti, leggieri, poltroni, immondi, fe. dizıof, ladri, ingrati, incorreggibili, vendicativi piu d'ogni al. tra Nazione: di si groffa pafta, cbe fi dubito, fe evano ragionevoli: barbari, befiali, condotti, come i bruti, dai laro appetiti ec. ec. Qusfto medefimo è il linguaggio del Sig. de PaW, e d'altri umaniffimi Europei: licchè pare, che quefti uomini non $f i$ credano obbligati in ciò, che riguarda $i$ Popoli del nuovo Mndo, di rifpettar la verità, nè d'offervare le leggi dolla carità fraternale, pubblicate dallo fteffo Figliuol di Dio nell' antico Continente.

Ma a qualunque Americano fornito d'un mediocre inge: gno, e di qualche esudizione, che voleffe render la pariglia a quefti Scrittori (ficcome abbiamo altrove detro d'un Filofofo Guineo) gli farebbe $f_{a}$ cile di comporre un'opera con quefto Storia Antica del $N_{i} e$ Jjico Tom. IV. C c tito-

(X) Tra le altre memorabili fravaganze di que' molti, che in quefti ultimi anni fi fono ammazzati, in Inghilterra, fo da perfona, che fi trovava allora in Londra, che un nomo ivi in morendo lafcio feritto, che fi dava la morte per liberarfi dalla moleftia di veftirfi e fpogliarfi ogni giorno.

(Y) Sappiamo, che nella fola Citta di Parigi in uno di quefti ultimi anui 6' ammazzarono da fe ftefli cencinquanta uomini incirca. 
sitolo: Ricerche flofoficbe $f u$ gli abitanti dell antico Continent te. Egli, tenendo lo fteffo metodo del Sig. de P., raccoglierebbe ciò, che troverebbe fcritto di paefi fterili del Mondo antico, di montagne inacceffibili, di pianure pantancle, di boIchi impenetrabili, di deferti arenofi, e di cartivi climi: di rettili ed infetti fchifori e nocevoli, di ferpi, di rofpi, di fcorpioni, di formiche, di ragni di fcolopendre, di fcarafaggi, di cimici, e di pidocchi : di quadrapedi irregolari, piccoli, fcodati, difetrofi, e pufillanimi : di genti degenerate, di brutto colore, di ftatura irregolare, di fattezze deformi, di cativya compleffione, d'animo dappoco, d'ingegno otturo, e d' indcle crudele. Quando veniffe all' articolo dei vizi, che immenta copia di materiali non avrebbe egli per la fua opera! Che efempi di viltà, di perfidia, di crudelià, di fuperftizione, e di diffoluzione! Che ecceffi in ogni forta di vizi! La fola Storia dei Romani, la più celebre Nazione, del Mondo antico, gli fornirebbe un' incredibile quantià delle più orrende rcellerdrezze. Riconofcerebbe pure, che fiffatti difetti, e vizi non erano comuni nè a tutti i paef, nè a tutti gli altri abitatori dell' antico Continente; ma non importa, mentre dovea egli fcrivere fullo fteffo modello del Sig. de P., e fervirfi della fteffa logica . Queita opera farebbe fenz' altro affai più pregevole, e più degna di fede, che non è quella del Sig. de $\mathrm{P}$., perchè laddove quefto Filorofo non cita contra l'America, e gli Americani, fe non gli Autori Europei, quello Scritrore Americano per l'oppolto non fi prevarrebbe per la fua curiofa opera, fe non degli Autori nativi del medefimo Continente, contro cui egli fcriverebbe.

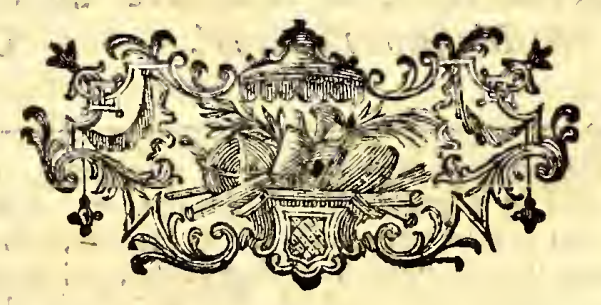




\section{DISSERTAZIONE VI.}

SU LA COLTURA DEI MESSICANI.

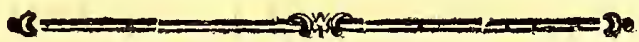

TL Sig. de PaW, ognora Aizzito ed infuriato contra il nuoz vo Mondo, appella baibari e felvaggi tutti gli Americani, e gli reputa inferiori in fagacita ed induftria ai più groffolani e rozzi Popoli deli'antico Continente. Se egli fi' foffe contentato di dire, che le Nazioni americane erano in gran parte incolte, barbare, e beftiali nei lor coltumi, ficcome erano gia ftate anticamente molte Nazioni delle più colte d'Europa; come fono prefentemente parecchi Popoli d'Afia, d'Africa, e anche della fteffa Europa : che le Nazioni più civili d'America erano di lunga inferiori in coltura alla maggior parte delle Nazioni eurnpee: che le loro arti non s'erano tanto perfezionate, oè le lor leggi erano sì buone, e sì bene ordinate: e che i lor facriñzi erano inumani, e alcune delle loro ufanze fravaganti, non avremmo ragione di contrdddirgli. Ma trattare i Mefficani ed i Peruani come i Caribi, e gl' Iroquefi, mettere in non cale la loro indultria, fcreditar le loro arti, difpre. giar in tutto le loro leggi, e mettere quelle induftriofe $\mathrm{Na}$ zioni fotto $\mathrm{i}$ piedi dei più groffolani Popoli dell'antico Continente, non è ciò oftinarfi nell'impegno d'avvilire il nuovo Mondo, ed i fuoi abitatori in vece di ricercare il vero, come dovrebbe giufta il titolo della fua opera?

Barbari e felvaggi diciamo oggid quegli uomini, che condotti più dal capriccio e dalle voglie naturali, che dalla ragione vè vivono congregati in focietà, nè hanno leggi pel loro governo, nè Giudici che aggiultino le loro differenze, nè Superiori che invigilino fu la loro condotta, nè efercitano le arti neceffarie per rimediare ai bifogni e miferie della vita, quelli finalmente che non hanno idea della Divinità, o almeno non hanno fabilito il culto con cui debbono onorarla. Ora i Mefo 
ficani, e tutte le altre Nazioni d'Anahuac, ficcome pure i Pesuani, riconofcevano un Effere fupremo e onnipotente, beuche la loro credenza foffe, come quella d'altri Popoli idolatri, con mille errori e fupertizioni viziata. Aveano pure un filtema fiffo di religione: aveano Sacerdoti, tempi, facrifizi, e riti or. dinati al culto uniforme della Divinita. Aveano Re, Gover. natori, e Magiftrati: aveano tante Città, e popolazioni sì grandi, e si bene ordinate, come faremo vedere in un' altra differtazione. Aveano leggi e coftumi, la cui offervazione zelavano i Magiftrati, e Governatori A veano commercio e prendevano una gran cura dell' equità e giuftizia nei contratti. A. veano diftribuite le terre, e afficurata a ciafcun particolare la proprietà, e la poffeffione del fuo terreno. Efercitavano l'agricoltura, ed altre arti, non, che le neceffarie alla vita, ma an. cor quelle che fervono foltanto alle delizie, e al luffo. Che dunque fi richiede davvantaggio acciocchè quelle Ndzioni non fieno reputate barbare e felvagge? La moneta, dice il Sig. de P., l'ufo del ferro, l'arte di fcrivere, e quelle di fabbricar vafcelli, di coftruir ponti di pietra, o di far la calcina. Le loro arti erano imperfette e groffolane : le lor lingue fcarfiffi= me di voci numerali, e di termini atti a efprimere le idee univerfali, e le lor leggi poffono dirfi niune; perchè non va ne poffono effer leggi, dove regna l'anarchia, e il defpotismo o. Tuti quefti articoli richiedono un particolar efame.

\section{F.}

\section{Su la mancanza di Moneta:o.}

If Sig: de $\mathbf{P}$. decide che niuna Nazione d'America era colta e civile; perchè niuna ufava di moneta, e per convincer queft'afferzione allega un paffo-del Montefquieu "Ari3. ftippo, dice quefto Politico, (a) avendo fatto naufragio, arri3. vò a nuoto alla vicina fpiaggia: vi vide delineate nell' are-

(a) L'Efprit des Loix lib. I8. c. 23. 
\# na alcune figure di Geometria, e s'empi di giubilo perfua: " dendofi d' effere arrivato preffo un Popolo greco, e non pref-

"fo una Nazione barbara. Immaginatevi che per qualche ac" cidente giungete ad un paefe incognito: fe vi trovate qualche moneta, non dubitiate, che fiete arrivato preffo un Po"polo colto., Ma fe Montefquieu conchiude bene dall'ufo della moneta la coltura d'un Popolo, il Sig. de P. inferifce affai male il difetto di coltura dalla mancanza di moneta. Se per moneta fi vuol intendere un pezzo di metallo coniato coll'impronta del Principe, o del Pubblico, egli è certo, che la mancanza d'effa in una Nazione non dimoftra barbarie. "Gli Ateniefi, dice il citato Montefquieu, perchè non avea"no verun ufo dei metalli, fi fervirono per moneta di buoi, "ficcome i Romani di pecore, e quindi ebbe origine, come fanno tutti il nome pecunia, mentre i Romani mifero nella prima moneta che coniarono l'impronta delle pecore, di cui fi fervivano innanzi per li loro contratti. I Greci erano fen. za dubbio una nazione affai colta ai tempi d'Omero, poichè non era poffibile, che in mezzo ad una Nazione incolta s'allevaffe un uomo capace di comporre l' Iliade, e l' Odiffea quei due poemi immortali, che dopo ventifette fecoli tuttora s'ammirano, ma non fi fanno imitare. Eppure i Greci a quei tempi non conofcevano la moneta coniata, come appare dalle fterfe opere di quel rinomato Poeta, il quale dovunque vuol fignificare il valore di qualche cofa non l'efprime altrimenti, che pel numero di buoi, o di pecore che valeva: ficcome fa nel lib. 7. dell' Hiade, dove dice che Glauco diede le fue arme d'oro, che valevano cento buoi, per quelle di Diomede, ch'erano di rame, e non valevano più di nove buoi. Dovunque: poi fa menzione di qualche acquifto per contratto, non ne parla d'altro, che di quello del cambio, o permuta. E però in quella controverfia antica eccitatafi tra i Sabiniani, ed i Proculiani, due ferte di Giureconfulti, quei primi foltenevano, che poteva farfi vera vendita e compra fenza prezzo, allegando per cio certi paffi d'Omero, nei quali fi dicono comprare e: wendere quelli che non altro faceano, che permutare. I Lace: 
demonj erano un Popolo civile della Grecia, contuttochè non ufaffero moneta, e tra le leggi fondamentali pubblicate da Licurgo fi foffe quella di non commerciar altrimenti, che per via di permutazione. (b) I Romani non ebbero moneta coniata infino al tempo di Servio Tullo, nè $i$ Perfiani infino al regno di Dario Iftafpe; e non però debbono dirfr Nazioni barbare nei tempi che precedettero quelle epoche. Gli Ebrei erano civili almeno infin dai tempi dei loro Giudici, eppur non fappiamo che forfe preffo loro in ufo la moneta improntata fi. no ai tempi dei Mdccabei. Dunque il difetto di moneta coniata non è argomento di barbarie.

Se per moneta s'intende un fegno rapprefentativo del va lor di tutte le merci, ficcome la definifce il Montefquieu, (c) egli è certo e indubitabile, che Mefficaní, e tutte le altre Nazioni d'Anahuac . fuorchè i barbari Cicimechi, ed Oromiti, fi fervivana della moneta nel loro commercio. Che cofa era il Caccao, di cui conftantemente fi fervivano per procacciarfi nel Mercata tutto cid, di cui aveano bifogno, fe non un fegno rapprefentativo del valor di tutte le merci? Il Caccao avea il fuo valor fiffo, e fi dava per numero, ma per rifparmiarfi la moleftia di contare qualo. ra le merci imporravano molte migliaja di mandorle, fapeano gia, che ogni facco di certa grandezza conteneva tre Xiquipilli, o fia venti quattro mila mandorle. Or chi non vede, che il Caccan è affai migliore per fervirfene in vece di moneta, che non i buoi, e le pecore, di cui fi fervivano anticamente i Greci, ed i Romani, e il fale, di cui fi fervono oggidì gli Abiffini? I buoi e le pecore non poteano fervire per acquiltar le merci minuie e di poca valura, e qualunque in. fermità, o altra difgrazia che fopravveniffe a quegli animali porrebbe impoverir coloro che non avevano altro capitale. "Si adopra il metallo per moneta, dice il Montefquieu, ac" ciocchè fia più durevole il fegno. " "Il Sale di cui fi fers) vono.

(b) Emi fingula non pecunia, fed compenfatione mersism jufft. Juftin. lib. 3 . (c) L'Esprit des Loix. 
9) vono gli Abiffini ha il difetto di andarfi continuamente di"minuendo "Il caccao per l'oppofto poteva fervir per qualunque merce, fi trafportava, e cuitodiva più facilmente, e i coniervava con manco pericolo, e con minor diligenza.

I.' ufo del caccao nel commercio di quelle Nazioni parrà forle a qualcuno un mero cambio; ma non era così; poichè effendovi parecchie fpezie di caccao, non ufavano come moneta il Tlalcacabuatl, o caccao minuto, il quale adopravano nelle cotidiane loro bevande, ma piuttofto altre fpezie d'inferior qualita, e men urili per cibarlene, le quali giravano in. ceffantemente come la moneta, $(d)$ e non aveano quafi altro ufo, che quello d'adoprarfi nel commercio. (e) Di quelta fpezie di moneta fanno menzione tutti gli Storici del Meffico tanto Spagnuoli quanto Indiani. Delle altre quattro Tpezie, di cui abbiam ragionato nel lib. 7 . della Storia, confta per la teltimonianza di Cortès, e di Torquemada. Corrès afferma nella fua ultima lettera all' Imperatore Carlo V., che avendo egii fatto delle ricerche intorno al Commercio di quelle Nazioni, trovo che in Tlachco, e inaltre Provincie commerciavano con moneta. Se egli non aveffe intefo parlare della moneta coniata, non avrebbe riftretto l' ufo d' effa, a Tlachco, ed a qualche altra Provincia; poichè ben fapeva, fenza che gli fuffe $d^{\text {' }}$ uopo di far nuove ricerche, che ne' mercati di Meffico, e di Tlafcalla, a' quali era Rato fpeffe volte prefente, fi fervivano, come di moneta, del cascao, di certe piccole tele di bambagia appellate $\mathrm{d} a$ loro Patolquacbtli, e dell' oro in polvere meffo dentro di penne d'Oche. Lo pe:ò fofperto, non oltante ciò che ho detro in quel lungo della Storia, che v' era ancora della moneta coniata, e che ranto qualle pezze fortili di ftagno, di cui $f_{a}$ menzione lo fteffo Corrès, quanto quelle di rame fatre in forma di $\mathrm{T}$, di cui parla il Torquemada, $(f)$ come di fpezie di

mo-

(d) Hernandez Rerum medicarim N. Hifpania Thefauri lib. 3. cap. $4^{6}$

(e) Nella ferta Capitale di Meffico, nella quale fi coniano annualmente fino a diciotto, o venti milioni di fcudi (pefos fuertes) in oro ed argento, ado. pra finora la pente povera il Caccao rer acquiltas nel mercato alcune coluccie.

(f) Monaribia Indiana lib. 14. cap. I4. 
moneta, aveano qualche impronta autorizzata dal Sovrano, 0 da' Signori feudatari .

Per impedir poi ogni frode nel Commercio niente, fuorchè gli ordinari viveri, fi poteva vendere fuor della piazza del Mercato, nel qual era, ficcome abbiam detto fu la depofizione di molti teltimoni oculari, il più bell' ordine che poffa im. maginarf. V' erano delle mifure prefcritte da' Magiftrati, de' Commeffari che giravano inceffantemente offervando quanto vi avveniva, e-de' Giudici di Commercio incaricati di conofcere le liti inforte tra i Negozianti, e di punire i delitti, che vi fi commettevano. E ciò non oftante dovrà dirfi che $i$ Mefficani erano inferiori in induftria a' Popoli più groffolani dell' antico Continente, tra $i$ quali vi fono alcuni tanto roz. $z i$, e tant' oftinati nella lor barbarie, che non ha baftato in tanti fecoli l' efempro delle altre Nazioni del lor Continente per dar loro a conofcere i vantaggi della moneta?

\section{II.}

\section{Sopra l'uso del Ferro.}

L' ufo del Ferro è una di quelle cole, che il Sig. de P. richiede per chiamar colta una Nazione; e per mancanza d'effo eglı crede barbari tutti gli Americani. Sicchè fe Iddio non aveffe creato quel metallo, tutti gli uomini dovrebbono effer per forza barbari fecondo il fentimento di quefto Filofofo. Ma nello fteffo luogo della fua opera, dove rinfaccia la barbarie agli Americani, ci fornifce tutti que' materiali, che potrebbon defiderar per ribatterlo. Egli afferma che in tutta l' eftenfione dell'. America $\int_{i}$ trovano affai pocbe miniere di ferro $e$ quello cbe $v$ ' $\dot{e}, \dot{e}$ si inferiore in qualita a quello dell' altro Continente, cbe $n \dot{e}$ anche può adoprarfi per farne dé cbiodi. Eglı ci dice, che gli Americani polJiedevano il Segreto, gia perduto nell' antico. Continente, di dave al Rame una tempra ugisale a quella cbe riceve l'Acciajo: che il Sig. Godin mandó nel 1727. (vorrà forfe dire nel 1747; poichè nel 172\%. non era ancor andato nel 
Perù il Sig. Godin) al Conte de Maurepas una veccbia foure di rame peruano indurito, e che avendola offervata al Conte de Cayliss, riconobbe, cbe quafi s'agguagliava in durezza alle anticbe arme di rame, di cui fi fervirono gia i Greci, ed $i$ Ro: mani, i quali non adoperavano il ferro in molte di quelle opere, in cui noi l'adopriamo prefentemente, o percbè allora eva piue raro, o percbè il lor rame temperato era migliore in qualita del loro acciajo. Finalmente loggiugne, che il C. de Caylùs maravigliato di quell'arte fi perfuafe (benchè in quefto fia impu. gnato dallo iteffo Sig. de Paw ) che quello ftrumento non era opera di quei Peruani imbeftati, che vi trovarono gli Spagnuo. li, ai tempi della conquifta, ma d'un' altra Nazione più antica e più induftriofa.

$\mathrm{Da}$ tutto quefto, che ne dice il Sig. de Paw io cavo quattro confeguenze importanti. I. che gli Americani ebbero l'onore d'imitare nell' ufo del rame le due Nazioni più celebri dell' antico Continente. 2. Ch' effi fi portarono faggiamente non fervendofi d'un ferro sì cattivo, che non può effere urile nè anche per farne dei chiodi, ed ufando un rame, al quale davano la tempra dell' acciajo. 3. Che fe non teppero l'arte comuniffima di lavorare il ferro, poffiedevano quella fingolariffma di temperare il rame come l'acciajo, che non hanno potuto reltaurare i Fifici europei del fecolo illuminato. 4: che tanto s' ingannò il C. de Caylùs nel giudizio che fece dei Peruani, quanto il Sig. de P. in quello che ha fatto di tutti gli Americani. Quefte fono le confeguenze legitrime che debbon dedurfi dalla dottrina del noltro Filofofo full' ufo del ferro, e non quella della mancanza d'induftria, che egli pretende dedurne. Vorrei da effo lui fapere, fe vi vuole maggior induftria per lavorare il ferro, come lo lavorano gli Europei, che per lavorar fenza ferro ogni forta di pietra e di legno, per fabbricar parecchie fpezie d'armi, e per far fenza ferro, come faceano gli Americani, i più curiofi lavori d'oro, d'argento, e di gemme. L'ufo precifo del ferro non prova una grande induftria negli Europei. Inventato effo dai primi uomini facilmente palsò daghı uni agli altri, e come gli Americani mo; Storia Antica del Mejsico Tom. $I V$. D d derni 
derni lo ricevettero dagli Europei, così gli antichi Europei l' ebbero dagli Aliatici. I primi popolatori dell' America conofcevano fenz' altro l' ufo del ferro, poichè l'invenzion d'effo fu quafi coetanea al Mondo; ma può crederfi che accadefle quello, che congetruriamo nella prima Differtazione, cioè che non avendo coloro trovate da principio le miniere di quel metallo nei paefi fettentrionali dell' America, dove allord fi tabilirono, fe ne perdette preffo i loro difcendenti la memoria.

Ma finalmente fe fon barbari quegli, che non hanno l'ufo del ferro, che faranno coloro, cui manca l' ufo del fuoco? Ora in tutta la vafta eltenfion dell'America non fi è trovata una Nazione, nè anche una tribù quantunque rozza, la quale non abbia faputo la maniera di far fuoco, e di fervirfene per gli ufi comuni della vita: ma nel Mondo antico $f i$ fon trovati dei Popoli tanto barbari, che non aveano nè uro, nè cognizione del fuoco. Tali fono tati gli abitatori delle ifole $\mathrm{Ma}$ riane, ai quali era affatto incognito quell' elemento prima che vi approdaffero gli Spagnuoli, ficcome ne fanno fede gli Srorici di quelle ifole. E vorra contuttociò perfuaderci il Sig. de PaW, che i Popoli americani fono plù felvaggi di tutti i felvaggi del Mondo antico?

Del refto tanto sbaglia il noftro Ricercatore in quello che dice del ferro americano, quanto in ciò che penfa del rame. Nella N. Spagna, nel Chile, e in molti altri paefi d'America $G$ fono fcoperte infinite miniere di busn ferro, e fe ron vi folfe proibito il lavorarle per non pregiudicare al commercio di Spagna, potrebbe l'America fornire all' Europa tutto il ferro neceffario; come la provuede dell'oro, e dell'argento. Se i) Sig. de P. aveffe faputo far le fue ricerche intorno all' America, avrebbe trovato preffo il Cronichilta Herrera; $(g)$ che anche nella Ifola. Spagnuoia evvi del ferro miglior di quello di Bifaglia. Aurebbe altresi trovato (b) preffo il medefimo Autore, che in Zacatula Provincia maricrima del Meffico, ev-

(g) $\mathrm{DCC}+\mathrm{lb}$ 6. $\mathrm{CHp} .7$.

(b) Heriera Difcrizione delle Indie Occideni. cap. Io. 
"vi del rame di due forti : l'uno duro, del quale fi fervívano in vece di ferro, per fare fcuri, accette, cd altri Atrumenti di guerra, e d'agricultura, e l'altro ordinario e pieghevole, il quale adopravano in pignatie, carini, ed altri vala per gli uff dimeftici : ficchè non a veano bifogno del vantato fegreto $d$ ' indurire il rame. La mia fincerità mi obbliga del pari a difendere i veri progıtefi dell'indu!tria americana, ed a rigettare quelle immaginarie invenzioni che s'attribuifcono alle Nazioni di quel nuova Mondo. Il fegreto che veramente poffedevano gli Americani, fi è quello, che fi legge preffo l'O viedo teftimonio oculare, e molto pratico e intendente di metalli : "Gli "Indiani, dice, (i) fanno dorare affai bene i vafi di rame, o " d'oro baffo, e dar loro un sì eccellente e sì accefo colore, " che fembra oro di ventidue carati e più : ciò che eglino fan" no con certe erbe. Quefto lavoro riefce così bene, che fe " qualche Orefice di Spagna, o d'Italia aveffe quefto fegreto, "fi crederebbe affai ricco.,

\section{§. II I .}

Su le arti di fabbricar Vafcelli e Ponti, e di far la Calciná:

Se ad altre Nazioni può forfe rinfacciarfi l' ignoranza dell' arte di conftruir vafcelli, quefto rimprovero non dee certamen. te farfi ai Mefficani; perchè non effendofi effi renduti padroni delle maremme, fe non fe negli ultimi tempi della lor Monarchì, non ebbero bifogno, nè occafione di penfare a fiffatta coftruzione. Alle Nazioni poi, che occupavano le fpiagge d'ambidue i Mari, prima che fe ne impadroniffero i Mefficani, baftavano quelle barche, che erano in ufo preffo loro per la pefca, e pel commercio colle vicine Provincie; perchè libere dall'ambizione, e dall'avarizia, le quali fono ftate per la più le cagioni delle navigazioni lunghe, nè cercavano d'ufurpar gli Stati da altre Nazioni legittimamente poffeduti, nè voleano traD d 2 \{por-

(i) Sommario della Stor. Natur, delle Indie Orcident. cap. 84. 
fportar da lontani paefi i preziofi metalli, di cui non abbifo. gnavano. I Romani, con tuttochè aveffero fondato la lor metropoli così vicino al mare, ftettero nulla di meno anni cinquecento fenza conftruir vafcelli $(k)$, finchè l' ambizione d'ampliare i loro dominj, e impadronirfi della Sicilia, lor fece $f_{d} b$. bricar delle navi per valicar quello ttretto. Che meraviglia dunque, fe quelle Nazioni americane, che non fencivano tali ftimoli per abbandonar la loro patria, non inventarono dei vafcelli da poterfi trafportar con manco rifchio nei paefi diftanti. Egli è certo, che il non avere inventati vafcelli non arguifce mancanza d'induftria in coloro, che non aveano verun intereffe in tale invenzione.

Non è per altro così nell' invenzione dei Ponti. Il Sig. de P. afferma, $(l)$ che non v'era un fol ponte di pietra in tutta l'America, allorcbè fu fcoperta, perchè gli Americani non fapevano fabbricar degli archi : e cbe il fegreto di far calcina fu a) Solutamente ignorato in tutta l'America. Ecco tre propofizioni, che fono altrettanti errori groffiftimi . I Mefficani fapeano far ponti di pietra, e tra gli avanzi dell' antica loro architettura fi veggono anche oggidi nel frume di Tula $i$ grandi $e$ forti pilaftri, che foftenevano il ponte che $v^{2}$ era. Gii avanai poi degli antichi palazzi di Tezcuco, e molto pì̀ i lor $T e$. maxcalli, o Ipocaufti, danno a divedere l'ufo antico degli archi, e delle volte preffo i Mefficani, e le altre Nazioni d'Anahuac. Didaco Valadès, il quale andò nel Meflico pochi anni dopo la conquifta, e vi dimorò anni trenta, ci fa vedere nella fua Rettorica Crifiana l'immagine d'un piccol tempio, che egli vi vide, il quale non ci lafcia verun dubbio in quefta materia.

Intorno all' ufo della calcina bifogna aver tutto l'ardire del

(k) Appio avera ufato tutta la diligenza poffibile per venire in foccorfo or a'Mamertini. Si trattava per riuf́cirvi di paffare lo Atretto di Meflina, e "' 'ंimprefa era temeraria, anzi pericolofa, ed anche fecondo tutte le pir̀ pru\%denti apparenze, impoffibile. Non avevano i Romani armata navale, ma 2) Folamente barche groffolanamente fabbricate, le quali potrebbono parago" narfi alle canoe degli Indiani. "Rollin Stor. Rom. lib. Ix.

(i) Recherch. Pbilofoph. part. s. lect. I. 
del Sig. de PaW per porer affermare, come egli $f_{a}$, che ilfegreto di far la calcina era affolutamente ignorato in tutta l'A. 'merica; poichè confta non meno per la depofizione de' Conquiftatori Spagnuoli, che per quella de' primi Miffionarj, che non folamente ufavano le Nazioni del Mefico la calcina; ma che imbiancavano affai bene, e rendevano curiofamente lif́cie e forbite le mura delle cafe, e de' tempj. Confta per le Storie di Bernal Diaz, di Gomara, d'Herrera, di Torquemada, e d'altri, che a' primi Spagnuoli, che entrarono nella Citta di Cem. poalla, parvero d'argento i muri del palazzo principale, perchè erano pulitamente imbiancati, e rifplendenti. Contta finalmente per le pitture de' tributi, che fono nella Raccolta di Mendoza, che le Città di Tepejacac, Tecamachalco, Quecholac \&c. erano obbligate a pagare annualmente al Re di Meffico quattro mila facchi di calcina. Ma ancorchè ci mancaffero tutti quefti documenti, bafterebbono a dimoftrar la verità di quanto diciamo, ed a confondere la temerità del Sig. de P. gli a vanzi degli antichi edifizi, che ancor $f i$ veggono in $\mathrm{Te}$ zcuco, in Mictlan, in Guatufco, e in molti altri luoghi di quel regno.

Per quello poi che riguarda il Perù, avvegnachè il $\mathrm{P}$. Acofta confeffi, che non v'era in ufo la calcina, e che quella Nazione non fabbricava nè archi, nè ponti di pietra, e quefto baftaffe al Sig. de P. per dire giufta la fua fcellerata logica, che l'ufo della calcina era ignorato in tutta l'America; cona tutto cid lo fteffo Acolta, il quale non era un uomo volgare, nè efagerante, nè parziale degli Americani, loda affai la maravigliofa induftria de' Peruani ne'loro ponti di totora, o fia giunco nella sboccatura del lago di Titicaca, e in altri luoghi, dove la fomma profondità oon permette fare ponti di pietra, - la Atraordinaria rapidità de' fumi rende pericolofo l' ufo delle barche. Egli teftifica d'effer paffato per tali ponti, e vanta la facilità, e la ficurezza del paffaggio. Il Sig. de Paw s'avanza a dire, che i Peruani non conofcevano l'ufo delle barche, che non feppero far fineftre negli edifizj, e anche fofpetta, che le loro cafe foffero fenza tetto. Spropofiti i più groffolani che pof- 
214 DISSERTAZION E VI.

fano faltare in tefta ad uno Scrittore d'A nerica. Egli dà a divedere, che non fa che cofa fiano i bejucos de' ponti peruani, e che non ha formato idea giulta de' fumi dell' America Meridionale. Vi fono molte cofe da opporre al Sig. de Po intorno a quefo foggetto; ma le tralafciamo per venire ad altri articoli più effenzıali .

\section{S. I V.}

Sit la mancanzan di Lettere.

Niuna Nazione d'America conofceva l'arte di fcrivere, fe per effa $s^{\prime}$ intenda l'arte di efprimere in carta, in pelli, in rela, $O$ in altra fimile materia, qual fi fia forta di parole colla differente combinazione d'alcuni caratteri; ma fe l'arte di fcrivere fi prende per quella di rapprefentare, e dar ad intendere qualfivoglia cofa agli affenti ed alla pofterita con figure, geroglifici, e caratteri, egli è certo, che una tal arte era conofciuta, $e$ in grand'ufo preffo i Mefficani, gli Acolhui, i Tlafcallefi, e tutre le altre Nazioni dirozzate d'Anahuac. Il C. de Buffon per dimoftrare, che l'America era una terra veramente nuova, e nuovi fimilmente i Popoli, che l'abitavano, allega, ficcome abbiam detto altrove, che ancbe quelle Nazio$m i$, le quali viveano in focietà, ignoravano l'arte di tramandare i fatti alla pofterità per mezzo di fegni durevoli, contuttocbè aveffero trovata l'arte di comunicarfi da lontano, $e$ di fcriverji annodando cordoni. Ma quella fteffa arte, di cui fi prevalevano per trattar cogli affenti, non dovea anche fervire per parlare alla pofterita? Che erano le pitture ftoriche de' Mefficani, fe non fegni durevoli per tramandare la memoria degli avvenimenti tanto a'luoghi, quanto a' fecoli remoti? Il $C_{\text {. de Buf- }}$ fon fi moftra in vero tanto ignorante della Storia del Meffico, quanto dotto nella Storia naturale. Il Siga de P., benchè accordi a' Mefficani quell'arte, che lor nega a torto il C. de Buftun, nondimeno per ifcreditarla allega parecchie ragioni, ed innumerabili Spropofiti, che non poffiamo diffimulare. 


\section{DISS ER T A Z IO N E VI. 2 IS}

Dice dunque, che i Meflicani non ufavano di gernglifici: che le loro pitture non erano altro, che difegni groffolani degli obbietti: che per rapprefentare un albero pignevano un albero: cbe nelle loro pitture nun $f_{i}$ fcorge veruna traccia del cbia. rofcuro, nè alcuna idea di profpettiva, o d'imitazione della Natura: che non aveano fatto alcun progreffo in quell' arte, per mezzo della quale effi s'adopravano a perperuare la memoria delle cose pafJate, e degli avvenimenti: che l'unica copia di pittura ftorica de' M flicani fottratta dall'incendio, che ne fecero i primi Miffionari, fi è quella, che il primo Vicerè del Meffico mandò a Carlo V. la quale pubblicarono poi Purchàs in 1nghilterra, e Tevenot in Francia: che quefta pittura è tanto groffolaria, e si mal efeguita, che non fi può difcernere, fe tratta, come dice l'Interprete d'effa, d'otto Re di Meffico, ovvero d'otto concubine di Morezuma \&c.

In tutto quefto dà a divedere il Sig. de P. la fua ignoranza, e da effa nafce la fuz temerità nello fcrivere. M 1 dovrà darfi maggior fede a un Filofofo Pruffiano, il quale ha veduto le fole groffolane copie del Purchàs, che a coloro $\mathrm{i}$ quali hanno vedute, e diligentemence itudiate moltiffime pitrure originali de' Meillcani? Il Sig. de P. non vuole, the i M fincani fienfi ferviti di geroglfici, perchè non fi penfi, che conceda loro qualche fomigilanza cogli anuchi Egzz. Il P. Kiker, quel celebre ricercatore, e lodatore delle antichilà egiziane nella fua opera incicolata Edipus Aggptiacus, e Adriano Walton ne' prolegomeni della Biblia Poliglotta fono del medefrimo fenumento del Sig. de P., e la loro opinione non ha altro appoggio, che quelio della fuddetra copia del Purchàs; ma Motolinia, (m) Sahagun, Valadès, Torquemada, Arrigo

Mar.

(nu) Torilio di Motrlinia ne' fuoi MSS. rpezialmente nell' elpofizione del Calendario Mefficano. Bermardian Sabagun nel tho Dizionario univerfale della lingua neflicana. Ditaco Valates nella fua Retrorica Criftiana Aamrata in Perugia, e dedicara a t'apa Gregorio XIII. I'anno 1579. Arrigo Martinez nella fila Storia della N. Spagna . Siguenza nella fua Ciclografia mefficana, e nell' opera intitulata: Teatro di virtì politiche. Torquemada nella fua Munarchia Indiana. Valades praticò i Melficani anni trenta, Torquemada più di qua- 


\section{DISSERT A Z IO NE VI.}

Martinez, Siguenza, e Borurini, i quali fepperola lingua Mefficana, conferirono cogl' Indiani, videro, e con diligenza Atudiarono moltiffme pitture antiche, dicono, che tra diverfe maniere, che aveano i Mefficani di rapprefentar gli obbietti era quella de' geroglifici, e delle pitture limboliche. Lo fteffo vien teltificato da Acofta, e Gomara nelle loro Storie, dal Dott. Eguiara nell' erudita prefazione della Biblioteca Meflicana, e da que' dotti Spagnuoli, che pubblicarono con nuove aggiunte l'opera di Gregorio Garcia $\int u$ l'origine degl' Indiani. Il P. Kircher fu affai bene impugnato dal Dott. Siguenza nella fua 0 pera intitolata: Teatro di virti politicbe. Egli è certo, che il Kirker fi contraddice apertamente; poichè nel primo tomo della medefima opera Edipus Egyptiacus, laddove confionta la religione de' Mefficani con quella degli Egizj, confeffa fchiettamente, che le parri di cui componevafi l'immagine del Dio Huitzilopochtli, aveano molte arcane, e mifteriofe fignificazioni . Acofta, la cui Storia è giuftamente pregiata dal Sig. de P. nella defcrizione, che fa di quell' immagine dice cosi: Tutto quefto ornato, cbe abbiam detto. e il refto, cb'era affai, aveva le fue particolari fignificazioni, fecondo cbe dicbiaravano i Meffacani : e nella defcrizione dell' Idolo di Tezcatlipoca s'efprime in quefti termini: "I fuoi capelli erano legati con una cordel" la d'oro, dalla cui eftremità pendeva un orecchio dello ftef" fo metallo con certi vapori di fumo in effo dipinti, $i$ quali " fignificavano le preghiere de' tribolati, e dei peccatori, " che erano da tal Dio afcoltati, allorchè fi raccomandava"no a lui... Nella man finiftra aveva un ventaglio d'oro " orlato di belle penne verdi, turchine, e gialle, si rilu"Iucente, che pareva uno fpecchio: nel che davano ad inten"dere, che in quello fpecchio vedeva tutto cio, che accade"va nel mondo... Nella deftra aveva quattro faette per fi. " gnificare il gaftigo che dava a' deliquenti per li loro misfat, ti "

ranta, Motolıì quarantacinque, e Sahazun fefranta. Quefti fu l'uomo più infruito ne' fegreti di quella Nazione. Vi vuole un grande orgoglio per deferir più a' propri fcarfi lumi, che a quelli di tanti uomini incomparabilmente più illuminati. 
i) ti "ec. Che fono tutte quefte, ed altre fiffatte infegne degl" Idoli mefficani, di cui abbiam fatta menzione nel libro VI. della Storia, fe non fimboli e geroglifici affai tomiglianti a quelli degli Egizj?

-Il Sig. de P. dice, che i Mefficani non faceano altro, che pignere un albero per rapprefentare un albero; $\mathrm{ma}$ mi dica per grazia, che pignevano per rapprefentare il Giorno, la Notte. il Mefe, l'Anno, il Secolo, i nomi di quelle perfone, che volevano dare ad intendere, \&c.? Come poteano rapprefentare il Tempo, ed altre cole, che non hanno figura fenza prevalerfi di fimboli, o di caratteri? "Aveano i Meflicani, dice il gia " lodato Acolta, le loro figure e geroglificı, co'quali rappre"Sentavano le cofe in quefta maniera: cioè, quelle cofe, che " aveano figura, le rapprefentavano colle proprie lor figure; " per quelle poi che non hanno immagine propria, fi prevale" vano d'altri caratseri fignificativi di quelle: cosi rapprefenta" vano quanto volevano: e per accennare il tempo, nel quale " avveniva qualfifia cola, fi fervivano di quelle ruote dipinte, " ciafcuna delle quali comprendeva un lor fecolo d'anni cin" quantadue \&c. "( $n$ )

Ma ecco quil un' altra pietra di fcandolo per l'ignoranza del Sig. de P. Egli fi beffa delle Ruote fecolari de' Mefficani, la cui efpofizione, dice, osò dar Carreri tenendo dietro a un Profeffor Caftrgliano, appellato Congara, il quale non ardì di pubblicare l'opera, che nvea promefso intorno a quefto foggetro; percbì $i$ fuoi parenti, ed amici, l' affecurarono, cb' effa conteneva molti errori. Pare, che il Sig. de P. non fappia fcrivere fenza errare. Quel Profeffore, cui tiene dietro Carreri, o fia Gemelli, non era Caftigliano, ma Creoglio nato nella feffa Città di Meffico: nè s'appellava Còngara, ma Siguenza, $e$ Gòngora: non iftamoò la fua Ciclografia Mefficana, che fu l'ope$x$ a di cui fi fervì Gemelli, non già perchè temeffe la cenfura del Pubblico, ma per cagione delle ecceffive fpefe della $f_{a} m$ $p^{a}$ in que' paefi, le quali hanno parimente impedito la pubbliStoria antica del Melfico TomoIV.

E e

ca-

(n) Stor. Nat. e Mor. delle Indie lib. 6. cap. 7. 
cazione di tante altre eccellenti opere, tanto del medefimo Siguenza, quanto d'altri uomini dotriffini. Il dire, che i parenti, e gli amici del Siguenza lo diftolfers da! $l_{a}$ pubblicazione di quell'opera, perchè vi trovarono degli errori, nemmen quefto è uno sbaglio, cagionato da qualche inavvedutezza, ma una manifefta bugia archicettata a bella pofta per abbagliare il Pubblico. Chi ha comunicato al Sig. de P. un sì ftrano aneddoto, affatto ignorato nella N. Spagna, ove è sì cara la memoria, e sì celebre la fama di quel grand' uomo, ed ove i Letterati fi lamentano della perdita di quella, e d'altre pregevoliffime opere del medefimo Autore? Che poteva temere il Siguenza dalla pubblicazione delle Ruote mefficane, pubblicate già in Italia dal Valadès più d'un fecolo prima di lui, e defcritte da Motolinìa, da Sahagun, da Gomara, da Acofta, da Herrera, da Torquemada, e da Marrinez tutri Europei, e dagli Storici Mefficani, Acolhui, e Tlafcallefi Ixthixochitl, Chimalpain, Tezozomoc, Niza, Ayala, ed altri? Tutti quelti Autori fon d'accordo con Siguenza in ciò, che riguarda le Ruote mefficane del Secolo, deli Anno, e del Mefe, $e$ foltanto difcordano intorno al principio dell' Anno, ed a' nomi d'alcuni Mefi per le cagioni da noi accennate nel libro Vi. della Storia. Del refto zutti gli Autori, che hanno !critto di quefta materia, tanto Spagnuoli, quanto Americani, i quali fono moltiffimi, convengono nel dire, che i Mefficani, e le altre Nazioni di que' paefi . If prevalevano di tali Ruote per rapprefentare il loro Secolo, il loro Anno, e il loro Mefe: che il lor fecolo conflava d'anni cinquanta dise, il loro Anno di giorni trecento feffanta cinque, diftribuiti in mefi diciotto di giorni venti l'uno, ed inoltre in cinque giorni, che appeilavano Nemontemi: che nel lor fecolo contavano periodi quattro d'o anni tredici, e che anche $\mathbf{i}$ giorni fi contavano per periodi di tredici : che i nomi e caratteri degli anni erano foltanto quattro, cioè quelli del Coniglio, della Canna, del Selce, e della Cafa, i quali fenza interruzione s'alternavano ognora con diverfi numeri \&ce.

Non può effere, dice il Sig. de P. ; perchè un tal ufo fup: 
porrebbe una lunga Serie d' offervazioni aftronomicbe, e di cognizio. ni affai precife per regolare $l$ anno folare, o quefte non poffono accordarfi con quella prodigiofa ignoranza, in cui erano immerfi que Popoli. Come avrebbon pointo perfezionar la loro cronologia coloro, cbe non aveano voci per numerare oltre a dieci? Sta bene. Dunque fe i Mefficani ebbero infatti quella maniera di regolare il tempo, non dovranno dirfi barbari e felvaggi, ma piuttofto colti e coltif. fimi; perchè non può effere che una Nazione coltiffima, quella che ha una lunga ferie d'offervazioni e di cognizioni precife d'Aftronomia. Or la certezza di tal regolazione del tempo preffo i Mefficani è tale da non poterfene dubitare; imperciocchè fe l'unanime teltimonianza degli Scrittori Spagnuoli intorno alla comunione de' Mefficani non permette dubitarne, íccome afferma in un altro luogo il Sig. de $P_{0},(0)$ come potrà dubitarfi del metodo, che aveano quelle Nazioni nel computare i fecoli, e gli anni, e della conformità d'effo col corfo folare, venendo e l'uno, e l'altro uusnimemente teftificato da turti gl Aurori Spagnuoli, Mefficani, Acolhui, e Tlafcallefi? Oltrechè la depofizione degli Spagnuoli in quefta materia èd' un pefo affai più grande, mentre egíno s'impegnarono piuttolto al dir del Sig. de P., nello fcreditare le Nazioni Americane fino a mettere in dubbio la loro razionalità. Bifogna dunque credere ciò, che dicono gli Storici di qualle Ruore, e conferfare, che i Meflicani non erano immerfi in quella profonda ignoranzd, che finge il Sig. de P. Quanto poi a ciò, ch' egli dice della fcarfezza di voci numerali nella lingua mefficana, dimoftreremo altrove il fuo errore, e la fua ignoranza.

Non pud faperfa, ripiglia il Sig. de P. il contenuto delle pitture de Mefficani; perchè gli Spagnuoli non potevano intenderle fenza che foffero loro efpofte da' Mefficani, o niuno $d{ }^{2}$ E e 2

que-

(o) ", Je vous avoue, que le confentement de tous les Hiftoriens Efpagnols 2, ne permet gueres de douter, que ces deux peuples Americains ( $i$ Meffaca" ni, ed $i$ Peruani) n'eufent dans la fumme immenfe de leurs fupertitions "groflieres, de quelques u「ages quı ne differoient pas beaucoup de ce qu' on "nomme la Communion parmi nous "Recb. Pbilof. tom. 2. lettre Io a Mr"* sur la Religion des Americains. 
quefit ba Sapito finora quello che bafta per tradurre un libro? O quanti fpropofiti in si poche parole! Acciocchè gli Spagnuo. li poteffero intendere le pitture Mefficane non bifognava, che i Mefficani fapeffero la lingua fpagnuold; poichè baltava, che gli Spagnuoli capiffero la mefficana: nè per efporre una pittura richiedef tanto, quanto vi vuole per tradurre un libro. Il Sig. de $P$. dice, che a cagione della rozzezza della lingua mefficana non v'è Pato finora uno Spagnuolo, che poffa pronunciarla, e che per cagione dell' incapacità de' Mefficani, neffuno di loro ha imparato finora la lingua fpagnuola; ma l'uno, e l'altro è affai lontano dal vero. Della lingua mefficana ragioneremo a fuo luogo. La Caftigliana ed Atata fempre mai comuniffima preflo i Mefficani, e vi fono moltifimi che la parlano così bene, come gli fteff Spagnuoli. Molri di loro fcriffero in caftigliano la loro ftoria antica, e quella ancora della conquifta di Meffico, alcuni de' quali fono tati da me lodati nel Catalogo degli Scrittori, che ho premeffo alla mia Storia. Altri poi traduffero dé libri latini in caftigliano, de"caftigliani in mefficano, e de"mefo frani in caftigliano: tra $\mathrm{i}$ quali fon degni di particolar menzio. ne D. Ferdinando d'Alba Ixtlilxochitl, tante volte da noi cirato, D. Antonio Valeriano d'Azcapozalco, Maeftro in lingua mefficana dello Storico Torquemada, e da lui con gran lodi celebrato, D. Giovanni Berardo da. Huexotzinco, D. Francefco Battifta Contreras da Quauhnahuac, Ferdinando Ribas, e Stefano Bravo da Tezcuco, Pietro de Gance, Didaco Adriano,e Agoftino de la Fuente da Tlatelolco. ( $p$ ) Sappiamo dalla Storia della Conquifta, che la celebre Indiana Donna Marina imparò con fomma preftezza e facilita la lingua caftigliana, e che parlava affai bene la Meflicana, e la Maya pil fra loro diverfe, che la Francefe, l'Ebraica, e l'Illirica. Effendo dunque fati in tutti i tempi moltiffrmi gli Spagnuoli, che hanno imparato il mefficano, $_{2}$ ficcome poi dimoftreremo, e moltiffimi ancora i Mefficani,

(p) Intorno a ciò che diciamo di quegl Indiani traduttori poftono confultarf Torquemada nella Monarcbia Indiana, Pinelo nell' epitome della Biblioteca Ocm cidentalie, il Dotr. Eguiara nella Biblioteca Mefjcana, e Betaquru nel Teatre atedicano. 
ni, che hanno imparato lo fpagnuolo, perchè non avranno po: tuto $\mathrm{i}$ Mefficani inftruir gli Spagnuoli nella fignificazione delle lor pitture?

Quanto alle copie delle pitture mefficane pubblicate dal Purchàs, e dal Tevenot, è vero, che in effe non fi vedono offervate le proporzioni, nè le leggi della Profpettiva; ma effendo ftate quelle groffolane copie intagliate in legno, pud effere, che quegli Autori accrefceffero i difetti degli originali: nè dobbiamo maravigliarci, fe eglino forfe tralafciarono qualche cofa appartenente alla perfezione di quelle pitture, mentre fappiamo che ommifero affat to le copie delle pitture 2 I., e 22. di quella Raccolta, e le immagini delle Città nella maggior parte delle altre, ed oltracciò cambiarono le figure degli anni corrifpondenti a' Regni d' Ahuizotl, e di Motezuma II., ficcome abbiam detto, laddove ragionammo delle diverfe raccolte di pitture mefficane nel tomo I. della Storia. Il Cav. Boturini; il quale vide in Meffico delle pitture originali di quegli annali, e di quella matricola de' tributi, che fi contengono nelle copie pubblicate dal Purchàs, e dal Tevenot , fi lamenta de' gran difetti trafcor fi in quefte edizioni. Infatti bafta paragonar le copie pubblicate in Meffico l'anno 1770 . da Monfig. Lorenzana con quelle pub. blicate in Londra dal Purchàs, $\mathrm{e}$ in Parigi dal Tevenot per conofcere il gran divario, che v'è tra le figure delle une, e delle altre. $\mathrm{Ma}$ io non $\mathrm{m}$ ' impegno in foftenere la perfezione delle pitture originali copiate dal Purchàs; anzi non dubito, che fiano ftate imperfette, come erano quafi tutte le pitture ftoriche, nelle quali contentandofi i Pittori dei contorni, e del colorito degli obbierti, non fr curavano delle proporzioni, nè del chiarofcuro, nè della profpertiva. Neppur era poffibile, che fi offervaffero quelle leggi dell' arte, attefo la ftraordinaria lo. ro preftezza nel far tali pitture, di cui fanno fede Cortès, e Bernal Diaz, teftimoni oculari. Ma veggiamo le confeguenze, che ne deduce il Sig. de P. Ecco i fuoi argomenti : I Mefficani non offervavano le leggi della profpettiva nelle lor pitture; dunque non potevano per mezzo d'effe perpetuar la me. moria dei loro avvenimenti: I Mefficani erano cattivi Pitto.

ri ; 
yi durque non poteano effere buoni Storici. Ma qualora fi vog id adoprare una logica di quefta fatta, dovrà ancora dirfi, che tutti quelli, che nello fcrivere non fanno fare un buon carattere, non poffono effere buoni Storigi; poichè quelln, che fono le lettere per li noltri Storici, erano le figure per gli Storici Mefficani: e come poffono fcriverfi buone Storie con un cattivo carattere, cosi poffono rapprefentarfi bene i fatti con pitture groffolane: bafta, che gli uni e gli altri Storici fi facciano intendere.

Ma quelto appunto è quello, che il Sig. de P. non fa trovare nelle copie del Purchàs. Egli protefta, che avendo confrontato in diverfe maniere le figure d'effe coll' interpretazione aggiuntavi, non potè mai fcoprire verun rapporto: che come s'interpretano d'otto Re di Meffico, così potrebbono interpretarfi d'otto concubine di Motezuma. Ma quefto fteffo potrebbe dire, fe gli foffe prefentato il libro Cbun-yum del Filofofo Confucio, fcritto in caracteri Chinefi, colla fua interpretazione a canto in lingua francefe. Egli confronterebbe in vari modi quei caratteri coll' interpretazione, e non vi fupponendo trovare alcun rapporto, potrebbe dire, che come interpretano quel libro delle nove condizioni, che debbe avere un buon Imperatore, così potrebbono interpretarlo di nove concubine, ovvero di nove Eunuchi di qualche Imperatore antico; poichè quafi tanto s'intende egli di caratteri Chinef, quanto di figure mefficane. $\mathrm{Se}$ io poteffi abboccarmi col Sig. de $\mathrm{PaW}$, gli farei vedere il rapporto, che hanno quelle figure colla loro interpretazione; ma perchè lo ignora, dee tare al giudizio degl' intendenti.

Egli crede, ó vuol farci credere, che quelle fole pittu. re, la cui copia pubblicò il Purchàs, fieno fcampate dall'incendio, che ne fecero i primi Miffionari; ma queito è falfifimo, ficcome abbiam fatto vedere contro il Robertfon nel principio del tomo I. Le pitture fcampate da quell' incendio furono tante, che effe fomminittrarono 12 maggior parte dei mareriali per la Storia antica del Meffico non meno agli Scrittori Spagnuoli, che agli fteffi Mefficani: Tutte le opere di D. Ferdinando d'Alba Ixtlilxochitl, di D. Domenico Chimalpain, 


\section{IS SER T A Z IO NE VI.}

di D. Ferdinando Alvarado Tezozomoc, di D. Taddeo de Niza, di D. Gabrielle d'Ayala, e degli altri nominati nel Catalogo degli Scrittori meffo nel principio del noftro primo tomo, fono ftate fatte coll'ajuto d'un gran numero di pitture antiche. L'infaticabile Sahagun fi prevalfe di molutfime pitture per la fua Sioria della N. Spagna. Torqueinada cita fpeffo le pitture da lui confultate per la fuz opera. Siguenza ereditd i MM. SS. e le pitture d' Ixtlilxochitl, e fe ne procacciò molte altre a grandi fpefe, e dopo efferfene fervito, le lafciò in morendo infieme colla fua preziofa libreria al Collegio di $S$. Pietro, e S. Paolo dei Gefuiti di Meffico, nella cui librerìa io vidi e ftudiai alcune delle dette pitture. Nei due fecoli paffati fi prefentavano fovente dagl' Indiani nei tribunali del Meffio co delle pitture antiche, come titoli di proprietà, o di poffeffone d'alcune terre, e però $v$ 'erano degl' Interpreti inftruiti nella fignificazione di tali pitture. Gonzalo d'Oviedo $f_{a}$ menzione di quell' ufo nei tribunali ai tempi di Monfig. Sebaftiano Ramirez di Fuenleal, Prefidente della Real Udienza di Mefo fico: e perchè importava affai l'intelligenza di sì farti ritoli per la decifione di parecchie liti, era già nell' Univerfità di Meffico un Profeffore incaricato d' infegnare la fcienza delle pitture, dei geroglıfici, e dei caratteri mefficani. Le molte pitture raccolte pochi anni fa dal Cav. Boturini, ed accennate nel Catalogo del fuo Mufeo ftampato in Madrid l'anno 1746., ficcome quelle ancora da noi altrove citate, dimoltrano, che non così poche, come penfano il Sig. de Paw, e il Dort. Ro. bertfon, ne fcamparono dall'incendio dei Miffionari .

Finalmente per confermar vieppiù quanto abbiamo fcritto nella noltra Storia, e per fare intendere al Sig. de P. la varietà delle pitture mefficane, accenneremo quì in riftretto ciò che ne lafciò fcritto il Dott. Eguiara $(q)$ nell' erudita prefazio.

ne

(q) Il Dott. Eguiara degno di perperuarfi nella noftra memoria per la fua indole amabiliffina per la fua impareggiabile modeftia, rer la fua gran letteratura, e per lo zelo con cui ftico fino alla fua morte in fervizio della fua patria, nacque in Meffico verfo la fine del fecolo paffato. Fu molti anni proe feffo- 
ne della fua Biblioteca mefficana. V'erano, dice, tra le pit: ture mefficane dei Lunari, appellati da loro Tonalamatl, nei quali pubblicavano $\mathrm{i}$ loro pronoftici intorno alle mutazioni del tempo. Una di quefte pitture adduffe il Dott. Siguenza nella Eua Ciclografia Mefecana, ficcome egli ne fa teltimonianza nell' opera intitolata Libra Aftronomica. Altre contenevano gli orofcopi dei bambini, nelle quali fi rapprefentavano $i$ loro no$\mathrm{mi}$, il giorno, e il fegno della lor nafcita, e la loro ventusa: di queta fatta di pitture fa menzione Girolamo Roman nella fua Repubblica del Mondo part. 2. tom. 2. Altre erano dogmatiche, contenenti il filtema della lor religione, altre fto. riche, altre geografiche ec. E' vero, foggiugne il lodato Autore, che quelle pitture, che fi facevano per l'ufo comune e familiare erano chiare e s'intendevano facilmente da chic heffia; ma quelle, che contenevano gli arcani della religione, erano piene di geroglifici, il cui fenfo non potevafi dal volgo comprendere. 'V'era pure una gran diverfità tra le pitture, tanto per riguardo agli Autori, quanto a ciò, che apparteneva al modo di farle, e al fine, e all' ufo d'effe. Quelle, che facevanfi per l'ornato dei palazzi, erano perfetce; ma in altre, che contenevano un fenfo arcano, $f i$ vedevano certi caratteri, e alcune figure moltruofe ed orribili. I Pittori erano molti; ma lo fcrivere dei caratteri, il comporre Annali, e il trattar di materie concernenti la religione, e la politica erano impieghi propri dei Sacerdoti. Fin quì il Dott. Eguiara. Sappia dunque, il Sig. $P_{0}$, che nelle pitcure mefficane al-

cune

feffore di Teologì in quella Univerfità, e vi fampo alcuni Trattati Teologici affai pregiati in un tomo in foglio. Fu Rettore, e finalmente Cancelliere della medefima Univerfita, e Dignità di quella Chiefa Metropolitana, amato fempre, e riverito da ogni forta di perfone per la fua immacolata vita, e per la rua dottrina. Dopo aver riculato il Vefcovado di Jucatan, a cui fudeftinato dal Re Catrolico per li fuoi rilevanti meriti, pubblico in Meflico un tomo in fog̣lio della Biblioteca Mefficana: per la qual opera, oltre all immenfa fatica nel raccogliere, nell'ordinate, e nel perfezionare $i$ materiali, fi fece venire a grandi frefe da Parigi una famperia copiofa, e ben fornita di caratieri Romani, Greci, ed Ebraici. La morte di lui accaduta nel i 7 Ka $_{3}$ non ci permife di veder termikata quell' upera, che avrebbe fatto un grande onose alla rua patria. 
cune erano mere immagini degli obbietti : v' erano ancora dei caratteri, non gì componenti parole, come i noftrali, ma fignificativi di cofe, come quelli degli Altron mi, e deglı Algebrifti. Alcune pitture erano deftinate a efprimere precifamente le cofe ovvero $i$ concetti, e per dirlo cosi, a fcrivere: e in quefte non fi curavano le proporzioni, nè la bellezza, perchè fi facevano in fretta, e col fine d'initruir la mente, non gia di piacere agli occhi: $\mathrm{Ma}$ in quelle, nelle quali fi cercava d'imitar la natura, e che fi efeguivano con quella lentezza, che richieggono le opere di quelta fatta, vi fi offervavano le pro. porzioni, le dittanze, le attitudini, e le regole dell'arte, benchè non con tutta quella perfezione, che ammiriamo nei buosi Pittori d'Europa. Del relto io vorrei, che il Sig. de P. mi moftraffe alcur Popolo rozzo o mezzo dirozzato dell' antico Continente, che abbia adoperato tanta induftria, e diligenza, quanta i Mefficani, ad eternar la memoria dei fuoi avvenimenti.

Il Dott. Robertfon, laddove ragiona della coltura dei Mef ficani nel libro 7. della fua Storia, efpone i progreffi, che fa l' induftria umana per arrivare all' invenzione delle lettere, colla combinazione delle quali poffa efprimere tutti i differenti fuoni della favella. Quefti fucceffivi progreffi fono, fecondo lui, dalla pittura attuale al femplice geroglifico, da quefto al fimbolo allegorico, quindi all' arbitrario carattere, e finalmente all' alfabero. Se qualcuno poi cerchi nella ftoria di lui di fapere fino a qual grado fieno giunti i Mefficani, non potrà certamente indovinario; perchè quel ragionatore ftorico ne parla con tal ambiguita, che talvolta feinbra, che gli crede appena giunti al fecondo grato, cioè a quello del fermplice geroglifico; e talvolta pare, che gli giudichi avanzati fino al quarto del carattere arbitrario. Ma checchè ne dica, egli è certo, che tutti $\mathrm{i}$ fudJetti moti di rapprefentare $\mathrm{i}$ concetti, fuorchè quello dell' alfabeto, erano in ufo preff, i Mefficani. I loro caratteri numerali, e quelli fignificativi della notte, del giorno, dell'anno, del fecolo, del cielo, della terra dell' acqua, della voce, del santo \&c. non erano forfe veri caratteri arbituari, o di Storia Anticadel MeJJico Tom. IV. F $f$ con: 
convenzions? Ecco dunque giun:i i Mellicani, fin dove fono arrivati dopo tanti fecoli di coltura i famofi Chinefi. Non v'è altro divario tra gli uni, e gli altri, fe non che $i$ caratteri Chinefi fonofi a tal ecceffo moltiplicati, che non bafta la vita d'un uomo per impararli.

Lo iteffo Dott. Robertfon lungi dal negare, come fa temerariamente il Sig. de Paw, le ruote fecolari de' Mefficani, confeffd il loro metodo nel computo de' tempi, e dice, che avendo coloro offervato, cha ne'diciotto mefi di giorni venti l'uno, non rimaneva completo il corfo del Sole, aggiunfero i cinque giorni nemontemi. "Quelto ftretto avvicinamento-alla " filofoñca accuratezza, foggiugne, moltra affai chiaro, che da" 2) Melficani erafi preftata quell' attenzione alle fpeculative ricer" che, alle quali gli uomini nel loro ruvido ftato non fono "mai foliti di rivolgere il lor penfiero.", (r) Che avrebbe egli detto, fa aveffe faputo, come noi fappiamo non meno per la graviffima teltimonianza del Dott. Siguenzz, che per le proprie noltre offervazioni fu la cronologia mefficana, che non folamente contavano i Mefficani giorni trecento feffantacinque nel loro anno, ma eziandio accortifi dell' ecceffo d'ore quafi fei dell' anno folare fopra il civile rimediarono a un tal divario per mezzo de' giorni tredici intercalari, che aggiugnevano al tor fecolo d'anni cinquantadue?

\section{V.}

Su le Arti dei Mefjicani.

Dopo aver fatto il Sig. de P. una ignominiola defcrizion del Perù, e della barbarie de' fuoi abitatori, parla del Meffico, del cui Stato, dice $(\rho) f_{i}$ fon raccontate tante faljita, $e$ maraviglie, quante del Perì; ma egli è certo, foggiugne, che quefte due Nazioni erano a un dipreffo uguali, ofi paragoni la

(r) Stor. dell' America lib. 7 .

(I) Recherch. Philofoph. part. 5. fect. I. 
loro polizia, of confiderino le lore arti, $e$ i loro frumenti: L' agricoltura era preffo loro abbandonata, e l'architettura me. fchina: le loro pitture erano groffolane, e le loro arti affai im. perfette: le loro fortificazioni, i loro palazzi, e i loro tempi fono mere finzioni degli Spagnuoli. "Se i Mefficani, dice, "aveffero avuto delle fortificazioni, $f$ farebbono meffi al co2) perto da'mofchetti, e que' fei mefchini cannoni di ferro, che 2) porto feco Cortès, non avrebbono rovinato in un momento " tanti baluardi, e trinciere... Le mura de' loro edifizi non 》) erano altro, che faffi grandi, meffi gli uni fopra gli altri . "Il vantato palazzo, dove dimoravano i Re di Meffico, era " una capanna: per lo che Ferdinando Cortès, non trovando "abitazione proporzionata in tutta la Capitale di quello. Sta"to, che avea di frefco conquiftato, fu coftretto a fabbricare "in fretta un palazzo, il quale finora fuflifte. "Non è facile di numerar g! i ' Propofiti del Sigo de P. in quelta materia: tralafciando però quelli, ehe appartengono al Perù, efaminia. mo quanto egli fcriffe contra le arti de' Mefficani .

Dulla loro agricoltura abbiamo ragionato in altri luoghi, laddove abbiam fatto vedere, che i Mefficani non folo coltivavano con fomma diligenza tutte le terre del lor Imperio, ma eziandio crearonfi con maravigliofa induftria nuovi terreni da coltivare, formando nell' acqua quegli orti, e que' campi galleggianti, che fono ftati con tante lodi celebrati dagli Spagnuoli, e dagli Stranieri, e che fino ad ora fono ammirati da quanti navigano in que' laghi. Abbiamo altresi dimoftrato, fus la depofizione di molti teltimoni oculari, che non folo le piante utili al foitentamento, al veftito, e alla falute, ma i fori ancora, ed altri vegetabili, che fervono unicamente alle delizie della vita, erano da loro diligentiffimamente coltivate. Ferdinando Cortès nelle fue lettere a Carlo V., e Bernal Diaz nella fua Storia parlano con iftupore de' famofi orti d'Iztapalapan, e di Huaxtepec da loro veduti, e ne $f_{a}$ anche menzio. ne nella fua Storia Naturale il Dott. Hernandez, il quale vide quegli orti quaranta anni dopo. Il medefimo Corrès in una fua lettera a Carlo V. de' 30 . Ottobre 1520. dice così : E'ca. F f 2 
si grande la moltitudine d' abitatori in quefi paefi, che non $v$ 'd neppure un palmo di terreno, che non fia coltivato. Bifogna effere troppo teflereccio par negar feda all' unanime teft". monianza degli Autori Spagnuoli.

Abbiamo fimilmente efpofto fu la fede di coftoro la gran diligenza de' Mefficani nell' allevare ogni forta d'animali: nel qual genere di magnificenza forpalsò Motezuma, come abbiam detto altrove, tutil i $\operatorname{Re}$ del Mondo. I Mefficani peraltro non poteano allevare una si ftupenda varietà di quadrupedi, di rettili, e d'uccelli, fenza avere una gran cognizione della lor natura, del loro iftinto, della lor maniera di vivere \&c.

La loro architertura non era paragonabile, con quella degli Europei, ma era cerramente di molto fuperiore a quella della maggior parte de'Popoli Afiatici, ed Affricani Chi oferà pareggiare alle cafe, a' palazzi, a' tempj, a'baluardi, agli acquidot:i, e alle frade degli antichi Mefficani non gia le miferabili capanne de' Tartari, de' Siberiani, degli Arabi, e di quelle trifte Nazioni, che vivono tra il Capo Verde, e quel: lo di Buona Speranza; ma neppur le fabbriche dell' Etiopia, d' una gran-parte dell'India, e dell' Ifole dell' Afia, e dell' A. frica, tranne quelle del Giappone? Bafta confrontare ciò, che hanno fcritto delle une, e delle altre, quegli Autori, che le videro, per ifmentire il Sigo de P., il quale ha avuto ardire di pubblicare, che turte le Nazioni americane erano inferiori in induftria e fagacità a' più groffolani Popoli dell' antico Continente .

Egli dice, che il vantato palazzo di Motezuma non era altro, che una capanna; ma Cortès, Bernal Diaz, e il Con: quiftatore Anonimo, i quali tante volte lo videro, affermano tutto l'oppofto. "Avea, dice Cortès ragionando del Re Mote: "zuma, in quefta Città ( di Meffico) delle cafe per fux abi"tazione tali, e tanto maravigliofe, che non crederei di po" ter mai efprimere l' eccellenza, e la grandezza: perlochè al"tro non dirò, fe non che non ve ne fono uguali in Ifpagna." Cosi Icrive quefto Conquiltatore al fuo Re fenza paura d'effere fmentito da'fuoi Capitani, e Soldati, i quali aveano anche 
effi fotto i lor occhi i palagi Mefficani. Il Conquiftatore Anonimo nella fua curiofa, e fincera Relazione, ragionando degli edifizi di Melfico, dice così: "V'erano belle cafe di Signori ") tanto grandi, e con tanti appartamenti e giardini alti e baf" fi, che ci rendevano attoniti per l'ammirazione. Io entrai " per curiofita quattro volte in un palazzo di Motezuma, ed "avendo girato per effo fino a ftancarmi', nol vidi mai tutto. "Ufavano avere d' intorno ad un gran cortile camere e fale "grandiffime; tna fopra tutto una ve n' era così grande, che " dentro di effa vi poteano ftare fenza incomodo più di tre " mila perfone: era tale, che nel corridojo, che v'era fopra, " fi formava una piazzetta, nella quale trenta uomini a ca"vallo aurebbono potuto giocare alle canne. "Simili efpreffioni fr leggono nella Storia di Bernal Diaz. Confta per la depofizione di tutti gli Storici del Meffico, che l'efercito di Cortès, compofto di fei mila, e più di quattrocento tra Spagnuoli, Tlafcallefi, e Cempoallefi, s'allogiò tutto nel palazzo, che era ftato del $\operatorname{Re}$ Axacajatl, e ve ne avanzd ancora per l'abitazione del $\operatorname{Re}$ Motezuma, e de' fuoi familiari, oltre a' magazzini, ne' quali fi guardava il teforo del Re Axajacatl. Confta per la depofizione de' medefimi Storici della magnificenza, e bellifima difpofizione del palazzo degli uccelli, e Cortès aggiunge, che negli appartamenti, che v' erano poteano a!bergarfi agiatamente due gran Principi con tutta la loro Corte, e defcrive minutamente i fuoi portici, le loggie, e i giardini . Lo fteffo Cortès dice a Carlo V., che nel palazzo del Re Nezahualpilli in Tezcuco alloggiò con feicento Spagnuoli, e quaranta cavalli, e che era tanco grande, che ve ne potrebbono ancora ftare agiatamente altri feicento. Similmente parla del palazzo del Signor d'Iztapalapan, e d'altre Cirtà, lodandone la Aruttura, la bellezza, e la magnificenza. Tali erano le capanne de'Re, e de' Signori Mefficani.

Il dire, che $\mathrm{f}_{2}$ il Sig. de P., che Cortès fecevi coftruire in fretta quel palazzo, perchè non trovava abitazione proporzionata in tutta quella Capitale, è un errore, o per dir meglio, e parlar con maggior proprietà, è una gran bugìa. E' $\begin{gathered}\text { vero, } \\ \text {. }\end{gathered}$ 
vero, che Cortès durante l'affedio di M ffico, bruciò, e ro. vinò la maggior parte di quella gran Gittà : come egli medefimo ne fa fede, e a tal fine dimandò, ed ottenne da' Cuoí Alleati alcune migliaja di Contadini, che non aveano altro impiego, che quello d'andar rovinando gli edifzi fecondochè gli Spagnuoli vi fi avanzavano, acciocchè non reftaffe alle loro fpalle veruna ca. $\mathrm{fa}$, da cui poteffero danneggiarli i Mefficani. Non farebbe dun. que da maravigliare, fe Cortès non aveffe trovato alcuna abitazione proporzionata in una Citta, che egli medefimo avea diftrutta; ma non fa la rovina tanto generale, che non reltaffe un gran numero di buone cafe nel quartiere di Tlatelolco, nelle quali avrebbono potuto comodamente alloggiarfi tutti gli Spagnuoli con buon numero dei loro Alleati. Dappoicbe piacque a Nofro Signore, dice Cortes nella fua ultima lettera a Car. lo V., cbe quefta gran Città di Temixtitan folfe conquiftata, non mi parve bene di rifedere in effa per cagione di: molti inconvenienti: ficcbè me ne andai con tutta la mia. Gente a faré in Cuyoacan. Se foffe vero ciò, che dice il Sig. de $\mathrm{PaW}$, baftava dire, che non reftò in Meffico, perchè non v' erano cafe dove ftare. Il palazzo di Cortès fi fabbricò nel medefimo firo dove era già quello di Motezuma. Se Cortès non aveffe rovinato quefto palazzo, avrebbe potuto abitar comodamente in effo, come vi abitava quel Monarca con tutta la fua Corte. E. poi fallo, che fuffitta prefentemente il palazzo fabbrica a to da Cortès; poichè quefto fu bruciato nel 1692 . in una fe. dizione popolare. Ma foprattutto è falfffimo, che le mura degli edifizi mefficani non foffero altro, che faffi grandi polti gli uni fopra gli altri fenza veruna unione, ficcome fi convince: per la teftimonianza di tutti gli Storici, e per gli avanzi degli edifizi antichi, di cui a fuo luogo ragioneremo. Sicchè non evvi in turto il paffo gia citato del Sig. de P. neppur una propofizione, che non fia un: errore.

Non contento il Sig. de P. d'annichilar le cafe dei Mefficani, fi mette anche a combattere i loro tempi, e fdegnato contra il Solis, perchè afferma, che i tempi di Meffica erano mon meno di due mila tra grandi, e piccoli, dice così ${ }_{22}$. Non 22. è $\operatorname{ltan}_{2}$ 
" è ftato mai un sì gran numero d' edifizi pubblici in alcuna "Città da Roma fino a Pekin: perlochè Gomara men teme"rario, o più favio del Solis, dice, che computando fette " cappelline, non vi fi trovarono più d'otto luoghi deftinati "a riporvi gl'Idoli di Meffici., "(s) Acciocchè fi veda, quan. ta fia l'infedeltà del Sig. de P. nel citar gli Autori, voglio dar quì il paffo di Gomara, allegato da lui: "Vi erano, dice " quetto Autore nel cap. 80. della fua Cronaca della N. Spa" gna, molti tempj nella Città di Meffico fparfi per le Parroc. " chie o contrade colle loro torri, nelle quali erano le cap" pelle, e gli altari da riporvi gl'Idoli ... Quafi tutti aveano " una fteffa forma: ficchè quello, che diremo del tempio prin" cipale, balterà per dare a conofcere turti gli altri," e dopo aver fatta una minuta defcrizione di quel gran tempio, nella quale vanta la fua aliezza, la fua ampiezza, e la fua bellezza, foggiunge:"Oltre a quefte torri, che formavanfi colle lo" ro cappelle fopra la piramide, v' erano altre quaranta e più "torri tra piccole e grandi in alcri Teocalli minori, $(u)$ che " v'erano dentro il recinto di quel tempio principale, i quali "tutti erano della medefima forma di quello... Altri Teocal"Ii o Cues v'erano in altri luoghi della Citta... Tutti que. "Iti tempj aveano le loro cafe proprie, i loro Sacerdoti, e i "L loro Dei, con tutto il bifognevole al loro culto e fervi"zio." Sicchè quel medefimo Gomara, che al dir del Sig. de P., non numera in Mefflico più d'otto luoghi deftinati a riporvi gl'idoli, comprefevi fette cappelline, annovera chiaramente più di quaranta tempj dentro il recinto del tempio principale, oltre a molti altri fparfi per le Parrocchie, o Contrade. Chı potrà mai fidarfi del Sig. de $P$. dopo una sì manifefta faluficazione?

\section{$E^{\prime}$}

(t) Recherch. Philof. part. 5. rect. I.

(u) Tecalli, cioè Cafa di Dio, era il nome, che davano i Mefficani a' loro temri Tra gli Spagnuoli alcuni gli appellarono Tempi, altri Adoratorj, altri Mefcbite, come quelli, che erano avvezzi al linguaggio de'Saracini, ed

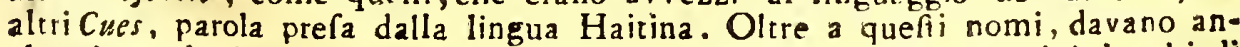
che a'tempi piccoli quelli di Sacrificaderos, e d'Humilladeros, cioè luoghi di facrifizi, e di adorazione. 
E'vero, che Solis fi moftò poco avveduto nel mettey come certo quel numero di tempj, che $\mathrm{i}$ primi Storici efpreffero foltanto per congettura; ma il Sig. de $\mathbb{P}$. fi dà ancora a divedere poco accorto nel comprendere tra gli edifizi pubblici anche quelle cappelline, che gli Sngnuoli appellarono Tempj. Di quelti ve n'erano innumerabili: Tutti coloro, che videro quei paefe prima della Conquifta, teftificano concordemente, che tanto nei luoghi abitati, quanto nelle Atrade, e nelle montagne fi vedevano dappertutto si fatti edifizi, i quali, benchè piccoli, ed affatto diverfi dalle noftre Chiefe, furono pure chi:mati tempi, perchè erano confacrati al culto degl'Idoli . Cosi dalle lettere di Cortès, come dalla Storia di Bernal Diaz fappiamo, che appena faceano un paffo $i$ Conquiftatori nelle loro fpedizioni, che non fi abbatteffero in alcun tempio, o cappella. Cortès dice d'avere egli numerati più di quattrocento tempj nella fola Citta di Cholulla. Ma v'era un gran divario quanto alla grandezza tra gli uni, e gli altri tempi. Alcuni non erano altro, che piccoli terrapieni poco alti, fopra i qua. li v'era una cappellina per l'idolo titolare. Altri poi erano d'una grandezza ed ampiezza ftupenda. Cortès, laddove parla del tempio maggior di Meffico, protelta a Carlo V., che non è facile defcrivere le fue parti, la fua grandezza, e le cofe, che vi fi contenevano, che effo era tanto grande, che dentro il recinto di quella forte muraglia, che il circondáva, vi potea capire un borgo di cafe cinquecento. Non parlano altrimenti di quefto, e di altri tempi di Mcflico, di Tezcuco, di Cholulla, e di altre Città, Bernal Diaz, il Conquittatore Anonimo, Sahagun, e Tobar, che gli videro, e gli Storici Mefficani, e Spagnuoli, che fcriffero dopo, a fe ne informarono bene, come fono Acolta, Gomara, Herrera, Torquemada, Siguenza, Betancurt ec. ec. Hernandez defcriffe ad una ad una le fettantotto parti, di cui fi componeva il tempio maggiore. Cortès foggiugne, che tra le alte torri dei tempj che abbellivano quella gran Capitale, ve n' erano quaranta così elevate, che la minor d'effe non era inferiore in altezza alla famola 
Giralda $\left(^{*}\right)$ di Siviglia. D. Ferdinando de Alba Ixtlilxuchitl fa menzione nei fuoi MM. SS. di quella torre di nove piani, che il fuo celebre arcavolo Nezahualcojotl edificò al Creator del Cielo, il quale fembra effere tato quel famofo tempio di Tezcutzinco, che con tante lodi innalza il Valadès nella fua Rettorica Criftiana.

Tutta quefta nube di teftimoni depone contro il Sig. de P. Contutrociò egli non vuol credere qualla gran moltitudine di tempj in Meflico; perchè Motezuma 1. fu, dice, quegli, che diede a quel villaggio la forma di Citta: dal regno di quefto Monarca fino all arrivo degli Spagnuoli non erano forfi piì di quarantadue anni: il quale $\int p a z i o$ di tempo non bafic certamente per fabbricare due mila tempi. Ecco tre afferzioni, che fono altreitanti errori. I. 'E' fallo che Motezuma I. deffe a Meffico la forma di Città; poichè fappiamo dalla ftoria, che quella Corte avea forma di Cirta infin da' tempi del primo Re A. camapitzin. 2. E' falfo altresi, che dal regno di Motezuma I. fino all' arrivo degli Spagnuoli non trafcorfero più di quaranta due anni. Motezuma cominciò a regnare, fecondochè abbiam fatto vedere nella feconda Differtazione, l'anno 1436 ., e fini di vivere il 1564., e gli Spagnuoli non giunfero a Meffico prima del i519.: dunque dal cominciamento di quel regno fino all'arrivo degli Spagnuoli trafcorfero anni ottantatre, e dalla morte di qual $\operatorname{Re}$ anni cinquantacinque. 3. Il Sig. de PaW fi moltra affdto ignorante della ftruttura de' tempi mefficani, nè fa, quanto grande fofle la moltitudine d'operai, che concorreva alla fabbrica degli edifizi pubblici, e quanta la preftezza lo. ro nel fabbricarli. Si è veduto talora nella N. Spagna fábbricare in una fola notte un villaggio intero (benchè compotto di capanne di legno coperte di fieno) e condurvi i nuovi coJoni le loro famiglie, i loro animali, e tutta la lor roba.$(x)$ Per ciò poi, che riguarda lé fortificazioni, egli è certo, e Storia Antica del Meffico Tom. IV. G g indu-

(*) Campanile alcifimo, e rinomato del Duomo di Siviglia.

(x) Vedafi ciò, che-narra il Torquemada nel lib. 3. cap. 33. della Monara sbia Indiana. 


\section{D IS S E R T A Z I O N E VI.}

indubitabile per la depofizione di Cortès, e di tutti coloro, che videro le antiche Citta di quell' Imperio, $(y)$ che i Meflicani, e tutte le altre Nazioni viventi in focietà, ufavano muraglie, baluardi, fteccati, foff, e trinciere. Ma ancorchè niuno di tanti teftimoni oculari ne faceffe fede, balterebbono le fortificazio-

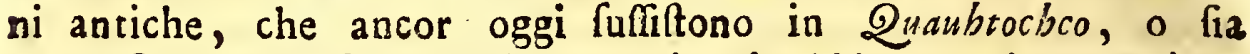
Guatufco, e preffo a Molcaxac, di cui abbiam ragionato altrove, a dimoftrar l'errore del Sig. de Paw. E' vero, che tali fortificazioni non erano paragonabili con quelle deli' Europa; perchè nè la loro architettura militare s' era tanto perfezionata, nè eglino aveano d'uopo di metterfi al coperto dell' artiglieria della quale non aveano veruna contezza; ma diedero a divedere abbaltanza la loro indultria nell' inventar tante forte di ripari per difenderfi da' lor ordinari nemici. Chiunque peraltro legga l' unanime depofizione de' Conquiltatori, non dubiterà del grande loro ftento nell' efpugnare i foffi e le trinciere de' Mefficani nell'affedio della Capitale, contuttochè aveffero un sì ecceffivo numero di truppe alleate, ed $i$ vantaggi delle armi da fuoco, e de'brigantini. La terribile fconfitca, che ebbero gli Spagnuoli, allorchè fi vollero ritirar da Meffico, non permetterà mai, che fi dubiti delle fortificazioni di quella Capitale. Elfa non era circundata da muraglie, perchè la fua fieuazione la rendeva abbaftanza ficura mercè que' foffi, che erano nelle tre Arade, per dove poteano affalirla i nemici; ma altre Città, che non erano pofte in una sì vantaggiofa fituazione, aveano muraglie, ed altri ripari per la loro difefa. 11 medefimo Cortès fa una efatta defcrizione delle muraglie di Quauhquechollan.

Ma perchè perdere il tempo nell' accumulare teftimonian. ze, ed altre prove deli' architettura de' Meflicani, mentre coftoro ci hanno lafciato nelle tre famofe ftrade, che coltruffero den-

tro

(y) Delle antiche fortificazioni fanno fpeffillimo menzione Cortes nelle fue lettere a Carlo V. Pietro Alvarado, e Didaco Godoy nelle lor lettere a Ferdinando Cortès, Bernal Diaz nella fua Storia, il Conquiftatore Anonimo nella fua Relazione. Alfonfo de'Ojeda nelle fue Memorie, e Sahagun nella fua Storia, tutti teftimoni oculari. 
tro lo fteffo lago, e nell'antichiffimo acquidotto di Chapolte: pec un monumento immortale della loro induftria?

Quagli Ateffi Autori, che fanno fede dell' architettura de' Mefficani, teltificano pure l' eccellenza de' lor Orefici, de loro Teffitori, de'loro Intagliatori di gemme, e de' loro lavoratori d'opere di piuma. Furono molti gli Europei, che videro sì fatti lavori, e fi maravigliarono dell' abilità degli artefici americani. I loro lavori di getto furono ammirati dagli Orefici di Europa, fecondochè affermano parecchj Autori Europei allor viventi, e tra gli altri lo Storico Gomara, il qual ebbe quelle opere nelle fue mani, e fentì il parere degli Orefici Sivigliani, che non fi credevano capaci d'imitarle . (z) E dove trovar mai, chi fia capace di far que'lavori maravigliofi da noi accennati nel lib. VII. \$1. della noftra Storia, e da moltiffrmi Scritiori concordemente teftificati, ficcome quello per efempio di far. di getto un pefce, che abbia le fquame alternatamente l'una d'oro, e l'altra d'argento? Corrès dice nella feconda fua lettera a Carlo V. che le immagini d'oro, e di piuma erano cosi ben lavorate da' Mefficani, che niun Artefice d'Europa potrebbe farle migliori: che quanto alle gioje non fi potea comprendere, con quali Arumenti fatte foffero opere tanto perfette: e che $i$ lavori di penne erano tali, che nè in cera, nè in feta fi potevano imitare. Nella fua terza lettera al medefimo Carlo V. laddove parla del bottino di Meffico, gli dice, che tra le fpoglie de' Mefficani vi trovò certe rotelle d'oro, e di penne, ed alıri lavori della fteffa materia così maravigliofi, che non effendogli poffibile di darne una giufta idea per ifcritto, gli manda a Sua Maeftà, acciocchè co' fuoi propri occhi poffa renderfi ficura della loro eccellenza e perfezione. Io fon cerro, che Cortès non avrebbe parlato cosł al fuo Re di que'lavori, che gli mandava, affinchè co' fuoi occhj gli vedeffe, fe non foffero tali, quali egli gli rapprefentava. Quafi negli feffi termini, che Cortès ne parlano tutti quegli Autori, che videro si fatte opere come Bernal Diaz, il Conquiftatore Anonimo, Gomara, Hernan-

$$
\text { G g } 2
$$

(z) Cronaca della N. Spagna cap. 39. e 79. 


\section{$23^{6}$ DISSERTAZIONE VI.}

dez, Acofta, ed altri da' quali abbiamo prefo tutto ciò, che intorno a quefto argomento abbiamo fcritro nella Storia.

Il Dott. Robertfon $(A)$ riconofce bensì l' unatime depolizione degli antichi Storici Spagnuoli, e crede, che efsi non ebbero veruna intenzione d'ingannarci; ma afferma, che tutti furono indotti ad efagerare dall' illufione della lor mente, cagiona. ta dal calore della loro immaginazione. Esco una bella foluzione, della quale ognuno potrebbefi prevalere per negar fede a tutte le Storie umane. Tutti dunque s' ingannarono, fenza eccettuare neppure il chiariflimo Acofta, nè il dotto Hernandez, nè gli Orefici di Siviglia, nè il Re Filippo II., nè il Sommo Pontefice Sifto V., amnaratori tutti, e lodatori di que' lavori mefficani? (B) Tutti ebbero l'immaginazione rifcaldata, ancor quelli, che fcriffero alcuni anni dopo la fcoperta del Meffico? St, tutti, foltanto lo Scozzefe Robertion, e il Pruffiano Paw hanno, dopo due fecoli e mezzo, quel temperamento nella fantafia, che fi richiede per formare un' idea giufta delle cofe, forfe perchè il freddo de'loro paef́ aṽì rallentato il calor della loro immaginazione. "Non fi dee però decidere, foggiunge il Robertfon, " del grado del loro merito ( de'lavori mefficani) da quefte medefime defcrizioni; ma bensì confiderando i faggi delle loro "arti, tali guali fi vedono preferwati ancora... Molti de' loro " ornamenti d'oro, e d'argento, come pure diverfi attrezzi " impiegati nella vita comune, fono depofitari nel magnifica " gabinetto di cofe naturali e artificiali, aperto ultimamente " dal Re Cactolico: e perfone al giudizio, ed al gufto dielle 9) quali io poffo fidarmi, mi hanno afficurato, che quefti van" tati sforzi di loro arte, fono goffe rapprefentazioni d'oggetti " comuni, o immagini di forme umane, o d'alcuni animali, " prive di grazia, e di proprietà, „E nella noła dice così: " Nell'armeria del palazzo reale di Madrid fi moftrano delle " Ferie d'armi, che fi dicono di Motezuma. Sono compofte di " fottili laftre di rame tirato a pulimento. Nell' opinione di "giu-

(A) Storia dell' Anerica lib. 7 .

(B) Cid rileggafi, che abbiamo fcritto nel lib. VII. \$.g Iodella noftra Storia. 
" giudici intendenti fono manifeftamente orientali. Le forme degli ornamenti d'argento, che vi fi vedono fopra, rapprefentanti dragoni, fi poffono confiderare, come una conferma dell'opinione medefima. In genere di fattura fono infinita. mente fuperiori a qualunque altro sforzo dell' arte americana... Il folo indubitabile faggio, cha io abbia veduto dell' arte meflicana nella Gran Bretagna, e una coppa d'oro finiffimo, che fi foltiene apparteneffe a Motezuma... E' rapprefentata in quelta coppa la faccia d' un uomo. Da una parte il vifo piano, dall' altra il profilo, e dalla terza il didie. " tro della tefta... Le fattezze fono rozze, ma tollerabili, e " certamente ruvide troppo per fupporla fattura Spagnuola . "Quefta coppa fu comprata da Odoardo Conte di Orford, "quando era nel porto di Cadice. "Fin qui il Robertion ai cui argomenti noi rifpondiamo I. Che non havvi ragione di credere, che quei rozzi lavori fieno veramente mefficani. 2. Che nemmeno fappiamo, fe quelle perfone, al cui giudizio credette di doverfi fidare il Robertfon, fieno ftate tali da meritar la noftra fede; poichè abbiamo offervato, che il Robertfon li fida fpeffo alla teftimonianza del Gages, del Corral, dell' Ibagnez, e d'altri si fatti Autori, affatto indegni d'effer creduti. Potrebbe effere ancora, che quelle perfone, che giudicarono di tali lavori, aveffero l'immaginazione rifcaldata; poichè è più facile affai, fecondo la condizione della noAtra natura guafta, di rifcaldarfi l'immaginazione contra una Nazione, che in 'favor d'effa 3 . Che è affai più probabile, che quelle armi di rame credute da giudici intelligenti manifeftamente orientali, fieno veramente mefficane; perchè fiamo ficuri per la teftimonianza di turti gli Scrittori del Meffico, che quelle Nazioni ufavano si fatte laftre di rame nella guerra, e che con effe procuravano di coprirfi il petto, le braccia, e le cofcie per difenderfi dalle frecce, laddove non fappiamo, che effe fieno ftate mai in ufo appreffo gli abitatori delle Ifole Fulippine, $(C)$ o appreffo verun altro Popo-

(C) Il Dott. Roberton dice, che li Spagnuoli ebbero probabilmente quelle armi dalle Irole Filippine. 


\section{DISSERT AZION E VI.}

1o, che con effi commerciafle. I dragoni rapprefentati in quelle armi in vece di confermare, come crede il Robertfon, l'opinione di coloro, che le credono orientali, confermano piuttofto la noftra opinione, poichè non vi fu mai veruna Nazione al Mondo, preffo la quale fieno ftate tanto in ufo nelle fue armi le immagini d'animali terribili, quanto preffo $i$ Mefficani. Nè dee recar maraviglia, che coftoro aveffero idea dei dragoni, mentre ebbero pure quella dei grifoni, ficcome ne $f_{a}$ fede il Gomara. (D) 5. Che quantunque fieno goffe le immagini formate nei lavori d'oro, e d'argento, quefti potrebbono effer peraltro eccellenti, maravigliofi, e inimitabili ; perchè in quei lavori debbono confiderarfi due arti affatto diftinte, e non conneffe, quella del difegno, e quella del getto: ficchè potrebbe quel pefce, del quale abbiamo fopra favellato, effere mal formato quanto alla figura, e nulladimeno effere maravigliofa e forprendente quell'alternazione di fquame d'oro e di argento, fatta di getto. 6. Finalmente il giudizio d'alcune perfone affatro incognite fopra quei pochi lavori dubbiofi, che fono nel Real gabinetto di Madrid, non può prevalere all' unanime depofizione di tutti gli Scrittori antichi, i quali videro innumerabili lavori certamente meflicani.

Da tutto cid, che finora abbiamo efpofto, fi rende manifefto il gran torto, che ha fatto il Sig. de Paw ai Mefficani, credendogli inferiori in induftria è fagacità ai più rozzi Popoli dell' antico Continente. If P. Acofta, laddove ragiona della induftria dei Peruani, dice così :"Se quefti uomini fono beftie, "giudichilo chi voglia; poichè io fon ficuro, che in quello, a ") che effi fi applicano, ci fuperano d'affai., (E) Quelta ingenua confeffione d' un Europeo di tanta Critica, di tanta pratica, e di tanta imparzialità non vale affai più di tutte le invettive d'un Filofofo Pruffiano, di tutti i ragionamenti d'uno Storico Scozzefe, l' uno e l'altro o male inftruito delle cofe dell'

(D) Alcuni Sigmori awevano nelle loro arme sn griffone volante e portante fra. gli artigli un iervo Cronaca della N. Spagna cap. zx $^{\mathrm{x}}$

(E) Stor. Nate e Moral. lib. 6. cap. 8. 
dell' America, o prevenuto contra gli Americani? Ma ancorchè concedeffimo al Sig. de PaW, che l'induftria degli Americani nelle arti fia inferiore a quella degli altri Popoli del Mondo, nulla quindi dovrebbe conchiuderfi contro le anime degli Americani, o contro il clima dell'America; mentre è certo e indubitabile, che le invenzioni, ed i progrefit delle arti nella maggior parte fono piuttofto dovute alla forte, alla neceffirà, e all' avarizia, che all' ingegno. Gli uomini più in. duftriofi nelle arti non fempre fono i più ingegnofi, ma fovente i più bifognofi, o i più portati per l'oro. "La fterilità del"la terra, dice bene il Montefquieu, $(F)$ fa gli uomini indu"Atriofi... bifogna, che eglino fi procaccino ciò, che lor non " tributa la terra. La ferrilità d'un paefe porta feco infieme " colla facilità di Coftentarfi la defidia "*, La neceffità, dice il "Robertfon, è lo ftimolo, e la guida del genere umano pér "le invenzioni. "I Chinefi non farebbono certamente tanto indultriofi, fe l'ecceffiva popolazione del loro paefe non rendeffe loro difficile il proprio foltentamento: nè in Europa fi farebbono fatti tanti progreffi nelle arti, fe vi foffe mancato l'allettamento dei premi, o la fperanza negli artigiani di migliorare la loro fortuna. Nulladimeno i Mefficani poffono van. tare molte loro invenzioni capaci d'immortalare il lor nome, quali fono, oltre a quelle delle famofe lor opere di getto, e dei mufaici di penne e di conchiglie, quella della Carta; $(G)$ quella di tignere di colori indelebili, di filare, e di teffere il pelo più fo:tile dei Conigli, e delle lepri : quella di fare i rafoi d'Itztla: $(H)$ quella d'allevare si induftriofamente la Cocciniglia per fervirfene nei colori : quella dello fmalto nei pavimenti delle lor cafe, e mille altre non meno pregevoli, che poffo-

(F) L'Erprit des Loix lib. 18. cap. 4.

(G) Vedafi cid che diciamo nel lib 7. della Storia delle diverfe forti dicarta Mefficana, cioè di cotone, di maguei, di palma montana, e di feta. L'invenzione della carta è fenz' altro più aniica in America, che in Egito, donde fi comunicò all' Europa. E'vero, che la carta de'Mefficani non era paragonabile nella finezza con quella degli Europei; ma fi dee avveltire, che coloro non la faceano per ifcrivere, ma per dipignere.

(H) Vedafi cio, che diciamo nel lib.7.9.56.della Storia intorno a quell' arre. 
poffono vederfi nella noftra Storia, e nelle opere degli altri Sto: rici del Meffico, ficcome le arti dei Peruani nelle opere dell' Acolta, e dell' Inca Garcilaffo, e nelle Lettere Americane del ch. Sig: C. Carli. Ma che maraviglia, che tali invenzioni fi trovaffero preffo quelle Nazioni civilizzate, mentre anche preffo altri Popoli Americani men dirozzati furono trovate delle arti fingolariffime? Che invenzione per efempio più fingolare e maravigliofa, che quella d'addimelticare i pefci marini, e fervirfene per dar la caccia ad altri pefci grandi, come faceano gli abitatori delle Ifole Antille? Quefta fola arte, di cui fanno menzione Oviedo, ( $I$ ) Gomara, ed altri Autori non bafterebbe a fmentire l'ingiuriofe invertive del Sig. de Paw contra l'induftria degli Americani?

\section{VI.}

\section{Su la lingua Me Jacana.}

"Le lingue dell' America, dice il Sig. de P., fono tanto 3) riftrette, e cosi farfe di parole, che non è poffibile efpri$\Rightarrow$ mere in effe verun concerto metafifico. Non vi è niuna di "quefte lingue nella quale $f i$ pofja numerare olite a tre. $(L)$ "Non è poffibile tradurre un libro, non già nelle lingue de"gli Algonquini, e dei Guaranì o Paraguajefi, ma neppure in " quelle del Meffico, o del Perù per cagione di non aver effe " una copia fufficiente di termini propri per enunciare le no"zioni generali." Chiunque legga quefte decifioni magiftrali del Sig. de P., fi perfuaderà fenza dubbio, che egli decide co-

(I) Oviedo Stor. Gener. e Natur. delle Indie lib. 13.cap. ro. e Sommariodella stor. delle Indie cap. 8. Gomara Storia Generale delle Indie cap. 20. La fpezie di pefce, di cui fi prevalevano gl'Indiani per dar la caceia a pefci grandi, come ti fervono in Europa de'falconi per cacciare altri uccelli, era affai piccola, da loro appellata Guaicàn, e dagli Spagnuoli Keoerfo. Vedafi nella Storia di Oviedo la maniera di fervirfene.

(L) Nella Neffa rezione I. della parte 5. delle Ricerche Filofoficbe, nella quale afferma, che non $v$ 'è niuna lingua Americana, nella quale fi poffa numerate oltre a tre, dice che i Mefficani contayano fino a dieci. 
S dopo d'aver viaggiato per tutta l'America, d'aver trattato con tutte quelle Nazioni, e d'avere efaminate tutse le loro lingue; ma non è così. Il Sig. de P. fenza ufcir dal fuo gabinetto in Berlino, fa meglio le cofe d'America, che gli ftefte Americani, e nella cognizione di quelle lingue fupera coloro, che le parlano. Io imparai la lingua mefficana, e la fentii parlar dai Mefficani molti anni; eppur non Capeva, che effd foffe cos̀ farfa di voci numerali, e di termini fignificanti le idee univerfali, finattantochè non venne a illuminarmi il Sig. de $P_{0}$ Io fapeva, che i Mefficani impofero il nome Centzontli, (400) - piuttolto quello di Centrontlatale (colui, che ha voci quattrocento ) a quell' uccello tanto rinomato per la fingolar dolcezza, e per l'incomparabile varietà del fuo canto. Lo fapeva altresi, come i Mefficani contavano anticamente per xiquipilli - le mandorle di caccao nel loro commercio, e le loro truppe nella guerra : che xiquipilli valeva otto mila: ficchè per dire, che un efercito fi componeva, per efempio di quaranta mila uomini, dicevano, che aveva cinque xiquipilli. Io fapeva finalmente, che $\mathrm{i}$ Mefficani aveano voci numerali per efprimere quante migliaja, e milioni volevano; ma il Sig. de P. fa tutto il contrario, e non vi è dubbio, che il faprà meglio di me; perchè io ebbi la difgrazia di nafcere fotto un clima meno favorevole alle operazioni intellettuali. Nulladimeno io voglio per compiacere alla curiofitz dei miei Lettori metter qul forto la ferie dei nomi numerali, di cui fi fon ferviti fempre mai $i$ Mefficani . $\left({ }^{*}\right)$ Nella quale fi vede, che coloro, che al dir del Sig. de P. non aveano voci per numerare oltre a tre, ne aveano pure a difpetto di lui per contáre almeno fino a quarantotto milioni. Sinvilmente potremmo convincere l' errore de'Signori de la Condamine, e de P. in molte altre lingue Storia Antica del Neffico Tom. IV. H h d'A-

(*) NOMI NUMERALI DELLA LINGUA MESSICANA.

Ce

Ome

Jei

Nabui

\begin{tabular}{l|l}
3 & Mocuilli \\
3 & Cbicuace \\
Cbicome \\
| Cbicusi
\end{tabular}


d'America anche di quelle, che fono timate le più rozze; poichè trovanfi prefentemente in Italia delle perfone pratiche di quel nuovo Mondo, e capaci di dar piena contezza di più di feffanta lingue Americane; ma non vogliamo ftancar la pazienza de' Lettori. Tra i materiali raccolti per quefta mia opera, ho i nomi numerali della lingua Araucana, la quale contuittochè fia la lingua d'una Nazione più guerriera, che civile, ha pure delle voci per efprimere anche de' milioni. $(M)$ Non è minor l' errore del Sig. de P. nell' aftermare, che fono

Mailacti

Con quefte voei diverfamente fra loro combinate, e infreme con quefti tre nomi Pobualli, o fia Poalli 20., Tzontli 400, e Xiquipilli 8000 , elprumono qualfivoglia quantità. Cosł

Cem poalli

Ompoalli

Epoalli

Matlacpoalli ( dieci volte 20 )

\section{0}

40 60
Naubpoalli
Macuilpoalli
Cbicuacempoalli
80

100 $120 \& 6$. 200 300

\section{0}

2000 2400 \&C. 4000 6000

$\left\{\begin{array}{l}\text { Naubtzontli } \\ \text { Macuiltzontli } \\ \text { Cbicuacentzontli }\end{array}\right.$ 2200

Etzontli

Matlactzontli ( dieci volte 400 )

Caltoltzontli (quindici volte 400 . )

Cosi fi reguita fino 28000 .

Ce-xiquipilli

Onxiquipilli

Exiquiplli

8000

16.000

24.000

Matlacxiquipilli ( 10 volte 8000 )

Caxtuixiquipilli ( Is volte 8000 )

Cempoalxiquipilli ( 20 volte 8000 )

Ompoalxiquipilli ( 40 volte 8000 )

Centzon-xquipilli ( 400 volte 8000 )

Ontzcnxiquipilli ( 800 volte 8000 )

Matlactzonxiquipilli ( 4000 volte 8000 )

Naubxiquipilli

Macuilxiquipilli

32.000

40.000 Cbicuacenxiquipilli 48.00086. 80.000 120.000 160.000 320. 000 \&4. 3.200 .000

6.400 .000 32.000 .000

Caltoltzonxiquipilli ( 6000 volte 8000 ) $48.000 .000 \&<$. Diffi, che avevano voci per contare fino a 48 . milioni almeno; perchè ve ne fono ancora per portar più oltre la numerazione, ma bifogna fervirfi di parole più lunghe, e le foprallegate baftano a fmentire il Sig. de $\mathbf{P}$.

(M) Mari in lingua Araucana vale dieci, Pataca cento, Huaranca mille, $\boldsymbol{p}_{\dot{\alpha}}$ tacbuaranca cento mila, Maripatacabuaranca un milione. Dopo terminata quefta Differtazione ho acquiftato ancora la ferie de'nomi numerali in lingua $\mathrm{O}$. tomira. Quantunque quefta lingua fia ftimata una delle più rozze del Meffia 60 , ha tuttaria delle roci per efprimere guante migliaja a rogliono. 
fono tanto fcarfe le lingue Americane, che non fono capaci d'efprimere un concetto metafíico; la qual lezione egli impasò dal Sig. de la Condamine. "Tempo, dice quefto Filofofo; "ragionando delle lingue degli Americani, Durazione, Spazio, Effere, Softanza, Materia, Corpo, tutte quefte parole, e mol"te altre non hanno voci equivalenti nelle lor lingue: e noa "folo i nomi degli efferi metafifici, ma nè pure quelli degli " efferi norali, poffono da loro efprimerfi, fe non impropria"mente, e per Junghe circonlocuzioni., Ma il Sig. de la Cconda. mine fapeva tanto delle lingue Americane, quanto il Sig. de $P$. , ed egli prefe fenz'altro informazione da qualche uomo i. gnorante, ficcome accade fpeflo a'Viaggiatori . Noi fiamo affatto ficuri, che molte lingue Americane non hanno quella farfezza di voci, che penfa il Sig. de la Condamine; ma tralafciando ora ciò, che riguarda le altre, difcorriamo foltanto della meflicana, la qual è il principal foggetto della noftra contefa.

E' ben vero, che i Mefficani non aveano voci per efprimere i concetti della materia, della foltanza, dell' accidente, e frmili; ma egli è parimente certo, che niuna lingua, 0 dell' Afia, o dell' Europa avea tali voci, prima che i Greci cominciaffero ad affottigliare, ad aftraere le loro idee, ed a crear nuoe vi termini per efprimerlo. Il gran Cicerone, il qual fapeva tanto bene la lingua latina, e fiorì a que' tempi, in cui effa era nella fua maggior perfezione, contuttochè la ftimaffe piùt copiofa della greca, egli nondimeno ftenta fpeffo nelle fue ope. re filofofiche a trovar voci corrifpondenti alle idee metafifiche de' Greci. Quante volte non fu egli coftretto a crear nuove voci equivalenti in qualche modo alle greche, perchè non le trovava tra le voci ufate da'Romani? Ma ancor oggidi, dappoichè quella lingua $f u$ arricchita di molte parole inventate $e$ da Cicerone, e da altri dorti Romani, che ad efempio di lui fi diedero allo ftudio della Filofofia, le mancano pur termini da efprimere molti concetti metafifici, fe non $f_{1} f_{a}$ ricorfo al barbaro linguaggio delle fcuole. Niuna di quelle lingue, che parlano i Filofof dell' Europa, avea parole fignificative della materia, della fuftanza, dell'accidente, e d'altri fimili concetti;

$\mathrm{H} \mathrm{h} 2$

e pe. 


\section{DISSERT A Z ION E VI.}

e pero fu neceffario, che i Filofofanti adottaffero le voci tati: ne, oppur le greche. I Mefficani antichi, perchè non s' impiegavano nello ltudio della Metafifica, fono fcufabili di non avere inventăte voci da efprimere quelle idee; non è però tanto fcarfa la loro lingua di termini fignificativi di cofe metafifiche, e morali, quanto afferma il Sig. de la Condanine, che fon quelle dell'America Meridionale : anzi affermo, che non è facile di trovare und lingua più atta della Mefficana a trattar le materie metaffiche; poichè è difficile di trovarne un'altra, chetanto abbondi, quanto quella di nomi aftratti; mentre pochi fono in effa $\mathrm{i}$ verbi, da' quali non fi formino verbali corrifpondenci a quelli in io de' Latini., e pochi fono ancora i nomi fuftantivi, o addiettivi, da' quali non fi formino nomi aftratti efprimenti l' effere, o come fi dice nelle fcuole, la quiddit delle cofe: i cui equivalenti non poffo trovar nell' Ebraico, nel Greco, nel Latino, nel Francefe, nell' Iraliano, nell' Inglefe, nello Spagnuolo, o Portoghefe: delle quali lingue mi pare d'aver quella cognizione, che fi richiede per farne il paragone. Or per dare qualche faggio di quefta lingua, e per compiacere alla curiofità de' Lettori, metterò quì fotto i lor occhj alcune voci fignificanti concetti metafifici, e morali, e intefe anche dagl' Indiani più rozzi. $\left(^{*}\right)$

L'ecceffiva abbondanza di fiffatte voci è ftata la cagione d'efferfi efpofti fenza gran difficoltà nella lingua mefficana i più alti nuifteri della religione criftiana, e d' efferiz ben tradotti in effa

(*) SAGGIO: DI VOCI MESSICANE SIGNIFICANTI CONCETTI: METAFISICI E MORALI.

\begin{tabular}{lc|l} 
Tlamantli & Cora: & Nejolnònotzaliztli \\
Jeliztli & Errenza & Tlacbtopaittaliztli \\
Qualloti & Bontà. & Nejoltzolzonaliztli \\
Neltiliztli & Verità & Tlalnamiquiliztli \\
Cetiliztli & Unità & Tlalcabualiztli \\
Ometiliztli & Dualià & Tlazotlaliztli \\
Jeitiliztli & Trinià \&c. & Tlacocoliztli \\
Tectl & Dio & Tlamaubtiliztli \\
Teojotl & Divinità & Netemacbiliztli
\end{tabular}

Rifleffion

Previfione

Dubbio

Ricordo

Obblio

Amore

Odio

Timore.

Speranza:

Sulos- 
effa alcuni libri della Sacra Scrittura, e tra gli altri quelli de' Proverbj di Salomone, e de' Vangelj. i quali, ficcome quelli dell' imirazione di Crifto di Tommafo Kempis, ed altri fimili traslatati anch' effi in mefficano, non poffono certamente tradurfi in quelle lingue, che fono fcarfe di termini fignificativi di cofe morali, e metafifiche. Sono tanti i libri pubblicati in mefficano fu la Religione, e fu la morale Critiana, che d'effi foli fi potrebbe formare una buona libreria. Noi daremo dopo quefta Differtazione un breve Catalogo de' principali Autori, di cui ci ricordiamo, non meno per confermare quanto diciamo, che per moltrare la noftra gratitudine alle loro fatiche. Alcuni d' effi hanno pubblicato un gran numero d'opere da me vedute. Altri poi per agevolare agli Spagnuoli l'intelligenza della lingua mefficana, ne hanno compolto delle Grammatiche, e de' Dizionarj. Quello, che diciamo del mefficano, potremmo in gran parte affermarlo d'altre lingue, che fi parlavano ne' dominj de'Me[ficani, ficcome l'Otomita, la Matlazinca, la Mixteca, la Zapoteca, la Totonaca, e la Popoluca : poichè fi fon parimente compofte Grammatiche, e Dizionari di tutte quefte lingue, $e$ in

tutce

\begin{tabular}{|c|c|c|c|}
\hline \multicolumn{2}{|c|}{$\begin{array}{l}\text { Tloque } \\
\text { Nabuaque }\end{array}$ Colui che ha preffo } & $\begin{array}{l}\text { Necocoliztli } \\
\text { Nejoltequipacboliztli } \\
\text { Ellebutliztli }\end{array}$ & $\begin{array}{r}\text { Dolore } \\
\text { Pentimento } \\
\text { Defiderio }\end{array}$ \\
\hline Amacicacaconi & Incomprenfibile & & Virtù \\
\hline Cemicacjeni & $\begin{array}{r}\text { Eterno } \\
i \quad \text { Eternita }\end{array}$ & $\begin{array}{l}\text { Jectibuani } \\
\text { Aquallotl }\end{array}$ & Malizia \\
\hline Cabsitl & Tempo & Tolsbicabualiztli & Fortezza \\
\hline $\begin{array}{l}\text { Cenjocojani } \\
\text { Oenbuelitini }\end{array}$ & $\begin{array}{l}\text { Creator di tutto } \\
\text { Onnipotente }\end{array}$ & $\begin{array}{l}\text { Tlaixjejecoliztli } \\
\text { Jollomacbiliztli }\end{array}$ & $\begin{array}{r}\text { Temperanza } \\
\text { Prudenza }\end{array}$ \\
\hline $\begin{array}{l}\text { Cenbueliciliztli } \\
\text { Tlacatl }\end{array}$ & $\begin{array}{r}\text { Onnipotenza } \\
\text { Perfona }\end{array}$ & $\begin{array}{c}\text { Tlamelabuacacbicx- } \\
\text { bualiztli }\end{array}$ & Giuftizia \\
\hline Tlacajotl & Perfonalità & Jolbueiliztli & Magnaminità \\
\hline Tajotl & Paternità & Tlapaccaibijobuiliztli & $i \quad$ Pazienz \\
\hline Nanjotl & Maternità & Tlanematiliztli & Liberali \\
\hline Tlalticpad & $\mathrm{Um}$ & Paccanemiliztli & Manfuetudir \\
\hline$T e j o$ & & & Ben \\
\hline Teixt & $\mathrm{Me}$ & Necnomatiliztli & \\
\hline 1 & Sapienza & Tlazocamatilizli & Gratirud \\
\hline nacbiliztli & & Nepobualizli & \\
\hline Ixaxiliztli & Hone & Teojebuacatiliztli & Avarizia \\
\hline Tlaiximat & one & Nexicoliztli & \\
\hline Tlanimilizt & & Tlatzibuiliztli & DIg \\
\hline
\end{tabular}




\section{DIS SER T A Z IONE VI.}

tutte fi fon pubblicati trattati di religione, come faremo veder nel promeffo Catalogo.

Quegli Europei, che hanno imparato il meflicano, tra' i quali vi fono degl' Iraliani, de' Francefi, de' Fiamminghi, de'Tedefchi, e degli Spagnuoli, che hanno celebrata con gran lodi quella-lingua, ed a tal fegno vantata, che da alcuni è ftata ftimata fuperiore alla latina, e alla greca, come abbiam detto altrove. Il Cav. Borurini afferma, cbe nella urbanita, nella pulitezza, e nella fublimità delle efprefsioni non v'èniuna lingua sbe poffa paragonarfi colla mefficana. Quefto Autore non era Spagnuolo, ma Milanefe: non era uomo volgare, ma erudito e critico: fapeva affai bene almeno il Latino, l' Italiano, il Francefe, e lo Spagnuolo, e del Mefficano feppe quanto baftava per poter farne il giudizio comparativo. Riconofca dunque il Siga de P. il fuo errore, ed impari a non decidere in quelle materie, the ignora .

Tra le prove alle quali vuole il $\mathrm{C}$. de Buffon appoggiare il fuo filtema della recente organizzazione della materia nel nuovo Mondo, dice, che gli organi degli Americani erano rozzi, e la lor lingua barbara. "Vedafi, foggiunge, la lifta de' loro ", animali, $i$ loro nomi fon tanto difficili da pronunziare, che " è da maravigliare, che vi freno ftati degli Europei, i quali "Fienfi prefa la fatica di fcriverli. "Ma io non mi maravi. glio tanto della lor fatica nello fcriverli, quanto della lor trafcuraggine nel copiarli. Tra tanti Autori europei, che hanno fcritto la Storia civile o naturale del Meflico in Európa, non ne ho trovato nemmen uno, che non abbia alterati, e sfigurati i nomi delle perfone, degli animali, e delle Città mefficane, e alcuni lo hanno fatto a tal fegno, che non è poffibile indovinare ciò, che vollero fcrivere. La Storia degli animali del Meffico pafsó dalle mani del fuo Autore il Dott. Hernandez a quelle di Nardo Antonio Recchi, il quale non Gapeva niente del Meficano: dalle mani di Recchi pafsò a quelle degli Accademici Lincei di Roma, i quali la pubblicarono con note e differtazioni: e di quetza edizione fi fervì il C. de Buffon. Fra tante mani d'Europei, ignoranti della lingua meflica- 
na, non poteano a meno di non effere alterati $i$ nomi degle animali. Per renderfi certo chi voleffe dell'alterazione, che effi foffrirono nelle mani del C. de Buffon, bafta confrontare i nomi melficani, che fi leggono nella Storia naturale di quel Filofofo, con quelli dell' edizione romana dell'Hernandez. Del refto egli è certo, che quella difficolta, che troviamo, nel pro. nunziare una lingua, alla quale non fiamo alfuefarti, e maffimamente fe l'articolazione d'effa è troppo diverfa da quella della noftra propria lingua, non convince, che quella fia barbara. Quella medefima difficolta, che fenté il C. de Buffon nel pronunziare i nomi melficani, fentirebbonla i Meifrcani nel pronunziare $i$ nomi francefi. Coloro, che fono avvezzi alla liagua fpagnuola, fentono gran difficolta nel pronunziar la lingua tedefca, e la pollaca, e pajono loro le più afpre e più duro di tutte. La lingua mefficana non è ftata quella dei miei Genitori, nè io la imparai da fanciullo: eppur turti i nomi mefficani d'animali dal C. de Buffon prodotri, come argomento della barbarie di quella lingua, mi fembrano più facili fenza paragone da pronunziare, che molti altri prefi da alcune lin. gue europee, i quali egli adopra $(N)$ nella fua Storia Natura. le: forfe parrà cosi a quegli Europei, che non fono affue. facti alle une, nè alle altre lingue; e non vi mancherà, chì fi maravigli, che il C. de Buffon fiafi prefa la fatica di fcrivere quei nomi capaci di far paura ai più coraggiofi fcritrori. Finalmente in ciò, che riguarda le lingue americane, deefi fta. re al giudizio di quegli Europei, che le feppero anzichè all' $O_{\text {: }}$ pinione di coloro, che non ne fanno nulla.

§. VII.

(N) Legganfi i nomi reguenti d'animali adoprati dal C. di Buffon e para goninfi coi Meflicani da lui melli ed alterati:

Baurd-mannet-jes

Brand-birts

Chemik-skarzeclek Udgiers-diur
Mifzorzecbovva

Stacbel-scbuvein

Scebeujcblafer

Sterzeczlock $\mid \begin{aligned} & \text { Niedzoviedz } \\ & \text { Przavviaská } \\ & \text { Mcer-Jcbvoeio } \\ & \text { Sczurbz \&c. }\end{aligned}$ 
6. VII:

\section{Su le leggi dei Mefjeani.}

Volendo il Sig. de Paw impugnar quell' antichita, che ato tribu' Gemelli per isbaglio alla corte dei Mefficani, allega l'anarcbia del loro governo, e la fcarfezza delle loro leggi: e trattando del governo dei Peruani dice, che non poffono effere del") le leggi in uno Stato difpotico: e calo che vi fieno un tem" po ftate, non è poffibile prefentemente di farne l'analifi, per9) chè non le conofiamo: nè poffiamo conofcerle, perchè non 3) furono mai fcritte, e la loro memoria dovea mancare nella "morte di coloro, che le fapeano."

Nefluno avea fatto menzione dell anarchia del regoo di Meffico; prima che veniffe al Mondo il Sig. de P., il cui cervello aver lembra una particolar organizzazione per intender le cofe al contrario di tutí gli altri uomini. Non vi è alcuno si ignorante della Storia del Meffico, che non fappia, che quei Popoli erano fottopolti a particolari Signori, e turto lo Stato ad un fupremo Capo, che era il Re di Meffico. Tutti gli Storici vantano la grande autorita di quel Sovrano, e il fommo rifpetto che gli portavano i fuoi vaffalli : Se quefto è anarchía, faranno fenza dubbio anarchici tutti gli Stati del Mondo.

Il difpotifmo non fu introdotto in Meffico fino agli ultimi anni della Monarchì. Nel tempo addietro aveano fempre i Monarchi rifpettato le leggi promulgate dai loro Anteceffori, ed aveano zelata la lor offervanza. Anche ai tempi di Mo. rezuma II., il quale fu l'unico $\mathrm{Re}$ veramente difporico, i Magiftrati giudicavano fecondo le leggi del regno, e lo fteffo Motezuma puniva feveramente i trafgreffori, non abufando del fuo potere, fe non in quello, che potea fervire all' accrefcimento della fua opulenza, e della fua autorità.

Quelte leggi non erano fcritte; ma fi perpetuavano nella memoria degli uomini non meno per la tradizione, che per le pitture. Non v' era fuddito, che non le fapeffe; perchè i $\mathrm{Pa}$ dri di famiglia non ceffavano d'iftruirne i loro figliuoli, ac: cioc: 
ciocchè evitando la trafgreffione, fchivaffero il gaftigo. Le co: pie delle pitture delle leggi erano fenza dubbio infinite; poichè quantunque effe foffero si furiolamen te perfeguitate dagli Spagnuoli, nondimeno io ne ho vedute molte. I.'intelligenza di tali pitture non è tanto difficile a chi ha cognizınne della maniera, colla quale rapprefentavano i Mefficani le cofe, dei caratteri da loro ufati, e della loro lingua, ma pel Sig. de $\mathbf{P}$. faranno tanto inintelligibili, quanto le leggi dei Chinefi, efpreffe nei caratteri propri di quella Nazione. Otracciò dopo la conquifta molti Mefficani bene intendenti fcriffero nei noitri caratteri le leggi di Meffico, d'Acolhuacan, di Tlafcalia, di Michuacan ec. Tra gli altri D. Ferdinando de Alba Ixtlilxochitl fcriffe in lingua Spagnuola le ottanta leggi pubbiicate già dal fuo famofo arcavolo il Re Nezahualcojotl, ficcome abbiam derto nella Storia. Gli Spagnuoli poi ricercarono le leggi ed i coftumi antichi di quelle Nazioni con maggior diligenza, che qualunque altro articolo della Storia; perchè la loro cognizione importava affii al governo criftiano, tanto civile, quanto Ecclefiaftico, maffimamente in riguardo dei maritaggi, delle prerogative delia Nobiltà, della qualità del Vaffallaggio, e della condizione degli [chiavi. S' informarono a bocca dagl' Indiani meglio iftruiti, e ftudiarono le loro pitture. Oltre ai primi Miffionar, $i$ quali faticarono fruttuofamente in quefta imprefa, D. Alfonfo Zurira, uno dej principali Giudici di Meffico, dotto in materia di Legge, e pratico di quei paefi, ne fece diligenti ricerche per ordine del Re Cattolico, e compore quell' utiliffima opera, di cui abbiam fatto menzione nel Catalogo degli Scrittori della Storia antica del Meffico. Ecco come poterono faperí le leggi antiche dei Mefficani fenza effere da loto foritte.

$\mathrm{M}_{\mathrm{d}}$ che Leggi ? degne molte di effe, dice l'Acofta, della noftra ammirazione, e fecondo le quali doveano quei Popoli reggersi ancbe nel loro criftionefimo. Imprima la coltituzione del loro ftato in ciò, rhe riguarda la fucceffione alla Corona, non poteva effere meglio intefa, come quella, nella quale del pari sfuggivanfi gl'inconvenienti della fuccelfione ereditaria, e quelStoria Amita del Meffico TomolV.

I i li 
li dell' elettiva. Dovea eleggerfi un individuo della famiglia Reale per confervar così lo fplendore della corona, e impedire, che il trono foffe giammai occupato da un uomo di baffa mafcita. Non fuccedendovi il figliuolo, ma il fratello non v'e. ra pericolo, che un sì eminente, e sì importante impiego foffe efpofto all' indifcrezione d'un giovane inefperto, o alla ma. lignità d' un Reggente ambiziolo.

Se i fratelli poi aveffero dovuto fuccedere fecondo l'ordine della lor nafcita, farebbe neceffariamente talvolta toccata la corona ad un uomo inetto al governo, ed avrebbe altresi potuto accadere, che l'Erede prefuntivo macchinaffe contra la vita del Sovrano per anticiparfi la fucceffione. All' uno e all'altro inconveriente fi ovviava coll' elezione. Gli Elettori fceglie. vano tra $i$ fratelli del $R e$ morto, e mancando effi, tra $i$ figli dei $\operatorname{Re}$ anteriori, il più idoneo a comandar la Nazione. Se foffe tato di balia del $\operatorname{Re}$ il nominar gli Elettori, avrebbe egli potuto Isegliere coloro, che foffero più favorevoli ai fuoi diffegni, e procacciarfi i loro fuffragi in favor di quel fratello, che foffe a lui più caro, e forfe anche in favor del figliuolo, non curando le leggi fondamentali dello Stato: ma non era così; poichè i medefimi Elettori erano eletti dal corpo della Nobiltà, la quale in loro comprometteva i fuffragj di tutta la $\mathrm{Na}$. zione. Se l' impiego degli Elettori foffe ftato perpetuo, avrebbono potuto coltoro, abufando della lor autorita, divenir pa. droni della monarchia; ma ficcome finiva nella prima elezio. ne la lor voce elettorale, e fi eleggevano allora nuovi Eletto. ri per la feguente, cos' non era tanto agevole all' ambizione l' ufurpar l'autorità. Finalmente per ifchivare altri inconvenien. ti, $i$ veri Elettori non erano più di quattro, uomini della prima nobiltà, di gran prudenza, e di notoria probità: E' vero, che nè anche dopo tante precauzioni poteanfi impedire tutti i difordini; ma qual governo fu mai tra gli uomini, che non forfe efpofto a maggiori mali?

La Nazion Nefficana era guerriera, e però abbifognava d'un capo intendente, ed efperto nel meftier della guerra : or qual configlio potea prenderfi più confacente a tal fine, che quel- 
quello di non eleggere $\mathrm{Re}$ colui, che non aveffe per li fuoi meriti ottenuta la carica di General d'efercito, e di non coronar colui, che dopo la fua elezione non $f i$ foffe nella guerra procacciate le virtime, che fecondo il loro fiftema di religione doveano facrificarfi nelle fefte dell' Incoronazione?

Quella prontezza, colla quale i Mefficani fcoffero il giogo de Tepanechi, e quella gloria, che fi guadagnarono le loro armi nella conquifta d' Azcapozalco, doveano naturalmente eccitare la rivalità, e la diffidenza de'loro vicini, e fpezialmente quella del $R e$ d'Acolhuacan, il qual era ftato, ed era anche allora il maggior $\operatorname{Re}$ di quella terra, ed effendo peraltro ancor vacillante il trono di Meffico, abbifognava d' un forte appoggio, che lo fofteneffe. Il $\operatorname{Re} d^{2}$ Acolhuacan, il qual avea di frefco ricuperata coll' ajuto de' Mefficani la corona, ufurpatagli già dal Tiranno Tezozomoc, dovea temere, che qualche fuddito poderofo, feguendo l'orme di quel Tiranno, non eccitaffe alla ribellione una parte del fuo regno, e lo privaffe, come fuo Padre, del!a corona, e della vita. Il $\operatorname{Re}$ di Tlacopan, il qual occupava un trono nuovamente ftabilito, e poco confiderabile, avea più da temere. Ciafcun di quefti $\mathrm{Re}$ era per fe folo poco fi. curo, e dovea diffidarfi degli altri due; ma uniti tutti e tre infieme poteano formare una potenza invincibile. Or che fanno? Formano una triplice alleanza, la qual renda ciafcun ficuro rifpetto agli altri due, e turti e tre rifpetto a'lor fudditi. Quefta fu quell' alleanza, che raffodò i troni d'Acolhuacan; e di Tlacopan, e che agevold a' Mefficani la loro conquifta: alleanza tanto ferma, e cosi ben ordinata, che non fí fconcerto giammai fino all'arrivo degli Spagnuoli . Queito fol colpo di politica bafta a dimoltrare il difcernimento, e la fagacita di quelle Nazioni; ma ve ne furono tanti altri fimili, che fe vo. leffima rapportarli tutti, farebbe d'uopo di copiare una buona parte della Storia.

La forma giudiziale de' Mefficani, e de'Tezcucani ci fomminittra parecchie lezioni utili di Politica. Quella diverfità di gradi ne'Magittrati ferviva al buan ordine: la lor affiduità ne' tribunali dallo fpuntar del di fino alla fera abbreviava il corfo 1 i 2

delle 


\section{D ISSER T A Z ION E VI.}

delle caure, e gli diftoglieva da molte pratiche clandeftine, le quali avrebbono potuto prevenirli in favor d'alcuna delle parti. Le pene capitali prefcritte contro i prevaricatori della giufizia, la puntualità della loro efecuzione, e la vigilanza de' Sovrani tenevano in freno $i$ Magiltrati, e quella cura, che fi avea di fomminiftrar loro a conto del $\operatorname{Re}$ tutto il bifognevole, gli rendeva inefcufabili. Quelle radunanze, che fi tenevano o. gni venti giorni innanzi al Sovrano, e particolarmente quell' affemblea generale di tutti i Magiltrati ogni ottanta giorni per terminar le caufe pendenti, oltre allo Cchivare que' gran mali, che cagiona la lentezza de'giudizj, faceano, che i Magiftrati fi comunicaffero reciprocamente i loro lumi: che il Re conofceffe meglio coloro, i quali egli avea coltituiti depofitarj della fua autorità: che l'innocenza aveffe più ricorfi, e che l'apparato del giudizio rendeffe più rifpettabile la giuftizia. Quella legge, che permetteva l' appellazione dal tribunale del Tlacatecatl a quello del Cibuacoatl nelle caufe criminali, e non nelle civili dà a divedere, che i Mefficani, rifpettando le leggi dell' umanità, riconofcevano, che î richiedeva più per credere un uomo delinquente, che per dichiararlo debitore. Ne' giudizj de' Mefficani non fi ammetteva alcra pruova contro il Reo, che quella de'teltimonj. Non fu mai veduto appo loro adoprar la tortura per far colpevole per forza de'tormenti l'innocente, nè prevalerfi di quelle barbare pruove del duello, del fuoco, dell' acqua bogliente, e fimili, che furono già sì frequenti in Euro$\mathrm{pa}$, ed oggi fono da noi lette con iftupore nelle ftorie. "Non " vi farà chi non fi maravigli, dice fopra quefto argomento "il Montefquieu, $(O)$ che i noftri Maggiori facefiero dipender "L'onore, la fortuna, ed i beni de' Cittadini da certe cofe, le 9) quali erano meno della giurifdizione della ragione, che di "quella della forte: e che adoperaffero inceffantemente quelle ", pruove, che nulla provavano, e non erano conneffe nè coll" ", innocenza, nè col delitto. "Ciò che ora diciamo di quelle pruove, dirà nell'avvenire la noftra pofterità della tortura, e non

(O) L' Esprit des loix lib. 28. cap. 27. 


\section{DIS SER T A Z IONE VI. 253}

non cefferanno mai di maravigliarfi, che si fatta pruova fia fta: ta generalmente in ufo per tanti fecoli nella parte più illuminata del Mondo. Il giuramento era prova di gran momento ne'giudizj de' Mefficani, ficcome abbiam detto altrove; imperocchè ficcome erano perfuafi de' terribili gaftighi, che doveano infallibilmente efeguire gli Dei ne' pergiuri, cosi credevano, che neffuno oferebbe ifpergiurare; ma non fappiamo, che fi permetteffe tal prova agli Attori contra il Reo, ma folamente al Reo per purgarfi dal delitto.

Punivano feveramente i Mefficani tutti que' delitti, che fono particolarmente ripugnanti alla ragione, o pregiudiziali allo Stato, il crimenlefe, l'omicidio, il furto, l'adulterio, l' incefto, e gli altri ecceffi in quefta materia contra natura : il fa. crilegio, l'ubbriachezza, e la bugìa. Si conduffero faviamente, non lafciando impuniti tali misfatti; ma peccarono nella quantità della pena, la quale in alcuni delitti era ecceffiva, e crudele. Io non pretendo di fcufare i falli di quella Nazione; ma nemmen poffo diffimulare, che di quanto è riprenfibile nella loro legislazione troveranfi efempj ne' più famofi Popoli dell' antico Continente, e tali da far comparire affai miti le leggi de' Mefficani, e più conformi alla ragione. Le celebri leggi delle dodici tavole fon piene, dice il Montefquieu, $(P)$ di difpofizioni crudeliffime... vedefi in effe il fupplizio del fuoco, e le pene Sempre capitali. Eppur quelta è quella lodatiffma compilazione, che fecero i Romani del meglio da lor trovato preffo i Popoli Greci. Or fe il neglio della coltiffima Grecia era tale, che farà ftato ciò, che non era così buono? Qual farà ftata la legislazione di que' Popoli, che erano da loro chiamati barbari? Qual legge più inumana e crudele di quella delle dodici tavole la qual permetteva a' creditori di sbranare (Q) il debitore, che non pagava, e di portarfene ciafcuno la parte

(P) L'Esprit des Loix lib. 14. cap. 15 .

(Q) Si plures forent, quibus reus effet judicatus, fecare fi vellent, eque partiri corpus addiati fabi bominis permi erunt. Aul. Gell. Noct Attic. lib. 20. cap. 1. So bene, ciò che dicono parecchj Giurifti per giuftificar quefta legge, ma fo ancora che non fon riufciti. 


\section{DISSERTA Z IO N E VI.}

fua per foddisfazione del credito? E quefta legge non fi promulgò io Roma ne' rozzj principj di quella sì rinomata Città, ma anni trecento dopo la fua fondazione. Qual legge per lo contrario più iniqua di quella del famofo Legislatore Licurgo, la qual permerteva il furto a' Lacedemonj? I Mefficani caltigavano quefto delitto tanto perniciofo alla Società ma non procedevano a pena capicale, fe non quando il Ladro non era in iftato di foddisfare, e pagare l' offefa colla fua libertà, e co' fuoi beni. Non era così rapporto al furto efeguito ne' feminati; perchè quefti, effendo per la lor firuazione più efpofti alla rapina, aveano maggior bifogno della cuftodia delle leggi; ma quella medefima legge, che prefcriveva pena capitale contra colui, che vi rubava un certo numero di frutti, o di piante, permetteva a'viandanti bifognofi di mangiarvi, quanto lor foffe d'uopo, per rimediare alla prefente neceffità. Quanto più ragionevole non era quefta legge di quella delle dodici tavole, la quale condannava fenza diftinzione ad effere impiccato chiunque prendeva qualche cofa da' feminari altrui? $\left.l^{*}\right)$

La bugła, quel peccato tanto perniciofo alla Società, fi lafcia per lo più impunita in moltiffini prefi dell'antico Coneinente, e nel Giappone fi caftiga feffo con pena capitale. I Mefficani fi allontanarono ugualmente dall' uno, e dall'altro eftremo. I loro Legislatori, ben confapevoli del genio, e delle inclinazioni della Nazione, s' accorfero, che le non prefcrive. vano pene gravi contro la bugia, e l'ubbriachezza, farebbe mancato negli uomini il giudizio per foddisfare a' rifpettivi lor obblighi, la verità mancata farebbe ne"giudizj, e la fede ne" contratti. La fperienza ha fatto conofcere, quanto pregiudiciale fia a quelle Nazioni l'impunità di quefti due peccati .

$\mathrm{Ma}$ in mezzo alla lor feverità ebbero cura i Mefficani di non involgere gl' innocenti nel gaftiga de'colpevoli. Molre leggi dell' Europa, e dell' Afia prefcriffero la fteffa pena al reo d'alto tradimento, ed a tutta la fua famiglia. I Mefficani puniva-

$\left(^{*}\right)$ Qui frugem aratro quefitam furtim nox pavit fecuitve Jufpenfus cereri mecator. 
nivano tal delitto con pena capitale; non però privavano di vita i parenti del reo, ma foltanto della libertà : e non già tutti, ma folamente coloro, che effendo confapevoli del tradimento, e non avendo voluto rivelarlo, s' erano renduti anch' effi colpevoli. Quanto più umana fi è quefta legge, che non quelle del Giappone? Quelle leggi delle quali dice il Monterquieu, $(R)$ cbe caftigano per un fol delitto tutta una famiglia, - tutto un quartiere: quelle leggi, che non fanno trovare in. nocensi, dove fono de' colpevoli. Non fappiamo, che i Mefficani prefcriveffero alcuna pena contra coloro, che fparlavano del governo: pare, che eglino non faceffero gran capitale di quello sfogo dell' amor proprio de'fudditi, che santo fi teme in altri paefi.

Le lor leggi concernenti i maritaggj erano renza dubbio più onefte e più decorofe di quelle de' Romani, de'Greci, de Perfiani, degli Egizi, e d'altri Popoli dell' antico Continente. I Tartari fi ammogliano colle lor figlie: gli ancichi Petfiani $e$ gli Affrj prendevano le fleffe lor Madri : gli Ateniefi, e gli $\mathrm{E}$. gizj le lor forelle. Nel Meffico era feveramente proibito ogni maritaggio tra perfone congiunte nel primo grado di confanguinità, e di affinità, fuorchè tra i cognati, allorchè il frateilo in morendo lafciava alcun figliuolo. Quella proibizione dà a dive. dere, che i Mefficani giudicavano meglio del matrimonio, che tutte le mentovate Ndzioni. Quell' eccezione dimoltra i loro fentimenti d'umanità. Se una Vedova paffa a feconde nozze, ha fovente il difpiacere di vedere i fuoi figliuoli poco amati da un Padre, che non diede loro la vita: il fuo nuovo Marito, poco rifpettato da quegli fteffi figliuoli, che il riguardano come ftrano: ed i figliuoli dell' uno, e dell' altro matrimonio cosi fra loro difuniti e difcordi, come fe foffero nati da diverfe madri. Or qual miglior configlio, ( parlo fecondo le regole della Politica umana, dalle quahi diriggevanfi qualle Nazioni, che non aveano cognizione delle (ante leggi del Critianefimo ) qual miglior configlio, dico io, poreano prender i Mefficani per rimediare

(K) L'Efprit des Loix lib. 14. cap. 15. 


\section{ig6 DISSER T A Z I O NE VI.}

diare a que'mali troppo comuni, chequello di maritar la ve: dova col cognato? Molte Nazioni antiche dell' Europa, imitate da non pochi Popoli moderni dell' Afia, e dell' Africa, compravano le lor mogli, e però efercitavano fopra loro un' autoritz affai più grande di quella, che lor concedetre l'Autor della Natura, e trattavanle più da fchiave, che da compagne. I Mefficani non acquiltavano le lor mogli, che per mezzo di lecite e decorofe pretenfioni: e benchè prefentaffero de' doni a' Genitori, quelli non erano per conto di prezzo della figlia, che pretendevano, ma folo un offequio per conciliarf la loro benevolenzı, e pie. gar la loro volontà al contratto. I Romani, contuttochè non aveffero fcrupolo di preftar le loro mogli, $(S)$ aveano cid nulla oftante dritco fecondo la legge di toglier loro la vita, qualora foffero colte in adulterio. Quefta iniqua legge, la quale coltituiva il marito giudice nella propria caula, ed eziandio efecutore della fua fentenza, in cambio d'impedire gli adulterj, aumentava i parricidj. Preffo i Meflicani non era permeffo a'Mariti queli' infame commercio delle lor mogli, nè aveano. verun' autorita fopra la lor vita. Era punito con pena capitale colui, che toglieva la vita alla fua moglie, ancorchè la cogliefle in adulterio. Quefto è, diceano, ufurpar l' autorità de' Magiftrati, a' quali tocca conofcer de' delitti, e caftigarli giufta il tenor delle leggi. Prima che foffe fatta da Augulto la leg. ge Julia de Adulteriis, non fappiamo, dice il Vives, $\left({ }^{*}\right)$ che foffe mai in Roma tenuto alcun giudizio nella caufa d'adulterio: vale a dire, che mancò a quella celebre Nazion la giuftizia in un punto sl grave, e si importante per più di fette fecoli. Se dopo aver fatto il paragone delle leggi, fi vuol fare anche

(S), In Roma, dice il Montefquieu, era permeffo al marito di preftare ad „, un altro la fua moglie. Il dice efpreframente Plutarco. Si fa che Catone " preftò la fua moglie ad Urtenfio, e Catone non era capace di violar le "leggi della fua patria "L'ESprit des Loix lib. 29.

(*) Not. in cap. 5. lib. 3. de civit. Dei. Molti Giurifti dicono, che a' mariti fu tolta quella podeftà fulla vita delle lor mogli adultere dalla Legge Cornelia de Sicariis; ma checchè ne fia, egli è certo che quelta legge fu fatta da Silla verf́c il fine del fecolo fettimo di Roma: ficchè quanto al tempo non $v$ 'è gran divario tra queita legge a quella d' Auguto. 
anche quello de'riti nuziali di quefte due Nazioni, troveraffe preffo amendue molta fuperfizione, ma del refto vi fi vedra una gran diverfità : quei de'Mefficani erano onelti e decenti, quei de' Romani ofceni ed infamı, come altrove vedremo.

Per ciò che riguarda le leggi della guerra, egli è difficile che effe fieno giufte preffo un Popolo guerriero: la grande Atima, che effo ha del valore, e della gloria militare, gii fa aver in conto di nemici quei, che nol fono, e l'ambizione di conquiftare lo fpinge a trapaffare i termini prefcritti dalla giuftizia. Nulladimeno nelle leggi de' Mefficani fi vedono tali trati d'equità, che farebbon onore alle Nazioni più colte. Non fi potea dichiarar la guerra fenza averne prima difaminate in pieno conGglio le ragioni, e fenza che foffero ftate approvate dal Sommo Sacerdote. Oltracciò vi fi doveano premettere delle ambafciate, e fpetfo replicate, dirette a coloro, cui fi deliberava di far la guerra, per ottenere pacificamente per via di qualche accomodamento ciò che fi voleva, prima di venire alla rottura. S) fatti indugi davano tempo a' lor nemici d'apparecchiarfi alla difefa; ma oltrechè fervivano alla lor giuftificazione, contribuivano altresi alla lor gloria; mentre da loro ftimavafi vilta il far la guerra a' nemici fprovveduti, e fenza averli prima folennemente sfidati, acciocchè la vittoria non poteffe mai ad altro afcriverfi, che alla lor bravura. E' vero, che quefte leggi non erano fempre offervate; ma non erano per ciò men giufte: e fe vi fu dell' ingiultizia nelle conquifte de'Mefficani, non fu certamente minore in qualle de' Romani, de' Greci, de' Perfiani, de'Goti, e d'altre celebri Nazioni. Uno de'gran mali, che fuol portar feco la guerra, è quello della fame per cagion delle oftilisà, che fi fanno nelle campagne. Non è poffibile impedire affatto quefto male; ma fe v'è ftata mai qualche cola capace di moderarlo, i fu fenza dubbio quell' ufanza de' Mefficani, e degli altri Popoli d'Anahuac d'aver in ogni provincia un luogo affegnato per campo di battaglia. Non era men conforme alla ragione, ed all' umanità quell' altra lor ufanza d'avere in tempo di guerra ogai cinque dỳ un giorno intero di tregua, e di ripolo.

Storia Anticadel Meffico Tom. IV. K k $\quad$ Avez: 


\section{DISSERTAZIONE VI.}

Aveano quelle Nazioni formata una fpezie di Jus Gen: tium, in virtù del quale, fe il Signore, la Nobiltà, e la Plebe tigettavano le propofizioni fatte loro da un altro Popolo, o Nazione, e rimeffane la decifione alle armi, reftavano vinti, il Signore perdeva il dritto Sovrano, la nobiltà il dominio ottimo, che avea fu le fue poffeffioni, la Plebe era fottopofta al fervizio perfonale, e tutti coloro, che erano fati fatti prigioni nel calor delia zuffa, erano privati quafi ex delifto della libertà, e del dritto alla vita. Ciò s'oppore fenz'altro a quelle idee, che noi abbiamo, dell umanità; ma la general convenzione di que' Popoli rendeva men biafimevole quell' inumanità; e gli efempi affai più atroci delle più colte Nazioni dell' antico Continente fanno fparir quel ribrezzo, che a prima vilta ci cagiona la crudeltà di que' Popoli americani . Prefso $i$ Greci, dice il Montefquieu, $(T)$ gli abitatori d'una Citta', prefa a forza d'armi, perdevano la liberta, ed erano venduti come fcbiavi. Non è certamente da paragonare quell'inumanita, che i Mefficani elercitavano verfo i loro prigionieri nemici, con quella, che gli A teniefi ufavano verfo i proprj lor Cittadini. Una legge d'Atene, dice il fuddetto Autore, ordinava, che quando la Città foffe affediata, fi facefle morir tutta la gente inutile. Non potrà trovarfi nè preffo i Mefficani, nè preffo verun' altra Nazione del nuovo Mondo alquanto dirozzata, una legge tanto barbara, quanto fi è quella del Popolo più colto dell antica Europa; anzi la maggior premura de' Mefficani, e di tutte quelle Nazioni d'Anahuac, allorchè dovea effere affediata qualche loro Citrà, era quella di porre in ficuro $\mathrm{i}$ lor figliuoli, le donne, e gl'invalidi, o mandandogli ad altre Città, 0 pur alle montagne. Cosi fottraevano quella debol gente dal furor de' nemici, ed impedivano per altro la foverchia confumazion de'? viveri .

Il tributo, che fi pagava a'Re d'Anahuac era ecceffivo; ed erano altresì tiranniche le leggi, che il prefcrivevano; ma quefte leggi furono confeguenze del difpotifmo, introdottovi ne-

(T) L'Esprit des Loix lib. 20. cap. 34. 
gli ultimi anni della Monarchia mefficana : il qual nel fuo -maggior aumento non giunfe a quell' ecceffo d'impadronirfi del: le terre dell' Imperio, e de' beni de' fudditi, che giuftamente biafimiamo ne' Monarchi afiatici: nè fu mai fentito, che da' Sovrani d'Anahuac foffero pubblicate leggi fu i tributi tanto ftravaganti, e dure, quanto fono ftace moiciffime pubblicate nel Mondo antico, come per efempio quella dell' Imperatore Anatafio, il qual impofe gravezza anche fu la refpirazione: wt unufquifque pro baufu aeris pendat.

Ma fe centuriamo nelle leggi fa i tributi la tirannica am. bizione di que' lvionarchi, non pofflamo a meno di non lodare ed ammirare nelle lor leggi ful commercio la coltura di quelle Nazioni, e la faviezza de' loro Legislatori. L'avere in ogni Cittz o Borgo una piazza, deftinata pel commercio di tutte le cofe, che poteano fervire a' bifogni, ed alle delizie della vita, giovava a riunirvi sutti i Mercatanti pel più pronto fpaccio delle merci, e gli metteva fotto gli occhi degli Ifpettori, o Commeffari, acciocchè fi evitaffe ogni frode, e difordine ne' contratti. L'avere ogni merce il fuo luogo determinato contribuiva al buon ordine, e al comodo di coloro, che volevano provvederfene. Il Tribunal di Commercio, ftabilito nella medefima piazza del mercato per aggiuftar le differenze inforte tra $i$ negozianti, e per punir prontamente qualunque ecceffo vi foffe, confervava inviolabili i dritti della giuftizia, ed afficurava la pubblica tranquillità A quefte favie difpofizioni fi dovette quell' ordine maravigliofo, che in mezzo ad un sì ecceffivo numero di negozianti vi ammirarono i primi Spagnuoli :

Finalmente nelle leggi fu gli fchiavi furono i Mefficani fuperiori alle più colte Nazioni dell' antica Europa. Se vuol farfi il paragone delle leggi de' Meficani, con quelle de' Romani, de' Lacedemoni, e d'altri celebri Popoli, tofto fi vedrà in quefte una tal barbarie e crudeltà, che $f_{a}$ ribrezzo, e in quelle una grande umınita, e un gran rifpetto alla legge della Natura ( non parlo ora de' prigionieri di guerra, di cui poi ragionerò.) Qual legge più umana di quella, che faceva nafcer li$\mathrm{K} \mathrm{k}_{2}$ beri 
beri turti gli uomini anche da genitori fchiavi: che lafciava allo fchiavo il dominio della roba fua, e di quello che acquiftava colla propria induftria o fatica: che obbligava il padrone a trattar lo fchiavo come uomo, e non come beftia; non gli permetteva verun'autorità fu la vita di lui, e anche lo privava della facoltà di poter venderlo nel mercato, fe non dopo aver Fatto conftar giuridicamente dell' indocilità d'effo lui? Or quanto diverfe da quefte erano le leggi de'Romani? Coltoro per la fomma autorità loro accordata dalle leggi erano padroni non che di rutto ciò, che gli fchiavi acquiftavano colla loro fatica, ma eziandio della lor vita, $(V)$ della quale gli privavano giu. fta il lor capriccio, gli trattavano colla maggior inumanità, e lor faceano tollerare $i$ più atroci tormenti : ed acciocchè fì veda l'indole inumana di quefta Nazione, mentre tanto ampliavano l'autorità de' Padroni contro gli fchiavi, la riftrignevano. pure in ciò, che era in favor di coltoro. La legge Fufia Caninia vietava a' Padroni il manomettere per reftamento oltre ad un certo numero di fchiavi. Nella legge Silaniana, e in altre era prefcritto, che ogni volta, che foffe uccifo un Padrone fi faceffero parimente morire tutti que fuoi fchiavi, che abitaffero dentro la medefima cala, $O$ in luogo ad effa vicino, donde fentir fi poteffe la fua voce. Se egli era uccifo in "qualche viaggio, doveano morire tutti quegli fchiavi, che foffero con lui reftati, e fimilmente turti quelli, che fe ne foffero fuggiti, quantunque manifefta foffe la loro innocenza. La legge Aquilia comprefe fotto una medelima azione la ferita fatta ad uno fchiavo e quella fatta a una beftia altrui. A cotal ecceffo giunfe la barbarie de coltiffimi Romani. Non furono in vero più umane le leggi de' Lacedemonj, le quali non concedevano agli fchiavi verun" azione in giudizio contra coloro ${ }_{4}$ che gl' infultavano, 0 ingiuriavano.

(V) Che maraviglia che i Romani accordaffero quella barbara auterità a' Pa droni fu gli Schiavi, avendola conceffa anche a' Padri di famiglia fopra i lor figli legittimi? Endo liberis jufis jus vite, necis, venumdandique poteffas Patri. . Quefa fola legge pubblicata in Roma da'primi $R e$, e inferita poi da'Decemviti nelle dodici Tavole balta per dare a divedere, che la legislazione de'MerGcani fu più umana. 


\section{DISSERT AZIO NE VI. 26:}

Se oltre il detto fin qui fi vuol paragonare il fiftema di educazione, che v'era preffo i Mefficani con quello de'Greci, riconofcerafi, che non eta si grande l'iftruzione de' Greci a' lor figliuoli nelle arti e nelle fcienze, come quella, che aveano i fanciulli, ed i giovani Mefficani ne' coftumi da' lor genitori . I Greci s'adoperavano più ad illuftrare la mente, i Mefficani a rettificare il cuore. Gli Ateniefi proftituivano i lor giovani alla più efecranda ofcenità in quelle medefime fcuole, che erano deftinate ad iftruirli nelle arti. I Lacedemonj ammaeftrava. no $i$ lor figliuoli, fecondo il prefcritto di Licurgo, nel rubare per rendergli fcaltri e fvelti, e gli sfetzavano forte mente, quando gli coglievano in qualche furto, caftigando in loro non quel peccato; ma la poca induftria nel farlo si, che vi foffero colti. $\mathrm{Ma}$ i Mefficani infegnavano a' lor figliuoli infieme colle arti la religione, la modeftia, l'oneftà, la fobrietà, la vita laboriofa, l' amor della verità, e il rifpetto a' maggiorì .

Quefto è un breve, ma vero faggio della coltura de'Mefficani prefo dalla loro Storia antica, dalle lor pitture, da' rag: guagli de' più efatti Storici Spagnuoli. 'Cosi fi reggevano que' Popoli, i quali il Sig. de PaW crede i piì felvaggi del mondo. Cosi fi reggevano que' Popoli inferiori quanto alla induftria, ed alla Sagacità a più rozzi Popoli dell' antico Continente. Così fi reggevano que' Popoli, della cui razionalità vollero dubitare alcuni Europei .

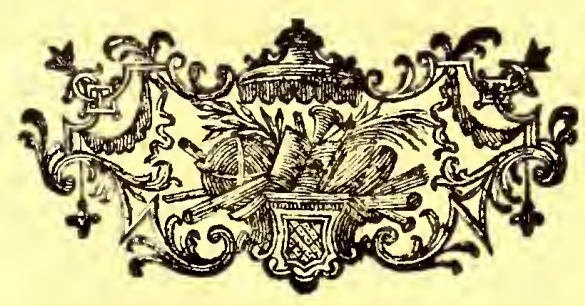

CA: 


\section{A T A L O G O}

D'ALCUNI AUTORI EUROPEI E CREOGLI, CHE HANNO SCRITTO DELLA DOTTRINA E MORALE CRISTIANA NELLE LINGUE DELLA NUOVA SPAGNA.

Za A vale Agoftiniano, il D. Domenicano, la F. Francefcano, il G Ge. fuita, il P. Prete fecolare $L^{\prime}$ afterifco denota, che l'Autore ftampò alcune opere.

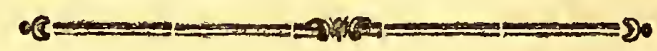

IN LINGUA MESSICANA.

* A Goltino de Betancurt F. CreoA glio.

Alfonfo de Efcalona F. Spagnuolo. Alfonfo de Herrera F. Spagn.

* Alfonfo Molina F. Spagn. Alfonfo Rangel $F$. Spagn. Alfonfo de Truxillo F. Creoglio . Andrea de Olmos F. Spagn. Antonio Davila Padilla D.Creoglio. Ant. de Tovar Motezuma P. Cr. Arnaldo Bafface F. Francefe. Baldaffare del Caftillo F. Spagn. Baldaffare Gonzalez G. Cr. Barnaba Paez A. Cr. Barnaba Vargas P. Cr. Bartolommeo de Alba P. Cr. Benedetto Fernandez D. Spagn. Bernardino Pinelo P. Cr. Bernardino de Sahagun F. Sp.

* Carlo de Tapia Centeno P. Cr. Filippo Diez F. Sp.

Francefco Gomez F. Sp. Francefco Ximenez F. Sp. Garcia de Cifneros F. Sp. Giov. de la Anunciacion A. Sp.

* Giov. de Ayora F. Sp.

* Gior. Battilta F. Cr.
Giov. di S. Francefco F. Sp. Giov. Focher F. Francefe. Giov. de Gaona F. Sp. Giov. Mijangos. Giov. de Ribas F. Sp. Giov. de Romanones F. Sp.

* Giov. de Torquemada F. Sp. Giov. de Tovar G. Cr. Girolamo Mendieta F. Sp.

* Giufeppe Perez F. Cr. Ignazio de Paredes G. Cr. Luigi Rodriguez F. Martino de Leon D. Cr. * Maturino Gilbert F. Francefe. Michele Zarate F.

* Pietro de Gante F. Fiammingo. Pietro de Oroz F. Sp.

* Toribio de Benavente F. Sp. IN LINGUA OTOMITA.

Alfonfo Rangel. Barnaba de Vargas.

- Francefco de Miranda G. Cra Gio: di Din Caftro G. Cr. Orazin Carnchi G. Milanefe Pietro Palacios F. Sp. Pietro de Oroz. Sebaftiano Ribero F. N. Sanchez P.Cr. 
IN IINGUA TARASCA:

Maturino Gilbert.

Gio: Battifta Lagunas F.

* Angelo Sierra F. Cr.

\section{IN LINGUA ZAPOTECA:}

Bernardo de Alburquerque D.Sp. e Vefcovo di Guajaca. Alfonfo Camacho D. Cr. Antonio del Pozo D. Cr. Criftofano Aguero D. Cr.

IN LINGUA MIZTECA.

Antonio Gonzalez D. Cr.

- Antonio de los Reyes D. Sp. Benedetto Fernandez D. Sp.

\section{IN LINGUA MAYA.}

Alfonfo de Solana F. Sp. Andrea de Avendaño F. Cr. Antonio de Giudad.Real Sp. Bernardino de Valladolid F. Sp. Carlo Mena F. Cr.

Giufeppe Dominguez Pr. Cr.

IN LINGUA TOTONACA.

Andrea de Olmos.

Antonio de Santoyo P. Cr.

Criftofano Diaz de Anaya P. Cr.

IN LINGUA POPOLUCA.

Francefco Toral F, Sp. e Vefcoso di Jucatan.
IN LINGUA MATLAZINCA.

Andrea de Caftro F. Sp.

IN LINGUA HUAXTECA:

Andrea de Olmos.

* Carlo de Tapia Centeno.

IN LINGUA MIXE.

* Agotino Quintana D. Cl.

IN LINGUA KICHE':

Bartolommeo de Anleo F. Cr: Agnotino de Avila F.

\section{IN LINGUA CAKCIQUEL:}

Bartolommeo de Anleo.

Alvaro Paz F. Cr.

Antonio $\mathrm{Saz}$ F. Cr.

Benedetto de Villacañas D. Cr'

IN LINGUA TARAUMARA.

Agoftino Roa G. Sp.

IN LINGUA TEPEHUANA'

Benedetto Rinaldini G. Napol.

Ve ne fonn altre lingue, come pure moltiffimi altri Scrittori; ma noi non accenniamo, fe non alcuni di coloro, le cui opere fono ftate ftampate, $o$ almeno particolarmen: te pregiate dagl' intelligenti. 


\section{AUTORI DI GRAMMATICHE E DIZIONARI DELLE SUDDETTE LINGUE.}

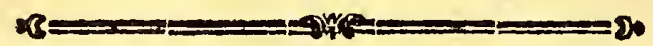

DELLA MESSICANA

GRanc. Ximenez Gram. e Diz.

1 Andrea de Olmos Gram. e Diz. Bernardino de Sahagun Gram. e Diz.

* Alfonfo de Molina Gram. e Diz.

* Garlo de Tapia Centeno Gram. e Diz.

Alfonfo Rangel Gram.

* Antonio del Rincon G. Cr. Gram.

* Orazio Carochi Gram. Bernardo Mercado G. Cr. Gram. Ant. Davila Padilla Gram.

- Agoltino de Betancurt Gram. Barnaba Paez Gram. Ant. de Tovar Motezuma Gram.

* Ignazio de Paredes Gram.

* Antonio Caftelu P. Cr. Gram.

* Giufeppe Perez Gram. Gaetano de Cabrera P. Cr. Gram.

- Agolt. de Aldana y Guevara P. Cr. Gram.

Giov. Focher F. Francele Gram.

- Antonio Cortes Canal, P. India. no Gram.

DELLA OTOMITA.

Giov. Rangel Gram.

Pietro Palacios Gram.

Orazio Carochi Gram.

N. Sanchez Diz.

Sebaftiano Ribero Diz.

Giov. di Dio Caftro Gram. e Diz.

DELLA TARASCA.

* Maturino Gilbert Gram. e Diz.

* Angelo Sierra Gram. e Diz.
Gio. Batt. de Lagunas Gram. DELLA ZAPOTECA. Antonio del Pozo Gram. Criftofano Aguero $D^{\prime} z$.

NELLA MIZTECA.

Ant. de los Reyes Gram.

DELLA MAYA.

Andrea de Avendaño Gram.e Diz. Ant. de Giudad.Real. Diz.

Luigi de Villalpando Gram. e Diz.

- Pietro Beltran F. Cr. Gram.

DELLA TOTONACA.

Andrea de Olmos Gram. e Diz.

Criftofano Diaz de Anaya Gram.'

e Diz.

DELLA POPOLUCA.

Franc. Toral Gram. e Diz.

DELLA MATLAZINCA. Andrea de Caftro Gram. e Diz:

DELLA HUAXTECA.

Andrea de Olmos Gram. e Diz. Carlo de Tapia Gram. e Diz.

DELLA MIXE.

Agoftino Quintana Gram. e Diz.: DELLA CAKCHIQUEL.

Benedetto de Villacañas Gram. e Diz.

DELLA TARAUMARA.

Girolamo Figueroa G. Cr. Gram. e Diz.

Agoftino de Roa Gram.

DELLA TEPEHUANA.

Girolamo Figueroa Gram. e Dir: Tommafo de Guadalaxara G. Gr. Gram.

Benetto Rinaldini Gram. 


\section{DISSERTAZIONE VII.}

SOPRA I CONFINI, E LA POPOLAZIONE DEI REGNI DI ANAHUAC.

$28=2 \%$

Cli sbagli di molti Scrirtori Spagnuoli intorno ai confini T dell' Imperio Mefficano, e gli fpropofiti del Sig. de PaW, e a altri Autori Straneri intorno alla popolazione di quei paefi, mi hanno coltretto a far quefta Differiazione per mettere in chiaro il vero: lo che procurerò di fare con tutta la bre: virà poffibile.

$$
\text { 5. I. }
$$

\section{Sopra i confini Dei Regni di Anabuac.}

Il Solis, tenendo dietro a parecchi Scrittori Spagnuoli mal informati, afferma, che l'Imperio Mefficano fi ftendeva dall'Iltmo di Panamà fino al Capo Mendocino nella California. Il P. Touron, Domenicano Francefe, volendo nella fua Storia General d'America ampliare ancor più quei termini, dice, che tutti i paefi fcoperti nell' America Settentrionale erano fottopofti al Re di Meflico: che l'eftenfione di quell' Imperio da levante a ponente era di leghe cinquecento, e da tramontana a mezzogiono di leghe dugento, o dugento cirquania: che $\mathrm{i}$ fuoi termini erano a tramontana l'Oseano Atlantico, a ponente il golfo d'Anian, a mezzogiorno il mar Pacifico, ed a levante l'lftmo di Panamà; ma oltre agli errori gengrafici, che havvi in quefta defcrizione, v'è ancora della contraddizione; poichè fe mai foffe vero, che quell' Imperio fi ftendeva dall' litmo di Panamà fino al golfo, o piutrofto ftresto d'Anian, la fua eftenfione non farebbe ftata di fole cinquacento leghe, ma an. cor di mille, mentre non vi farebbono ftati comprefi meno di cinquanta gradi.

Storia Antica del Mefjico Tom. IV. I 1 I2 
La cagion di tali errori fi è, perchè érano perfuafi quefti Autori, che in Anahuac non v'era altro Sovrano che quello di Meffico: che i Re di Acolhuacan, e di Tlacopan foffero fudditi di lui, e che $\mathrm{i}$ Michuacanefi, ed $\mathrm{i}$ Tlafcallefi appartenenti anch'effi a quella Corona, fi foffero poi ribellati. Ma non è così: poichè niuno de' fopraddetti Stati appartenne mai al regno di Meffico, ficcome confta dalla depofizıone di tutti gli Storici Indiani, e di tutti quegli Scrttori Spagnuoli, che da effo loro prefero informazione, come Motolinid, Sahagun, e Torquemada. Il Re d'Acolhuacan era ttato fempre alleato di quello di Meffico infin dall' anno 1424; ma non ne fu mai fuddito. E' vero, che quando vi gunnfero gli Spagnuoli, il $\mathrm{Re}$ Cacamatzin pareva dipendere da Motezuma fuo zio; perchè a cagione della prepotenza del fuo fratello Ixtlilxochitl abbifognava dell' ajuto de'Mefficani. Gli Spagnuoli poi videro Cacamatzin venir loro incontro, come Ambafciatore del Re di Meffico, e fervire anche a coltui da bracciere. Videro altresi condurlo prigione a Meffico per ordine di Motezuma. Tutro ciò rende fcufabile per molti capi l'errore degli Spagnuoli ; ma egli è certo, che quelle dimoltrazioni fatre da Cacamatzin a Motezuma non erano fervizj di vaffallo verfo il fuo Re, ma offequj di Nipote verfo il fuo Zio: e che Motezuma nel farlo pigliare per compiacere agli Spagnuoli s'arrogó quell' autorità, che non gli conveniva, e fece a quel Re un graviffimo torto, del qual ebbe poi a pentirfi. Quanto al Re di Tlacopan è vero, che egli fu creato $\mathrm{Re}$ dal $\mathrm{Re}$ di Meffico; ma gli fu ac. cordato un perfetto dominio e piena fovranità ne'fuoi Stati colla fola condizione d'effere perpetuo alleato de' Mefficani, e di dar loro ajuto colle fue truppe, ogni volta che bilognaffe. Il Re di Michuacan, e la Repubblica di Tlafcalla furono mai fempre rivali, e nemici capitali de'Mefficani, e non v'è memoria, che nè l'uno, nè l'altro Stato fofle mai fottopolto alla Corona di Meffico.

Lo fteffo dobbiamo dire di molti altri paefi, che dagli Storici Spagnuoli furon creduti provincie dell' Imperio mefficano. Come era polfibile, che una Nazione, che era ridotta ad 
una fola Città fotto il dominio de' Tepanechi, foggiogaffe in meno d'un fecolo tanti Popoli, quanti ve n' erano dall' Iftmo di Panamà fino alla California? Tutto ciò, che in realtà fece. ro i Meflicani, quantunque affai meno di quello, che dicono i fuddetti Autori, fu una cofa in vero forprendente, e non farebbe credibile la rapidicà delle loro conquilte, fe non veniffe confermata con tanti innegabili documenti. Del refto nè dalla narrazione degli Storici Indiani, nè dall' enumerazione degli Stati conquiftati da'Re di Meffico, che trovafi nella Raccolta di Mendoza, nè dalla matricola delle Città tributarie, efpofta nella medefima Raccolta, fi può aver niun fondamento da confermare quell'arbitraria ampliazione de' Dominj mefficani; anzi confta tutto il contrario dal ragguaglio di Bernal Diaz. Quefti nel cap. 93. della lua Storia dice così: "Avea il gran Mo"tezuma molti prefidj, e gente di guerra nelle frontiere de' "fuoi Stati. Uno ne avea in Soconufco per difenderfi da Gua"timala, e da Chiapa: un altro per difenderfi da' Pauuchefi tra "Tuzapan e quel luogo, che noi appelliamo Almeria: un altro " in Coatzacualco, e un altro in Michuacan. " (a)

Siam dunque ficuri imprima, che i Dominj mefficani non fi ftendevano verfo Scirocco di la da Xoconochco, e che niuna di tutte quelle Provincie, che oggidi fon comprefe nelle tre Diocef di Guatimala, di Nicaragua, e di Honduras apparteneva all' Imperio Meflicano. Nel libro 4. della Storia abbiam detto, che Tliltototl, celebre General Mefficano, negli ultimi anni del $\operatorname{Re}$ Ahuitzotl porto le fue armi vittoriofe fino a Quauhtemallan: ma quivi ancora aggiugniamo, che non fi $f a$, che reftaffe allora quel paefe fottomeffo alla Corona di Meffico:anzi dalla Storia appare tutto l'oppofto. Torquemada nel lib. 2. cap. 8 I. fa menzione della Conquifta di Nicaragua fatta da'Mefficani; ma quello fteffo, che nel citato luogo afferma d'un efercito meflicano a' tempi di Motezuma II., attribuifce nel lib.z. cap. 10. ad una colonia ufcita molti anni prima per ordine degli

$$
\text { L. } 12
$$

Dei

(a) Per intender meglio ciò, che diciamo intorno a' confini de'regni di Anahuac, converrà metterfí fotto gli occhi le noltre carte geograficbe. 
Dei dalle vicinanze di Xoconochco: per lo che non fi dee far conto del fuo ragguaglio .

Lo Ateffo Bernal Diaz tanto nel luogo citato, quanto nel cap. 66 . afferma efpreffimente, che i Chiapanefi non furono mai foggiogati da'Mefficani; ma ciò non può intenderfi di tutto il paefe de'Chiapanefi, ma d'una fola parte; poichè fappiamo dal Remezal, Cronichifta di quella Provincia, che i Mefficani aveano prefidio in Tzinacantla, e ci confta dalla Matricola de' triburi, che Tochtlan, $\left(^{*}\right)$ ed altre Città di quel paefe erano tributarie de' Mefficani.

Dalla parte di greco non fi avanzarono i Mefficani oltre a Tuzapan, come confta dal paffo citato di Bernal Diaz: e fap. piamo di certo, che i Panuchefi non furono mai fottopofti a' Meficani. Dalla parte di Levante abbiam fiffari i confini nel fiume Coatzacualco. Bernal Diaz dice, che il paefe di Coatza. cualco non era provincia di Meffico: da un altro canto trovizmo tra le Citta tributarie di quella Corona Tochtlan, Michapan, ed altri luoghi della fopraddetca provincia. Noi pertanto fiamo perfuafi, che i Mefficari poffedevano tutto cid, che era a ponente del fiume Coatzacualco, non però ciò, che v' era a levante, e che quel fume era per quella parte termine del loro Impesio. Verfo tramontana era quefto riftrerto dal paefe degli Hudxtechi non mai foggiogato da' Mefficani. Verfo greco non fi itendeva l'imperio oltre alla provincia di Tulla: tutto qued gran tratto di terra che vi era di là da quella provincia, era occupato da'barbari Osomiti, e Cicimechi, i quali nè avéano veruna popolazione, nè ubbidivano a verun Sovrano. Dalla parte di ponente fi fa che terminava l'Imperio in Tlaximalojan, frontiera del regno di Michuacan; ma nelle maremme frno all' eftremità occidentale della provincia ài Coliman, e non più oltre. Nel Catalogo delle Cirtà tributarie vedonfi Coliman: ed altri luoghi di quella provincia, e niuno di quelli, che fo.

no

(*) V'erano, e vi fono tre luoghi almeno appellati Tocbtlan ( preflo gli SpaEniroli Tmfla) il primo nella Provincia di Cbiapa, il fecondo in quella di Xoa conosbo, a Soconurca, e it terzo in quella di Coatzacualco. 
no di là da effa: e nemmeno fe ne fa menzion nella Storia di Meflico. I Mefficani non aveano che fare colla California, nè poteano afpettar verun vantaggio dalla conquifta d'un paefe tanto lontano, il più fpopolaro, e il più miferabile del Mondo. Se quell'arida e faffola penifola foffe giammai ftata provincia dell' Imperio Meflicano, vi fi farebbono trovate alcune popolazioni; ma egli è certo, che non vi fi trovò ne anche una cafa, nè verun avanzo o traccia d'effa. Finalmente dalla parte di mezzogiono s'erano impadroniti i Mefficani di tutti qua grandi Stati, che v'erano dalla Valle Mefficana infino al mar pacifico. Ora ftendendofi le maremme mefficane da Xoconochco fino a Coliman, quivi appunto era la maggior lunghezza de? lor dominj.

Il Dott. Robertfon dice, che "i territorj appartenenti ai " Capi di Tezcuco, e di Tacuba appena cedevano in eftenfio. "ne a quelli del Sovrano del Meffico., (b) $\mathbf{M}_{\text {a }}$ quefto è troppo lontano dal vero, e contrario altresi a ciò, che ne dicono tutti gli Storici del Meflico. Il regno di Tezcuco, o fía d'A. colhuacan era a ponente riftretto parte dal lago di Tezcuco, e parte da Tzompanco, e da altri Stati Meficani, ed a levante dai Dominj di Tlafcalla: ficchè non potea avere da ponente a levante più di feffanta miglia: a mezzogiorno era riftretto dallo Stato di Chalco appartenente anch'effo a Meffico, ed a tramontana dal paefe indipendente degli Huaxtechi. Or dalla frontiera di quefto paefe a quella di Chalco vi fono dugento miglia incirca. Ecco tutta l'eftenfione del regno d'Acolhuacan, la quale non fa nè anche l'ortava parte di quella dei Dominj mefficani. Gli Stati del Regolo di Tlacopan, o Tacuba, erano sì piccoli, che non meritavano il nome di Regno; poichè dal lago mefficano a levante fino alla frostiera di Michuacan a ponente, non avea più d'ottanta miglia, nè più di cinquanta dalla Valle di Toloccan a mezzogiono al paefe degli Otomiti a tramontana. E' dunque un errore il parax gone

(b) Storia dell America lib. I. 
270 DISSER T AZIONE VII.

gone fatio del Robertfon dei Dominj d'Acolhuacan e di Tlacopan con quelli di Melfico.

La Repubblica di Tlafcalla, circondata dai Dominj meffie cani e tezcucani, e dagli Stati d'Huexotzinco, e di Cholulla, era sì riftretta, che da levante a ponente appena avea miglia cinquanta, e da mezzogiorno a tramontana trenta in circa. Non ho trovato verun Autore, che dia maggior eftenfione a quelio Stato, fe non Cortès, il qual dice, che i Dominj di Tlafcalla aveano leghe novanta di circuito; ma quefto è un manifefto sbaglio.

Intorno al regno di Michuacan neffuno, che io Cappia, ne ha accennati tutti gli antichi confini, fuorchè il Cav. Bo. turini. Quefto Autore dice, che l'eftenfione di quel regro, dalla Valle d'Ixtlahuacan preffo a Toloccan fino al mar pacifico, era di leghe cencinquanta, e da Zacatóllan fino a Xichù di leghe centofeffanta: e che nei Dominj Michuacanefi erano comprefe le Provincie di Zacatollan, di Coliman, e quella, che gli Spagnuoli appellarono Pravincia d'Avalos, fituata a maeltro di quella di Coliman. $\mathrm{Ma}$ in tutto ciò s'ingannò il citato Cavaliere; poichè fi fa ficuramente, che il regno di Michuacan non a veva i fuoi confini in Ixtlahuacan, ma in Tlaximalojan, fin dove arrivavano i Dominj mefficani. Si fa dalla matricola dei tributi, che le provincie marittime di Zacatollan, e di Coliman appartenevano a Meffico. Finalmente non poteano i Michuacanefi ampliare i fuoi Dominj fino a Xichù fenza foggiogar prima i barbari Cicimechi, che occupavano quella contrada; ma di quefti lappiamo, che non furono foggiogati, che dagli Spagnuoli molti anni dopo la conquifta di Meffico. Non era dunque tanto grande il regno di Michuacan, quanto credette il Cav. Boturini. La fua eftenfione non com: prendeva, che tre gradi in circa di longitudine, e poco più di due di laticudine.

Quanto finora abbiam detto giova a dimoftrare l"efartezza della noftra defcrizione, e delle noftre carte geografiche intorno ai confini di quei regni, fondata fu la fteffa Storia, fu la matricola dei tributi, e fu la teftimonianza degli Storici antichi . \$. II 
๑. II.

\section{Sopra la Popolaxione di Anabuac.}

Non pretendo di ragionar qui della popolazione di tutta l'America; perchè tal argomento farebbe troppo vafto, ed alieo no altresì dal mio propofito, ma folamente di quella del Meffico che appartiene alla mia Storia. Nell' America vi erano, e vi fono dei paefi molto popolati, e vi fono ancora dei valti deferti : e non meno s'allontanano dal vero coloro, che s'immaginano i paefi del nuovo Mondo tanto popolati, quanto quelli della China, che quegli altri che gli credono tanto fpopolati, quanto quelli dell' Africa. Tanto incerto è il calcolo del P. Riccioli, quanto quelli di Sufmilch, e del Sig. de PaW Il P. Riccioli conta nell' America trecento milioni d'abitatori. Gli Aritmetici politici non ve ne contano, dice il Sig. de Paw, più di cento : Sufmilch in un luogo della fua opera vi computa cento, $e$ in un altro cencinquanta milioni. Il Sig. de P., il qual rapporta turti quefti calcoli, dice, che non vi fono di veri Americani, che da trenta in quaranta milioni. Ma tutri quefti calcoli fono, torno a dire, incertiffimi, e non fi appoggiano a verun fondamento; imperocchè fe non fi fa finora, neppure a un dipreffo la popolazione di quei paefi, nei quali fonofi ftabiliti gli Europei, come quelli del Meffico, di Guatimala, del Perù, del Quito, di Terraferma, del Chile ec. chi farà capace d'indovinare il numero degli abitatori delle immenfe contrade niente o poco conofciute dagli Europei, come quelle che fono a tramontana e a maeftro della Coahuila, del nuovo Meffico, della California, e del fiume Colorado, o fia roffo nell' America Settentrionale? Chi potrà numerar gli abitatori del nuavvo Mondo, mentre non $f_{1} \cdot f_{a}$, nè fi può $f_{1}$ peIs neppur il numero delle provincie, e delle Nazioni, che vi fi contengono? Lafciando dunque fiff $f_{t t i}$ calcoli, i quali non poffono intraprenderfi fenza temerità, ci contenteremo d'efaminare ciò, che dicono il Sig. de Paw, e il Dott. Robertfon fu la popolazione del Meffico. 
"La popolazione del Meffico e del Perù è Itata, dice il s) Sig. de Paw, indubitatamente efagerata dagli Scrittori Spa9) gnuoli, avvezzi a dipignere gli oggetri con proporzioni fmi9) furate. Tre anni dopo la conquifta del Meffico ebbero d'uo9 po gli Spagnuoli di farvi paffar gente dalle ifole Lucaje, e 9) pofcia dalle cofte dell' Africa per popolare il regno di Meff" co. Se quelta Monarchia conteneva nel I 18 . trenta milio"ni d'abitatori, perchè nel 152I. era fpopolata?", Io non ne. gherò mai, che tra gli Scrittori Spagnuoli vi fieno ftati alcuni elageratori, ficcome ve ne fono ancora Ptari tra' Pruffiani, tra' Francefi, tra gl'Inglefi, e tra gli altri Popoli; perchè la fmo. derara brama d'aggrandir le cole, che fi defcrivono, è una paflione affai comune a tutte le Nazioni del Mondo: dalla quale certamente non s'è prefervato $\|$ Sig. de $\mathrm{P}$., come il fa palefe in tutta la fua opera: ma tacciarne curti gli Spagnuoli all' ingroffo è fare un gravifimo torto a quella Nazione, la quále ha, come tutte le altre, del buono, e del cattivo. Io almeno dopo aver letti migliori Storici delle Nazioni colte d'Europa non ne ho trovari due, i quali ni pajnno paragonabili quanto alla fincerita (c) coi due fpagnuoli Mariana. ed Acolta, fommamente ftinati perc̀, ed a cagione lodari anche dai nemici della lor Nazione, e della lor Religione. Tra gli antichi Storici del Meflico vi fono ftati alcuni, ficcome l'Acofta, il Bernal Diaz, e lo fteflo Cortès, della cui fincerisa non fi può dubitare. Ma avvegnachè cialcheduno di quegli Autori non foffe ftaso fornito di quelle qualità, che richiedonfi per meritar la noftra fede, tuttavia l'uniformità delle loro teftimonianze formerebbe un efficaciffimo argomento in favor della verica: del loro ragguaglio. Gli Autori poco veritieri non $f_{1}$ accordano mai fra loro, fe non quando copiano gli uni dagli altri; ma çio non avvenne ai notri Storici, i quali intenti folamente a fc:ivere quello, che aveano veduto coilor occhi, o aveano trovato vero per le loro informazioni, non fi curarono di ciò, che

(c) Parlò qui colamente della fincerità perchè fa al mio pronofiro: del re fto que' due Storici hanno altri pregj, che gli rendono oltremodo fimabili. 
che aveano fcritto gli altri; anzi apparifce dalle fteffe lor opere, che allorchè fcrivevano, non aveano fotto gli occhi gli fcritti altrui. Lo fteffo Sig. de P., ragionando in una fua lette. ra (d) di quel rito, che aveano i Mefficani, di confacrare e mangia:e la ttatua di pafta del Dio Huitzilopochtli, da lui appellato Vitzil.pultzi, e di quello de' Peruani nella fefta Capac.rai$m e$, dice cosi al fuo corrifpondente: Io vi confeffo, cbe $l u$ nanime teftimonianza di tutti gli Scrittori Spagnuoli non ci permerte dubitarne $\mathcal{O}_{c}$. Ora fe il confenfo degli Storici Spagnuoli intorno a ciò, che non videro co'lor occhj, non permette dubitarne, come potrà dubitarfi di quello, che eglino depongono come teftimonj oculari?

Vediamo danque, che cofa dicano della popolazione del Meffico gli artichi Scrittori Spagnuoli. Turti concordano nell' affermare, che que' paefi erano affai popolati, che v'erano moltiffeme Città grandi, e infiniti borghi, e cafali : che ne'mercati deile Cirta poprolofe concorrevano molte migliaja di negozidnti: che metrevano in piede eferciti numerofiffimi \&c. Cortès nelle fue letrere a Carlo V., il Conquiltartore Anonimo nelia fua Relazione, Alfonfo d'Ojeda, ed Aifinio de Mata nelle lo. ro Memorie, Monfig. de las Cafas nell' upera, che porta il titolo Della Difruzione delle Indie, Brndi Diaz nella fua Sto. ria, Motolinia, Sahagun, e Mendieta ne'loro furitti, tutti teftimoni oculari dell'antica popolazione del Meffico: Herrera, Gomara, Acofta, Torquemada, e Martinez tutu fon d'accordo intorno alla gran popolazıone di que' psefr. Non può allegarmi il Sig. de $P_{a}$ w neppure un fol Autore antico, che nol confermi colla fua teltimunianza, laddove io poffo citargli pa. recchj Scrittori, che non fanno menzione di quel rito luperitiziofo de' Meflicani, come Cortès, Bernal Diaz, e il Conquiftatore Anonimo, i tre più antichi Storici Spagnuoli del Meffico. Contuttociò afferma il Sig. de Paw, che non può dubitarfi di tal rito per cagione dell' unanime teftimonianza degli altri Spagnuoli: perchè dunque vorrà dubitare della gran popolazione Storia Antica del MefJlco Tom. IV. M m del

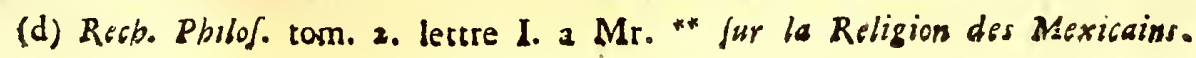


del Meffico, anzi negarla arditamente contra l' uniforme depo: fizione di tutti gli Storici antichi? Ma fe era sì grande la popolazione del Mieffico nel 1518., perchè nel 152 I. bifognò condurvi gente dalle Ifole Lucaje, e pofcia dalle colte dell' Africa per popolarlo? Confeffo ingenuamente, che non poffo legger queta obbiezione del Sig. de P. fenza fdegnarmi in vedendo una tal arditezza nell' affermare ciò, che è affolutamente falfo, e contrario affatto al ragguaglio degli Aucori. Ove mai ha letto il Sig. de P., che per popolare il Meffico foffe bifogno di trafportarvi gente dalle Lucaje? Lo sfido a produrmi un fol Autore, che il dica; anzi da molti Scrittori fappiamo tutto il contrario. Sappiamo dal Cronichifta Herrera, e da altri Scrittori, che dal 1493. nel qual anno fi At abilirono gli Spagnuoli nell' ifola di $S$. Domenico f́so al I496. perì per la guerra, e per altri gravifimi difagi la terza parce degli abitatori di quella grand'ifola. (e) Nel 1507. non v'era rimafto più della decima parte degl' Indiani, che v' erano nel 1493. come ne $f a$ fede Monfig. de las Cafas teftimonio oculare, $(f)$ e d'allora innanzi fi andò fcemando a tal fegno la popolazione di quell' ifola, che nel $154^{\circ}$. appena vi reltavano dugento India. ni: per lo che fin dal principio del fecolo XV. cominciarono gli Spagnuoli a tirare migliaja d' Indiani dalle Lucaje per rifar la popolazione del!a Spagnuola; $m a$ effendo ancor quefti periti cominciarono prima della conquitta del Meffico a condurvi popolatori da Terraferma, e da altri paefi del Continente d'America, fecondo che s'andavano fcoprendo. Si fa da una lettera fcritta al Configlio delle Indie dal primo Vefcovo di Meffico, allegata all' Imperatore Carlo V. da Monfig. de las Cafas, che il crudele Nugno Guzman, Governatore di Panuco, mandò quindi ventotto vafcelli carichi d'Indiani fchiavi da vendere nelle ifole: ficchè è tanto lontano dal vero, che gii Spagnuoli

(e) Dec. I. lib. 2. cap. I 8.

(f) Della Difruzione delle Indie. Tutto ciò, che diciamo quì, confta non meno per la teftim rnianza dello ftefo Monfig. de las Cafas nellopera intitolata: Il fupplice Schiavo. Indiáno,e in altre, che per quella del Cronichifta Her rera nelle fue Decade. 
gnuoli conduceffero gente dalle ifole per popolare il Continen: te dell' America fettentrionale, che anzi da quefts trafferogente per popolar le ifole, ficcome il dicono efpreffamente $i$ due citati Autori, ed altri. E' bensi vero, che dopo la conquifta del Meffico vi furono trafportati fchiavi dall' Africa; non però perchè vi foffe bifogno di popolatori; ma perchè gli Spagnuoli fe ne volevano fervire per la fabbrica dello zucchero,e per li lavori delle miniere, a'quali nón poteano coltrignere gli Americani, attefo le leggi allora recentemente pubblicate. E' dunque falfo, e contrario alla depofizione de' fuddetti Autori, che il Meffico foffe tanto fpopolato tre anni dopo la conquifta, che foffe d'uopo di farvi paffar gente dalle Lucaje, edall' Africa per tornarlo a popolare; anzi fiamo ficuri per lo contrario, che da' paefi fottopofti già al Re di Meffico, e alla Repub. blica di Tlafcalla, fi mandarono colonie alcuni anni dopo la conquilta per popolare altri paefi, ficcome Zacatecas, S. Luigi Potosi, il Saltillo \&c.

Ma vediamo, che cofa dicano in particolare della popola. zione del Meffico quegli antichi Scritturi. Io non fo, che alcun di loro abbia avuto l'ardire d' efprimere il numero degli abitatori dell' Imperio Meficano: fe effo conteneva, o no trenta milioni, ciò porea folamente faperfí da' Re di Meffico, e da' lor Miniftri: e benchè da coltoro fe ne poteffero informar gli Spagnuoli, niuno, che io fappia, fe ne informò. Ciò, che parecchj di quegli Autori affermarono, fi è, che tra i Feudatarj della Corona di Meffico ve n'erano trenta, ciafcheduno de'quali avea intorno a cento mila fudditi, ed altri tre mila Signori, i quali aveano un minor numero di vaffalli. $(g)$ Lorenzo surio afferma $(b)$ ciò conftare da' documenti, che erano nell' archivio Reale di Carlo V. Cortès nella fua prima lettera allo fteffo Imperatore gli dice così: „E' sì grande la moltitudine "d'abitatori in quefti paef, che non v'è neppur un palmo $M$ m 2 "di

(g) Vedanfi Gomara nel cap. 76. della Cronaca della N. Spagna, ed Hersera nella Dec. 2. lib. 7 . cap. 12.

(b) Surius in Commentario brevi rerwm in Orbe geftarkm ab anno 1500. ad I 568. 


\section{DISSERT AZ I O NE VII.}

"di terreno, che non fia coltivato; ma contuttocio v'è mol"ta gente, che per mancanza di pane va mendicando per le " cafe, per le ftrade, e per li mercati. "Simile idea ci danno in generale della popolazione del Meffico Bernal Diaz, il Conquiltatore Anonimo, Motolinia, ed altri teftimoni oculari.Venendo ora a'paefi particolari d'Anahuac fiam ficuri per la de. pofizione de'fuddetti Scrittori, e di quafi tutti gli antichi, della gran popolazione della Valle Mefficana, de'paefi degli Oromiri, de' Matlatzinchi, de' Tlahuichi, de' Cohulxchı, de' Miztechi, de' Zapotechi, e de' Cuitlatechi, della Provincia di Coatzacualco, de' regni d'Acolhuacan, e di Michuacan, e degli Stati di Tlafcalla, di Cholulla, d' Huexotzinco \&c.

La Valle Mefficana, contuttochè i laghi ne occupaffero una gran parte, era almeno tanto popolata, quanto il più po. polato paefe d'Europa. V'erano quaranta Città confiderabili, da noi altrove nominate, $e$ mentovate ancora dagli antichi Scrittori : gli altri luoghi abitati d'effa erano innumerabili, i nomi de' quali farebbono quì da noi efpreffi, fe non temeffimo di annojare i Lettori. Il finceriffmo Bernal Diaz defcrivendo nel cap. 88. della fua Storia ciò, che andavano vedendo nel fuo cammino per la valle mefficana verfo la Capitale, dice cosi: "Allorchè vedemmo cofe tanto maravigliofe, non fapeva. "mo che dirci, nè fe era vero quello, che avevamo forto gli "occhi: perchè vedevamo tante gran Citta polte in terra fer= " ma, e molte altre nel lago, e tutto pieno di barche. "Di. ce inoltre, che alcuni foldati fuoi compagni oltremodo maravigliari in vedendo tante e s'̀ belle popolazioni, dubitavano, fe erano fogni, o cofe d'incantefimo quelle, che vedevano. Quefta, e molre altre confeffioni fincere di Bernal Diaz baftano per rifpondere al Dott. Roberton, il qual fi prevalfe di certe parole di quell' Autore da lui mal intefe per far credere a' fuoi Lettori, che la popolazione del Meflicn non era sì gran. de, come fi vuole.

Intorno alla popolazione dell' antica Capitale v'è una gran varietà di pareri : nè pud avvenire altrimenti, qualora $f$ vuol giudicare ad occhio della popolazione d" una gran città; ma 
tutti gli Scrittori, che la videro, o furono informati da teft:monj oculari, fon d'acordo nel dire, che effa era affai grande. Il Cronichifta Herrera dice (i), che era grande al doppio di Milano: Cortès afferma, che era tanto grande come Siviglia, e Cordova: (k) Lorenzo Surio (l) citando certi documenti, che erano nell' Archivio Reale di Carlo V., dice, che la popolazione di Meflico fi componeva di I30. mila cafe: Torquemada, tenendo dierro a Sahagun, e ad alcuni Storici Indiani, vi numera 120 . mila cafe, $(m)$ e foggiugne, che in ciafcheduna cafa v' erano da quattro in dieci abitatori. Il Conquiftatore A. nonimo ne parla così: "Può avere quefta Città di Temiftitan ") più di due leghe e mezza, o preffo a tre, poco più o me. " no di circuito: la maggior parte di coloro, che l'hanno ve. "duta, giudica, che vi fieno più di feffanta mila fuochi, e "piutrofto più, che meno.", Quefto calcolo adottato da Gomara, e da Herrera mi par quelío, che più s'accofta al vero, attefo. la eftenfione de!la città, e la maniera d'abitar di quelle genti.

Ma tutto quefto vien contraddetto dal Sig. de Paw. Egli appella ", ecceffiva e ftravagante la defcrizione fattaci di quelta "Città americana, la quale conteneva, al dir d'alcuni Auto. "ri, fettanta mila Cafe ai tempi di Motezuma II : ficchè avrà " allora avuti trecento cinquanta mila abitatori, laddove è no" torio, che la Città di Meffico, confiderabilmente accrefciutz "Sotto la dominazione degli Spagnuoli, non ha prefentemen"te, che feffanta mila abitanti, comprefivi venti mila Negri, "e Mulati.," (n) Ecco un altro paffo delle Ricercbe Filofoficbe, che farà ridere $\mathrm{i}$ Mefficani. Ma chi non riderà in veden. do un Filofofo Pruffiano, tanto impegnato nello fcemar la po. polazione di quella gran Città americana, e fdegnato contra coloro, che la rapprefentaro più grande di quello, che egli vuo-

(i) Dec. 2. lib. 7. cap. 13.

(k) Lettera $I_{n}$ all' Imper. Carlo V.

(l) Surius in Commentario brevi $\omega_{\text {i }}$

(m) Monaxchia Indiana lib. 3. cap. 24.

(a) Rech. Pbilof. part. s. Tect. 


\section{D I S S E R T A Z I O N E VII.}

vuole? Chi per altro non fi maraviglierà nel fentire, che \& notorio in Berlino il numero degli abitanti di Meffico, mentre in Meffico non l'era poco tempo fa neppure agli fteffi Parrochi, che ogni anno ne faceano la numerazione? Io però vo. glio dare al Sig. de P. alcune notizie ficure di quella Citta americana, affinchè poffa nell'avvenire fchivar quegli errori, ne'quali è incorfo in parlando della popolazione di effa.

Sappia dunque egli, che Meffico è la Città più popolofa di tutte quante ne ha il Re Cattolico ne' fuoi valti dominj. Dalla nota de' nati, e de' morti in Madrid, e in Meffico pubblicata ne' Diarj dell' una, e dell' altra Cirtà apparifce, che il numero degli abitatori di Madrid è più d'un quarto minore di quello di Meffico: (o) ciò̀ fe Madrid per efempio ha 160. mila abitanti, Meffio ne ha fenza dubbio più di 200. mila. V'è ftata una gran diverfira d'opinioni intorno al numero d'anime della moderna Citià di Meffico, ficcome vi fu intorno all' antica, e ficcome parimente v'è intorno ad altre Citrà di primo ordine, ( $p$ ) ma effendovifi fatta in quefti ultimi anni con maggior diligenza la numerazione tanto dalla parte dei Parrochi, quanto da quella dei Magittrati, s'è trovato, che gli abitatori di quella capitale oltrepaffano $i$ dugento mila, benchè non fe ne pofa fapere appunto l'ecceffo. Si pud aver qualche idea della popo-

la-

(o) Egli è certo, che a proporzione dell' ecceffo d'una città fopra un 'altra nel numero de'nati, e de' morti, ne farà ancor l'ecceffo del numero degli abitatori: e non v'è mezzo più ficuro di ritrovare a un dipreffo il numero degli abitatori d' una città troppo grande, che quello di fapere il nnmerode' na$t i$, e de' morti in effa, purchè fi adoprino quelle precauzioni, che vi fi richieggono.

(p) Bafta fapere la diverfità d'opinioni, ch' v'è tra i moderni Scrittori, intorno alla popolazione di Parigi: chi vi conta cinquecento mila abitanti, chi fettecento mila, chi un milione. Parimente diverfe fono fate le opinioni intorno alla moderna Meffico. Lionnel Waffer, celebre Viaggiatore Inglefe del fecolo paffato, credette, che vi foffero trecento mila abitanti: al Viaggiatore Gemelli parvero centomila, ed al Miffionario Tallandier feffanta mila : un moderniffimo Viaggiatore Europeo, il qual andò nel Meffico dopo aver viaggiato per l'Europa, e per li principali paefi dell' Afia, fu di parere, che non v'era meno d'un milione e mezzo d'abitanti. Quefti fpropofito per eccefo, e Tallandier per difetto. 
lazione di quella Città dalla quantità di pulque, $(q)$ e di tabacco, che quotidianamente vi fi confuma. ( $r$ ) Ogni giorno vi entrano più di fei mila arrobas di pulche, cioè cento novanta mila libbre romane: nell' anno I774. vi entrarono $2.214 .294 \frac{1}{2}$. arrobas, cioè più di fettanta tre milioni di libbre romane; ma in quefto computo non fi comprende quello, che vi fi introduce di contrabbando, nè queilo, che vendono gl'Indiani elenti nella piazza principal della Città. Quefta sì gran quantità di pulche confumafi preffochè dai foli Indiani, e Mulati, il cui numero è forpaffato da quello dei bianchi Europei e Cieogli; tra i quali non fon molti quelli, che ufano abirualmente quella bevanda. Il dazio fopra effa afcende annualmente nella lola capitale a dugento ottanta mila fcudi (pefos fueries) in circa. It confumo di tabacco da fumare in quella capitale importa ogni giorno 1250. Fcudi in circa: ciò che in un anno forma la forn. ma di fcudi quattrocento cinquanta mila e più. Ma bilogna fapere, che tra gl' Indiani fon rari quelli, che ufano il tabacco: tra i Creogli e gli Europei fon moltifimi coloro, che non l'u. fano, e tra i Mulati alcuni. Or chi farà, che voglia dar maggior fede al calcolo del Sig. de PaW, che alle Ateffe matricole di quella Capirale, e che pregi più il giudicio d'un moderno Pruffiano tanto Itravagante intorno all' antica popolazione di quella Corre, che quello di tanti antichi Scrittori, che la videro coi proprj lor occhi?

Per ciò, che riguarda la Città e Corte di Tezcuco, fafo piamo dalle lettere di Cortès a Carlo V., che effa avea intorno a trenta mila cafe; ma ciò debbe intenderfi della fola Coro te; poichè prefa infieme colle altre tre città di Coatlichan, Huexotla, ed Arenco, le quali, come teftifica lo fteffo Corrès, parevano formare una fola popolazione, era di lunga mano più

gran.

(q) Il Pulque ( in ital. direno Pulche) è il vino, o piuttofto birra, piu ufuale de'Mefficani, fatro dal rugo fermentato del Maghei, come abbiamo detto nella Storia. Quefta bavanda non pudguardarfi per un altro giorno: e perd ogni giorno fi confuma tutta quella, che vi fi introduce.

(r) La nota del confumo diario di pulche, e di tabacco in Meffico è prefa da una lettera d'uno de'principali Computilti di quella dogana fcritta ai 23. Febbrajo 57 s. 
grande di Meffico. Torquemada, tenendo dietro a Sahagun, ed ai ragguagli degl' Indiani, afferma, che la popolazione di quelle quattro Citta conteneva cento quarantamila cafe: dal qual numero ancorchè voleffimo levar la metà, refterebbe nulla di meno una popolazione affai grande. Niuno Storico ci ha detro quanta folfe la popolazione della Corte di Tlacopan, benchè tutti affermino, che era confiderabile. Di quella di Xocbimilco Cappiamo, che era la nuaggior di tutte dopo le Corti. Di quella d'Iztapalapan afferma Cortès, che avea da dodici in quindici mila fuochi: di Mixcoac dice, che ne avea fei mila incirca, d'Huitzilopocbco, da quattro in cinque mila, d'Acolman, e d'Otompan ciafcheduna quattro mila, e di Mexicaltzinco tre mila. Chalco, Azcapozalco, Cojoacan, Quaubtitlan erano prù grandi fenza paragone di queite ultime Citrà. Tutte quelte, e molriffime altre popolazioni erano comprefe nella fola Valle Mefficana: la veduta delle quali cagionò non minore ammirazione, che paura ai Conquiftatori, allorchè offervarono la prima volta dalle cime de'monti quella deliziola valle. Lo fteffo accadde loro, quando videro la popolazione di Tlafcalla. Cortès nella fua lettera a Carlo V. parla così di quella Citt̀̀ :, Effa è così grande, e maravigliofa, che benchè io trala" fci molto di ciò, che ne potrei dire, quel poco, che ne di, rò, credo, che farà incredibile; perchè e affai più grande, $e$ " più popolata di Granata, allorchè fu tolta ai Mori, affai piu "forte, di sl buoni edifizj, ed affai più abbondante di rutto. Similmente ne parla il Conquiftatore Anonimo: "Vi fo"no, dice, di gran Citrà, e tra le altre quella di Tlafcalla, "la quale in alcune cofe s'affomiglia a Granata, $e$ in altre a "Segovia; ma è più popolofa di alcuna di effe.," Dl Tzimpantzinco, Città di quella Repubblica, afferma Cortès, (s)che effendovifi fatta per ordin fuo la numerazione, vi fi contaro. no più di venti mila cafe. D' Huejotlipan, luogo ancor effo della medefima Repubblica, dice, che avea da tre in quattro mi-

(f) Cortès parla di quefta Citrà fenza nominarla; ma dal contefto appare che ar foffe Tzimpantzinco, e Torquemada lo dice efpreffamente. 
mila fuochi. Di Cholulla afferma lo fteffo Cortès, che avea intorno a venti mila cafe, e quafi altrettante in quei luoghi circonvicini, i quali erano come i fuoi fobborghi. Huexotzinzco, e Tepejacac erano emule di Cholulla nella grandezza. Quefte fono alcune popolazioni di quelie, che videro gli Spagnuoli prima della Conquilta tralafciando ancora molte altre, della cui grandezza conlta per la depofizione di-queitı, e d'altri Scrittori.

Non meno fi può dare a divedere la gran popolazione di quei paefi dəgl' in rumerabili concorfi, che vedevanfi nei mercati, dai numerofiffimi eferciti, che fi levavano, ogni volta che $v$ ' era d'uopo, e dal torprendente numero di battefimi, che vi fu dopo la conquifta. Intorno ai concorfi nei mercati, ed agli eferciti ne abbiamo detto abbaftanza nella Storia fu la fede di molti teltimonj oculari. Potrebbe fofpettarfi, che i Conquiftatori aveffero efagerato il numero delle truppe Indiane per render più gloriofe le loro conquifte, ma ciò potrebbefi da loro fare in parlando delle truppe nemiche, non però in contando le truppe con loro confederate; poichè quanto più foffe accrefciuto il numero di quefte, tanto men difficili, e men gloriofe comparir doveano le lor conquite. Eppure il Conquiftatore Ojeda numerò cencinquanta mila uomini di truppe alleate di Tlafcalla, Cholulla, Tepejacac, e Huexotzinco nella raffegna che fe ne fece in Tlafcalla per andare a por l'affedio a Meffico. Il medefimo Cortès afferma, che le truppe alleare, che l'accompagnarono alla guerra di Quauhquechollan, oltrepaffavano li cento mila, e che quelle, che l'ajutarono nell' affedio della Capitale oltrepaffarono di lunga mano li dugento mila. Dd un'altra parte gli affediati erano tanti, che effendone morti durante l'affedıo più di cencinquanta mila, ficcome abbiain detto nella Storia, ciò non oftante allorchè fu prefa dagli Spagnuoli quella capirale, e fu ordinato, che ne ufciftro tutti i Mefficani, fi videro tre giorni e tre notti continue piene le tre ftrade della gente, che ne ufciva per andare a ricoverarfi in altri lurghi, come ne fa fede Bernal Diaz, teltimonio oculare. Quanto poi al numero dei battefimi, fiam ficuri Storia Amrica del Melfico TomolV.

$\mathrm{N}$ n per. 


\section{DISSERTAZIONE VII.}

per la teftimonianza di quei medefimi Apoltolici Religiofi; che s'impiegarono nella converfion di quei Popoli, che i fanciulli e gli adulti battezzativi dai Coli Padri Francefcani ( $t$ ) dall' anno 1524 . fino al 1540 . furono più di fei milioni : i quali erano per la maggior parte degli abitatori della Valle Mefficana, e delle Provincie circonvicine. Ora in quefto numero non fon comprefi coloro, che furono-battezzati dai Preti, dai Domenicani, e dagli Agoltiniani, rra i quali ed i Francefcani fu allora compartita quell'abbondantiffima meffe, e per altro è certo, che furono innumerabili quegl' Indiani, che fi mantennero oftinati nel loro gentilefimo, o non ricevettero la fede Criftiana, che molti anni dopo. Sappiamo inoltre dalle ftrepitofe controverfie eccitatevi da alcuni Religiofi, e riportate al Ro. mano Pontefice Paolo III., che per cagione dell ftraordinaria, e non mai veduta moltitudine di Catecumeni furono coftretri i Miffionarj ad ommettere alcune ceremonie del Battefimo, e tra le altre quella della faliva, mentre dal tanto trarne, fi Seccavano loro la bocca, la lingua, e le fauci.

Dalla fcoperca del Meffico in quà è andato ognora diminuendofi il numero degl' Indiani. Oltre alle infinite migliaja perite nel primo contagio del vajuolo portatovi nel 1520 , e nella guerra degli Spagnuoli, nell' epidemia del 1545. ne morirono ottocento mila, e in quella del 1576. più di due milioni nelle fole diocefi di Melfico, Angelopoli, Michudcan, e Guaxaca: il che fi feppe dalla nota dei morti d'ogni Parrocchia prefentata al Vicerè. Contuttociò il Cronichutta Herrera, i) quale fcriffe verfo la fine del fecolo XV1., rapporta fu la fede dei documenti autentici, mandatigli dal Vicerè del Meflco, che nelle fole diocefi, d'Angelopoli, e di Guaxaca, e in quelle provincie della diocel di Meffico, che erano circonvicine alla Capitale, vi fi contavano allora 655 . Jusghi principali d'Indiani, e innumerabili altri minori da quelli dipendentı :

nei

(t) Toribio di Benavente, o fa Motolinì, uno di quegli Apoftolici Religiofi, battezzò più di quattrocento mila Indiani, il conto de'quali lafcio f́critto di rua mano. 
nei quali erano novecento mila famiglie d'Indiani tributari . (u) $\mathrm{Ma}_{\mathrm{b}}$ bifogna fapere, che tra i tributari non fon comprefi i Nobili, nè i Thafcallefi, nè altri Indiani di quelli, che ajutarono gli Spagnuoli nella Conquifta; poichè in riguardo alla lor nafcita, o ai fervizj preftati ai Conquiftatori, furono efia efenti dai tributi. Lo fteffo Herrera affai bene iftruito di quefto foggetto, afferma, che a quei tempi contavanfi nella Capitale quattro mila famiglie di Spagnuoli, e trenta mila cafe d'Indiani. Dd allora innanzi fi andò fempre diminuendo il numero degl' Indiani, e accrefcendo quello degli Spagnuoli, o fia Bianchi .

Il Sig. de P. rifponderà fecondo il fuo ftile, che tutti i documenti, da noi addotti per dimoftrar la gran popolazione del Meffico, valgono meno, che niente; perchè fono ftati prefi da Soldati rozzi e fcellerati, o da Religiof ignoranti e fuperftiziofi; ma quantunque foffero tali tutti gli Scrittori da noi allegati, ciò che è affatto falfo, tuttavia farebbe di gran momento la lor reftimonianza a cagione della loro uniformità. Chi poi potrà perfuaderfi, che Cortès, e gli Uffiziali Regi, che con effo lui fottofcriffero le fue lettere, ardiffero d'ingannare i lor Re, potendo sì facilmente effere fmentiti da tante centinaja di teltimonj, e da non pochi nemici? Sarebbe mai poffibile, che tanti Scrittori si Spagnuoli, come Indiani, tutti fi accordaffero nell' efagerare la popolazione di quei paefi, e che tra loro non vi foffe neppure uno, che rifpetıaffe la pofterità? Della veracità dei primi Miffionarj non fi può dubitare. Eglino furon uomini di vita efemplare, e di gran dottrina, fcelti tra molti per piantare il Vangelo in quel nuovo Mondo: alcuni di loro erano ftati Lettori nelle più celebri Univerfita d'Europa, aveano ottenute le prime cariche nel lor Ordine e fi erano meritate la grazia e la confidenza dell' Imperatore Carlo V. Quegli onori, ai quali rinunziarono in Europa, e quelli, che non accettarono in America $(x)$, danno chiaramente a $\mathrm{N}$ n 2 dive.

(u) Defcriz. delle Indie Occident. cap. 9. e ro.

(x) Tra i quindici primi Miflionari Francelcani vi furono fei i quali efren. do Atati nominati Vefcovi da Carlo V. non accettarono quella dignità 


\section{D ISS E R T A ZIO N E VIr.}

divedere il loro zelo difintereffato: la volontaria, e rigida lor povertà, il continuo lor tratıo con Dio, le indicibili loro $\mathrm{fa}_{\text {. }}$ tiche in tanti viaggi sì lunghi, e sì malagevoli, fatti a piedi, e fenza viatico, e in tanti sì penofi minifteri, e foprattutto l'efimia lor carità verlo quelle afflitte Nazioni piena di compaffione e di dolcezza, renderanno maifempre venerabile la lor memoria in quel Regno a difpet to del Sig. de P., e di qualunque altro maligno Scrittore, cui bafta riconofcere in un Autore la qualità di Religiofo per difpregiarlo, ed ingiuriarlo. Negli fcritti di quegli uomini immortali fi fcorge un tal carattere di fincerità, che non permette fofperti contra la verità dei lor racconti. E' vero, che effi commifero un gran peccato al giudizio del Sig. de P., bruciando come fuperftiziofe la maggior parte delle pitture foriche dei Mefficani. Io pregio più, che il Sig. de P., quelle pitture, e mi rincrefce affai più $l_{3}$ loro perdita; ma non però difpregio gli Autori di quel deplo. rabile incendio, nè biafimo la lor memoria; perchè quel ma. le, al qual furono allora portati da un zelo troppo ardente, $e$ non bene informato, non è da paragonare col gran bene, che per altro vi fecero: oltrechè eglino fteffi cercarono di riparar quella perdita colle lor opere, fpezialmente Motolinia, Saha: gun, Olmos, e Torquemada.

Ma il Sig. de P. fi è tanto impegnato nel diminuire la popolazione di quei paefi, che è giunto ad affermare (chi il crederebbe?) in tuono decifivo e magiftrale, che in tutte quelle contrade non v'era altra Città, che quella di Meffico. Sentiamolo difcorrere per divertirci un poco:,"Siccome non fi fco. " prono, dice egli, in turto il regno di Meffico alcuni vefti" gj d'antiche Città Indiane, egli è manifefto, che non v'era " più d'un fol luogo, che aveffe qualche apparenza di Cirta, e " quefto era Meffico, it qual vollero gli Scrittori Spagnuoli ap"pellare la Babilonia delle Indie; ma è già un gran pezzo, " che non ci ingannano $i$ nomi magnifici da loro dati ai mio " ferabili villaggi d'A merica."

Ma tutri quanti gli Autori, che hannofcritto del Meffico, una: nimemense afferinano, che turte le Nazioni di quel valto Im. perio 
perio viveano in focietà, che aveano popolazioni molte, grandi; e bene ordinate, nominano le città da loro vedute $(y)$; e coloro, che hanno viaggiato per quelle regioni due fecoli e mezzo dopo la conquifta, hanno vedute coi lor occhi le fuddette popolazioni nei medefimi luoghi da quegli Autori accennati : ficchè o il Sig. de P. fi perfuade, che quegli Scrittori annunziarono profeticamente le future popolazioni, o dee confeffare, che fin d'allora v' erano, ove fono prefentemente. E' vero, che gli Spagnuoli vi fondarono molte popolazioni, ficcome le cittá d'Angelopoli, di Guadalaxara, di Vagliadolid, della Veracroce, di Zelaja, del Potosi, di Cordova, di Leone ec.; ma le po. polazioni, da loro fondate nel diftretto dell' imperio Mefficano, rapporto a quelle fondate dagl'Indiani non fono neppur nella proporzione d' uno a mille. I nomi mefficani impofti alle popolazioni, i quali fino ad ora fi confervano, dimoftrano chiaramente, che non furono già gli Spagnuoli, ma gl' Indiani i loro fondatori. Or che quei luoghi, dei quali facciamo fpeffo menzione nella Storia, non fieno ftati per lo più miferabili villaggi, ma Città, e popolazioni grandi, e ben formate, come quelle d'Europa, ciò confta dalla depofizione di tusti quegli Scrittori, che gli videro.

Il Sig. de Paw vorrebbe, che gli fi moftraffero i veftigi delle Citta antiche; ma noi gli moltriamo d'avvantaggio queile fteffe antiche città ancor fuffiftenti. Ciò non oftante $\mathrm{fe}$ egli vuol veftigi, vada a Tezcuco, a Otumba, a Tlafcalla, a Cholulla, ad Huexotzinco, a Cempoalla, a Tulla ec., a ve ne troverà tanti, da non poter dubitare dell'antica grandezza di quelle Città americane.

Quefto gran numero di città, e di luoghi abitati, con tut-

to

(y, Cortès nelle quattro fue lunghe lettere a Carlo V. Bernal Diaz del Cafilto nella fua Storia della Conquifta: il Conquiftatore Anonimo nella fua curiofa Relazione: Motolonia, Sabagun, e Mendięta ne' loro manofcritti: Monfig de las Cafas in alcune delle fue opere : Pietro Alvarado, Didaco Godoi, e Nugno Guzman nelle lor lettere, le quali trovanfi nella Raccolta del Ramufio, tutti teftimsni oculari, a'quali debbono aggiugnerfi tutti gli Storici Mefficani, Acolhai, e Tlafcallefi, principal nente quelli da noi meffi nel catalogo degli Autori della Storia antica del Melfico. 
to che annualmente vi periffero tante migliaja d'uomini nei facrifizj, e nelle continue guerre di quei popoli, dà chiaramente a divedere la gran popolazione dell'Imperio Mefficano, e degli altri paef d'Anahuac; ma fe nulla di quanto abbiam detto ba. fta a convincere il Sig. de P., io caritatevolmente gli configlierei di farfi cóndure ad uno Spedale.

Ciò che abbiamo addotto contra il Sig. de $\mathrm{P}$. giova parimente a ribattere il Dott. Robertfon, il qual vedendo tanti te!timonj contrarj al fuo fentimento, ricorre a un futrerfugio fimile affacro a queilo del calor dell' immaginazione, di cui fi prevalfe per negar fede agli Scrittori Spagnuoli intorno a ciò, che dicono dell' eccellenza dei lavori mefficani di getro. Egli dunque ragionando di quella maraviglia, che cagiond agli Spagouoli la vifia delle Citrà del Meffico, dice cosł nel lib. 7 . della fua Storia:" Nel primo fervor della lor maraviglia para" gonarono Cempoalla , benchè citrà folamente di fecondo, o " di terzo rango, ad alcune di maggior nota nel proprio lor \% paefe. Quando poi videro fucceffivamente Tlafcalla, Cholul" la, Tacuba, Tezcuco, e Meffico fteffa, s'accrebbe di tanto ") il loro ftupore, che portarono le idee della lor grandezza e 9) popolazione a quel che confina coll'incredibile... Conviene " per tal ragione, che fi faccia una gran tara alla loro nume. s razione degli abitanti delle città mefficane, e dovrà fiffarfi " ad una rata più baffa il modello della lor popolazione ., Cos) comanda il Robertfon; ma io non fon difpofto ad ubbidir10. Se gli Spagnuoli aveffero fcritto le loro Storie, Lettere, o Relazioni nel primo fervor della lor maraviglia, potrebbe allo. ra ben fofpertarf, che lo ftupore gli aveffe portati ad efagera. re; ma non avvenne cosł. Cortès, il più antico di quegli Scrituori, non ifcriffe la prima fua lettera a Carlo V., che un anno e mezzo, dappoi che era arrivato in quel paefe: il Conquiltatore Anonimo fcriffe alcuni anni dopo la conquifta: Bernal Diaz dopo più di quaranta anni di continua dimora ia quelle contrade, e cosi gli altri. E' mai polfibile, che duraffe uno, venti, ed anche quaranta anni quel primo fervor della maraviglia? Ma donde nacque in loro tal maraviglia? Sentia. molo 


\section{DISSER T A Z IO NE VII.}

molo dallo fteffo Dott. Robertfon:, Gli Spagnuoli, dice, av\#, vezzi a quefto modo d'abitazioni (delle capanne ifolate) tra " tutte le felvagge tribù, delle qualı erano già informati, re" Atarono attoniti all' entrar nella N. Spagna, e al ritrovatvi " i Nazionali, che rifedevano in città grandi fomiglianti a "quelle d'Europa.", Ma Cortès, e i fuoi compagni, prima d'andar nel Meffico, fapeano già, che quei Popoli non erano tribù felvagge, $e$ che le loro cale non erano capanne: 2veano già fentito da tutti coloro, che un anno innanzi aveano fatto quel viaggio col Grijalva, che v'erano belle popolazioni, fornite di cafe ben fatte di pietra e calcina, e d'alte torri, ficcome ne fa fede Bernal Diaz, teftımonio oculare. Non era dunque quella la cagione della lor maraviglia; ma bensi la vera grandezza e moltitudine delle citta, the vi videro." Non è gran fatto, foggiugne il Robertfon, che Cor$\rightarrow$ tès, e $\mathrm{i}$ fuoi compagni, portati potentemente a magnificar 2) le cofe per efaltare il merito delle loro fcoperte, e conqui " fte, foffero caduti in quefto errore comune d'innalzar le de. "fcrizioni molto al difopra del vero." Ma Cortès non era fciocco, e vedeva bene, che l'efagerare il numero dei fuoi Alleati, anzichè ad efaltare il merito, ferviva a diminuir la glo. ria delle fue conquitte. Eppure egli fpeffo confeffa, che era ajutato nelle fue conquilte or da ottanta, or da cento mila, or da più di dugento mila uomini: e ficcome quefte ingenue confeffioni fanno palefe la fua fincerita, così quei tanto numerofi eferciti dimoftrano la gran popolazione di quel paefe. Oltracciò il Dott. Robertfon fuppone, che quanto fcriffero gli Autori Spagnunli intorno al numero delle cafe delle cirtà mefficane fu folamen. te efpreffo da loro per congettura, e fecondo il giudizio che formarono ad occhio; $\mathrm{ma}$ non pafsò così: poichè lo fteffo Cortès teftifica nella fua prima lettera all' Imperatore Carlo V. d'aver egli farto fare la numerazion delle cafe, che erano nel diftretto della Repubblica di Tlafcalla, e d'averne trovato più di cencinquanta mila, e nella fola citzà di Tzimpantzinco piùs di venti mila. 


\section{8 \\ DISSER TAZIONE VIII.}

SU LA RELIGIONE DEI MESSICANI.

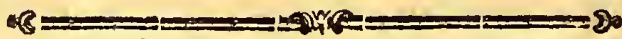

TOn ho che fare in quefta differtazione, come nelle altre; I col Sig. de $\mathrm{PaW}$; poichè egli ingenuamente riconofce la fomiglianza, che v'è tra i deliri degli Americani, e quelli d'altre Nazioni dell'antico Continente in materia di Religione ., Siccome le fuperftizioni religiofe dei Popoli d'America, " dice egli, (a) hanno avuto una fomiglianza fenfibile con quel" le, che hanno ufato le Nazioni dell' antico Continente, non " s'è parlato di quefti fpropofiti, che per farne il paragone, e 3) per dare a divedere, che malgrado della diverfita dei climi, " la debolezza dello fpirito umano è ftata coftante ed invaria") bile." Se collo fteffo giudizio aveffe difcorfo in altri punti, ci aurebbe rifparmiate parecchie contefe, ed aurebbe preferva: ta la fua opera da quelle gravi e forti cenfure, che ne hanno farte alcuni uomini faggi della medefima Europa. Io però in: dirizzo quefta differtazione a coloro, che per ignoranza di quanto è paffato, e paffa prefentemente al Mondo, o per mancanza di rifleffione, hanno tanto fchiamazzats in leggendo nella Storia del Meffico la crudeltà, e la fupertizione di quei Popoli, come fe foffero cofe non mai fentite tra $i$ mortali. Farò dunque palefe il lor errore, e dimoltrerò, che la Religione dei Mefficani fu, men fuperftiziofa, meno indecente, men puerile, e meno irragionevole, che quella delle più colte Nazioni dell'antica Europa, e che della crudeltà di coloro vi fono ftati efempi, e forle più atroci in quafi tutii i Popoli del Mondo.

Il Giftema della Religione Naturale dipende principalmente da quell' idea, che fi ha della Divinità. Se il fupremo Effere fi con-

(a) Nella prefaz. delle Ricercbe Filoofofiche. 
concepifce, come un Padre pieno di bontà, la cui provvidenza invigila fu le fue creature, nelle pratiche religiofe fi fcorgerd dell' amore, e del rifpetto. Se per lo contrario s'immagina, come un Tiranno ineforabile, il culto farà fanguinario. Se fi crede Onnipotente, farà ad un folo diretta la venerdzione; ma fe fi giudica riftretto il poter di lui, nun potranno a meno di non moltiplicarfi gli obbietti del culto. Se fi riconofce la fan. tità e perfezione del fuo effere, fi cercherà la fua protezione con un culto puro e fanto; ma fe fi reputa loggiacente alle imperfezioni e vizi degli uomini, la ftef $a$ Religione conáacrerà i delitti.

Paragoniamo dunque l'idea, che aveano i Meffican dei loro Dei con quella, che aveano dei loro Numi i Greci, i Romani, ed altre Nazioni, da cui coloro impararono la Religione, e tolto riconofceremo i vantaggi dei Mefficani in quelta materia fopra quelle Nazioni antiche. E' vero, che i Meffica. ni compartivano tra vari Numi il potere, imnaginando rittretta a certi limiti la giurifdizion di ciafcheduno., Io non du"bito, diceva il $\operatorname{Re}$ Motezuma al Conquiltatore Cortès in " una conferenza di Religione, io non dubito della bontà del "Dio, che adorate; ma le egli è buono per la Spagna, i no. "Itri Dei fono parimente buoni pel Meffico."

"Il noftro Dio Camaxtle, diceano al medefimo Cortès i "Tlafcallefi : ci concede la vittoria contra i noftri nemici: la "noftra Dea Matlalcueje ci manda la pioggia ai noltri campi "neceffaria, e ci difende dalle inondazionı del Zabuapun. A " ciafcuno dei noltri Dí fiamo debitori d'una parte della feli" cità della noltra vita;" ma non credettero mai sì imporenti i loro Dai, quanto i Greci ed i Romani. I Mefficani nonaveano più $d$ un Nume fotto il nome di Centeorl per la cura della campagna, e dei feminati, e conturtochè foffero tanto amanti dei loro figliuoli, fi contentavano d'un fol Dio per la loro protezione. 1 Romani oltre alla Dea Cerere impiegavano bel folo frumento una gran folla di Dei, $(b)$ e nella cura ed Storia Antica del Meffico Tom. IV.

0 educa-

(b) Seja era iacaricata del grano feminato, Proferpina del grano nato, Noo doto 


\section{0 DIS S R TAZION E VIIT.}

educazione dei loro figliuoli più di venti, oltre a quei molti; che erano occupati nella generazione, e nella nafcita dei bambini. (c) Chi crederebbe, che abbifognaffero di tre Dij per la fola guardia della porta? Forculo era incaricato delle impolte, Carna dell' arpione, e Limentino della foglia. Ita, efclarna quà Sant' Agoftino (d) ita non poterat Forculus fimul fores, $\mathcal{O}$ cardinem limenque fervare. Tanto mefchino era a giudizio dei Romani il poter dei loro Dei! Anche i nomi, coi quali erano alcuni d'effi appellati, danno a divedere il cattivo concetto, in cui erano preffo i loro adoratori. Quali nomi più indegni della Divinirà, che quelli di Jupiter Piftor, Venus Calva, Pecunia, Caca, Subigus, e Cloacina? Chi fi perfuaderebbe mai, che una ftatua trovata da Tazio nella principal cloaca di Roma doyeffe divenir Dea col nome di Cloacina? Non è forfe quefto beffarfi della propria lor Religione, e render vili e fprezzabili quegli fteffi Dei, che adoravano?'(e) $\mathrm{Ma}$ in niun'altra cofa moltrarono meglio $i$ Greci, ed $i$ Ro-

doto de' nodi del futto; Volatina degli occhi, o gemne, Patelena delle foglie già rp.egate, Flora de' fiori, Oftilina della fpiga, Segefa de' nuovi grani, Lattanzia del grano ancor latteggiante, Matuta del grano maturo, Tutano e Tutiline del grano guardato nel granaro: a'quali debbono aggiugnerfi il Dio Stereulio, il qual badava al letaminamento de campi; Priapo, il qual difendeva il grano dagli uccelli: Rubigo, il qual l) prefervava dagli infetti, e le $\mathbf{N i n f e}$ Napee, le quali aveano cura del fugo nutritivo.

(c) La Dex Ope era incaricata di dare ajuto al bambino nafcente, e d'accoglierlo nel fuo grembo, Vaticano d'aprirgli la bocca nel pianto, Levana d'alzarlo da terra, Cunina di guardar la culla, le Carmenti d'annunziare il tuo deftino, Fortuna di favorirlo ne fuoi avvenimenti, Rumina d'incrodurte il capezzolo della poppa materna nella bocca del bambino, Potina della fua bevanda, Educa della fua pappa, Faventia di fargli bau, Venilia d'avvivar la fua fperanza, Volupia di prender cura de' ruoi piaceri, Agenoria di badarealle fue operazoni, Stimula di farlo attivo, Strenua di fargli coraggio, Numeria di fargli impurare $\mathrm{i}$ conti, Camena d'ammaeftrarlo nel ranto, Confodi dargli configli, Sencia ai fargli prender rifoluzione, Juvenia avea cura del principio della gioventù, e la Fortuna barbata avea l' importantifimo impiego di far nafcere il pelo agli adulti.

(d) Aug. de Civ. Dei lib. 4. cap. 8.

(e) Q i ita religionum derifio elt? $S$ i earum defenfor effem quid tam graviter queri poffem, quam Deorum numen in ta.stum venifle contemptum, ut turpıllimis nominibus ludibrı habeatur? Qu's non rideat Fornacem Deam? Quis cum audiat Deam Muiam tenere rifum queat? colitur \& Caca \&c. La* etant. Inft. Divin. lib. 1. cap. 20. 
Romani l'opinione, che aveano dei lor Numi, che in quei vizi, che loro attribuivano. Tutta la lor Mitologìa è una lunga ferie di delitti : turta la vita dei lor Dei fi riduceva a rancori, vendette, incelti, adulteri, e ad altre paffioni baffe, capaci d'infamare anche gli uomini più vili. Giove, quel Padre onnipotente, quel principio di turte le cofe, quel Re degli uomini, e degli Dei, come l'appellano i Poeti, fi moftra talora travifato in uomo per trattar con Alcumena, talora in Satiro per goder d'Antiopa, talora in Toro per rapire Europa, talora in Cigno per abufar di Leda, e talora in pioggia d' oro per corromper Danae; talora prende altre forme per compir le fue ree voglie. Frattanto la gran Dea Giunone rabbiofa per la ge. losia non fa fare altro, che prender vendetta del fus sleal marito. Di quefto medefimo calibro erano gli altri Dei immorta. li, (pezialmente i Maggiori o fcelti, come erano appellati $\mathrm{d} z$ loro. Scelti, dice S. Agoftino, $(f)$ per la fuperiorità dei lor vizj, non già per l'eccellenza delle lor virtù. $E$ per dire il vero quali efempi buoni poteano contar dei loro Dei quelle Nazioni, che mentre fi vantavano d'infegnare agli uomini la virtù, non alcro confacravano nei lor Dei, che i vizj? Quali meriti aveano per ottenere, l'apoteofi preffo i Greci Leena, e preffo i Romani Lupa, Faula, e Flora, fe non quelli d' effere ftate famofe meretrici? Quindi nacque l' effere ftati varj Numi incaricati dei più infami e vergognofi impieghi. $(g)$.

Ma che diremo degli Egizj, $i$ quali furono $i$ principali Autori della fuperftizione? (b) Eglino davan culto non che al Bue, al Cane, al Lupo, al Gatto, al Coccodrillo, allo Sparviere, e ad altri si fatti animali, ma eziandio ai Porri, alle Cipolle, e agli Agli : ciò che diede morivo a quel bel motto di Giuvenale: O fanctas gentes, quibus bic nafcuntur in bor.

$$
\mathrm{O} 02 \text { tis }
$$

(f) Aug. de Civ. Dei lib. 7. cap. 33.

(g) Chi voleffe fapere i nomi, e gl'impieghi di que"Dei, gli troverà nel libro 6 cap. 2., e nel lib. 9. cap. 9. de Civit. Dei; poichè a me non bafta l'animo di metterli quil fotto gli occhi de'miei Leggitori.

(h) Nos in templa tuam Rcmana accepimus Is $n$ Semicanefque Deos, \& fifra moventia luabm. Lusanus. 


\section{DISSERTAZIONE VIII.}

is Numina! E non contenti di ciò, celebrarono anche l'apoteofi delle cofe più indecenti. Quella ufanza deteftabile di ammogliarfi colle loro forelle fi credeva autorizzata coll' efempio dei lor Dei .

Affai diverfa da quefta era l'idea, che aveano dei lor $\mathrm{Nu}$ mi i Mefficani. Non fi trova in tutta la lor Mitologia veru. na traccia di quelle fupende fcelleratezze, colle quali furono da quelle altre Nazioni infamati i loro Dei. I Mefficani onoravano le virtù, non i vizj, nelle loro Divinità: in Huitzilo. posboli la prodezza, in Cenreott, in Tzapotlatenan, in Opocbtli, $e$ in altri la beneficenza, e in Quetzalcoatl la caftità, la giuAtizia, e la prudenza. Benchè fingeffero Numi d'ambidue i feffi, non gli muritareno, nè gli credettero capaci di quei piace. ri ofceni, che fono ftati tanto comuni negli Di Greci, e Ro. mani. Supponevano $i$ Mefficani in effi una fomma avverfione ad ogni forta di delitti : onde il loro culeó s'indirizzava a piacar lo [degno dei Numi provocato coi peccati degli uomini, ed a procacciarfi la loro protezione col pentimento, e cogl' offequi religiofi.

Conformi affatto alli idea degli Dei erano i riti ufati da quelle Nazioni. La fupertizione era comune a tutte; ma quella de' Mefficani era minore e men pútrile, bafta fare il paragone de' loro augurj. Gli Aftrologi Melficani offervavano i fegni o caratteri de' giorni per li loro mariraggi, per li loro viage gi, \&c. ficcome gli Aftrologi Europei offervavano la pofizione degli aftri per indi predire la ventura degli uomini. Gli uni e gli altri temevano del pari gli ecliffi, e le comete, come precurfori di gran calamità; perchè quefta fuperfizione è ftata coa. mune a tuti $i$ popoli del Mondo. Tutti frmilmente s'impaurivano in fentendo la voce del gufo, o di qualche altro fiffate to uccello. Quefte, ed altre fimili fuperftizioni fono ftare gea nerali, e fono anche oggidì troppo comuni nel volgo dell' uno, - dell'altro Continente, anche nel centro della coltiflima Eua ropa; ma tutto ciò, che fappiamo di quelle Nazioni Americane in quefta materia, non è certamente da paragonare corr quello ${ }_{2}$, che ci dicono degli antichi Romani is propri loro Storici

Coe 
- Poti. Le opere di Livio, di Plinio, di Virgilio, di Sveto. nio, di Valerio Maflimo, e d'altri giudiziofi Autori (le qua. li non poffuno leggerfí fenza compaffione) fanno vedere fino a qual ecceffo arrivo la pueril fuperftizione de' Romani, ne'loro augurj. Non v'era animale ne tra i quadrupedi, né tra i retrili, nè tra gli uccelli, dal quale non fi prendeffe augurio dell' avvenire. Se l'uccello volava verfo la man finiftra, fe crocidava il corvo, fe fi fentiva la voce della cornacchia, fe il forcio affaggiava il mele, fe la lepre paffava a traverfo la ftrada, tutto cio fi ftimava pronoftico di qualche gran difgrazia. Si vide gia far l'efpiazione, o fia luftrazione di tutta Roma non per altro, fe non perchè entrò un gufo nel Campidoglio. (i) E non che gli animali, ma anche le cole più triviali e difpregevoli baltavano a cagionar loro un timor fupertiziofo, co. me per efempio fe ftando a tavola fi fpargeva il vino, o il fale, o cadeva in terra qualche particella delle vivande. Chi non fi farebbe maravigliato in contemplando i Signori Arufpici, perfone tanto autorevoli, feriamente occupati nell' offervare tutti i movimenti delle vittime, lo ftato delle lor vifcere, e il color del lor fangue per pronofticar da tali fegni i principali avvenimenti di quella famofa Repubblica? Mi maraviglio, diceva il gran Cicerone, $(k)$ che un Arufpice, non fe ne rida in vedendo un altro della medefima profeifione. Qual cofa in vero più ridicola di quella fpezie d'augurio, che appellavano Tripudium? Chi potrebbe perfuaderfi, che una Nazione da un canto tanto illuftra$\mathfrak{t a}$, e da un altro tanto guerriera, portaffe feco ne' fuoi eferciti, come cofa la più importante per la felicita delle fue armi, una gabbia di pollaftri e che fenza prima confultarli non ardiffe di dar la bartaglia? Se i pollattri non affaggiavano quella pafta, che lor fi metteva innanzi, era un cattivo fegno: fe oltre al non mangiarla, fcappavano fuor della gabbia, peggio: fe per contrario la mangiavano avidamente, ciò Atimavaíl l'augurio più felice. Sicchè̀:

(i) Bubo funebris, of maxime abominatus pu'licis precipue aufpiciis. . . Capitolii cellam ipfam intravit Sex. Papellio Iftro. L. Pedanio CofJ. propter quod: nonis Martiis Urbs lufrata efs eo anno. Plin. Hif. Nat. lib. IO. cap. I2.

(k) Miror quin rideat Harufpex, cum Haruspisem wideat. Cic. de Divizo. 


\section{D IS S E R T A Z I O N E VIII.}

il mezzo più efficace d'afficurarfi la vittoria farebbe ftato quello di far foffrir la fame a' pollaftri prima di confultarli.

A fiffatii ecceffi è facilmente portato lo fpirito umano, qualora fi abbandona a'proprj fuoi lumi . La fperienza di que groffolani errori, di quella ridicola puerilita, e di quelle moAtruofe abbominazioni, nelle quali fon incorfe le più colte $\mathrm{Na}$ zion del Gentilefimo, dà a divedere, che non dobbiamo afpettar la vera e fanta Religione, fe non da quel medefimo Dio, che adoriamo. A lui tocca rivelar le verità, che dobbiamo credere, e prefcrivere il culto, con cui dobbiam riverirlo. Se l'afo fare graviffimo della religione fi confida alla ragione umana, della cui deba'ezza abbiamo tanța fperienza, i maggiori affurdi sapprefenteranli alla noftra mente, come veri dogmi, e il culto dovuto al Supremo Effere farà difettofo per l'empietà, o ecceffivo per la fuperftizione. Iddio voleffe che quegli iteffi Filofofi dal noftro illuminato fecolo, che tanto vantano le forze della ragione, non ci deffero nelle medefime lor opere tante e sì chiare prove della loro imbecillità!

Ma alla fine Americani, Greci, Romani, ed Egizj tutti erano fuperftiziofi e puerili nella pratica della lor religione; non però casì nell' ofcenità de' lor riti; poichè ne' riti de'Mefficani non fi trova il menomo veftigio di quelle abbominazioni tanto comuni preffo i Romani, ed altre colte Nazioni dell'Antichità. Qual cofa più indecente delle fefte Eleufine, che faceano i Greci, di quelle che celebravano ad onor di Venere i Ro. mani nelle calende d'Aprile, e foprattutto quegli ofcenifimi giuochi, che fi faceano ad onor di Cibele, di Flora, di Bacco, e d'altri sì rei Numi, contro i quali inveirono fortiffimamente parecchj Padri della Chiefa, ed anche alcuni degli fteffi Ro. mani? Qual rito più ofceno di quello, che fi faceva nella fta. tua di Priapo tra le ceremonie nuziali? (l) Come poteana celebrar le fefte di quegli Dei inceftuofi ed adulteri, fe non con

(1) Vedafi ciò che diçono intorno a quello, e ad altri fiffatti riti Lattanzio Firmiano nell' opera De Divinis. Infritutionibus, e S. Agoftina ne' libri de Civit. Dei. 


\section{DIS S E R T A Z I O N E VIII.}

con tali abbominazioni? Come era poffibile, che fi vergognaffero di que'vizj, che vedevano confacrati nelle loro Divinita?

E' vero, che quantunque ne' riti de Mefficani non inter. veniffe veruna ofcenità, ve n'erano pure alcuni, i quali fuppoIta la divinità de' loro Numi farebbono ftati indecenti, come quello d"unger le labbra degl' Idoli col fangue relle vittime; ma non farebbe ftato affai più indecente quello di dar loro fchiaff, ficcome ne davano i Romani alla Dea Matura nelle fefte Matrali? Suppofto l'errore degli uni, e degli altri, erano cerramente meno irragionevoli i Meftiani, dando ad affaggiare a'lor Dei un liquore, il quale fecondo i principj della lor religione era da coloro gradito, che non $i$ Romani efeguendo verfo la loro Dea un'azione, la quale è Atimata gravemenie ingiuriofa preffo tutti i Popoli del Mondo.

Cid che finora abbiam derto, benchè fufficiente a dimoAtrare, che la Religion de' Meflicani era men biafimevole di quella de'Romani, de' Greci, e degli Egizj, può dirfi quafi niente, Te fi paragona con ouello, che ommettiamo per non re. car noja a' Lettori. $M_{i}$ peraltro veggo bene, che non dee farli il paragone folamente ne'fuddetti articoli; ma piuttofto in quello che riguarda la qualità de' facrifizj. Io confeffo, che la Religion de' Mefficani era troppo fanguinaria, che i loro facrifizj erano crudehıfrmi, e le loro autterià olcremodo barbare; ma ogni volta che mi metto a confiderare quello, che hanno fatto le altre Nazioni del Mondo, mi confondo in riconofsendo $P$ imbecillita della mente umana, e gli errori deplorabili, ne' quaii fi precipita, quando non è guidata dal lume della vera Religione, e rendo infinite grazie all' Aluffimo d'avermi prefervato da tan. ti mali.

Non v'è tata quafi alcuna Nazione al Mondo, la quale non abbia talvolta facrificate vitrime unane a quel $D i s$, che adorava. Sappiamo da'libri fatti, che gli Ammontti bruciavano alcuni de'lor figliuoli ad onore del loro Dio Moloch, e che lo fteffo faceano altri Popoli del paefe di Canadm, il cui efempio fu talora imitato dagl' Ifraeliti. Confta dal lib. 4. de' Re, che Achaz, e Manaffe Re di Giuda ufarono quel rito gentile: 


\section{DISSERT A Z I N E VIIT.}

fco di paffare $i$ lor figliuoli pel fuoco. L' efpreffione del facro teftó pare fignificar piuttolto una mera lultrazione, o confacrazione, che un olocaufto; ma il Salmo roj. non ci permette di dubitare, che gli Irraelici facrificavano veramente $i$ lor figliuoli agl' Iddii de' Cananei, ( $m$ ) non baltando a ritrarli da quella barbara fuperftizione gli ftupendi ed evidenti miracoli operati dal braccio onnipotente del vero Dio. Degli Egizj fappiamo per la teftimonianza di Manetone Sacerdote, e Storico celebre di quelIa Nazione, citato da Eufebio Cefarienfe, che ognigiono fi facrificavano tre uomini in Eliopoli alla fola Dea Giunone. Or ficcome gli Ammoniti facrificavano vitrime umane al loro Moloch, ed i Cananei al loro Beelfegor, cosł ne facrificavano i Perfiani al loro Mitra, o Sole, i Fenicj ed i Cartaginefi al loro Basl, o Saturno, i Cretefi a Giove, i Lacedemoni a Marte, i Focefi a Diana, i Lesbi a Bacco, i Teffali al Certauro Chirone, ed a Peleo, i Galli ad Efo, ed a Teutate, (n) i Bardi della Germania a Tuiftone, e così altre $\mathbf{N}$ dzıoni a' lor Dei tutelari. Filone dice, che i Fenicj nelle pubbliche lor ca: lami-

(m) Commifi funt inter gentes, $\sigma$ didicerunt opera corum, o Jervierunt fculptilibus eorum, G factum eft illis in fcantalum. Et immolaverunt filios juos, O flias Suas Damoniis. Et effuderunt Janguinem innocentem: fanguinem fliorum fuorum, $\mathcal{O}$ fliarum fuaium, quas immolaverunt fculptilibus Cbanaam, $\mathcal{O}$ infe\&a ef terra in fanguinibus. Pr. 105.

(n) Un certo Autore francefe, fpinto da un cieco amore alla fatria, nega arditamente, che foffero mai ftate facrificate vittime umane da' Galli; ma son allega veruna ragione da potere rmentire le teftimonianze di Cefare, di Plinio, di Svetonio, di Dindoro, di Stratone, di Lattanzio, di S. Agoftino. - d'altri gravi Autori. Bafta a confonderlo il teftimonio di Cefare, il quale, come quegli, che ebbe più pratica de' Galli, gli conofceva meglio: Natio ef. -mnis Gallorum, dice, admodum dedita religionibus, atque ob eam caufam qui funt affecti gravioribus morbis, quique in pralio periculifque verfantur, aut pro vidtimis bumines immolant, aut fe immolaturos vovent, adminifris ad ea facrificia Druidibus; quod pro vita bominis, niji vita bominis reddatur, non polfe aliter Deorum immortalium numen placari arbitrantur: publiceque ejufdem generis babent infituta facrificia. Alii immani magnitudine fimulacra bibent: quo rum contexta viminibus membra vivis bominibus complent, quibus fucceris circumventi fanma examinantur bomines. Supplicia eorum, qui in furto, aut latrocinio, aut aliqua noxa fint comprebenfi, gratiora Diis immortalibus effe arlitrantur. Scd cum ejus generis copia deficit, etiam ad inn centium fupplicia defcendunt. Lib. 6. de Bello Gallico cap. 5. Nel che fi vede, che i Galli furo. so ancor più crudeli de' Meflicani. 
lamità offerivano in facrifizio al lor inumano Baal $i$ più cari de'lor figliuoli, e Curzio afferma, che tal facrifizıo fu in ufo preffo i Tir; fino all' eccidio della famofa lor Città. Lo ftefo faceano i Cartaginefi lor nazionali ad onor di Saturno il crudele: così a ragione da loro appellato. Sappiamo, che effendo ftati vinti da Agatocle $\mathrm{Re}$ di Siracufa, per placare il loro Nume, il quale credevano fdegnato, gli facrificarono dugento $\mathrm{f}_{\mathrm{an}}$ ciulli nobili, oltre a trecento giovani, che fpontaneamente s' offerirono al facrifizio per moftrar la loro bravura, la lor pietà verfo gli Dei, e il lor amore verfo la patria: e fecondochè afferma Tertulliano, il qual come Africano, e poco polteriore a quell' epoca, di cui parlava, doveva faperlo bene; que' facrifizj furono ufati in Africa fino a' tempi dell' I mperator Tiberio, ficcome nelle Gallie fino ai tempi di Claudio, fecondochè ne fa fede Svetonio.

I Pelafgi antichi abitatori dell' Italia facrificavano per ub. bidire a un oracolo la decima parte de'loro figli, come racconta Dionifio Alicarnaffo. I Romani, i quali furono tanto fanguinari, quanto fuperftiziofi, non rifiutarono fiffatti facrifizj. Tutto il' tempo, che furono effi fotto i lor Re ufarono facrificar fanciulli alla Dea Mania, Madre de' Larj, per la felicita delle lor cafe, indotti, come dice Macrobio, da certo oracolo d'Apollo: e fappiamo da Plinio (o) che non vi fu interdetto facrificar vittime umane fino all' anno 657. di Roma; ma non per quefta proibizione ceffarono affatto gli efempi di quella barbara fuperAtizione, poichè Auguito, fecondo che affermarono alcuni Scrittori citati da Svetonio, dopo la prefa di Perugia, laddove s'era fortificato il Confole L. Antonio, facrifico ad onor del fun Zio Giulio Cefare, divinizzato già da' Romani, trecento uomini parte Senatori, e parte Cavalieri Romani, fcelti tra la gente d'Antonio, fopra un altare eretto a quel nuovo Nume. ( $p$ )

Storia Antica del Meffico Tom. IV. P P Lat-

(o) DCLVII. demum anno Urbis Cn. Corn. Lentulo. P. Liciniv CofJ. Senatusconfultum factum eff, ne bomo immolaretur. Plin. Hilt. Nat. lib. 30. cap. I.

(p) Pernfia capta in plurimos animadvertit: orave veniam, vel excufare fe conantibus una voce occurrens, Moriendum efse. Scribunt quidam, trecentos ex dedititiis electos utriufque ordinis ad ara:ii D. J:!lio exfruftam Idib. Martiis wis gimarum more makzatos. Svetonius in OAaviano. 
Lattanzio Firmiano, uomo affai bene iftruito nelle cofe de' $R$ a: mani, il qual fiori nel fecolo IV. della Chiefa, dice efpreffamente, che anche a'tempi fuoi f faceano in Italia que' racrifizj a Giove Laziale. (q) Neppur gli Spagnuoli fi prefervarono da quella barbara fuperftizione. Srrabone racconta nel lib. 3., che i Lultani facrificavano i prigionieri, tagliavan loro la deltra per confacrarla a' lor Dei, offervavano le loro vifcere, e le guardavano per li loro augurj: che turti gli abitatori delle montagne ufavano facrificare $i$ prigionieri infiems $c 0^{\circ}$ cavalli, offerendo a cento a cento tali vitrime al Dio Marte, e parlando in generale dice, che era proprio degli Spagnuoli il facrificarf per- li lo: ro amici. Non è alieno da quefto modo di pentare cid, che Silio Iralico racconta de' Betici fuoi maggiori, cioè che dopo aver paffata l'età giovanile, infaftiditi della vita, fi davano eglino Itelli la morte: ciô che eg!i loda come un' azione eroica. (r), Chi crederebbe, che queft antica moda della Betica doveffe a" noftri di rinnovarf in Inghilterra, $e$ in Francia. Venendo poi a' tempi polteriori, il P. Mariana in parlando de' Goti, che occuparono la Spagna dice cosi: $(\Omega)$ "Perchè erano perfuali, che 2) non riufcirebbe bene loro la guerra, qualora non offeriffero 2) fangue umano per l' efercito, facrificavano i prigionieri di 9) Guerra al Dio Marte, del qual erano principa!mente divoti: 2) ed ufavano ancora offerirgli le primizie delle fpoglie, e fo. 2) Ipender da' pedali degli alberi le pelli di coloro, che ammaz"zavano. "Se quegli Spagnuoli, che fcriffero la Storia del Meffico, non fi foffero dimenticati di ciò, che era gì accaduto alla loro penifola, non fi farebbero tanto maravigliati de' la: crifizj de' Melficani .

Chi ne volefie più efempi, puó confultare Eufebio di Cefarea nel lib. 4. de Preparatione Evangelica, laddove fa un lungo

(q) Nec Latini quidem bujus immanitatis expertes fuerunt : fiquidem Latralis Iupiter etian num Janguine colitur bumano. Lactant. Intit. Divin. lib. I. c. 23)

(r) Prodiga gens animo, $\mathcal{O}$ properare facillima mortem:

Namque ubi tranfcendit forentes viribus anwos

Impatiens avi fpernit veniffe feneitam.

(I) Stor. gener. di Spagna lib. S. 
go dettaglio delle Nazioni, preffo le quali fono ftati in ufo que barbari facrifizj: poichè a noi bafta quanto abbiamo accennato per dimoftrare, che i Mefficani non altro hanno fatto, che bato ter le orme delle più celebri Nazioni dell' antico Continente, e che $\mathrm{i}$ loro riti non furono più crudeli, nè più irragionevoli. Non è forfe maggior inumanità quella di facrificare i proprì Citradini, i propri figliuoli, e fe medefimi, come faceano per la maggior parte quelle Nazioni, che non i prigionieri di guerra, come era in ufo preffo i Meflicani? Non furono mai veduti i Mefficani facrificare i proprj lor Nazionali, fe non colo. ro, che per li loro delitti erano rei di morte, e talvolta le mogli de'Signori, acciocchè gli accompagnaffero ancora nell'altro mondo. Quella rifpofta, che diede Motezuma a Cortès, il quale gli rinfacciava la crudeltà de'loro facrifizj, dà a divedere, che quantunque $i$ lor fentimenti non' foffero giuft, erano pure meno irragionevoli di quelli d'altre Nazioni incorfe nella Atelifa fuperftizione. Noi, diffe, abbiam dritto di toglier la vita $a^{2}$ noltri nemici: potremo uccidergli nel calor della battaglia, come voi fate co' voltri nemici. Or qual ingiuftizia $v^{\prime}$ è nel far morire que' rei di morte ad onor de' noftri Dei?

La frequenza di cotali facrifizj non fu certamente minore in Egitro, in Italia, in Ilpagna, e velle Gallie, che nel Meffico. Se nella fola Citto d'Eliopoli fo facrificavano annualmenre, al dir di Manetone, più di mille vitrime umane alla fola Dea Giunone, quante faranno ftaze quelle, che fi facrificavano nelle aitre Citra d' Egitto alla famofa Dea Ifade, e agli altri innumerabili Numi, adorati da quella tanto fuperfiziofa Na. zione? Quanta ne farà ftata la frequenza tra i Pelasgi, i quali facrificavano a lor Dei la decima parte de"lor figliuoli? Qual numero d'uomini non farà ftato confunto in quelle ecatombe, - Cacrifizj centenari degli antichi Spagnuoli? E che diremo de' Galli, i quali dopo aver facrificati i prigionieri di guerra, ed i malfattori, faceano anche morir nel facrifzio gl'innocenti cittadini, come dice Cefare? Il numero poi de'facrifizi mefficanı è ftato certamente efagerato dalla maggior parte degliStorici del Meffico, come abbiamo detto altrove.

$$
P \text { P } 2
$$


Gli umaniffimi Romani, i quali aveano fcrupolo d'offer: var le umane vifcere, $(t)$ quantunque in capo di fei fecoli $e$ mezzo dopo la fondazione della famofa lor metropoli vietaffero finalmente di facrificare nomini, tuttavia permifero con troppa frequenza il facrifizio gladiatorio. Voglio cosi appellare quei barbari combattimenti, i quali fervendo al divertimento di quel Popolo feroce, erano per altro prefcritti dalla lor religione. Oltre al gran fangue umano, che fi fpargeva nei giuochi Circenfi, e nei conviti, non era in vero poco quello, che fi fpargeva nei funerali dei beneftanti, o combattendo fra loro i Gladiatori, o facendo morire alcuni prigionieri per placare i Mani del morto: ed erano sì perfuafi della necefità del fangue umano per un tal fine, che quando per mancanza di facoltà non fi poteano fopportar le fpefe dei Gladiatori, o dei prigiomieri, fi pagavano Prefiche, acciocchè colle unghie fi cavaflero fangue dalle gote. Quante dunque faranno ftate le vittime fatte morire dalla fuperftizion dei Romani in tanti funerali, maf. fimamente effendo fata tra loro dell' emulazione, mentre ciafcuno cercava di fuperar gli altri nel numero dei gladiatori, e dei prigioni, che doveano fervire alla funebre lor pompa? Quefto f́irito fanguinario dei Romani fu quello, che tante Atragi fece nei Popoli d' Europa, d'Afia, e d'Africa, e quello altresì, che inondò fpeffo Roma col fangue dei propri Cittadini, fpezialmente durante quelle orrende profcrizioni, le quali ofcurarono la gloria di quella famofa Repubblica.

Non folo furono inumani i Mefficani verfo i loro prigionieri, ma eziandio verfo di fe medefimi con quelle barbare aufterith, che abbiamo efpofte nella Storia. Ma il cavarfi fangue colle fpine del maghei dalla lingua, dalle braccia, e dale le gambe, come faceano tutti, e il forarfi la lingua con pezzuoli di canna, come ufavano alcuni più aufteri, parranno mortificazioni leggiere a lato di quelle fpaventevoli ed inaudite au. fterità efeguite dai Penitenti dell' India Orientale, e del Giappone, che non poffono leggerfi fenza orrore. Chi oferà para.

gona-

(t) Adspici bumana exta nefas tabetur. Plinius Hil. Nate lib. 28. cap. Io 


\section{DISSER T A IONE VIII. ¿OI}

gonare l'inumanità dei più famofi Tlamacazqui di Meffico, e di Tlafcalla con quella dei Sacerdoti di Bellona e di Cibele? (u) Quando fi vide, che $\mathrm{i}$ Mefficani fi fquarciaffero le mem. bra, fi Itrappaffero coi denti la carne, o fi caftraffero ad onor dei lor Dei, come faceano quei Sacerdoti ad onor della lor Cibele?

Finalmente i Mefficani non contenti di facrificar vittime umane, mangiavano ancora la loro carne. Io confeffo, che in ciò furono più inumani di quelle altre Nazioni; ma non fono ftati sì rari nell' antico Continente, anche preffo $\mathrm{Na}$ zioni colte, gli efempi di fiffatta inumanita, che debbano però annoverarfi i Mefficani tra i popoli affolutamente barbari. "Quell' orribile ufanza, dice lo Storico Sol's, di mangiarfi gli " uomini gli uni dagli altri, fi vide prima in altri barbari del " noltro Emisferio, ficcome lo confeffa nei fuoi Annali la Gal"Lizia."Oltre agli antichi Africani, i cui difcendenti fono in parte anche oggidì antropofaghi, egli è certo, che lo furono fimilmente molte Nazioni di quelle, che erano già conofciute col nome comune di Sciti, ed anche gli antichi popolatori della Sicilia, e del Continente dell' Italia, come dicono Plinio, ed altri Autori. Dei Giudei, che viveano ai tempi d'Antioco l'Illuftre, fcriffe Apione, Storico Egizio (non Greco, come dice il Sig. de Paw ) che foftentavano un prigionere Greco per mangiarlo in capo d'un anno. Del famofo Annibale dice Livio, che fece mangiar carne umana ai fuoi Soldati per in-

co-

(u) Dea Magne Sacerdoies, qui Galli vocabantur, virilia fibi amputabant, \& furore perciti caput rotabant cultrifque faciem mufculofque totius corparis diffecabant: morfibus quoque fe ipfos impetebant. Auguit. de Civ. Dei lib. 2. cap. 7 .

Ille viriles fbi partes amputat, ille lacertos fecat. Ubi iratos Deos timent, qui fic propitios merentur?.. Tantus eft perturbata mentis of fedibus fuis pulfa furor, ut fic Dii placentur, quemadmodum ne bomines quidem Saviunt teterrimi, $\mathfrak{G}$ in fabulas traditi crudelitatis Tyranni laceraverunt aliguarum membra; neminem $\int u x$ lacerare juferunt. In regice libidinis voluptatem caftrati funtquidam; fed nemo fibi, ne vir effet, jubente domino manus intulit. Se ip/s is templis contrucidant, vulneribus $\int u$ is ac fanguine fupplicant. Si cui inture $i$ vacet que facisnt. quaque patiuntur, inveniet tam indecora boxeftis, tam indigna liberis, tam dif:. fimilia fanis, ut nemo fuerit dubitaturus furere eos, $f$ cum paucioribus furerent; anuc fanitatis patrocinium infanientium turba eft. Seneca lib. de luperfitit. 
coraggirli alla guerra. Plinio rimprovera gravemente Greci dell' ulanza di mangiar tutte le parti del corpo umano per guazir di diverfe malatie. ( $x$ ) Or che maraviglia, che i Mefficami efeguiffaro per maffima di religione ciò, che $i$ Greci ufavaao per medicina? Ma no, non pretendo di far l'apologla dei Meficani in quefto punto. La lor religione in cid, che riguaida l'antropofagia, fu fenz' altro piu barbara di quella dei Ro. mani, degli Egizi, e di quelle altre Nazioni colte; ma del refto non pud dubicarfi, artefo ciò che abbiamo detro, che 6 la men fuperitiziola, men ridicola, meno indecente.

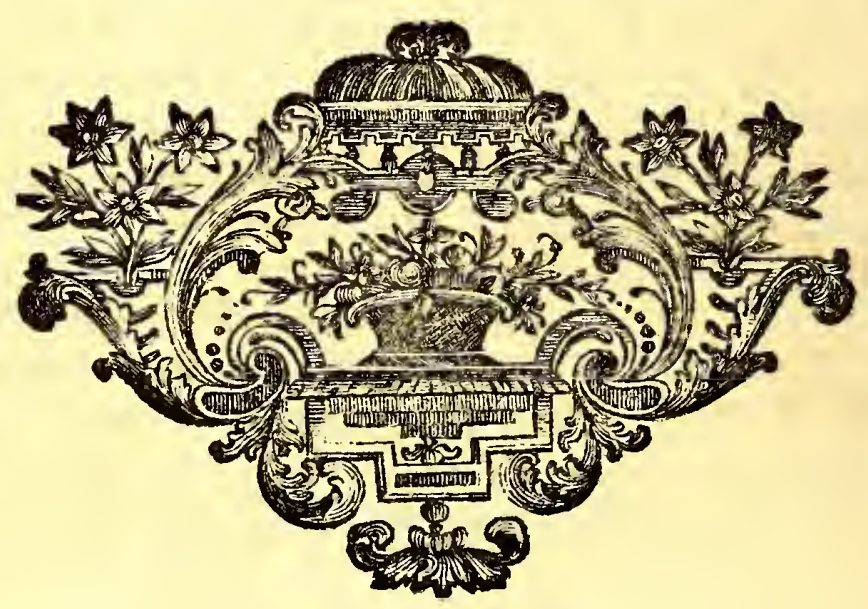

DIS.

(x: Quis invenit fingula membra bumana mandere? Qua conjectura inductus? Quim potef medicine ifta originem babuiffe? Quis venificia innocentiora fecit, quan remelia' ${ }^{2}$ Efo, barbari externique ritus invenerint; etiam-ne Graci fuas fecere bes artes? OC. Plinius Hif. Nat. lib. 28. cap. 8. 
 \\ DISSERTA ZIONE IX.}

SU L' ORIGINE DEL MALFRANCESE.

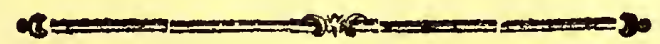

TElla prefente Differtazione non abbiamo a difputar fola: mente col Sig. de paw, ma eziandio con quafi turti gli Europei, i quali fon già generalmente perfuali, che il Malo francefe ebbe origine dall' America: poi hè efferdofi data vicendevolmente la colpa alcune Nazioni d' Europa per più di treno ta anni fu l'origiue d'una malattia sì vergngnofa, alla fine fi ace cordarono d'incolpare il nu vo Mondo. Noi dovremmo fenz dubbio efter tacciari di temerità in volendo combartere un'opinione si univerfale, fe gli argomenti, che fiamo per opporvi, - l'efempio di due moderni Europei non rendeffero fcufabile il noftro ardire. (a) Siccome tra i foltenitori della comune opinione il principale, il più rinomato, e colui, che ne ha fcrito to più copiofamente e più eruditamente ed il Sig. Aftru: dotto Medico Erancefe, cosł deffo fala da noi principalmente impus. gnato fervendoci per ciò in gran parte di quegli Ite@i materia: li, che egli ci fomminiftra nella fua opera. (b)

5. I.

(a) Quefti due Autori fono Guglielmo Becket, Cerufico di Londra, ed Ano tonio Ribero Sanchez. Becket fcriffe tre Difiertazioni, le quali furono inferite ne'volumi 30. e 3r. delle Tranfazismi Filofofiche, per provare, che il Malfrancefe era già conofciuto in Inghiltersa infin dal fecolo XIV. Ribero fcriffe una Differtazione, la quale fu fampata in Parigi nel I765.con quefto titolo: Differtation Jur l'origine de la maladie venerienne, dans la quelle on prouve qu clla n'd point eté portée de l'Amerique. Noi avendo letto il titolo di yueite Difertazione nel Catalogo di libri e manofcritti Spagnuoli aggiunto al romo IV. della Storia d'A merica del Dott. Robertfon, I'abbiamo cercaro qui, -in Ruma, in Genova, $e$ in Venezia, e non l'abbiamo potuto trovare: nè fappiamo, re l' Autore fia Spagnuolo o Portoghefe, come apparifce da' cognomi, o pur nato in Francia da Genirori Spagnno!i.

(b) De morbis venereis vol. 2. Mi fsia fervito dell' edizion di Venezia. 
Opinioni dei Medici anticbi intorno alla origine del Malfrancese.

Nei primi trenta anni, dappoi che comincio a fentirfi in Italia il Malfrancefe, non vi fu verun Autore, che attribuiffe la fua origine all' America, come poi diremo. Tutti gli Autori, che ne fcriffero prima del I525., ed alcuni anche di quelli, che fcriffero dopo, lo attribuirono a diverfe cagioni, la eui notizia recherà ai Lettori e compaffione e piacere.

Alcuni dei primi Medici allor viventi, come Corradino Gillini, e Gafpare Torella, fi perfuafero fecondo le idee di quei tempi, che il Malfrancefe era ftato cagionato dalla gran congiunzione del Sole con Giove, Saturno, e Mercurio nel fegno di Libra accaduta nel 1483 .

Altri, dietro al celebre Niccolò Leoniceno (c), l'attribuirono alle abbondantiflime pioggie, e alle inondazioni avvenute in Italia quell' anno, in cui comincio il contagio.

Giovanni Manardi, dotto Profeffore dell' Univerfità di Fersara, afcriffe l'origine di tal male al commercio impuro d' un Cavalier Valenzano infetto dalla lebbra con una meretrice; e Paracelfo al commercio d'un Lebbrofo francefe con una proftituta. Antonio Mufa Brafavola, dotto Ferrarefe, afferma, che il malfrancefe ebbe principio da una meretrice, che trovavafi nell' efercito dei Francefi in Napoli, la quale avea un afceffo nella bocca dell' utero.

Gabrielle Fallopio, celebre medico Modonefe, afferma; che gli Spagnuoli effendo pochi nella guerra di Napoli, ed i Francefi infiniti, avvelenarono una notte l'acqua dei pozzi, della quale doveano bere $i$ lor nemici, e che quindi ebbe origine il contagio.

An-

(c) Itaque dicimis, malun boc, quod Morbum Gallicum vulgo appellant inter epidemias debere connumerari... Illud fatis conftat, eo anne magnam aquarum per univerfam Italiam fuifje exuberantiam.... affivam autem ad illam veniffe intemperiem calidam Scilicet of bumidam \&6. Opufce de Morbo Gallico. 


\section{DISSER T A Z IO N E IX. 305}

Andrea Cefalpino, Medico di Clemente VIII. dice, avere egli faputo da quelli, che intervennero alla guerra di Napoli, che allorchè era affediata dai Francefi Somma, luogo nel vefuvio, dove è una grande ábbondanza d' eccellente vino greco, gli Spagnuoli fcapparono fegretamente una notte, lafciandovi una gran quantità di quel vino, mefchiato con fangue degli ammalati di San Lazzaro, e che entrandovi immedatamente i Francefi bevivero di quel vino, e tolto cominciarono a fentir gli effetti del mal venereo.

Leonardo Fioravanti dorto Medico Bolognefe, dice nella fua opera intitolata: Capricci Medicinali, avere egli faputo dal figliuolo di colui, che era ftato vivandiere dell' efercito d'Alfonfo Re di Napoli verfo l' anno 1456., che venendo a mancare $i$ viveri per la lunghezza della guerra tanto nell' efercito di quel $\operatorname{Re}$, quanto in quello de' Erancefi, $i$ vivandieri fomminiftravano agli uni, e agli altri della carne umana condita, e che da ciò prefe origine il Malfrancefe. Il celebre Cancelliere Bacone di Verulamio foggiugne, $(d)$ che la carne lor miniftrata era d'uomini ammazzati in Barberia, la quale conciavano, come il Tonoo.

Siccome neffuno feppe, nè potè fapere, chi fu in Europa il primo a patir quel gran male, cosi nemmeno fe ne pud fa: per la cagione; ma vediamo ciò, che porè avvenire.

\section{II.}

Il Malfrancefe poted comunicarfi all' Europa da altri paefa del Continente anrico.

Per dimoftrare, che il Malfrancefe pote comunicarfi per via di contagio all' Europa da altri paefi del medefimo Continente, bifogna, e bafta provare, che il fuddetto male fi pati gà in alcuni di que' paefi, e che effi aveano commercio coll Europa, prima che foffe fcoperto il nuovo Mondo. L'uno, e l'altro farà quì pienamente dímốtrato.

Storia Antica dal Melfico Tom. IV. $\quad$ Q q Va-

(d) Sylve Sylvorum centur. I. art. 26. 
Vatablo, il P. Pineda, il P. Calmet, ed altri Autori fo. ftennero, che tra le malattie, da cui fu travagliato il Santo Giobbe, fu una quella del Malfrancefe. Quefta opinione è tanto antica, che tolto che comparve quel male in Italia, alcuni lo appellarono il mal di Giobbe, ficcome ne fa fede Battifta Fulgofio, Autore allor vivente. (e) Il P. Calmet fi sforza $(f)$ di provare il fuo fentimento con una grande erudizione; ma ficcome nulla fappiamo dell' infermità di Giobbe, fuorchè quelo lo, che fi accenna nel facro libro, il quale può facilmente intenderfi d'altre malattie conofciute, o di qualcuna a noi affatto incognita, così non fi dee far conto di queft'opinione.

Andrea Thevet, Geografo Francefe $(g)$, ed altri . Autori affermano, che il Malfrancele era endemico nelle Provincie in. teriori dell' Africa fituate all' una, e all'altra riva del Senegal. Andrea Cleyer, Protomedico della Colonia Olandefe dell' Ifola di Java, dice, (b) che il Mal venereo era proprio e natural di quell ifola, e tanto comune, quanto la febbre cotidiaa. Lo fteffo avea affermato il Thuano. (i)

Giacomo Bonzio, Medico degli Olandefi nell' India Orientale, teftifica, $(l)$ che quel male era endemico in Amboino, e nelle Moluche, e che per contraerlo non vi bifognava. yerun previo commercio carnale. Cid venne in parte confermato dal ragguaglio de' compagni di Magallanes, i primi che fecero il giro del Mondo nel famofo vafcello $L$ a Vittoria, i quali teftificarono, fecondochè dice il Cronichifta Herrera $(m)$ di aver trovato in Timor, ifola dell' Arcipelago Moluco, un gran numero d'Ifolani infetti del Malfrancefé: il quale non vi fu certamente portato nè dagli Americani, nè dagli Europei già con. tagiati .

(e) Nell' opera iatitolata Difa factaque memordbilia lib. x. c. 4

(f) Differt. in Morbum Jobi.

(g) Cofmgoraphie univerfelle liv. I. chap. Ir.

(h) Epift. ad Chriftianum 'Mentzelium.

(i) Hiftor. fui temporis cap. $7 \mathbf{r}$.

(I) In Metbodo medendi, qua in Indiis Orientalibus oportet uti in cura morborum illic vulgo ac populariter graffantium.

(m) Dec. III. lib. IV. cap. I. 
Il P. Foureau Gefuita francefe, dotto, efatto, e pratico delle cofe della China, interrogato dal Sig. Aftruc $(n)$, fe i Medici della China reputavano il mal venero originario del lor paefe, o pur d'altronde portatovi, rifpofe, che i Medici Chinefi da lui confultati erano d'arvifo, che tal mal pativafi in quell' Imperio fin dalla più rimota antichità, e che in fatti i libri di medicina fcritti in caratteri chinefi, i quali ftimavanfi da loro antichi, nulla diceano intorno al cominciamento di quella malattia, anzi ne faceano menzione, come d'un mal antichiffmo anche in quel tempo, nel quale i fuddetti libri fi fcrivevano: che pertanto non era noto, neppur verifimile, che tal male vi foffe ftato portato da altri paefi.

Finalmente lo fteffo Sig. Aftruc dice, (o) a lui parere, dopo avere efaminate e ponderate le teftimonianze degli Autori, che il mal venereo non era proprio folamente dell' Ifola Haitì o Spagnuola, ma comune eziandio a molte regioni dell' antico Continente, e forfe a tutti i paefi equinoziali del Mondo, ne' quali regnava effo ab antico. Quefta ingenua confeffione d' un uomo, tanto iftruito in quefta materia, e peraltro tanto impegnato contra l'America, oltre a' teftimoni fopraccennati, va= le affai a dimoftrare, che ancorchè fupponghiamo il Malfrancefe anticamente efiftente in quel nuovo Mondo, nulla però può allegarfi in quefta materia dagli Europei contra l' America, che non poffa dirfi dagli Americani contra parecchj paefi del Mondo antico, e che fe era guafto, come vuole it Sig. de P., il fangue degli Americani non era più fano quello di tanti Afiatici ed Africani .

Il Sig. Aftruc foggiugne, che da que' paefi dell' Afia, e dell' Africa, ne' quali era endemico il Malfrancefe, effo potrebbe bensì comunicarfi pel commercio a' Popoli vicini, non però agli Europei; perchè a cagione d' effere fata creduta inabitabile, e inacceffibile la zona torrida, non vi era verun commercio tra que' paefi, e l'Europa. Ma a chi non è noto quel

$$
\text { Q q } 2 \text { gran }
$$

(n) Diflert. De origine morborum venercorsm inter sinas, ad calc. tom.. $x$.

(o) Da morbis oenereis lib. I. cap. גI. 


\section{DISSER TA Z IO N E IX.}

gran commercio, che ebbe per tanti fecoli l'Egitto da una parre co'paefi equinoziali dell' Afra, e da un' altra coll' Italia? Or perchè non avranno potuto i Negozianti afiatici portar dall'India infieme colle droghe il mal venereo in Egitto, e quindi portarlo in Italia i Veneziani, i Genovefi, ed i Pifani, i qua. li aveano da gran tempo un continuo commercio colla Città d'Aleflandria, ficcome altri Europei portarono in Italia dalla Sorla la lebbra, e dall' Arabia il vajuolo? Oltracciò tra que' molti Europei, che dal fecolo XII. innanzi intraprefero il viag. gio ne paefi meridionali dell' Afia, come Benjamino di Tudela, Carpini, Marco Polo, e Mandeville, tra i quali alcuni, come Marco Polo, s'innoltrarono fin nella China, non potè qualcun di toro portar nel fuo ritorno in Europa il contagio prefo in que' paefi afiatici? Qui non difcorriamo di quello, che in fatti avvenne, ma di ciò folamente, che poteva avvenire.

Non folo dall' Afia, ma ancor dall' Africa potè paffare in Europa il Malfrancefe, prima che folle fcoperta l' America; poichè i Portoghefi, trenta anni prima della gloriofa fpedizione di Colombo, aveano gia fcoperta una gran parte de paefi equinoziali deli' Africa, e vi aveano intavolato il commercio.Ornon potè qualche Portoghefe, quivi contagiato dal Malfrancefe, contagiare poi i fuoi Nazionali, e in feguito altre Nazioni d'Europa, ficcome forfe infatti avvenne fecondo quello, che appreffo diremo? Veda dunque il Sig. Aftuc in quante maniere potè comunicarfi il Malfrancefe all' Europa fenza intervento dell' America, contuttochè foffe dagli antichi creduta inacceffibile la zona torrida.

\section{I I I.}

\section{Il Malfrancefe poted venire in Europa fenza contagio.}

Prima di trattar quefto argomento bifogna dire un motto fu la natura, e la cagione fifica di quel male. Il Malfrancefe è fecondo i Medici una fpezie di cacheffia, nella quale la lin. $\mathrm{fa}_{\mathrm{a}}$, e maflimamente la parte fierola d'effa, prende una fingolar 
craffizie ed acrimonia. Il veleno venereo, dice il Sig. Aftruc, $(p)$ è di natura falfa, o piuttofto acidofalfa, corrofiva, e fiffa. Effo cagiona il condenfamento e l'acrimonia della linfa, e quindi nafcono le infiammazioni, $i$ porri, le ulcere, le erofioni, i dolori, e tutti gli altri orrendi fintomi noti a' Medici.

Quefto veleno comunicato a un uomo fano non dee confiderarfi, dice il fuddetto Autore, come un nuovo umore aggiunto agli umori naturali, ma piuttofto come una mera $d y \int c r a$ sìa, o viziofa qualità de'naturali umori, i quali degenerando dal natural loro ftato fi cangiano in acidofalc.

Or quafi tutti i Medici fi fon perfuafi, che quefto male non pud provenire altrimenti, che per via di contagio comunicato pel liquor feminale, o pel latte, o per la faliva, o pel fudore, o pel contatto delle ulcere veneree \&c. $\mathrm{Ma}$ io con buona pace di quefti Signori foftengo, che il Malfrancefe può affolutamente generarfi nell' uomo fenza verun contagio, o comunicazione coi contagiati; perchè effo può affolutamente generaŕa in quella fteffa maniera, in cui generoffi nel primo uomo che lo pat' : or coitui non l' ebbe per contagio, perchè allora non farebbe egli ftato il primo a patirlo, ma da un' altra cagione affai diverfa: dunque da fimil cagione, qualunque effa foffe, po. trebbe quella medefima cacheffia produrfi fenza contagio in altri individui della fpezie umana. Quefto è vero, dice il Sig. Aftruc in America, $O$ in altro fimil paefe, non però in Europa. E perchè l'Europa è s̀̀ privilegiata? perchè non vi concorrono rifponde il fuddetto Autore, quelle cagioni, che da principio po. terono cagionar quel male in America. $E$ quali fon quefte cagioni? Efaminiamole.

Imprima il Signor Aftruc dice, (q) che non dee annove. rarfi tra tali cagioni l'aria, la quale potè bensì cagionare altre

$\mathrm{ma}$ -

(p) De morbis venereis lib. 2. cap. 2.

(q) Videtur quidem e numero caufarum expungendus aer, qui in Hifpaniole morbos alios forsan inferre potuit, at vero luem veneream minime. Utique conAat, Europeos, qui eam infulam jam a 200. annis (immo pene 300 ) incolunt luen vineream ibider nunquarn contraxiffe nif contagione. Europ i tamen aerem ibidem ducunt of eundem, quem olim ducebant indignx, $O$ dubio procul eodem odo temperatum or confitutum. Afruc De Morbis Venereis lib. I. car. I2. 


\section{IO DISSERTAZIONE IX.}

malatic nell' Ifola Spagnuola, non però il mal venereo, perchè gli Europei, i quali fin da dugento anni e più abitano in quell' ifola, non vi hanno contratto mai quel male, fe non per via di contagio; eppur l'aria non v'è prefentemente diverfa da quella, che v'era trecento anni fa: e calo che prefentemente vi folfe diverfa, almeno non lo era nel principio del fecolo XV. Non dee dunque farfi conto dell' aria, ove fi tratta di fcoprir la prima origine del mal venereo. Contuttociò lo fteffo Sig. Aftrue dopo avere efclufa l'aria dal numero delle cagioni del Malfrancefe, fa ricorfo ad effa contraddicendofi apertamente, come appreffo vedremo.

Due fole cagioni vengono accennate dal Sig. Altruc, i cibi e il caldo. Quanto ai cibi dice, che gli abitatori della Spa. gnuola allorchè mancava loro il frumentone, il calave ec. fi cibavano di ragni, di vermi, di pipifrelli, e di s̀ fatti animaluzzi. Intorno al caldo afferma, che le donne nei paefi caldi fogliono effer travagliate da meftrui troppo acri e quafi virulenti, maffimamente fe ufano di cibi malfani. Ciò fuppofto difcorre cosł il lodato Autore:" Multis ergo \& graviffumis i) morbis indigena infula Haiti affici olim debuerunt, ubi nemo a mentruatis mulieribus fe continebat: ubi viri libidine

g2 impotentes in venerem obviam belluarum ritu agebantur: ubi 2) mulieres, quæ impudentiffimæ erant, viros promifcue admit2) tebant, ut teftatur Confalvus de Oviedo Hift. Indiar. lib. 5. 22 cap. 3. immo eoldem \& plures impudentius provocabant men2) Atruationis tempore, cum tunc incalefcente utero libidine ma2) gis infanirent pecudum more. Quid jgitur mirum varia, he2) terogenea, acria multorum virorum femina una confula, cum 2, acerrimo \& virulento menftrua fanguine mixta intra uterum 2) xltuantem \& olidum fpurciffimarum mulierum coercita, mo"ra, heterogeneitate, calore laci brevi computruiffe, ac pri" ma morbi venerei feminia conftituiffe, qua in alios, fi qui is fortè continentiores erant, dimanavere? ",

Ecco tutto il difcorfo del Sig. Aftruc fu la prima origine del mal venereo pieno da capo a piè di fallicà, ficcome appreffo dimoftreremo; ma fupponendo ora, che tutto foffe ve. 
ro, io affermo, che quello fteffo, che al dir di lui avvenne nella Spagnuola, potè fimilmente avvenire in Europa; perchè ficcome quegli Americani in mancando loro il frumentone, ed altri viveri ufuali, cibavanfi di ragni, di vermi ec. cus' gli Europei in mancando loro il frumento, ed altri buoni alımen. ti $f_{i}$ fon veduti talvolta mangiare forci, lucertole, e altri fiffatti animaluzzi, gli efcrementi di parecchi animali, ed anche pane fatto di farina d'offa umane, arrecando loro gravifima infermità. Bafta ricordarfi delle orrende fami, patite già in Europa, cagionate parte dal tempo, e parte dalla gue:ra. Vi fono poi mai fempre ftati degli uomini, i quali a guifa di beftie fi fon lafciati portar da una sfrenata libidine ai più efecrandi ecceffi. Vi fono fempre ftate delle donne sfacciate e fporchiffime, e fe ne potrebbe affermare ciò, che dice Plauto: Plus fcortorum ibi eft, quam mufcarum tum, cum caletur maxumb. Del refto non vi fon mancati mai nè fluidi feminali troppo acri, nè uteri eftuanti, nè meftrui virulenti. Potrebbono dunque tali cagioni produrre in Europa il Malfrancefe, come il produffero in America a detta del Sig. Altrue.

"No, rifponde quefto Autore, non è cosl; perchè effen. " do 'l'aria più temperata in Europa ( ecco il ricorfo all' aria "dopo averla efclufa dal numero delle cagioni del Malfrance" (e) non adeft eadem in virorum femine acrimonia, eadem in "menftruo fanguine virulentia, idem in utero mulierum fervor, "quales in infula Haiti fuiffe probatum eft: (le prove del Sig. Aftruc non fono altre, che quelle fopra efpofte) onde, fog" giugne, nè vi poteano mai produrfi quei fintomi dal concor" fo fimultaneo delle cagioni. E per dirlo in poche parole deefi " giudicar delle malattie, e delle lor cagioni, come della gene" razion degli animali e delle piante. Or ficcome in Europa " non generano i leoni, nè le f́cimie fi propagano, nè $i$ pap" pagalli fabbricano dei nidi, nè molte piante indiane o ame"ricane vengono in Europa, quıntunque vi fi feminino, cos̀ ") nè il Maifrancefe potè mai produrfi in Europa da quelle "cagioni, dalle quali, fecondo che abbiamo detto, fu gia " prodotio nella Spagnuola; perchè ciafcun clima ha la fua 


\section{2 \\ DISSERTAZIO NE IX.}

"particolar proprietà, e quelle cole, che in un clima vengo"no per fe fteffe, in un altro non polfono con verun' arte ves) nire, perchè, come dice il Poeta, Non omnis fert omnis "tellus."

Io voglio conceder molte cole al Sig. Aftruc, che da neffun altro certamente gli farebbono concedure. Io gli concedo, che non fia mai ftato in Europa nè quell'abufo delle donne meftruate, nè quell' acrimonia, nè quella virulenza nei fluidi del corpo umano, nè quel fervor nell' utero, che egli fuppone nell'ifola Spagnuola, quantunque dai libri di Medicina pubblicati da due mila anni in quà contti tutto il contrario. Io gli concedo, che non vi fi lieno mai veduti degli efempj della più sfrenata luffuria; perchè a lui par troppo il confeffar fiffatti efempi in Europa, $(r)$ e gli concedo inoltre, che tutte le donne e gli uomini dell' Europa fieno ftati faniffimi e caftifimi. Tueto ciò gli concedo, avvegnachè venga contradetto dalla Storia e dalla comun opinione dei medefími Europei. Contuttuciò io affermo, che il Malfrancefe potè affolutamente generarfi in Europa fenza contagio; perchè tutti quei difordini, che il Sig. Aftruc fuppone nell'ifola Spaguusla, poterono anche avvenire in Europa, ancorchè in realtà non vi foffero mai ltati. Quelle donne caltiffime poteano indotte da quelle ree paffioni, che fon comuni a tutti i figliuoli d'Adamo, divenir tanto incontinenti e sfacciate, quanto quell' Autore crede, che foffero le Americane della Spagnuola: Quegli uomini tanto fani poteano alimentarfi di cibi tanto nocevoli, quanto erano quelli degli Haitini. Lo fperma umano, il qual è da per fe molto acre, ficcome dice lo fteffo Sig. Aftruc, porè a cagione dei cibi malfani diventar più e più acre fino a prender quel grado d'acrimonia, che richiedefi pel mal venereo. I meftrui poteano diventar virulenti o dalla previa lor fuppreffione, o dalla pletora, 0 da molte altre cagioni morbofe tanto nei liuidi, quanto nei vafi. L'ute-

(r) Sed efo: demus in Elbropa venerem aque impuram, atque in Hifpaniola exerceri; neque enim contra pugnare placet, quanquam ea tamen nimia videantur. Aftruc De morbis venereis lib. I. cap. 12 . 


\section{DIS S E R T A Z I O N E IX. ZI}

I' utero poi poteva concepire un ardore ecceffivo dal fangue rifcaldato coi liquori gagliardi, o coi cibi troppo caldi. Non credo che vi fia verun Medico, che contraddica a quefte verità: e poichè il Sig. Aftruc confeffa, che il veleno venereo non है un nuovo umore aggiunto agli umori naturali, ma una mera depravazione degli fteffi umori, perchè quelle cagioni, che cagionarono al dir di lui tal depravazione nella Spagnyola, non ayranno potuto cagionarla anche in Europa? Perchè is Euro$\mathrm{pa}$, dice egli, l'aria è più temperata.

Quefto ì l'unico futterfugio, che refta al Sig. Aftruc; ma non gli giova niente; perchè egli è certo, che in molti paefi d'Europa, come in Italia, e particolarmente nella parte più meridionale di effa, l'aria è più calda nella ftate, che nella Spagnuola, e non v'è peraltro la ragion di credere, che fiz neceffario il calor di tutto l'anno, e non bafti quello d'alcuni mefi per cagionare quella depravazion degli umori. Ma chi ha penfato mai, che fia d'uopo del calor efterno dell' aria per caginnarfi quella Atraordinaria acrimonia e virulenza negli umori? Lo fcorburo è una cacheffia molto fimile a quella del mal venereo, ma più terribile, la quale porta feco una ltupenda acrimonia e corruzione nel fangue: or quefta forta di malattia viene tanto nelle regioni calde, quanto nei paefi, e nei mari fettentrionali e più fpeffo fi cagiona viaggiando nelle zone temperate o fredde, che nella Torrida: dunque non è neceffaria l' aria calda, acciocchè fi generi una ftupenda acrimonia e corruzion negli umori.

Finalmente il Sig. Aftruc vuole, che fi giudichi delle ma. lattie, e delle lor cagıni come della generazion degli animali; ed afferma, che ficcome i Leoni non generano in Europa, nè le fcimie vi fi propagano, così nemmeno vi fi può produrre il Malfrancefe da quelle cagioni, che il produffero nella Spagnuola. Ma che direbbe il Sig. Aftruc, fe vedeffe i Leoni divenir più forti in Europa, e le fcimie propagarvifi affai più, che in Africa? Direbbe fenz'altro, o almeno dovrebbe dire, che il clima dell' Europa era più atto e più confacevole di quel10 dell' Africa alla generazion di tali animali, ora il Malfane Storia Aintica del Meffico Tomo IV. R. R cefe 
cefe \& divenuto affai più forte in Europa, che in America; come lo confeffa il Sig. Aftruc, e anche il Sig. de Paw, $(f)$ e l'Oviedo $(t)$, cioè quell'Autore, che pud dirfi l'inventore di qual male in America, ed in oltre effo fi è propagato affai più in Europa, che in America, ficcome è noto a tutti coloro, che fono ftati in amendue quefte parti del Mondo, o fe ne fon bene informati: dunque giulta $i$ principi del Sig. Altruc il clima d'Europa è più atto e confacevole di quello d'America alla generazion del Malfrancele.

Finora abbiamo ragionato nella fuppofizione, che foffe vero cid, che rapporta il Sig. Altruc nel fuo difcorfo; ma oltre ad alcuni errori in materia di Fifica, fu'quali non conviene difcorrere, vi fono ancora dei fatti arbitrariam nte fuppofti, e contrari alla verita. Egli Cuppone I. che gl' Indiani della Spagnuola fi cibavano di vermi, di ragnı, ec.; ma quefto for re accadde alcuni anni dopo la fcoperta di quell'afola, allorchè gli Americani fuggendo dal furor dei Conquiltatori Europei, andavano fmarriti per li bofchi, mancando loro il frumento. ne, $e$ il carave, perchè nun gli aveano feminati in odio dei lor nemici, come ne $f_{d}$ fede Pietro Martire d'Anghiera (u), mangiavano quello, che vi trovavano; ma niun Autore antico afferma, che effi ufaffero di tali cibı, prima che vi approdaffero gli Spagnuoli; e per dimoltrare, che i cibi fuddetti ebbero qualche influffo nel Malfrancefe, bifngnerebbe provare, che l'ufo d'effi fu ameno tanto antico in quell' ifold, quanto lo era tal infermità a giudicio del Sig. Aftruc: ciò che egli non ha fatto, nè poteva fare. 2. Egli afferma, che nella ifola Spagnuola ne: mo fe a menftruatis mulieribus continebat; $\mathrm{md}$ io vorrei, che per ciò confermare aveffe allegato la teltimonianza di qualche antico Autore; poichè io non trovo chi lo dica, anzi veggo, che tra le cofe fingolari notate dagli Scrittori Europei negli Americani, anche nelle barbare tribù, fi è quella di non ufar delle

(f) Recberch. Pbilofopb. part. I.

(t) Stor. Gener. delle Indie lib. ro. cap. 2.

(u) Sommar. della Stor. delle Indie Occid. 
delle donne durante la periodica lor evacuazione. Il Sig. de Paw, quel nemico capitale di tutto il nuovo Mondo, e quel gran Ricercatore delle immondizie americane, dice così nella I. parte delle fue Ricerche:",Vi era una legge preffo tutti i "popoli felvaggi del nuovo Mondo di non accoftarfi alle don" ne nel tempa delle lor regole, o perchè il contatto del fluf. "fo fofle da loro ftimato perniciofo, o perchè il folo iftinto "lor infegnaffe un tal riguardo ." 3 . il Sig. Ailruc rapprefenta gli uomini, e le donne della Spagnuola oltremodo rifcalo dati ed agitati da una violenta e rabbiofa libidine; il C. de Buffon e il Siga de PaW (x) rapprefentano per lo contrario tuto ti gli Americani freddifimi, e infenfibili agli ftimoli dell' amo: re. Che vuol dire fiffatta contraddizione, fe non che quefti Autori fiftematici dipingono gli Americani con quei colori, che tornano loro a conto? Quando vogliono rilevar l'apatia o infenfibilità di quegli uomini, dicono, che effi fono freddiffimi; ma quando pretendono fcreditare i lor coftumi, 0 incolpargli del Malfrancefe, allora affermano, che effi fono oltremifura libidinofi. Il Sig. Aftruc allega il teftimonio di Gonzalo de Oviedo nel lib. 5. cap. 3. della fua Storia per convincere, che le donne Haitine erano troppo sfacciate e che proftituivanfi indiftintamente a tutti gli uomini; ma oltrechè la teftimonianza di quefto: Autore contra gli Americani vale meno, che niente, ficcome appreffo dimoftreremo, egli non dice ciò, che vuol farci credere il Siga Aftruc. Ecco quello, che ne dice nel citato luogo l' Oviedo: Le donne di quefta ifola erano continenti coi lor vomini, ma ai Criftiani faceano volentieri copia di fe ftefse. Ecco quello, che ne dice l'Herrera: $(y)$ Le donne erano continena ti coi lor Nazionali, e difonefte coi Caftigliani. Se elleno erano continenti coi loro Nazionali, non potea la lor incontinen. za cagionare il Malfrancefe, prima che vi approdaffero gli Spagnuoli. Se erano difonefte folamente coi Criftiani, fi dee cre.

$$
\text { R } r \text { de- }
$$

(x) Vedafi cid che dicono intorno alla freddezza degli Americani il $C$. de Buffon in vari luoghi della rua Storia. Naturale, e il Sig. de Pa nella pa I. delle fue Ricerche.

(y) Dec. 1. lib. 3. cap. 4. 


\section{DISSERTAZIONE IX.}

dere, che elleno erano più fpinte a tali difordini dalla impor. tunità o dalla paura dei lor Conquiftatori; che dalla propria libidine. Finalmente quanto afferma il Sig. Altruc intorno all'acrimonia dell' umore fpermatico, alla virulenza del fangue meAtruale, alla fporcheria delle Americane, e al loro fervor uterino è un difcorfo in aria, e fenza verun fondamento nella Storia .

Prima di rerminar quefto articolo non poffo a meno di non far menzione della non men fozza, che ftravagante opinione del Dott. Giovanni Linder Inglefe fu la cagione del Mal. francefe, affinchè fi vegga, fin dove fia giunto l'impegno di fcreditare in quefta materia gli Americani. Egli dunque afferma, che quel male ebbe origine dalla congiunzion carnale degli $A$. mericani coi Satiri, ovvero cercopitechi grandi; $(z)$ ma per fortuna degl' Indiani dell' Ifola Spagnuola, non erano in effa, nè in verun' altra di quelle Ifole Gercopitechi nè grandi, nè piccoli .

\section{IV.}

\section{Malfrancefe non venne da America.}

Abbiam di fopra accennato, che nei primi trenta anni, dappoi che fu feoperta l'America, neffuno afcriffe l'origine del Malfrancefe a quel nuovo Mondo. Io almeno, dopo aver confultati moltiffmi Autori, tanto medici, quanto Storici, che in quei primi tempi fcriffero di tal male, e della fua origine, non ne ho trovato neppur uno, che fofe di tal fentimento: nè il potè trovare il Sig. Aftruc, contuttochè aveffe cercato tra turti gli Scrittori Italiani, Francefi, Inglefz, Spagnuoli, e Tedefchi, chi patrocinaffe la fua opinione. Il primo, cui vense in tefa il penfiero d' incolpar l'America del Mlalfrancefe, fu

Gon-

(z) Originem duxit a Sodomia bomines inter o cercopitbecos magnos, five veterum Satyros aliquando exercita. Exercitat. de venenis cap. I. \& 10. Quo commento, dice il Sig. AAruc, ut nibil vanius et abfurdius, for nibil putidiss. confingi potwit. 
Gonzalo Hernandez de Oviedo, il qual nel Sommario della Storia delle Indie Occidentali, prefentato a Carlo V. nel 1525. affirmo, che gli Spagnuoli contagiati nell' ifola Spagnuola ritornati poi in If pagna coll' Almirante Colombo, e quindi paffati in Italia col Gran Capitano, attaccarono quel male alle $\mathrm{Na}$ politane, e quefte ai Francefi ec. Siccome quefto Autore era Letterato, e viffe alcuni anni in America, éercitando una carica onorevole, così la fua autorità traffe dietro a lui quafi tutti gli Scrittori; poichè da una parte tutti lo credevano bene informato, e da un' altra tornava a conto a tutti, che egli foffe creduro per liberar ciafcuno la fua Nazione dall' imputazione d'un male sì vergognofo. Ma prima di efaminare il fuo ragguaglio, bifogna dare a conofcere quefto Scrittore, la cui autorità è ftata il principale, o per dir meglio, l'unico appoggio della comun opinione.

Monfig. de las Cafas, il qual viffe in America mel medefimo tempo dell' Oviedo, e lo conofceva affai bene, nell' impugnazione del Dott. Sepulveda, il qual allegava I' autorità dell' Oviedo contra gl'Indiani, dice così: "Quello; che più pre" giudica alla perfona del Rev. Dottore preffo i prudenti e ti" morati, che hanno notizia oculare delle Indie, fi è l' alle"gare, che egli fa, come Autore irrefragabile l' Oviedo nella " fua falffifima ed efecranda Storia, effendo egli ftato uno dei "Tiranni ladroni e diftruttori delle Indie, come egli medefi" mo il confeffa nella prefazione della prima parte, e nel lib. "6. cap. 8., e per tanto capitale nemico degl'Indiani. Giu"dichino le perfone favie, fe tale Scrittore fia idoneo tefti" monio contro gl' Indiani. Eppur quefti ̀̀ appellato grave $e$ "diligente Cronichifta dal Dottore, perchè lo trovò confacevo"L al fuo intento; ma egli è certo, che quella Storia ha po. " co più di fogli, che di bugle, ficcome largamente proviamo. " in altri fcritti, e nell Apologì.," Infatti il Cronichilta Herrera, uomo giudiciofo ed imparziale, dice, che Monfig. de las. Cafas ebbe ragione di lagnarfi dell' Oviedo, e che coftui non fu troppo efatto in alcune notizie. Egli per altro promofe alcune opinioni Atravaganti, indotto dallo pirito d'adulazione, o 


\section{I8 DISSER TAZIONE IX.}

dalla vanità Bafta leggere il libro fecondo della fua Storia, nel quale oltre al dire, che i Trojani difendevano dagli Spagnuo. li, afferma, che le ifole Antille fon le Hefperidi degli Antichi, e che furono così chiamate da Helpero Re XII. di Spa. gna, il quale fu Signor d'effe 1658 . anni prima dell' era CriStiana. " In quefta maniera, foggiugne, con si antica ragione, "E per la via detta ritornò quefta Signoria alla Spagna in ca"po di tanti fecoli : e come cofa fua, pare, che abbia volu"to la Divina Giuftizia refticuirla ad effa, acciocchè la poffeg" ga per la fortuna dei due felici e Cattolici Re D. Ferdinan. " do, e Donna Ifabella a " $(A)$ Tal \&̀ l' Autore della comun opinione; efaminiamo ora il fuo ragguaglio.

Egli parla con qualche varietà nel Sommario della Sto. ria, e nella Storia medefima; ma ficcome quefta è l'opera fua principale, la più: ftefa, pubblicata alcuni anni dopo il Sommario, e lavorata con maggiore ftudio, così dobbiamo piuttofto ftare a ciò, che dice in effa, dovunque fia qualche divario. Egli dunque dice nel libo, 2a capo. 14. della. Storia. Generale delle Indie, che gli Spagnuoli ritornati con: Colombo in Ifpagna nel 1596. dal fecondo lor viaggio in America, vi riportarono dall'ifola Spagnuola il Malfrancefe infieme colle moAtre dell' oro delle famofe miniere di Cibao: e che alcuni d'effi gia contagiati, che paffarono in Iraliz col Gran Capitano Gonzalo. Hernandez de Cordova, contagiarono per mezzo delle Italiane i Francefi, che eran venuti col Re Carlo VIII. a impadronirfi del regno di Napoli: Ma quefto ragguaglio è affatto infuffiftente, e pieno d'anacronifmi; poichè Colombo ria tornd in Ifpagna dal fuo fecondo viaggio ai 3. Giugno 1496., e fappiamo da infiniti teftimoni oculari, che l' Europa era già. infetta dal Malfrancefe almeno infia dal 1495:- dunque: tal infezione non porè provenire: da quegli Spagnuoli, che allora ritorarano con Colombo. Per dimoftrar poi colla maggior evidenza ftorica, che i Francefi, che erano a Napoli col Re Cara lo,

(A) Il dotto. D. Ferdinando Colombo nel cap. 9. della fua Storia rinfaccia all'Oricdo la fravaganza delle fue opinioni; e l'infedelta nelle fue citazioni. 
lo VIII., non poterono effer contagiati dalle truppe Spagnuole, che vennero col Gran Capitano in Italia, bafta efporre femplicemente le date, come le troviamo preffo Guicciardini, Mariana, Mezeray, ed altri Storici Italiani, Spagnuoli, e Fran. ceíi. Il Re Carlo VIII. marciò col fuo efercito verfo l'I Italia nell' Agoito 1494.: gilunfe ad Afti, città lungo il Tanaro, ai 9. Settembre: encrò in Roma ai 31. Dic. e in Napoli ai 22. Febbrajo 1495. In queíta Città aon iftette più di tre mefi; perchè confapevole della gran confederazione fatta contro lui, fi affrettò per ritornare in Francia. Ufci da Napoli ai 20. Maggio, come ne $f_{a} n n c$ fede il Guicciardini, il Bembo, il Miria. na ec., ed avendo vinto ai 6 . Luglio la famola battaglia di Fornovo contra i Veneziani, fi ritiro precipitofamente alla fuz Corte, conducendo il fuo efercito contagiato dal mal venereo, come teltificano rutri gli Storici di quei tempi. Il Gran Capitano, trattenuto in Majorica, e in Sardignd da' venti contrari, non porè arrivare colla fua armata a Meifina prima de' 24. Maggio 1495. cicè quattro giorni dopo, che il Re Carlo fi era partito da Napoit col fuo efercito contagiato: dunque quefto non $\mathrm{fu}$, nè porè effer con'agiato da quelle iruope fpagnuole, fe già nou fi vunle, the queili iteff venu contrari, che impedivano l'armita del gran Capitano d' accoftarfi all' Italia, vi portaffero il contagio. Io mi maraviglio, che gli Autori della comun opinione non fi accorgeffero d' un si manifelto anacronifmo. Potrebbe qualcuno dire, the quel contagio non fu portato dalle truppe del gran Capitano: ma da altre truppe Spagnuole venute prima in Italia; $m_{d}$ oltrechè tanto l'Oviedo inventor della comun opinione, quanto gii altri Scrittori, che gli tengono dietro, atcrivono generalmente il contagio di Napoli alle truppe del $G$ an Capitano; io non ho potuto trovare avendone fatte diligenti ricerche, che dalla fcoperta dell' America fino all' arrivo del Gran Capitano fien venure altre truppe Spagnuole nel Continente dell' Italia; anzi dal ragguaglio del Mariana apparifce tutto il contrario: non furono dunque le truppe Tpagnuole quelle, che cagionarono il contagio di Napoli.

Per quello che abbiam detto di lopra non fi dee penfare; che 
che il Malfrancefe foffe folamente alcuni giorni anteriore in Iralia all' arrivo delle truppe Spagnuole; poichè fappiamo per Ia depofizione de' più bravi Medici di quel tempo, che tal male vi cominciò almeno alcuni mefi prima, che vi approdaffe l' armara Spagnuola. Gafpare Torella Valenzano, Medico di Papa Aleffandro VI. allor regnance, (B) Wendelino Hoock, dotto Tedefco, e Profeffore di Medicina a que' tempi in quelta Univerfita di Bologna (C), Giacomo Cattaneo di Lagomarfini, dotto Medico Genovefe $(D)$, Giovanni di Vigo, Genovefe, Medico e Cerufico di Papa Giulio II. (E); Quefti quatero Autori, oltre ad altri affai autorevoli, perchè dotti e bene intendenti di malatrie, e perchè ne furono tẹtimoni oculari, teftificano, che il Malfrancefe cominciò a fentirfi in Iralia fo dal 1494. Non vi è peraltro da maravigliare, che vi fia qualche divario tra gli Autori intorno al comiaciamento di quel male; porchè da alcuni fu più prefto, che dagli altri offervato, a cagione di non effere tato nel medefimo tempo fentito in tutti gli Stati di quefta penifola.

Ma potrebbe ancor dirfi, che benchè Oviedo frafi sbaglia. to nella Storia, affermando, che i primi, che portarono il Malfrancefe in Ifpagna furono quelli, che vi ritornarono col Colombo nel 1496., non cosi nel Sommario della medefima Sto: ria ,

(B) Gallis manu forti Italiam ingredientibus, of maxime regno Parbenopeo occufato, $\mathscr{O}$ ibi commorantibus, bic morbus detectus fuit. Tract. de Dolore in $\boldsymbol{P}$ udentagra in lucem edito anno isoo. Nel che fi vede, che il Malfrancefe. conninció in Italia, dacchè vi entrarono i Francefi, benchè ll fuo grande aumento foffe, dappoichè efli occuparono il regno di Napoli. I Francefi entrarono, ficcome abbiam detto, in Iralia nel Settembre 1494.

(C) Sicut evenit boc tempore, fcilicet $a b$ anno 1494. ufque ad pre(entem ansam 1503., quo morbus quidam contagiofus, qui Gallicus appellatur $\mathcal{F}_{c}$. Opulc. de Morbo Gallico typis edito anno 1502.

(D) Anno Virginei partus 1494. invadente Carolo VIII. Francorism Rege regnum Partbenopium, Alexandro vero VI. ea tempefate Summum Pontificatum gerente, exortus ef in Italia' monfrofus morbis nullis ante feculis vifus $\mathcal{O}^{\circ}$ : Tract. de Morbo Gallico elucubrato anno isos.

(E) Anno 1494. de Minfe Decembri, quo anno Serenifsmus ille Carolus Francorum Rex magna comitante caterva verfus Itali partes iter accepit at regnum Neapolitanum recuperandum, apparuit utique codem anno quoddam morbi genuo quaji per totam Itaiiam incognita natura, quem variis, of diverfis nominibus diverfa Nationes appellarunt. In Praxi Chirurgiz typis edita annorsia. lib. s. cap. $\mathrm{I}$. 
ria, pubblicato alcuni anni prima, nel quale dà chiaramente ad intendere, che anche tra coloro, che erano ritornati col medefimo Colombo in Ifpagna nel 1493. vennero alcuni contagiati; ina neppur quefto è vero, nè verilimile. Confta dal.e lettere dello fteffo Criftoforo Colombo, addotte dal fuo dotto figlio D. Ferdinando, che egli mife piede a terra la prima volta nell' Ifola Spagnuola ai 24. Dic. 1492., perchè gli fi ruppe in una fecca una caravella della lua miferabile armata: che tutti quei giorni, che vi ftette dai 24. Dic. fino ai 4. Gennajo, furono dalla fua poca gente implegati nel cavar dalla fecca il legname della caravella per farne una piccola fortezza, nella quale avendo lafciati quaranta uomini, s'imbarcò quello fteffo giorno col refto della fua gente per ritornare in llpagna a portar la nuova della fcoperta di quel nuovo Mondo. Tutte le circoftanze del loro arrivo in quell I fola non permettono di fofpettare, che gli Spagnuoli aveffero agio d'innolttarí a tanta familiarità colle Americane, quanta fi richiedeva per reftare inferti dal Malfrancefe. La vicendevole ammirazione cagionata agli uni ed agl' altri dalla veduta di tanti oggetri nuovi, e la breviffima dimora di foli undici giorni occupati nella gran fatica di eftrarre $i$ legnami della caravella, e di fabbricare in tanta fretta quella fortezza dopo $i$ difagı d' una navigazione la più lunga, e più pericolofa, che fi foffe farta fino allora, rendono affdtto inverifimile quefta congettura. Non meno inverifimile fi rende dal filenzio del medefimo Colombo, del fuo figlıo D. Ferdinando, e di, Pietro Martire d'Anghiera, i quali defcrivendo i gran dilagi di quella navigazione, nulla dicono di tal malattia.

Ma ancorchè concedeffino, che quegli Spagnuoli, che rio cornarono dal permo viaggio, vennero infetti dal Malfrancefe, tuttavia diremmo, che il contagio d'Europa non venne da loro, atrefo le reftimonianze d'alcuni Scrittori autorevoli allor viventi. Gafpare Torella, dotto Medico da noi fopra citato dice uella fua opera intitolata Apbrodyfiacum $(F)$, che il MalStoria Antica del Melfico Tom.IV.

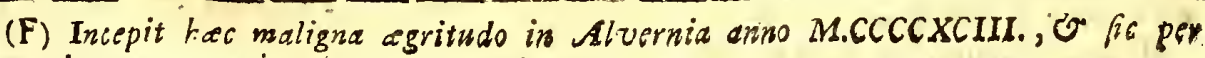
contagionem pervenit. $\mathcal{O} c$. 
francele cominciò in Alvernia, Provincia di Francia affai diAfante da Spagna, nel 1493. Battifta Fulgofio, o fid Fregofo, Doge di Genova nel 1478. nella fua curiofa opera intitolata: Dicta Factaque memorabilia, e ftampata nel 1509. afferma (G), che il Malfrancefe cominciò a conofcerf́ due anni prima, che il Re Carlo VIII. veniffe in Italia. Or quefti venne in Italiz nel Settembre 1494.: dunque quel male fu conofciuto fin dal 1492., O al più tardi nel comminciare il 1493., cioè alcuni mefi prima, che Colombo ritornaffe dal fuo primo viaggio. Gıvanni Leone, già Maomettano, natio di Granata in Ilpagna, volgarmente appellato Leone Africano, nella fua Defcrizione dell Africa, compofta in Roma fotto il Ponrificato di Leone X. dopo che egli fi era convertito, dice, che gli Ebrei, fcacciati da Spagna ai tempi di Ferdinando il Cattolico, portarono in Barberia il Malfrancele, e contagiarono gli Africani : che però fu quivi appellato Male Spagnuolo. $(\boldsymbol{H})$ Or l' editto dei Re Cattolici intorno alla efpulfione degli Ebrei fu pubblicato nel Marzo 1492. Ficcome dice il Muriana ( $I)$, concedendo loro von più di quattro mefi, acciocchè potelfero vendere $\mathrm{i}$ lor beni, fe non voleano portarli feco: e nel mefe feguente fu pubblicato un altro editto da Frà Tommafo Torquemada, in. quifitore Generale; nel qual fa proibì ai Criltianı fotto graviffrme pene di trattar cogli Ebrei, e di fomminiftrar loro dei viveri paffato quel termine dal Re prefcritto: ficchè tutti, fu.r. chè quelli, che fi fecero, o finfero di farfi Criftiani, furono

co.

(G) Biennio antequam in Italiam Carolus (VIII.) veniret, nova agrituto inter mortales detecta fuit, cui nec nomen, nec remedia $M$ edici ex veterum $\mathcal{A}$ iEorum difciplina invenielant, varie, ut regiones erant, appellate. In Gallia Neapolitanum dixerunt morbum, at in Italia Gallicum appellabant. : Lib. 1. cap. 4. 5. ultimo.

(H) Hujus mali ne nomen quidem ipfis Africanis notum erat antequam Hipaniarum Rex Ferdinandus Judxos onnes ex Hifpuvia prnfigaljet: qui wbi in patriam jam rediiffent, caperunt miferi quidam ac feeteratifsini etetbiop:'s cum illorum mulieribus babere commercium, ac fic tantem veluti pip manus poftis bac per totam fe fparft regionem, ita ut vix fit familia, que ab boc malo remanferit libera. Id autem fibi frmifime atque indubitale perfuaferunt ex Hifpania ad illos trafmigralfe. Quamobrem $\mathscr{\sigma}$ illi morbo Malum Hifpacicum (ne womine deo fitueretur) indiderunt. Lib. I.

(I) Stor. Gener. di Spagna lib. 26. cap. $x_{4}$ 
coftretti ad ufcirne, prima che Colombo andaffe a fcoprir l'A. merica; poichè quefti non falpò dal parto prima dei 3. Agofto di quell'anno: dunque il Malfrancefe cominciò in Europa, prima che foffe fcoperta l'America. Oltracciò troviamo tra le poesie di Pacifico Maffimo Poeta d'Afcoli pubblicate in Firenze nel 1479. alcuni verti, nei quali defcrive la gonorrea virulenta, e le ulceri veneree, che egli pativa, cagionate dai froi ecceffi. $(K)$

Non contento l' Oviedo d'affermare, che il Malfrancefe venne dalla Spagnuola, fi efibifce anche a provarlo. Ecco le fue prove 1. Col guajaco (legno abbondante nella Spagnuola) fi guarifce meglio, che con ogni altra medicina, di quell'orrenda infermità delle bolle: e la Clemenza Divina, dove permette per li noftri peccati il male, la per fua mifericardia provuede di rimedj. Se quefto argomento foffe buono, dovrebbe conchiuderfi, che l'Europa, anzichè l'Ifola Spagnuola, è la patria del Malfrancefe; poichè tutti fanno, che il rimedio piu efficace contro tal male è il mercurio, il quale effendo comune in Europa, non fi trovò nella Spagnuola, e neppur era dagl' In. diani conofciuto: Egli è certo, che appena, che comparve il morbo gallico in Europa, vi fi comincid ad adoprare il mercurio, e che l' ufarono Giovanni Berengario da Carpi, Galpare Torella, Giovanni Vigo, Wendenlino Hoock, ed altri famofr Medici di quel tempo, benchè fcreditato poi per l'indifcrezione d'alcuni Empirici, andaffe per qualche tempo in difufo. Il Guajaco non cominciò ad adoprarfi fe non fe nel I5I7., cioè anni venticinque dopo la fcoperta del Malfrancefe. La falfapariglia comincid ad ufarfi nel i 535. , la China verfo il medefimo tempo, e il faflafràs poco dopo.

L'altra pruova dell'Oviedo (poichè non ne allega piì di due ) fi è, che tra quegli Spagnuoli, che ritornarono col Co. lombo dal fuo fecondo viaggio nal 1496 . v' era $D$. Pietro Margarit, Cavalier Catalano 2 il qual, dice, andava cosi infermo, $S[2$, a fi

(K) Hecatalegii lib. 3. Ad Priapum, \& lib. 8. Ad Mentulam. Non capia mo qul $\mathrm{i}$ fuddetri rerf, perchè fono troppo indecenti. 


\section{DISSERT A Z IONE IX.}

5) e fi lamentava tanto, che ben mi credo, che effo fentiffe " quei dolori, che fentir fogliono coloro, che fon da quefta " paffione tocchi, benchè io non gli vedeffi mai veruna bolla " nel vifo. Indi a pochi mefi nel medelimo anno 96. comin. " cid a fentirfi quefta infermitz̀ tra alcuni Cortigiani; poichè " in quei principj andara quefto male tra perfone baffe... O. "ra fegui poi, che il Gran Capitano fu mandato in Italia 9) con una groffa e bell'armata.... e fra quegli Spagnuoli, ") che andarono in quelt' armata, vi furono alcuni infetti da "quella infermità: onde col mezzo delle donne ec., (L) Tali fono le prove dell' Oviedo, le quali neppur meritavano, che fo ne faceffe menzione.

Il Sig. de Paw fi crede d'aver vinto la lite, $(M)$ e d'aver dimoltrato la verità della comun opinione colla teftimonianza di Roderico Diaz de Isla, Medico di Siviglia, il qual da lui dicefi Autor contemporaneo, ficcome la fua teftimonianza Itimafi decifiva; ma nè Diaz fu Autor contemporaneo, poichè non ifcriffe, fe non feffanta anni dopo la fcoperta del Malfrancefe, nè il fuo ragguaglio merita alcuna fede. Egli dice, che i primi Spagnuoli, ritornati dalla Spagnuola col Colombo nel 1493. portarono il contagio a Barcellona, dove allora fi trovava la Corte: che quefta Città fu la prima ad infettarfi: che il male vi fece una tale ftrage, che fi fece ricorfo alle preghiere pubbliche, ai digiuni, e alle limofine per placar la colJera di Dio: che effendo l'anno feguente paffato in Italia il Re Carlo di Francia, certi Spagnuoli, che vi erano infetti, 0 molti reggimenti, fecondochè dice il Sig. de P., mandati da Spagra per opporfi all' invafione del Re Carlo, contagiarono i Francefi. Ma dalla Storia fappiamo, che niun reggimento nè conta. giato, nè fano, nè alcuni altri Spagnuoli furono mandati in ItaIia, prima che il Re Carlo ufciffe da Napoli col fuo efercito gia contagiato fer tornarfa in Francia. Per ciò, che riguarda il contagio di Barcellona, fappiamo, che quando vi arrivò il Colom. bo

(L) Stor. Gener. delle Indie lib. 2, cap. 14.

(M) Rech. Philor. part. 2. fet. 3. 
bo, vi fi trovava l'Oviedo. Or fe foffe vero quello, che racconta il Medico Sivigliano, Oviedo, il qual andava cercando prove per confermar la fua ftravagante opinione, avrebbe fenza dubbio alle. gato quella ftrage da lui veduta, quelle preghiere, quei digiuni, e quelle limofine, e non fi farebbe prevaluto delle miferabili prove del Guajaco, e delle lamentazioni del Margarit. Oltrechè il Malfrancefe è ancor più antico di quell' epoca in Europa, come già abbiamo detto.

Pare, che i Medici Sivigliani fieno ftati a quei tempi i più male informati intorno all' origine del Malfrancefe; poichè Niccolò Monardes, Medico anch' effo di quella Città, e contemporaneo del Diaz, ne $f_{a}$ un ragguaglio sì pieno di favole, che non può leggerfi fenza fdegno. Dice dunque, $(N)$ che l'an"no 1493. nella guerra, che il Re Cattolico ebbe in Napoli " col Re Carlo di Francia, venne Don Criftoforo Colombo " dal primo difcoprimento, che egli feee dell' Ifola di S. Da"menico ec. e conduffe feco da quell' ifola una gran moltitu"dine d'Indiani e d'Indiane, i quali menò a Napoli, dove "allora fi trovava il Re Cattolico finita la guerra. E perchè ") era pace tra $\mathrm{i}$ due $\mathrm{Re}$, e gli eferciti praticavano infieme, "giunto quivi il Columbo coi fuoi Indiani ed Indiane, co" minciarono ad ufare gli Spagnuoli colle Indiane, e gl' India"ni colle Spagnuole, e in tal maniera infettarono gl' In" diani e le Indiane l' efercito degli Spagnuoli, Italiani, e Tedefchi , ec. Chi fi perfuaderebbe, che un Letterato "Spagnuolo foffe per isfigurare a tal fegno i fatti pubblici della fua Nazione, non più d'ottanta anni innanzi accaduti, che non foffe nel fuo ragguaglio neppur una propofizione, che non foffe un groffo errore? Ma ove fi tratta di fcreditar l'America, non fi ha vucun riguardo alla verità. Egli è certo, e notorio, che non vi fu guerra tra Spagna e Francia nel 1493.: che il Re Cattolico non fi trovava allora in Napoli, ma in Barcellona, non ancora guarito dalle ferite, che ebbe da un paz-

(N) Dolle cofe, ibe viengono portate dalle Indie Occidentali perinenti all uso della Medicina. Part. x. cap. 9. 
pazzo: che Colombo non conduffe feco una gran moltitudine $\mathrm{d}^{2}$ indiani e $\mathrm{d}^{4}$ Indiane, ma foltanto dieci Indiani : che Colombo non venne mai in Italia dopo la fua gloriofa fpedizione: che gl" Indiani, da lui condotti, non videro mai l'I talia ec.

Io per lo contrario, dopo averne fatte le più diligenti ricerche, fon tanto lontano dal credere, cbe dall'America veniffe il Malfrancefe in Europa, che anzi mi fon perfuafo, che effo, non meno che il Vajuolo, fa portato in America dagli Europei . I. Perchè nè D. Criftofora Colombo nel fuo Diario, nè D. Ferdinanda Colombo nella Vita del fua famofo Padre, i quali videro quei paefi recentemente fcoperti, e vi notarono le lor particolarita, non fanno motto del Malfrancefe, contutrochè raccontino minutamente $i$ difagi e patimenti di quei primi viaggi. Nemmeno ne fece menzione nella Storia di quei medefimi paefi Pietro Martire d'Anghiera, Autor contempora. nea del Colombo, e bene informato, come quegli, che fu Pro. tonotario del Configlio delle Indie, e Abate di Giamaica. $\mathrm{O}$. viedo, il primo che attribuì quel maie all' America, non vi andd, fe non venti anni, dopo che l'ifola Haiti era abitata dagli Spagnuoli . Ciò, che diciamo del filenzio di quegli Autori intorno alle ifole Antille, poffiamo ancor dirlo di quello dei primi Storici d'altri paefi d'America . 2. Se l'America foffe ftata la vera patria del Malfrancefe, e fe gli Americani foffero tati i primi a patirlo, effo fi patirebbe più che altrove in America, e gli Americani foggiacerebbono più che verun' altra Nazione a ral male; ma non è cosi. Degl'Indiani delle Ifole Antille non poffiamo ora difcorrere; perchè fono ormai due fecoli che effi perirono affatto: ma nei prefenti abitatori di quelJe ifole è affai più raro il fuddetto contagio, che in Europa, non fi fente fe non in quei luoghi, dove è gran frequenza di Soldati e Marinaj Europei. Nella Capital del Meffica vi fono alcuni bianchi ed Indiani inferti dal mal venerea, $\mathrm{ma}$ pochiffmi rapporto al gran numero degli abitanci. In altre Citta grandi di quel valto regno fono rariflimi i contagiati, ed in altre non havvene niuno affatto; $\mathrm{ma}$ in quei luoghi di Americani, nei qualì non vì c̀ gran concorlo di bianchi, non 
è veduta, nè fentita mai tal malattia. Intorno all' America Meridionale me ne fono bene informato da perfone efatte, filleere, e pratiche molto di quei paefi, ed ho faputo, che tanto nelle Provincie del Chile, quanto in quelle del Paraguai, è rariffimo quel male tra i bianchi, e non mai veduto tra gl' Americani. Alcuni Miffionarj, i quali hanno dimorato chi venti, chi trenta anni tra differenti Nazioni d'Americani, fono d'ac= cordo nell' affermare, che non hanno mai veduto alcuno infetto da quel male, e nemmeno hanno faputo, che vi foffe. Quan= to alle Provincie del Perù e del Quito, il Sig. de Ulloa dice, $(0)$ che contutrochè in quei paefi fia tanto comune il mal venereo tra i bianchi, e tra altre razze d'uomioi, è non pertanto cola rariffluna il vedervi un Indiano inferto. Non è dunque l'America la parria di quel male, come volgarmente fi è creduto, nè tal male dee confiderarfi, ficcome vuole il Sig. de P., $(P)$ come un' affezione del fangue gualto, e del cattivo tempiramento degli Americant.

Qudl è cunque la vera parria del Malfrancefe; poichè effo non ebbe origine nè dall' Europa, nè dall' America? lo nol fo; $\mathrm{ma}_{\mathrm{f}} \mathrm{fe}$ in mazzo all' incertezza mi fi permette di fervirmi di congetture, io tofpetto, che quel contagio fia venuto dalla Guinea, o da altro paefe equinoziale dell' Africa. Di quelto medefino fentimento $f_{u}$ il dotritino Medico Inglefe Tommafo Sydenham (Q), e vien confermato da ciò, che afferma Batrifta Fulgofio, teftimonio oculare del cominciámento del Malfrance.

re

(O) Viage d la America Merid. part. x. Lit. 6. cat. 6. Pre, che qu Scritcore abbia confufo, adottando I'opinione del volgo, il Malfrancere collo Scorbuto; poichè jo fo, che it Ditt. Siu :o Rondoli Pefrêre, Medico famofo di Lima, affrimò ad una perfona au urevole, che tra molí infermi creduti infetti dal Malfrancefe, e da lini curati, quafi niuno avea trovato, che forfe travagliato da quel male; ma quafi tutti erann fcorbutici, ed egli erz siufcito nella lor curazione, adoprando i rimedj dello fcorbuto.

(P) Rech. Pbilof. part. $x$.

(Q) Sydenham afferma in una delle fue lettere (Epilt. 2. ref.) che il Matfrancele è tanto foreftiere in America, quanto in Europa, e che $v i$ fu porta. to da'Mori condotti rchiavi dalla Guinea; ma non è vero, che gli fteff Mori lo portaftero in America; poichè prima che effi folfero condotti nella Spt. gnuola avea già quefta ifola cominciato ad infetrarfene. 


\section{DISSERTAZIONE IX.}

fe in Europa. Coftui dice nell'opera gia citata $(R)$, che iI Malfrancefe fu portato da Spagua in Italia, e da Etiopia in If́pagna. Il Sig. Aftruc prerende, che Fulgofio voleffe fignifi. car l'America col nome d'Etiopia. Ecco un mojo curiofo di trarfi dalla difficolì. Ma chi mai ha appellato Etiopia l'America? Noi fappiamo per lo contrario, che era comune preffo gli Autori di quel Secolo il dare il nome d'Etiopia a qualunque paefe abitato da uomini neri, e chiamare Etiopi tali uomini : ficchè il fenfo naturale delle parole tel Fulgofio $f_{i} \dot{e}$, che il Mdifrancefe fu portato dai paefi equinoziali dell' Africa nella Spagna Lufitanica, o fia Portogallo. Io pertanto forpesto, che il primo paefe d'Europa a contagiarfi fu il Portogallo; ma non oferei affermarlo fenza far nuove ricerche, e procacciarmi documenti migliori.

\section{I $\quad \mathbf{F} I \mathrm{~N}$ E.}

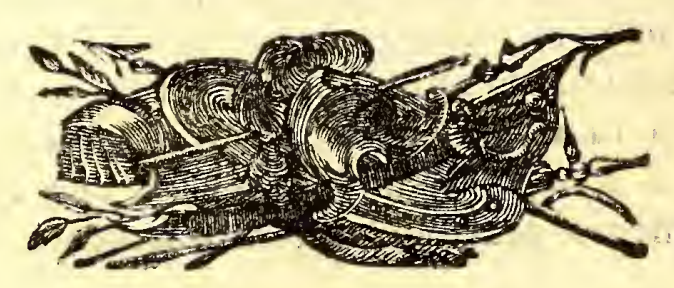

(R) Que peftis ( ita enim vifa ef ) primo ex Hifpania in Italiam allata: ad IHifpanos ex Etbiopia, brevi totum terrarum orber comprebendit. Fulgos Difor. FaÉt. que memorab. lib. 1. cap. 4. 


\section{N D I C E \\ D E L T O MO I V.}

ISSERT. I. SU LA POPOLAZIONE DELL'AMERICA E PARTICOLARMENTE SOPRA QUEJLA DEL MESSICO.

5. I. In qual tempo fi cominciò a popolar l'America? Pag. 9

f. II. Chi ne furono i primi Popolatori? pag. I4

6. III. Da qual parte, e come paffarono i Popolatori, e le beftie in America.

DISSERT. II. SU LE PRINCIPALI EPOCHE DELLA STORIA DEL MESSICO.

6. I. Sull' epoca dell' arrivo de'Toltechi, e di altre Nazioni nel paefe di Anahuac.

6. II. Su la corrifpondenza degli anni mefficani a' noftrali, e full' epoca della fondazione di Meffico.

๒. III. Su la cronología de' Re Mefficani .

5. IV. Sull' epoche degli avvenimenti della Conquifa pag. 55

DISSERT. III. SU IA TERRA DEL MESSICO -

6. I. Su la pretefa inondazione dell'A merica. $\quad$ pag. 65

O. II. Sul clima del Meffico.

f. III. Su la qualità della Terra del Meffico. $\quad$ pag. 92

DISSERT. IV. SU GLI ANIMALI DEL MESSICO.

6. I. Su gli animali propri del Meffico. pag. 105

5. II. Su gli animali europei trafportati in America • pag. I 37

CATALOGO DEI QUADRUYEDI AMERICANI.

9. I. Spezie riconofciute ed ammeffe dal C. de Buffon. pag. I5I

6. II. Spezie confufe dal C. de Buffon con altre diverfe. pag. ${ }_{15} 6$

6. III. Spezie ignorate, o negate a torto dal C. de Buffon. pag. I57

DISSERT. V. SU LA COSTITUZIONE FISICA E MORALE DEI MESSICANI.

6. I. Su le qualità corporali de' Mefficani.

f. II. Su le anime de'Meflicani.

5. II. Su le anime de' Mefficani.
DISSERT. VI. SU LA COLTURA DE' MESSICANI 177

pag. I 60

pag. I 62

pag. 203

pag. 204

. I. Su la mancanza di moneta.

§. II. Sopra l'ufo del Ferro.

pag. 208

§. III. Su le arti di fabbricar Vafcelli e Ponti, e di fár la calcina.

T t

pag. 2 II

๑. IV. 
6. IV. Su la mancanza di Lettere.

6. V. Su le arti de' Mefficani.

6. VI. Su la lingua Mefficana.

5. VII. Su le leggi de' Mefficani.

GATALOGO DI ALCUNI AUTORI EUROPEI E CREOGLI CHE HANNO SCRITTO DELL A DOTTRINA E MO. RALE CRISTIANA NELLE LINGUE DELLA N. SPAGNA.

AUTORI DI GRAMMATICHE E DIZIONARJ DELLE SUD. DETTE LINGUE.

DISSERT. VII. SOPRA I CONFINI E LA PÓPOLAZIONE pag. 264 DE' REGNI DI ANAHUAC.

๑. I. Sopra i confini de' Regni di Anahuac.

f. II. Sopra la popolazione d'Anahuac.

pag. 265 pag. $27 \mathrm{I}$ DISSERT. VIII. SU LA RELIGIONE DEI MESSICANI. pag. 288 DISSERT. IX. SU L' ORIGINE DEL MALFRANCESE. pag. 303 6. I. Opinioni de'Medici antichi intorno all'origine del Malfrancefe. pag. 304

§. II. Il Malfrancefe potè comunicarfi all'Europa da altri paefi del Continente antico. pag. 305

๑. III. Il Malfrancefe potè venire in Europa fenza contagio. pağ. 308 5. IV. Il Malfrancefe non venne da America. pag. 3 I 6

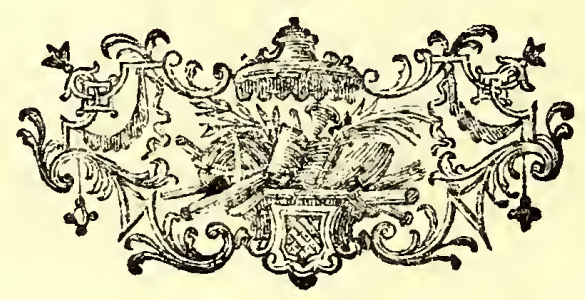




\author{
$E R R \wedge T A$. CORRIGE.
}

Pag. ro. N. per bofce die. sa invent... per bofce dies inventa

pag. 14. lin. I. da un altro canto ... dele altro

pag. 16. lin. 19. Hoba . : . . . Hobo

pag. 20. lin. 12. contenevano . . contavano

pag. 22. lin. 21. Xolbua . . . Xelbua.

lin. 21. Itancueitl . . . . . Ilancueitl

pag. 47. lin. 17. nel 1536. . ... nel 1636.

pag. 49. !in. 16. In Tula nove anni... In Tula nove anni, in Tepexic

in Tepexic

pag. 55 . N. nell'edizingne di Tedi .... nell' edizione di Tevenot, non già in Purchàs.

pag. 72. N. Canapis . . . . . Canapy

pag. 77. lin. 14. ributtati . . . . ribatiuti

pag. I 08. $N$. nel 1762. , e un altro... nel 1752, e un altro nel 1753. nel 1763 .

pag. I1 4. lin. 4. si prodigiofa . . . si prodiga

pag. 13+. lin. 3. Condori . . . . Condori

pag. 15r. lin. 13. Alleo . . . Allco

pag. 154. lin. 8. des Antillies . . des Antilles

pag. I56. N. gli Spagnuoli ed altri... gli Spagnuoli del Meffico, e di altr? paefí

pag. I58. lin. 3. CUL . . . . . CUI

pag. 196. lin. 13. nè parla - . ne parla

pag. 222. lin. 19. non vi fupponendo ... non vi fapendo

pae. 242. Chaxtolli $\cdot . \cdot \cdot . \cdot$. Caxtolli

pag. 246. lin. 5. che hanno celebrata... dele che

pag. 273. N. Parld . . . . . . Parlo

pag. 291. lin. 30. quibus bic ${ }^{\circ} \cdot$. quibus beo

pag. 309. N. indigna . . . . . . indigena

pag. $318 . \operatorname{lin}_{2} 20.1596$. . . . . 1496.

pag. 320 . N. montrofus $\dot{0}^{-} \cdot \ldots$ monftuofus

$\mathrm{V} \mathrm{i}$ fono altri errori della ftampa, maffimamente in cio che riguarda la punteggiatura, i qualı faranno fcufati da'cortefi Leggitori. 
Iidit pro Illuftrifs. ac Reverendifs. D.Vic. Gen. Bartolucci

Carolus Cat tani

I M P R I A T U R:

Jo: B. Bartolucci Vic. Gen.

Vidit pro Adnood. R. P. Vic. S. Offic. Cesenot

Comes Francifcus Fattiboni Confultor S. Officii.

I M P R I M A T U R.

F. Antonius Gatti Vic. S. Offic, Cælenæ. 



